\title{
Synthesis and Structural Characteristics of all Mono- and Difluorinated 4,6- Dideoxy-D-xylo-hexopyranoses
}

David E. Wheatley, Clement Q. Fontenelle, Ramakrishna Kuppala, Robert Szpera, Edward L. Briggs, Jean-Baptiste Vendeville, Neil J. Wells, Mark E. Light, Bruno Linclau*

School of Chemistry, University of Southampton, Highfield, Southampton SO17 1BJ, UK

bruno.linclau@soton.ac.uk

\section{Supporting information}

Table of Contents

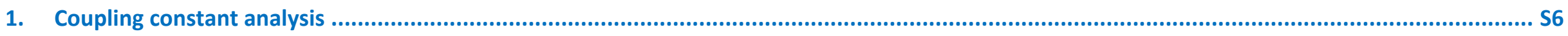

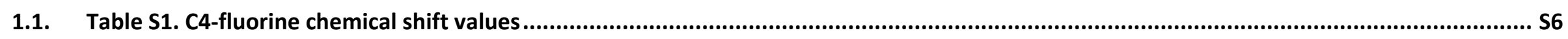

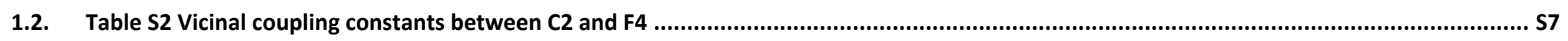

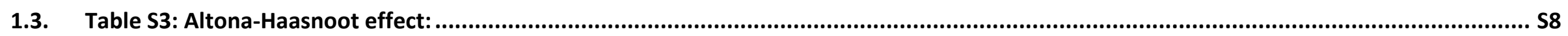

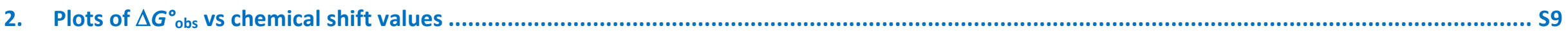

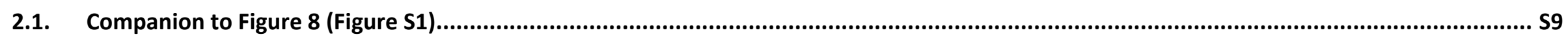

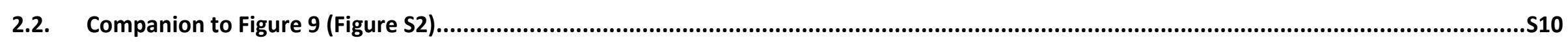

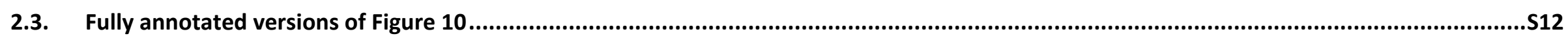




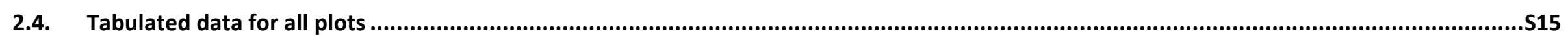

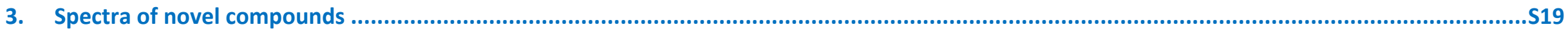

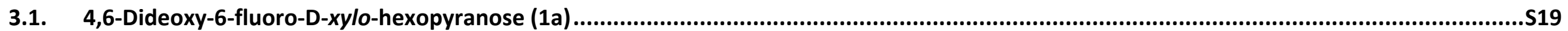

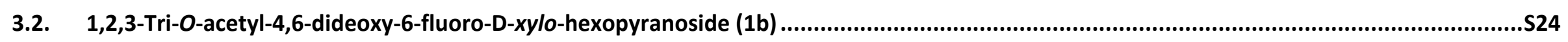

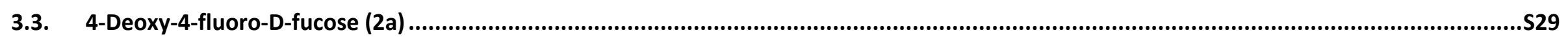

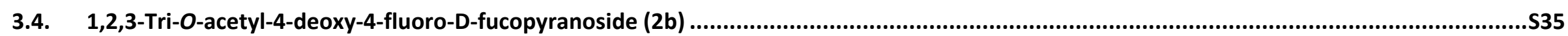

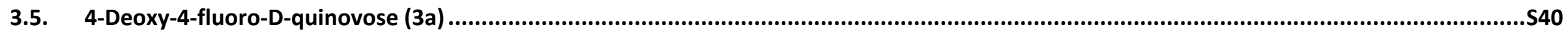

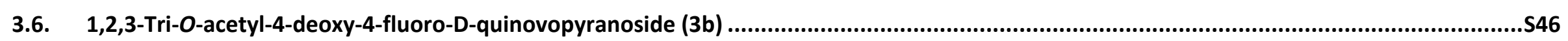

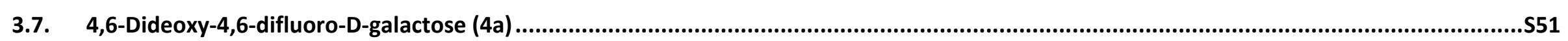

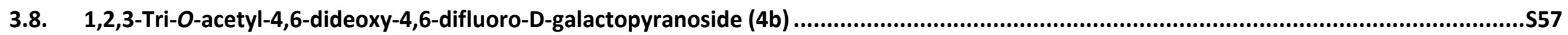

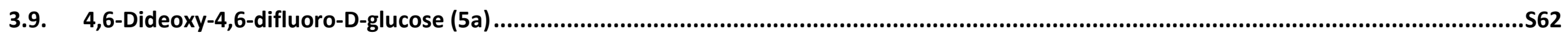

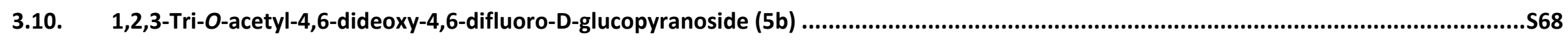

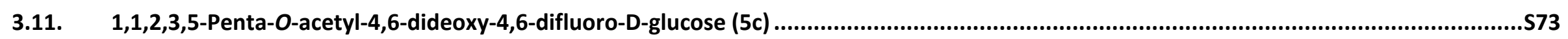

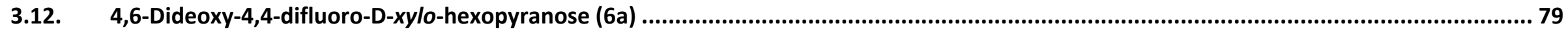

3.13. 1,2,3-Tri-O-acetyl-4,6-dideoxy-4,4-difluoro-D-xylo-hexopyranoside (6b) .............................................................................................585

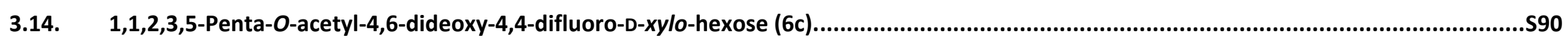

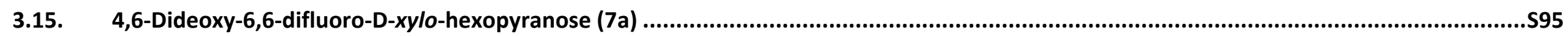

3.16. 1,2,3-Tri-O-acetyl-4,6-dideoxy-6,6-difluoro-D-xylo-hexopyranoside (7b) .............................................................................................5103

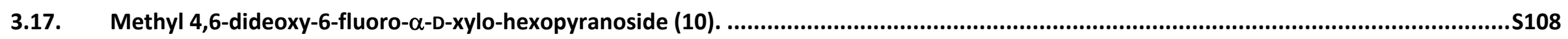

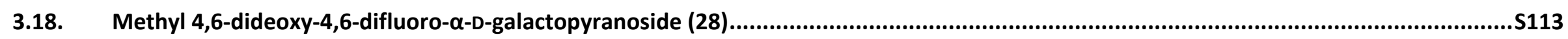

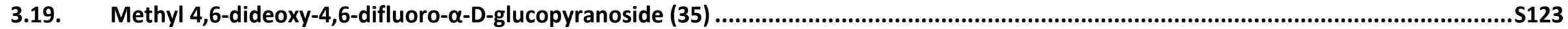

3.20. Methyl 2,3-di-O-benzoyl-4,6-dideoxy-4,6-difluoro- $\alpha$-D-glucopyranoside (39) ....................................................................................5132

3.21. Methyl 6-O-tert-butyldimethylsilyl-2,3-O-((2'R,3' $R)-2^{\prime}, 3^{\prime}$-dimethoxybutane-2' $3^{\prime}$-diyl)- $\alpha$-D-galactopyranoside (47): ..................................S137 
3.22. Methyl 6-O-tert-butyldimethylsilyl-4-O-phenoxythiocarbonyl-2,3-O-((2'R, $\left.3^{\prime} R\right)-2^{\prime}, 3^{\prime}$-dimethoxybutane-2', $3^{\prime}$-diyl)- $\alpha$-D-galactopyranoside (48): S140

3.23. Methyl 6-O-tert-butyldimethylsilyl-4-deoxy-2,3-O-((2'R, $\left.3^{\prime} R\right)-2^{\prime}, 3^{\prime}$-dimethoxybutane-2', $3^{\prime}$-diyl)- $\alpha$-D-xylo-hexopyranoside (49): .$S 143$

3.24. Mixture of Methyl 4-O-benzoyl-2,3-O-((2'R,3'S)-2',3'-dimethoxybutane-2', $3^{\prime}$-diyl)- $\alpha$-D-fucoopyranoside (52) and Methyl 6-O-benzoyl-4-

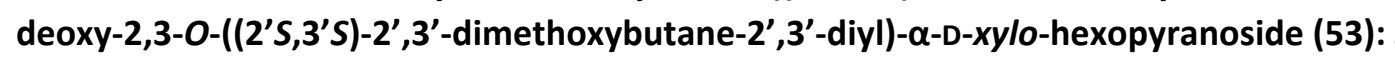

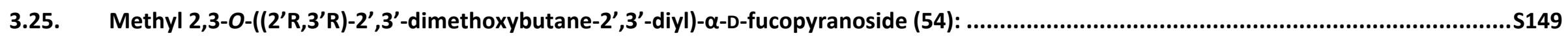

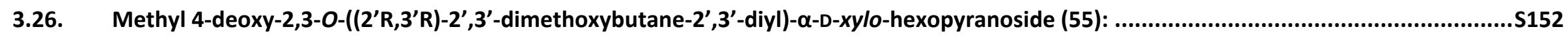

3.27. Methyl 4,6-dideoxy-6-fluoro 2,3-O-((2'R,3'R)-2', $3^{\prime}$-dimethoxybutane-2', $3^{\prime}$-diyl)- $\alpha$-D-xylo-hexopyranoside (56): .......................................S155

3.28. Mixture of Methyl 4-O-benzoyl-2,3-O-((2'S,3'S)-2', 3'-dimethoxybutane-2', $3^{\prime}$-diyl)- $\alpha$-D-fucoopyranoside (57) and Methyl 6-O-benzoyl-4-deoxy-

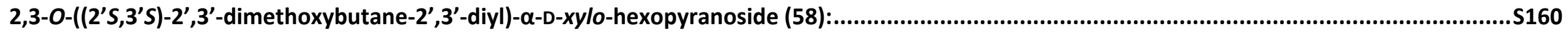

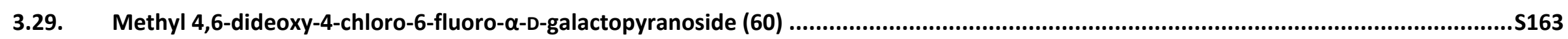

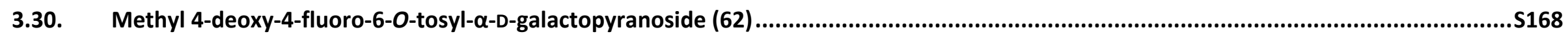

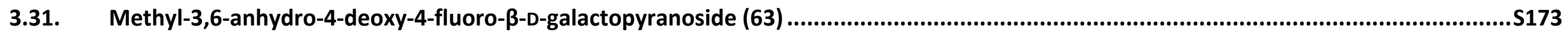

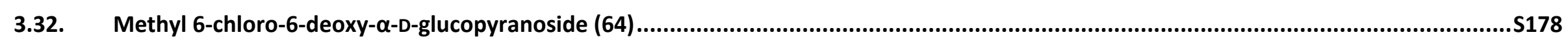

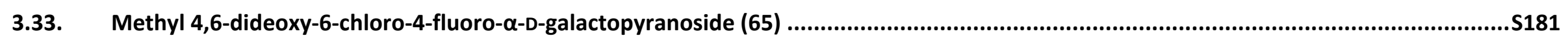

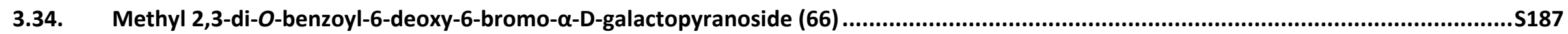

3.35. Mixture of Methyl 2,3-di-O-benzoyl-- $\alpha$-D-fucopyranoside (67) and methyl 2,4-di-O-benzoyl- $\alpha$-D-fucopyranoside (68) ...............................S190

3.36. Methyl 2,3-di-O-benzoyl-4,6-dideoxy-4-fluoro-6-bromo- $\alpha$-D-glucopyranoside (69)...........................................................................S193

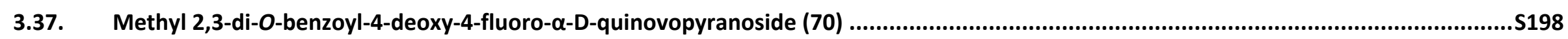

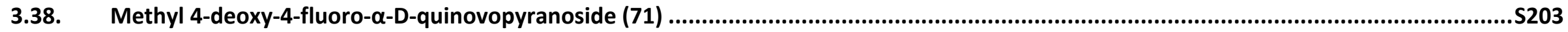

3.39. Methyl 6-O-p-toluenesufonyl-2,3-O-((2'R,3'R)-2',3'-dimethoxybutane $-2^{\prime}, 3^{\prime}$-diyl)- $\alpha$-D-galactopyransoside (72) ........................................S209

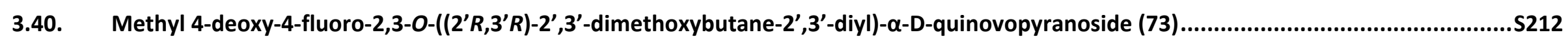

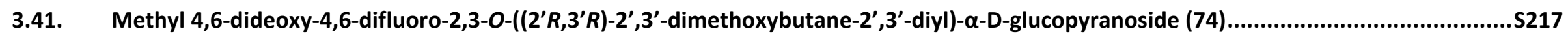

3.42. Methyl 4,6-dideoxy-4,4-difluoro-2,3-O-((2' $\left.R, 3^{\prime} R\right)-2^{\prime}, 3^{\prime}$-dimethoxybutane-2',3'-diyl)- $\alpha$-D-xylo-hexopyranoside (76) ....................................S222 
3.43. Methyl 4,6-dideoxy-4-fluoro-2,3-O-((2'R,3'R)-2',3'-dimethoxybutane-2',3'-diyl)- $\alpha$-D-erythro-hex-3-enopyranoside (77) ............................S227

3.44. Methyl 4,6-dideoxy-4-fluoro-2,3-O-((2'R,3'R)-2',3'-dimethoxybutane-2',3'-diyl)- $\alpha$-L-threo-hex-4-enopyranoside (78) ...............................S232

3.45. Methyl 4,6-dideoxy-6,6-difluoro-2,3-O-((2'R,3'R)-2', 3'-dimethoxybutane-2', 3'-diyl)- $\alpha$-D-xylo-hexopyranoside (80)..................................S237

3.46. Methyl 4,6-dideoxy-4,4-difluoro- $\alpha$-D-xylo-hexopyranoside (85) .................................................................................................S242

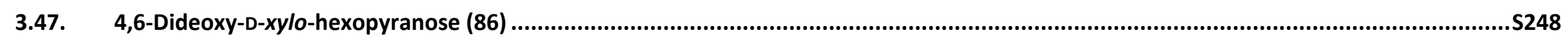

3.48. 1,2,3-Tri-O-acetyl-4,6-dideoxy-D-xylo-hexopyranoside (94) .............................................................................................S251

4. $\mathrm{D}_{2} \mathrm{O}$ NMR spectra of compounds $1 \mathrm{a}-7 \mathrm{7}$ a and $83,84,86,87,89,92$ \& 93 including qNMR experiments...................................................S254

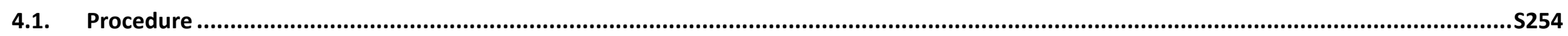

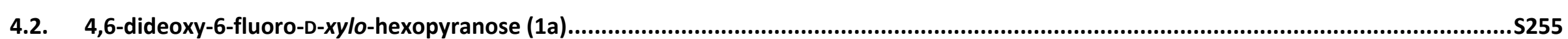

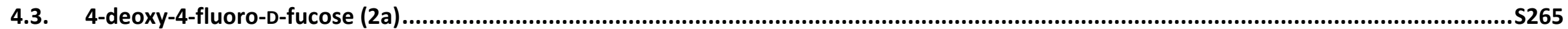

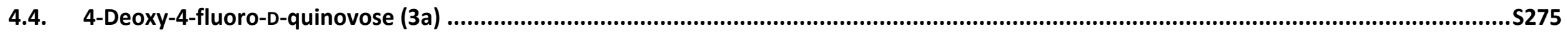

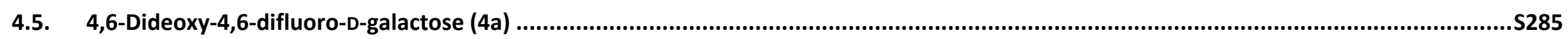

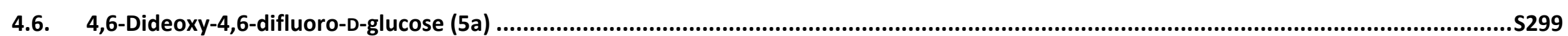

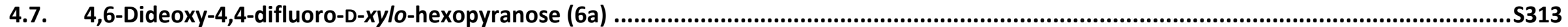

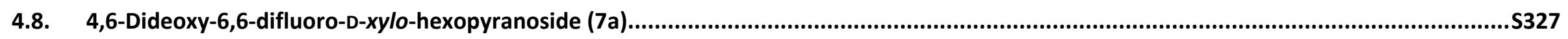

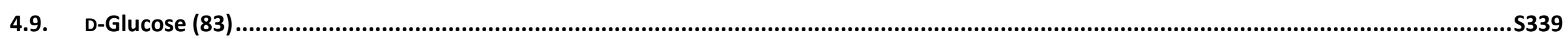

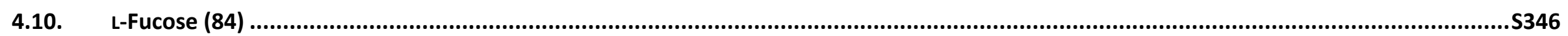

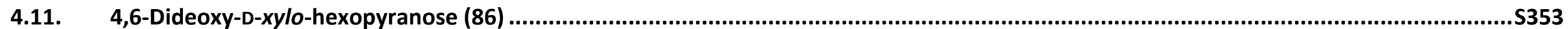

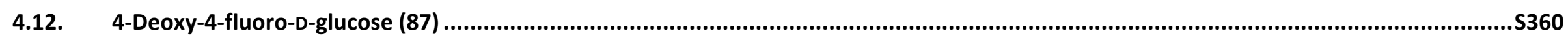

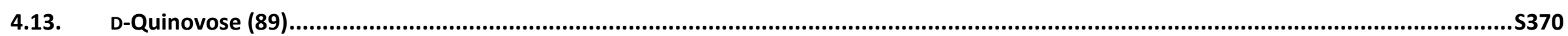

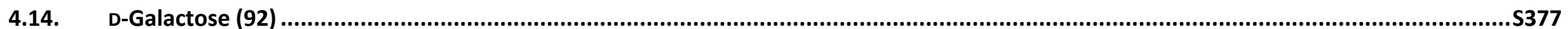

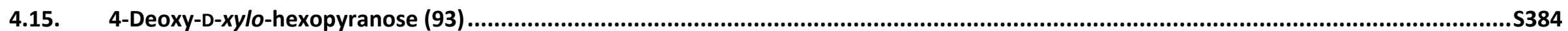

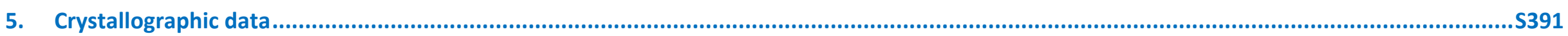




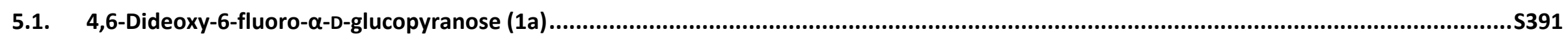

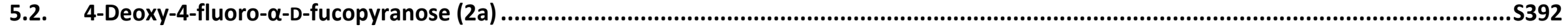

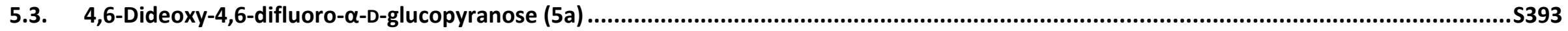

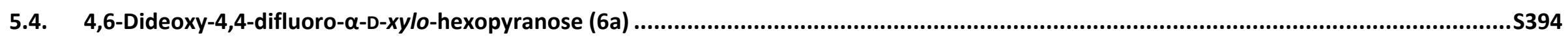

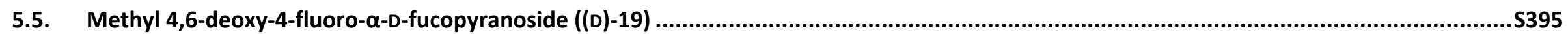

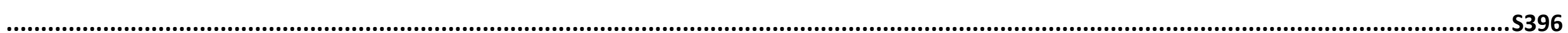

5.6. Methyl 4,6-dideoxy-2,3-di-O-benzoyl-6-bromo-4-fluoro- $\alpha$-D-glucopyranoside (69)........................................................................S396

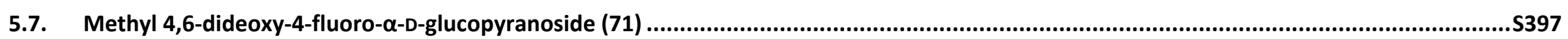

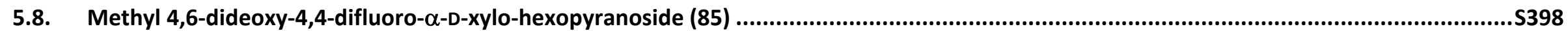

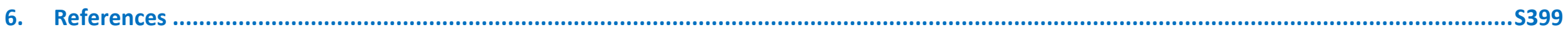


1. Coupling constant analysis

1.1. Table S1. C4-fluorine chemical shift values

Table S1: Tabulation of C4-fluorine chemical shift values.

\begin{tabular}{|c|c|c|c|c|c|c|c|c|}
\hline & & $x$ & ${ }_{\mathrm{HO}} \underbrace{S_{\mathrm{O}}^{x}}_{\mathrm{HO}}$ & $\mathrm{HO} \underbrace{\mathrm{S}_{\mathrm{O}}^{\mathrm{x}}}_{\mathrm{HO}}$ & & $\underbrace{\mathrm{FO}_{\mathrm{O}}^{\mathrm{x}}}_{\mathrm{HO} \text { OMe }}$ & $\left.\underbrace{\mathrm{F}}_{\mathrm{AcO}}\right|_{\mathrm{OAC}} ^{\mathrm{x}}$ & ${ }_{A c O} \underbrace{S_{O A C}^{X}}_{A C O}$ \\
\hline \multirow{5}{*}{$\begin{array}{c}\delta_{\mathrm{F} 4} \\
(\mathrm{ppm})\end{array}$} & $\mathrm{CD}_{3} \mathrm{OD}$ & $\mathrm{H}$ & -222.67 & -219.54 & $\mathrm{CDCl}_{3}$ & & -219.89 & -217.62 \\
\hline & & $\mathrm{F}$ & -221.9 & -219.0 & & & -219.75 & n.o. \\
\hline & $\mathrm{D}_{2} \mathrm{O}$ & $\mathrm{H}$ & -221.1 & -218.2 & & & & \\
\hline & & $\mathrm{F}$ & -219.2 & -216.9 & & & & \\
\hline & & & $\underbrace{\int_{\mathrm{OH}}}_{\mathrm{HO}}$ & ${ }_{\mathrm{HO}} \underbrace{S_{\mathrm{O}}}_{\mathrm{HO}_{\mathrm{O}}}$ & & ${ }_{\mathrm{HO}}^{\mathrm{S}} \underbrace{\mathrm{x}}_{\mathrm{HOMe}}$ & 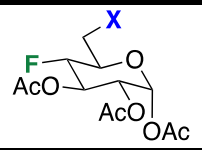 & 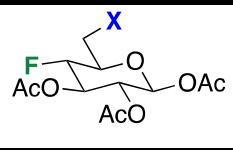 \\
\hline \multirow{3}{*}{$\begin{array}{c}\delta_{\mathrm{F} 4} \\
(\mathrm{ppm})\end{array}$} & $\mathrm{CD}_{3} \mathrm{OD}$ & $\mathrm{H}$ & -197.6 & -199.3 & $\mathrm{CDCl}_{3}$ & & -197.34 & -198.99 \\
\hline & & $\mathrm{F}$ & -199.5 & -201.3 & & -199.23 & -199.10 & -200.75 \\
\hline & $\mathrm{D}_{2} \mathrm{O}$ & & $\begin{array}{l}-196.3 \\
-198.4\end{array}$ & $\begin{array}{l}-198.3 \\
-200.5\end{array}$ & & & & \\
\hline
\end{tabular}




\subsection{Table S2 Vicinal coupling constants between $\mathrm{C} 2$ and F4}

For gluco-configured sugar derivatives, $\mathrm{C} 2$ and $\mathrm{F} 4$ are antiperiplanar, and for galacto-configured derivatives, there is a gauche-dihedral. Hence, the ${ }^{3} \mathrm{~F}_{\mathrm{F} 4 \mathrm{C} 2}$ values for the former are larger. For the 4,4-difluorinated derivative, there is no ${ }^{3} \mathrm{~F}_{\mathrm{F}-\mathrm{C} 2}$ value observed involving the axial fluorine.

Table S2: ${ }^{3} J_{\mathrm{F} 4-\mathrm{C} 2}$ values

\begin{tabular}{|c|c|c|c|c|c|c|c|}
\hline & & $X$ & $\int_{\mathrm{HO}}^{S_{\mathrm{HO}}}$ & $\underbrace{\int_{\mathrm{HO}}^{\mathrm{C}}}_{\mathrm{HO}}$ & & $\begin{array}{c}\text { Alfa- } \\
\text { peracetate }\end{array}$ & $\begin{array}{c}\text { Beta- } \\
\text { peracetate }\end{array}$ \\
\hline \multirow[t]{5}{*}{$3 J_{F 4-C 2}$} & $\mathrm{CD}_{3} \mathrm{OD}$ & $\mathrm{H}$ & $7.9 \mathrm{~Hz}$ & $8.3 \mathrm{~Hz}$ & $\mathrm{CDCl}_{3}$ & $8.8 \mathrm{~Hz}$ & $8.1 \mathrm{~Hz}$ \\
\hline & & $\mathrm{F}$ & $8.1 \mathrm{~Hz}$ & $8.4 \mathrm{~Hz}$ & & $8.1 \mathrm{~Hz}$ & $8.1 \mathrm{~Hz}$ \\
\hline & $\mathrm{D}_{2} \mathrm{O}$ & $\mathrm{H}$ & $8.1 \mathrm{~Hz}$ & $8.8 \mathrm{~Hz}$ & & & \\
\hline & & $\mathrm{F}$ & & & & & \\
\hline & & $x$ & $\frac{\mathrm{HO}}{\mathrm{HO}}$ & $\underbrace{\mathrm{F}}_{\mathrm{HO}}$ & & & \\
\hline \multirow[t]{2}{*}{${ }^{3} J_{F 4-C 2}$} & $\begin{array}{c}\mathrm{CD}_{3} \mathrm{OD} \\
\mathrm{D}_{2} \mathrm{O}\end{array}$ & & $7.3 \mathrm{~Hz}$ & $8.2 \mathrm{~Hz}$ & $\mathrm{CDCl}_{3}$ & $7.3 \mathrm{~Hz}$ & $8.1 \mathrm{~Hz}$ \\
\hline & & $x$ & $\underbrace{\mathrm{S}_{\mathrm{HO}}^{\mathrm{X}}}_{\mathrm{HO}}$ & $\underbrace{\sum_{\mathrm{HO}}^{\mathrm{x}}}_{\mathrm{HO}}$ & & & \\
\hline \multirow[t]{4}{*}{${ }^{3} J_{F 4-C 2}$} & $\mathrm{CD}_{3} \mathrm{OD}$ & $\mathrm{H}$ & $2.2 \mathrm{~Hz}$ & (s) & $\mathrm{CDCl}_{3}$ & $2.2 \mathrm{~Hz}$ & (s) \\
\hline & & $\mathrm{F}$ & $2.2 \mathrm{~Hz}$ & (s) & & $2.2 \mathrm{~Hz}$ & - \\
\hline & $\mathrm{D}_{2} \mathrm{O}$ & $\mathrm{H}$ & $2.2 \mathrm{~Hz}$ & $0.7 \mathrm{~Hz}$ & & & \\
\hline & & $\mathrm{F}$ & $2.2 \mathrm{~Hz}$ & (s) & & & \\
\hline
\end{tabular}




\subsection{Table S3: Altona-Haasnoot effect:}

${ }^{3} J_{\mathrm{H}-\mathrm{H}}$ is larger when there is an antiperiplanar electronegative group to either $\mathrm{H}$.

Table S3: ${ }^{3} \mathrm{~J}_{\mathrm{H2}-\mathrm{H3}}$ comparison of synthesized sugars in order to confirm Altona-Haasnoot effect.

\begin{tabular}{|c|c|c|c|c|c|c|c|}
\hline & & $x$ & $\underbrace{\mathrm{FOH}_{\mathrm{OH}}^{\mathrm{x}}}_{\mathrm{HO}}$ & $\underbrace{\mathrm{SOH}_{\mathrm{HO}}^{\mathrm{x}} \mathrm{OH}}_{\mathrm{HO}}$ & & $\underbrace{\sum_{\mathrm{OAc}}^{\mathrm{X}}}_{\mathrm{AcO}}$ & ${ }_{A c O} \underbrace{x}_{A c O} O A C$ \\
\hline \multirow{4}{*}{${ }^{3} J_{\mathrm{H} 2-\mathrm{H} 3}$} & \multirow{2}{*}{$\mathrm{CD}_{3} \mathrm{OD}$} & $\mathrm{H}$ & $10.2 \mathrm{~Hz}$ & $9.9 \mathrm{~Hz}$ & \multirow{2}{*}{$\mathrm{CDCl}_{3}$} & $10.9 \mathrm{~Hz}$ & - \\
\hline & & $\mathrm{F}$ & $10.2 \mathrm{~Hz}$ & $9.9 \mathrm{~Hz}$ & & $10.9 \mathrm{~Hz}$ & - \\
\hline & \multirow{2}{*}{$\mathrm{D}_{2} \mathrm{O}$} & $\mathrm{H}$ & $10.4 \mathrm{~Hz}$ & $10.0 \mathrm{~Hz}$ & & & \\
\hline & & $\mathrm{F}$ & $10.4 \mathrm{~Hz}$ & $10.0 \mathrm{~Hz}$ & & & \\
\hline & & & & ${ }_{\mathrm{HO}}$ & & & $\underbrace{}_{A C O}$ \\
\hline \multirow{2}{*}{${ }^{3} J_{\mathrm{H} 2-\mathrm{H} 3}$} & $\mathrm{CD}_{3} \mathrm{OD}$ & & $10.0 \mathrm{~Hz}$ & $9.8 \mathrm{~Hz}$ & \multirow{2}{*}{$\mathrm{CDCl}_{3}$} & \multirow{2}{*}{$10.7 \mathrm{~Hz}$} & \multirow{2}{*}{$10.2 \mathrm{~Hz}$} \\
\hline & $\mathrm{D}_{2} \mathrm{O}$ & & $10.2 \mathrm{~Hz}$ & $9.9 \mathrm{~Hz}$ & & & \\
\hline & & & & 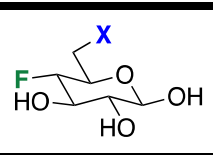 & & $\underbrace{S_{O A C}}_{A c O}$ & ${ }_{A c O}^{S_{A C O}} \int_{A C}$ \\
\hline \multirow{4}{*}{${ }^{3} J_{\mathrm{H} 2-\mathrm{H} 3}$} & & $\mathrm{H}$ & $9.4 \mathrm{~Hz}$ & $9.4 \mathrm{~Hz}$ & \multirow{2}{*}{$\mathrm{CDCl}_{3}$} & $10.4 \mathrm{~Hz}$ & $9.7 \mathrm{~Hz}$ \\
\hline & $\mathrm{CD}_{3} \mathrm{OD}$ & $\mathrm{F}$ & $9.6 \mathrm{~Hz}$ & $9.4 \mathrm{~Hz}$ & & $10.3 \mathrm{~Hz}$ & $9.4 \mathrm{~Hz}$ \\
\hline & \multirow{2}{*}{$\mathrm{D}_{2} \mathrm{O}$} & $\mathrm{H}$ & $9.8 \mathrm{~Hz}$ & $9.6 \mathrm{~Hz}$ & & & \\
\hline & & $\mathrm{F}$ & $9.9 \mathrm{~Hz}$ & $9.6 \mathrm{~Hz}$ & & & \\
\hline & & & $\underbrace{S_{\mathrm{O}}^{\mathrm{X}}}_{\mathrm{HO}_{\mathrm{OH}}}$ & 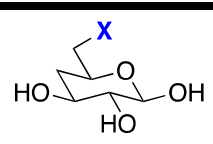 & & $\underbrace{S_{O A C}}_{A c O}$ & ${ }_{A c O}^{S_{A c O}^{x}} O A C$ \\
\hline \multirow{3}{*}{${ }^{3} J_{\mathrm{H} 2-\mathrm{H} 3}$} & & $F$ & $9.4 \mathrm{~Hz}$ & $9.0 \mathrm{~Hz}$ & \multirow{2}{*}{$\mathrm{CDCl}_{3}$} & $(\mathrm{~m})$ & $(\mathrm{m})$ \\
\hline & $\mathrm{CD}_{3} \mathrm{OD}$ & $F, F$ & $9.4 \mathrm{~Hz}$ & $9.1 \mathrm{~Hz}$ & & 10.3 & (m) \\
\hline & $\mathrm{D}_{2} \mathrm{O}$ & $\begin{array}{c}F \\
F, F\end{array}$ & $9.4 \mathrm{~Hz}$ & $9.1 \mathrm{~Hz}$ & & & \\
\hline
\end{tabular}

${ }^{a}$ Resonance not resolved. 


\section{Plots of $\Delta G^{\circ}{ }_{\text {obs }}$ vs chemical shift values}

2.1. Companion to Figure 8 (Figure S1)

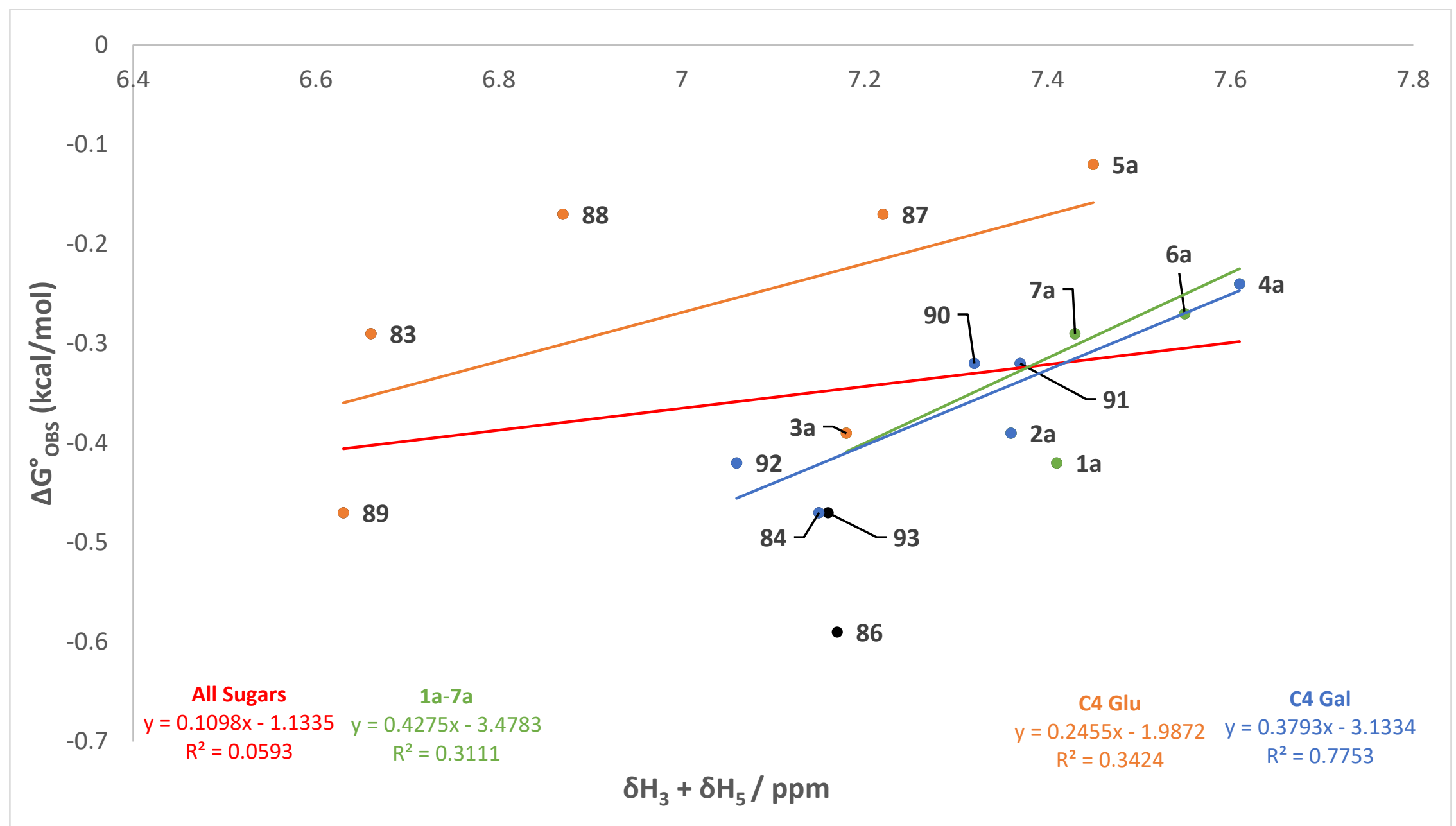

Figure S1: Plot of $\Delta G^{\circ}{ }_{\text {obs }}$ vs $\beta$-anomer chemical shift values of $1 \mathrm{a}-7 \mathrm{a}$ and $83,84,86-93$. Values for 88,90 and 91 taken from ref. ${ }^{1}$ 


\subsection{Companion to Figure 9 (Figure S2)}

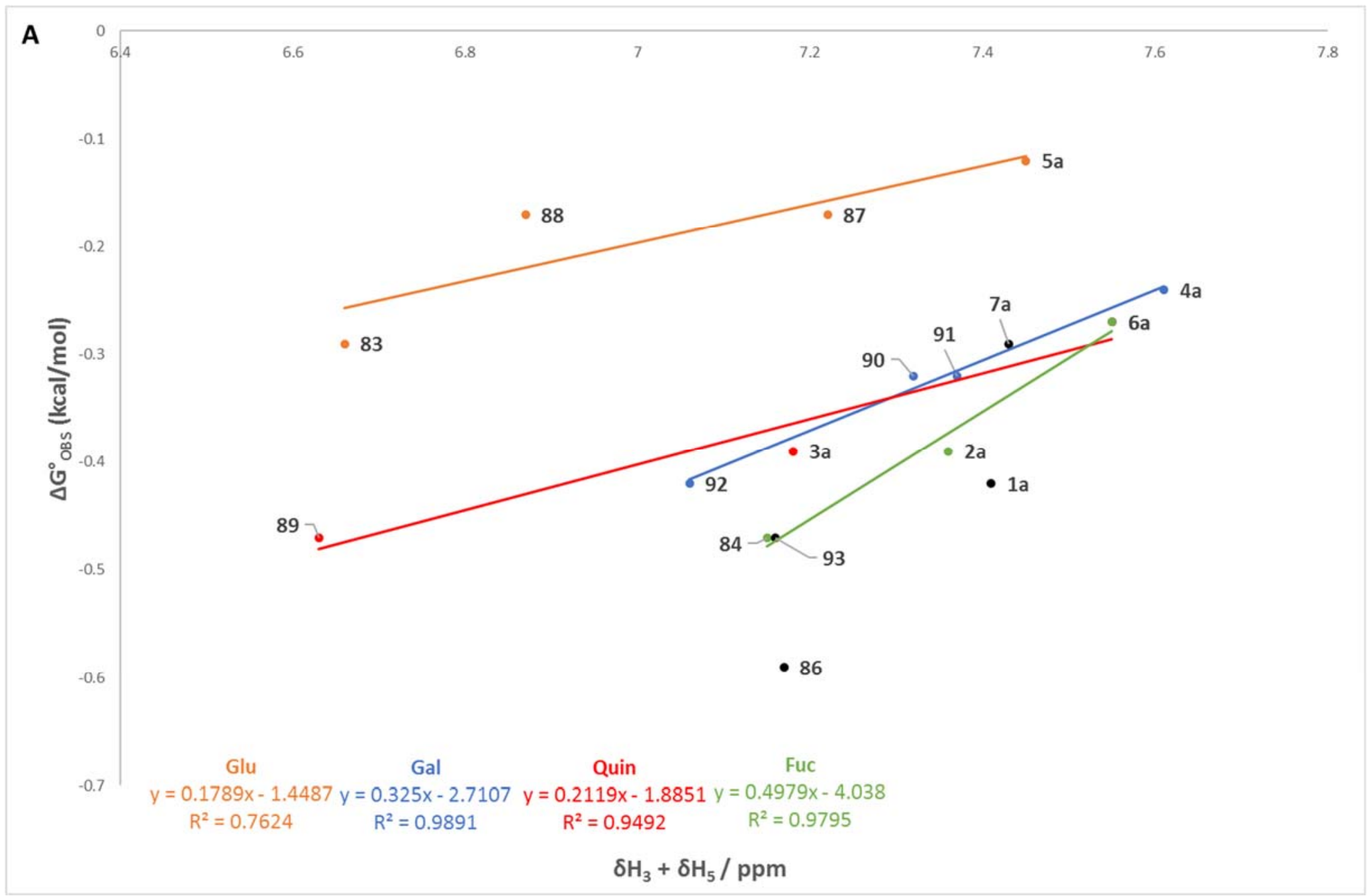




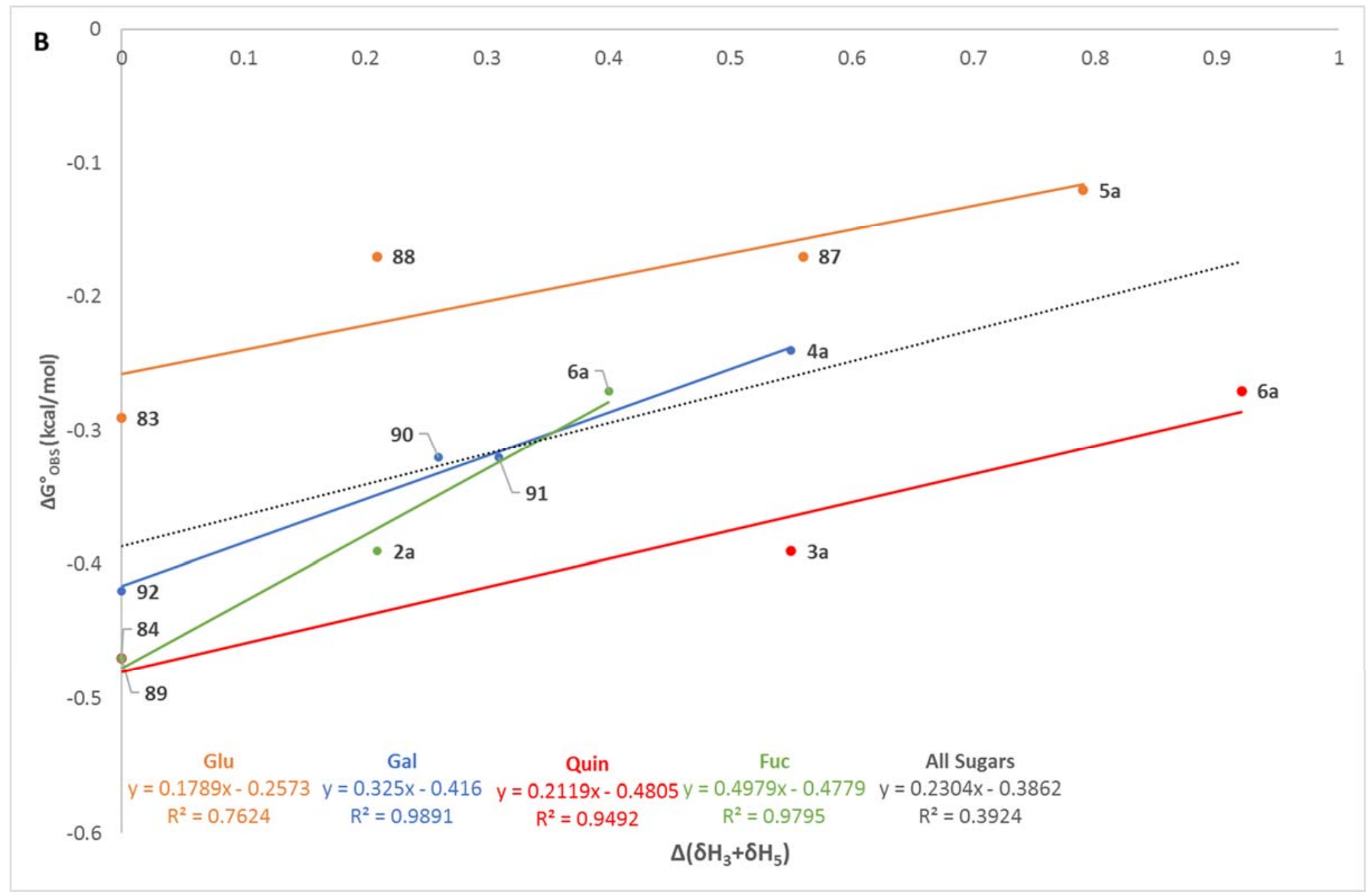

Figure S2: Plots of: A) $\Delta G^{\circ}{ }_{\text {obs }}$ vs $\beta$-anomer chemical shift values of $1 a-7 a$ and $83,84,86-93$ and B) $\Delta G^{\circ}{ }^{\circ}$ vs $v$-anomer chemical shift difference of $2 a-6 a$ and 83, 87-93 from the "parent" pyranose. Trendlines have been calculated for the four different parent series (Glu, Gal, Quin and Fuc), and for the whole dataset. Values for 88,90 and 91 taken from ref ${ }^{1}$. 


\subsection{Fully annotated versions of Figure 10}

2.3.1. Compound structures used in plots.

Galactose series

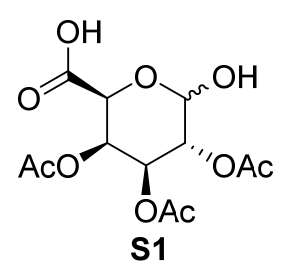

S1
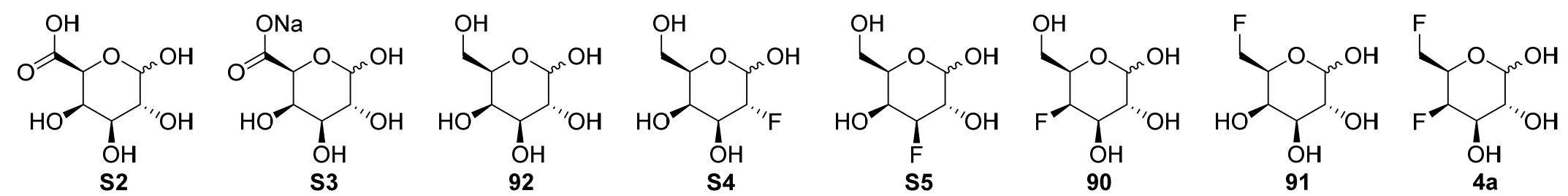

Glucose series
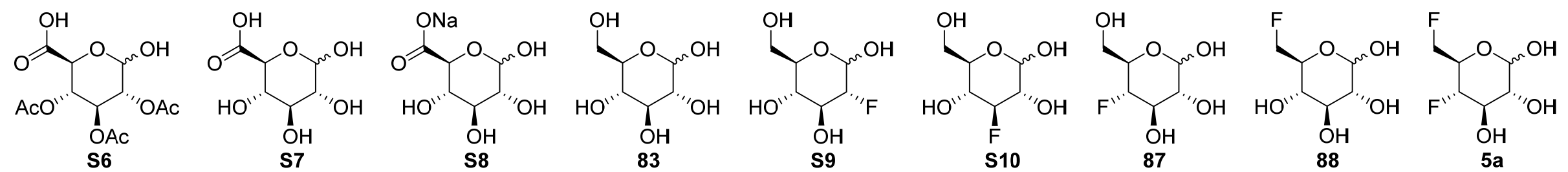

Figure S3: Structures of all sugars used for the plots in Figure S4 and Figure S5. 


\subsubsection{Annotated version of Figure 10 (Figure S4)}

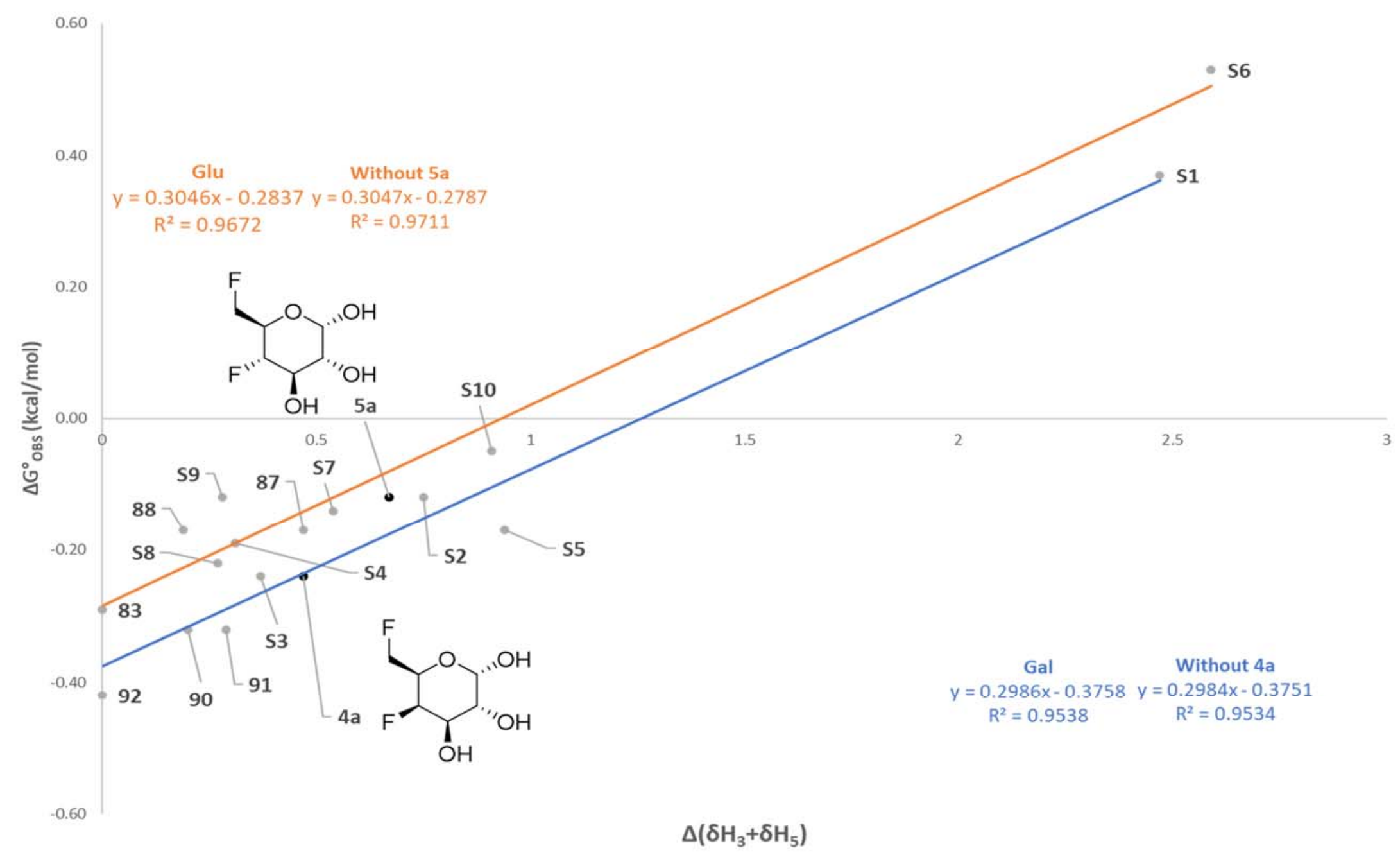

Figure S4: $\Delta G^{\circ}{ }_{\text {obs }}$ vs chemical shift difference for compounds $4 \mathbf{a}$ and $\mathbf{5 a}$ superimposed onto the Murphy ${ }^{100}$ data. Trendlines have been calculated with and without compounds $\mathbf{4} \mathbf{a}$ and $\mathbf{5} \mathbf{a}$. Only the $\alpha$-anomers are shown. 


\subsubsection{Companion to Figure 10 (Figure S5)}

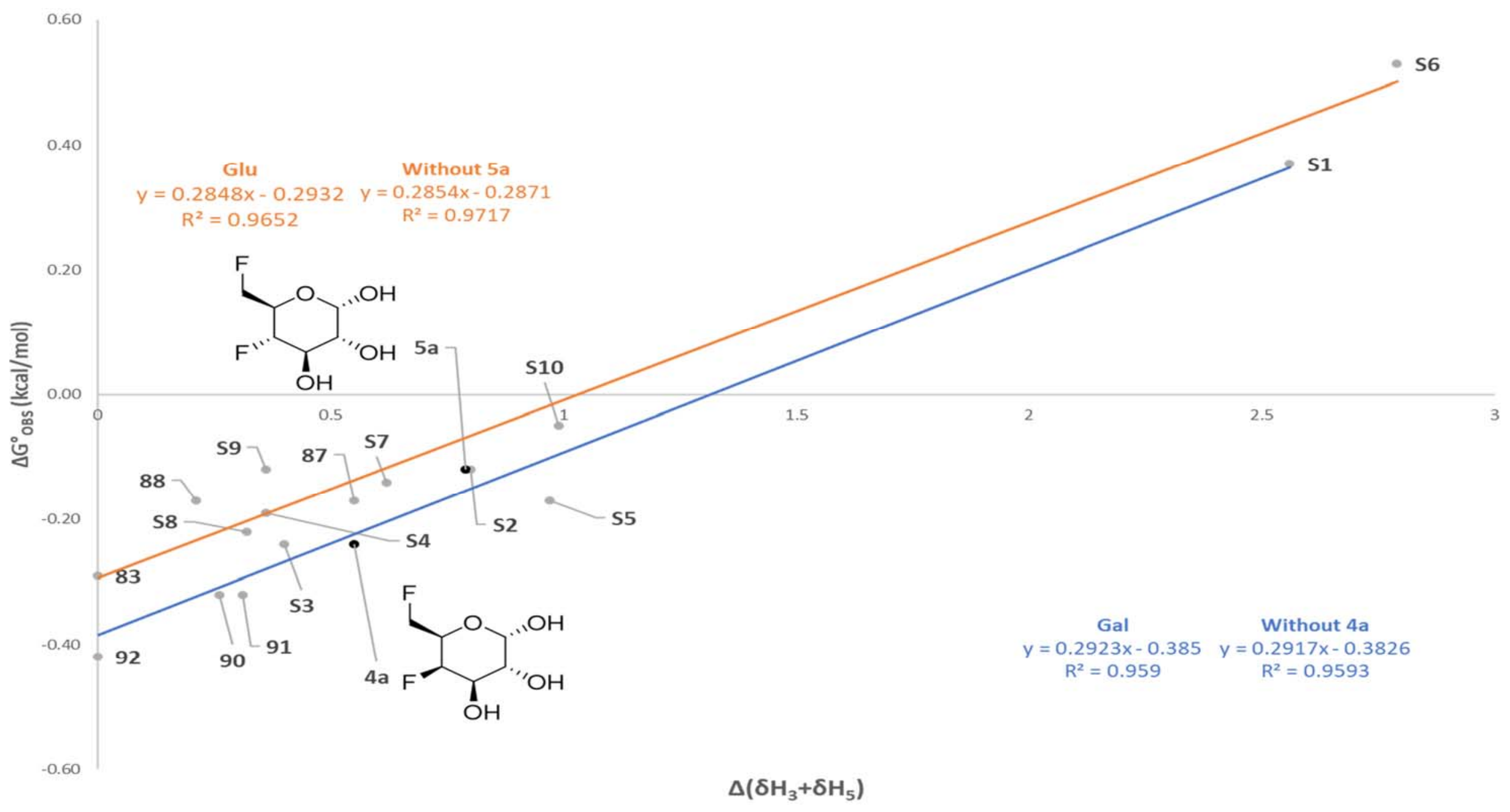

Figure S5: $\Delta G^{\circ}{ }_{\text {obs }}$ vs chemical shift difference for compounds $4 a$ and 5 a superimposed onto the Murphy ${ }^{100}$ data. Trendlines have been calculated with and without compounds $\mathbf{4 a}$ and $\mathbf{5 a}$. Only the $\beta$-anomers are shown. 


\subsection{Tabulated data for all plots}

Table S4: Chemical shift data used to generate graphs in this manuscript: Gluco-series

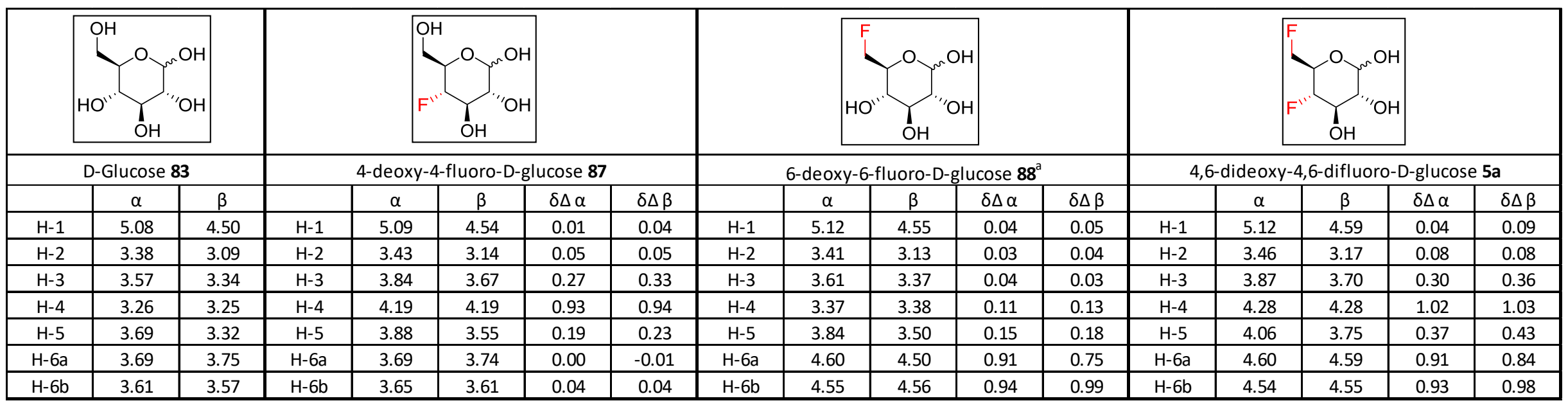

Table S5: Chemical shift data used to generate graphs in this manuscript: Galacto-series

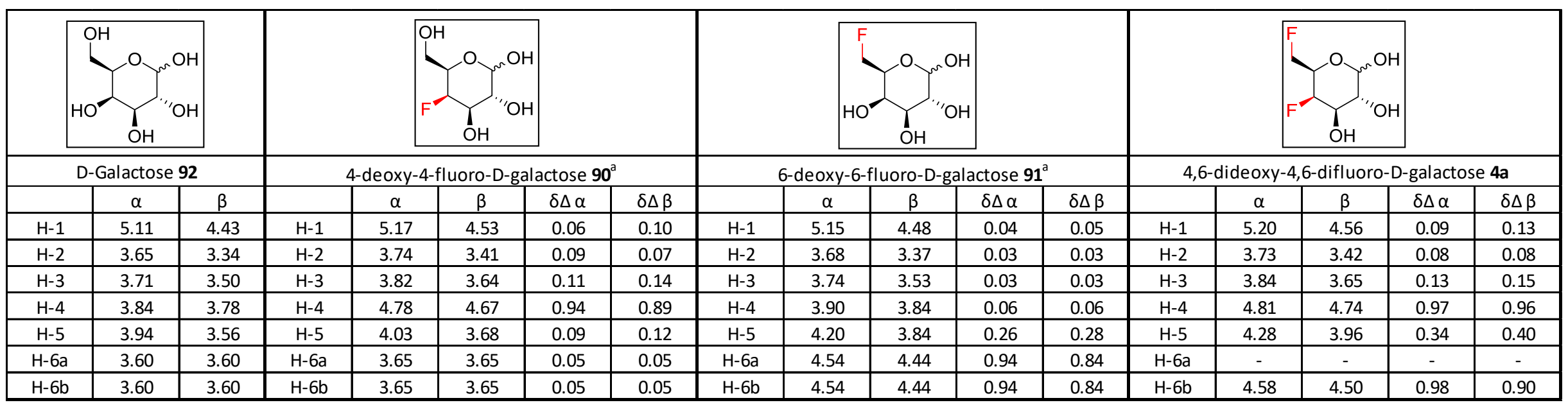


Table S6: Chemical shift data used to generate graphs in this manuscript: Quinovo-series

\begin{tabular}{|c|c|c|c|c|c|c|c|c|c|c|c|c|c|}
\hline & $\mathrm{OH}$ & & & & $\int_{1,0}^{n O}$ & & & & & $\mathrm{OH}$ & & & \multirow{9}{*}{ 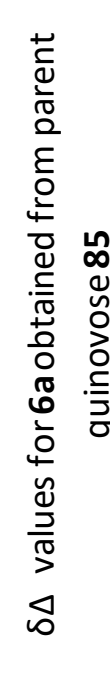 } \\
\hline \multicolumn{3}{|c|}{ D-quinovose 89} & \multicolumn{5}{|c|}{ 4-deoxy-4-fluoro-D-quinovose 3a } & \multicolumn{5}{|c|}{ 4,6-dideoxy-4,4-difluoro-D-xylo-hexopyranose 6a } & \\
\hline & $\alpha$ & $\beta$ & & $\alpha$ & $\beta$ & $\delta \Delta \alpha$ & $\delta \Delta \beta$ & & $\alpha$ & $\beta$ & $\delta \Delta \alpha$ & $\delta \Delta \beta$ & \\
\hline $\mathrm{H}-1$ & 5.03 & 4.48 & $\mathrm{H}-1$ & 5.03 & 4.52 & 0.00 & 0.04 & $\mathrm{H}-1$ & 5.11 & 4.60 & 0.08 & 0.12 & \\
\hline $\mathrm{H}-2$ & 3.39 & 3.10 & $\mathrm{H}-2$ & 3.44 & 3.15 & 0.05 & 0.05 & $\mathrm{H}-2$ & 3.59 & 3.29 & 0.20 & 0.19 & \\
\hline $\mathrm{H}-3$ & 3.51 & 3.28 & $\mathrm{H}-3$ & 3.77 & 3.60 & 0.26 & 0.32 & $\mathrm{H}-3$ & 3.91 & 3.75 & 0.40 & 0.47 & \\
\hline $\mathrm{H}-4$ & 2.99 & 3.01 & $\mathrm{H}-4$ & 3.91 & 3.92 & 0.92 & 0.91 & & & & & -3.01 & \\
\hline $\mathrm{H}-5$ & 3.75 & 3.35 & $\mathrm{H}-5$ & 3.94 & 3.58 & 0.19 & 0.23 & $\mathrm{H}-5$ & 4.16 & 3.80 & 0.41 & 0.45 & \\
\hline $\mathrm{H}-6$ & 1.11 & 1.14 & $\mathrm{H}-6$ & 1.15 & 1.18 & 0.04 & 0.04 & $\mathrm{H}-6$ & 1.13 & 1.16 & 0.02 & 0.02 & \\
\hline
\end{tabular}

Table S7: Chemical shift data used to generate graphs in this manuscript: Fuco-series

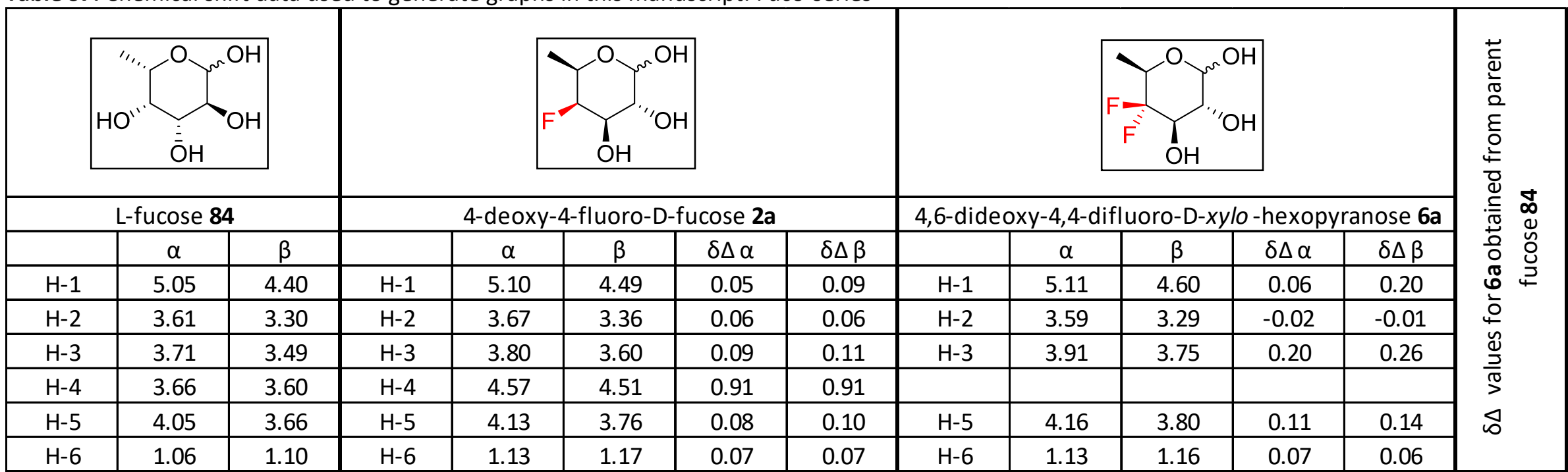


Table S8: Chemical shift data used to generate graphs in this manuscript: Data from Murphy, ${ }^{1}$ except for compounds $\mathbf{8 3}, \mathbf{8 7}$, and $\mathbf{9 2 .}$

\begin{tabular}{|c|c|c|c|c|c|c|c|c|c|c|c|c|c|}
\hline & & \multicolumn{2}{|c|}{$\mathrm{H}_{3}$} & \multicolumn{2}{|c|}{$\mathrm{H}_{5}$} & \multicolumn{2}{|c|}{$\delta \mathrm{H}_{3}+\delta \mathrm{H}_{5}$} & \multicolumn{2}{|c|}{$\mathrm{H}_{3}-\mathrm{H}_{3}[$ parent $]$} & \multicolumn{2}{|c|}{$\mathrm{H}_{5}-\mathrm{H}_{5}[$ parent $]$} & \multicolumn{2}{|c|}{$\delta \Delta\left(\mathrm{H}_{3}+\mathrm{H}_{5}\right)$} \\
\hline Substrate & $\Delta \mathrm{G}_{\mathrm{OBS}}^{\circ}(\mathrm{kcal} / \mathrm{mol})$ & $\alpha$ & $\beta$ & $\alpha$ & $\beta$ & $\alpha$ & $\beta$ & $\alpha$ & $\beta$ & $\alpha$ & $\beta$ & $\alpha$ & $\beta$ \\
\hline S1 & 0.37 & 5.31 & 5.15 & 4.81 & 4.47 & 10.12 & 9.62 & 1.6 & 1.65 & 0.87 & 0.91 & 2.47 & 2.56 \\
\hline S2 & -0.12 & 3.81 & 3.6 & 4.59 & 4.26 & 8.4 & 7.86 & 0.1 & 0.1 & 0.65 & 0.7 & 0.75 & 0.8 \\
\hline S3 & -0.24 & 3.76 & 3.55 & 4.26 & 3.91 & 8.02 & 7.46 & 0.05 & 0.05 & 0.32 & 0.35 & 0.37 & 0.4 \\
\hline 92 & -0.42 & 3.71 & 3.5 & 3.94 & 3.56 & 7.65 & 7.06 & 0 & 0 & 0 & 0 & 0 & 0 \\
\hline S4 & -0.19 & 3.99 & 3.81 & 3.97 & 3.61 & 7.96 & 7.42 & 0.28 & 0.31 & 0.03 & 0.05 & 0.31 & 0.36 \\
\hline S5 & -0.17 & 4.64 & 4.45 & 3.95 & 3.58 & 8.59 & 8.03 & 0.93 & 0.95 & 0.01 & 0.02 & 0.94 & 0.97 \\
\hline 90 & -0.32 & 3.82 & 3.64 & 4.03 & 3.68 & 7.85 & 7.32 & 0.11 & 0.14 & 0.09 & 0.12 & 0.2 & 0.26 \\
\hline 91 & -0.32 & 3.74 & 3.53 & 4.2 & 3.84 & 7.94 & 7.37 & 0.03 & 0.03 & 0.26 & 0.28 & 0.29 & 0.31 \\
\hline $4 a$ & -0.24 & 3.84 & 3.65 & 4.28 & 3.96 & 8.12 & 7.61 & 0.13 & 0.15 & 0.34 & $\begin{array}{l}0.4 \\
\end{array}$ & 0.47 & 0.55 \\
\hline S6 & 0.53 & 5.37 & 5.27 & 4.48 & 4.18 & 9.85 & 9.45 & 1.8 & 1.93 & 0.79 & 0.86 & 2.59 & 2.79 \\
\hline S7 & -0.14 & 3.6 & 3.41 & 4.2 & 3.87 & 7.8 & $\begin{array}{l}7.28 \\
\end{array}$ & 0.03 & 0.07 & 0.51 & 0.55 & 0.54 & 0.62 \\
\hline S8 & -0.22 & 3.59 & 3.37 & 3.94 & 3.61 & 7.53 & 6.98 & 0.02 & 0.03 & 0.25 & 0.29 & 0.27 & 0.32 \\
\hline 83 & -0.29 & 3.57 & 3.34 & 3.69 & 3.32 & 7.26 & 6.66 & 0 & 0 & 0 & 0 & 0 & 0 \\
\hline S9 & -0.12 & 3.83 & 3.66 & 3.71 & 3.36 & 7.54 & 7.02 & 0.26 & 0.32 & 0.02 & 0.04 & 0.28 & 0.36 \\
\hline S10 & -0.05 & 4.49 & 4.3 & 3.68 & 3.35 & 8.17 & 7.65 & 0.92 & 0.96 & -0.01 & 0.03 & 0.91 & 0.99 \\
\hline 87 & -0.17 & 3.85 & 3.66 & 3.88 & 3.55 & 7.73 & 7.21 & 0.28 & 0.32 & 0.19 & 0.23 & 0.47 & 0.55 \\
\hline 88 & -0.17 & 3.61 & 3.37 & 3.84 & 3.5 & 7.45 & 6.87 & 0.04 & 0.03 & 0.15 & 0.18 & 0.19 & 0.21 \\
\hline $5 a$ & -0.12 & 3.87 & 3.7 & 4.06 & 3.75 & 7.93 & 7.45 & 0.3 & 0.36 & 0.37 & 0.43 & 0.67 & 0.79 \\
\hline
\end{tabular}


Table S9: Chemical shift data used to generate graphs in this manuscript: Miscellaneous sugars not belonging to a specific series.

\begin{tabular}{|c|c|c|c|c|c|c|c|c|c|c|c|}
\hline \multicolumn{2}{|c|}{$\underbrace{\mathrm{OH}}_{\mathrm{OH}}$} & & \multicolumn{3}{|c|}{$\mathrm{F}$} & \multicolumn{3}{|c|}{${ }_{\mathrm{OH}}^{\mathrm{OH}}$} & & & \\
\hline \multicolumn{3}{|c|}{ 4-deoxy-D-glucose 93} & \multicolumn{3}{|c|}{$\begin{array}{c}\text { 4,6-dideoxy-6-fluoro-D-xylo- } \\
\text { hexopyranose 1a }\end{array}$} & \multicolumn{3}{|c|}{$\begin{array}{c}\text { 4,6-dideoxy-6,6-difluoro-D- } \\
\text { xylo-hexopyranose 7a }\end{array}$} & \multicolumn{3}{|c|}{ 4,6-dideoxy-D- $x y l o$-hexose 86} \\
\hline & $\alpha$ & $\beta$ & & $\alpha$ & $\beta$ & & $\alpha$ & $\beta$ & & $\alpha$ & $\beta$ \\
\hline $\mathrm{H}-1$ & 5.11 & 4.42 & $\mathrm{H}-1$ & 5.14 & 4.46 & $\mathrm{H}-1$ & 5.17 & 4.49 & $\mathrm{H}-1$ & 5.06 & 4.40 \\
\hline $\mathrm{H}-2$ & 3.31 & 3.00 & $\mathrm{H}-2$ & 3.32 & 3.02 & $\mathrm{H}-2$ & 3.35 & 3.05 & $\mathrm{H}-2$ & 3.29 & 2.98 \\
\hline $\mathrm{H}-3$ & 3.80 & 3.59 & $\mathrm{H}-3$ & 3.83 & 3.62 & $\mathrm{H}-3$ & 3.83 & 3.63 & $\mathrm{H}-3$ & 3.75 & 3.55 \\
\hline $\mathrm{H}-4 \mathrm{ax}$ & 1.29 & 1.28 & $\mathrm{H}-4 \mathrm{ax}$ & 1.87 & 1.84 & $\mathrm{H}-4 \mathrm{ax}$ & 1.43 & 1.44 & $\mathrm{H}-4 \mathrm{ax}$ & 1.22 & 1.24 \\
\hline $\mathrm{H}-4 \mathrm{eq}$ & 1.84 & 1.82 & $\mathrm{H}-4 \mathrm{eq}$ & 1.39 & 1.37 & $\mathrm{H}-4 \mathrm{eq}$ & 2.00 & 1.98 & $\mathrm{H}-4 \mathrm{eq}$ & 1.91 & 1.88 \\
\hline $\mathrm{H}-5$ & 3.95 & 3.57 & $\mathrm{H}-5$ & 4.14 & 3.79 & $\mathrm{H}-5$ & 4.14 & 3.80 & $\mathrm{H}-5$ & 4.02 & 3.62 \\
\hline $\mathrm{H}-6 \mathrm{a}$ & 3.52 & 3.53 & $\mathrm{H}-6 \mathrm{a}$ & 4.41 & 4.43 & $\mathrm{H}-6$ & 5.78 & 5.78 & $\mathrm{H} 6$ & 1.05 & 1.08 \\
\hline $\mathrm{H}-6 \mathrm{~b}$ & 3.43 & 3.45 & $\mathrm{H}-6 \mathrm{~b}$ & 4.33 & 4.32 & & & & & & \\
\hline
\end{tabular}

${ }^{a}$ Data taken from ref ${ }^{1}$. 


\section{Spectra of novel compounds}

\subsection{4,6-Dideoxy-6-fluoro-D-xylo-hexopyranose (1a)}

3.1.1. ${ }^{1} \mathrm{H}$ NMR $\left(400 \mathrm{MHz}, \mathrm{CD}_{3} \mathrm{OD}\right)$

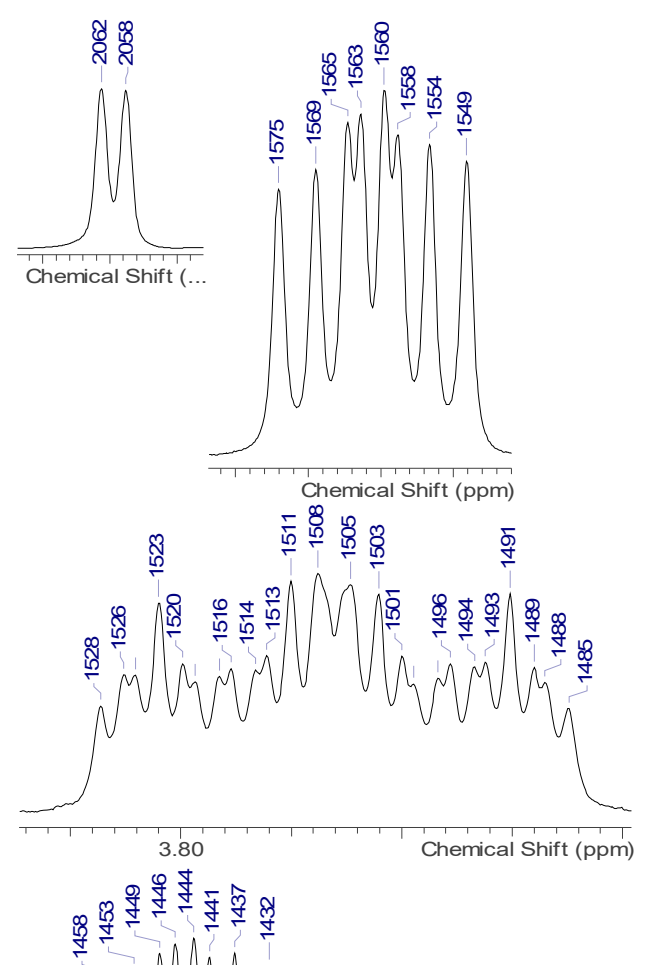

$$
\text { ह }
$$

METHANOL-d4
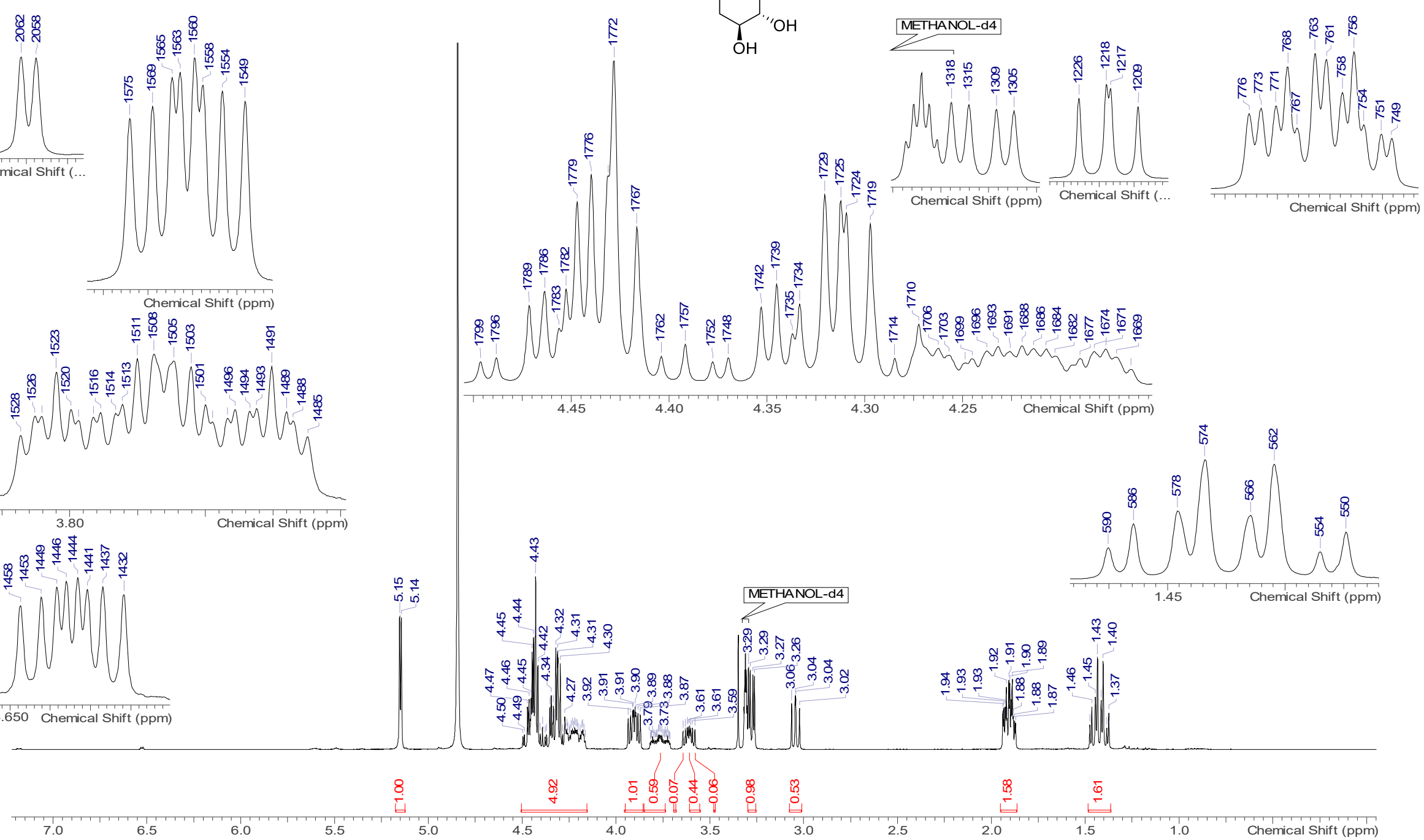
3.1.2. ${ }^{13} \mathrm{C}\left\{{ }^{1} \mathrm{H}\right\} \mathrm{NMR}\left(101 \mathrm{MHz}, \mathrm{CD}_{3} \mathrm{OD}\right)$
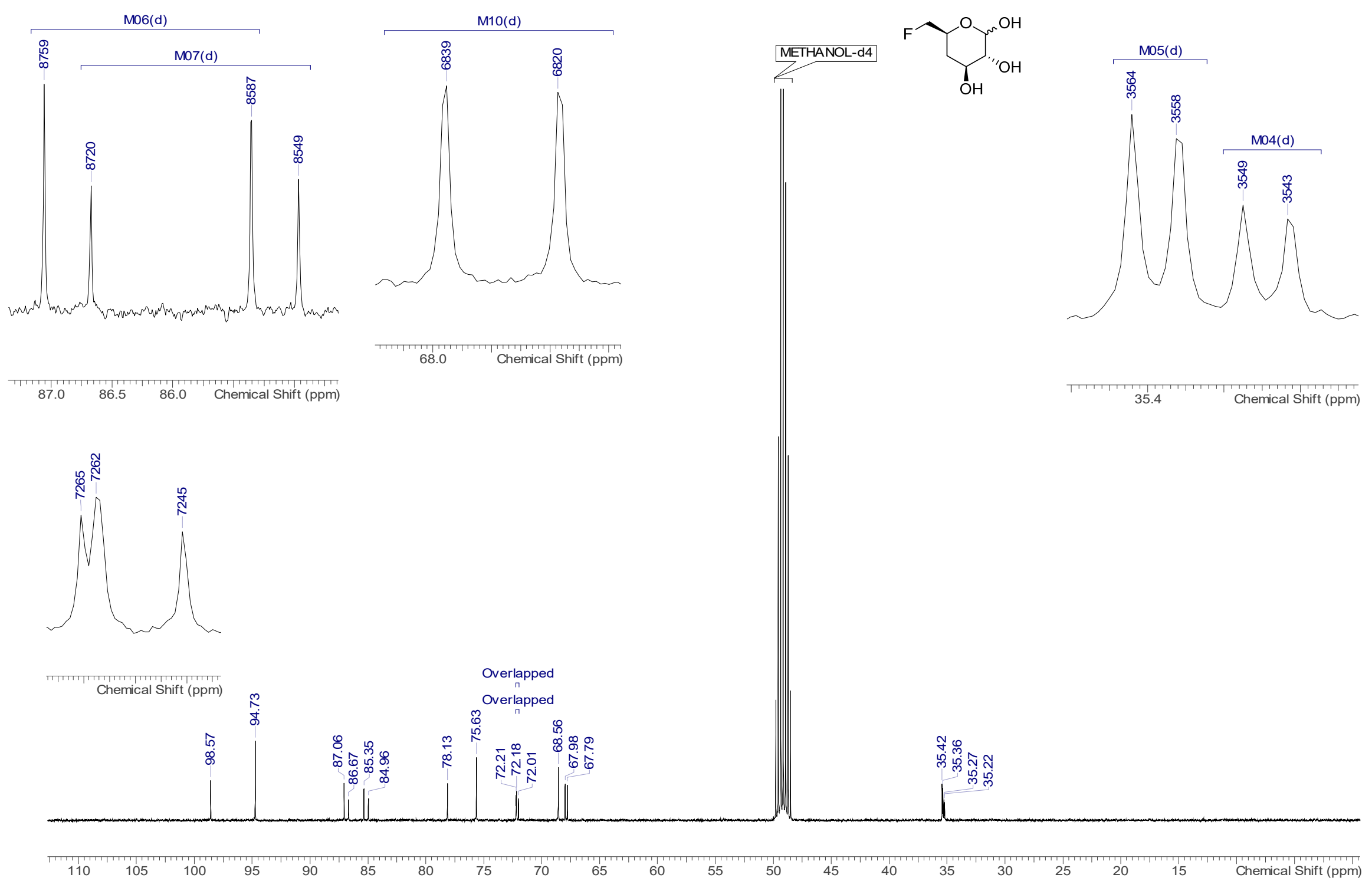
3.1.3. COSY, HSQC-DEPT \& $\mathrm{HMBC}\left(\mathrm{CD}_{3} \mathrm{OD}\right)$

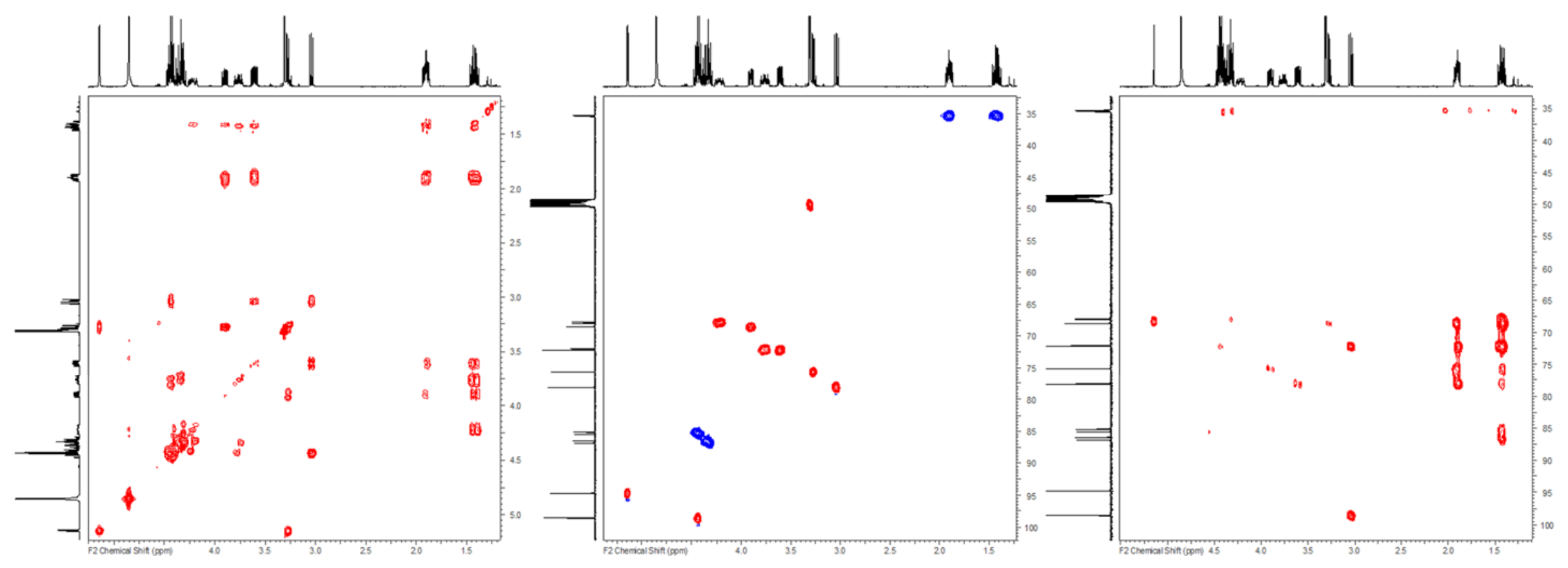


S 22

3.1.4. $\left.{ }^{19} \mathrm{~F} \mathrm{NMR} \mathrm{(376} \mathrm{MHz,} \mathrm{CD}_{3} \mathrm{OD}\right)$

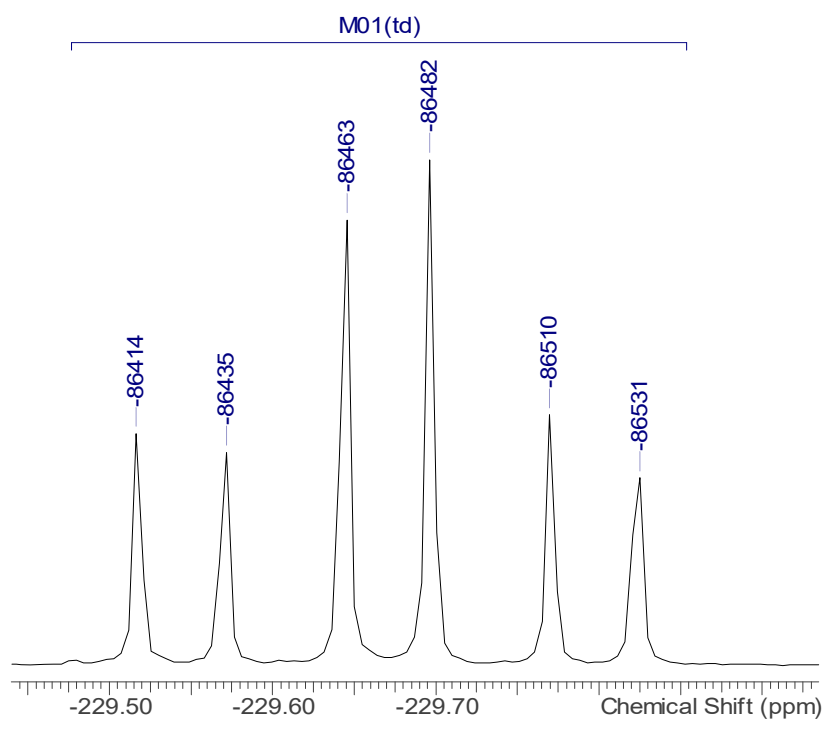<smiles>O[C@H]1C[C@H](CF)O[C@@H](O)[C@@H]1O</smiles>

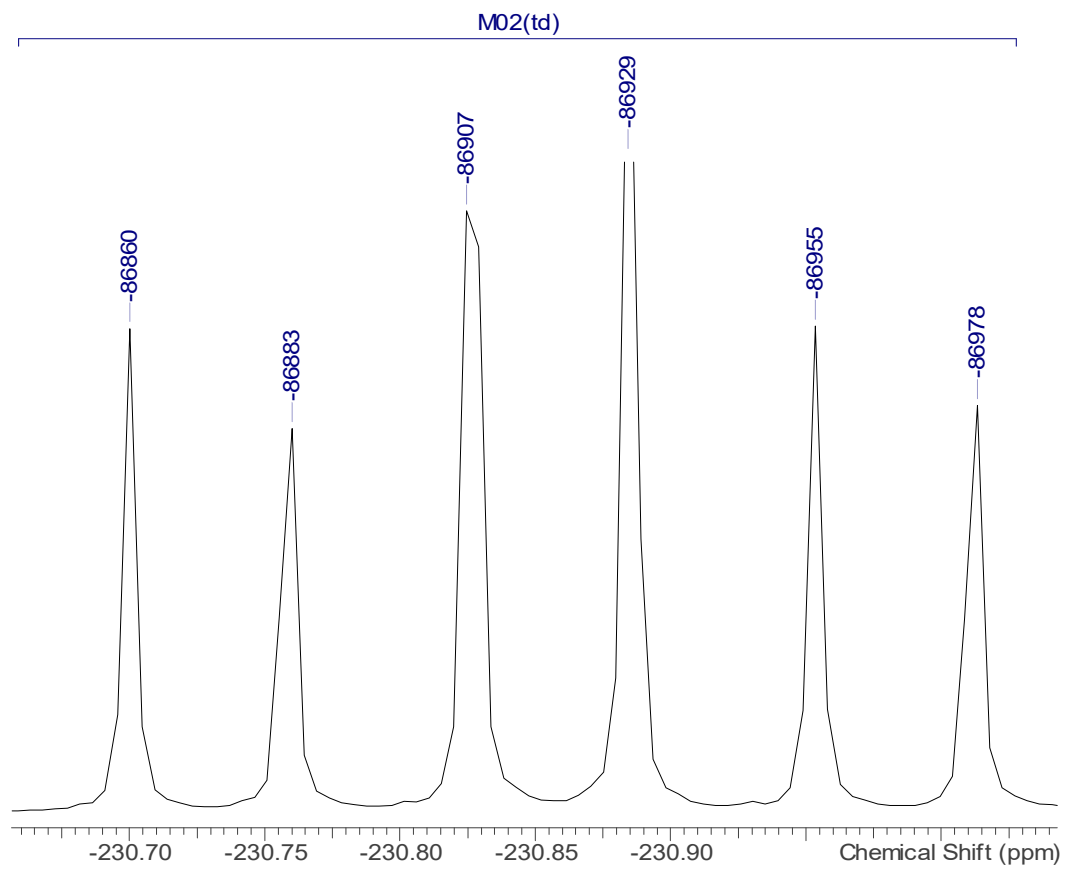

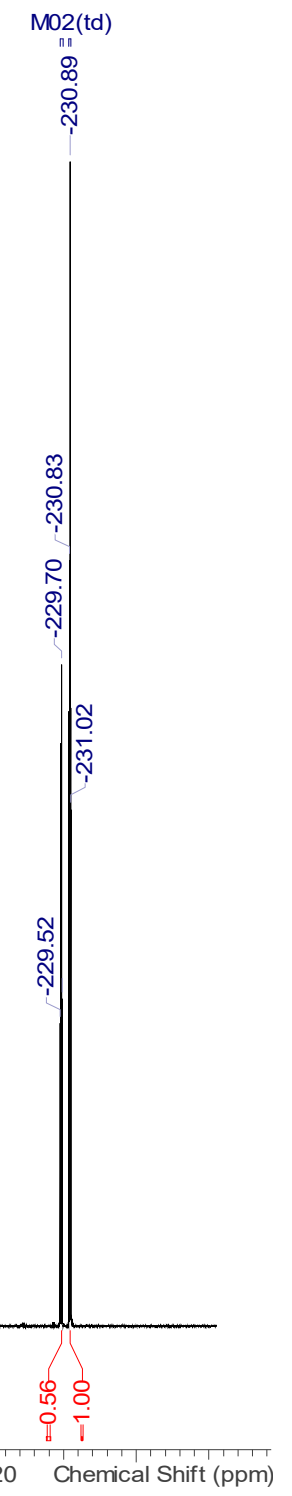


3.1.5. ${ }^{19} \mathrm{~F}\left\{{ }^{1} \mathrm{H}\right\} \mathrm{NMR}\left(376 \mathrm{MHz}, \mathrm{CD}_{3} \mathrm{OD}\right)$

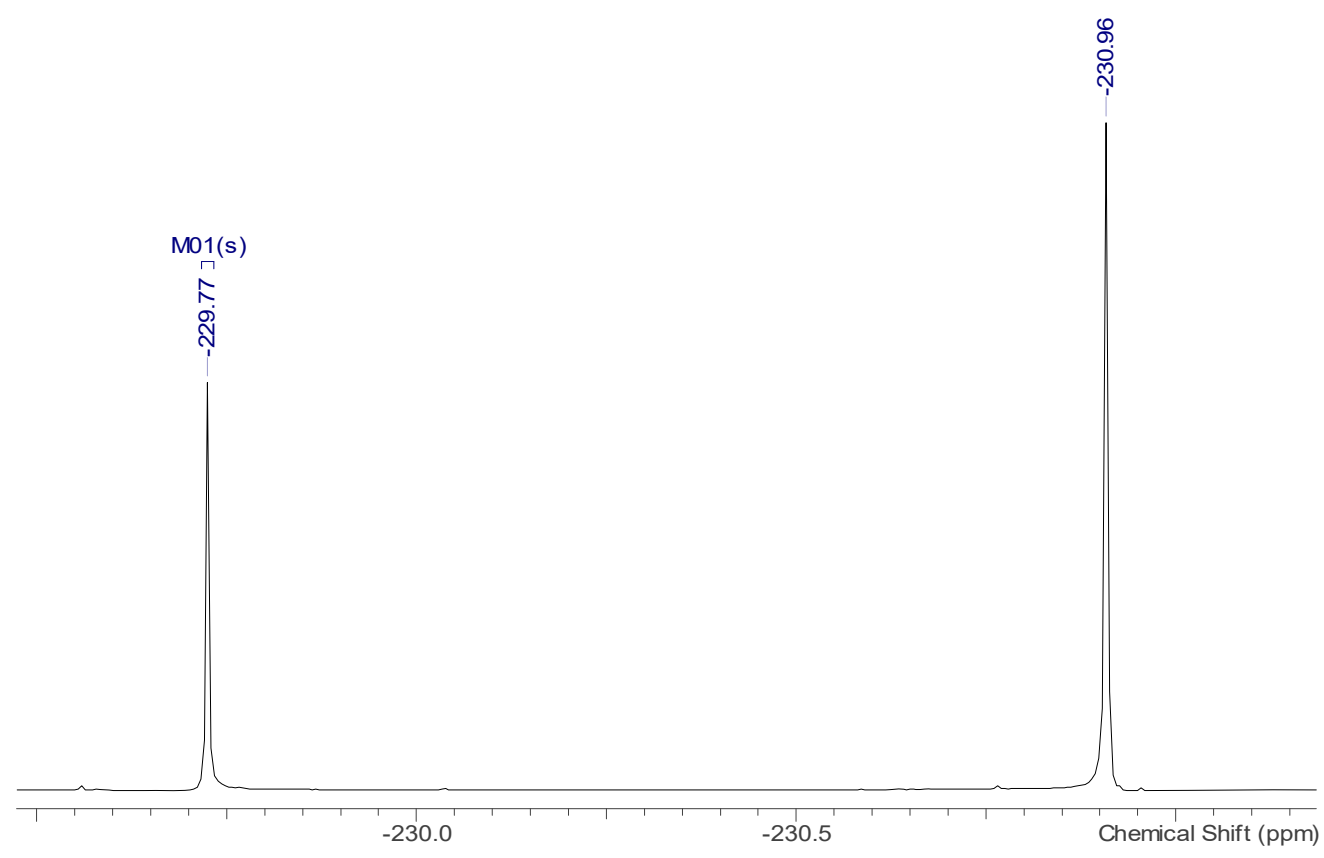

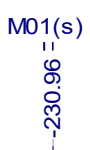

$\underbrace{\mathrm{O} / \mathrm{OH}}_{\mathrm{OH}}$

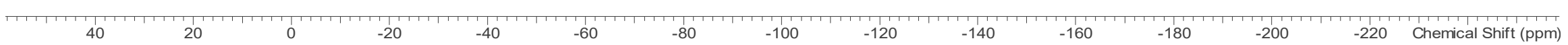




\subsection{1,2,3-Tri-O-acetyl-4,6-dideoxy-6-fluoro-D-xylo-hexopyranoside (1b)}

3.2.1. ${ }^{1} \mathrm{H} \mathrm{NMR}\left(400 \mathrm{MHz}, \mathrm{CDCl}_{3}\right)$

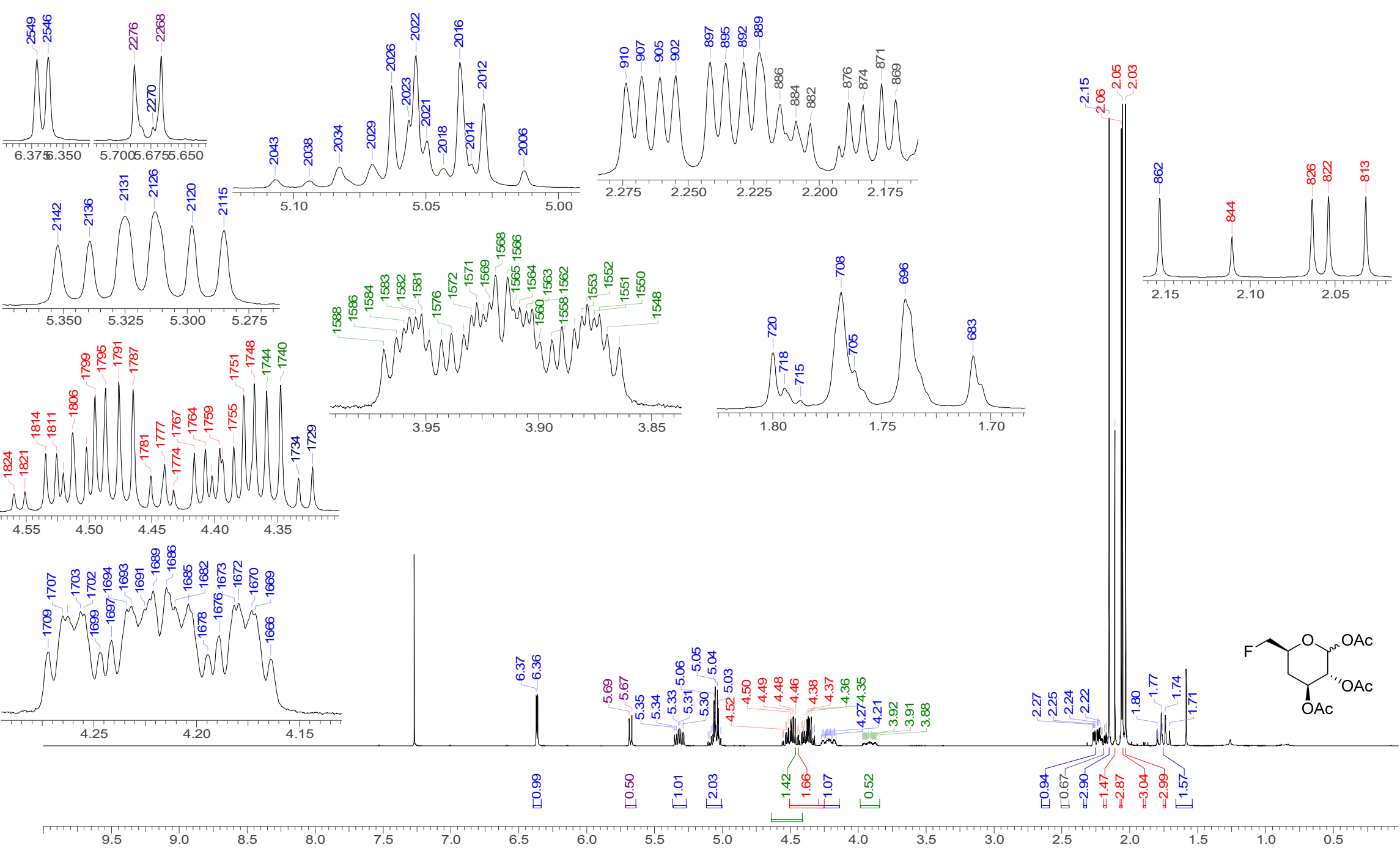




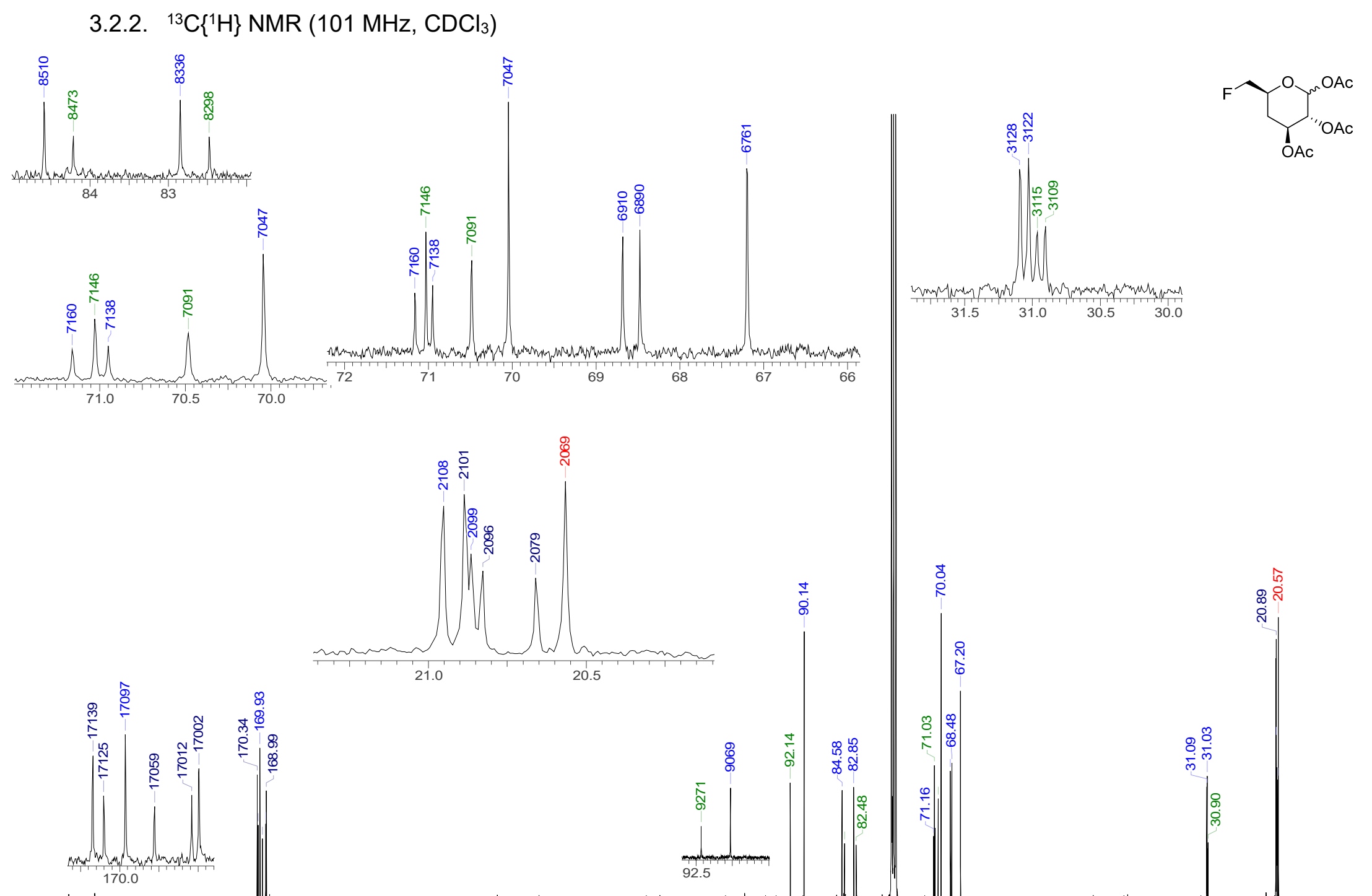

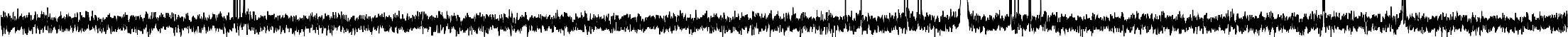

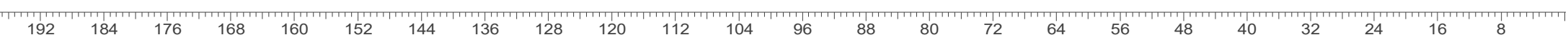


3.2.3. COSY, HSQC-DEPT \& $\mathrm{HMBC}\left(\mathrm{CDCl}_{3}\right)$

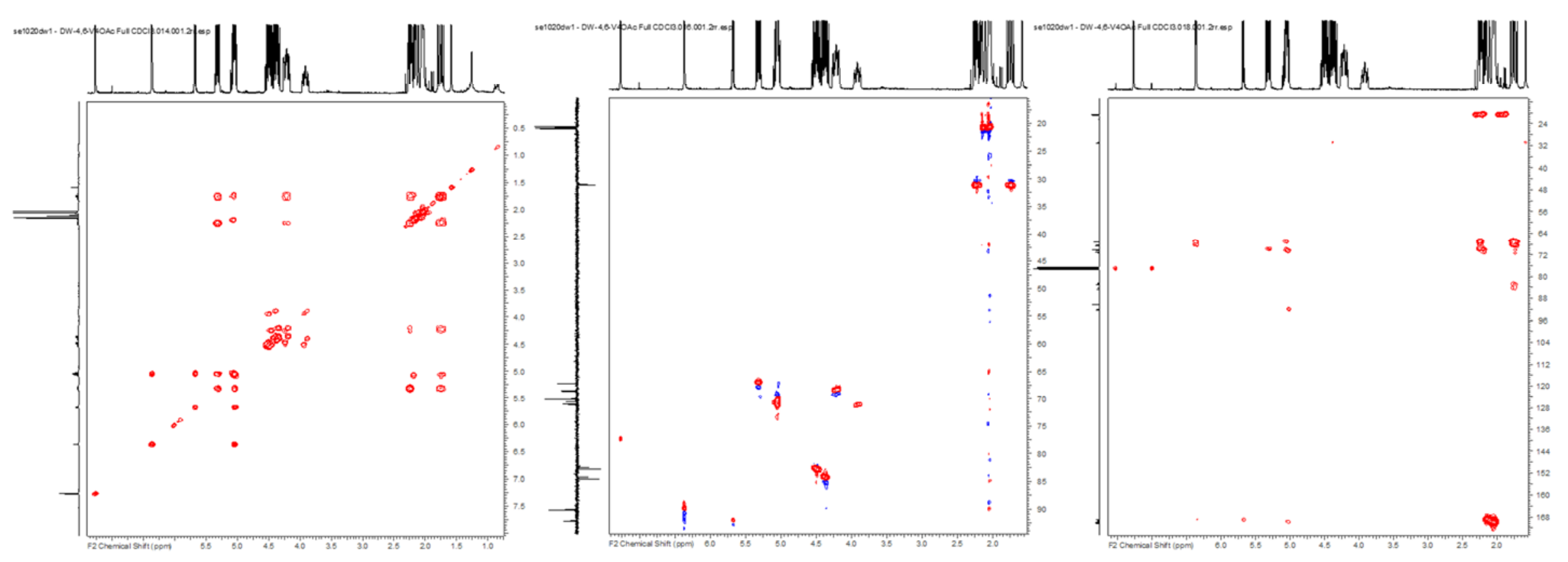


3.2.4. ${ }^{19} \mathrm{~F} \mathrm{NMR}\left(376 \mathrm{MHz}, \mathrm{CDCl}_{3}\right)$

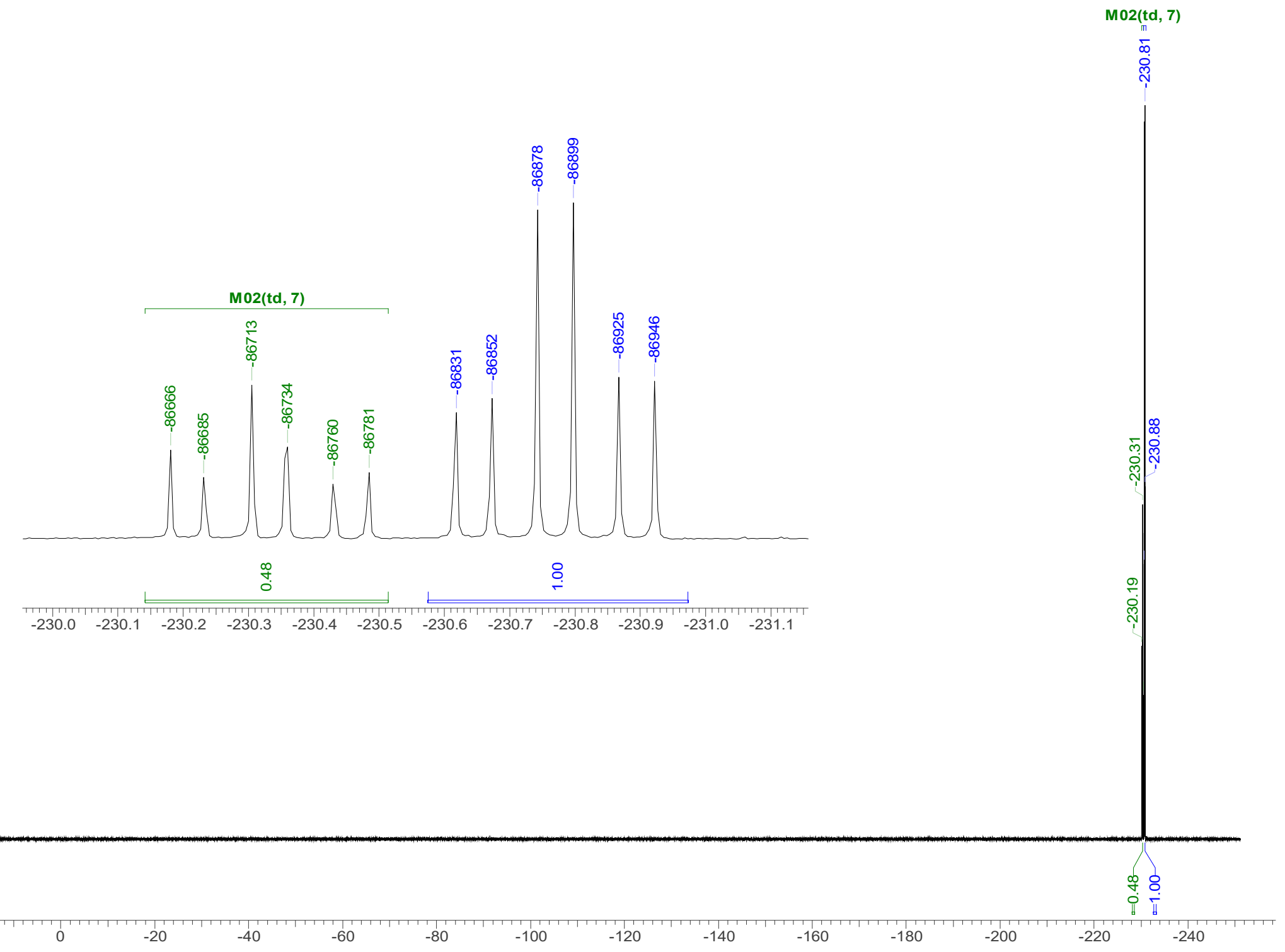


3.2.5. ${ }^{19} \mathrm{~F}\left\{{ }^{1} \mathrm{H}\right\} \mathrm{NMR}\left(376 \mathrm{MHz}, \mathrm{CDCl}_{3}\right)$

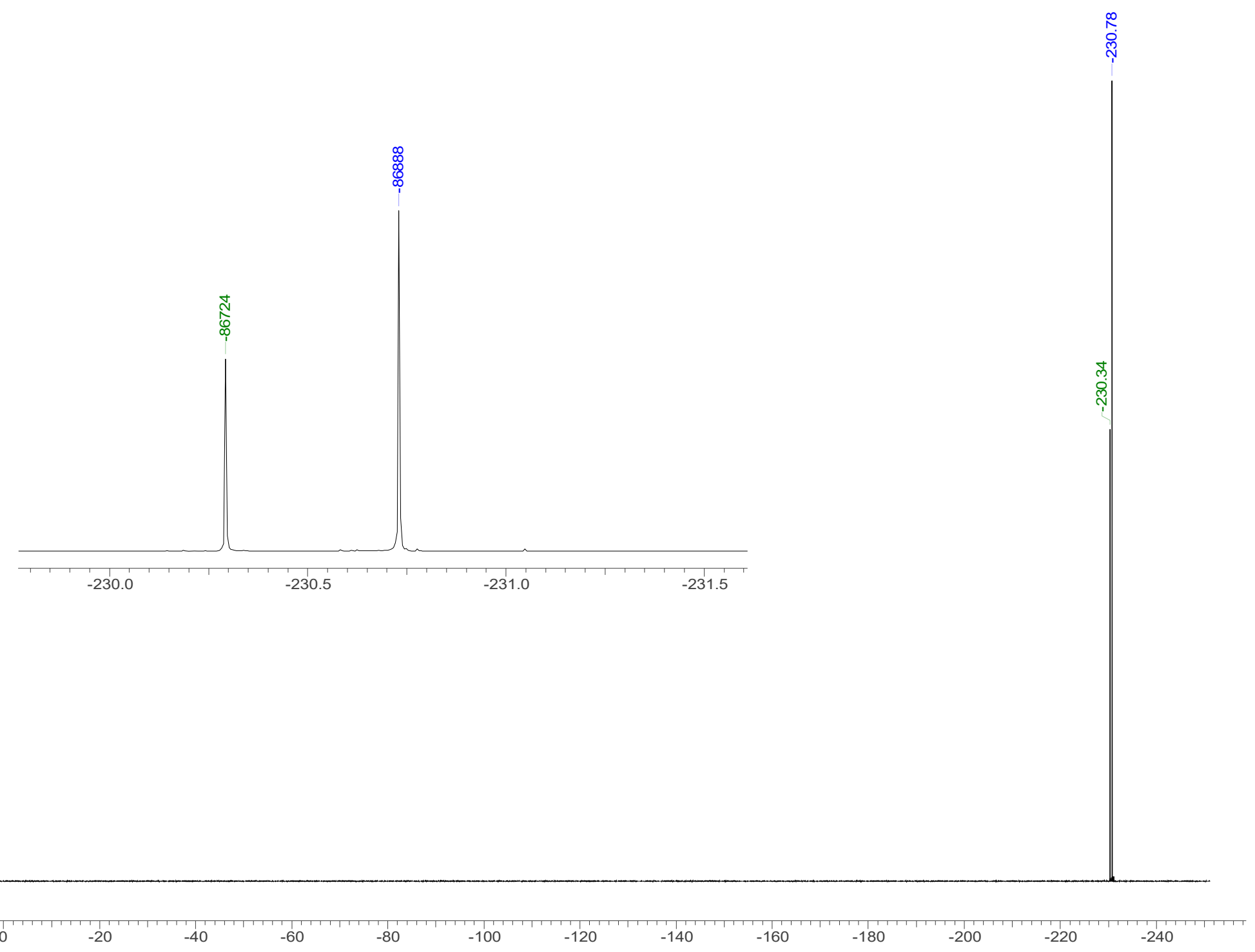




\subsection{4-Deoxy-4-fluoro-D-fucose (2a)}

3.3.1. ${ }^{1} \mathrm{H}$ NMR $\left(500 \mathrm{MHz}, \mathrm{CD}_{3} \mathrm{OD}\right)$

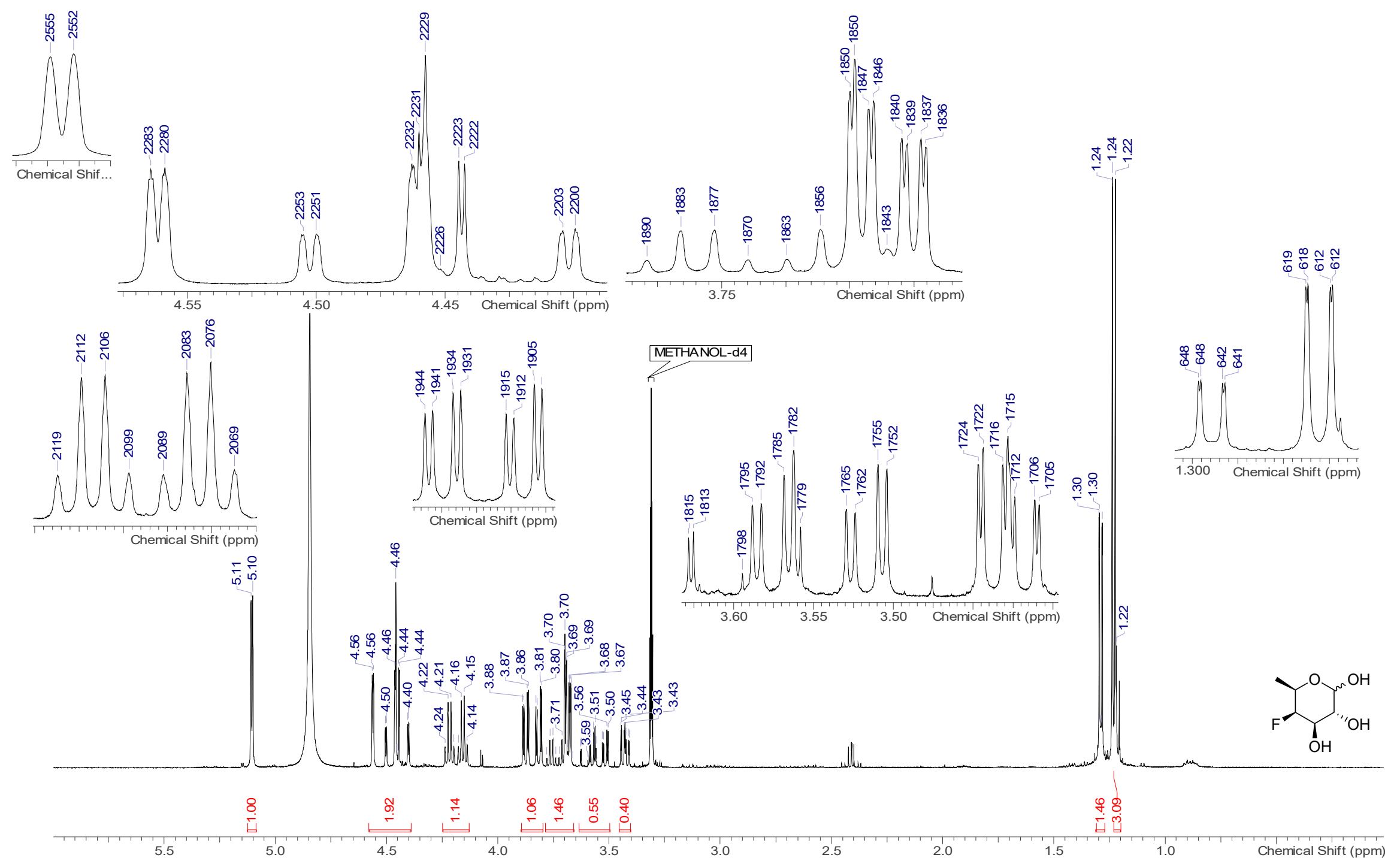




\subsection{2. ${ }^{1} \mathrm{H}\left\{{ }^{19} \mathrm{~F}\right\} \mathrm{NMR}\left(500 \mathrm{MHz}, \mathrm{CD}_{3} \mathrm{OD}\right)$}

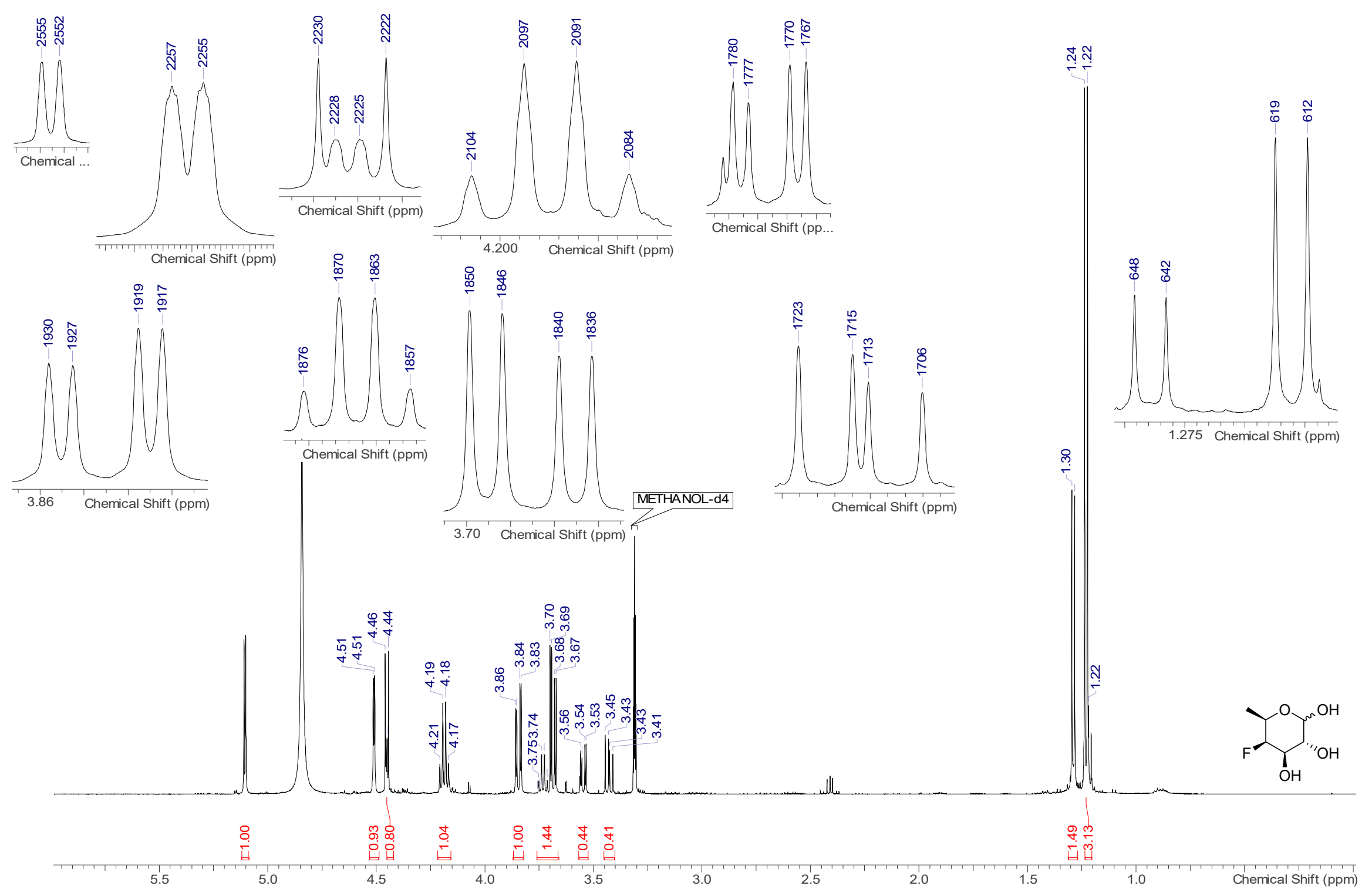


3.3.3. ${ }^{13} \mathrm{C}\left\{{ }^{1} \mathrm{H}\right\} \operatorname{NMR}\left(126 \mathrm{MHz}, \mathrm{CD}_{3} \mathrm{OD}\right)$

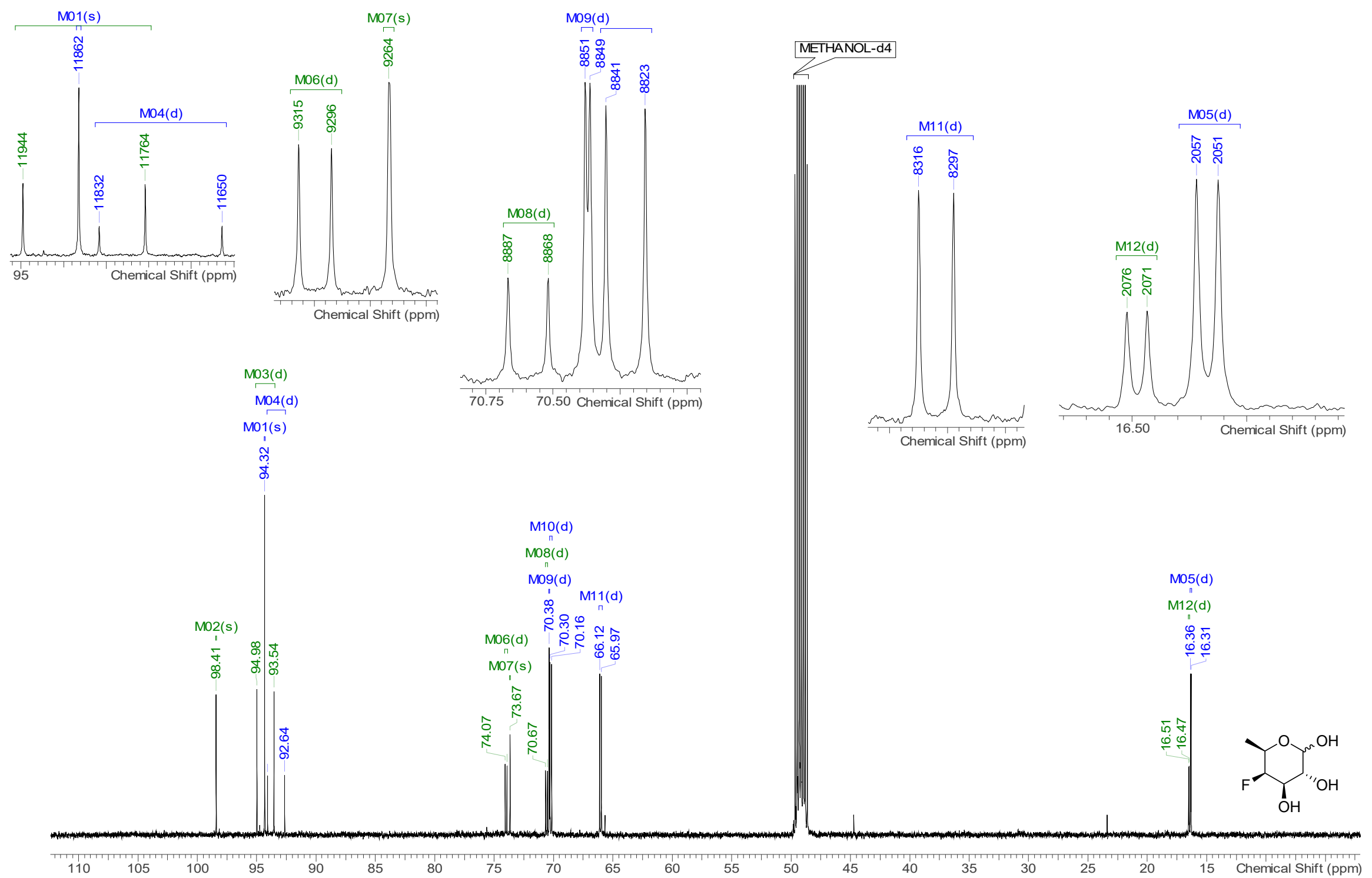


3.3.4. COSY \& HSQC-DEPT $\left(\mathrm{CD}_{3} \mathrm{OD}\right)$

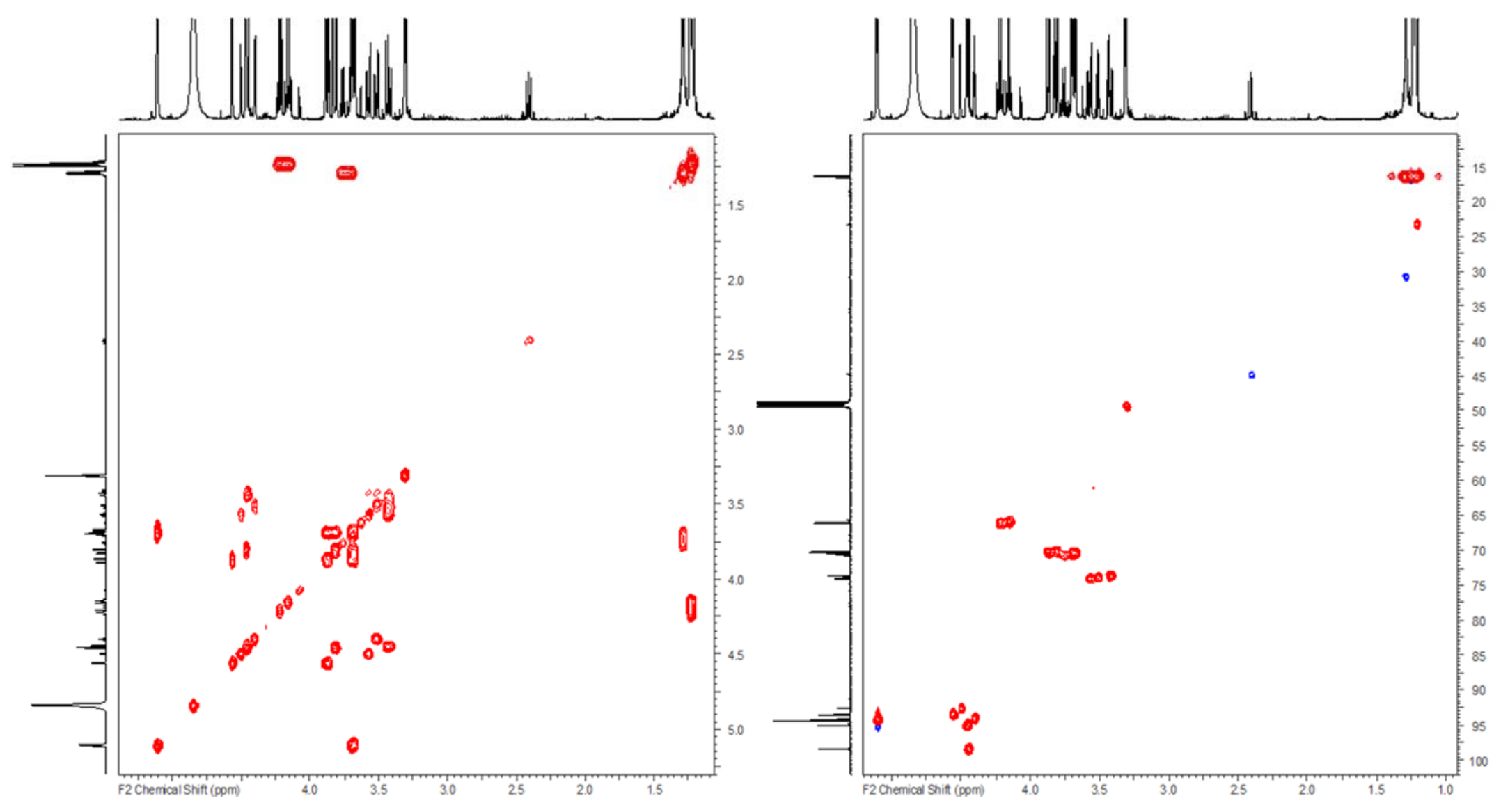


3.3.5. ${ }^{19} \mathrm{~F} \mathrm{NMR}\left(470 \mathrm{MHz}, \mathrm{CD}_{3} \mathrm{OD}\right)$

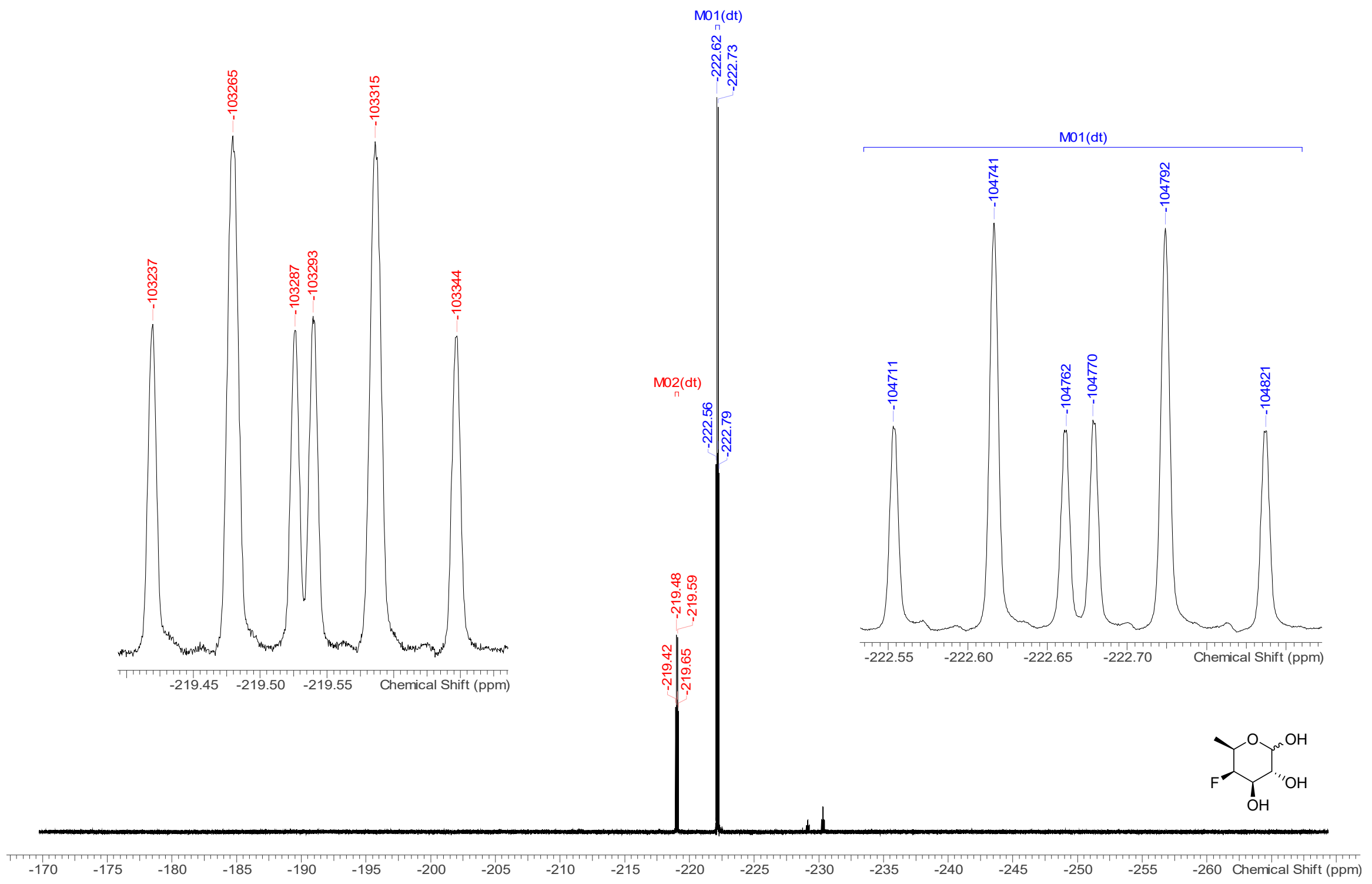


3.3.6. ${ }^{19} \mathrm{~F}\left\{{ }^{1} \mathrm{H}\right\} \mathrm{NMR}\left(470 \mathrm{MHz}, \mathrm{CD}_{3} \mathrm{OD}\right)$
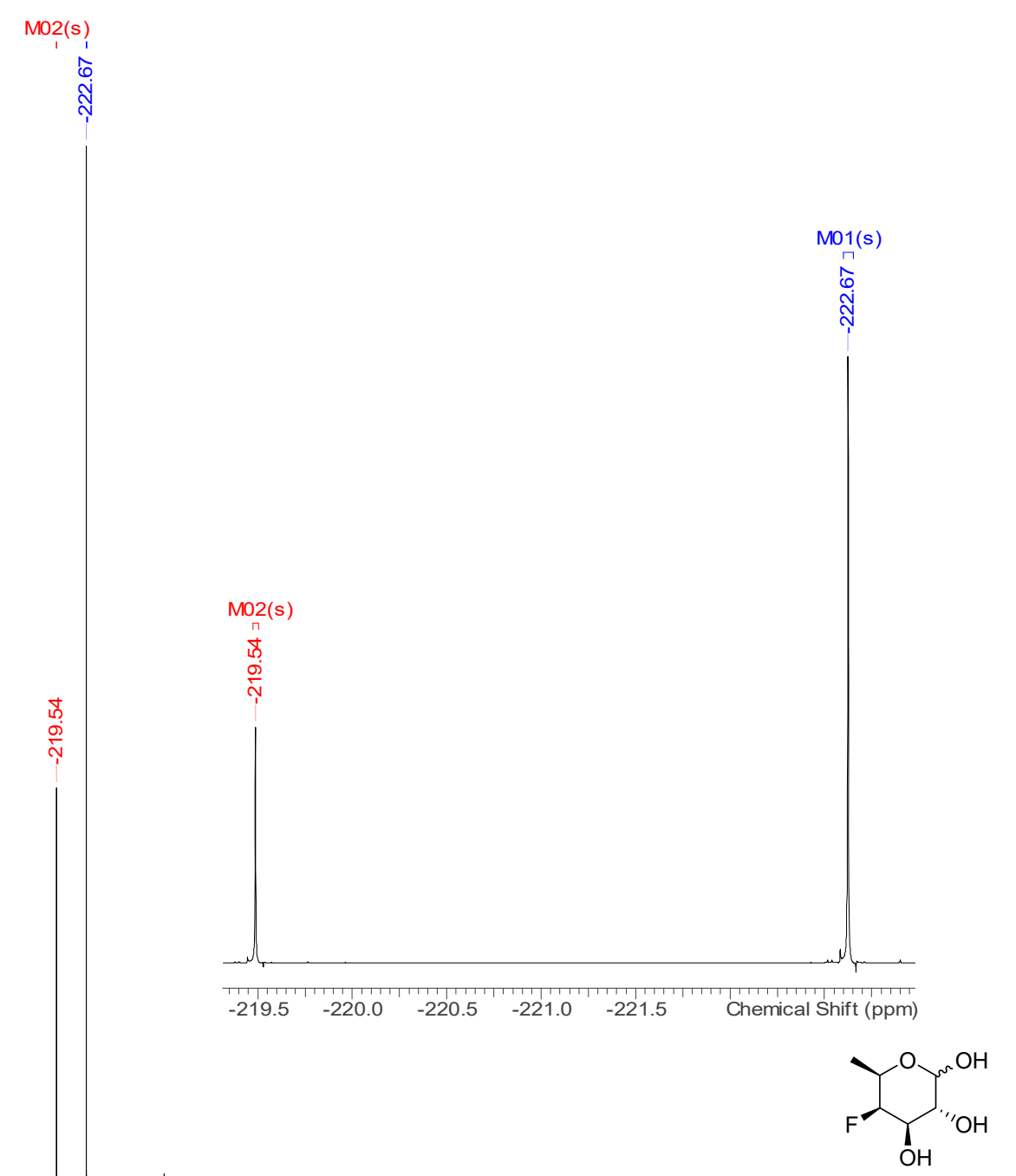

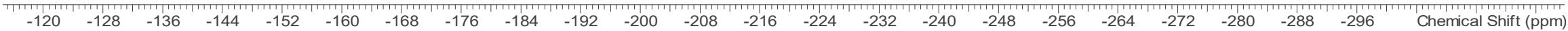




\subsection{1,2,3-Tri-O-acetyl-4-deoxy-4-fluoro-D-fucopyranoside (2b)}

3.4.1. ${ }^{1} \mathrm{H} \mathrm{NMR}\left(400 \mathrm{MHz}, \mathrm{CDCl}_{3}\right)$

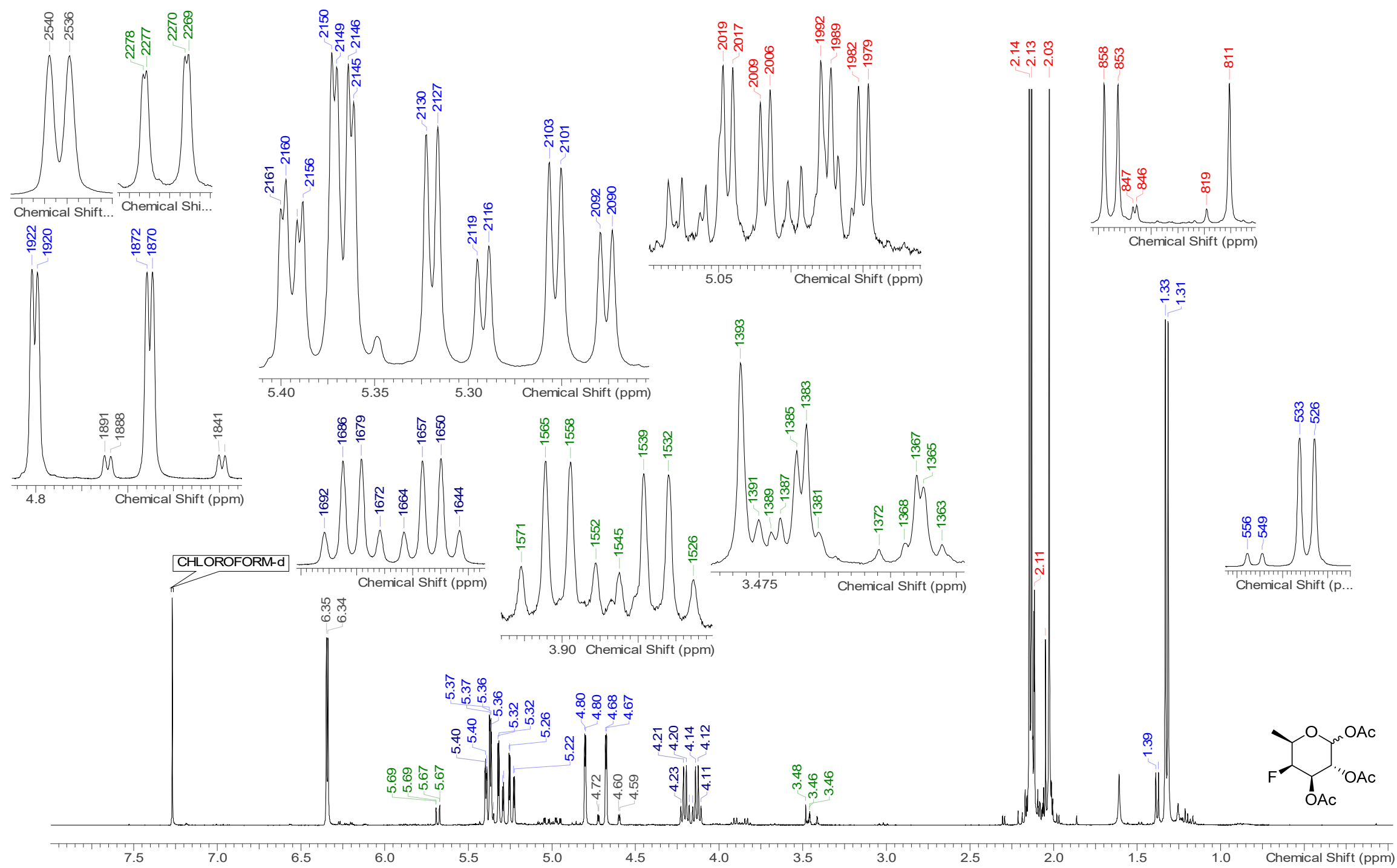


3.4.2. ${ }^{13} \mathrm{C}\left\{{ }^{1} \mathrm{H}\right\} \operatorname{NMR}\left(101 \mathrm{MHz}, \mathrm{CDCl}_{3}\right)$
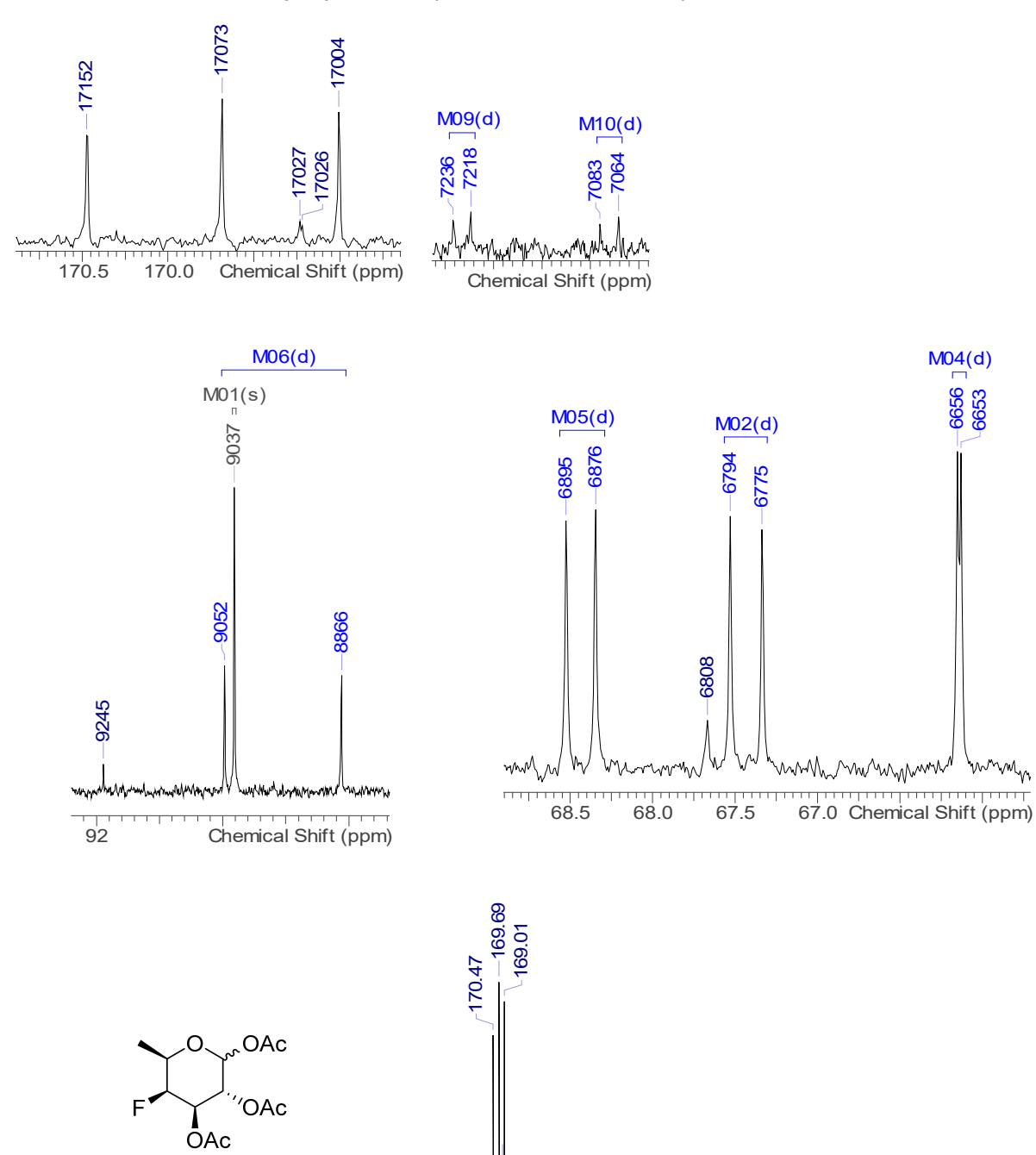

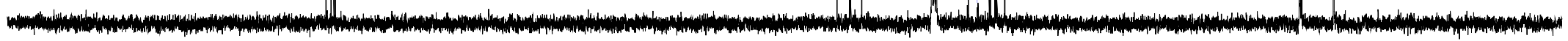

CHLOROFORM-d
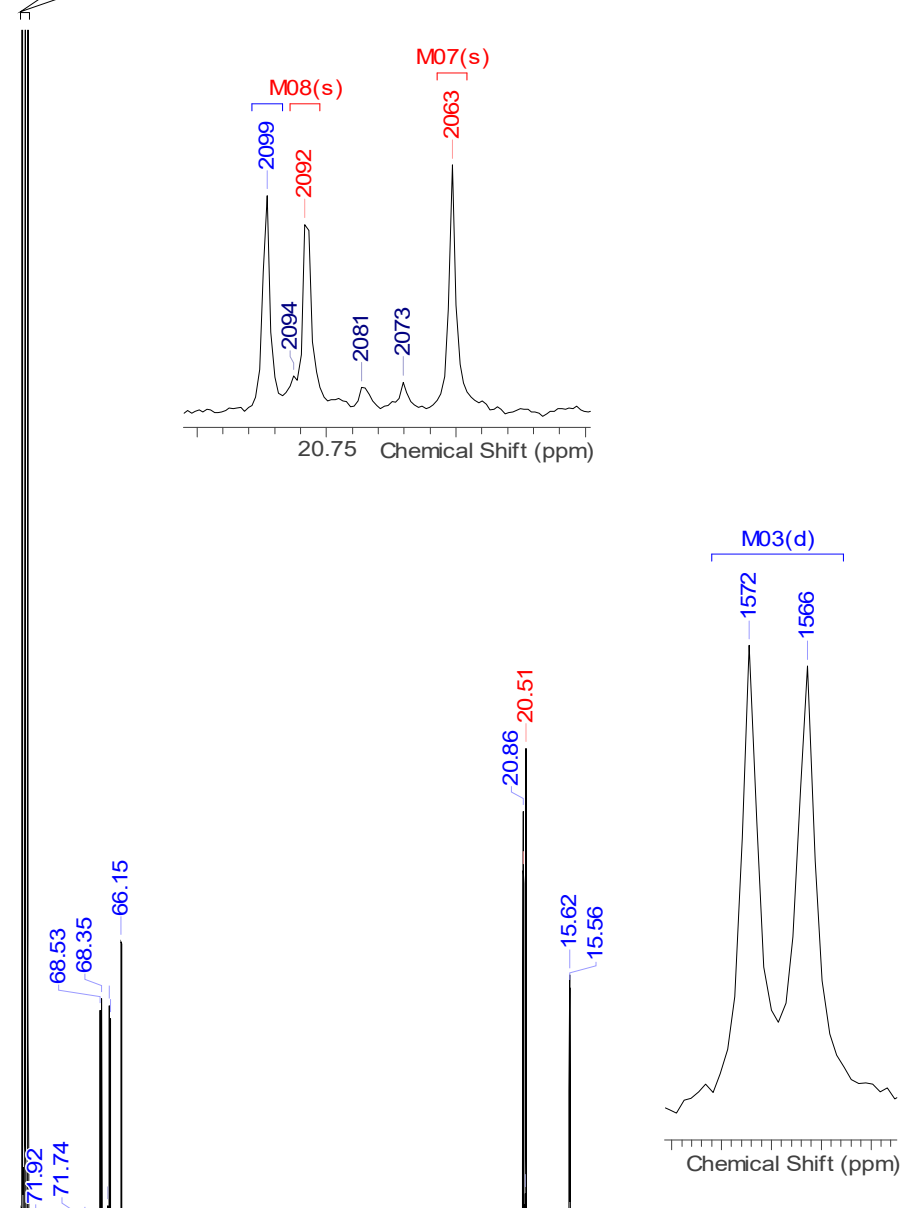

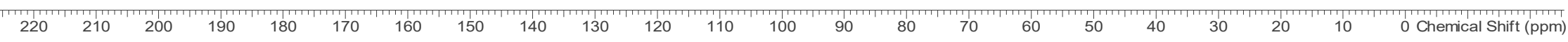


3.4.3. COSY \& HSQC $\left(\mathrm{CDCl}_{3}\right)$

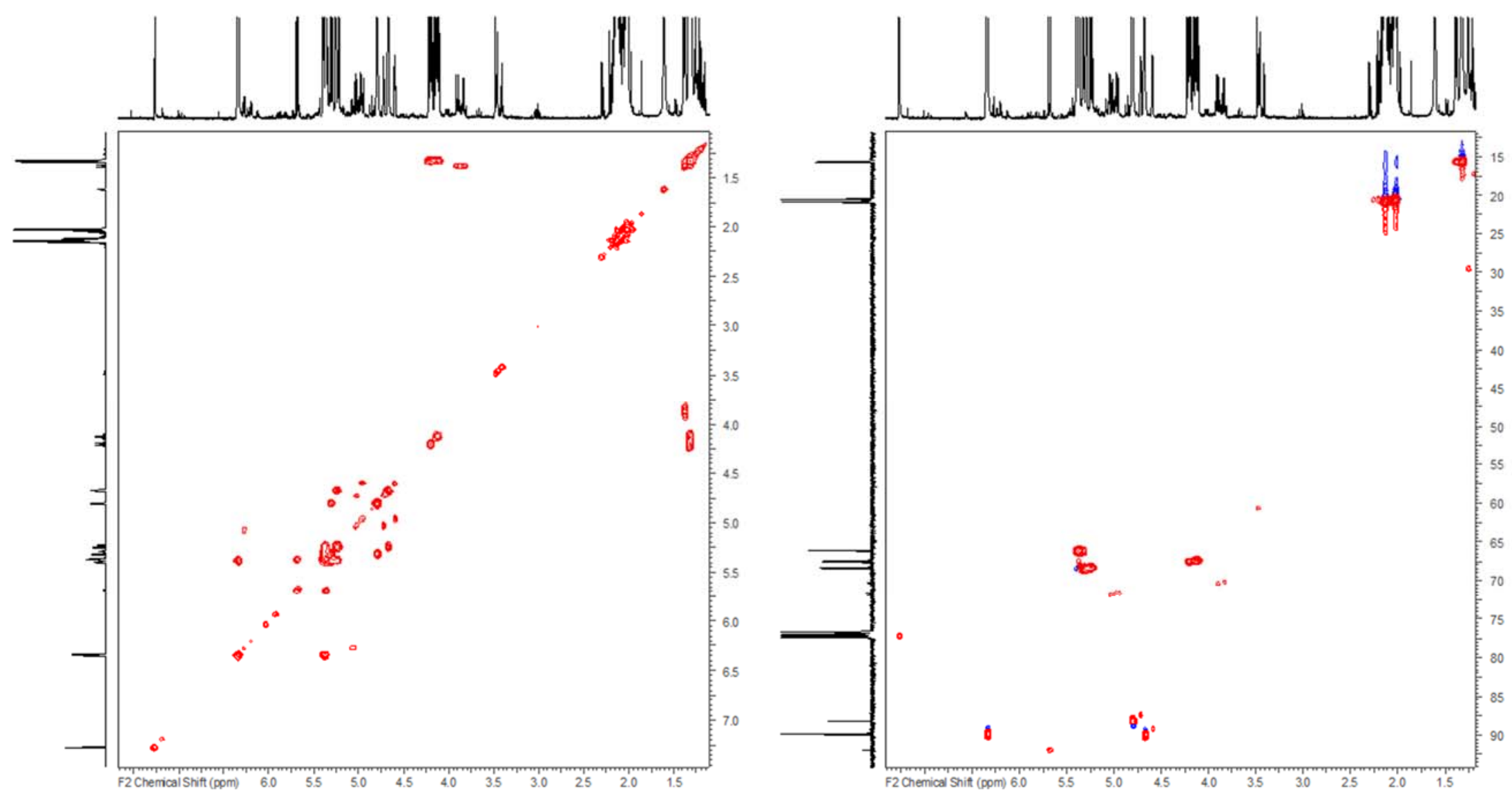


3.4.4. ${ }^{19} \mathrm{~F} \mathrm{NMR}\left(376 \mathrm{MHz}, \mathrm{CDCl}_{3}\right)$
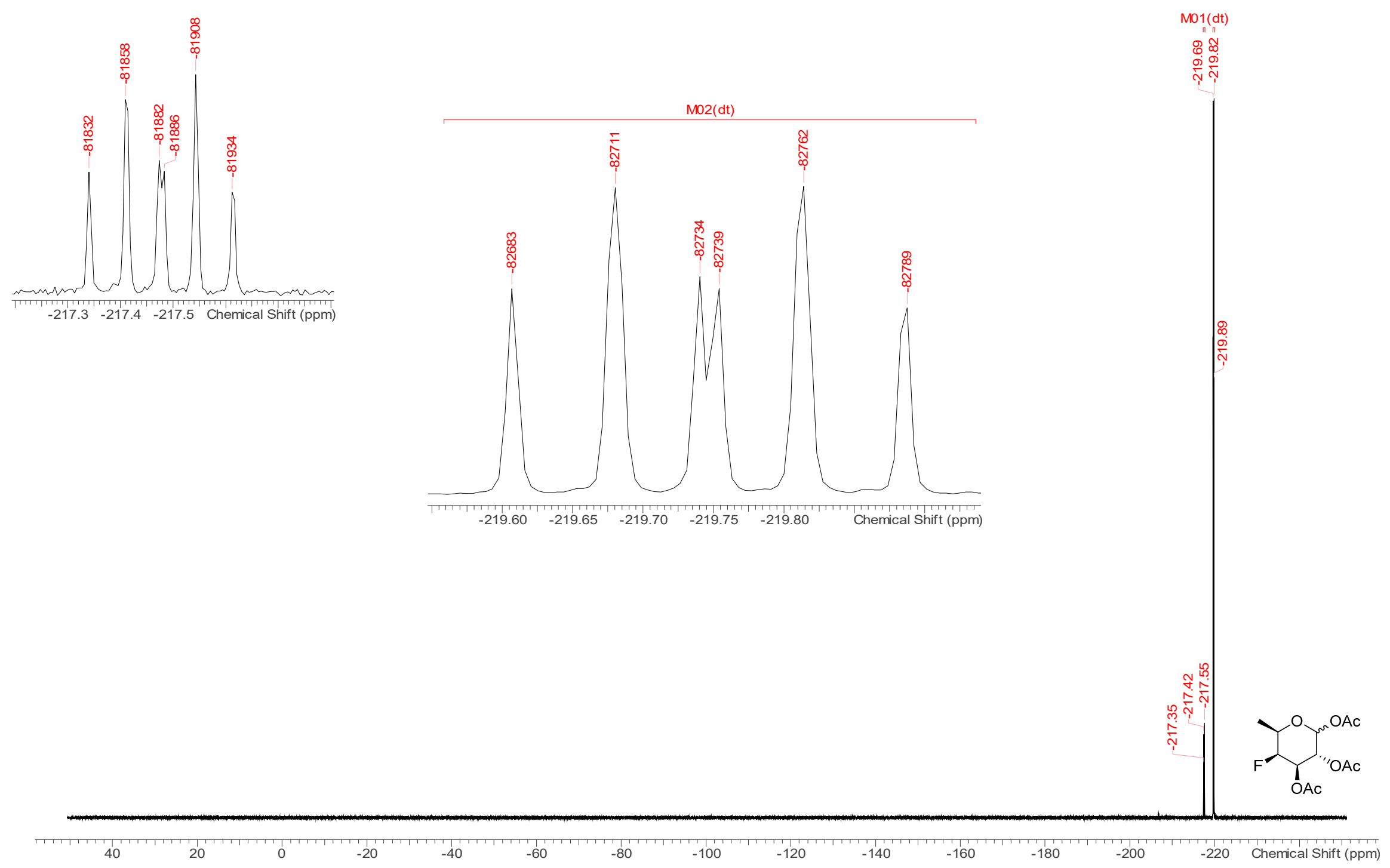
3.4.5. ${ }^{19} \mathrm{~F}\left\{{ }^{1} \mathrm{H}\right\} \mathrm{NMR}\left(376 \mathrm{MHz}, \mathrm{CDCl}_{3}\right)$

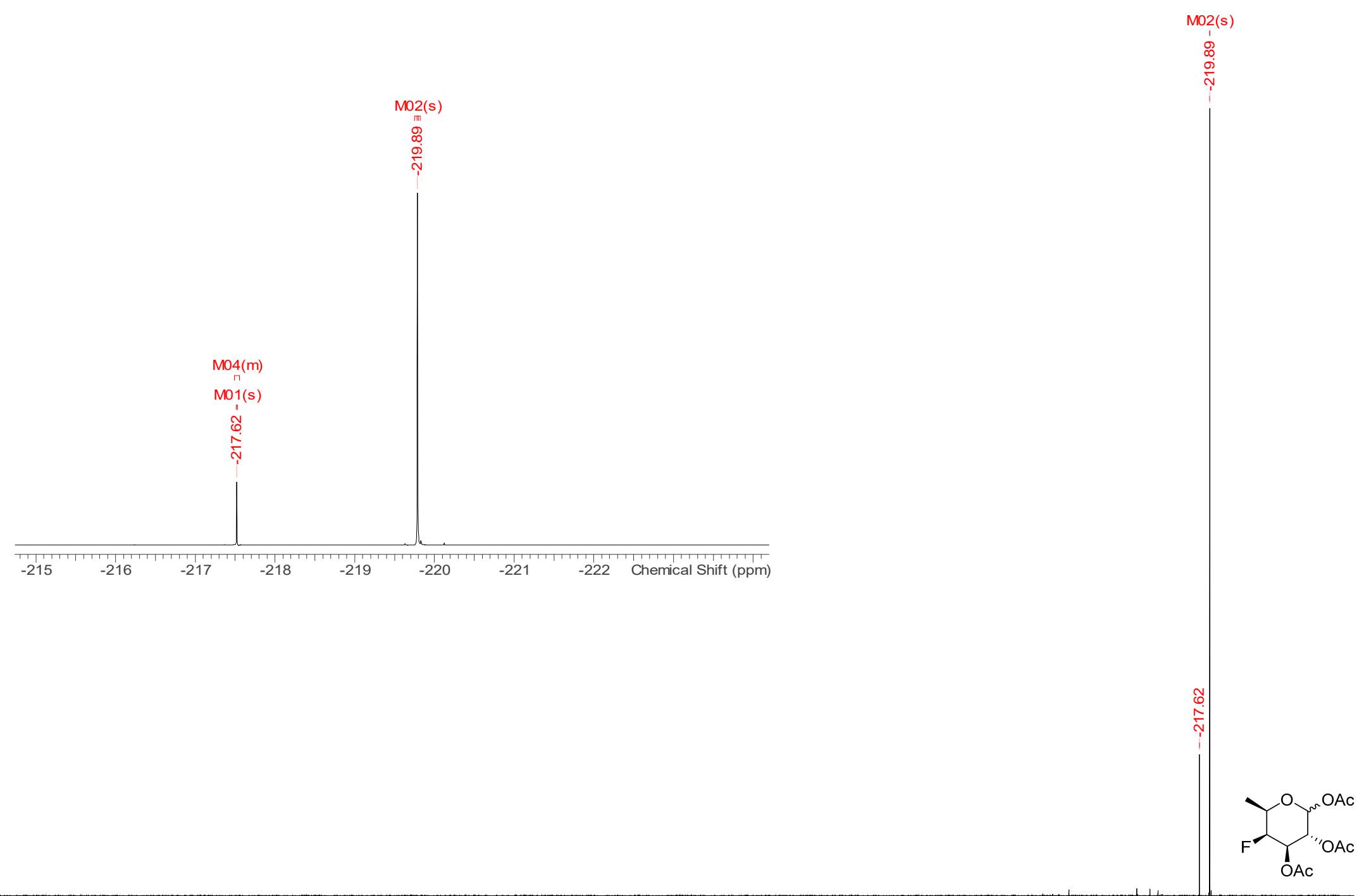




\subsection{4-Deoxy-4-fluoro-D-quinovose (3a)}

3.5.1. ${ }^{1} \mathrm{H}$ NMR $\left(500 \mathrm{MHz}, \mathrm{CD}_{3} \mathrm{OD}\right)$

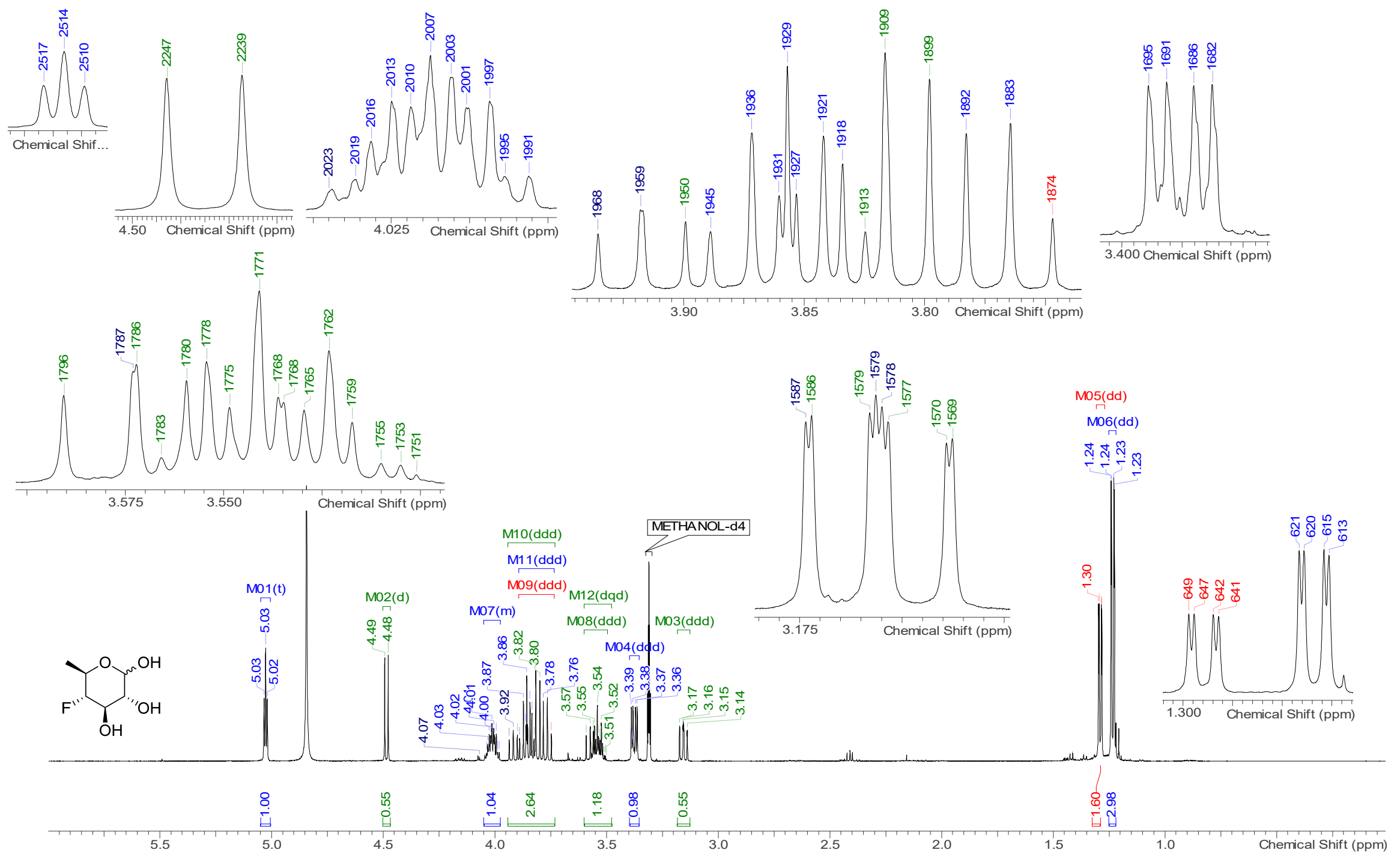




\subsection{2. ${ }^{1} \mathrm{H}\left\{{ }^{19} \mathrm{~F}\right\} \mathrm{NMR}\left(500 \mathrm{MHz}, \mathrm{CD}_{3} \mathrm{OD}\right)$}

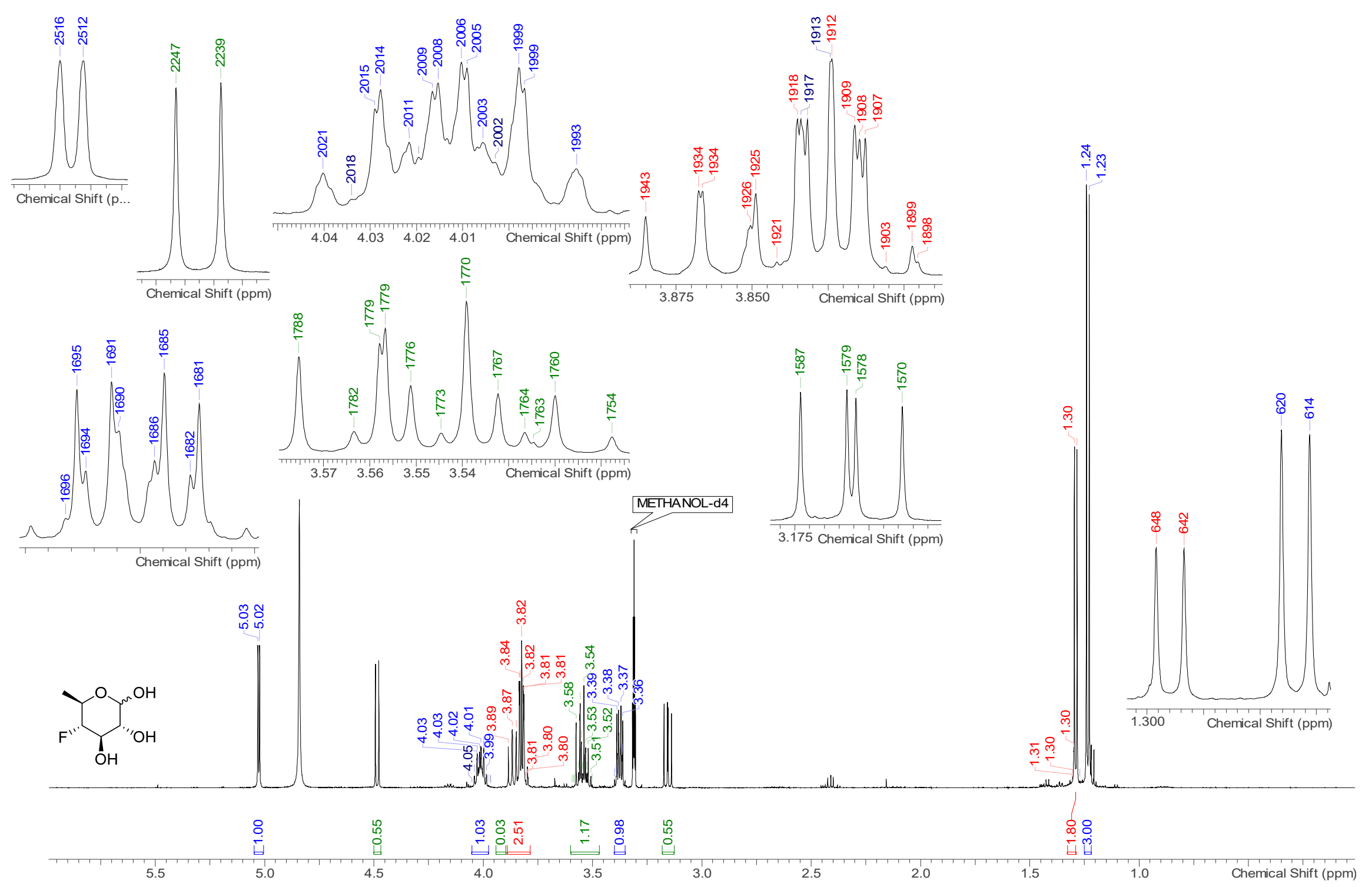


3.5.3. ${ }^{13} \mathrm{C}\left\{{ }^{1} \mathrm{H}\right\} \mathrm{NMR}\left(126 \mathrm{MHz}, \mathrm{CD}_{3} \mathrm{OD}\right)$
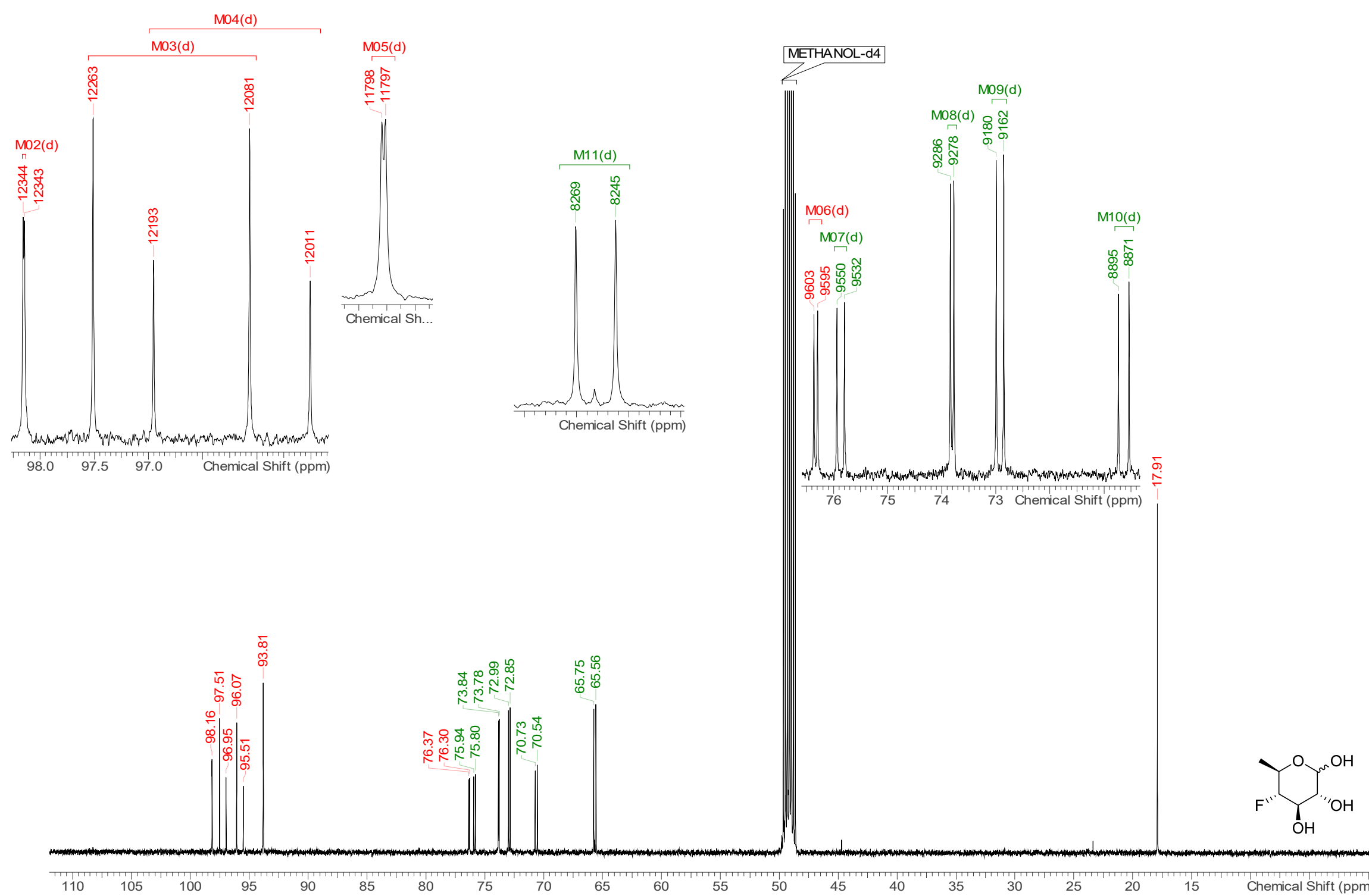
3.5.4. COSY, HSQC-DEPT \& $\mathrm{HMBC}\left(\mathrm{CD}_{3} \mathrm{OD}\right)$

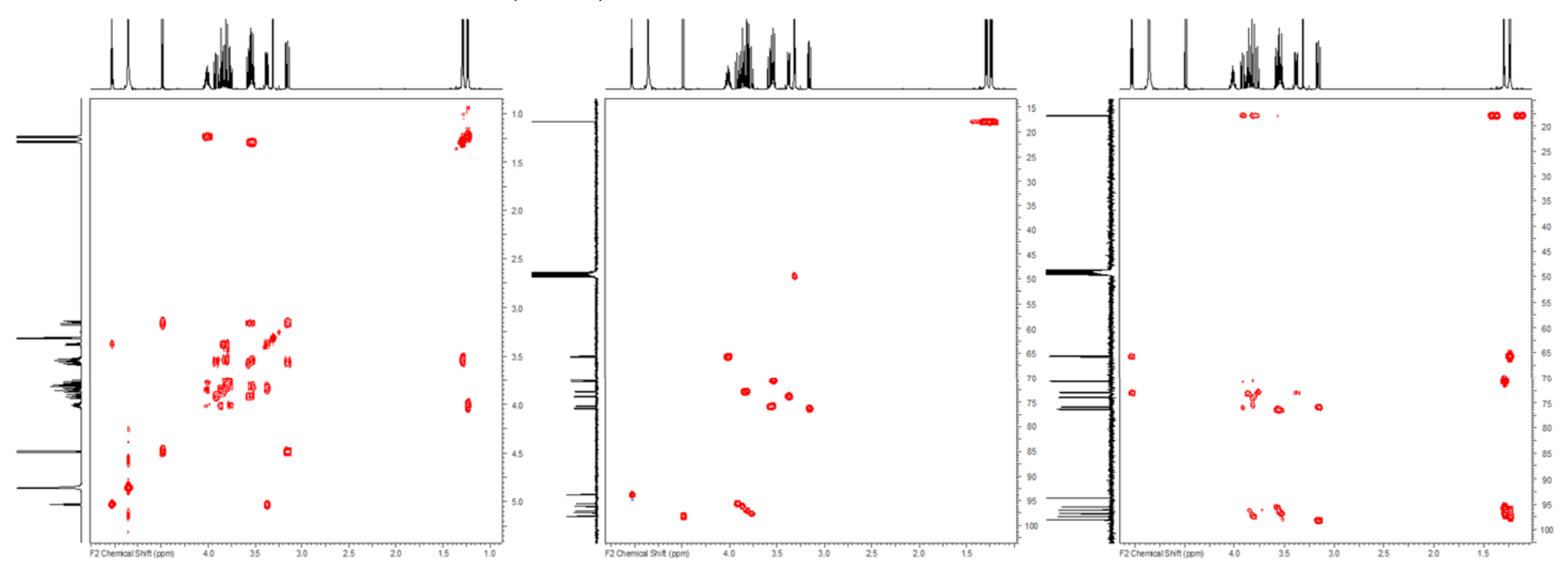


3.5.5. ${ }^{19} \mathrm{~F} \mathrm{NMR}\left(470 \mathrm{MHz}, \mathrm{CD}_{3} \mathrm{OD}\right)$

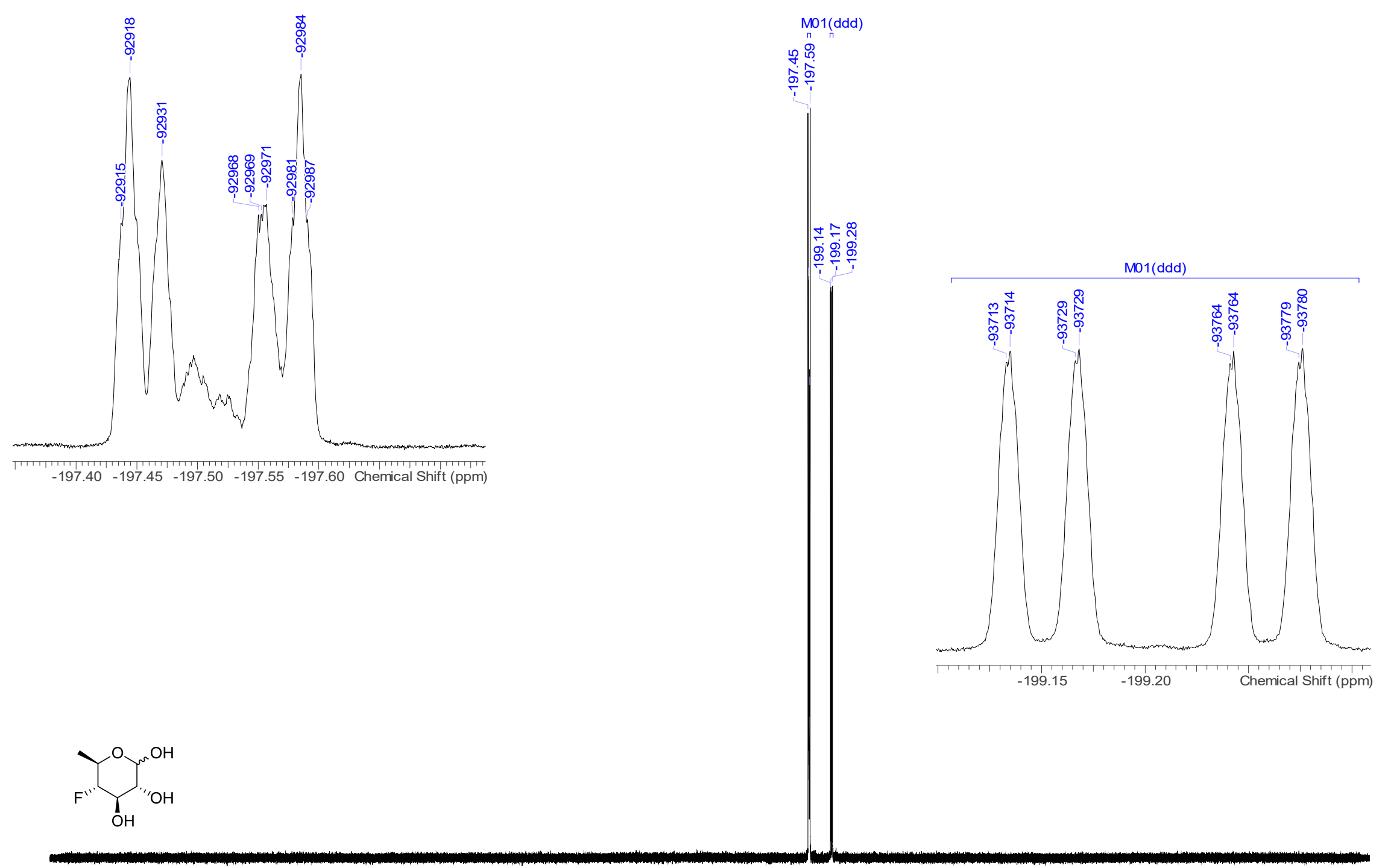

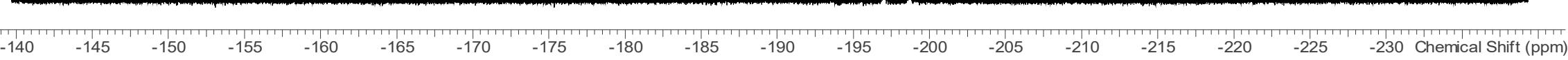


3.5.6. ${ }^{19} \mathrm{~F}\left\{{ }^{1} \mathrm{H}\right\} \mathrm{NMR}\left(470 \mathrm{MHz}, \mathrm{CD}_{3} \mathrm{OD}\right)$

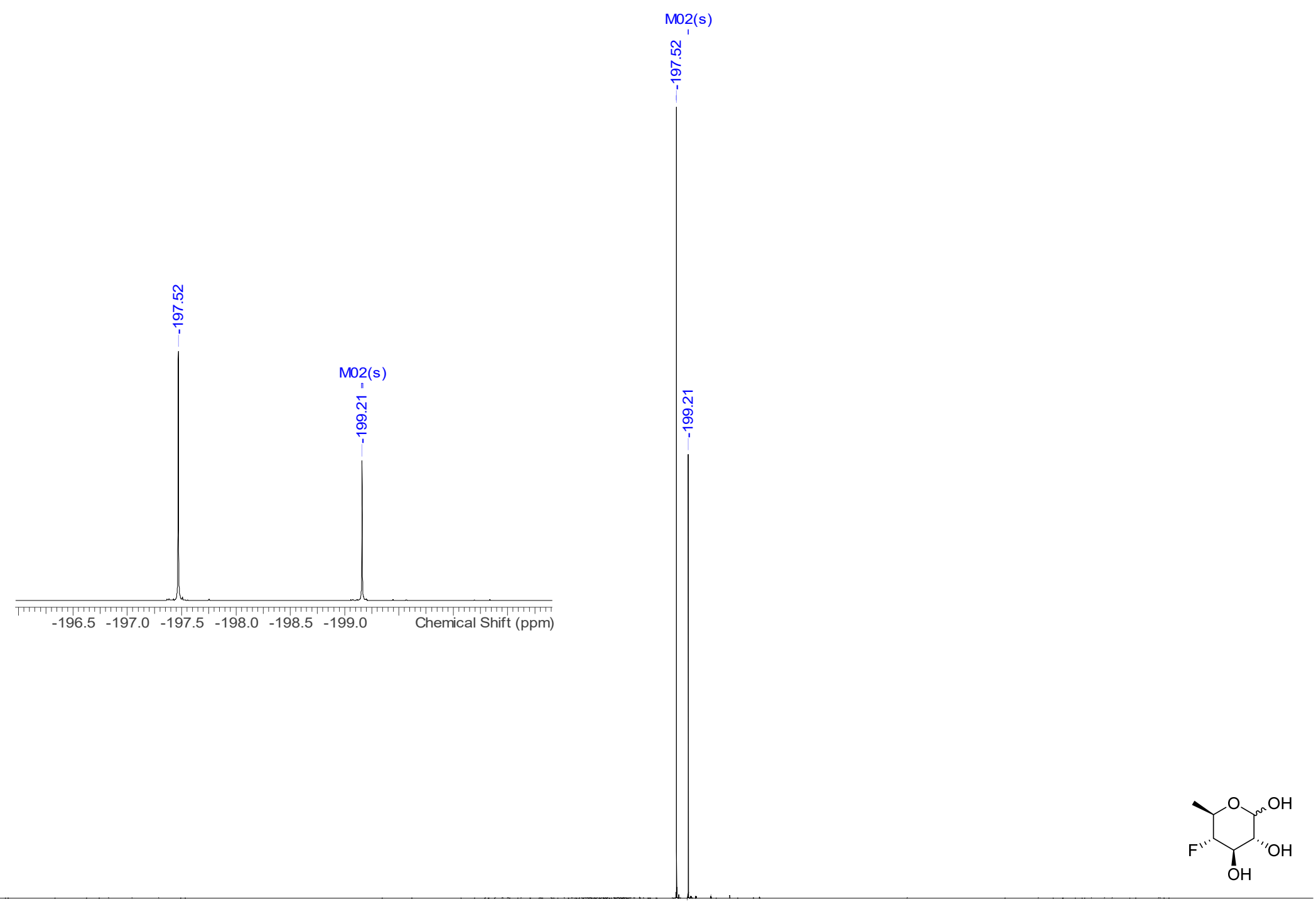

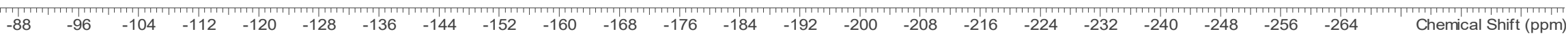




\subsection{1,2,3-Tri-O-acetyl-4-deoxy-4-fluoro-D-quinovopyranoside (3b)}

3.6.1. ${ }^{1} \mathrm{H} \mathrm{NMR}\left(400 \mathrm{MHz}, \mathrm{CDCl}_{3}\right)$

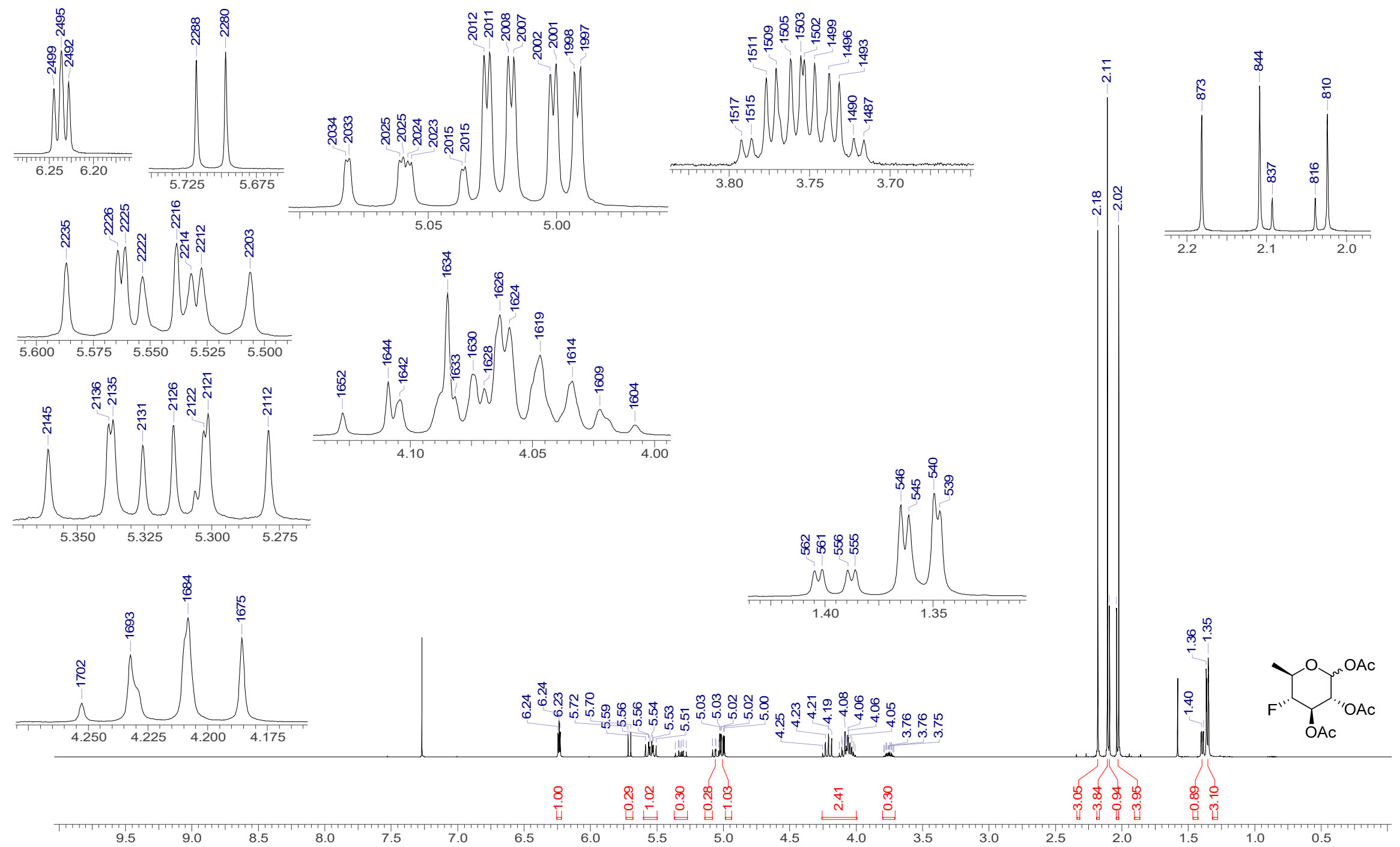


3.6.2. ${ }^{13} \mathrm{C}\left\{{ }^{1} \mathrm{H}\right\} \operatorname{NMR}\left(101 \mathrm{MHz}, \mathrm{CDCl}_{3}\right)$
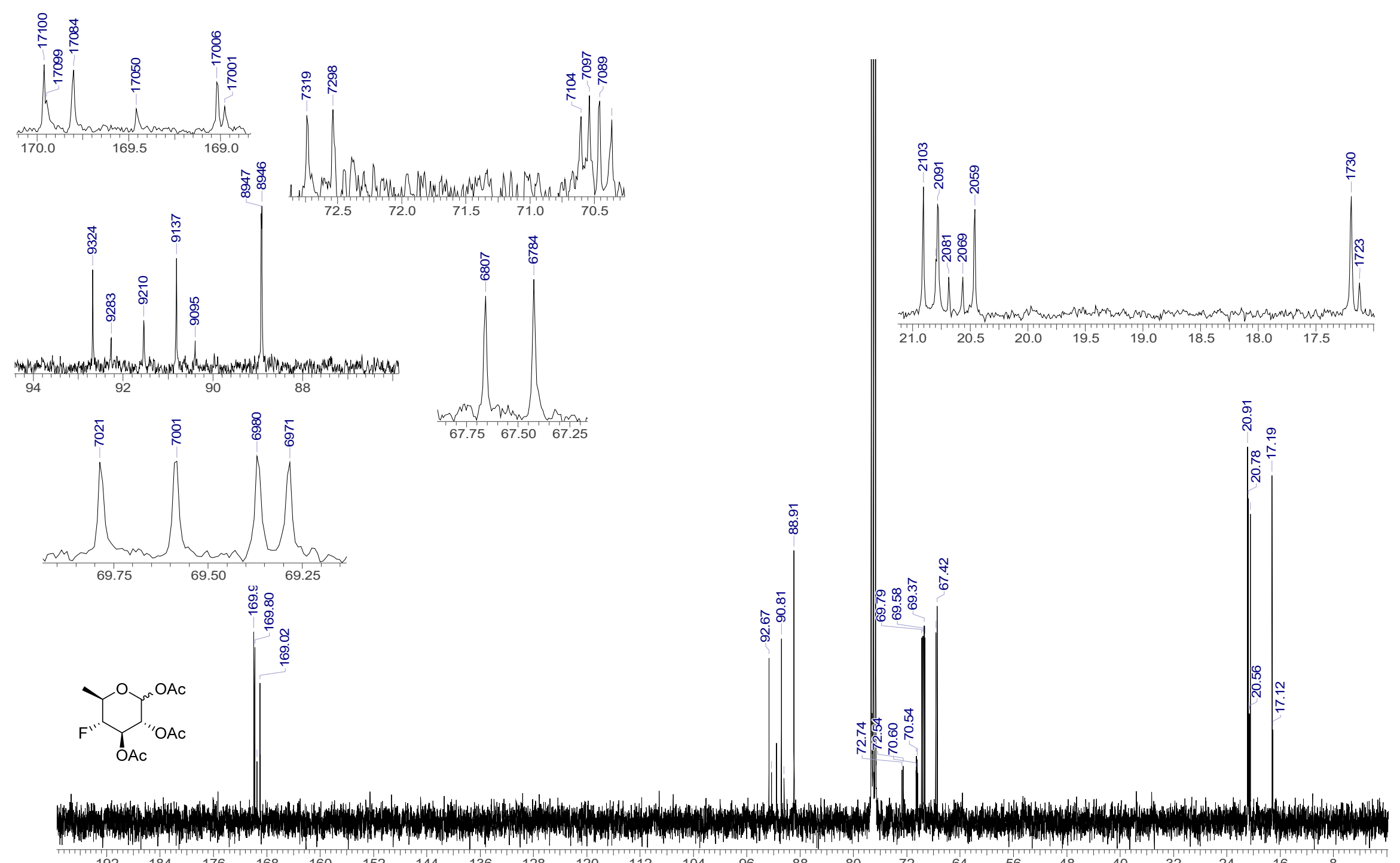
3.6.3. COSY \& HSQC $\left(\mathrm{CDCl}_{3}\right)$

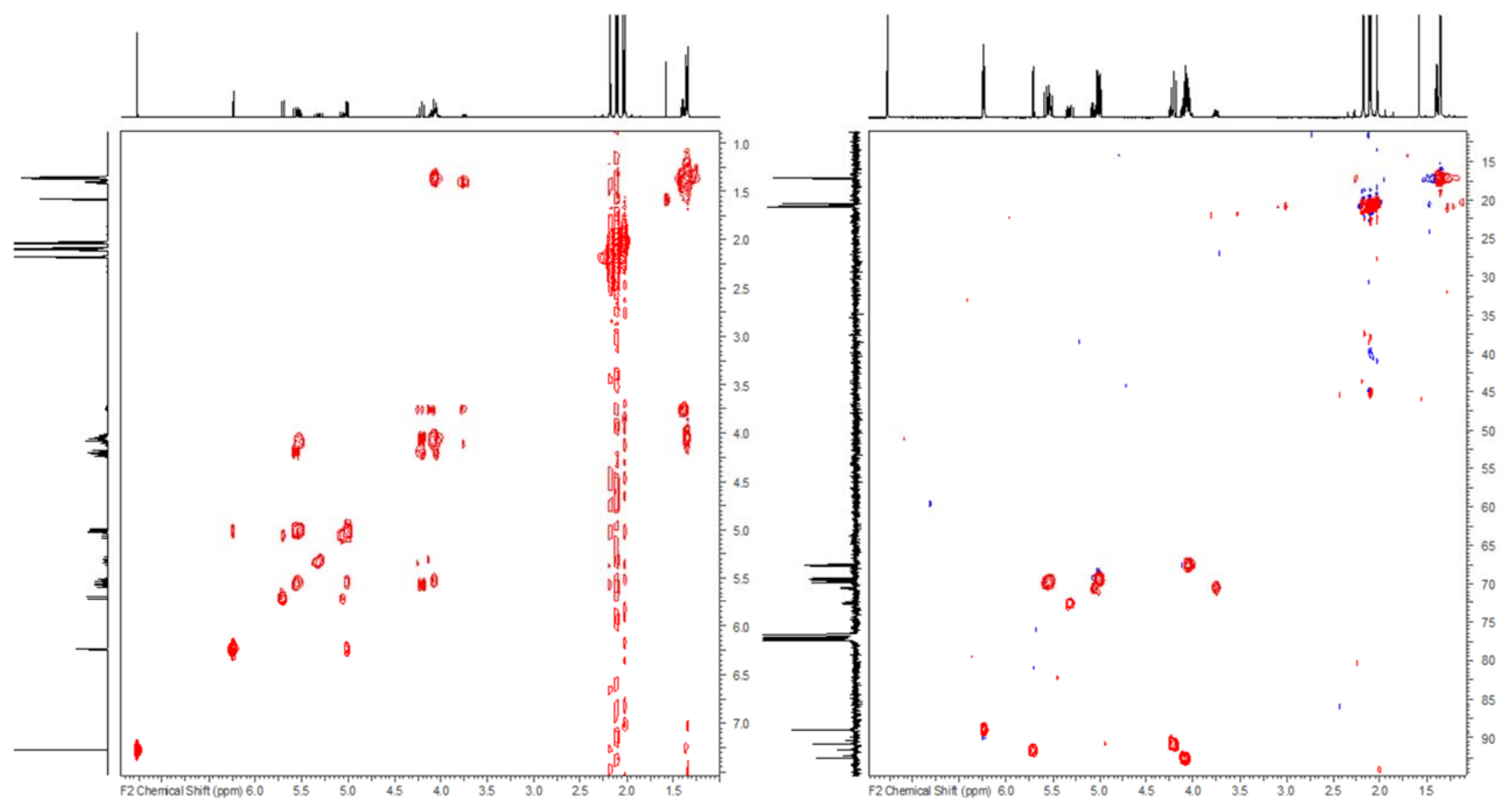


S 49

3.6.4. ${ }^{19} \mathrm{~F} \mathrm{NMR}\left(376 \mathrm{MHz}, \mathrm{CDCl}_{3}\right)$
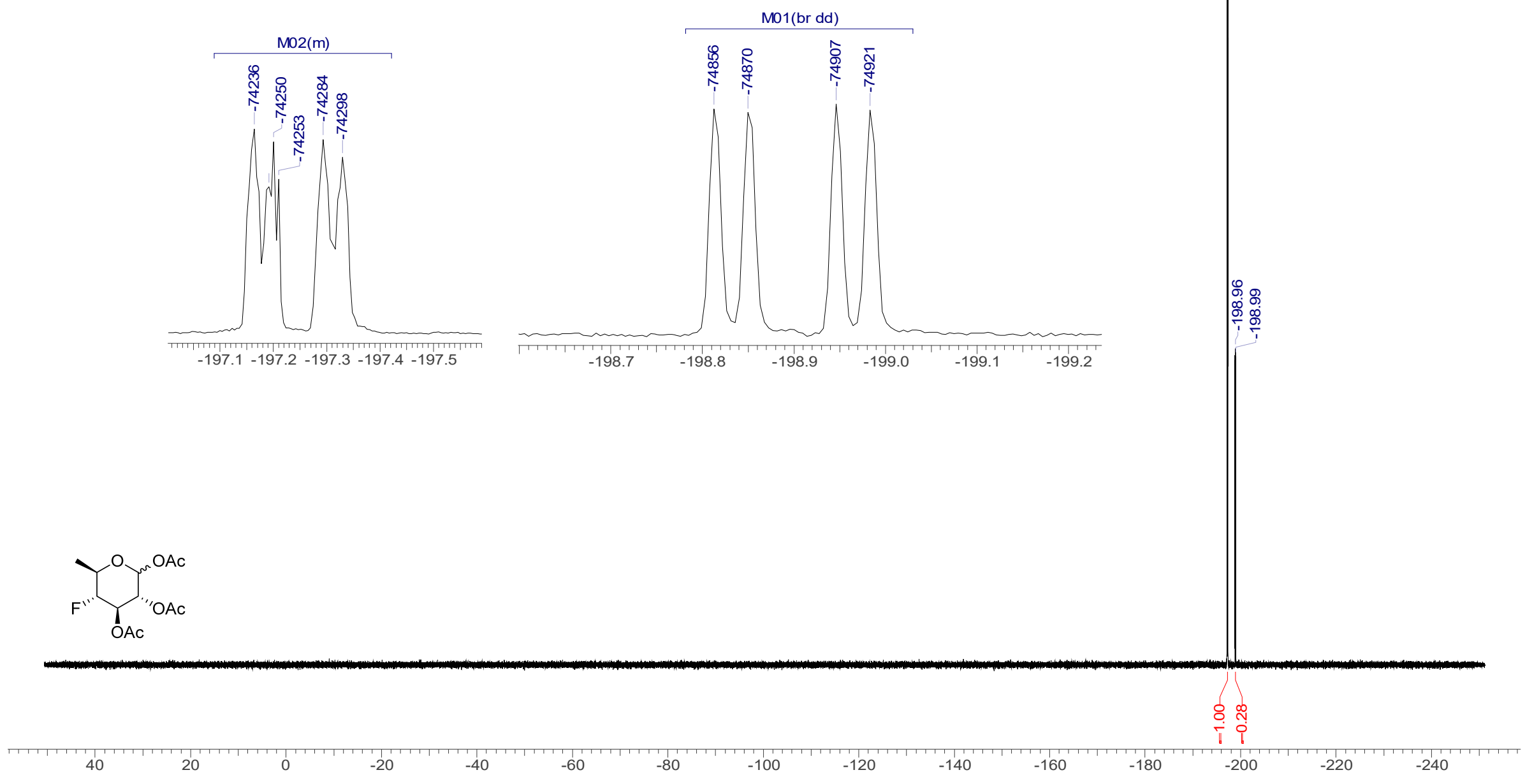
3.6.5. ${ }^{19} \mathrm{~F}\left\{{ }^{1} \mathrm{H}\right\} \mathrm{NMR}\left(376 \mathrm{MHz}, \mathrm{CDCl}_{3}\right)$

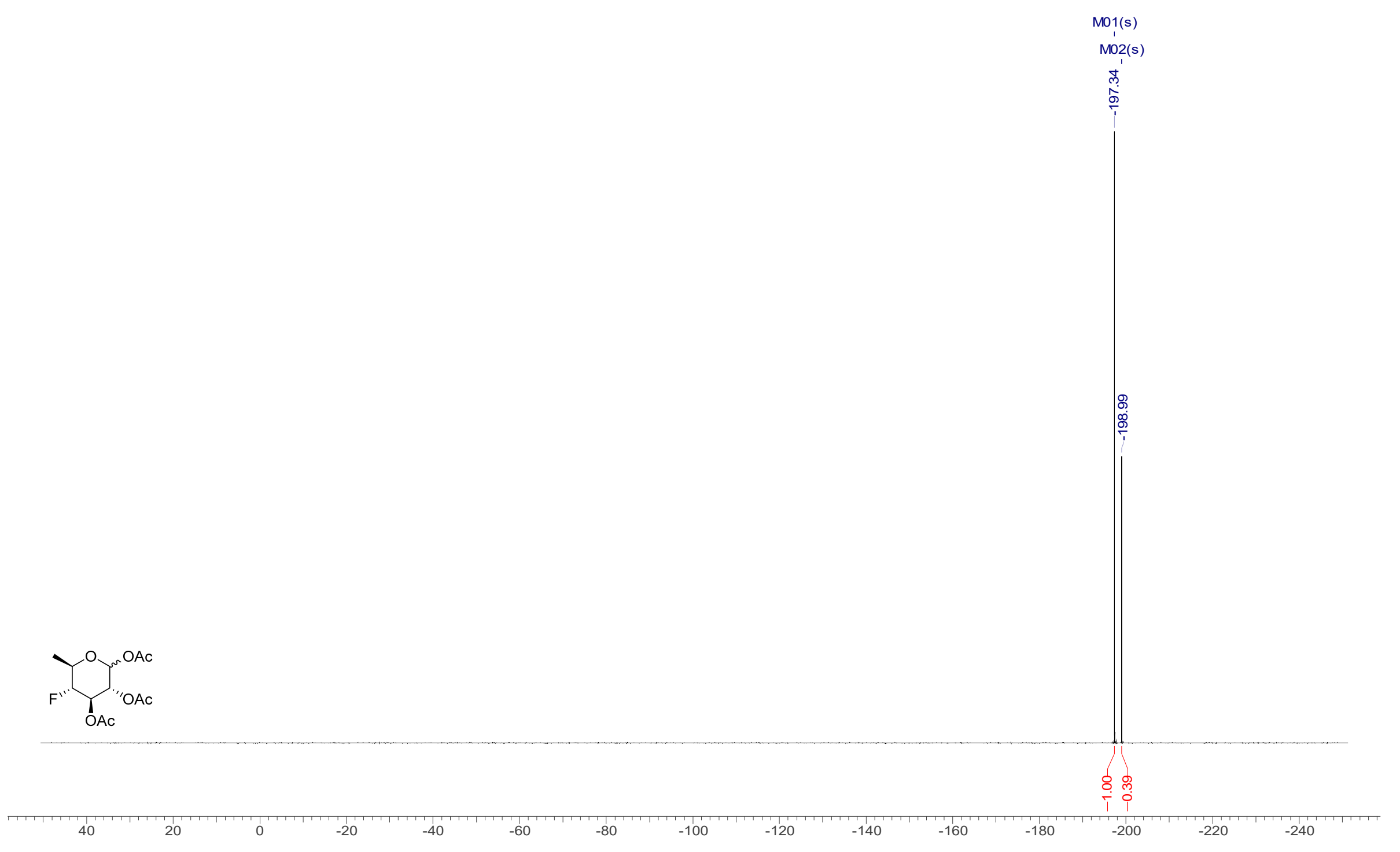




\subsection{4,6-Dideoxy-4,6-difluoro-D-galactose (4a)}

3.7.1. ${ }^{1} \mathrm{H}$ NMR $\left(500 \mathrm{MHz}, \mathrm{CD}_{3} \mathrm{OD}\right)$
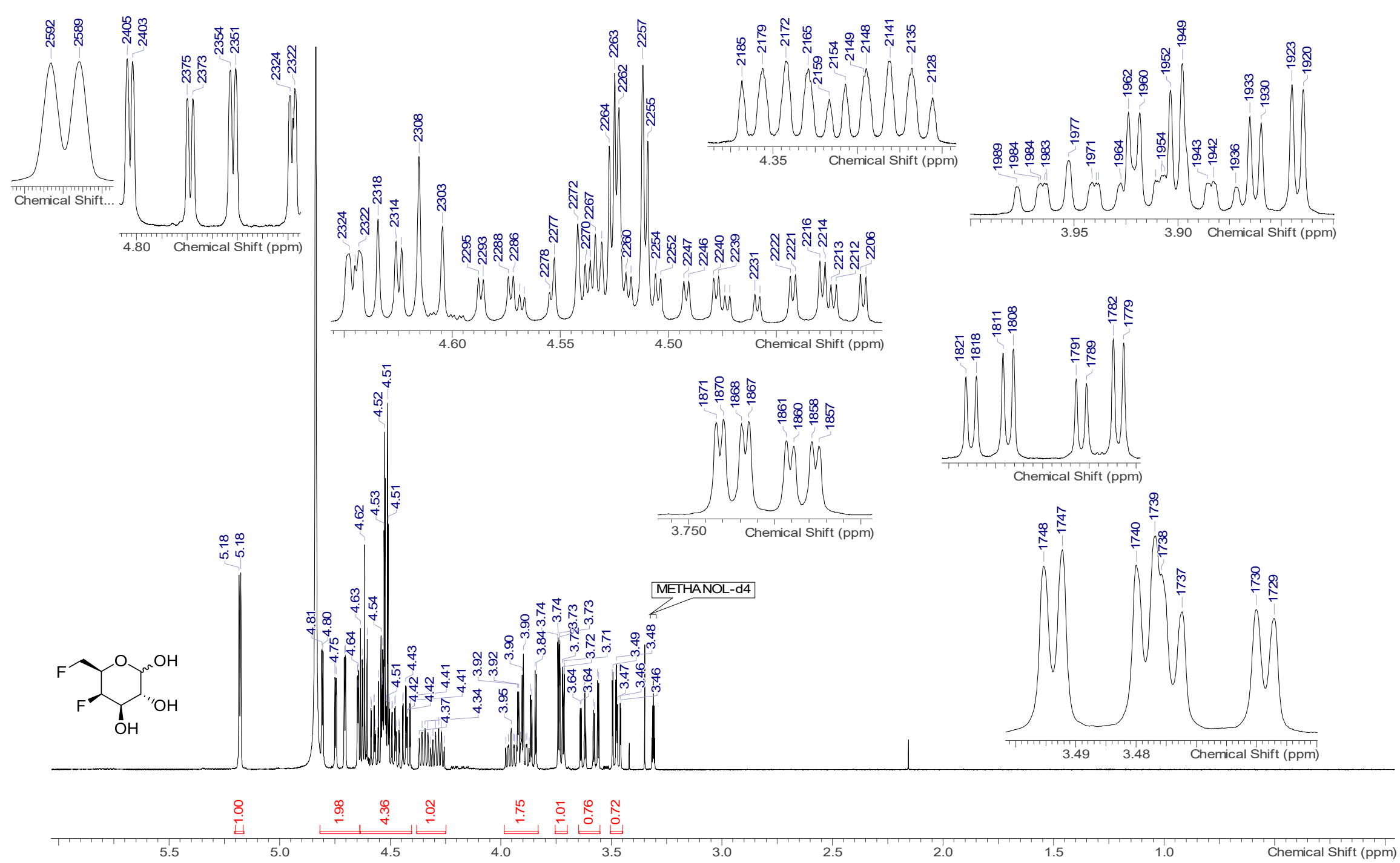
3.7.2. ${ }^{1} \mathrm{H}\left\{{ }^{19} \mathrm{~F}\right\} \mathrm{NMR}\left(500 \mathrm{MHz}, \mathrm{CD}_{3} \mathrm{OD}\right)$

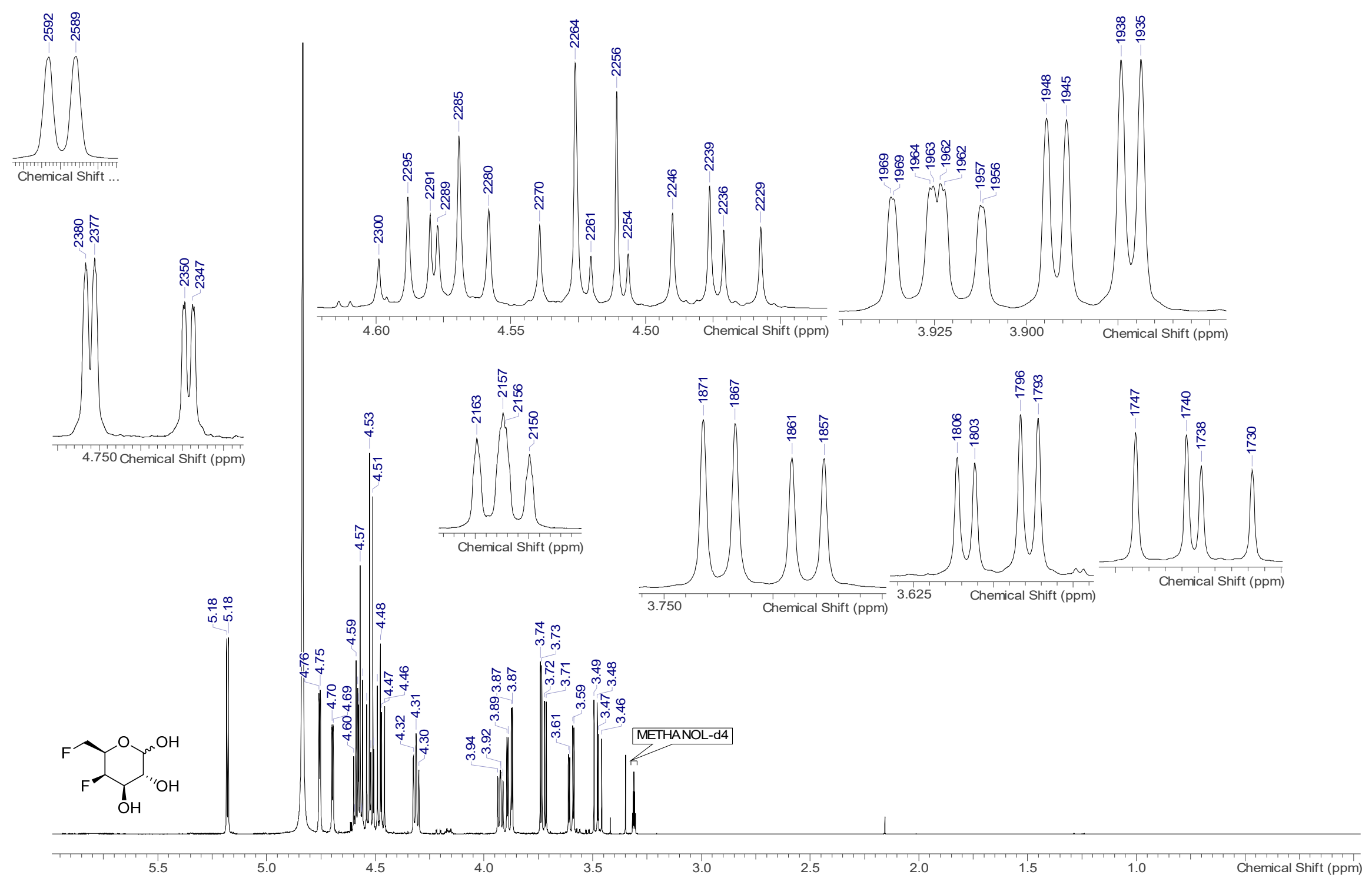




\subsection{3. ${ }^{13} \mathrm{C}\left\{{ }^{1} \mathrm{H}\right\} \mathrm{NMR}\left(101 \mathrm{MHz}, \mathrm{CD}_{3} \mathrm{OD}\right)$}
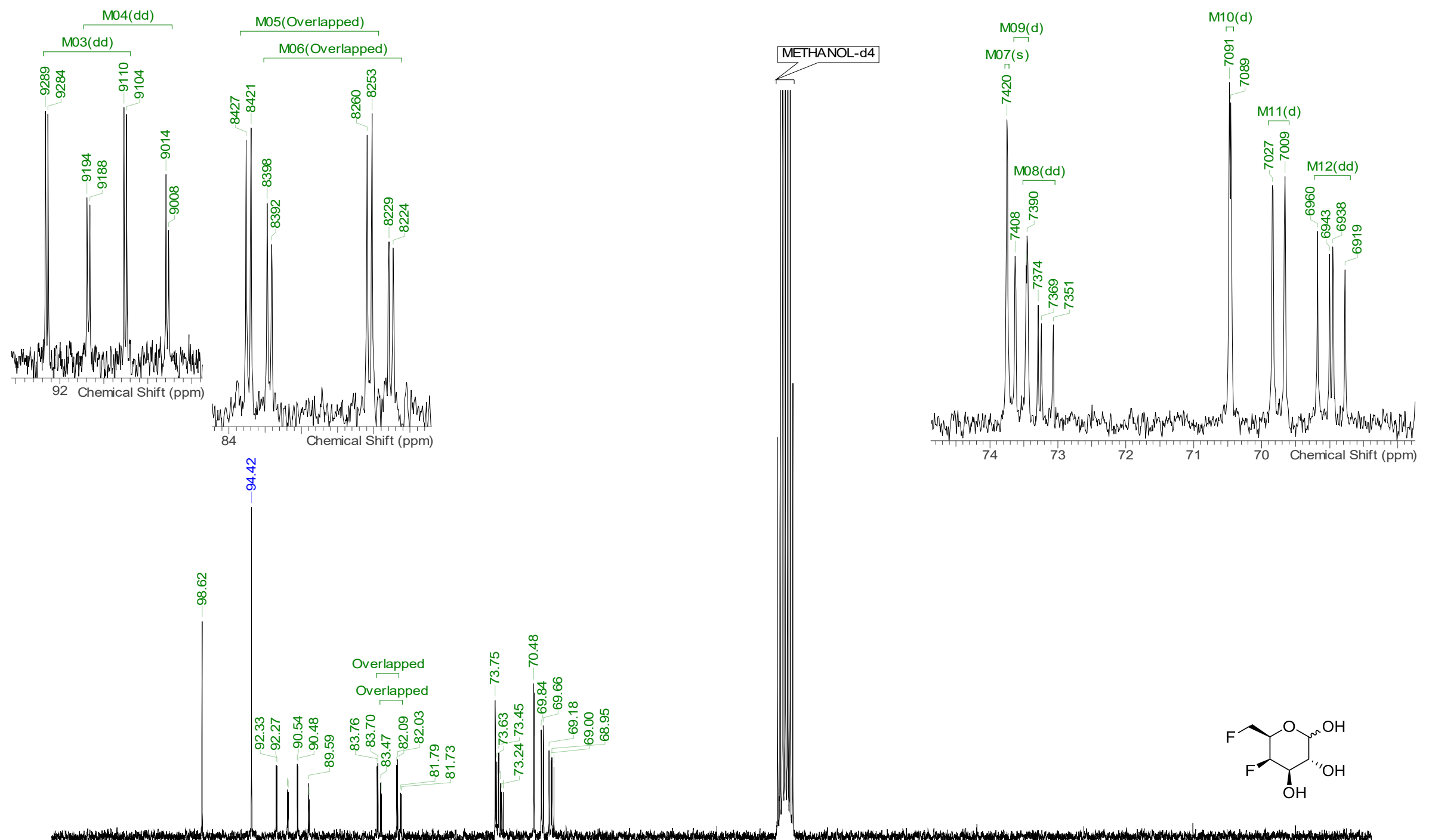

mits

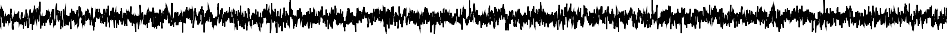

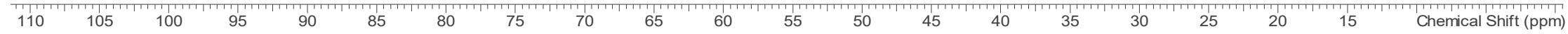


3.7.4. COSY \& HSQC-DEPT $\left(\mathrm{CD}_{3} \mathrm{OD}\right)$

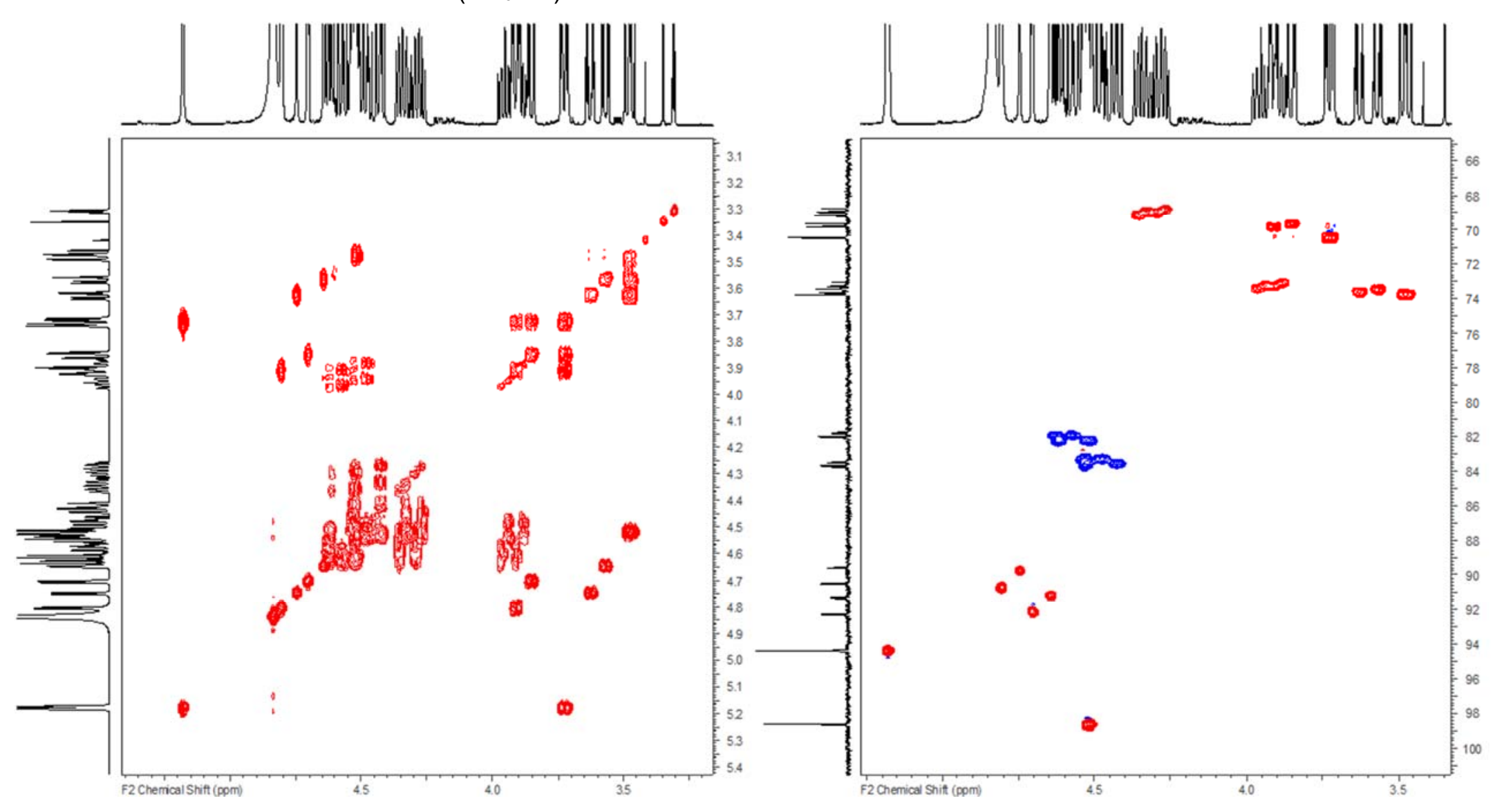


3.7.5. ${ }^{19} \mathrm{~F} \mathrm{NMR}\left(470 \mathrm{MHz}, \mathrm{CD}_{3} \mathrm{OD}\right)$

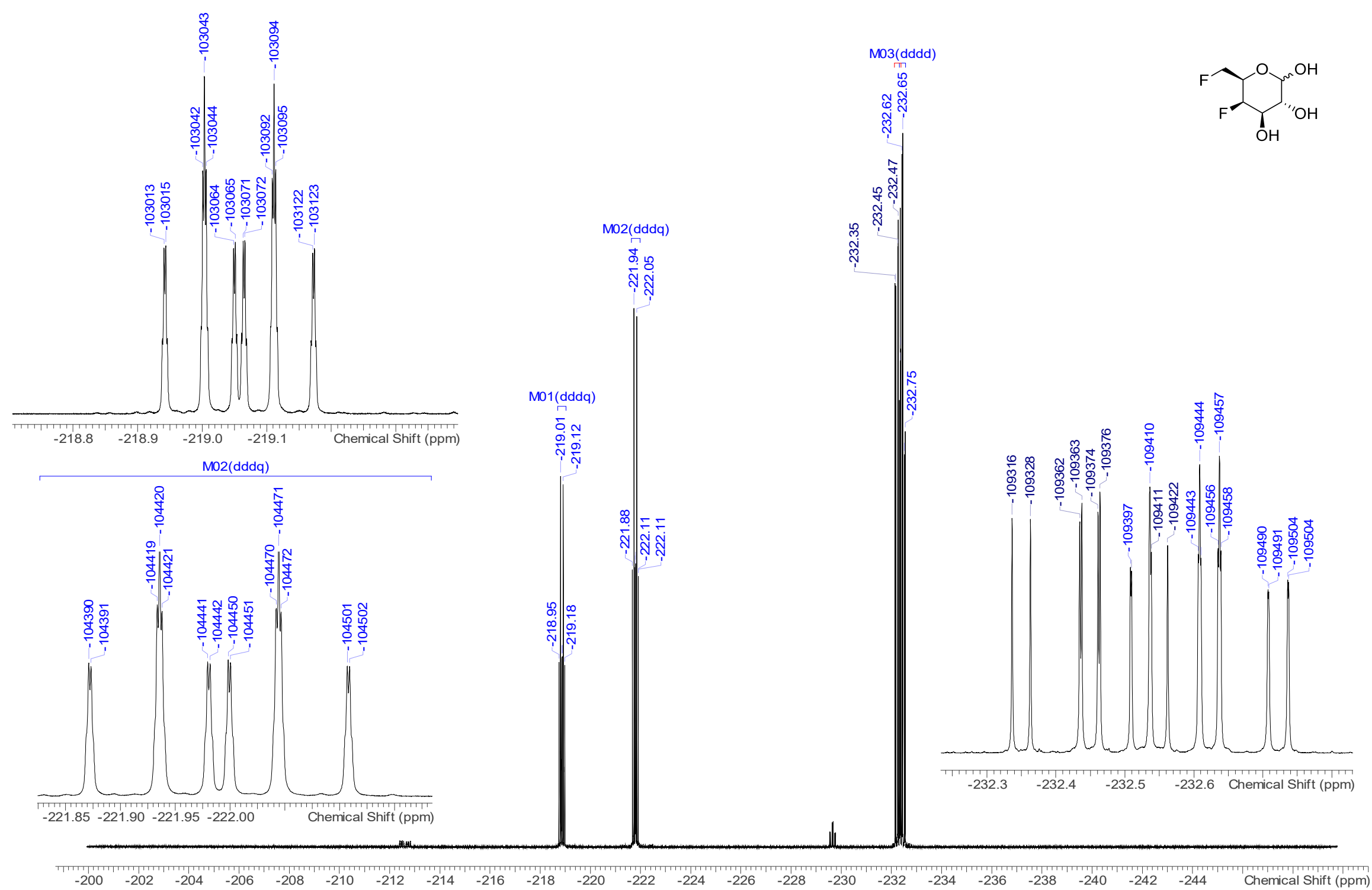


3.7.6. ${ }^{19} \mathrm{~F}\left\{{ }^{1} \mathrm{H}\right\} \mathrm{NMR}\left(470 \mathrm{MHz}, \mathrm{CD}_{3} \mathrm{OD}\right)$

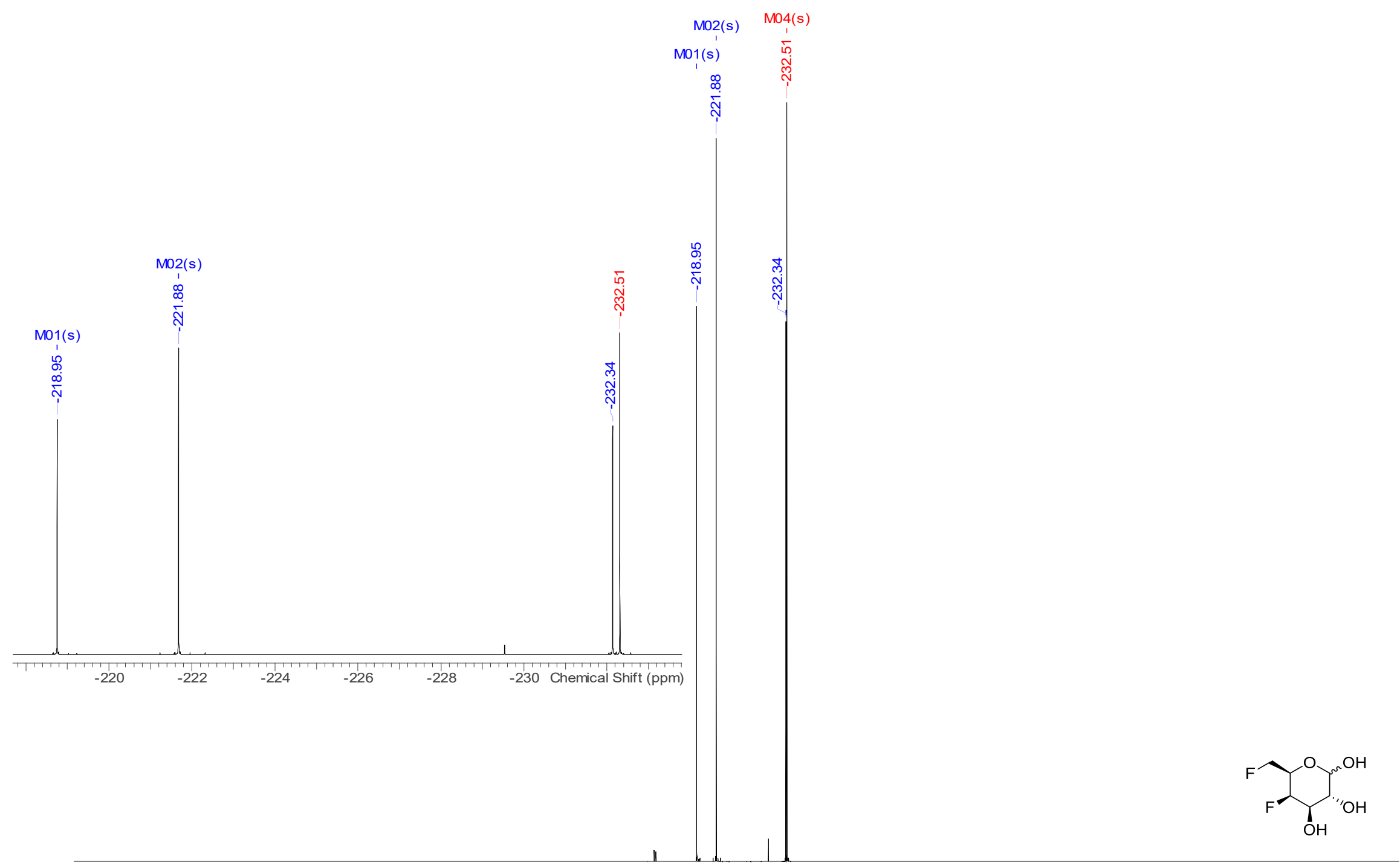

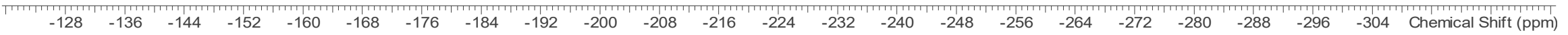




\subsection{1,2,3-Tri-O-acetyl-4,6-dideoxy-4,6-difluoro-D-galactopyranoside (4b)}

3.8.1. ${ }^{1} \mathrm{H} \mathrm{NMR}\left(400 \mathrm{MHz}, \mathrm{CDCl}_{3}\right)$

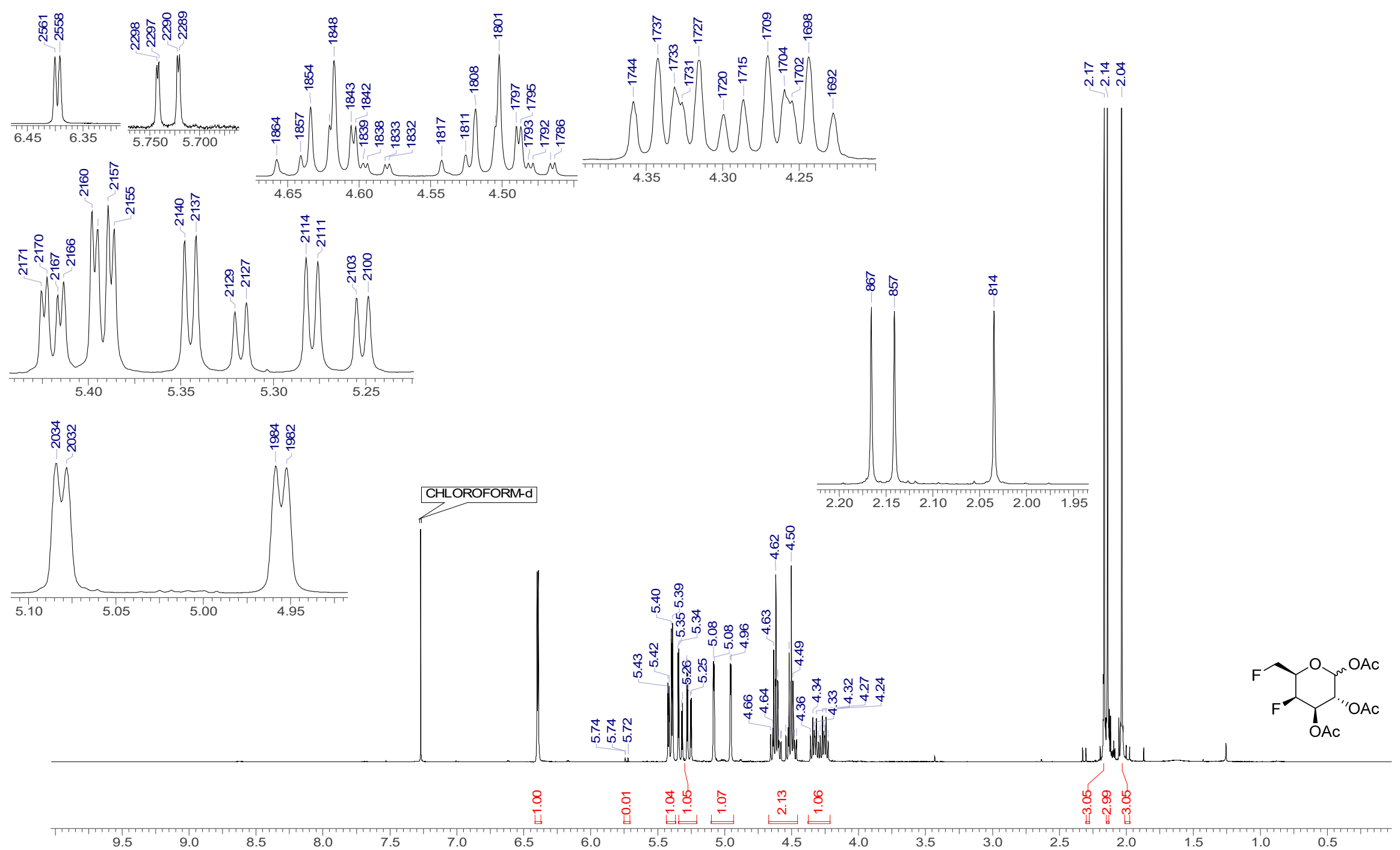


3.8.2. ${ }^{13} \mathrm{C}\left\{{ }^{1} \mathrm{H}\right\} \operatorname{NMR}\left(101 \mathrm{MHz}, \mathrm{CDCl}_{3}\right)$
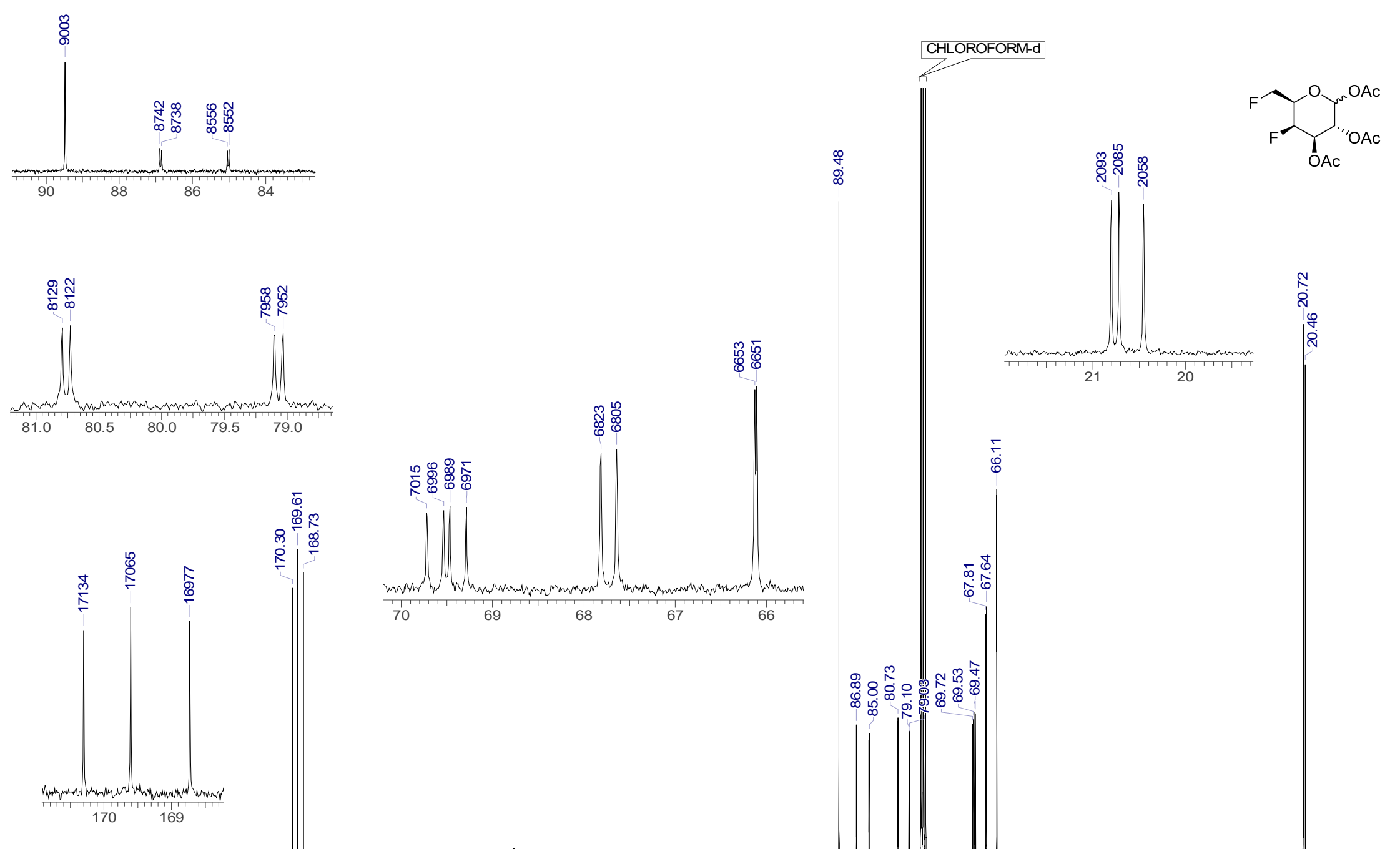

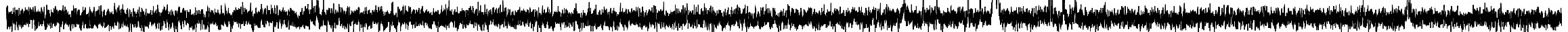

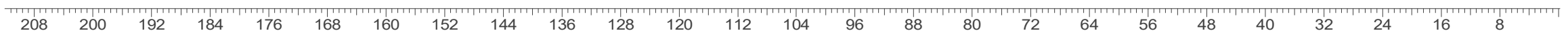


3.8.3. COSY, HSQC-DEPT \& $\mathrm{HMBC}\left(\mathrm{CDCl}_{3}\right)$

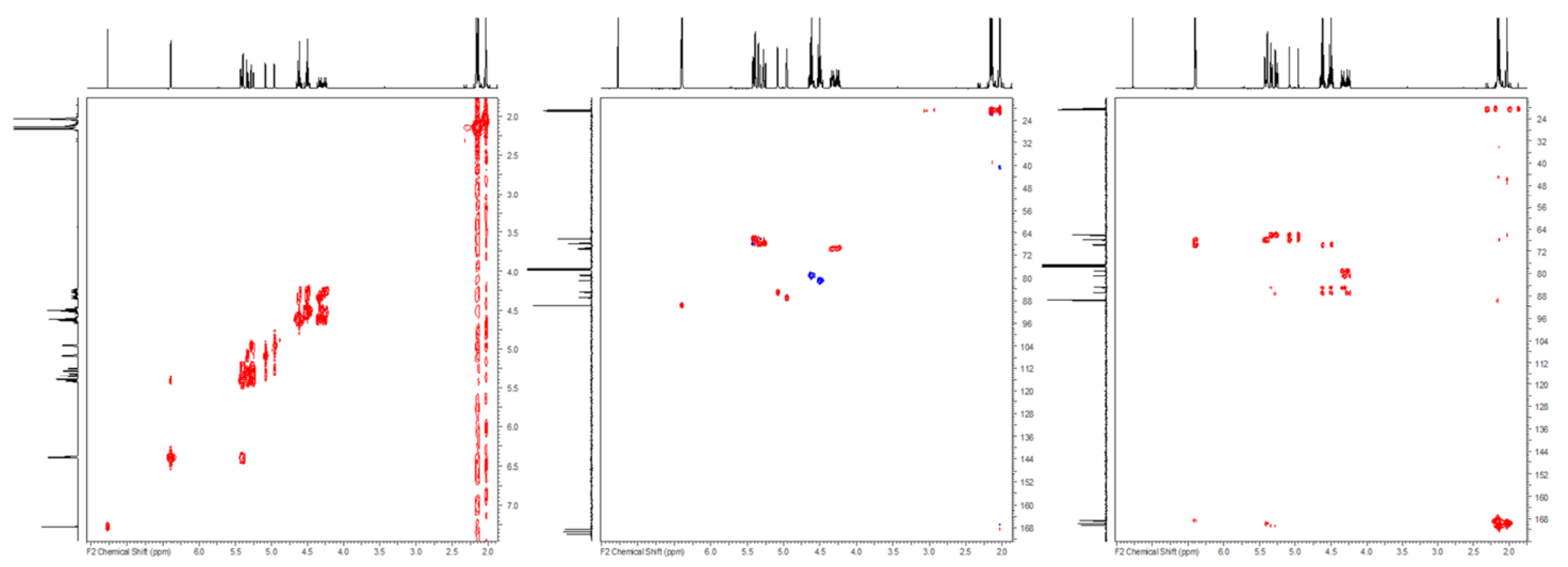


3.8.4. ${ }^{19} \mathrm{~F} \mathrm{NMR}\left(376 \mathrm{MHz}, \mathrm{CDCl}_{3}\right)$

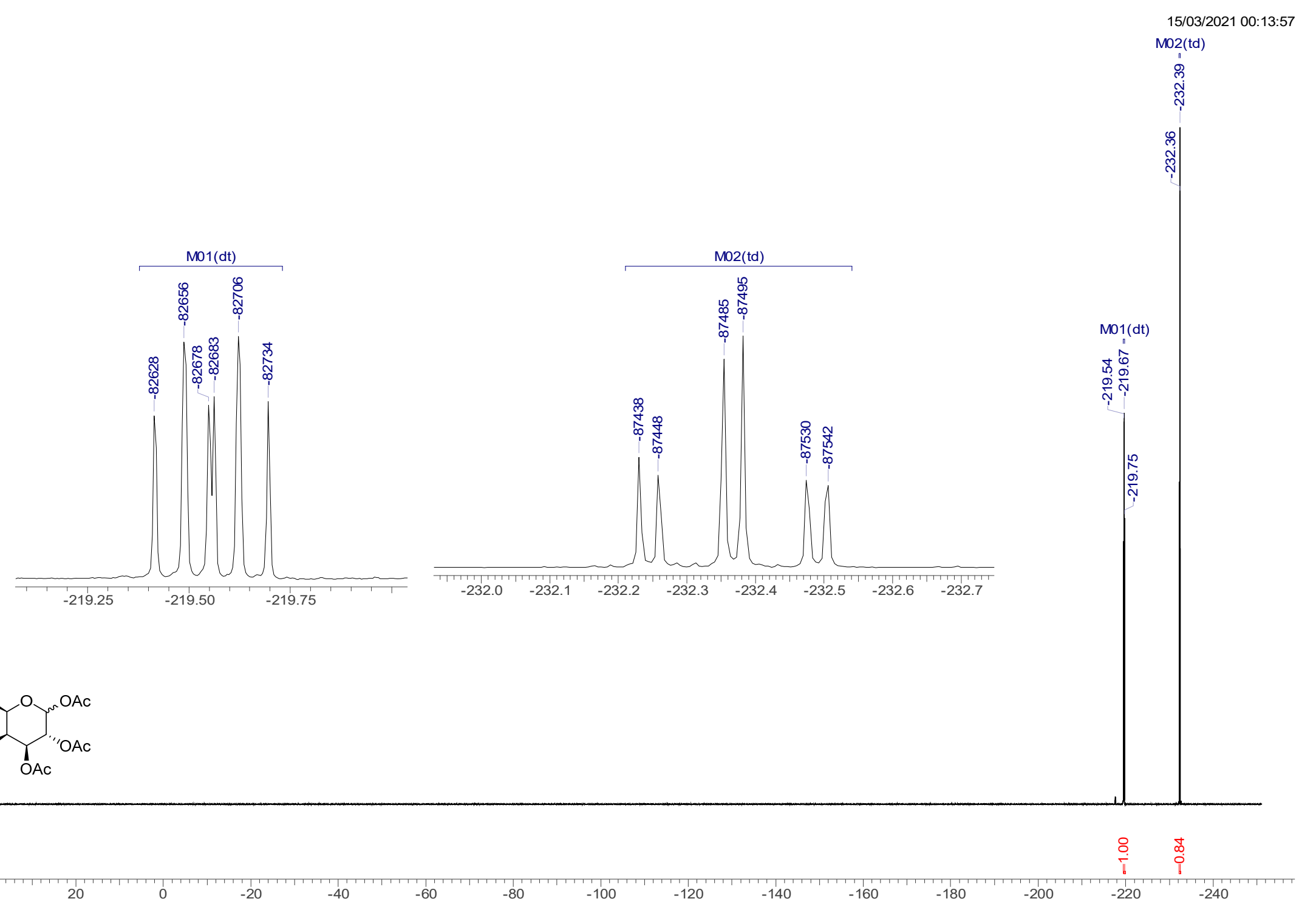


3.8.5. ${ }^{19} \mathrm{~F}\left\{{ }^{1} \mathrm{H}\right\} \mathrm{NMR}\left(376 \mathrm{MHz}, \mathrm{CDCl}_{3}\right)$

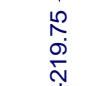

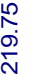

$\mathrm{MO2}$ (s)

ָָ ָิ

$\underbrace{O C_{1 \prime O A C} O A C}_{O A c}$ 


\subsection{4,6-Dideoxy-4,6-difluoro-D-glucose (5a)}

3.9.1. ${ }^{1} \mathrm{H}$ NMR $\left(500 \mathrm{MHz}, \mathrm{CD}_{3} \mathrm{OD}\right)$

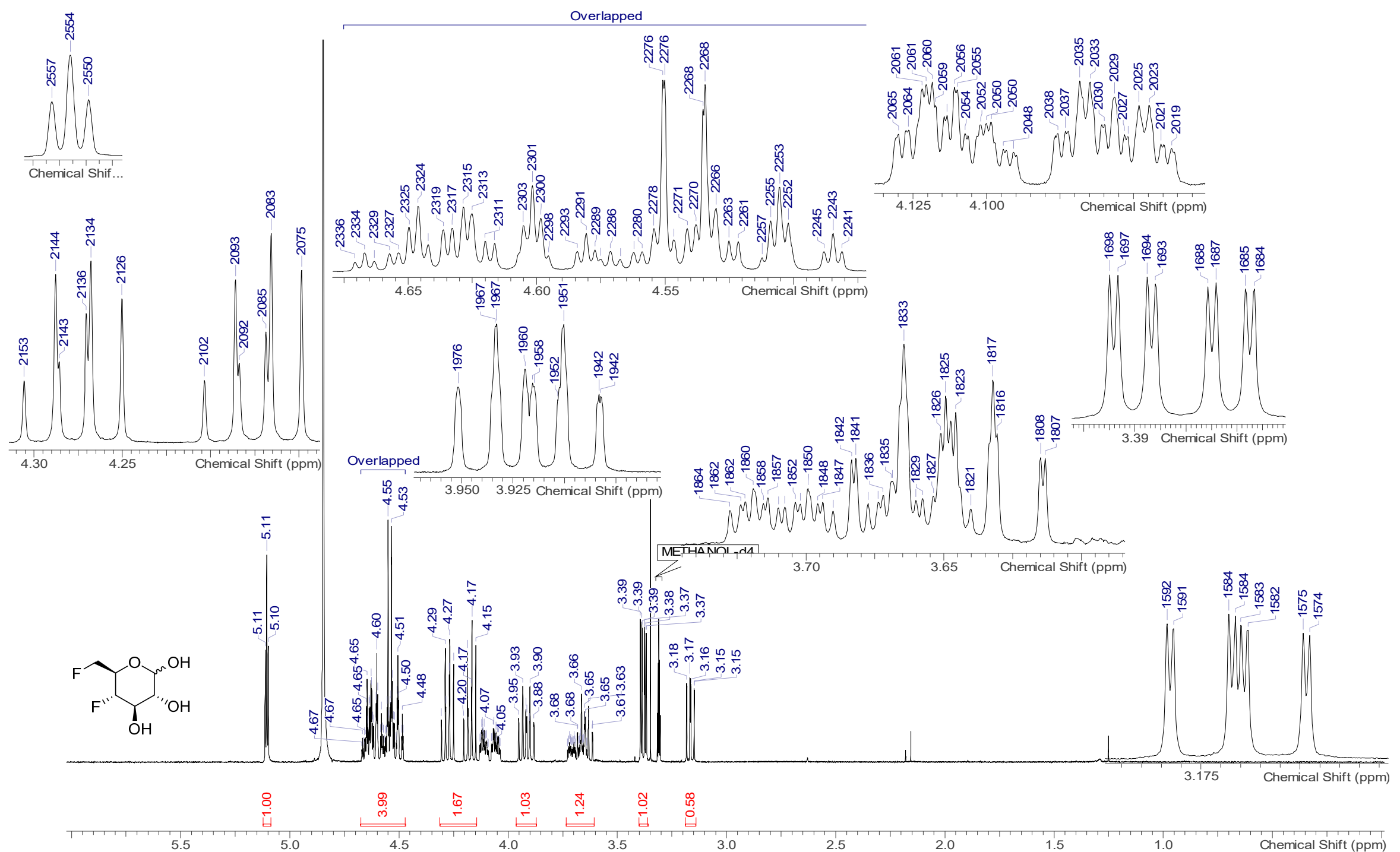




\subsection{2. ${ }^{1} \mathrm{H}\left\{{ }^{19} \mathrm{~F}\right\}$ NMR $\left(500 \mathrm{MHz}, \mathrm{CD}_{3} \mathrm{OD}\right)$}

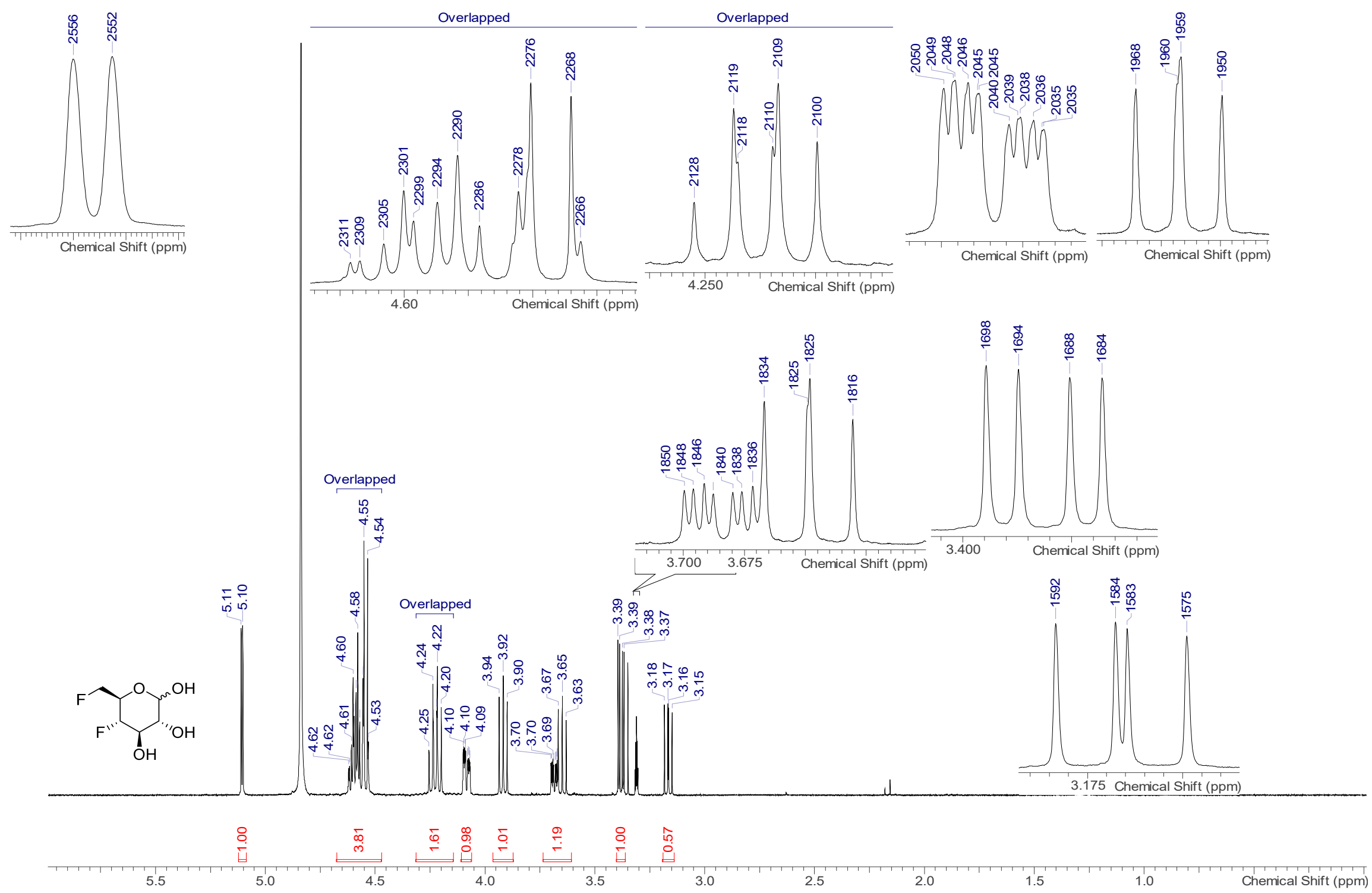


3.9.3. ${ }^{13} \mathrm{C}\left\{{ }^{1} \mathrm{H}\right\} \mathrm{NMR}\left(101 \mathrm{MHz}, \mathrm{CD}_{3} \mathrm{OD}\right)$

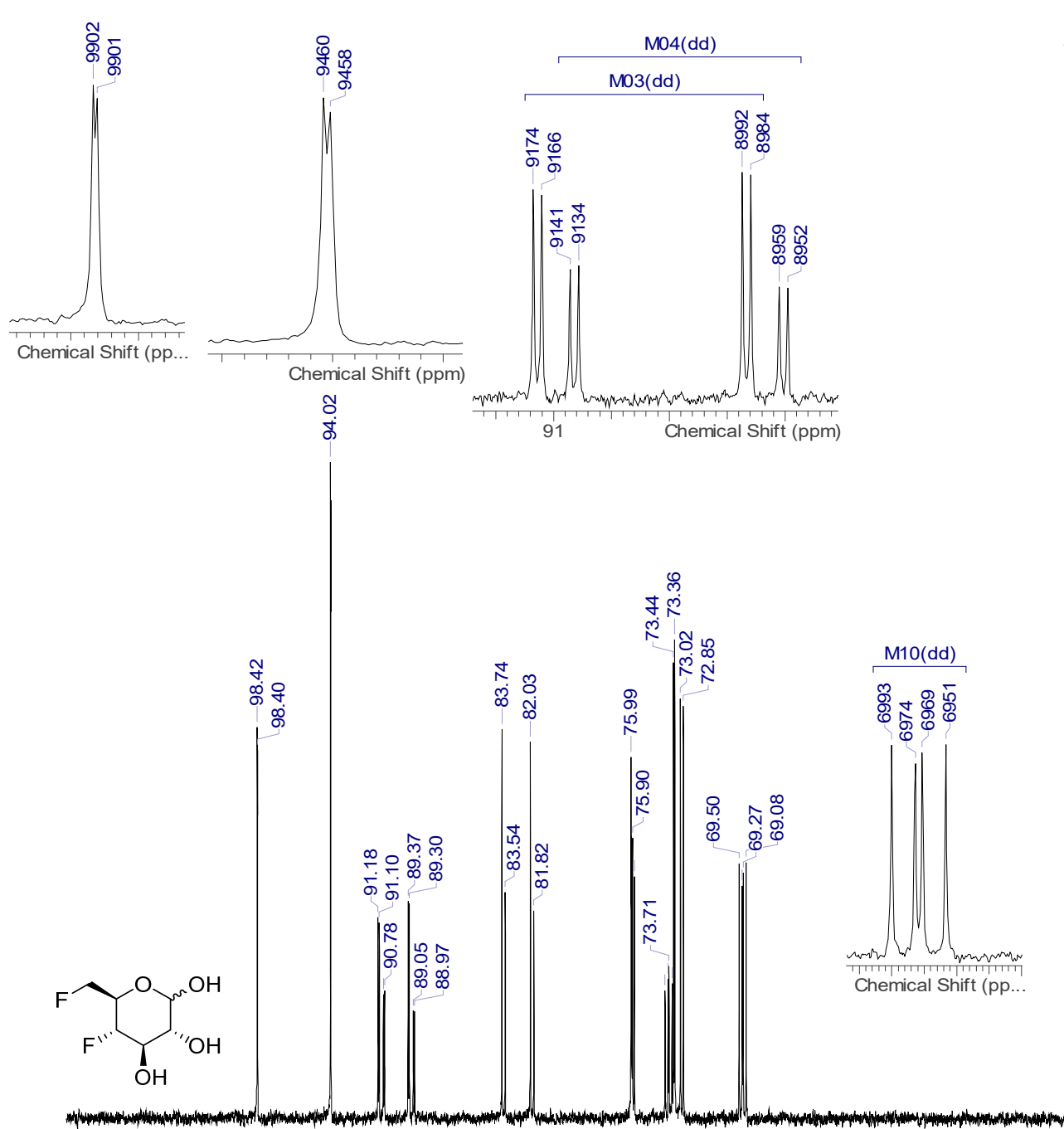

METHANOL-d4

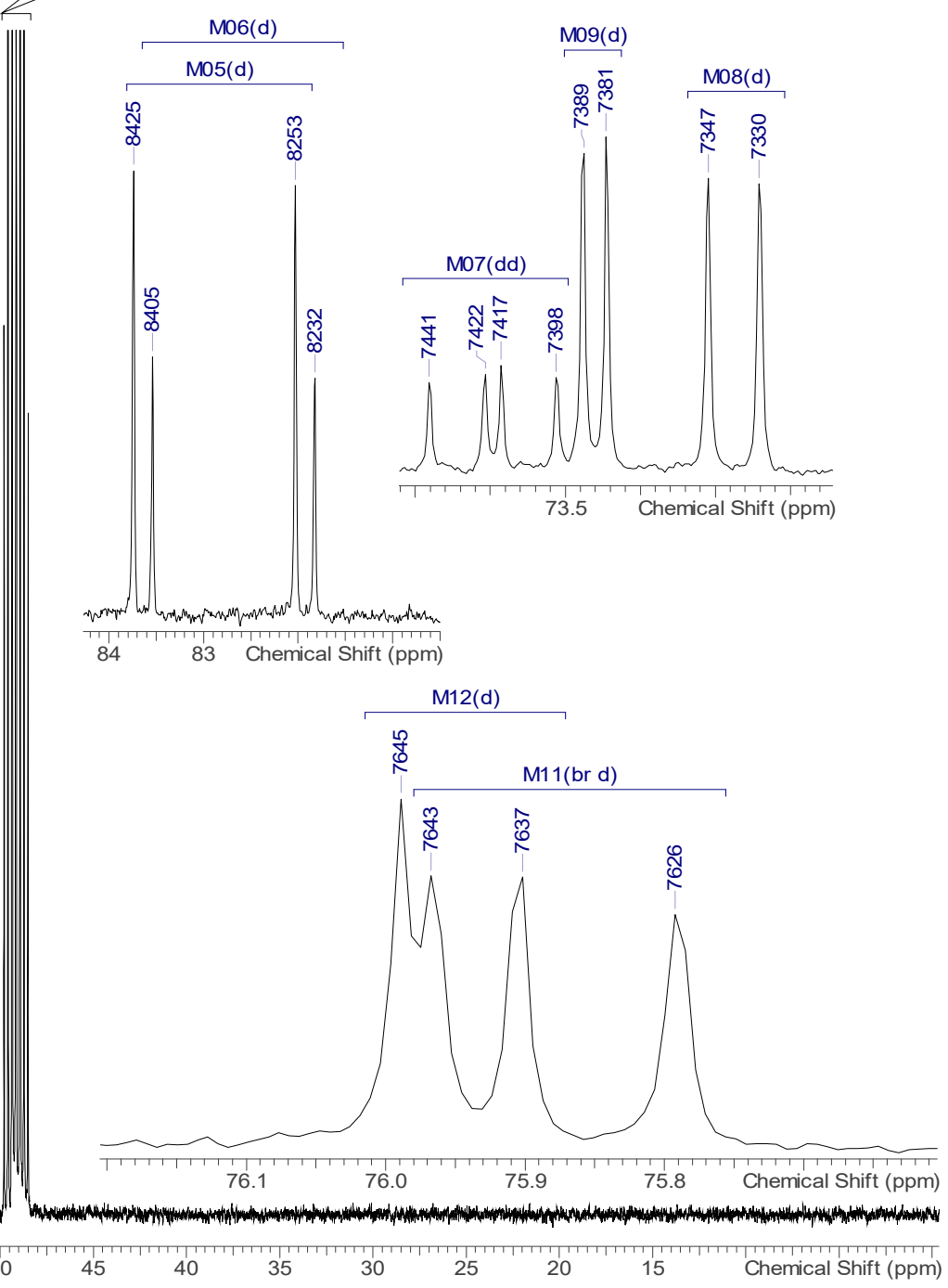


3.9.4. COSY \& HSQC $\left(\mathrm{CD}_{3} \mathrm{OD}\right)$

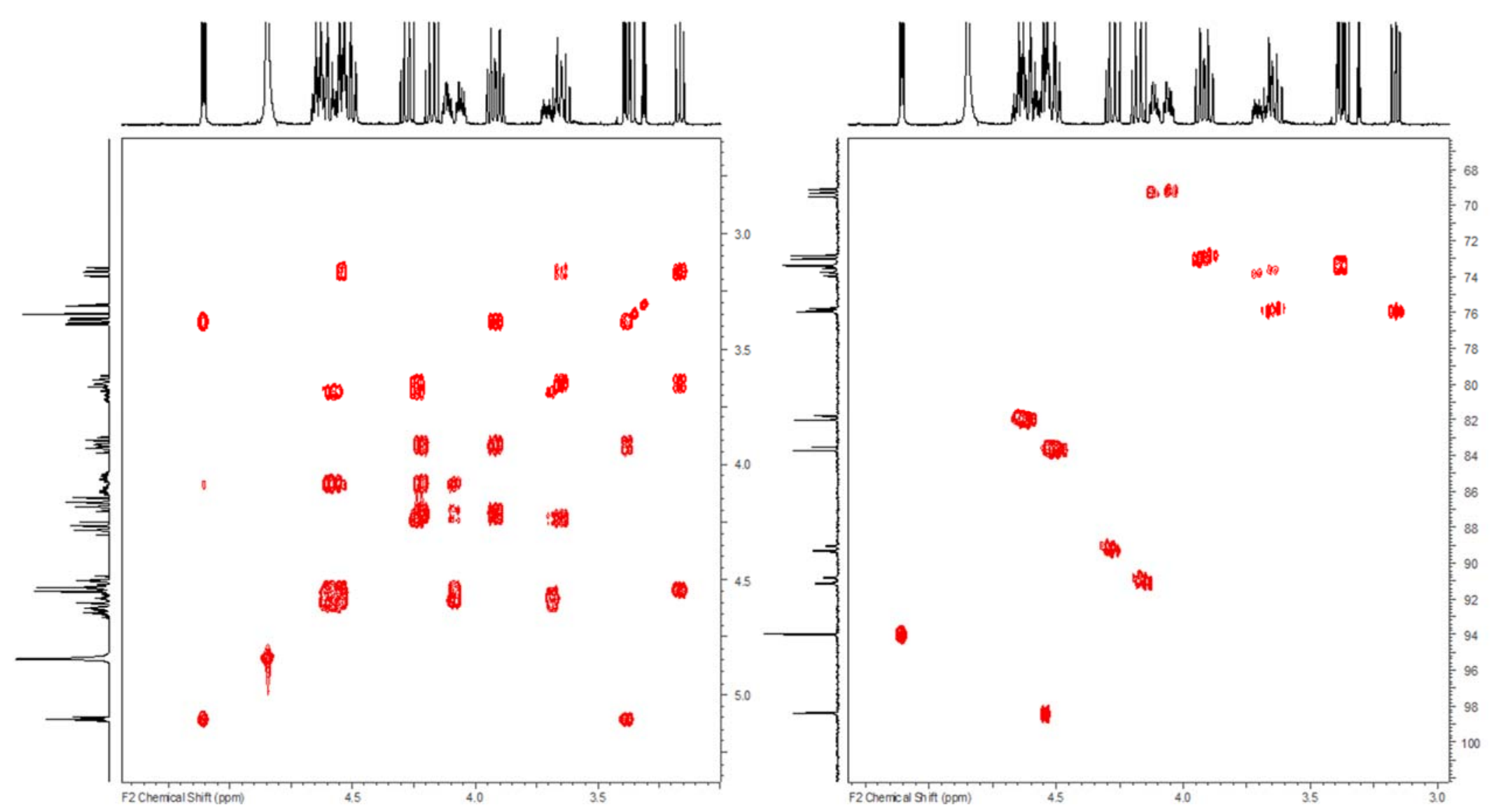


3.9.5. ${ }^{19} \mathrm{~F} \mathrm{NMR}\left(470 \mathrm{MHz}, \mathrm{CD}_{3} \mathrm{OD}\right)$

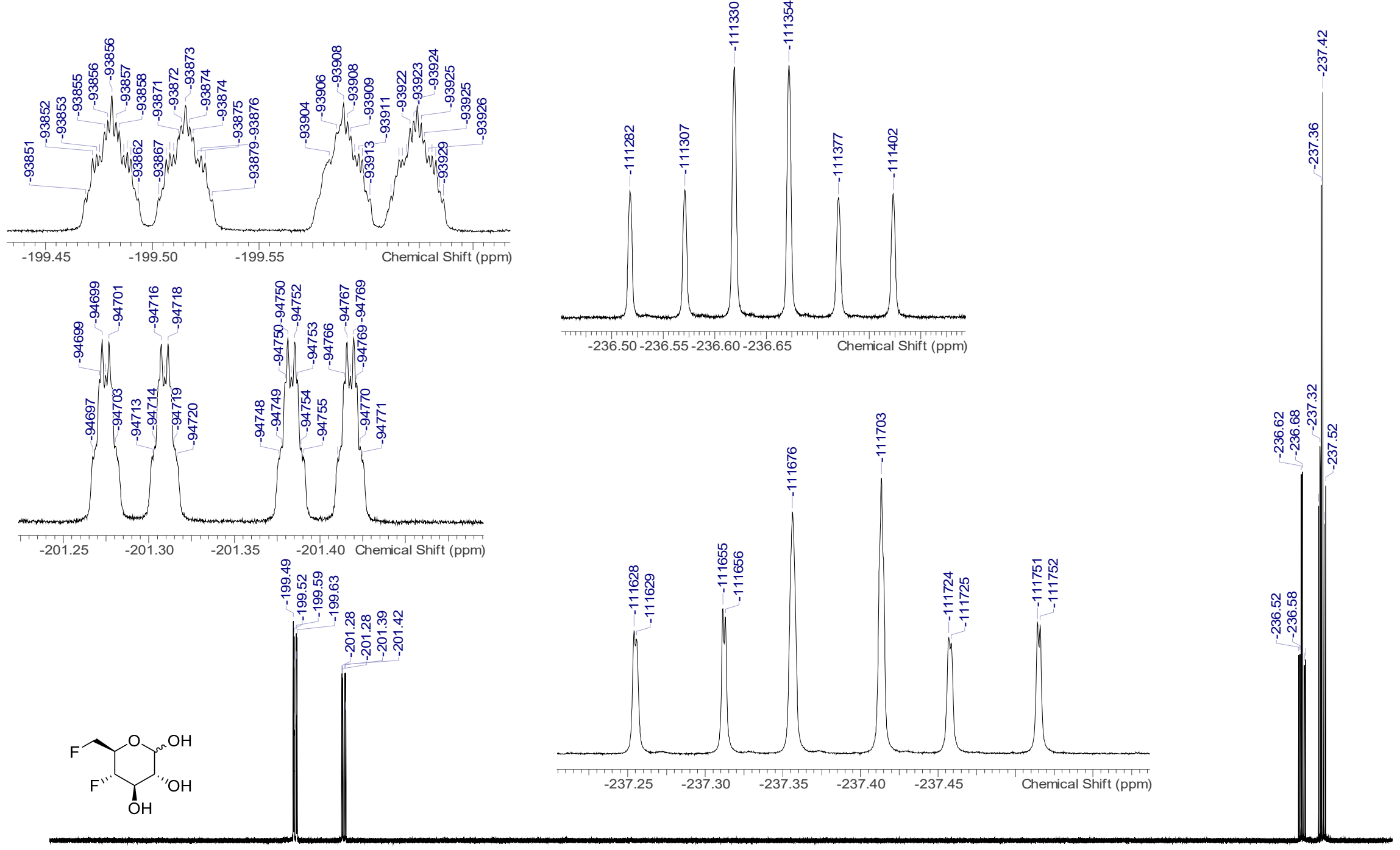

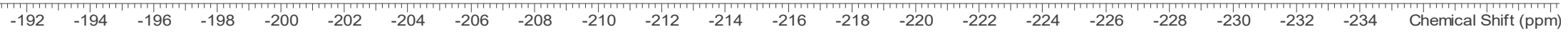


3.9.6. ${ }^{19} \mathrm{~F}\left\{{ }^{1} \mathrm{H}\right\} \mathrm{NMR}\left(470 \mathrm{MHz}, \mathrm{CD}_{3} \mathrm{OD}\right)$

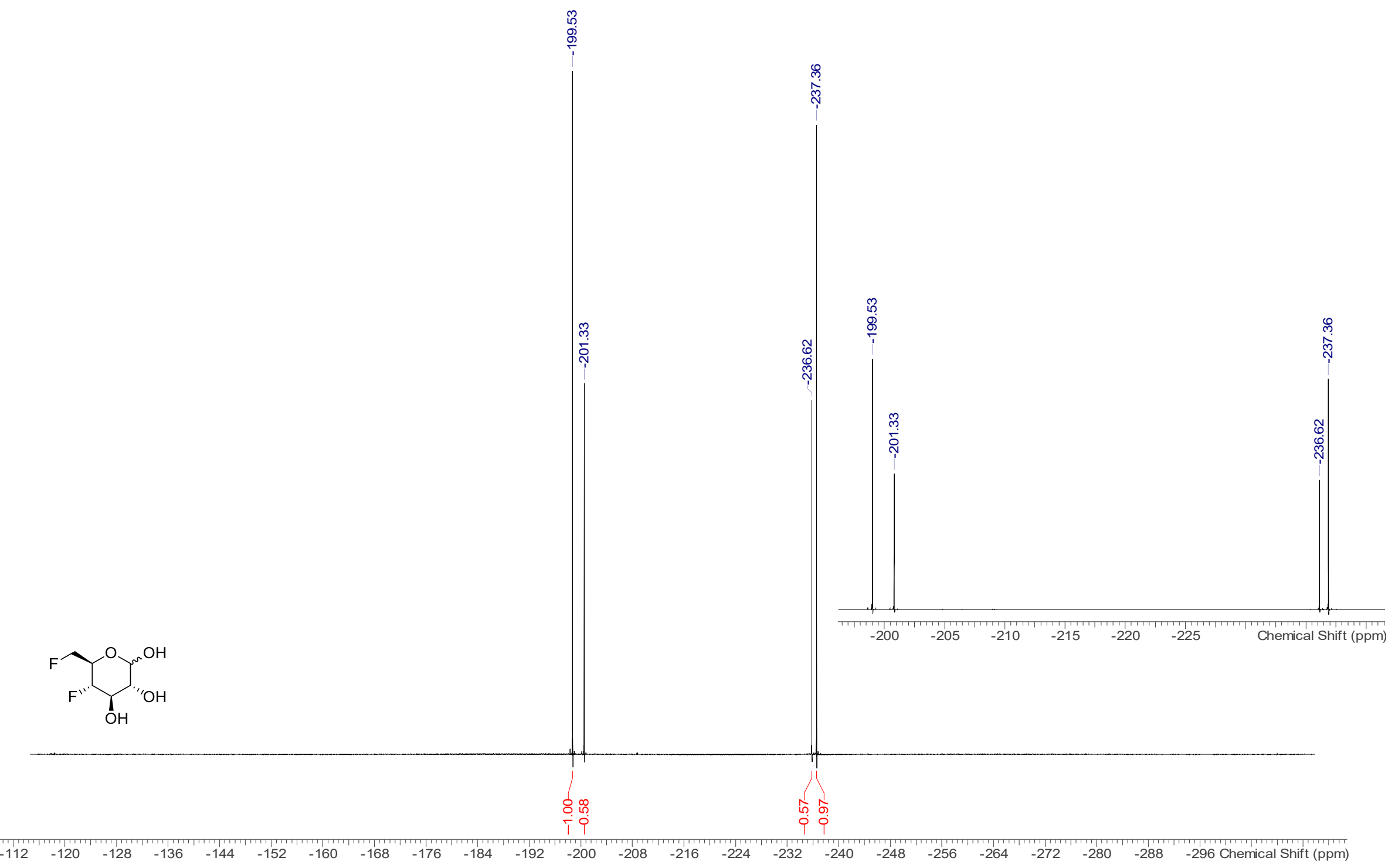


3.10. 1,2,3-Tri-O-acetyl-4,6-dideoxy-4,6-difluoro-D-glucopyranoside (5b)

3.10.1. ${ }^{1} \mathrm{H}$ NMR $\left(400 \mathrm{MHz}, \mathrm{CDCl}_{3}\right)$

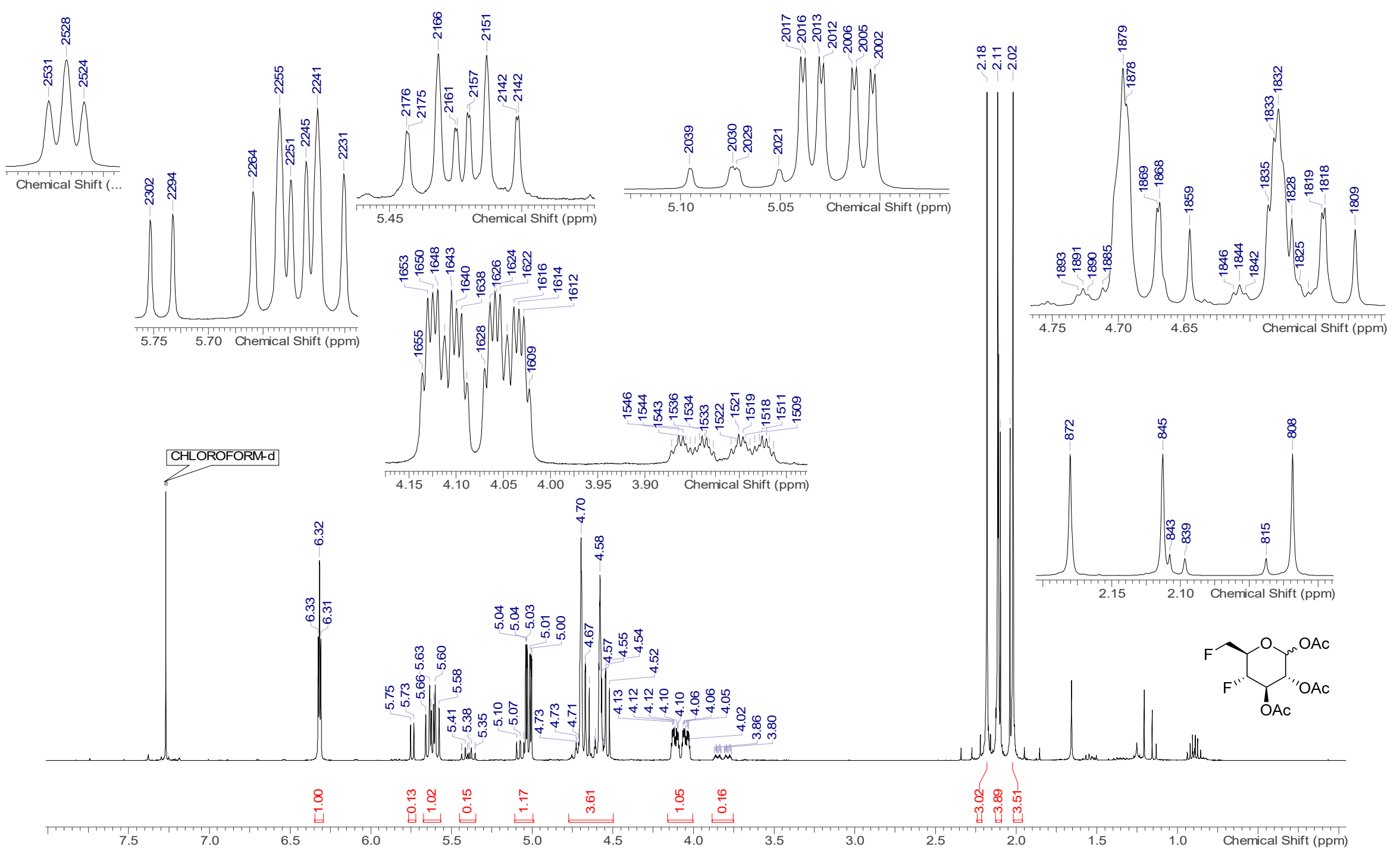


3.10.2. ${ }^{13} \mathrm{C}\left\{{ }^{1} \mathrm{H}\right\}$ NMR $\left(101 \mathrm{MHz}, \mathrm{CDCl}_{3}\right)$

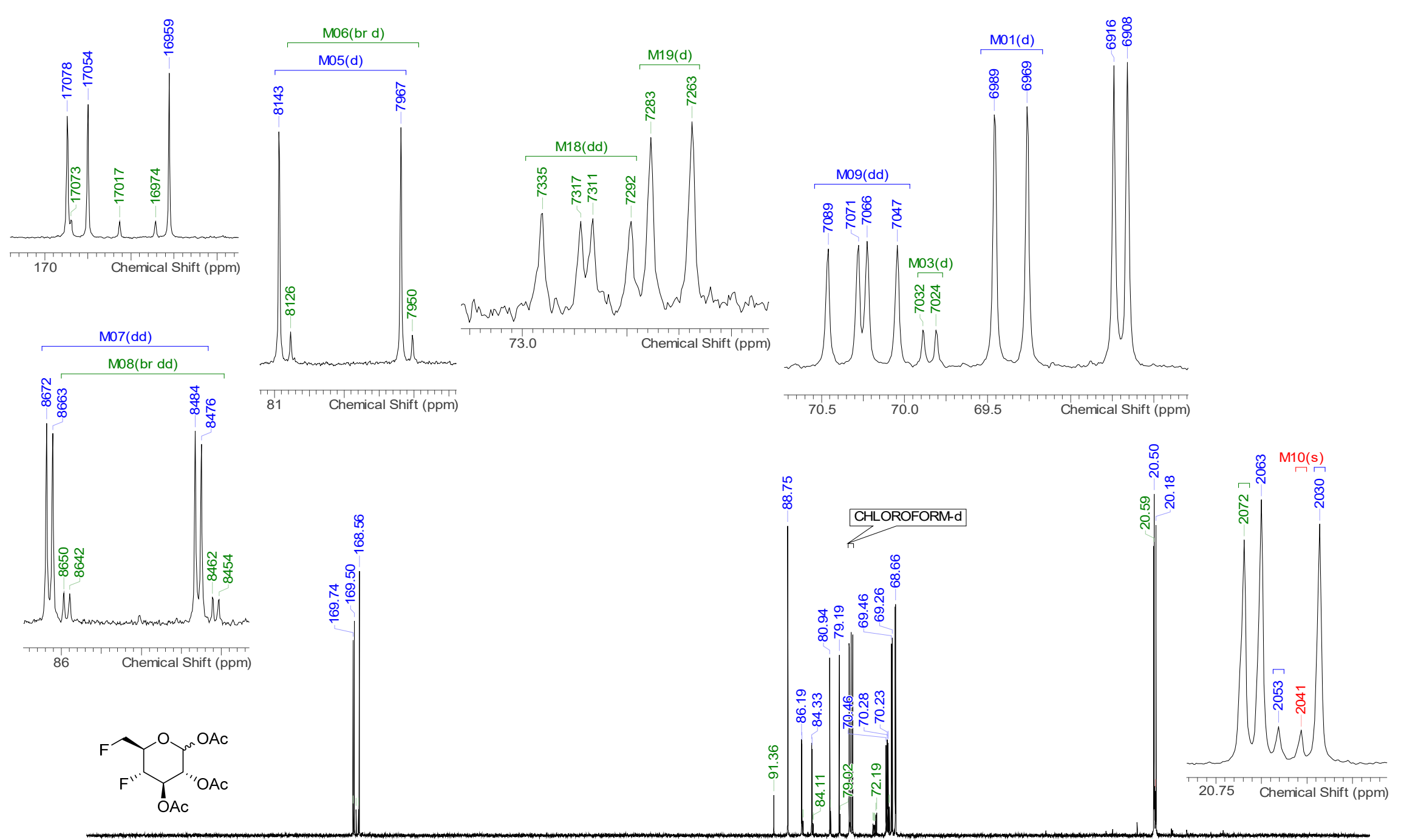

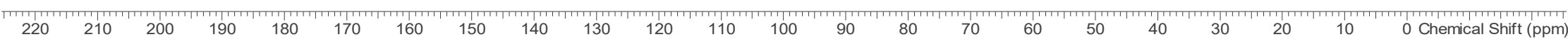


3.10.3. COSY, $\mathrm{HSQC} \& \mathrm{HMBC}\left(\mathrm{CDCl}_{3}\right)$

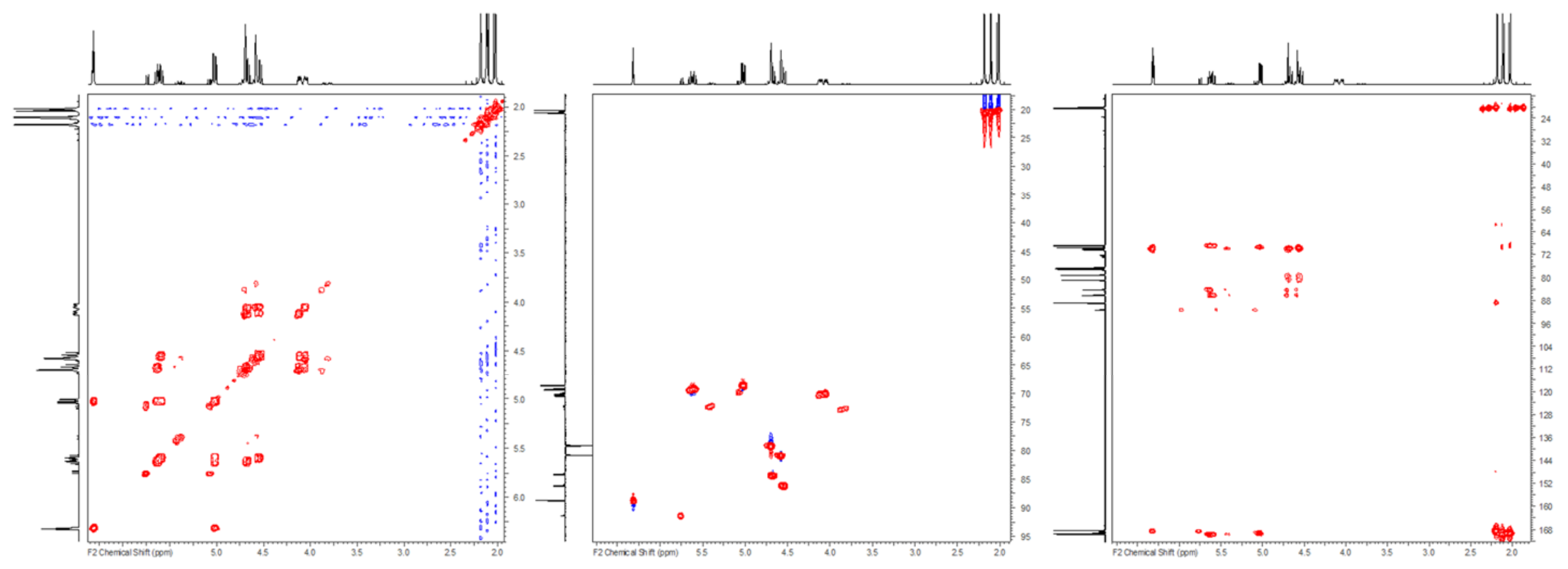


3.10.4. $\left.{ }^{19} \mathrm{~F} \mathrm{NMR} \mathrm{(376} \mathrm{MHz,} \mathrm{CDCl}_{3}\right)$
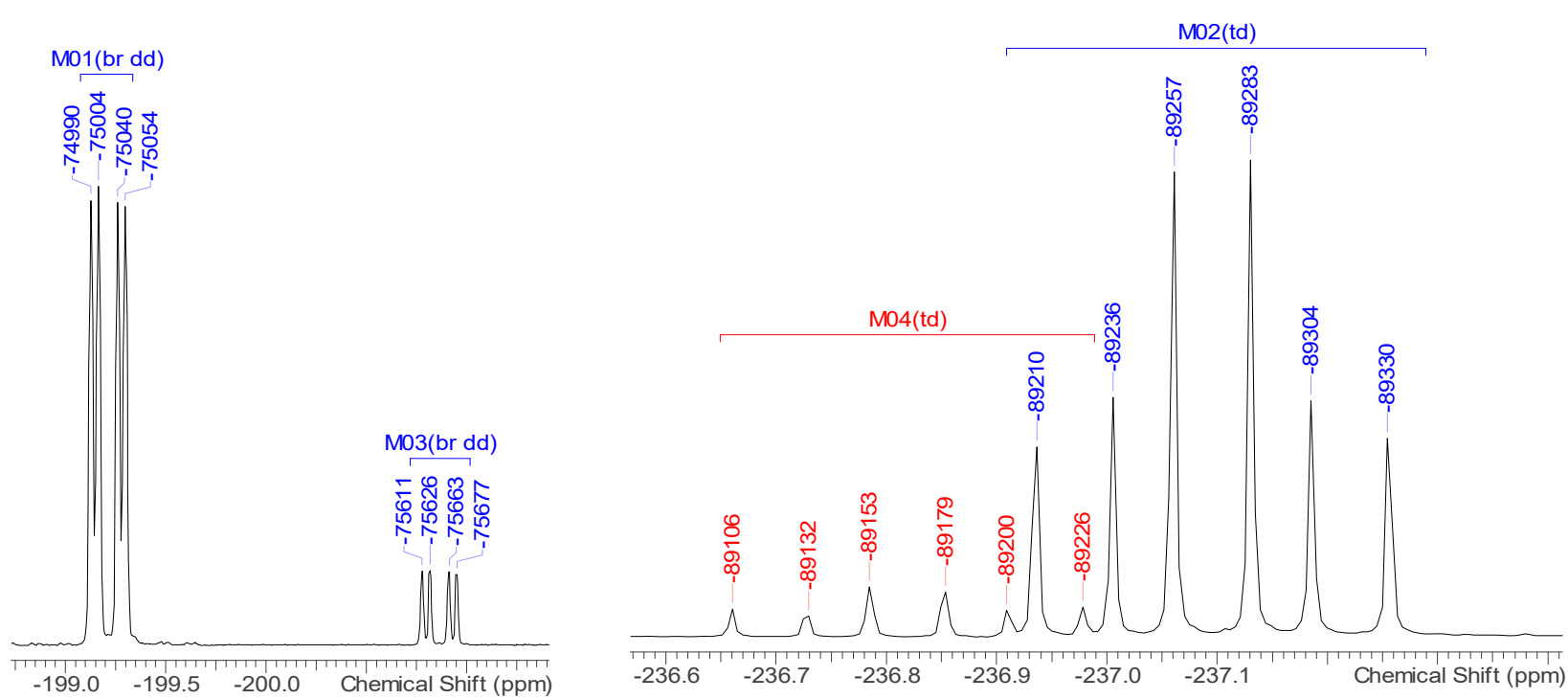

$$
\text { OCAc }
$$

M01(br dd) 
3.10.5. ${ }^{19} \mathrm{~F}\left\{{ }^{1} \mathrm{H}\right\} \mathrm{NMR}\left(376 \mathrm{MHz}, \mathrm{CDCl}_{3}\right)$

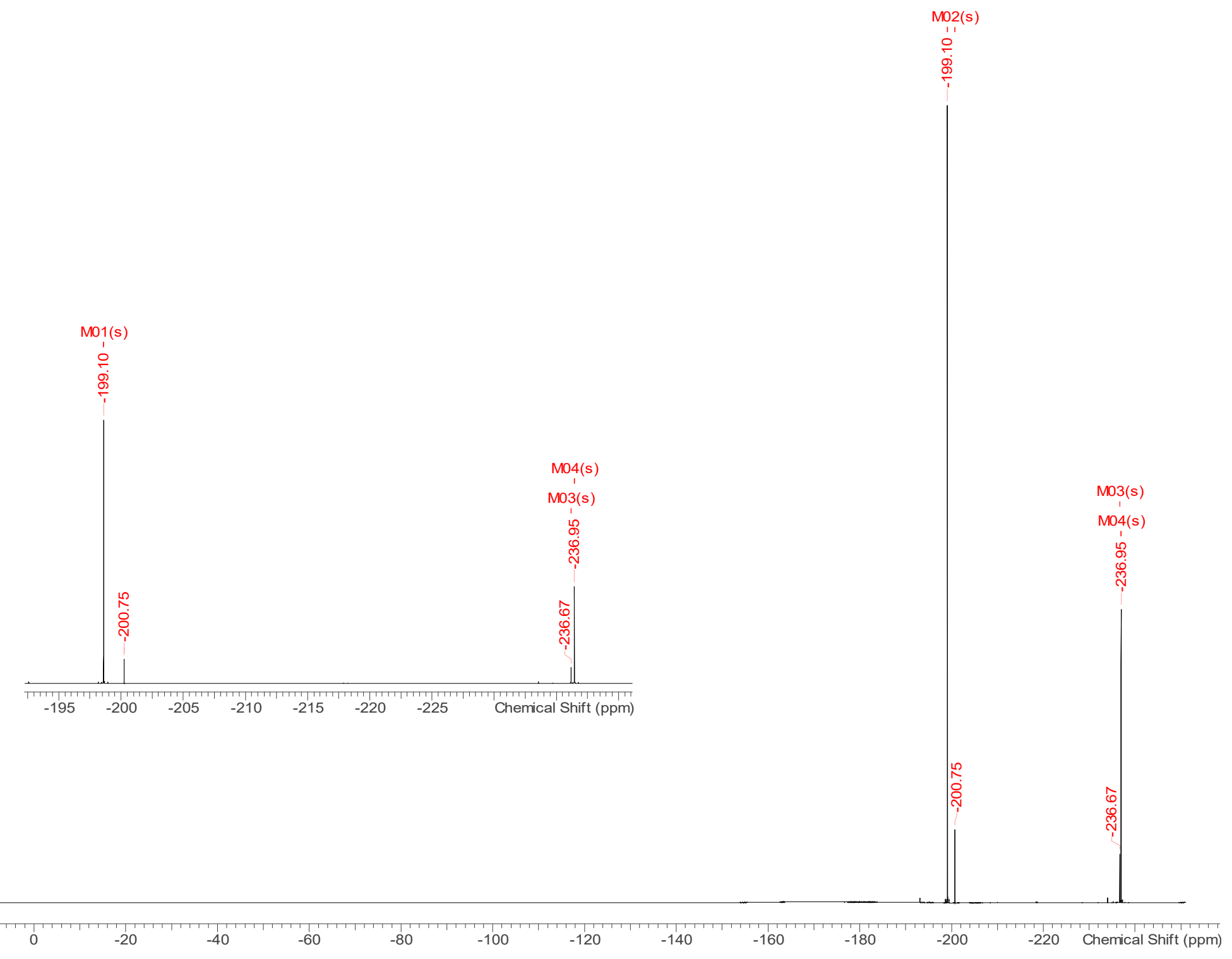




\subsection{1,1,2,3,5-Penta-O-acetyl-4,6-dideoxy-4,6-difluoro-D-glucose (5c)}

3.11.1. ${ }^{1} \mathrm{H} \mathrm{NMR}\left(500 \mathrm{MHz}, \mathrm{CDCl}_{3}\right)$

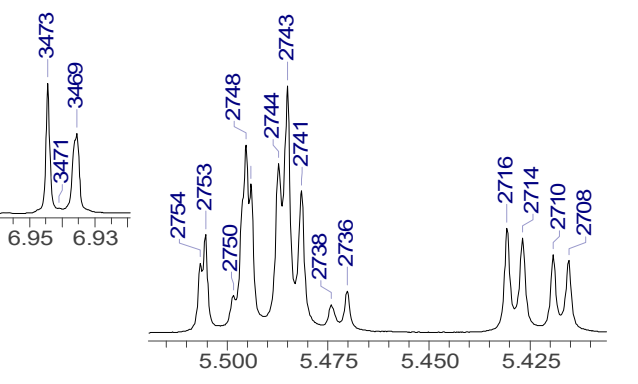

CHLOROFORM-
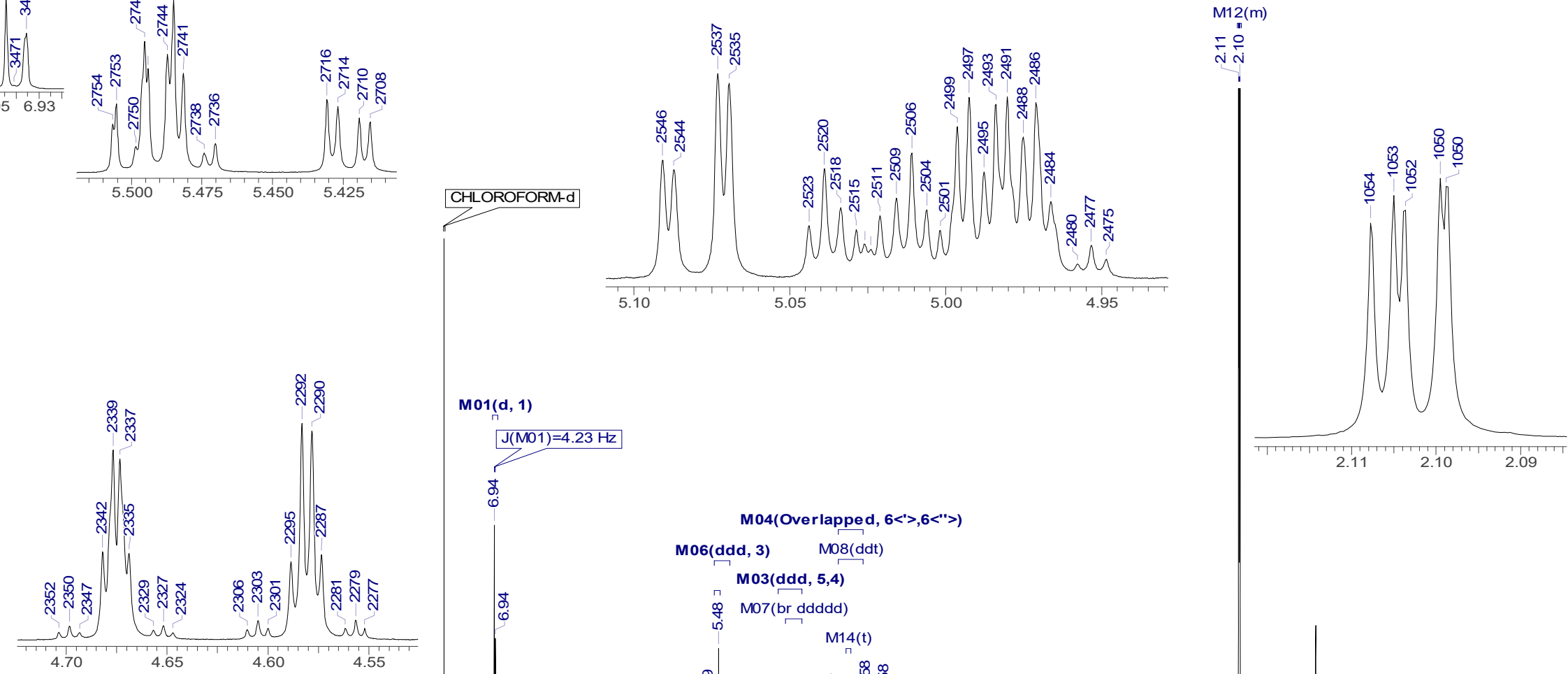

M01(d, 1)

$J(M 01)=4.23 \mathrm{~Hz}$

离

M04(Overlapped, 6<'>,6<">)

M06(ddd, 3) MO8(ddt)

M03(ddd, 5,4)

of MO7(br ddddd)

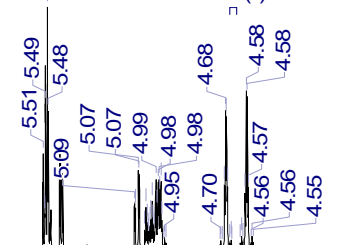

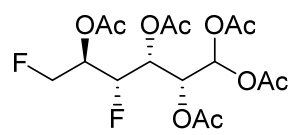

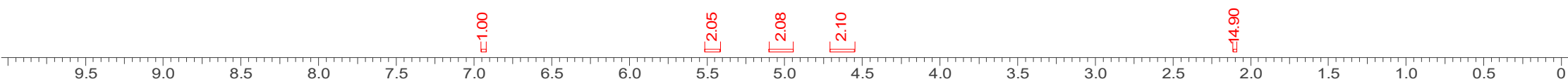


3.11.2. ${ }^{1} \mathrm{H}\left\{{ }^{19} \mathrm{~F}\right\}$ NMR $\left(500 \mathrm{MHz}, \mathrm{CDCl}_{3}\right)$

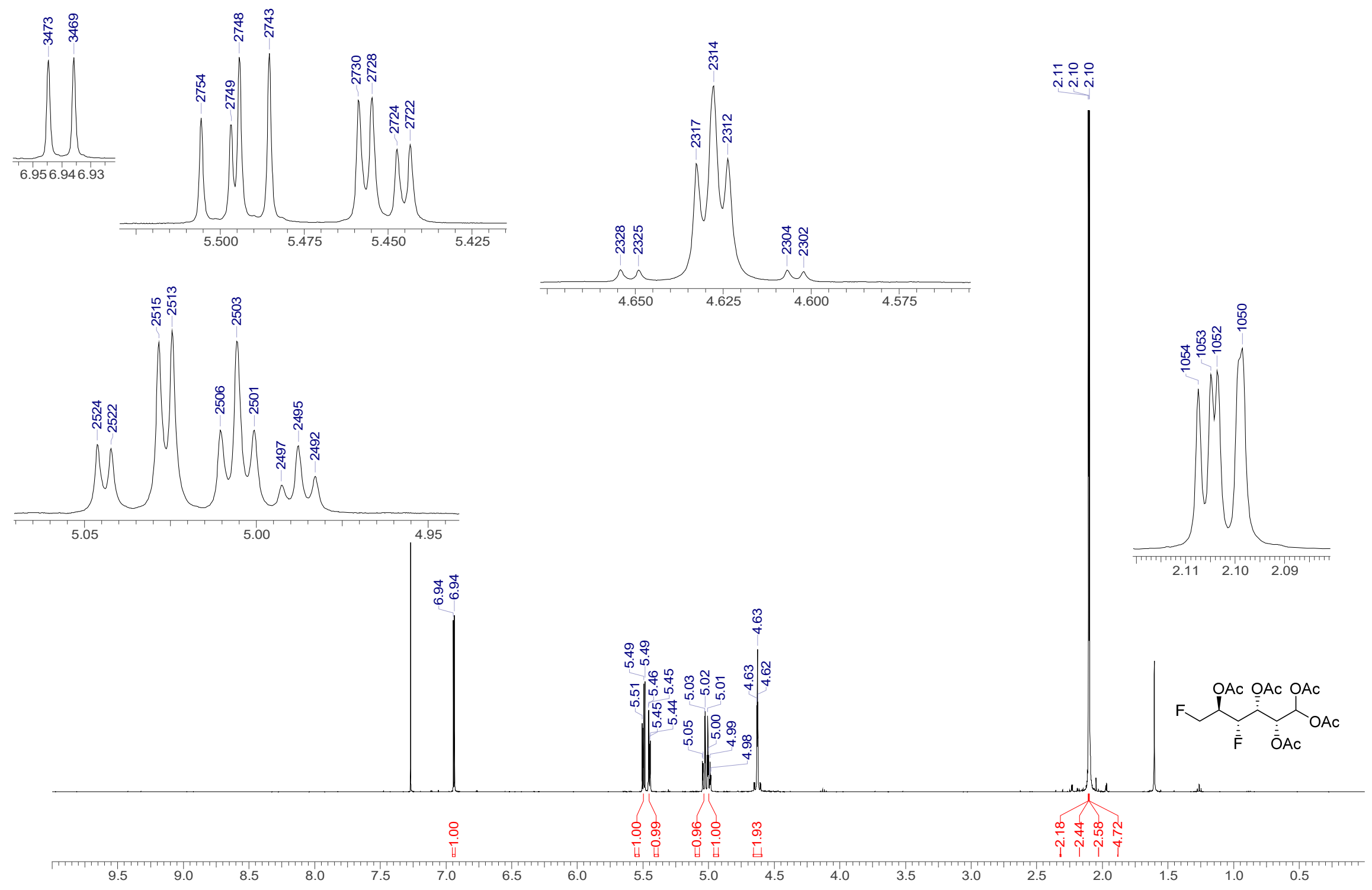


3.11.3. ${ }^{13} \mathrm{C}\left\{{ }^{1} \mathrm{H}\right\}$ NMR $\left(126 \mathrm{MHz}, \mathrm{CDCl}_{3}\right)$
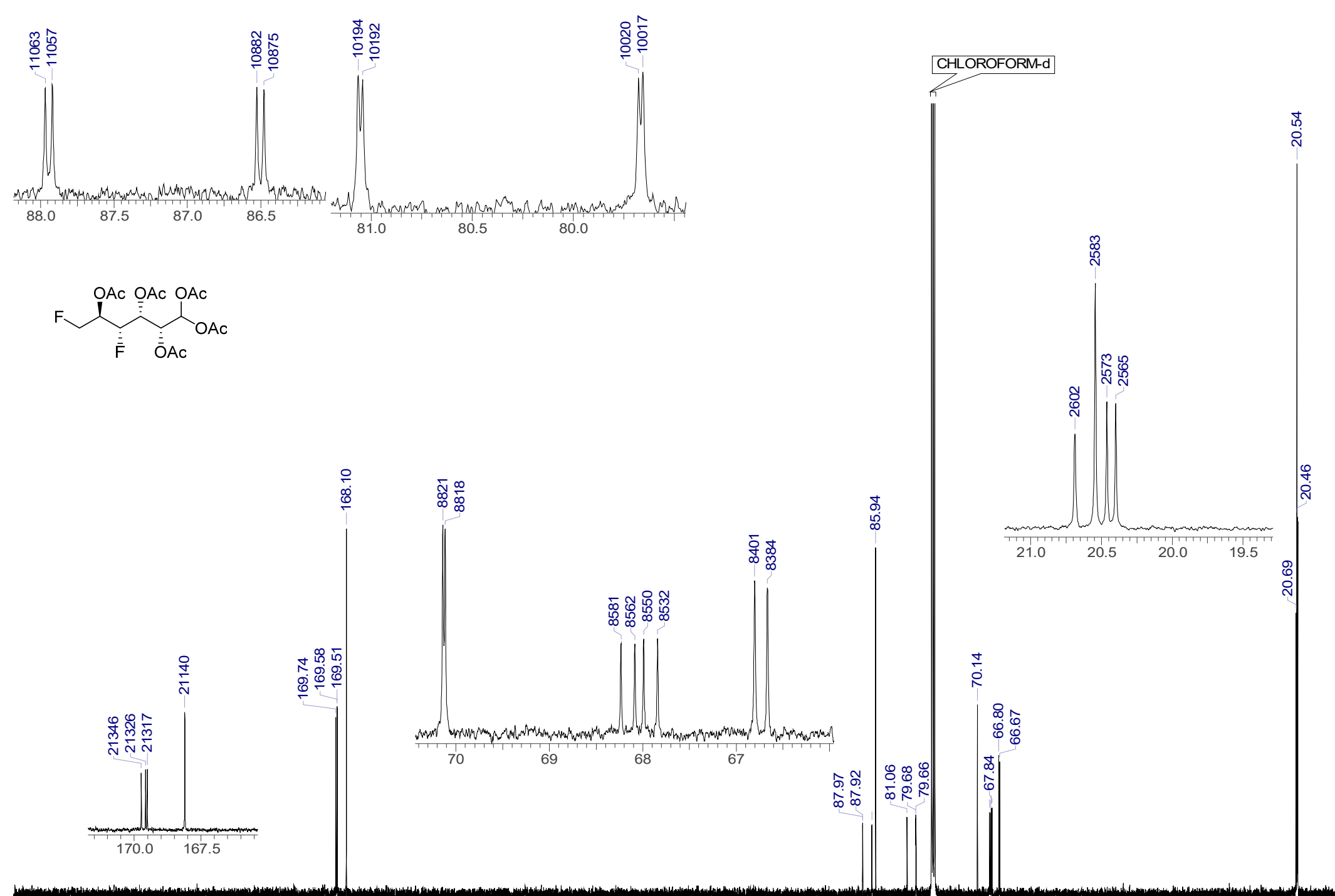

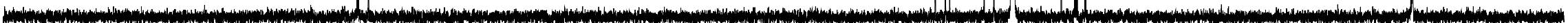

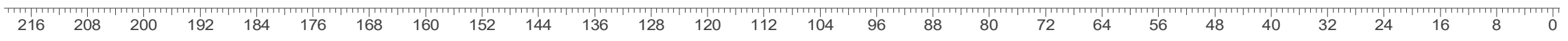


3.11.4. COSY, HSQC-DEPT \& HMBC $\left(\mathrm{CDCl}_{3}\right)$

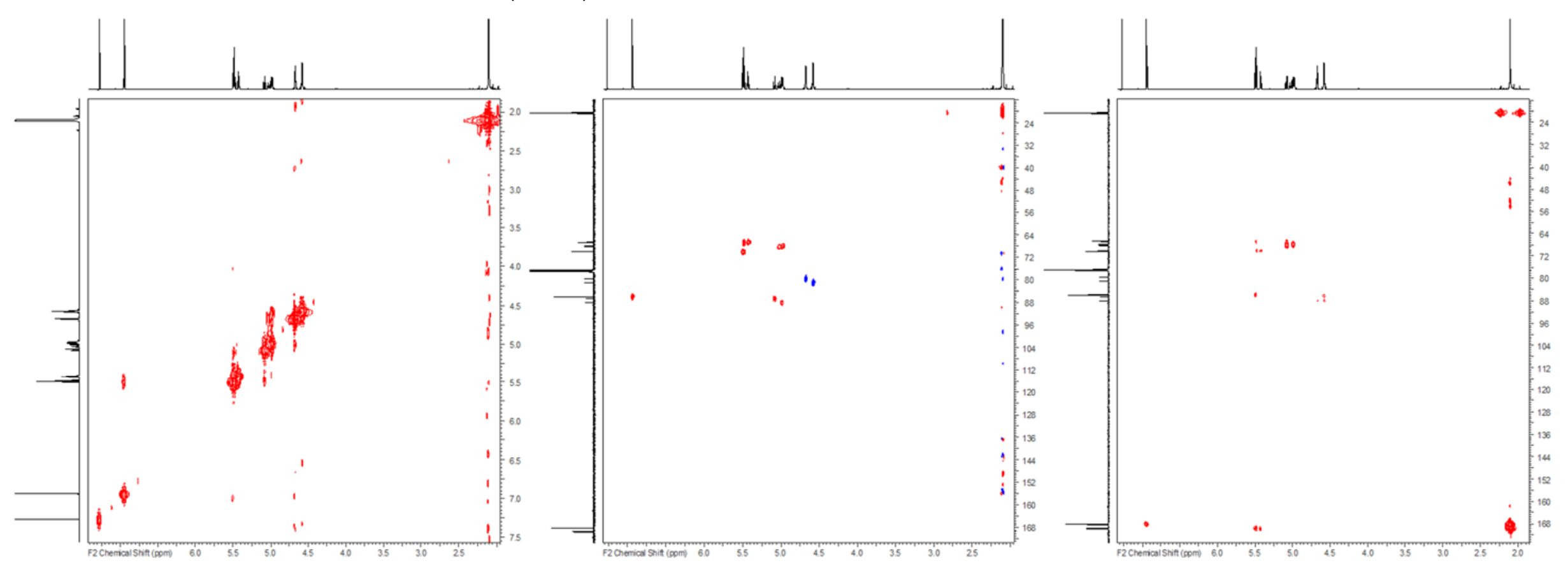


3.11.5. ${ }^{19} \mathrm{~F}$ NMR $\left(470 \mathrm{MHz}, \mathrm{CDCl}_{3}\right)$

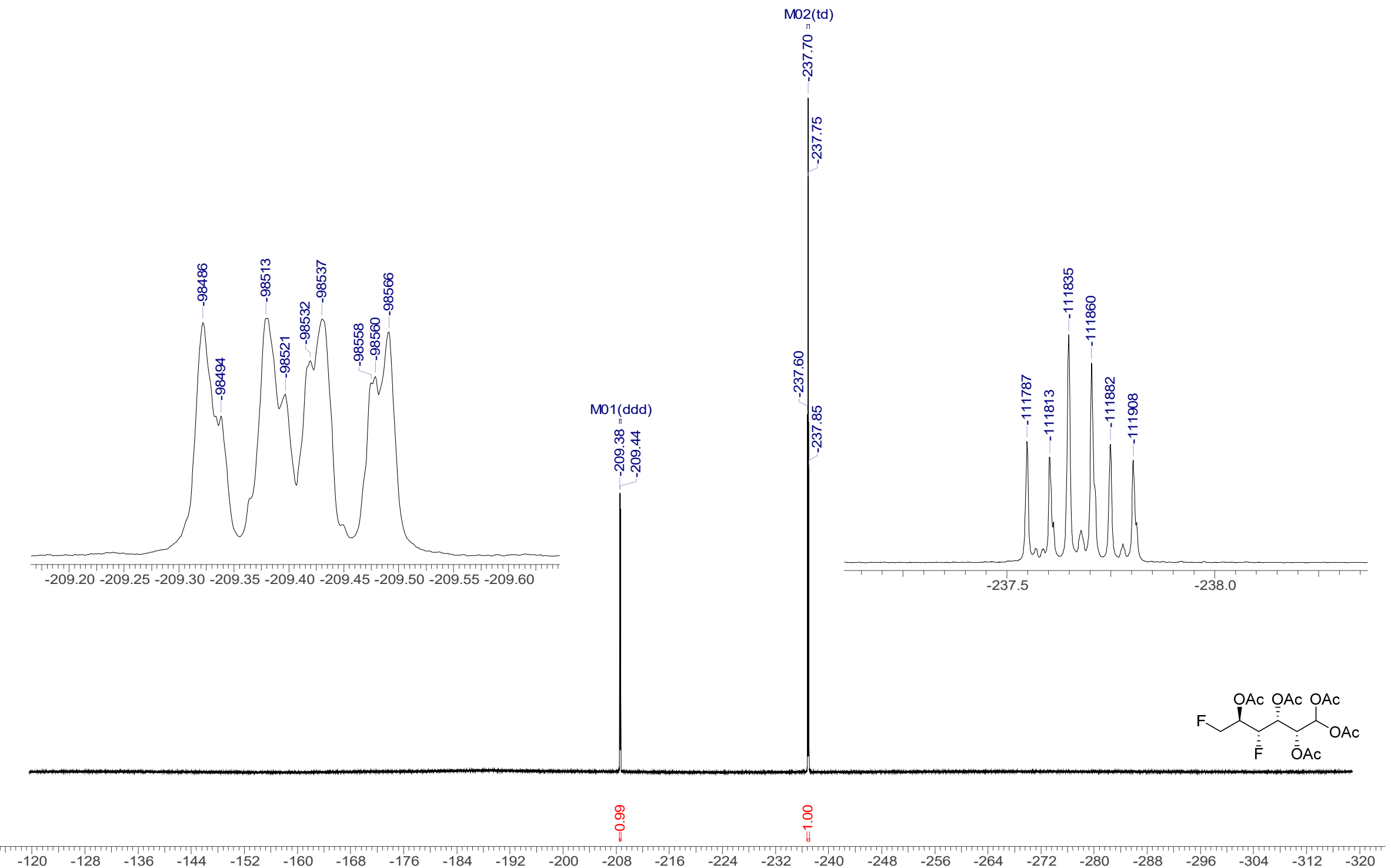


3.11.6. ${ }^{19} \mathrm{~F}\left\{{ }^{1} \mathrm{H}\right\}$ NMR $\left(470 \mathrm{MHz}, \mathrm{CDCl}_{3}\right)$

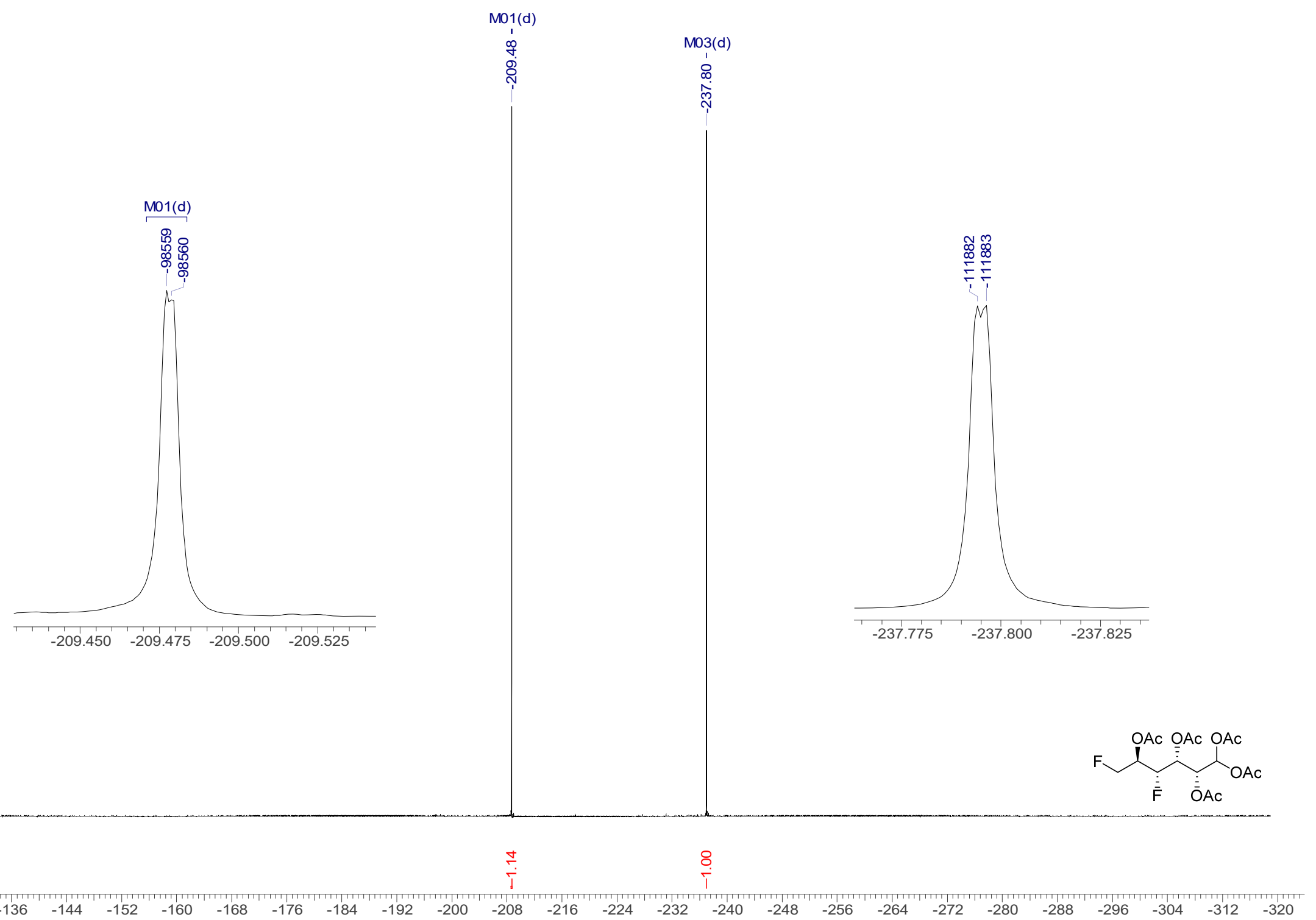




\subsection{4,6-Dideoxy-4,4-difluoro-D-xylo-hexopyranose (6a)}

3.12.1. ${ }^{1} \mathrm{H}$ NMR $\left(500 \mathrm{MHz}, \mathrm{CD}_{3} \mathrm{OD}\right)$

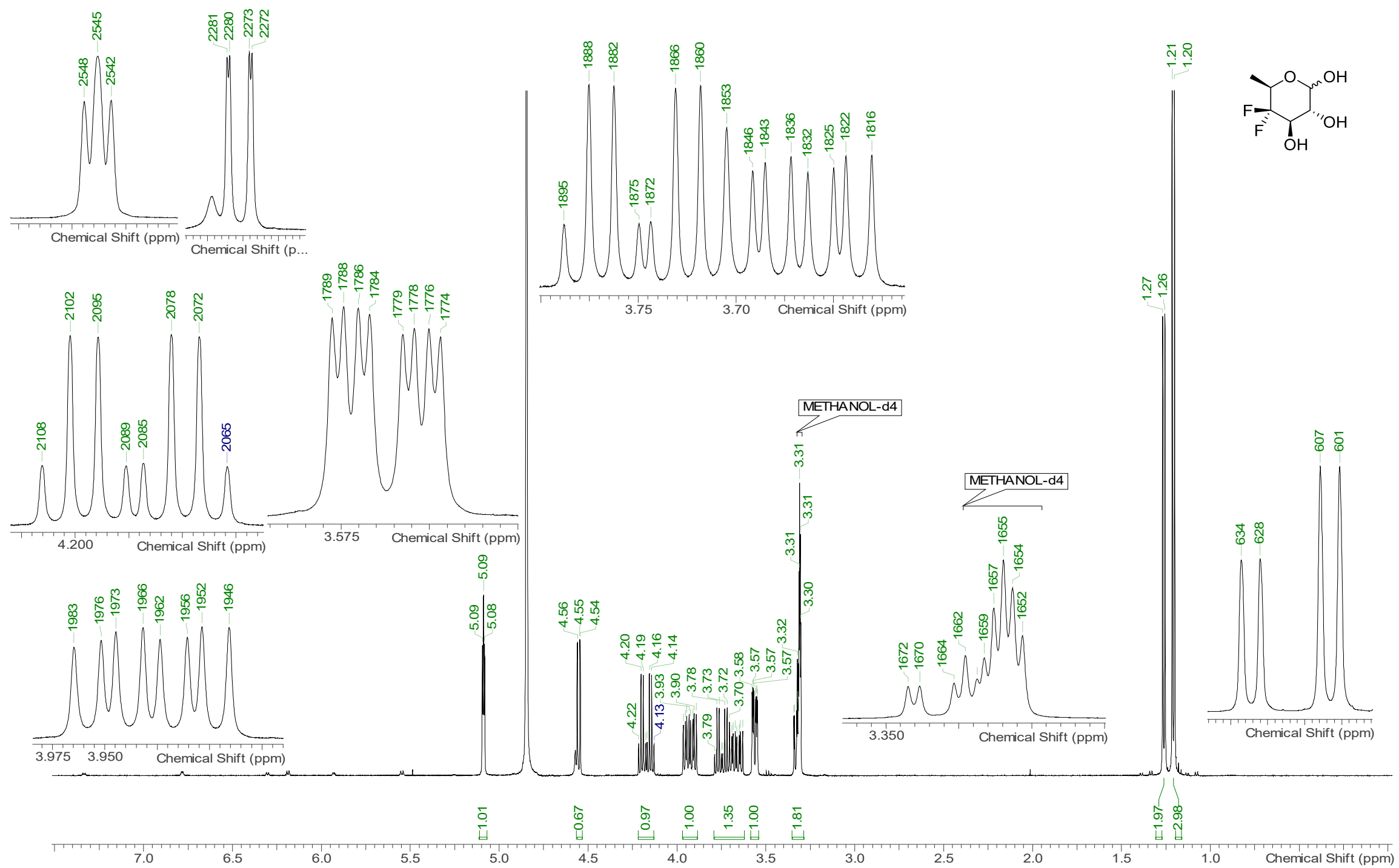


3.12.2. ${ }^{1} \mathrm{H}\left\{{ }^{19} \mathrm{~F}\right\}$ NMR $\left(500 \mathrm{MHz}, \mathrm{CD}_{3} \mathrm{OD}\right)$
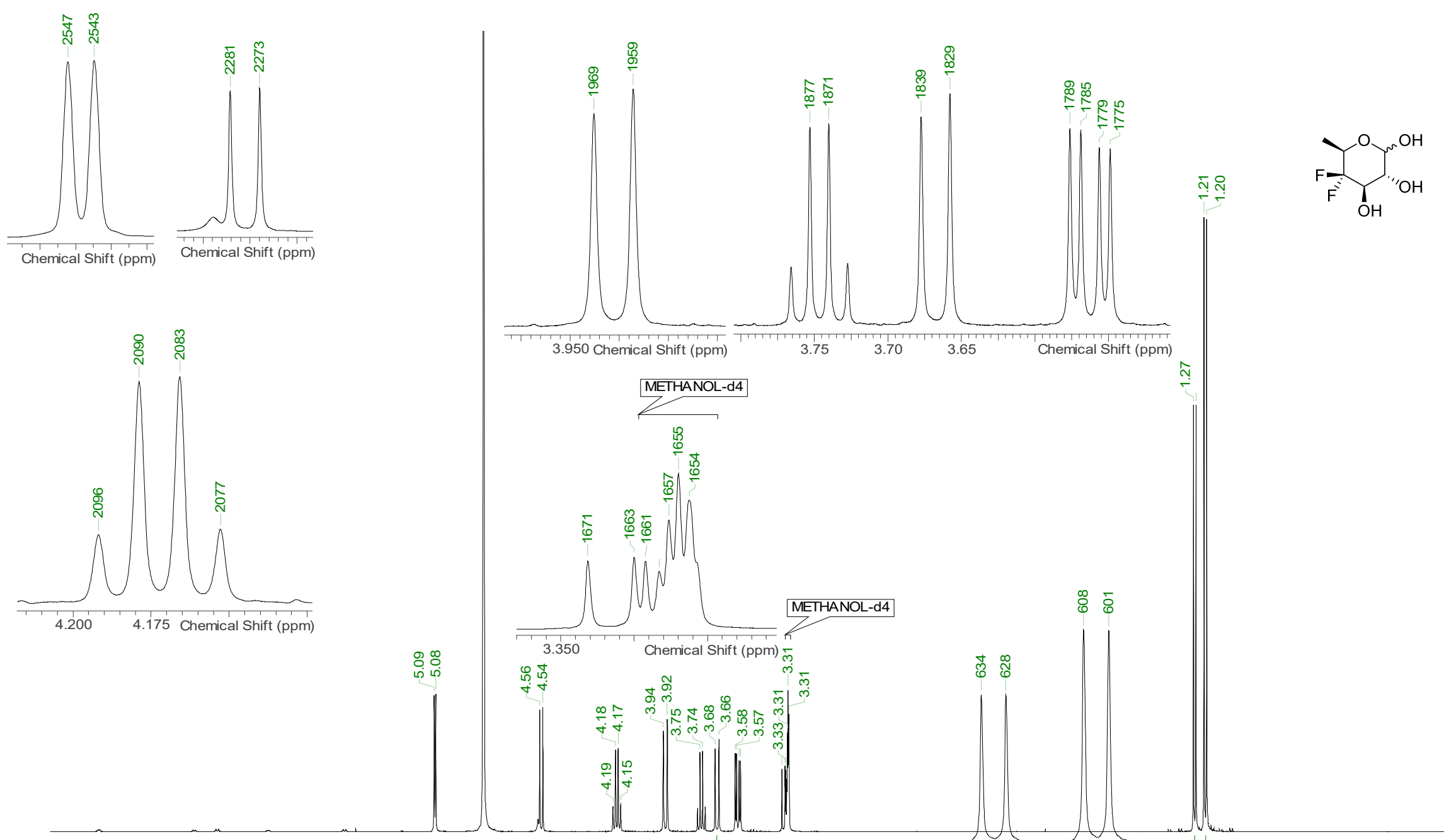

METHANOL-d4
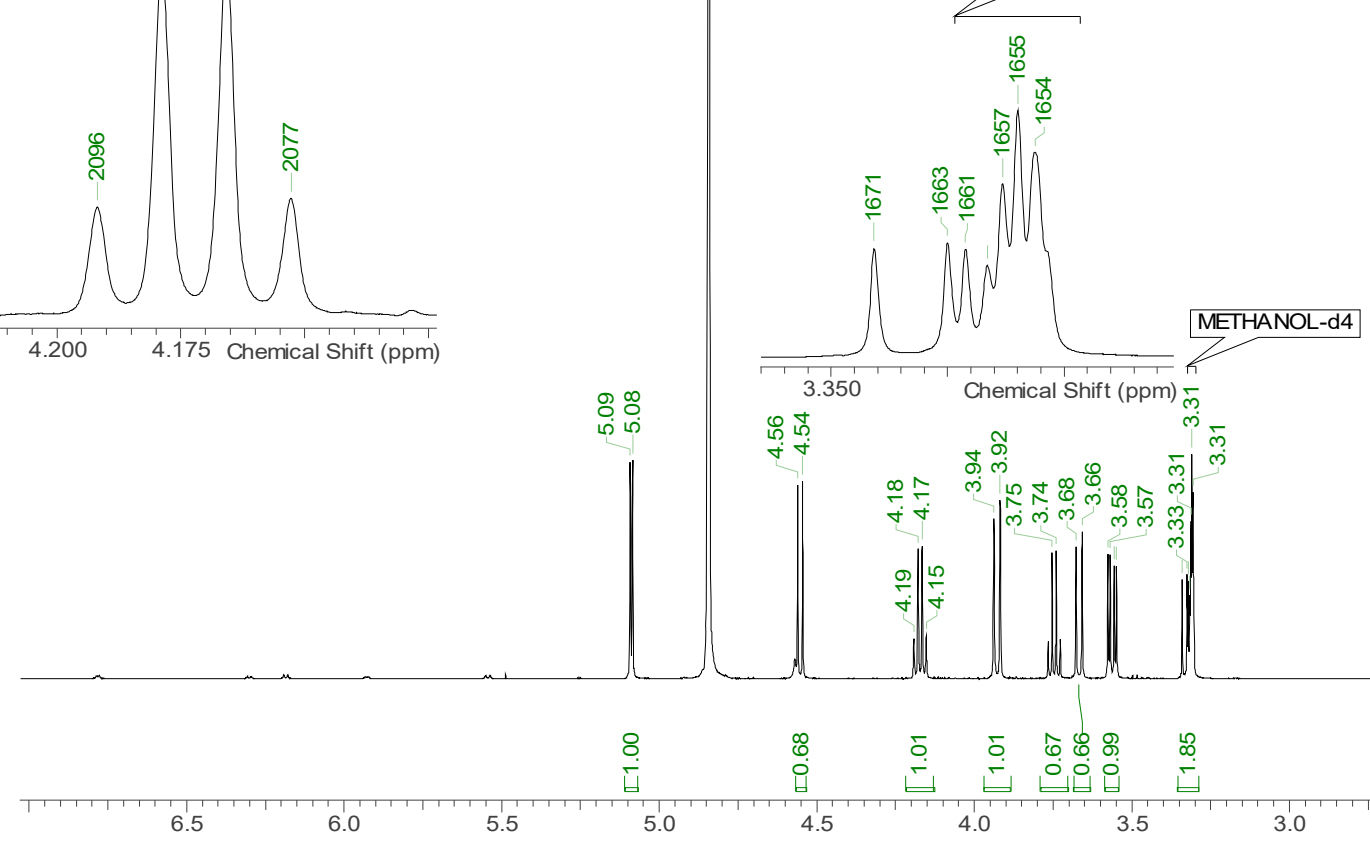

2.5

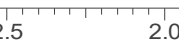

.25 Chemical Shift (ppm)

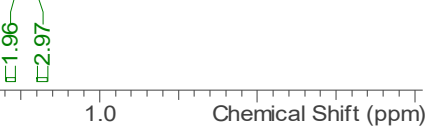




\subsection{3. ${ }^{13} \mathrm{C}\left\{{ }^{1} \mathrm{H}\right\}$-APT NMR (126 MHz, $\left.\mathrm{CD}_{3} \mathrm{OD}\right)$}

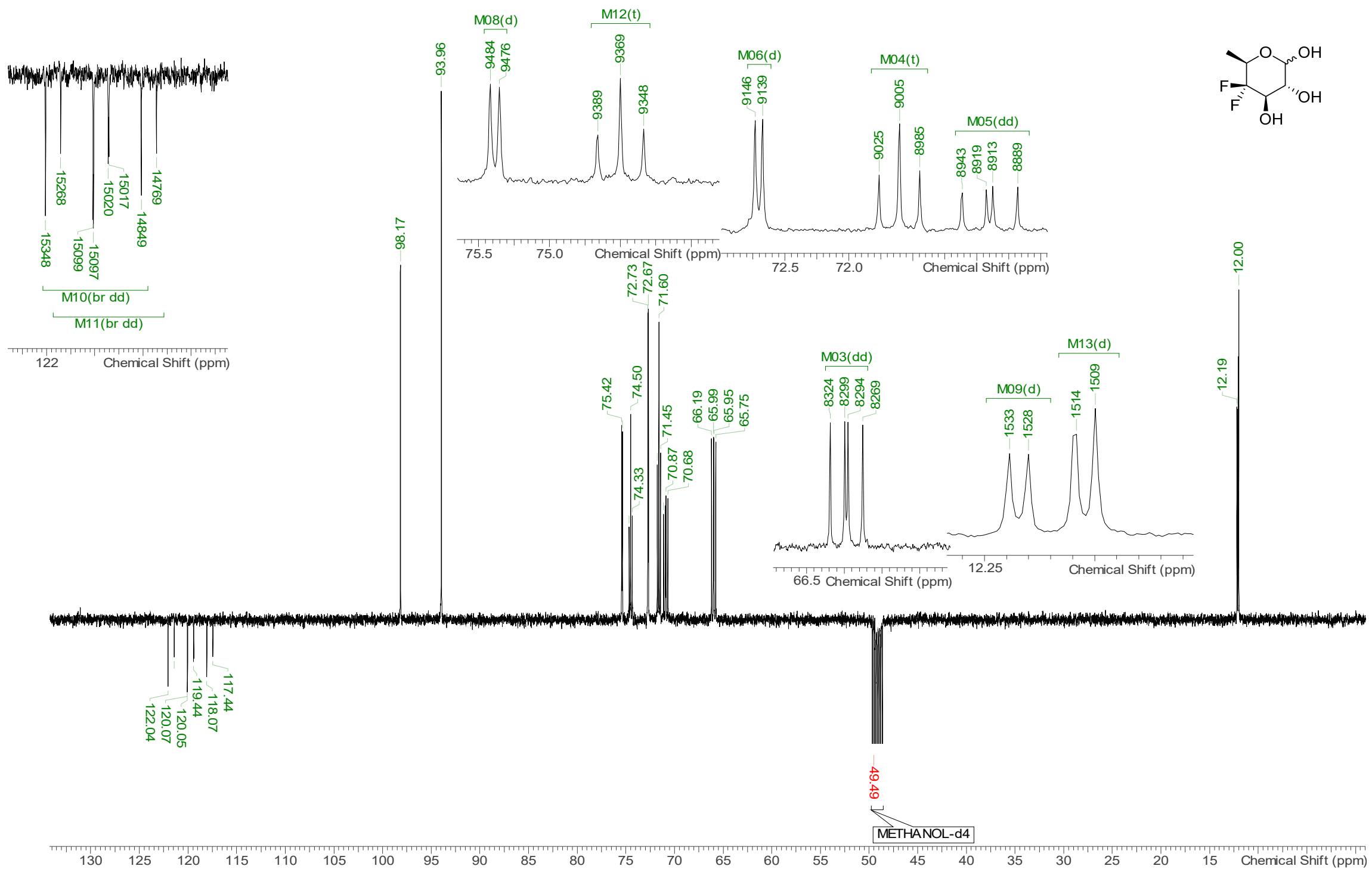


S 82

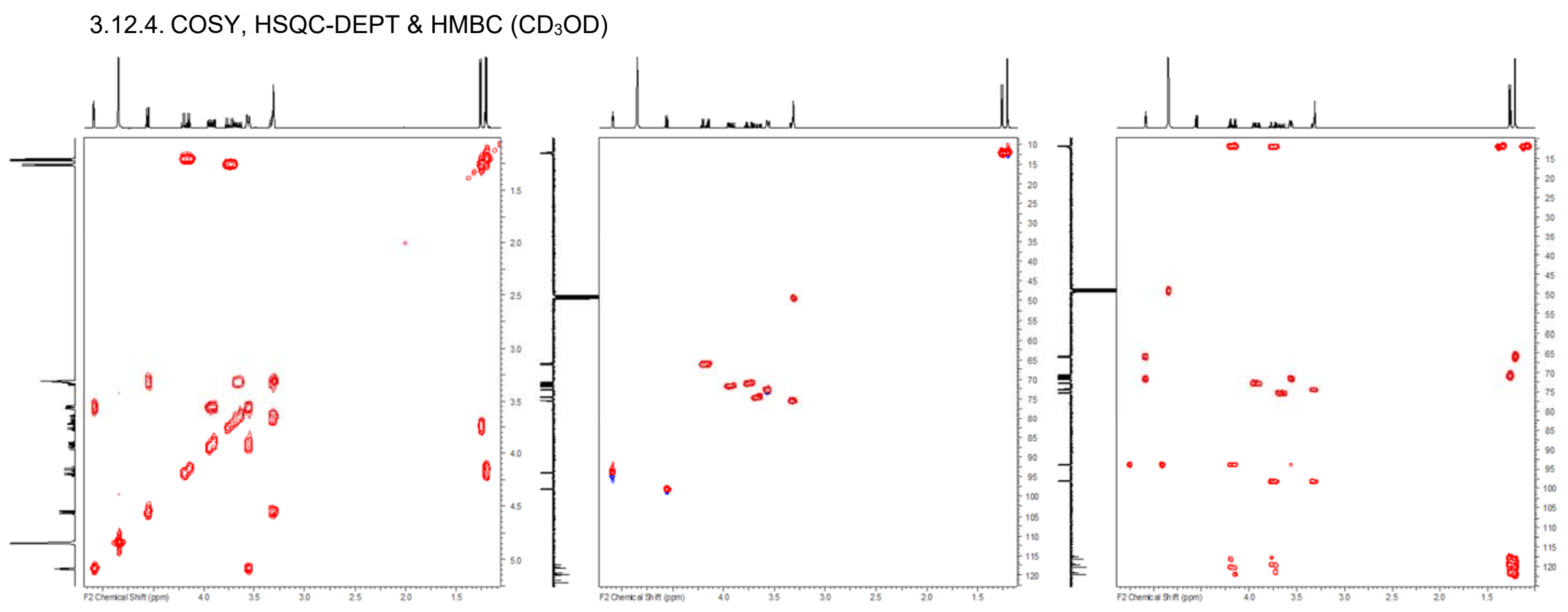




\subsection{5. ${ }^{19} \mathrm{~F}$ NMR $\left(470 \mathrm{MHz}, \mathrm{CD}_{3} \mathrm{OD}\right)$}

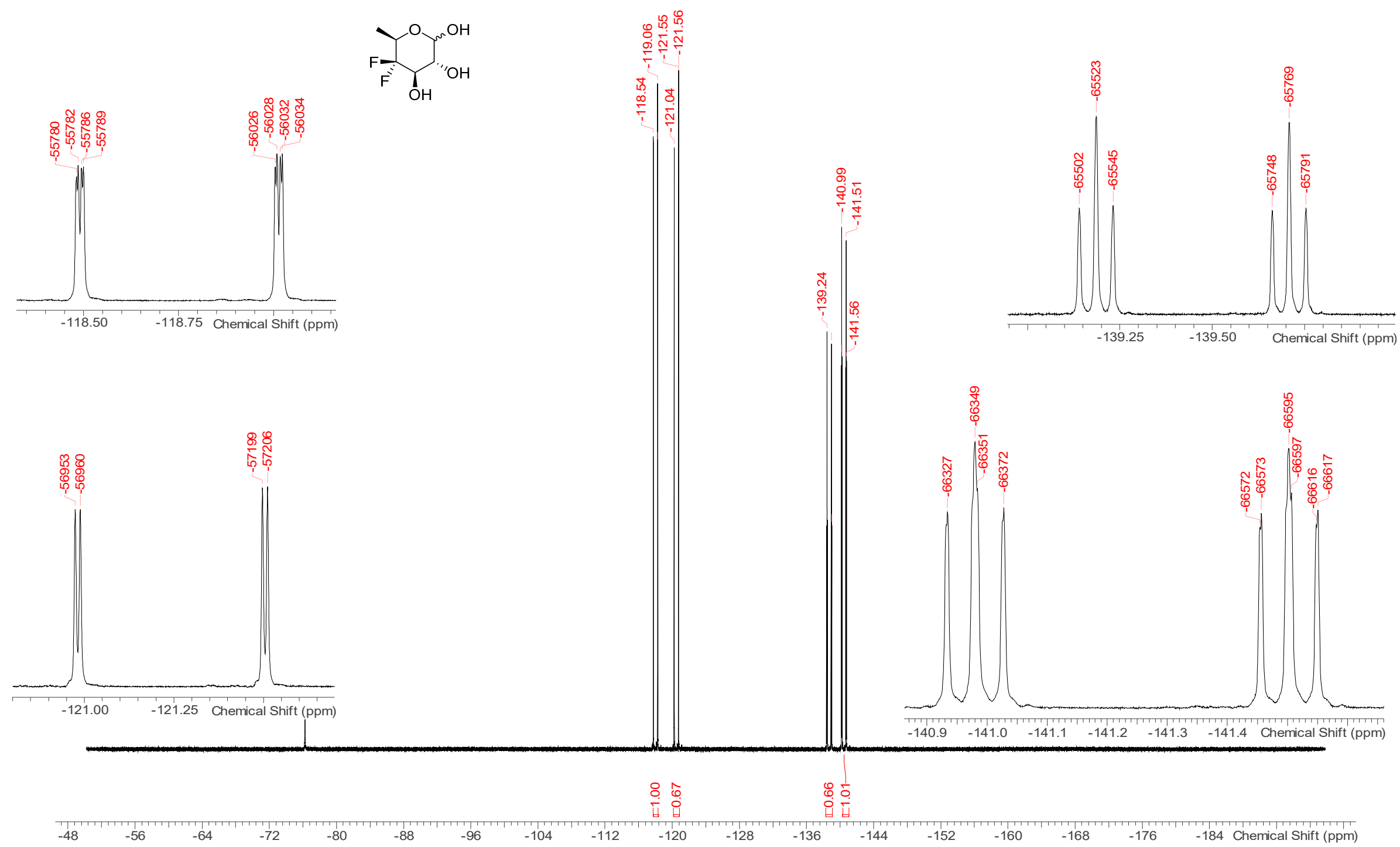


3.12.6. ${ }^{19} \mathrm{~F}\left\{{ }^{1} \mathrm{H}\right\} \mathrm{NMR}\left(470 \mathrm{MHz}, \mathrm{CD}_{3} \mathrm{OD}\right)$

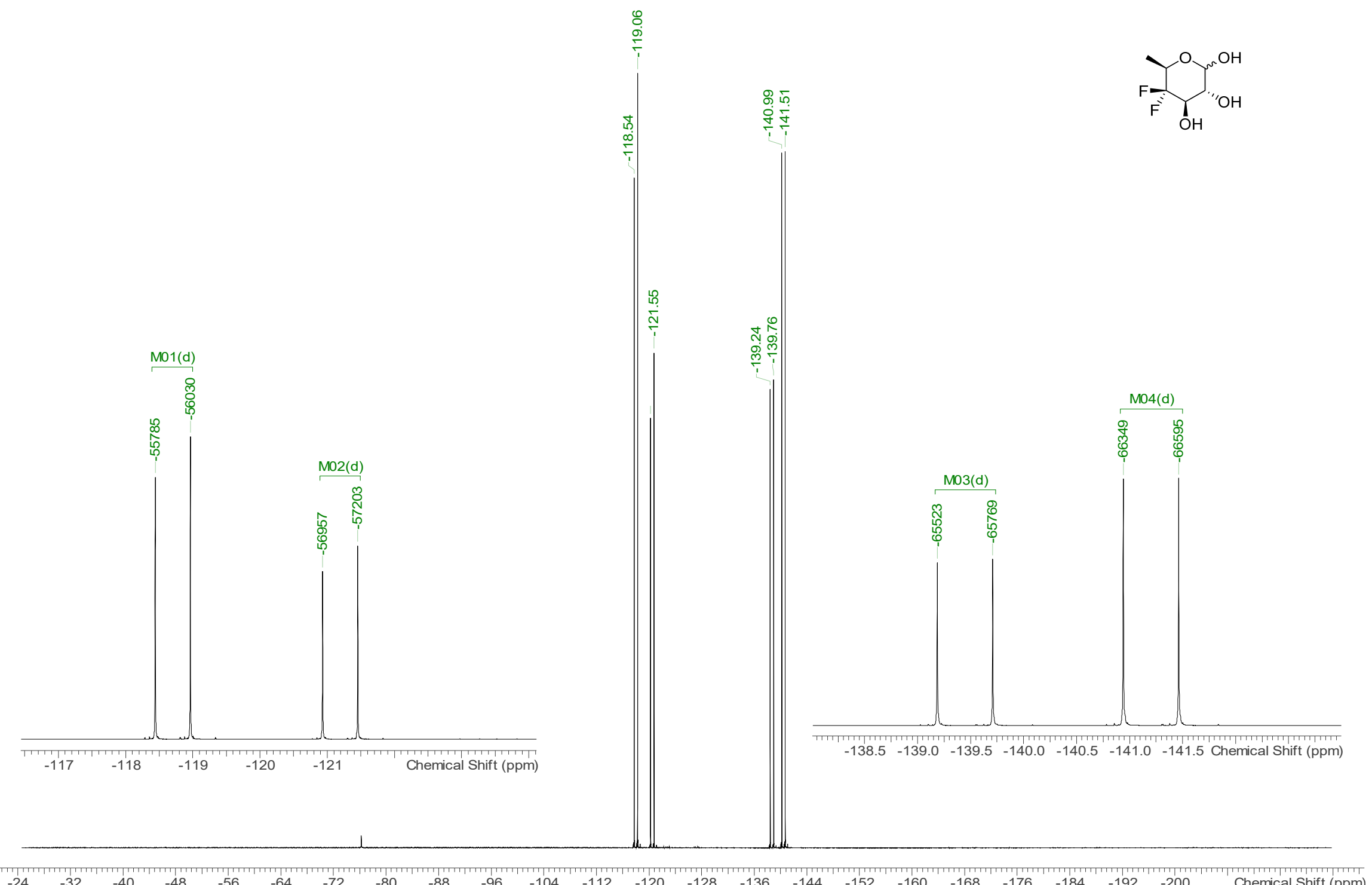




\subsection{1,2,3-Tri-O-acetyl-4,6-dideoxy-4,4-difluoro-D-xylo-hexopyranoside (6b)}

3.13.1. ${ }^{1} \mathrm{H} \mathrm{NMR}\left(400 \mathrm{MHz}, \mathrm{CDCl}_{3}\right)$

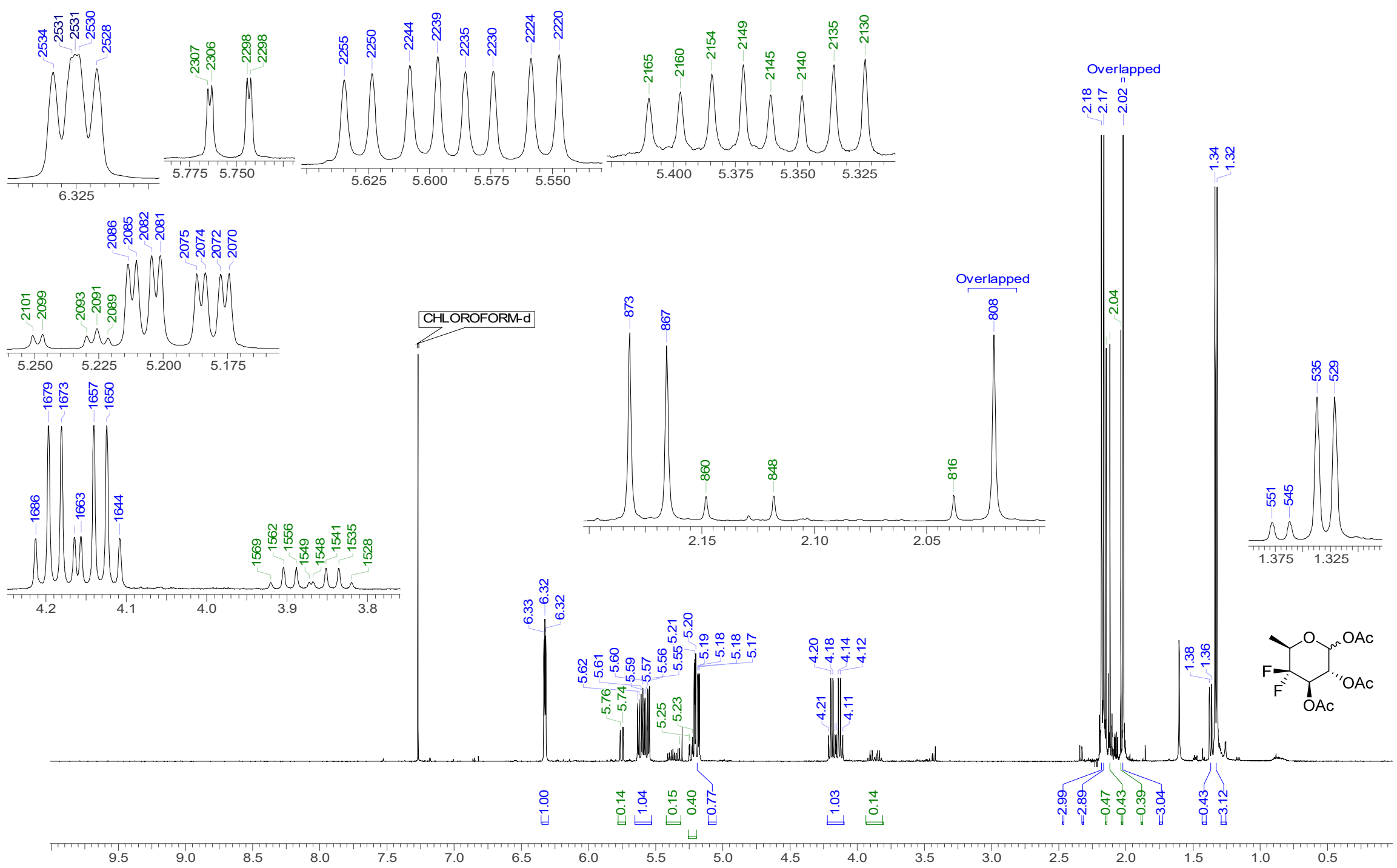


3.13.2. ${ }^{13} \mathrm{C}\left\{{ }^{1} \mathrm{H}\right\}$ NMR $\left(101 \mathrm{MHz}, \mathrm{CDCl}_{3}\right)$

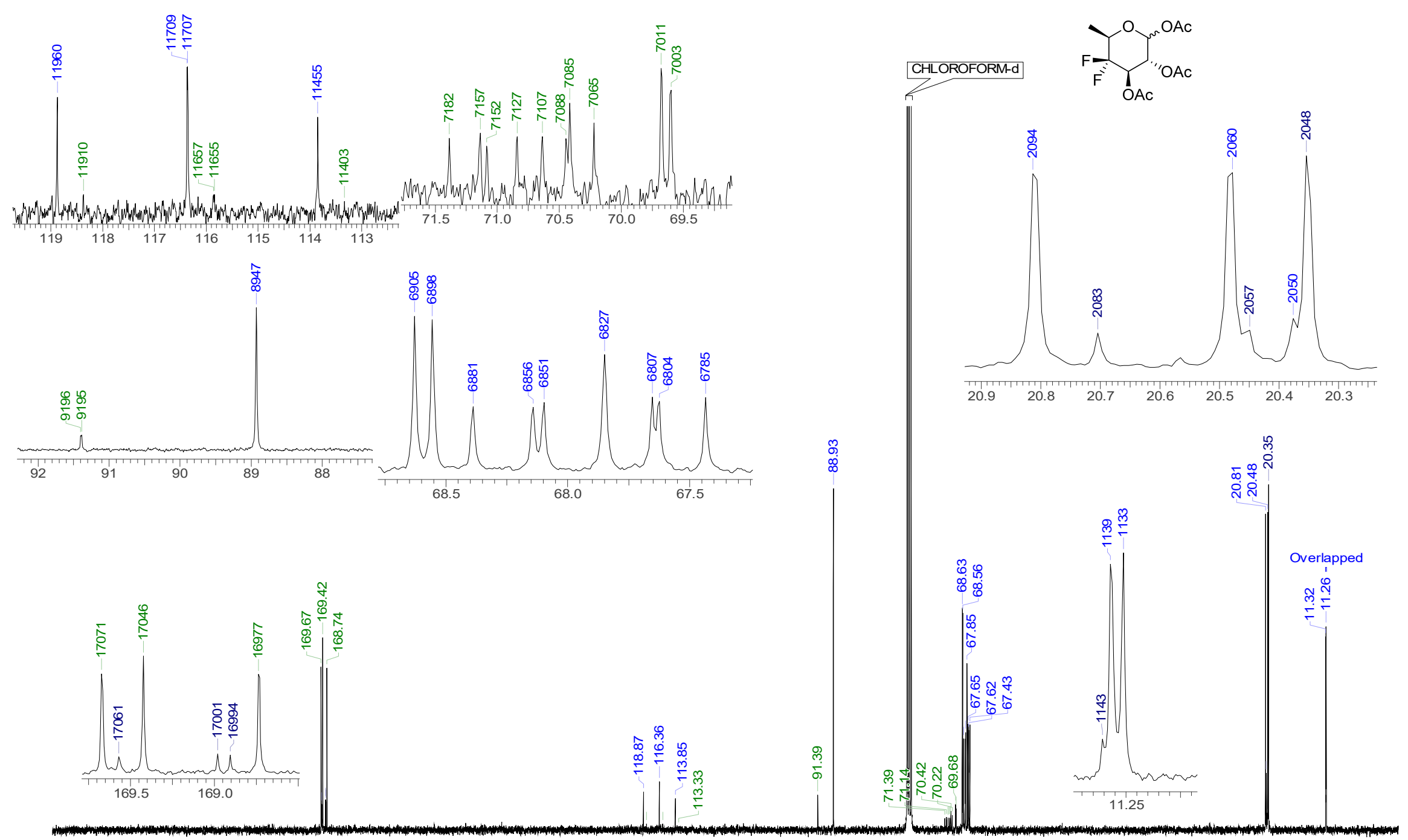

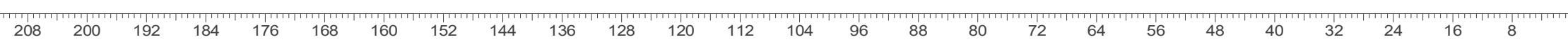




$$
t
$$


3.13.4. ${ }^{19} \mathrm{~F}$ NMR (376 MHz, $\mathrm{CDCl}_{3}$ )

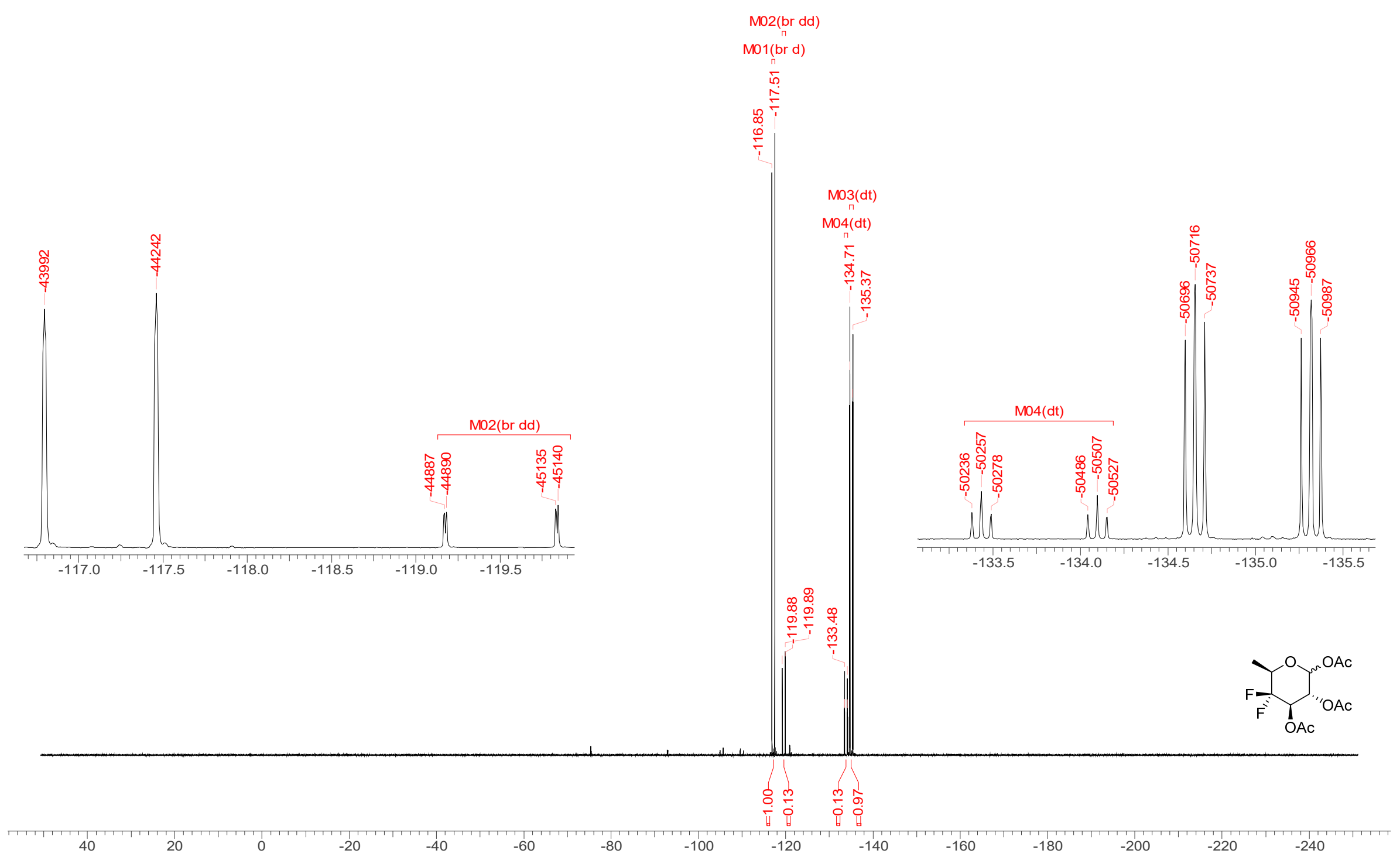


S 89

3.13.5. ${ }^{19} \mathrm{~F}\left\{{ }^{1} \mathrm{H}\right\} \operatorname{NMR}\left(376 \mathrm{MHz}, \mathrm{CDCl}_{3}\right)$
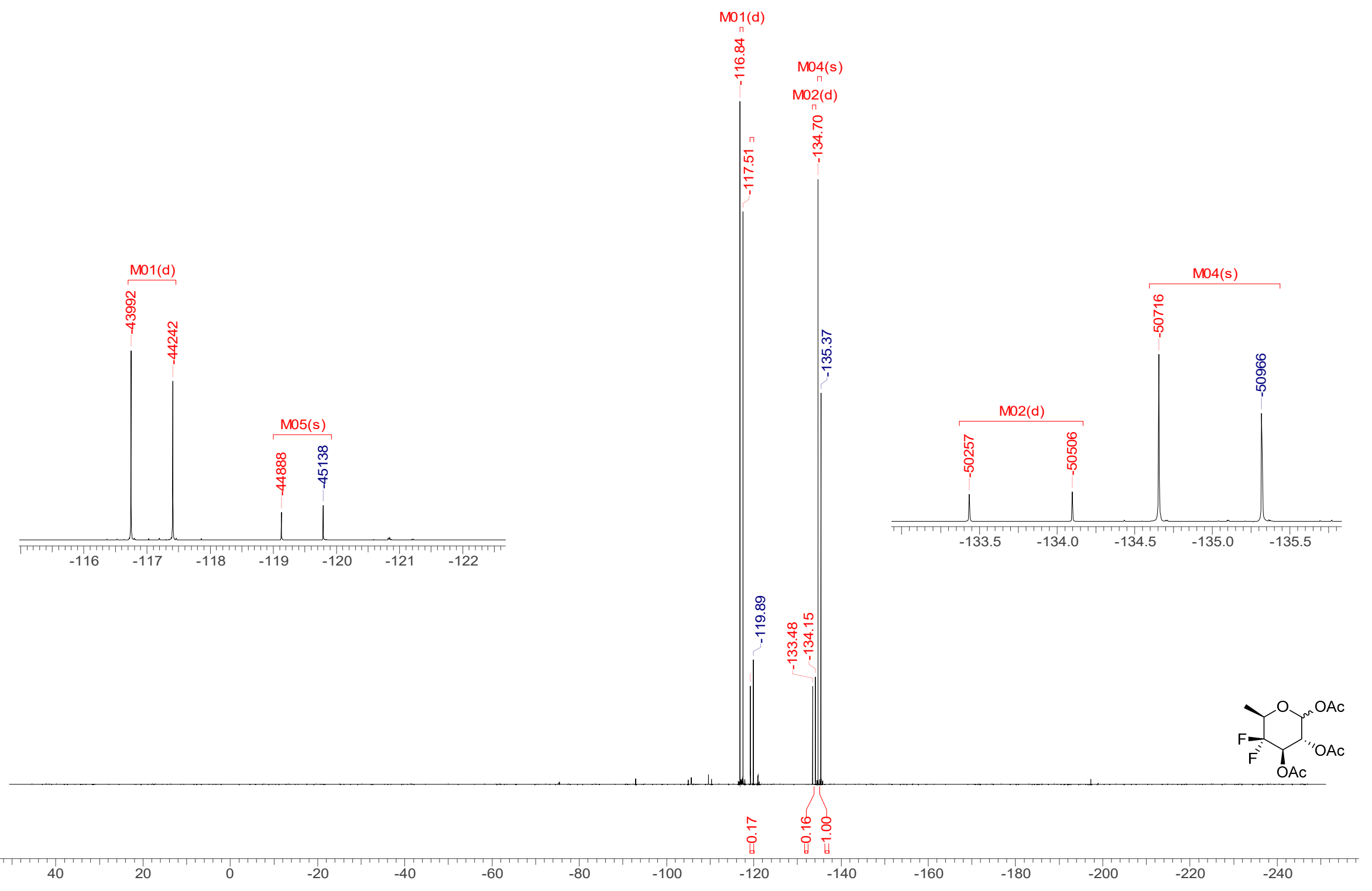
3.14. 1,1,2,3,5-Penta-O-acetyl-4,6-dideoxy-4,4-difluoro-D-xylo-hexose (6c).

3.14.1. ${ }^{1} \mathrm{H} \mathrm{NMR}\left(400 \mathrm{MHz}, \mathrm{CDCl}_{3}\right)$
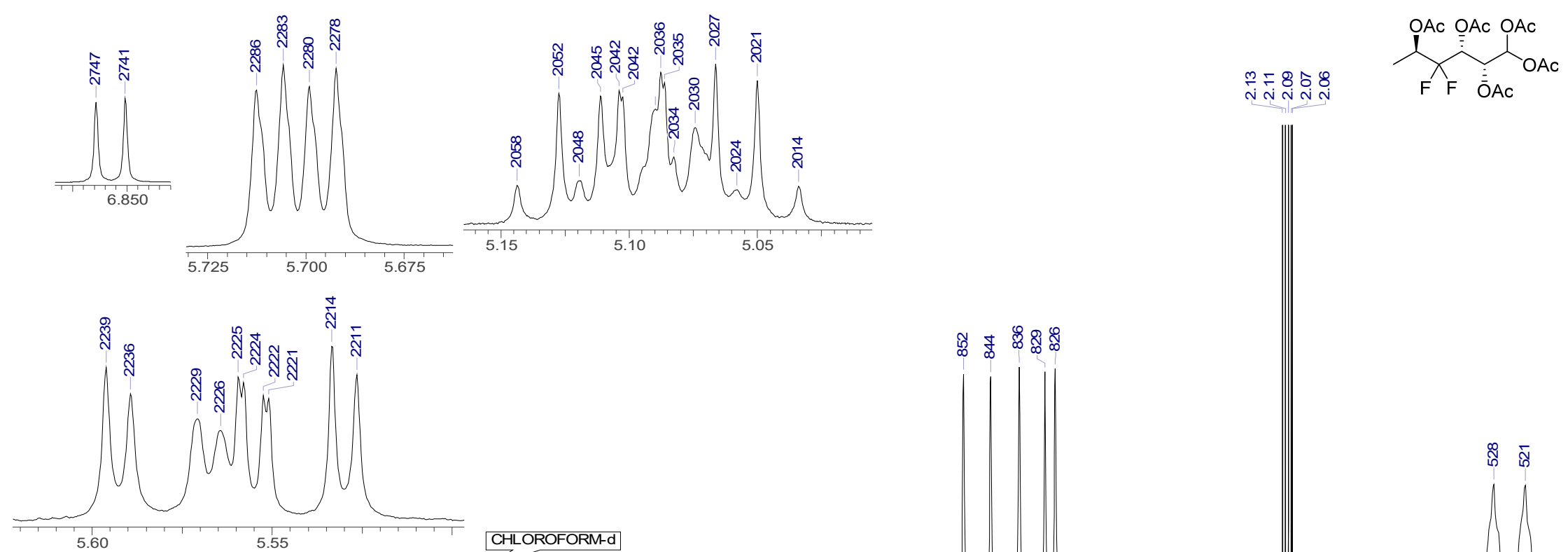

CHLOROFORM-d
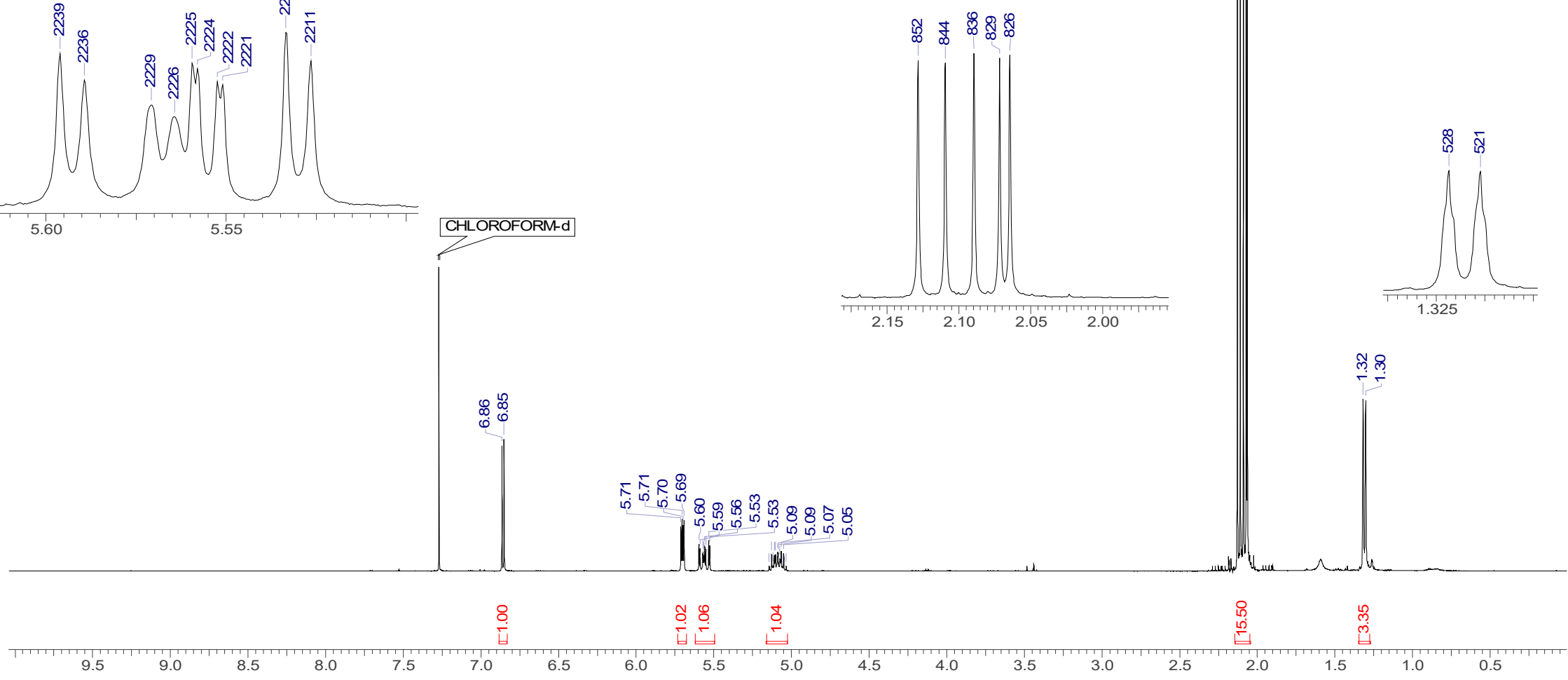
3.14.2. ${ }^{13} \mathrm{C}\left\{{ }^{1} \mathrm{H}\right\} \mathrm{NMR}\left(101 \mathrm{MHz}, \mathrm{CDCl}_{3}\right)$

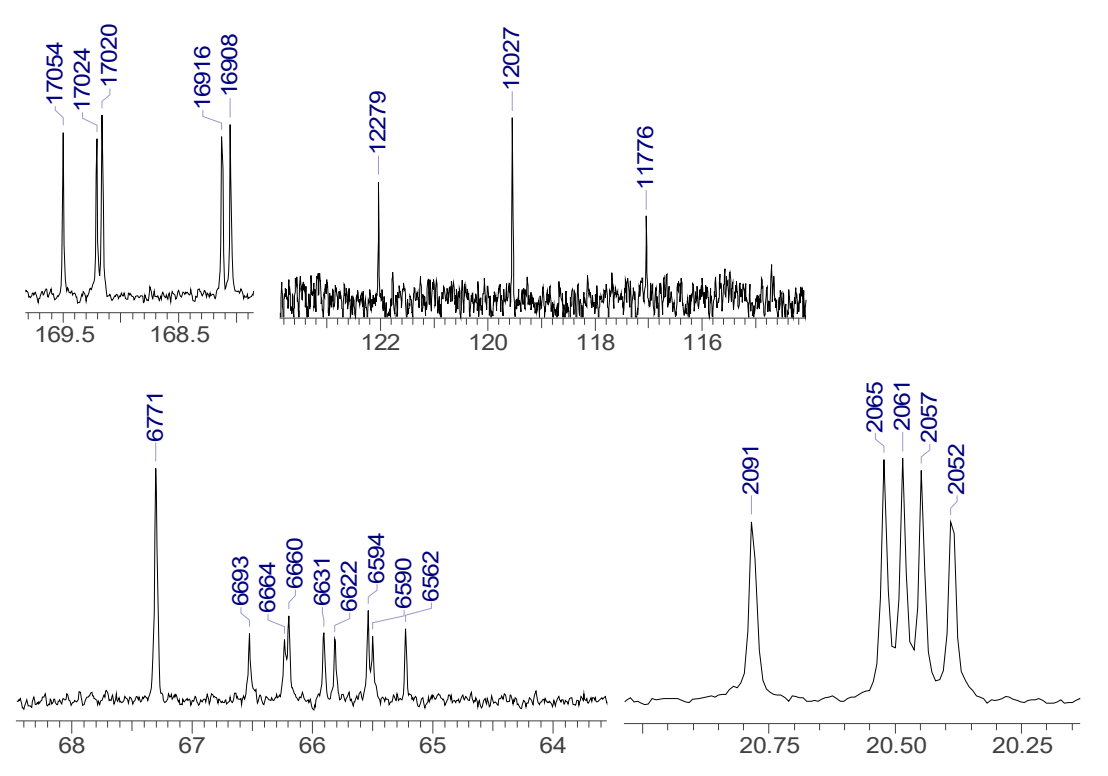

CHLOROFORM-d

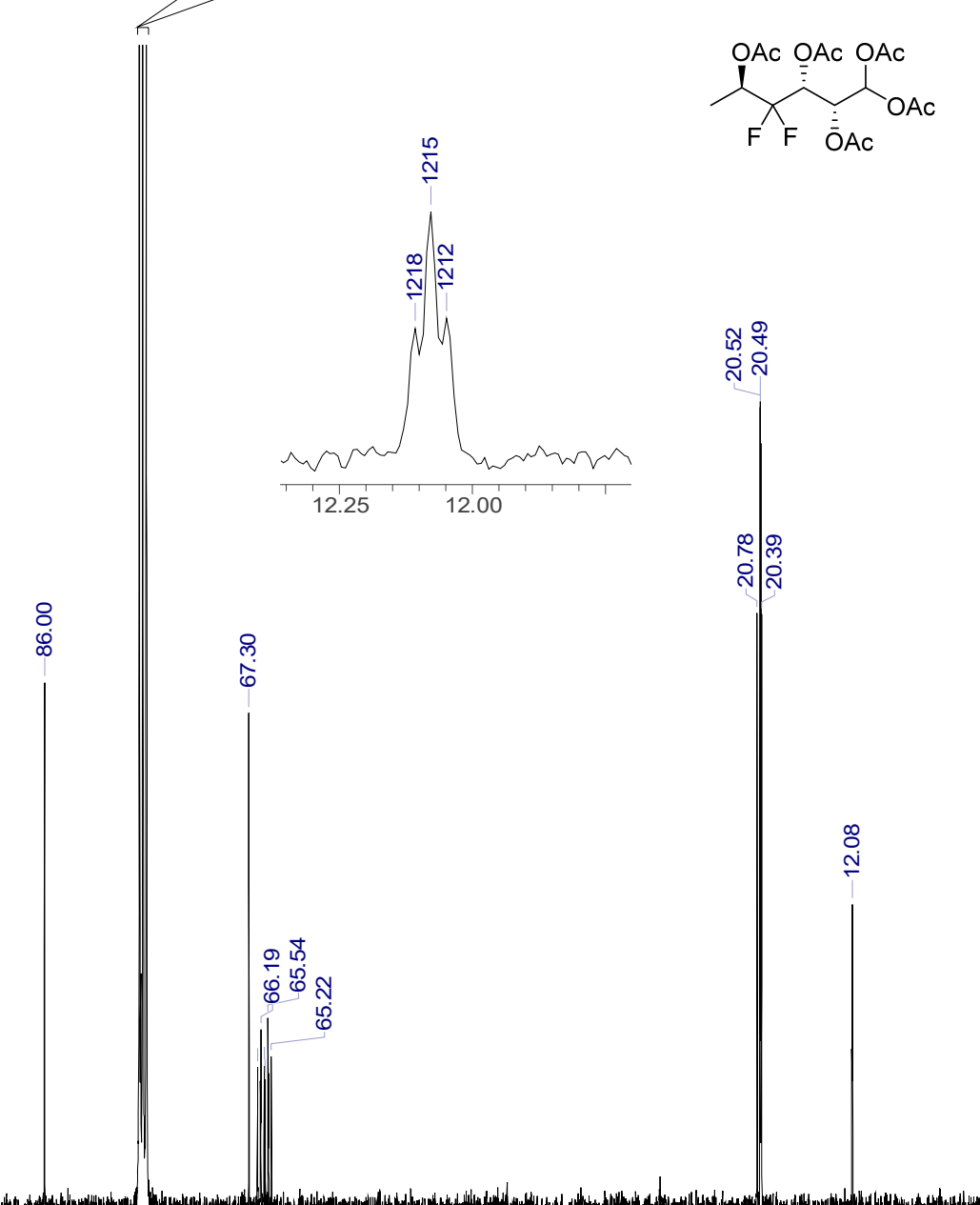

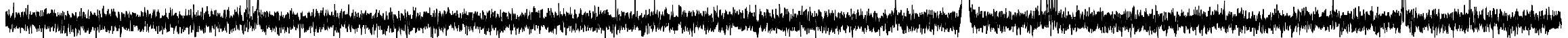

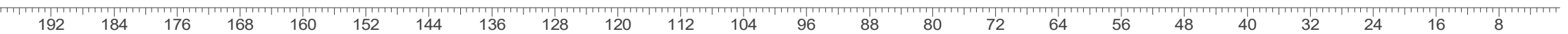


3.14.3. COSY, $\mathrm{HSQC} \& \mathrm{HMBC}\left(\mathrm{CDCl}_{3}\right)$

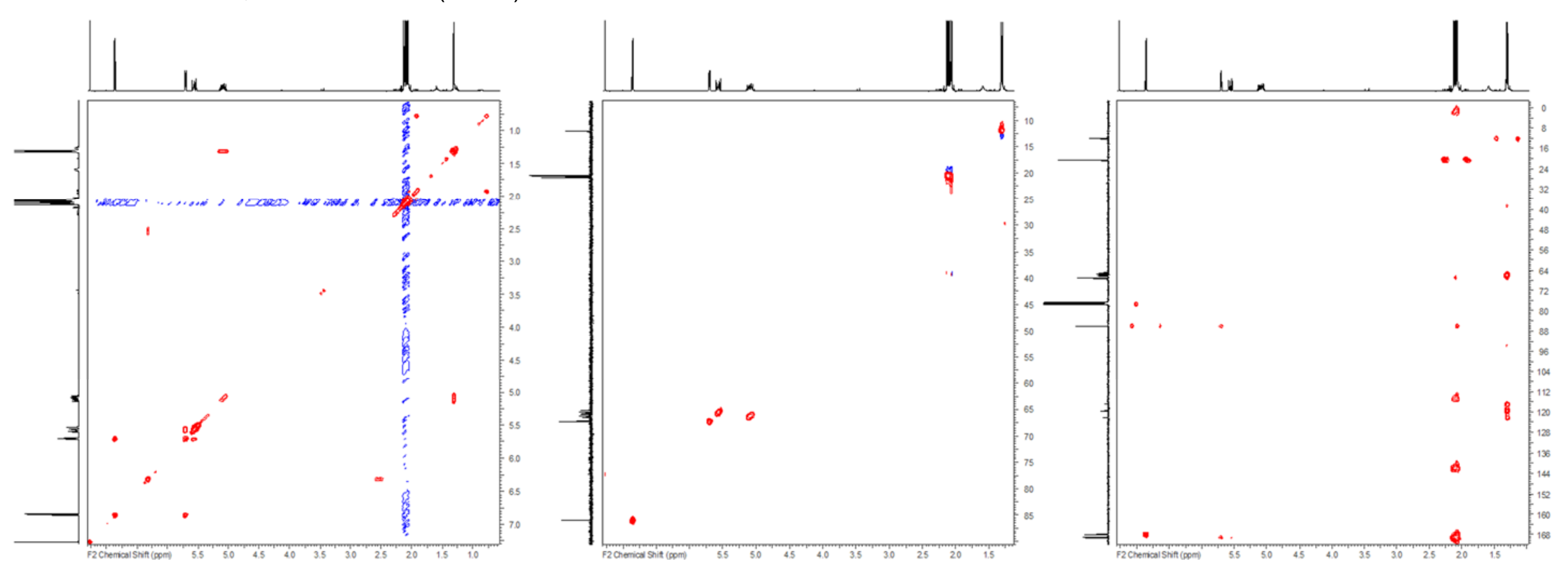


3.14.4. ${ }^{19} \mathrm{~F}$ NMR (376 MHz, $\mathrm{CDCl}_{3}$ )
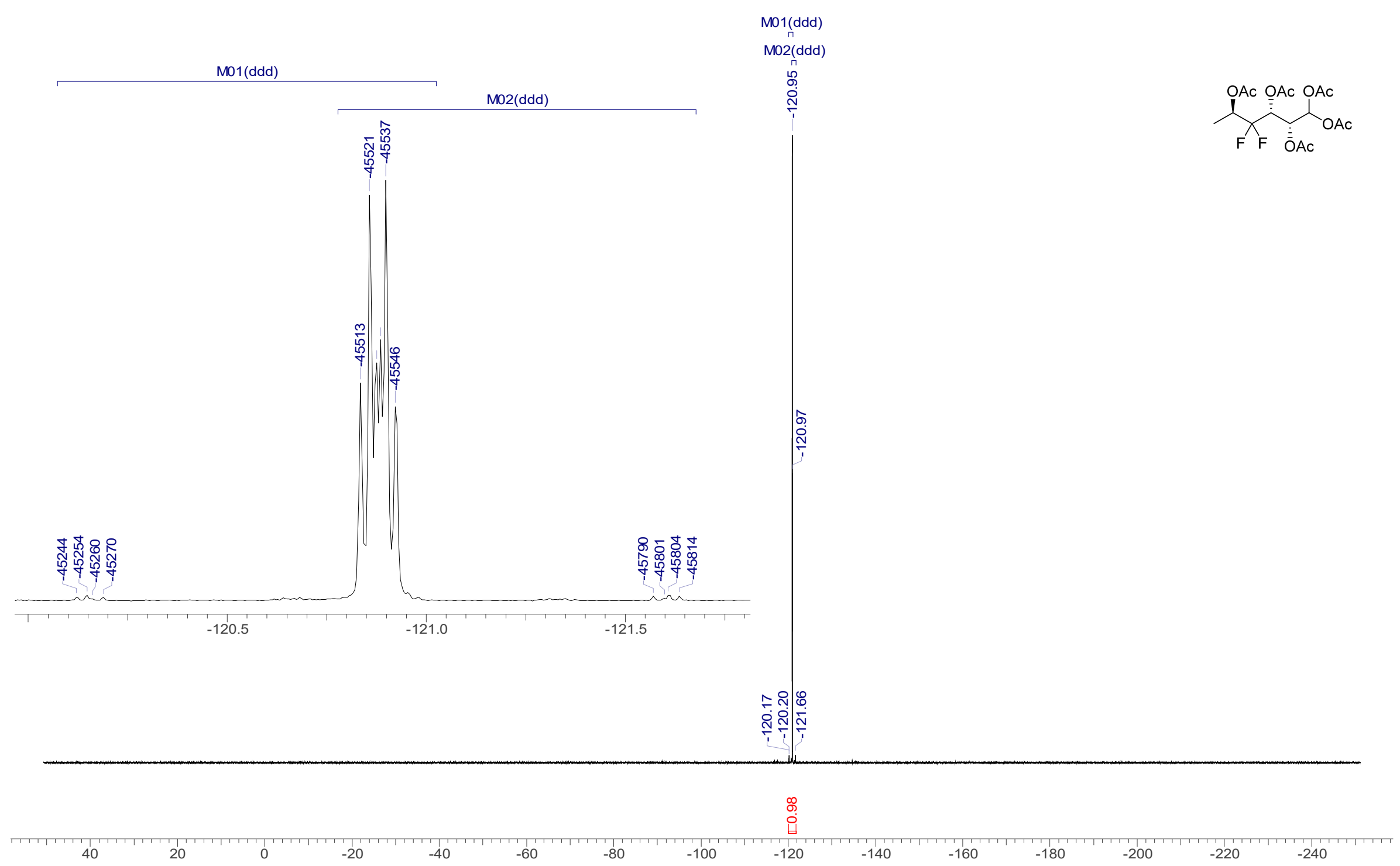
3.14.5. ${ }^{19} \mathrm{~F}\left\{{ }^{1} \mathrm{H}\right\} \mathrm{NMR}\left(376 \mathrm{MHz}, \mathrm{CDCl}_{3}\right)$

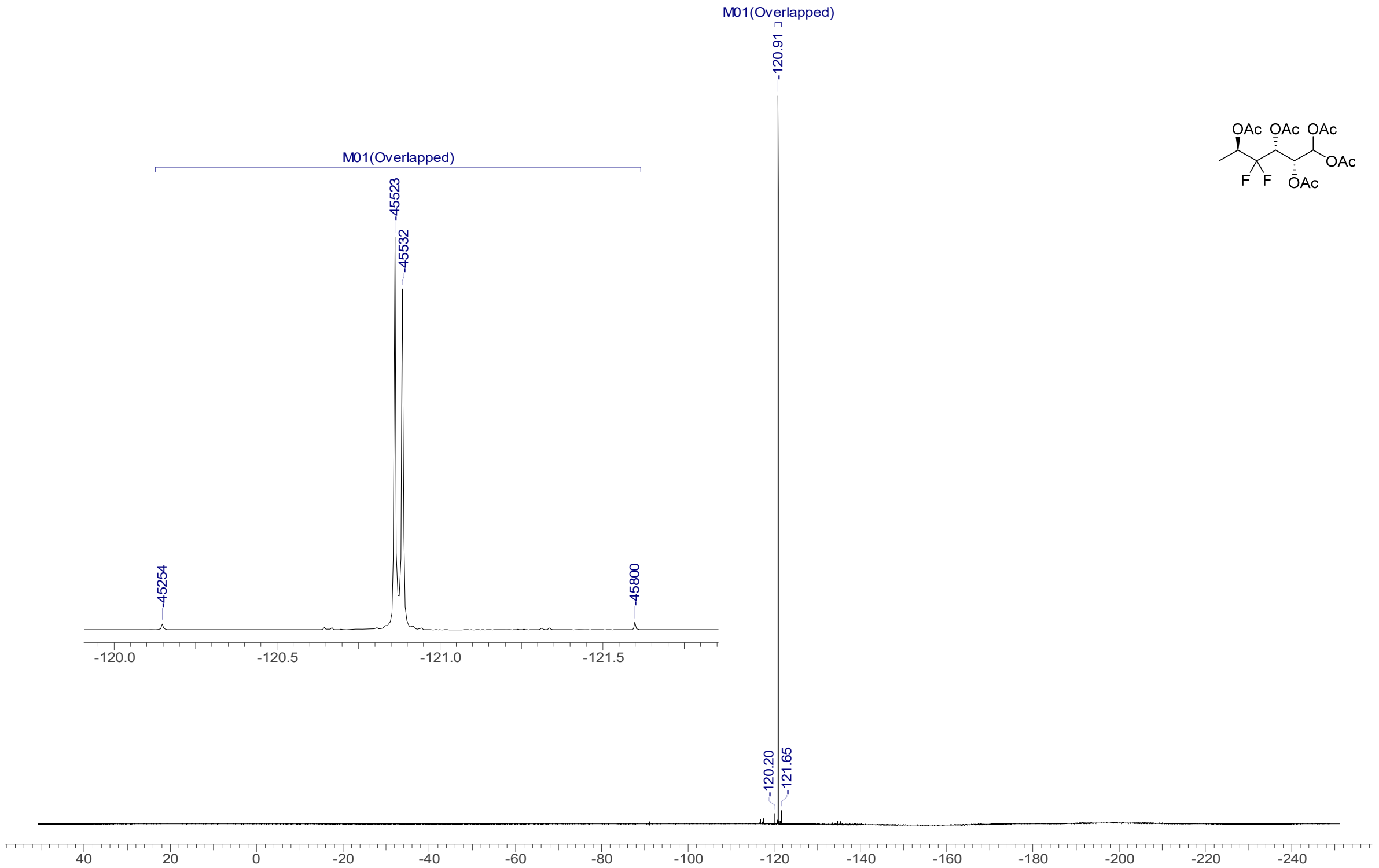




\subsection{4,6-Dideoxy-6,6-difluoro-D-xylo-hexopyranose (7a)}

3.15.1. ${ }^{1} \mathrm{H}$ NMR $\left(500 \mathrm{MHz}, \mathrm{CD}_{3} \mathrm{OD}\right)$

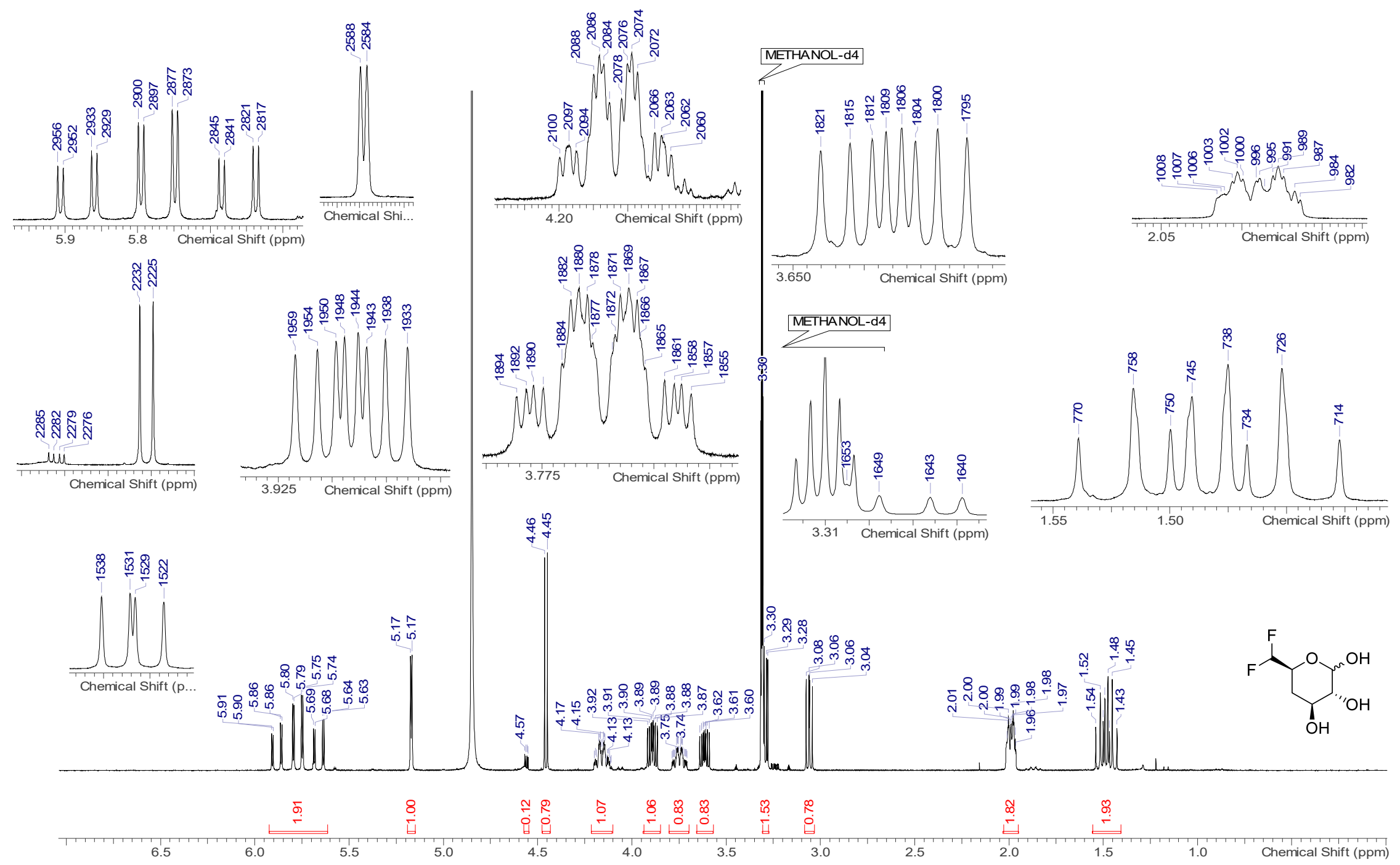




\subsection{2. $1 \mathrm{H}\{19 \mathrm{~F}\} \mathrm{NMR}\left(500 \mathrm{MHz}, \mathrm{CD}_{3} \mathrm{OD}\right)$}
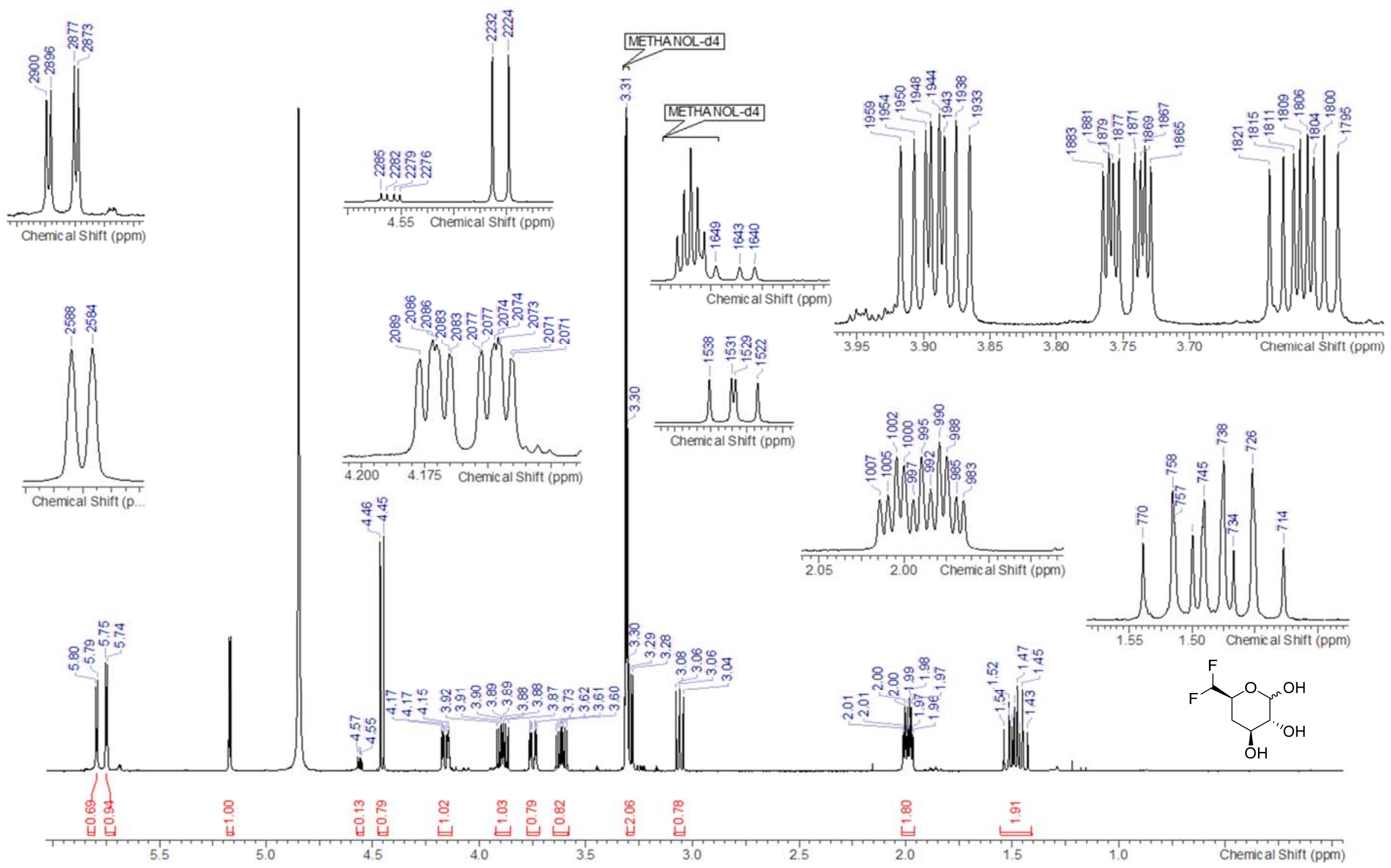
3.15.3. ${ }^{13} \mathrm{C}\left\{{ }^{1} \mathrm{H}\right\}$ NMR $\left(126 \mathrm{MHz}, \mathrm{CD}_{3} \mathrm{OD}\right)$
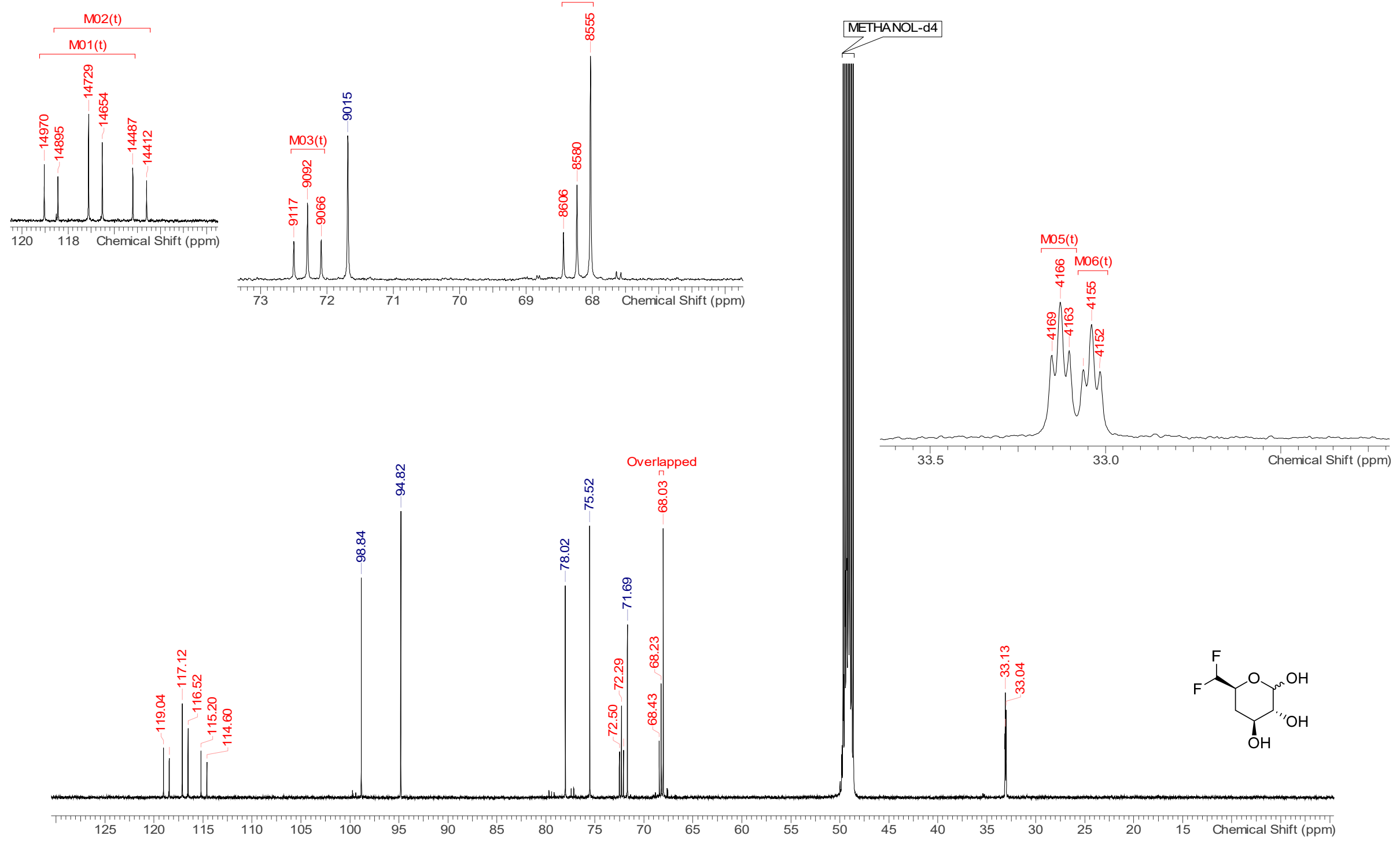
3.15.4. COSY \& HSQC-DEPT $\left(\mathrm{CD}_{3} \mathrm{OD}\right)$

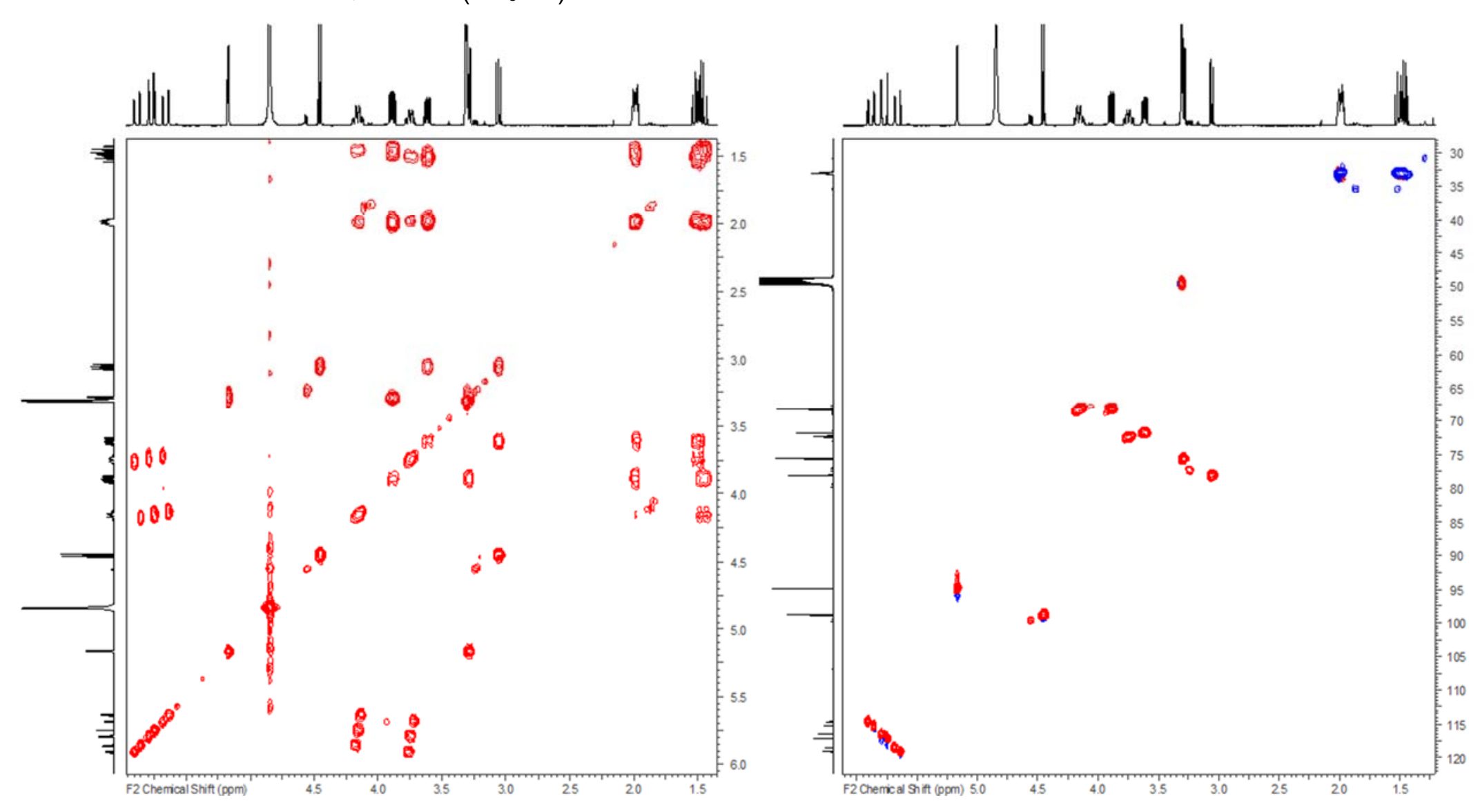


3.15.5. ${ }^{19} \mathrm{~F} \mathrm{NMR}\left(470 \mathrm{MHz}, \mathrm{CD}_{3} \mathrm{OD}\right)$

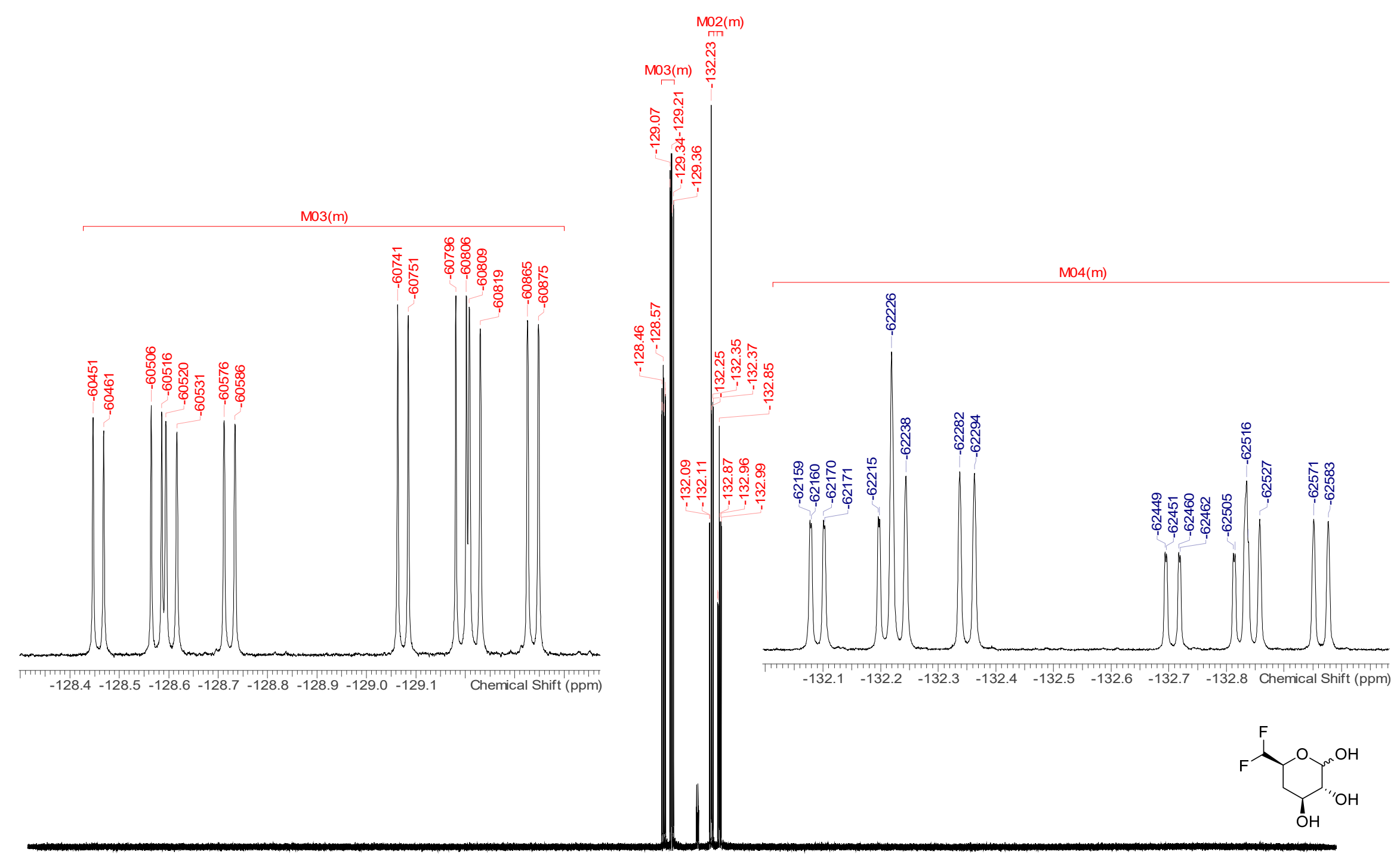

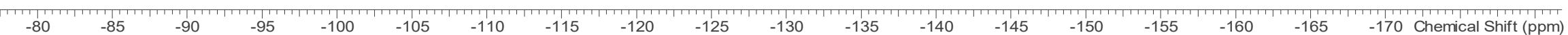


3.15.6. ${ }^{19} \mathrm{~F}\left\{{ }^{1} \mathrm{H}\right\} \mathrm{NMR}\left(470 \mathrm{MHz}, \mathrm{CD}_{3} \mathrm{OD}\right)$

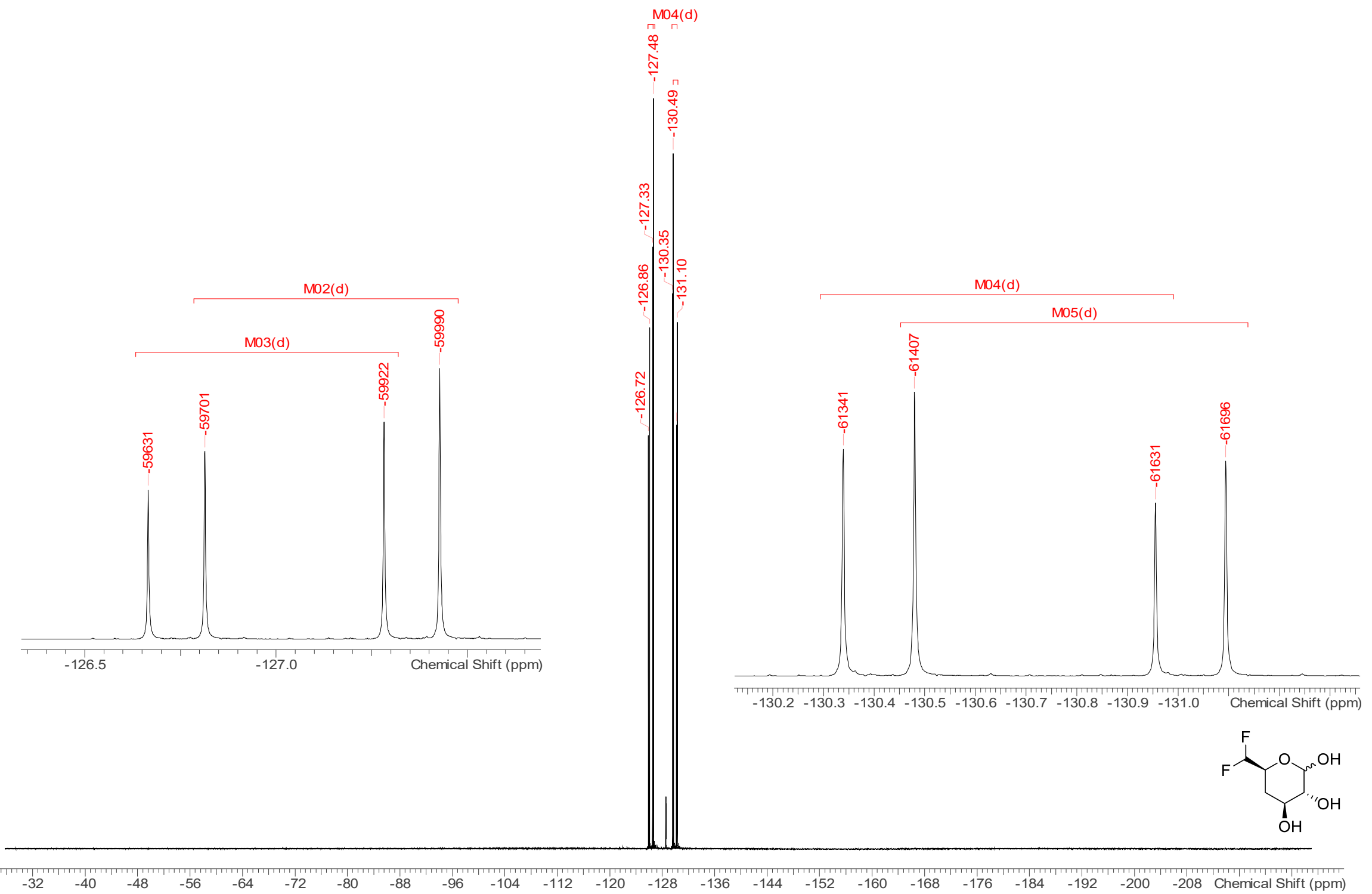


3.15.7. TOCSY @ $\mathrm{H}_{1 \alpha} 5.193$ ppm (500 MHz, CD $\left.{ }_{3} \mathrm{OD}\right)$
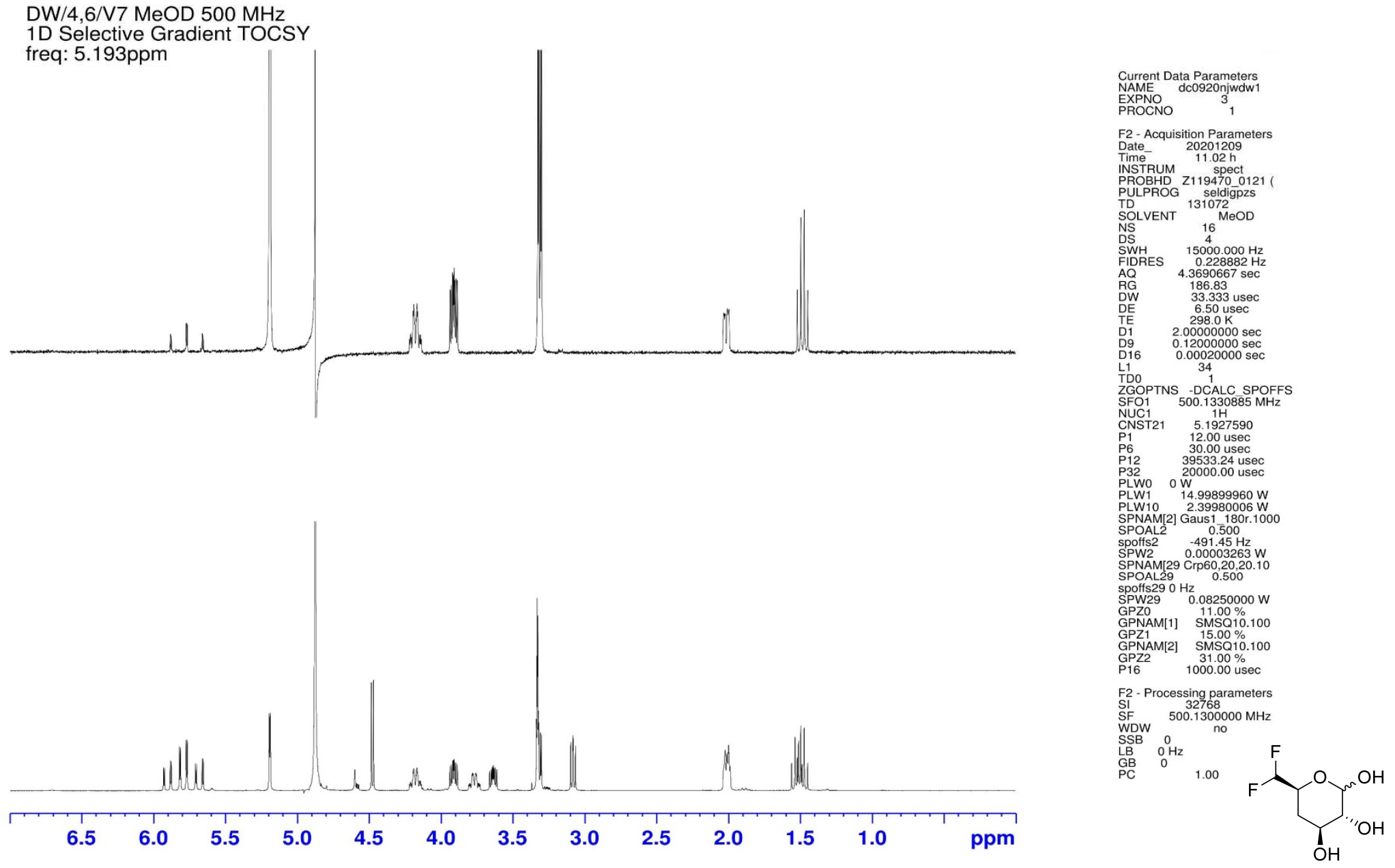
3.15.8. TOCSY @ $\mathrm{H}_{1 \beta} 5.193$ ppm (500 MHz, CD $\left.{ }_{3} \mathrm{OD}\right)$
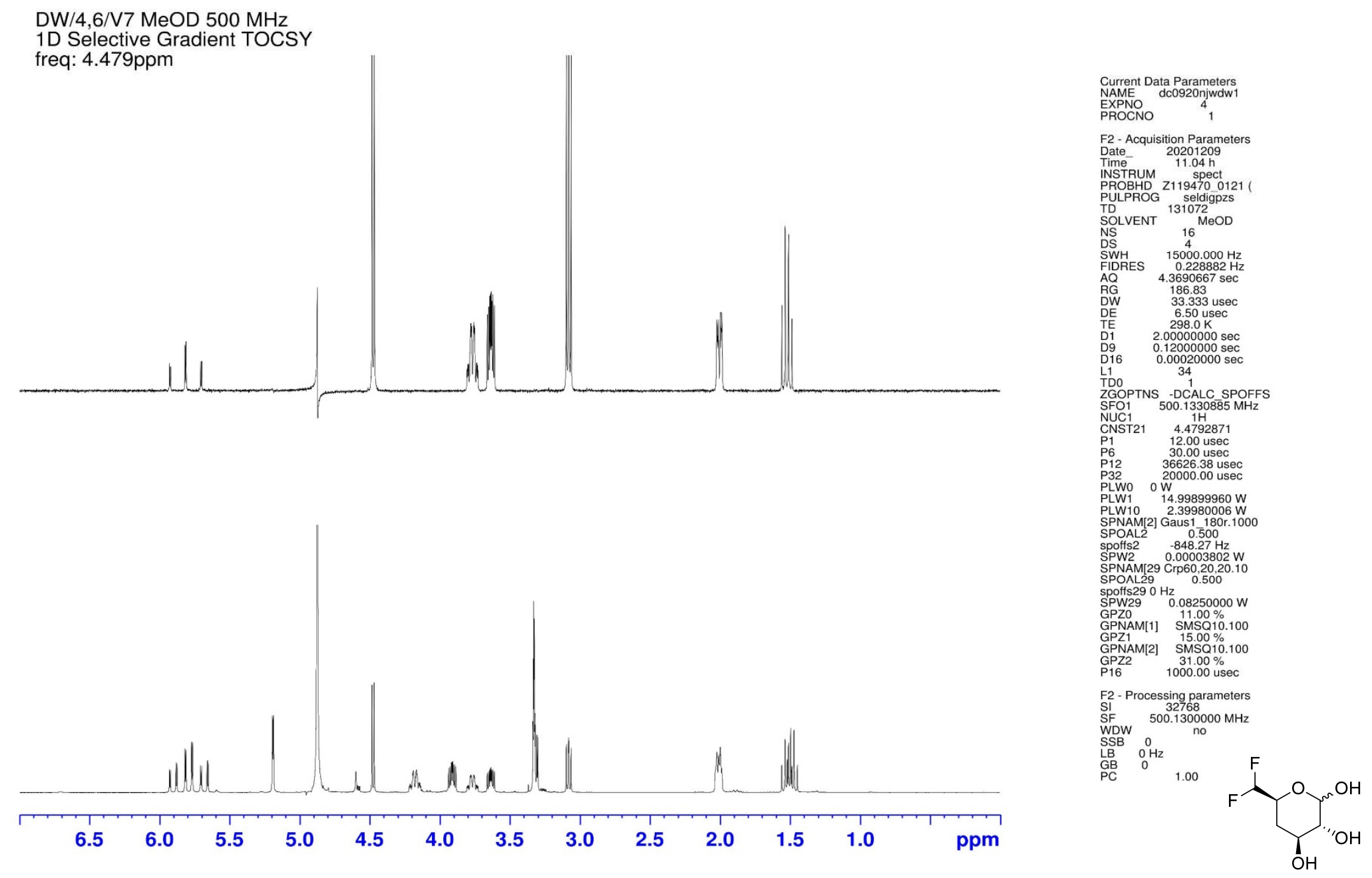
3.16. 1,2,3-Tri-O-acetyl-4,6-dideoxy-6,6-difluoro-D-xylo-hexopyranoside (7b)

3.16.1. ${ }^{1} \mathrm{H}$ NMR $\left(400 \mathrm{MHz}, \mathrm{CDCl}_{3}\right)$

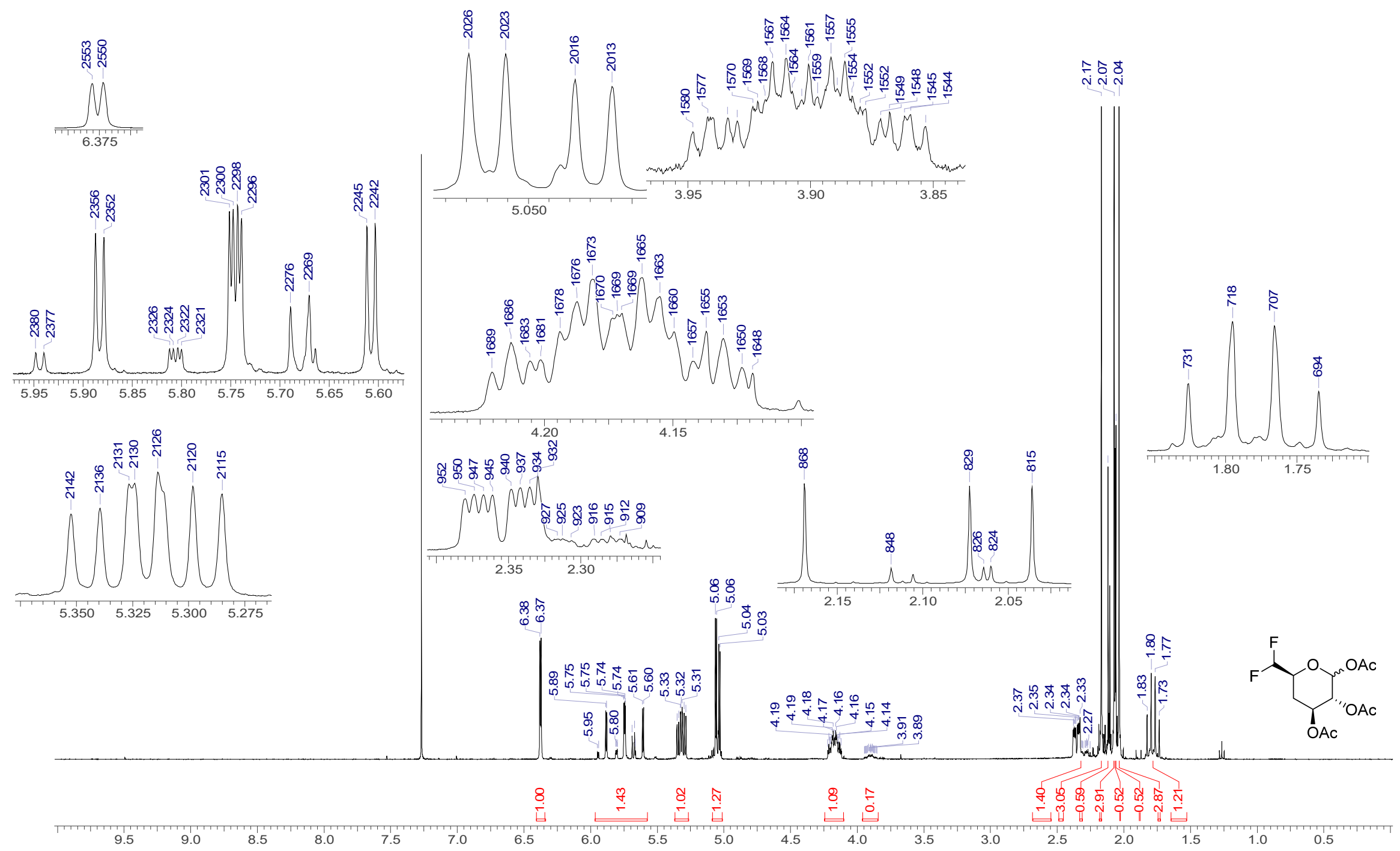


3.16.2. ${ }^{13} \mathrm{C}\left\{{ }^{1} \mathrm{H}\right\}$ NMR $\left(101 \mathrm{MHz}, \mathrm{CDCl}_{3}\right)$
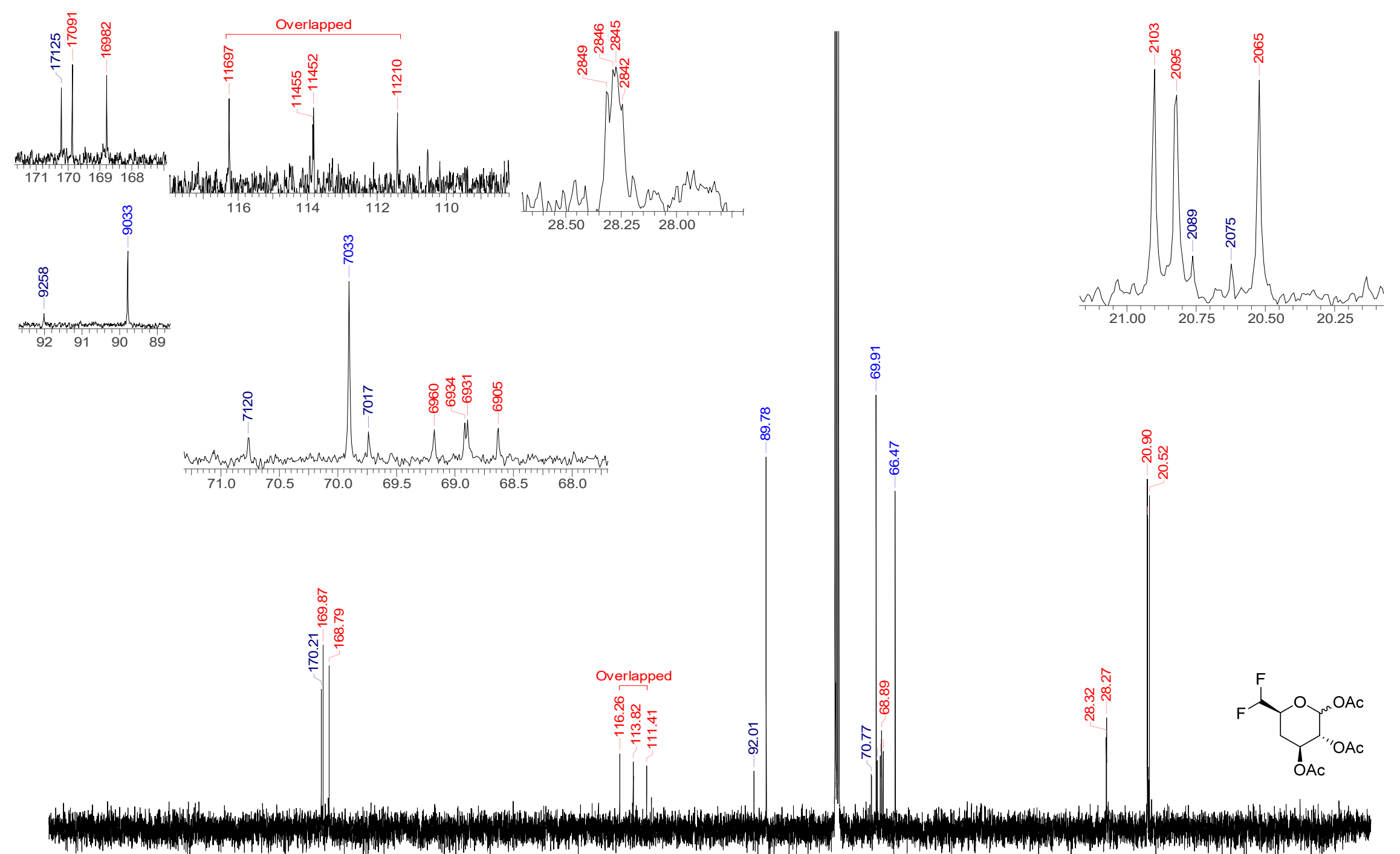

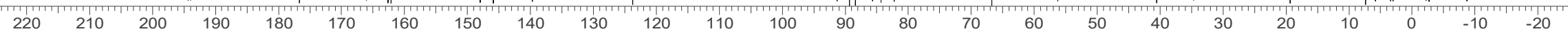


3.16.3. COSY, $\mathrm{HSQC} \& \mathrm{HMBC}\left(\mathrm{CDCl}_{3}\right)$

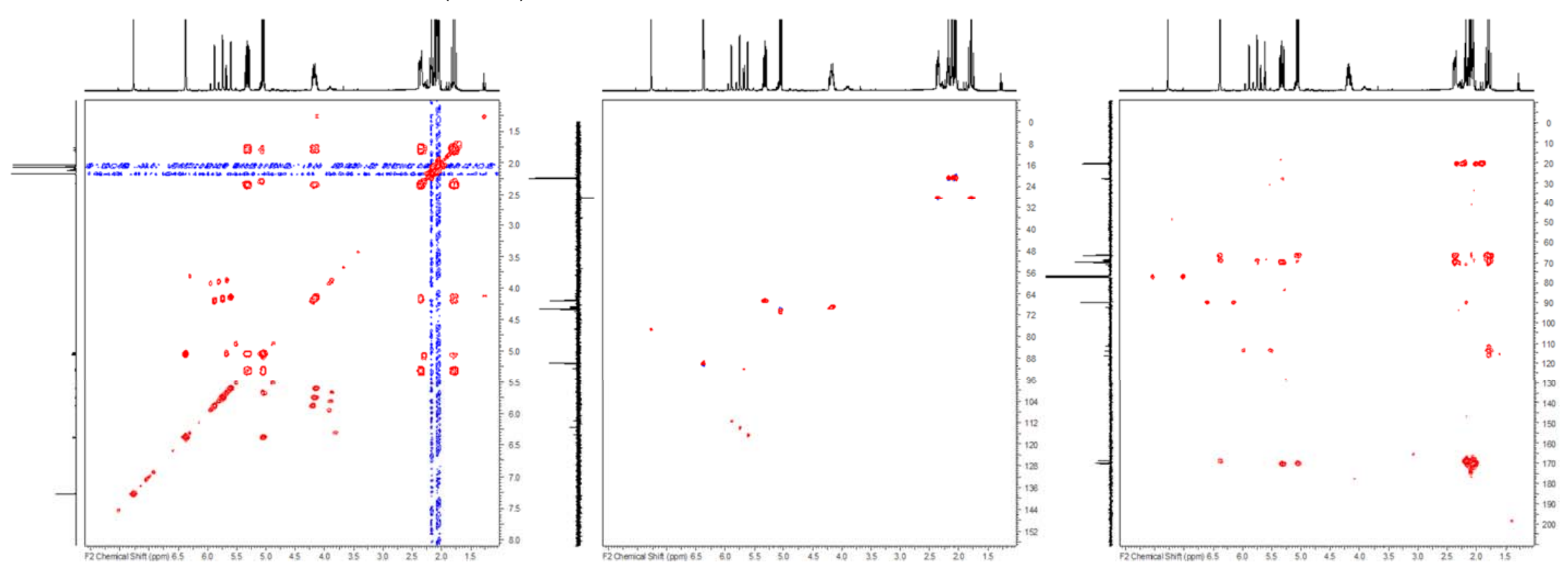


3.16.4. ${ }^{19} \mathrm{~F} \mathrm{NMR}\left(376 \mathrm{MHz}, \mathrm{CDCl}_{3}\right)$
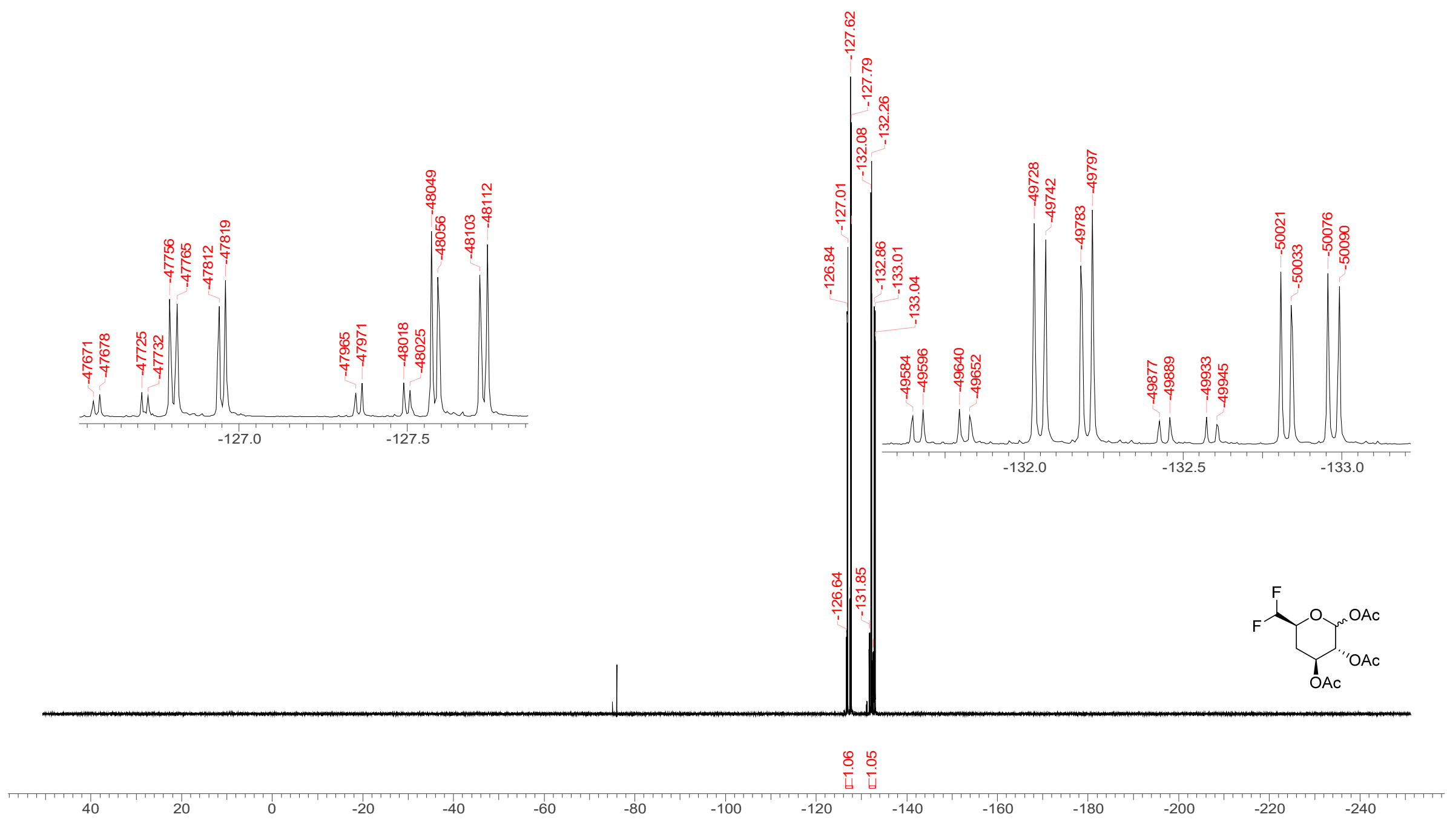
3.16.5. ${ }^{19} \mathrm{~F}\left\{{ }^{1} \mathrm{H}\right\} \mathrm{NMR}\left(376 \mathrm{MHz}, \mathrm{CDCl}_{3}\right)$

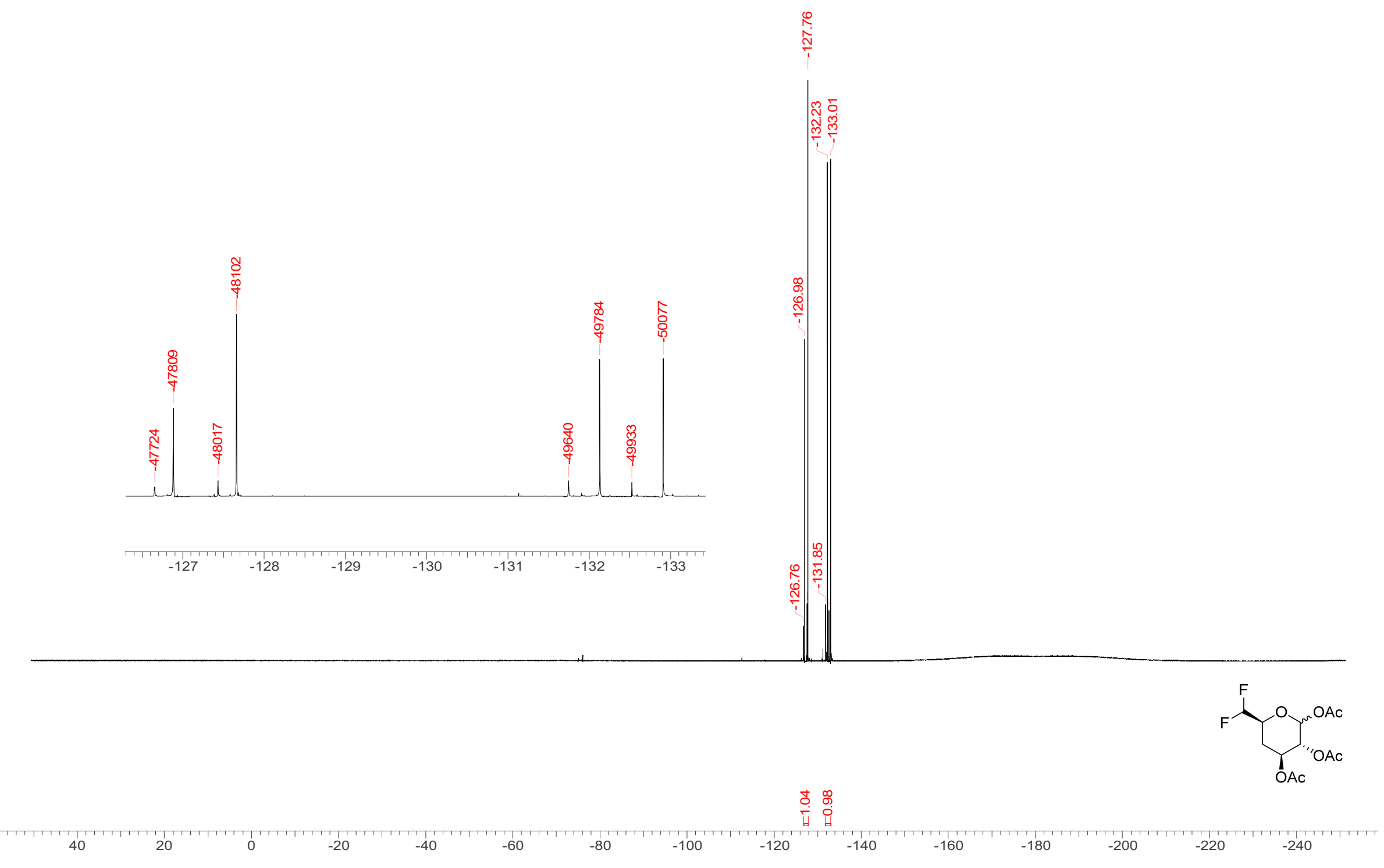


3.17. Methyl 4,6-dideoxy-6-fluoro- $\alpha$-D-xylo-hexopyranoside (10).

3.17.1. ${ }^{1} \mathrm{H} \mathrm{NMR}\left(400 \mathrm{MHz}, \mathrm{CDCl}_{3}\right)$
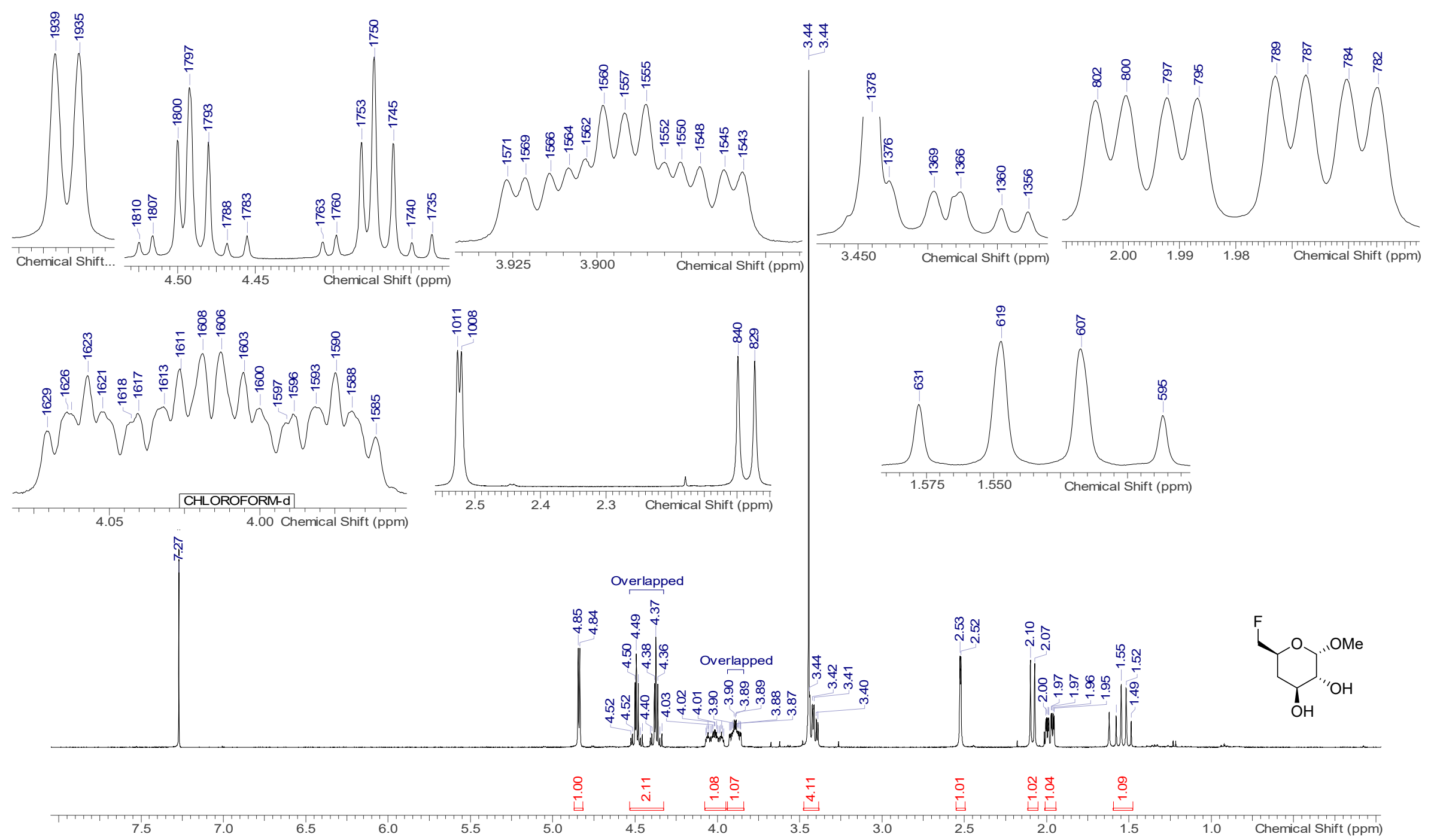
3.17.2. ${ }^{13} \mathrm{C}\left\{{ }^{1} \mathrm{H}\right\}$ NMR $\left(101 \mathrm{MHz}, \mathrm{CDCl}_{3}\right)$

Overlapped

㽞蛋

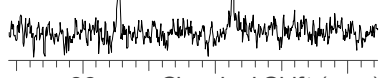

86 Chemical Shift (ppm)
CHLOROFORM-d

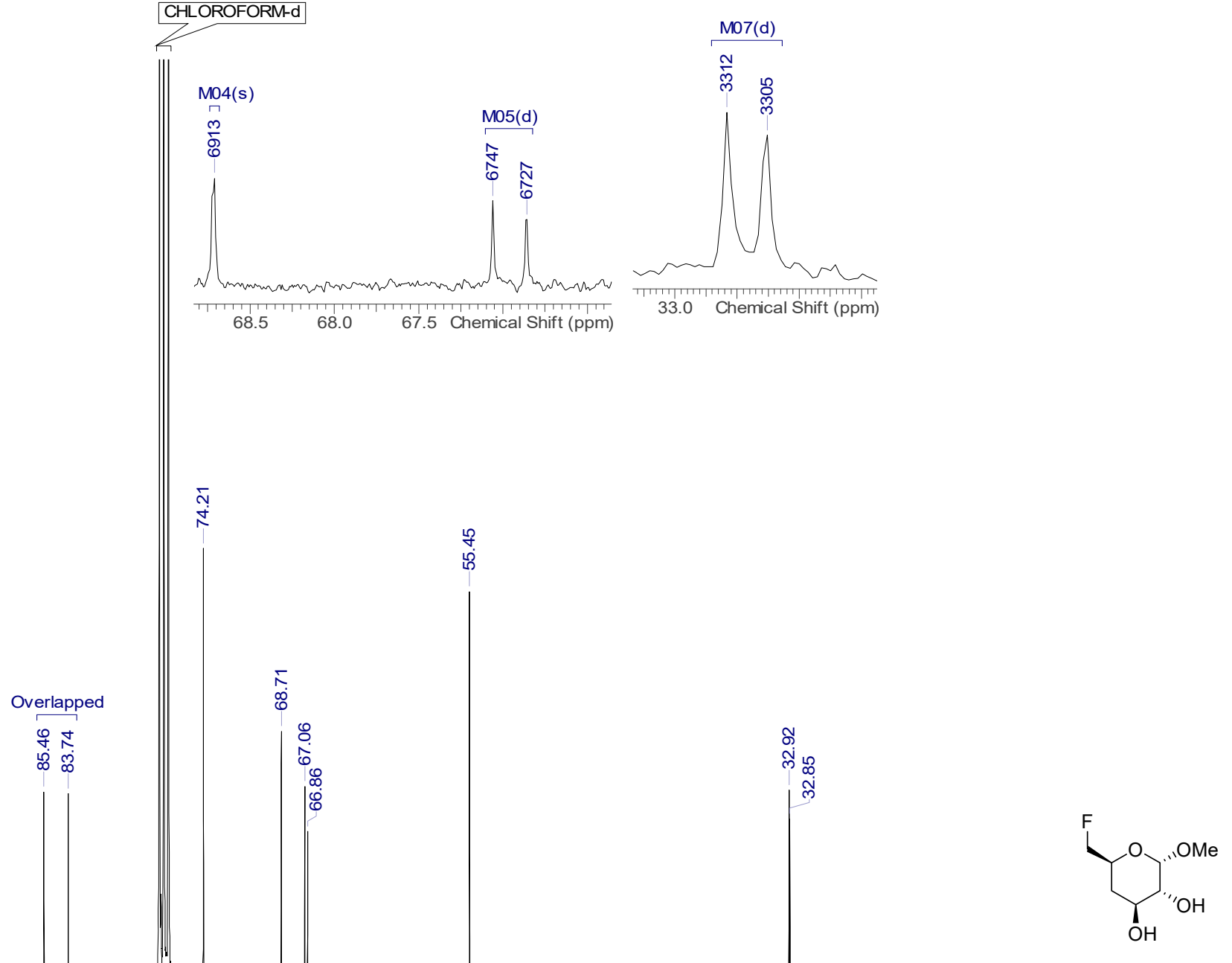

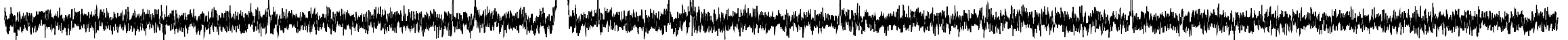

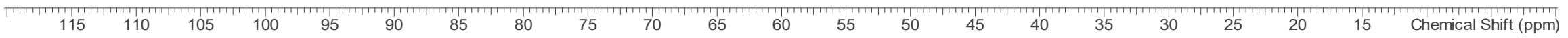




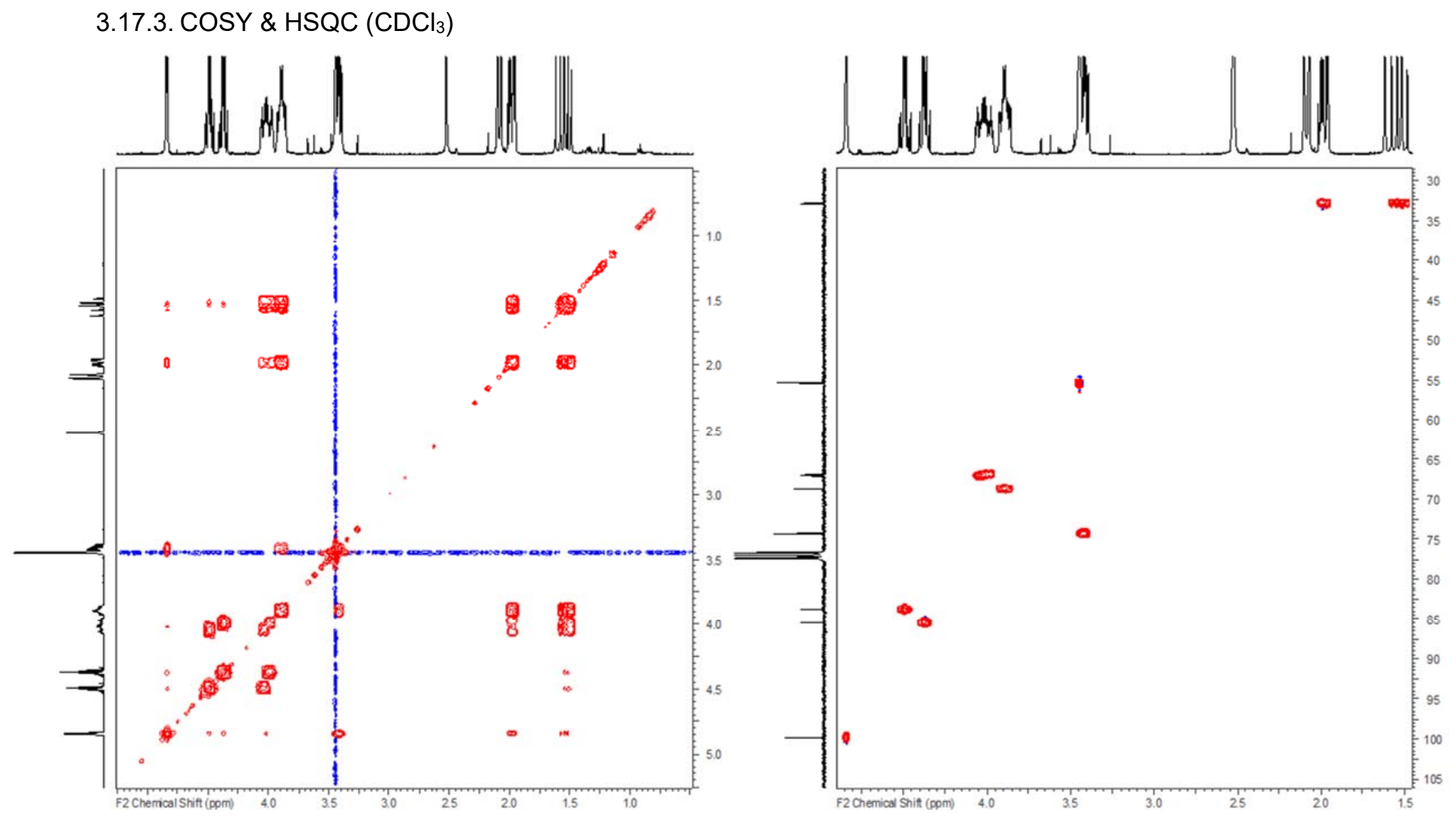


3.17.4. $\left.{ }^{19} \mathrm{~F} \mathrm{NMR} \mathrm{(376} \mathrm{MHz,} \mathrm{CDCl}_{3}\right)$

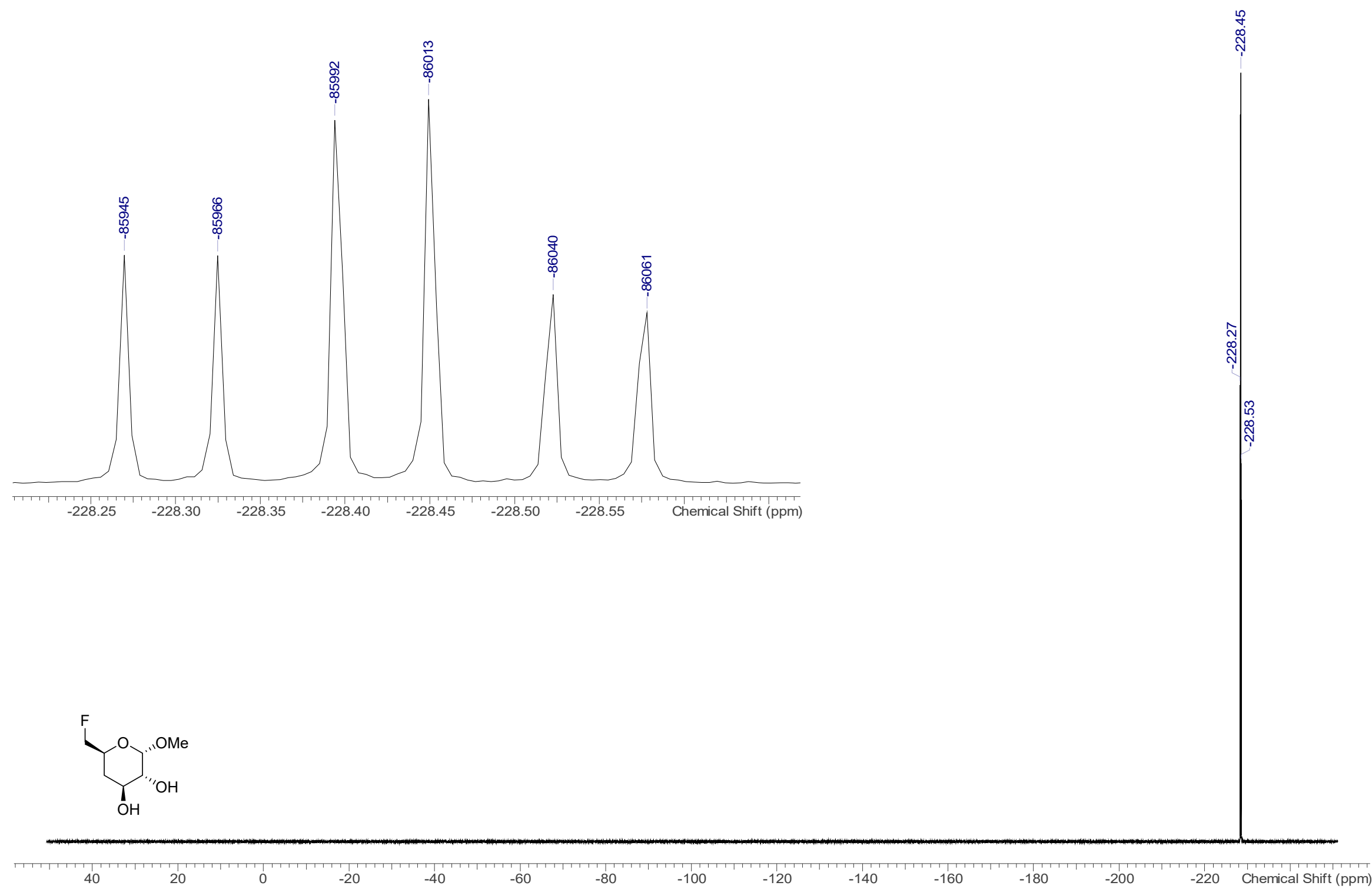


3.17.5. ${ }^{19} \mathrm{~F}\left\{{ }^{1} \mathrm{H}\right\}$ NMR (376 MHz, $\left.\mathrm{CDCl}_{3}\right)$

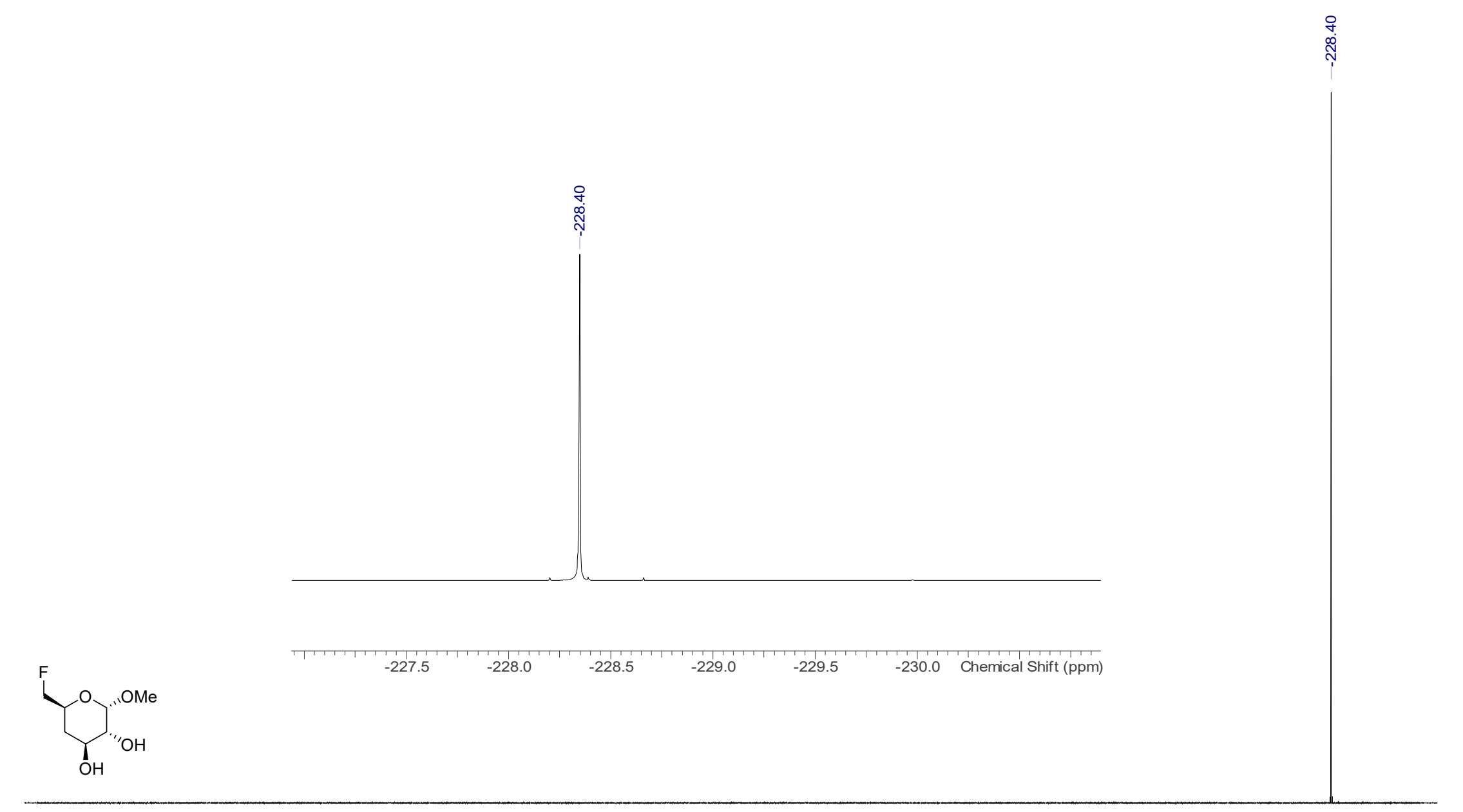




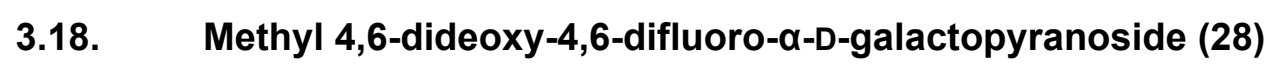

3.18.1. ${ }^{1} \mathrm{H}$ NMR $\left(400 \mathrm{MHz}, \mathrm{CDCl}_{3}\right)$
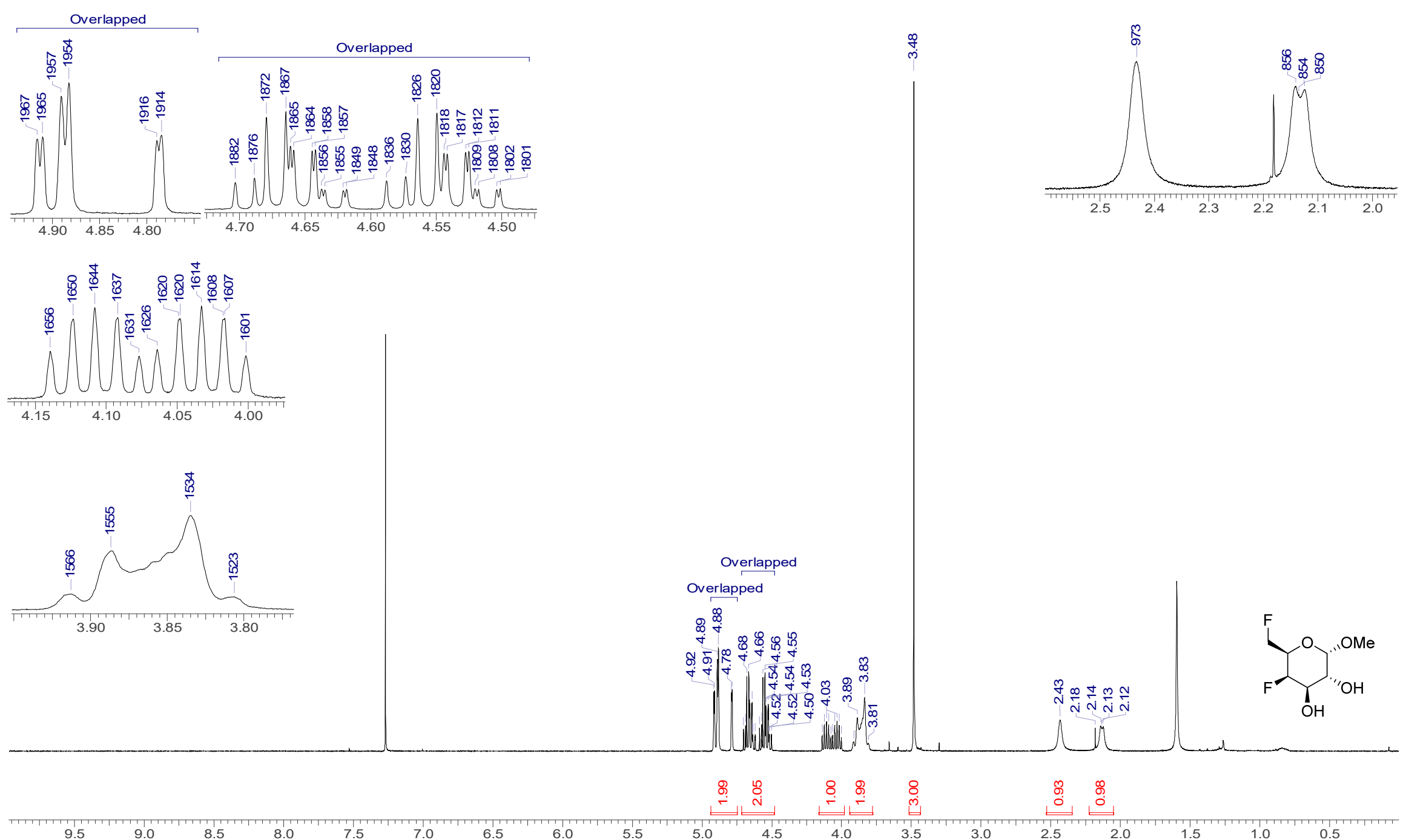
3.18.2. ${ }^{13} \mathrm{C} \mathrm{NMR}\left(101 \mathrm{MHz}, \mathrm{CDCl}_{3}\right)$
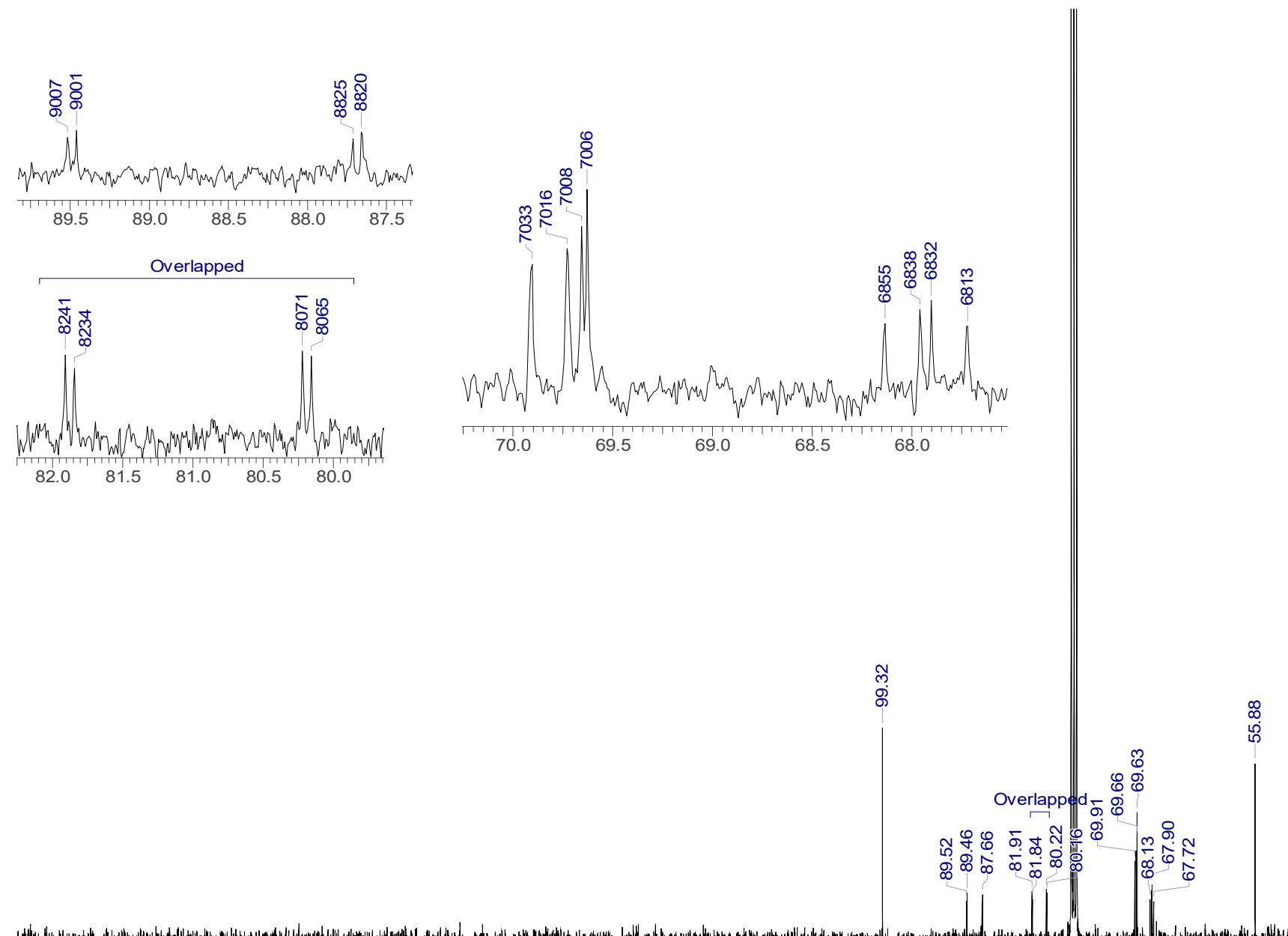

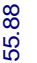

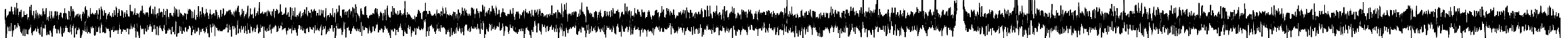


3.18.3. COSY \& HSQC $\left(\mathrm{CDCl}_{3}\right)$

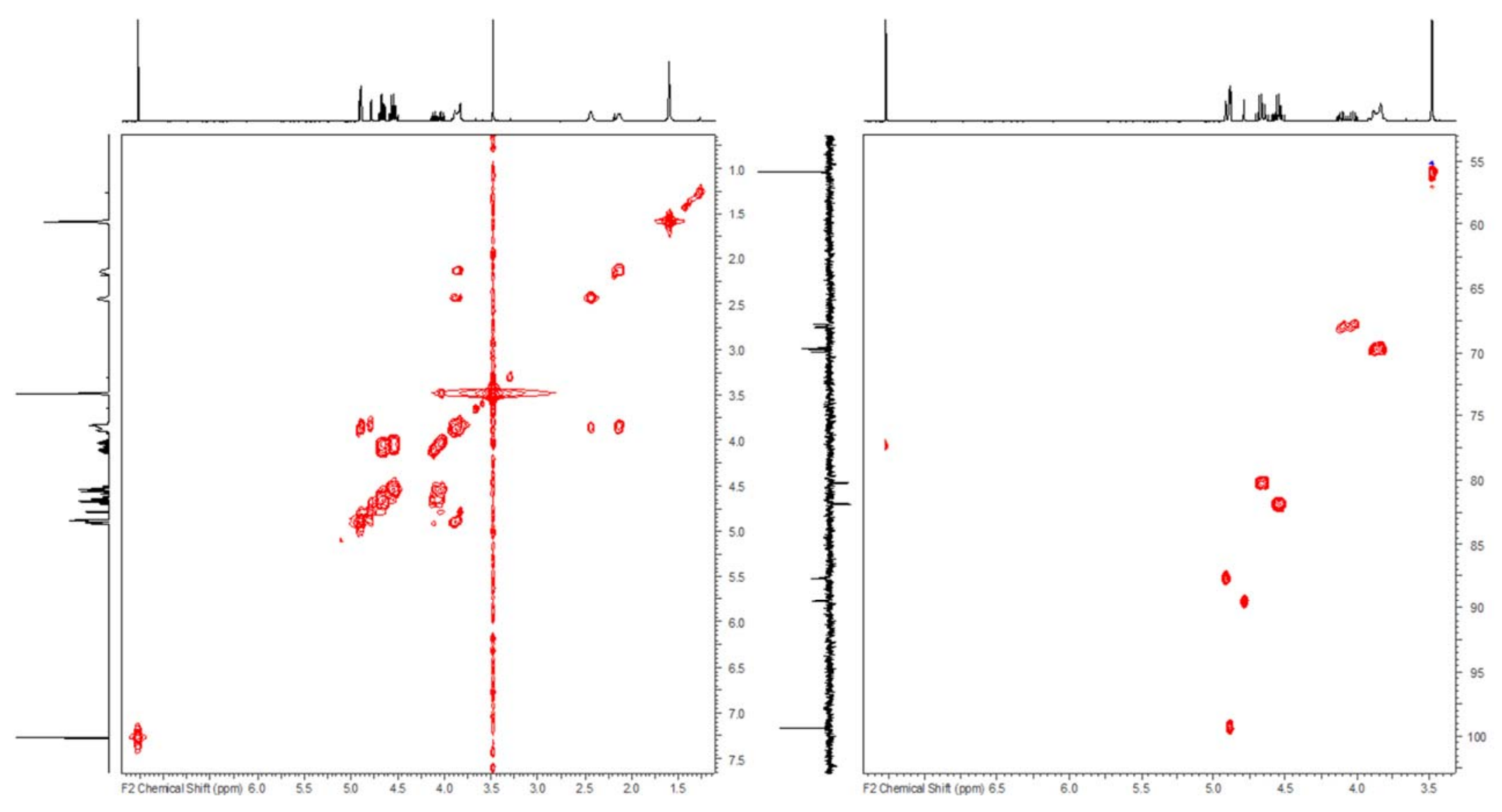


3.18.4. ${ }^{19} \mathrm{~F}$ NMR (376 MHz, $\mathrm{CDCl}_{3}$ )

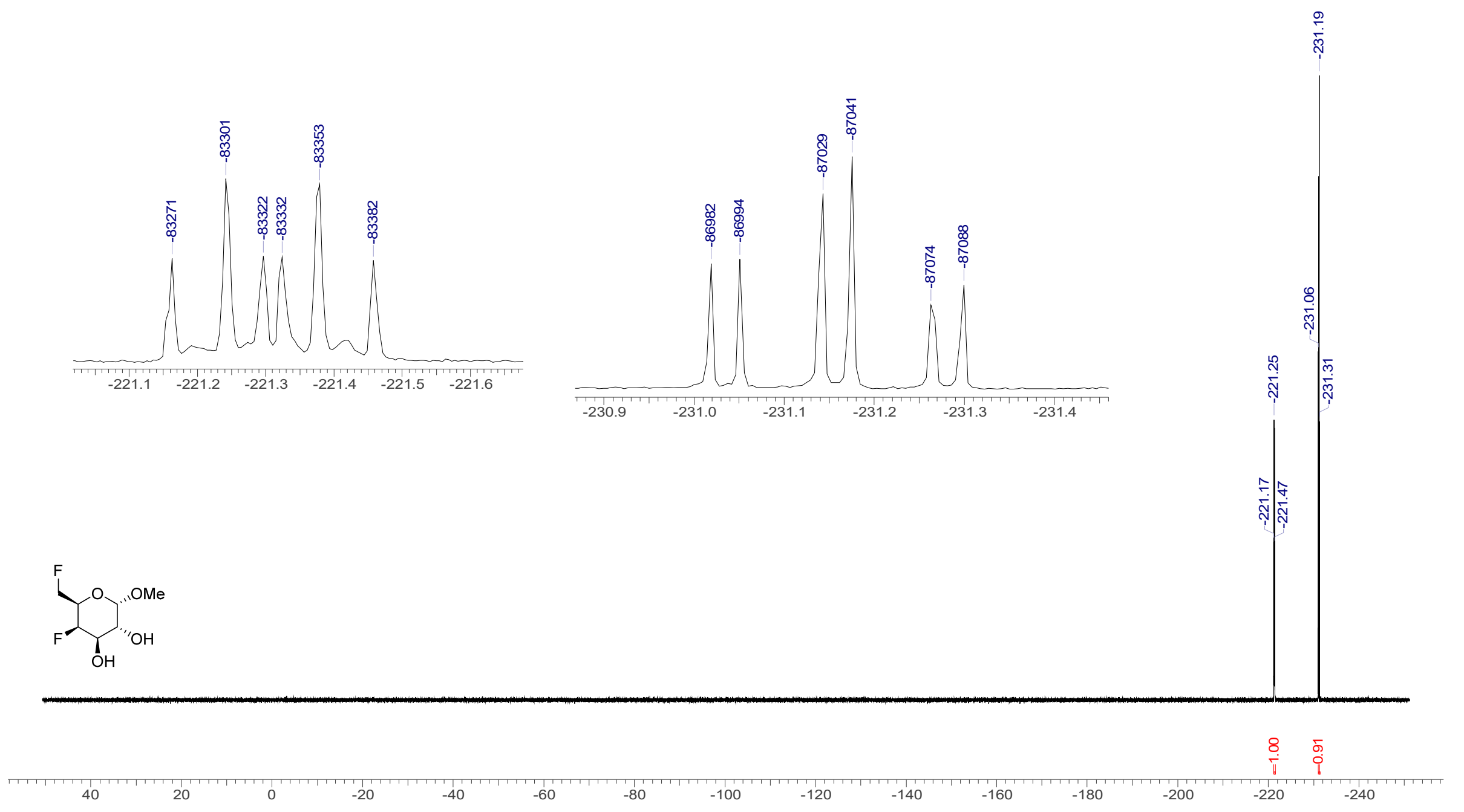


3.18.5. ${ }^{19} \mathrm{~F}\left({ }^{1} \mathrm{H}\right) \mathrm{NMR}\left(376 \mathrm{MHz}, \mathrm{CDCl}_{3}\right)$

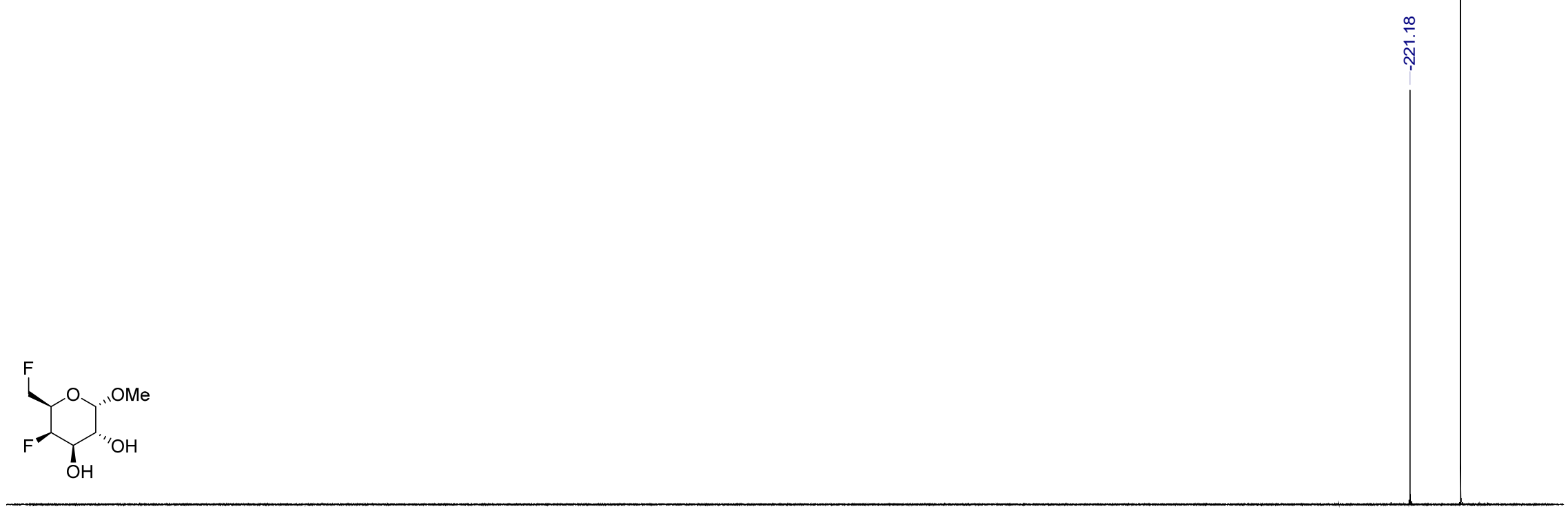

$40+1+1+20$ 20 o 11
-20 
3.18.6. ${ }^{1} \mathrm{H}$ NMR $\left(400 \mathrm{MHz}\right.$, acetone- $\left.\mathrm{d}_{6}\right)$

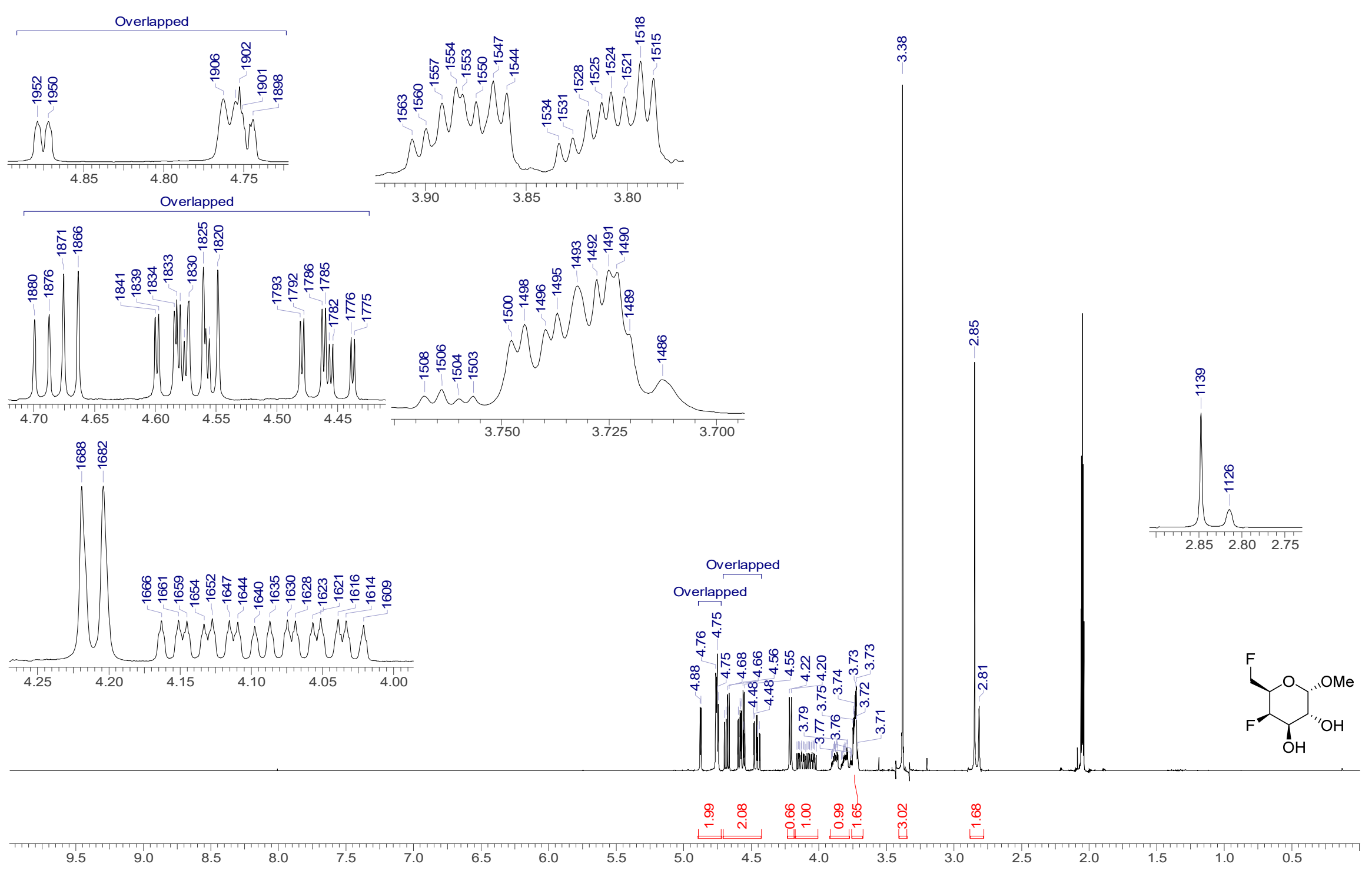


3.18.7. ${ }^{13} \mathrm{C}$ NMR $\left(101 \mathrm{MHz}\right.$, acetone- $\left.\mathrm{d}_{6}\right)$

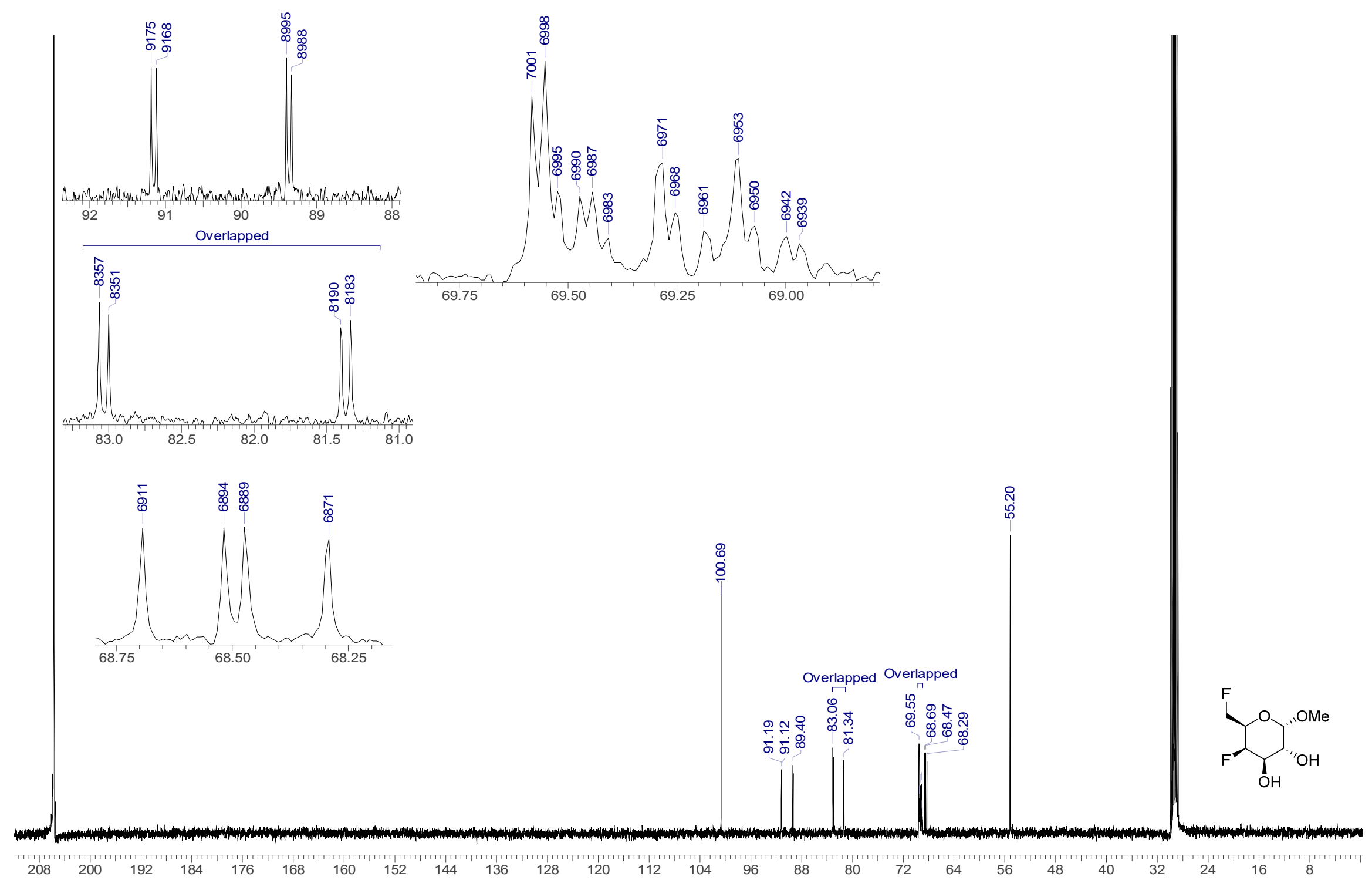


3.18.8. COSY, HSQC \& HMBC (acetone- $d_{6}$ )
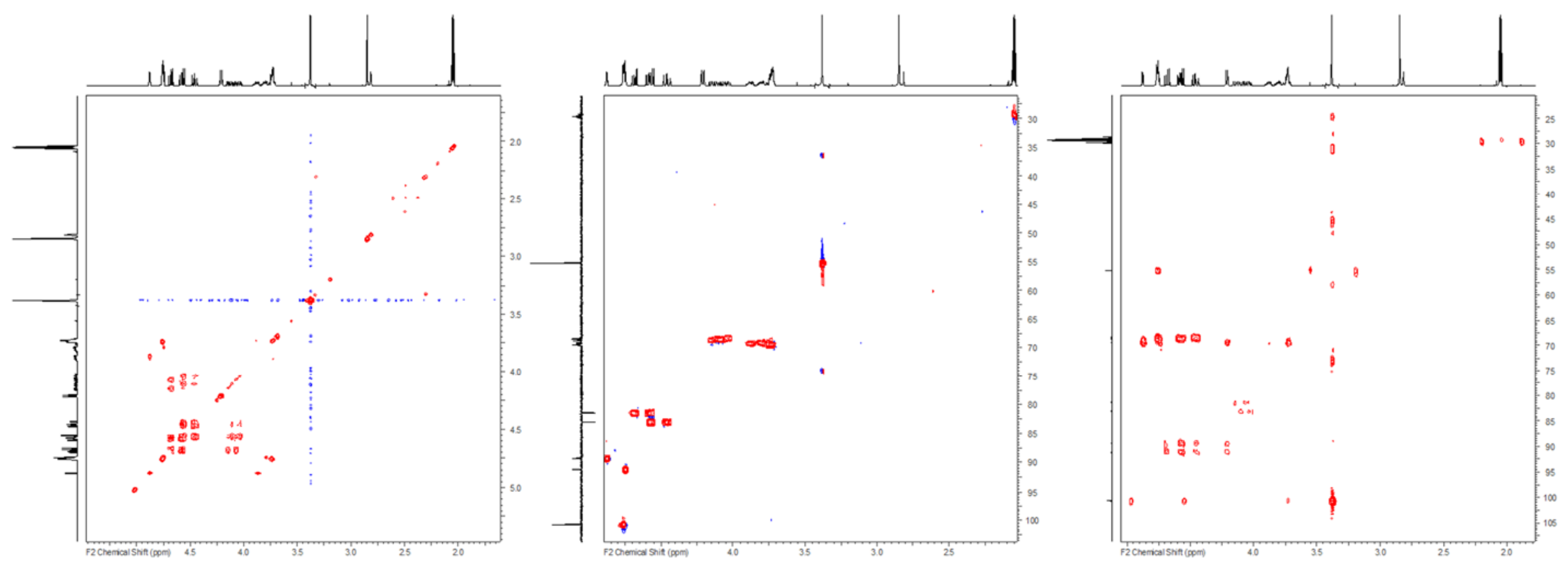
3.18.9. ${ }^{19} \mathrm{~F} \mathrm{NMR}\left(376 \mathrm{MHz}\right.$, acetone- $\left.\mathrm{d}_{6}\right)$
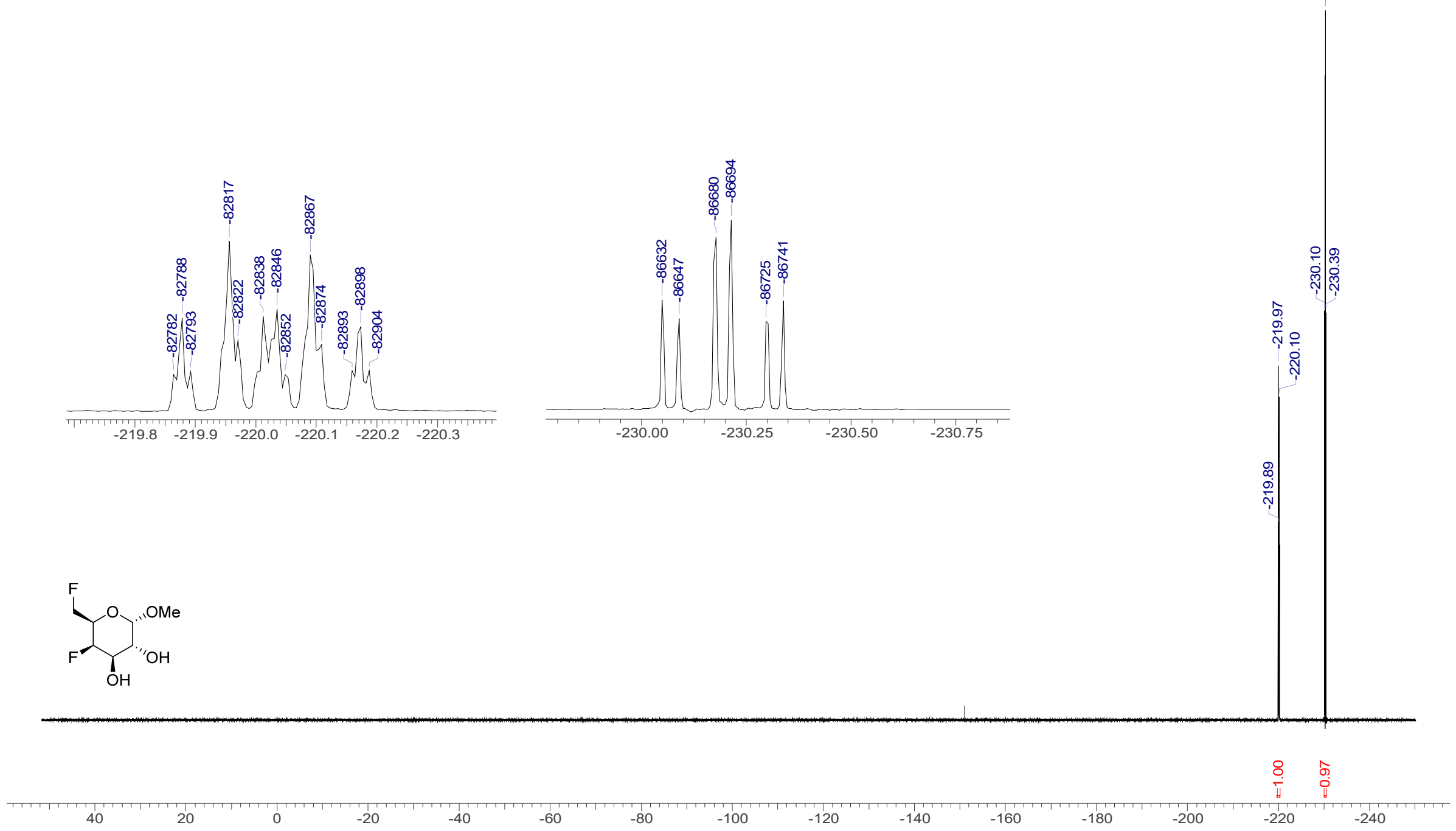
3.18.10. $\quad{ }^{19} \mathrm{~F}\left({ }^{1} \mathrm{H}\right)$ NMR $\left(376 \mathrm{MHz}\right.$, acetone- $\left.\mathrm{d}_{6}\right)$

ণัن

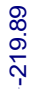

(OH

40

20

' 0

$-20$

$-40$

$-60$

$-80$

$-100$

$-120$

$-180$

$-200$ 


\subsection{Methyl 4,6-dideoxy-4,6-difluoro- $\alpha$-D-glucopyranoside (35)}

3.19.1. ${ }^{1} \mathrm{H}$ NMR $\left(400 \mathrm{MHz}, \mathrm{CDCl}_{3}\right)$

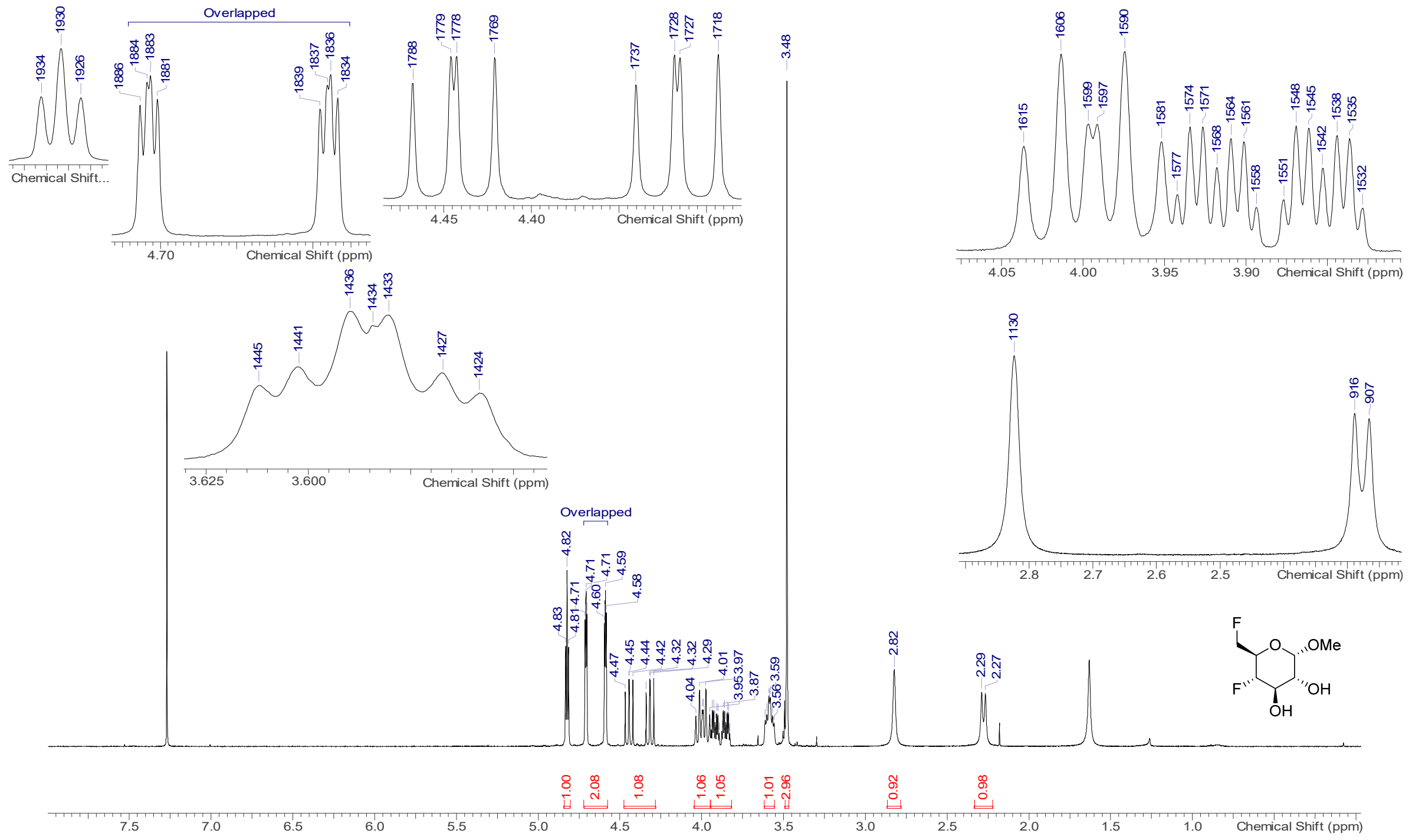


3.19.2. ${ }^{13} \mathrm{C}\left\{{ }^{1} \mathrm{H}\right\} \mathrm{NMR}\left(101 \mathrm{MHz}, \mathrm{CDCl}_{3}\right)$
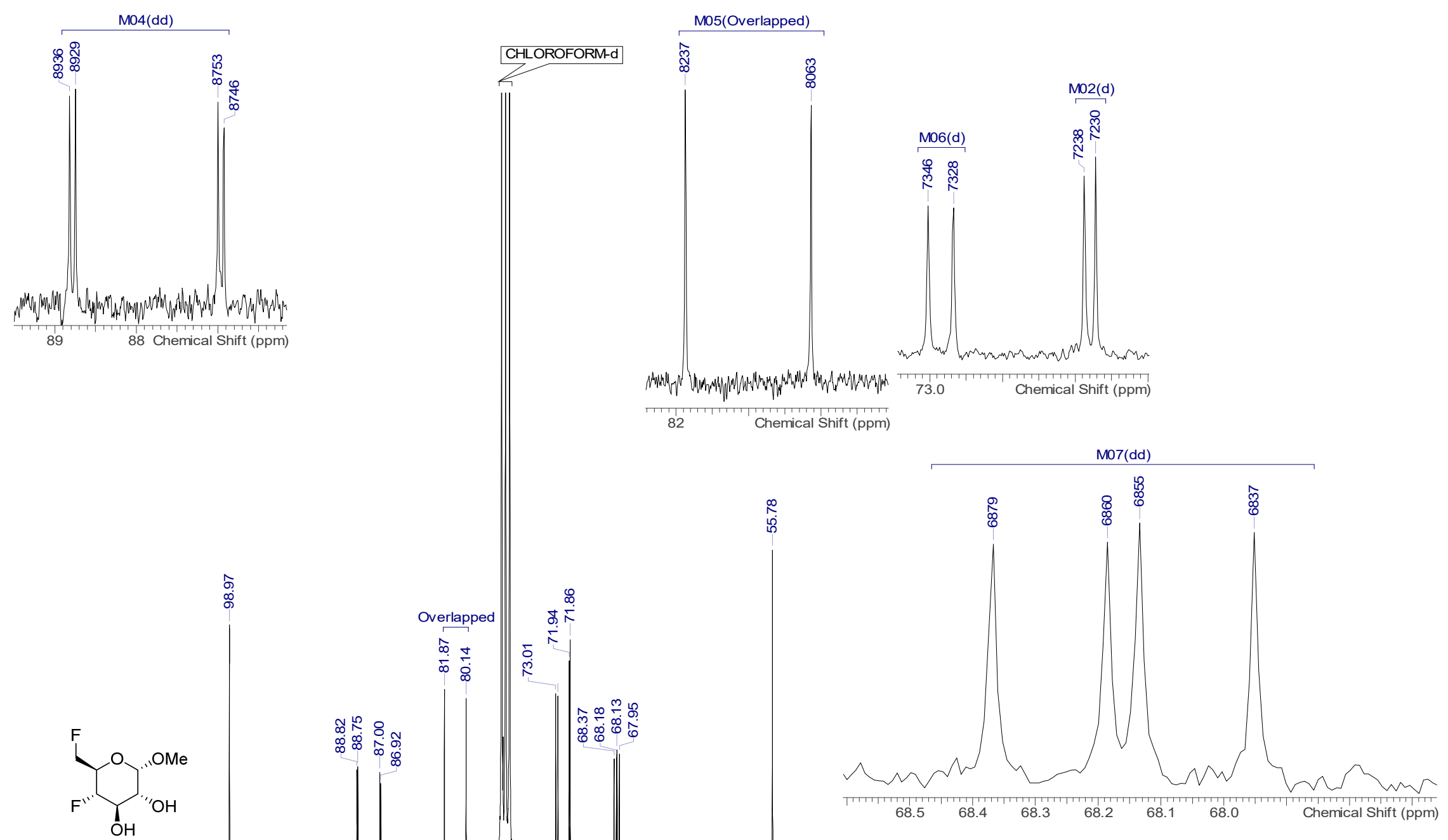

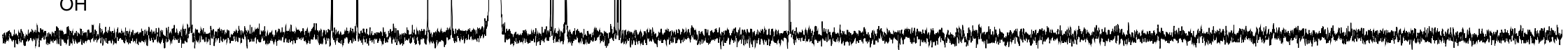

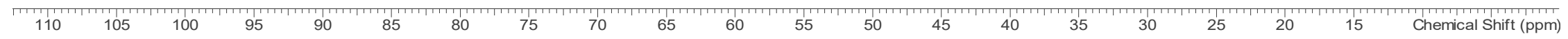


3.19.3. COSY \& HSQC $\left(\mathrm{CDCl}_{3}\right)$

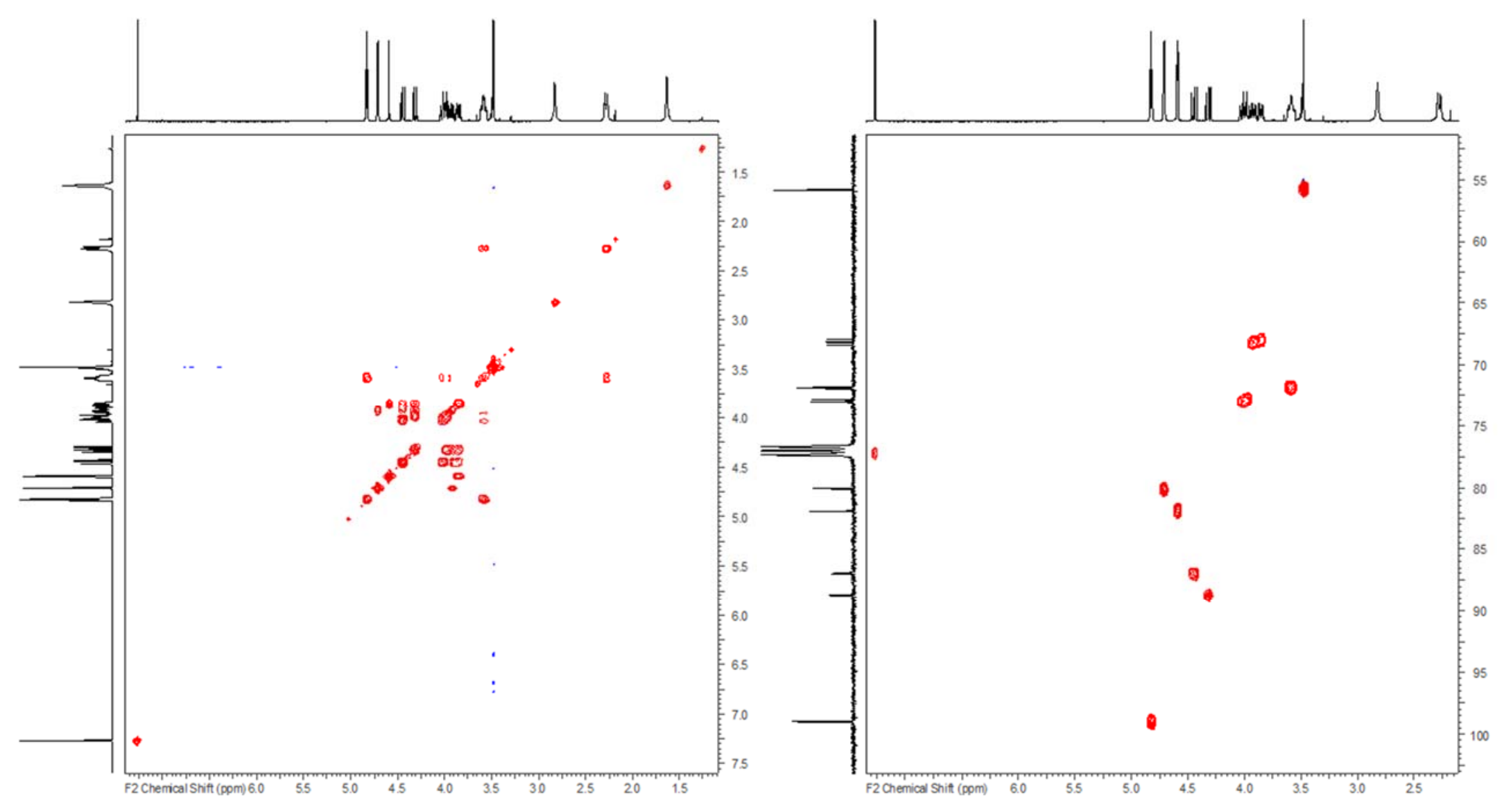


S 126

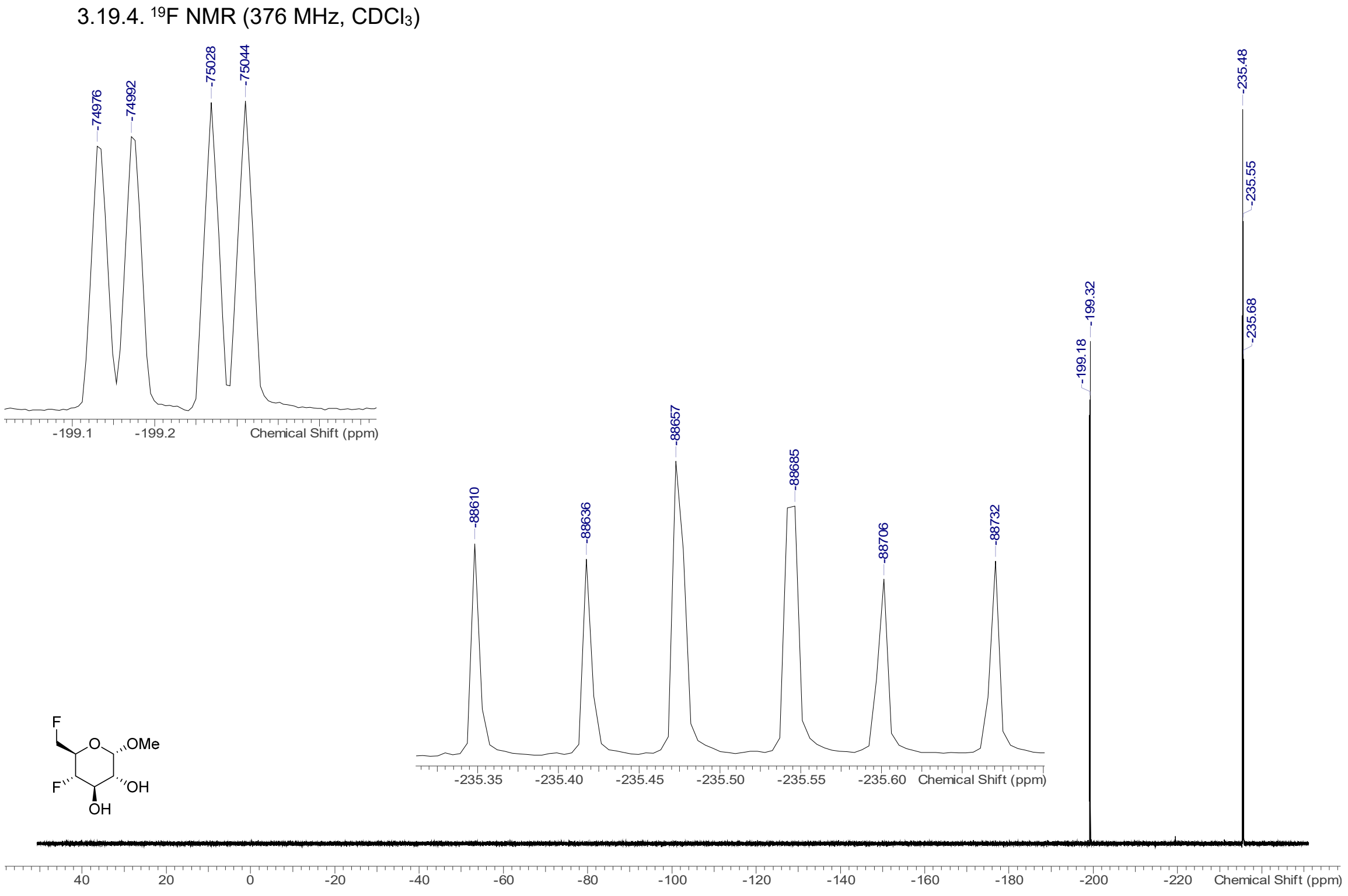




\subsection{5. ${ }^{1} \mathrm{H}$ NMR $\left(400 \mathrm{MHz}\right.$, acetone- $\left.\mathrm{d}_{6}\right)$}
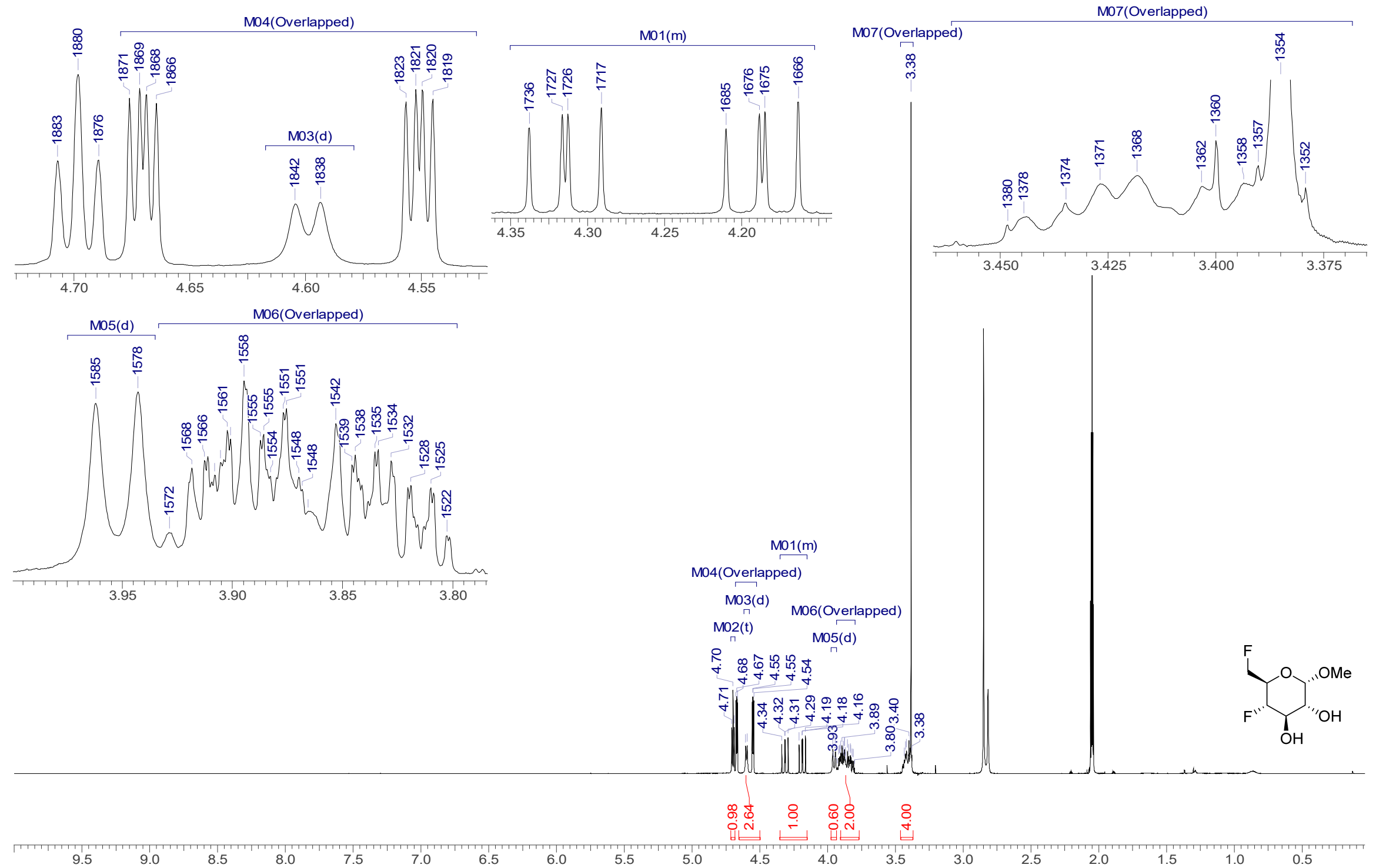
3.19.6. ${ }^{13} \mathrm{C}$ NMR $\left(101 \mathrm{MHz}\right.$, acetone- $\left.\mathrm{d}_{6}\right)$

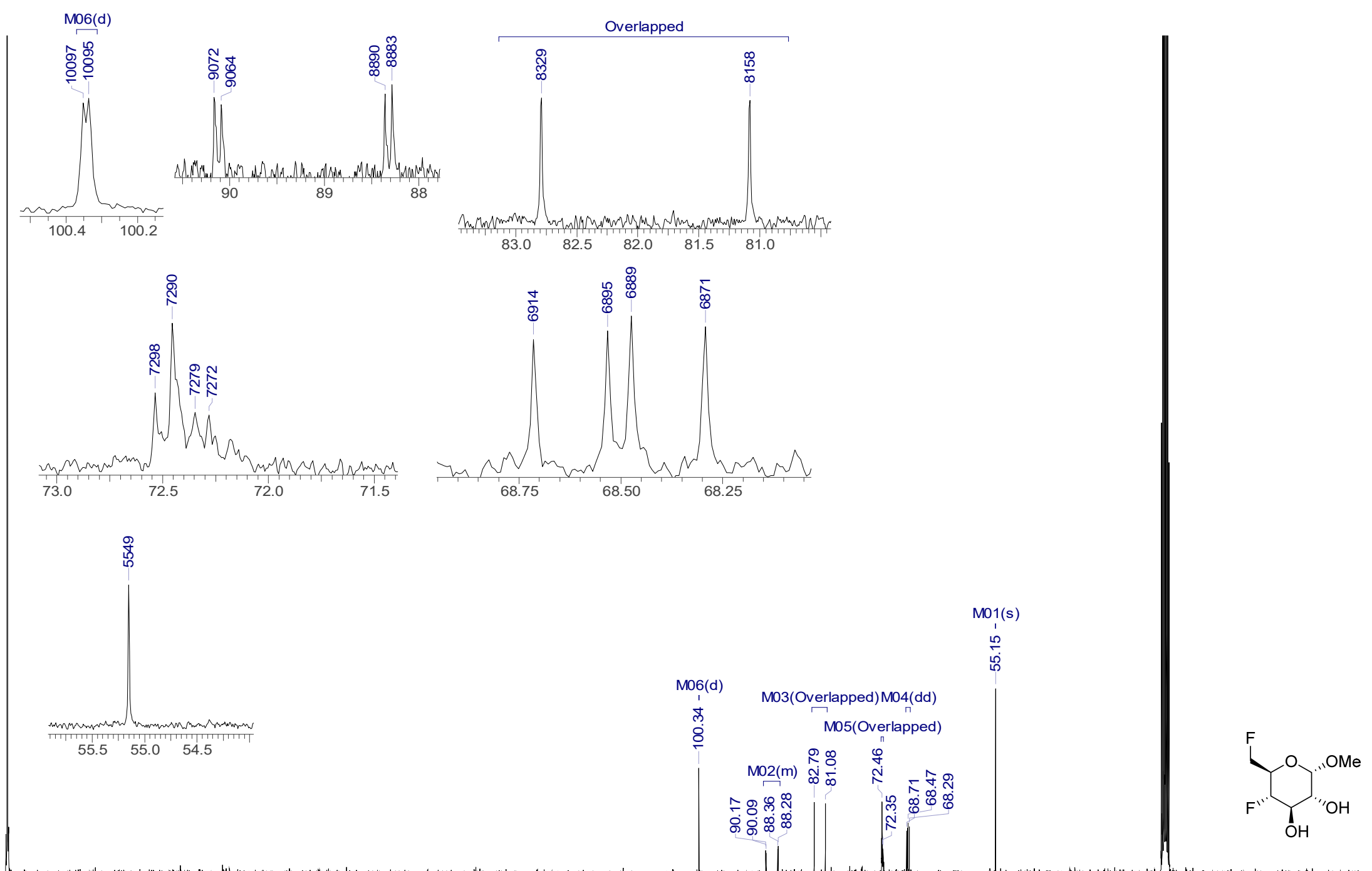

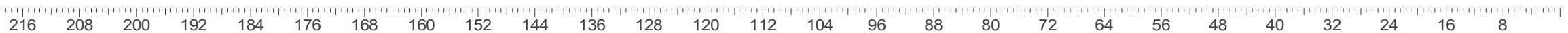


3.19.7. COSY, HSQC \& HMBC (acetone- $d_{6}$ )

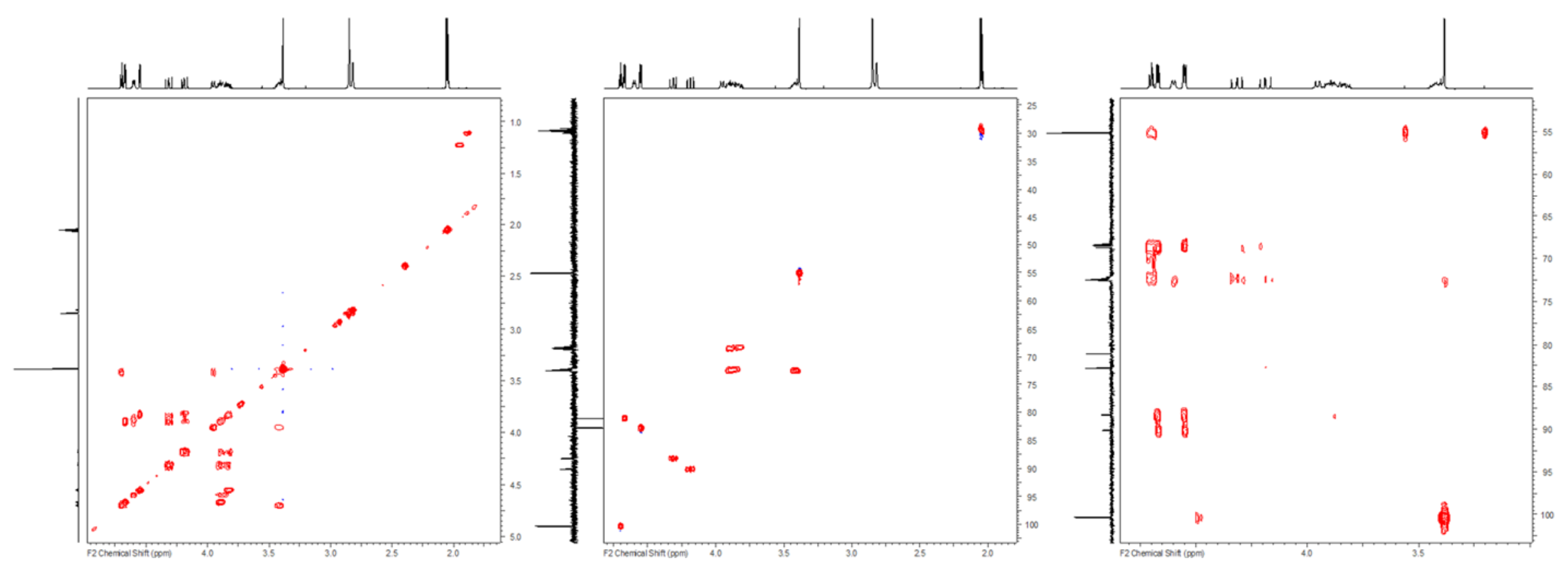


S 130

3.19.8. ${ }^{19} \mathrm{~F}$ NMR (376 MHz, acetone- $\left.\mathrm{d}_{6}\right)$
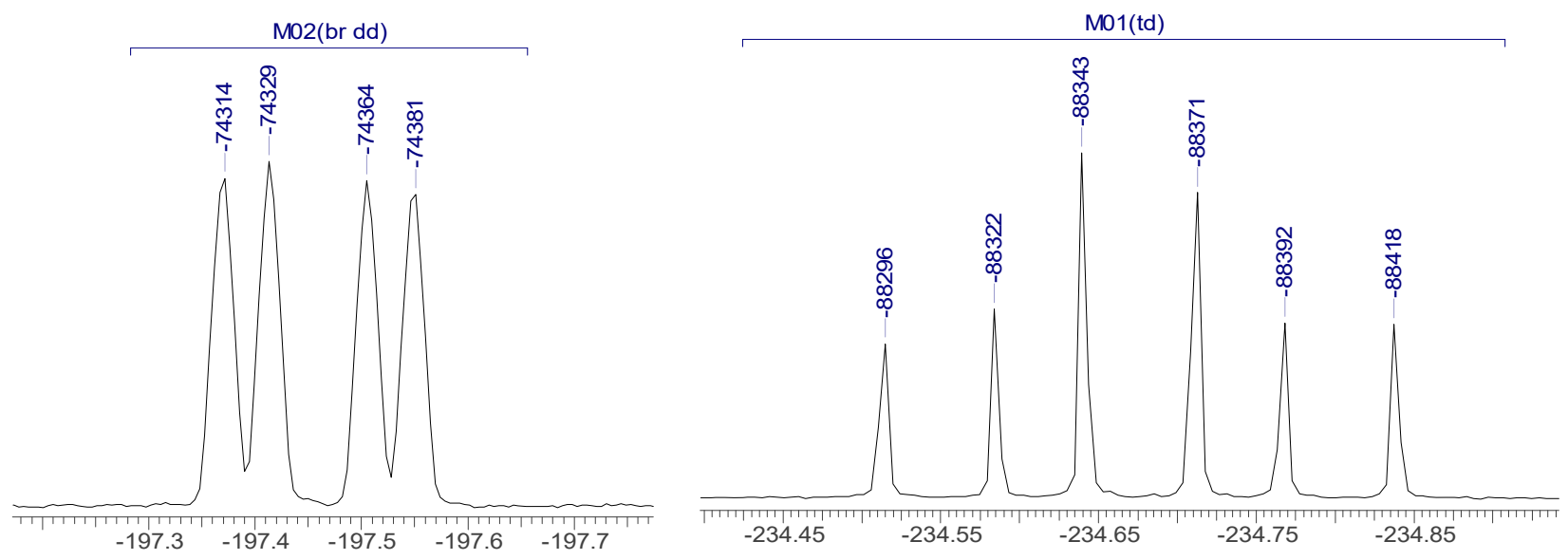<smiles>CO[C@H]1O[C@H](CF)[C@@H](F)[C@H](O)[C@H]1O</smiles>
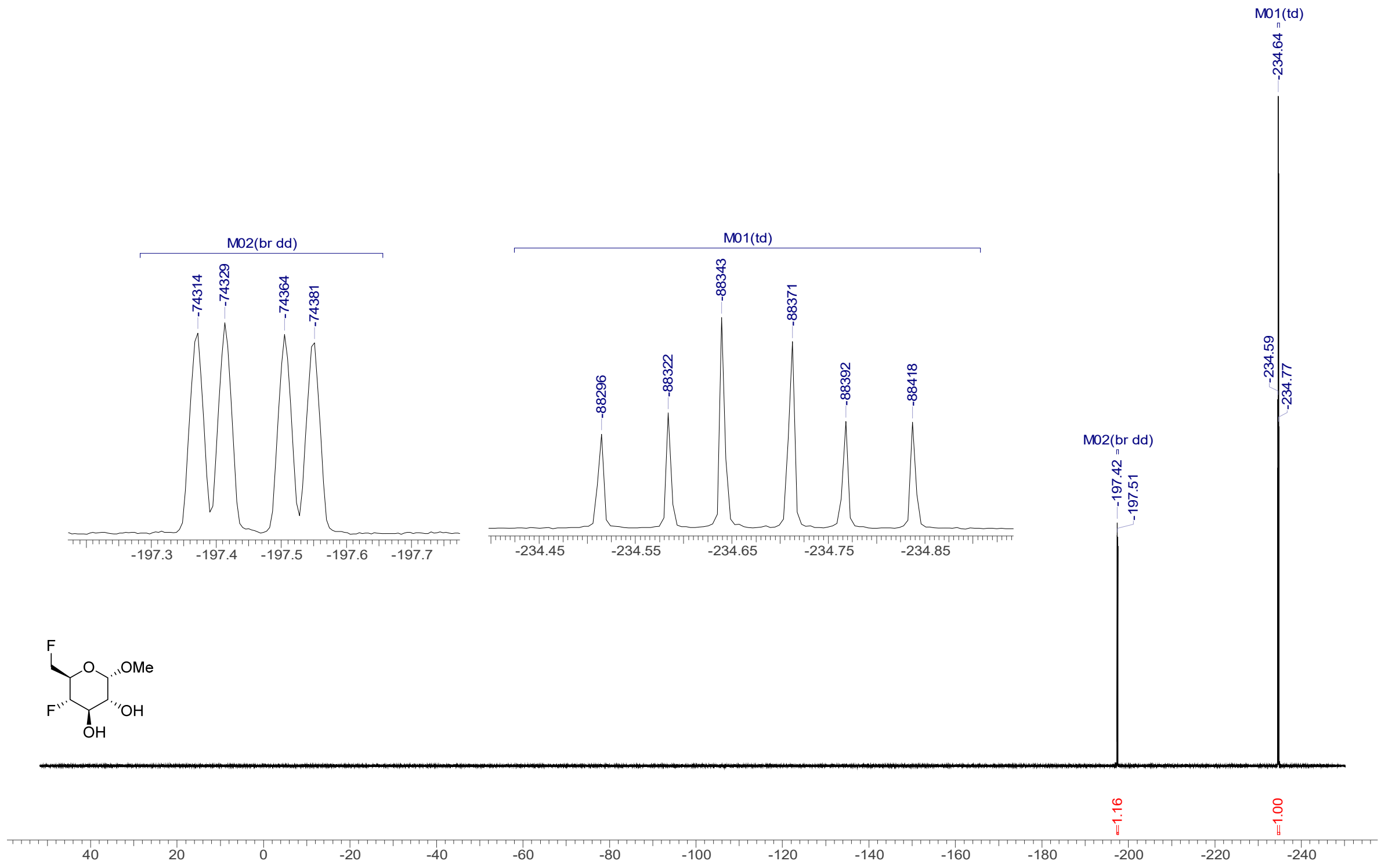
S 131

3.19.9. ${ }^{19} \mathrm{~F}\left({ }^{1} \mathrm{H}\right)$ NMR $\left(376 \mathrm{MHz}\right.$, acetone- $\left.\mathrm{d}_{6}\right)$

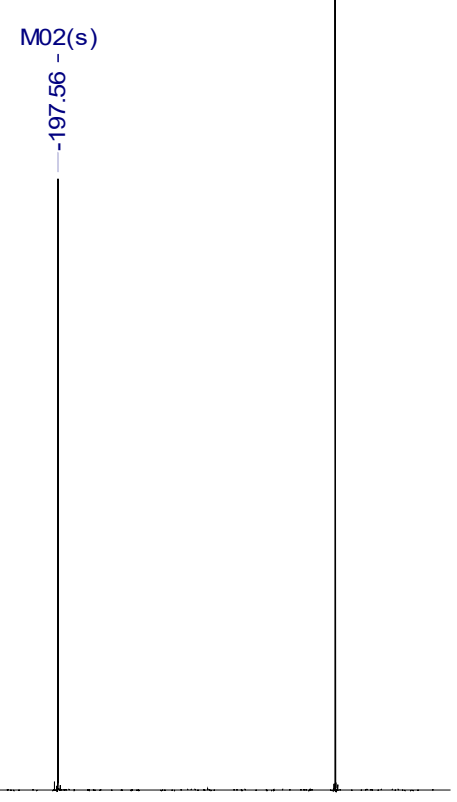

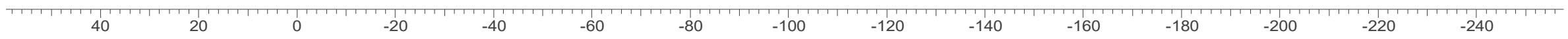


3.20. Methyl 2,3-di-O-benzoyl-4,6-dideoxy-4,6-difluoro- $\alpha$-D-glucopyranoside (39) 3.20.1. ${ }^{1} \mathrm{H}$ NMR $\left(400 \mathrm{MHz}, \mathrm{CDCl}_{3}\right)$
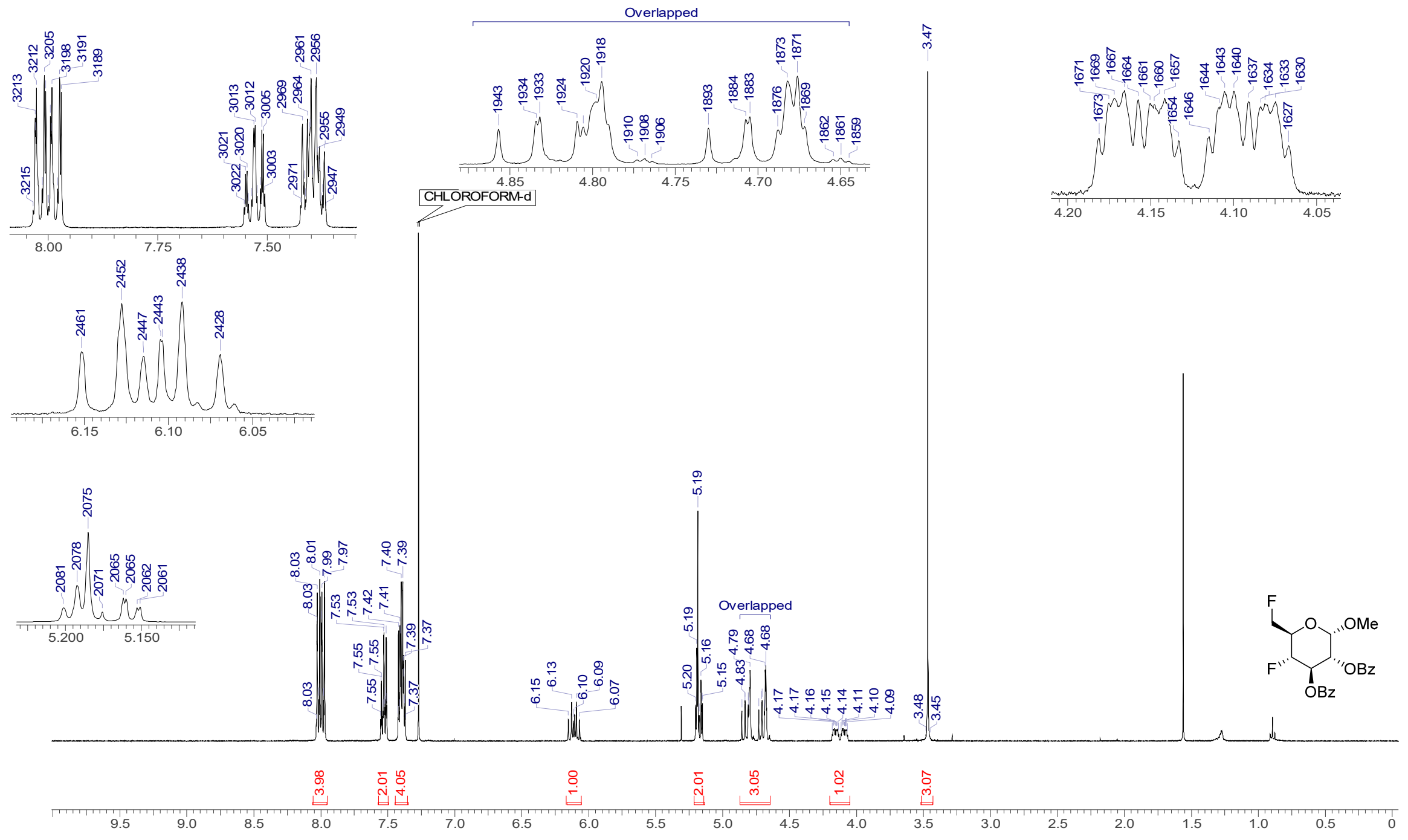
3.20.2. ${ }^{13} \mathrm{C}\left\{{ }^{1} \mathrm{H}\right\}$ NMR $\left(101 \mathrm{MHz}, \mathrm{CDCl}_{3}\right)$
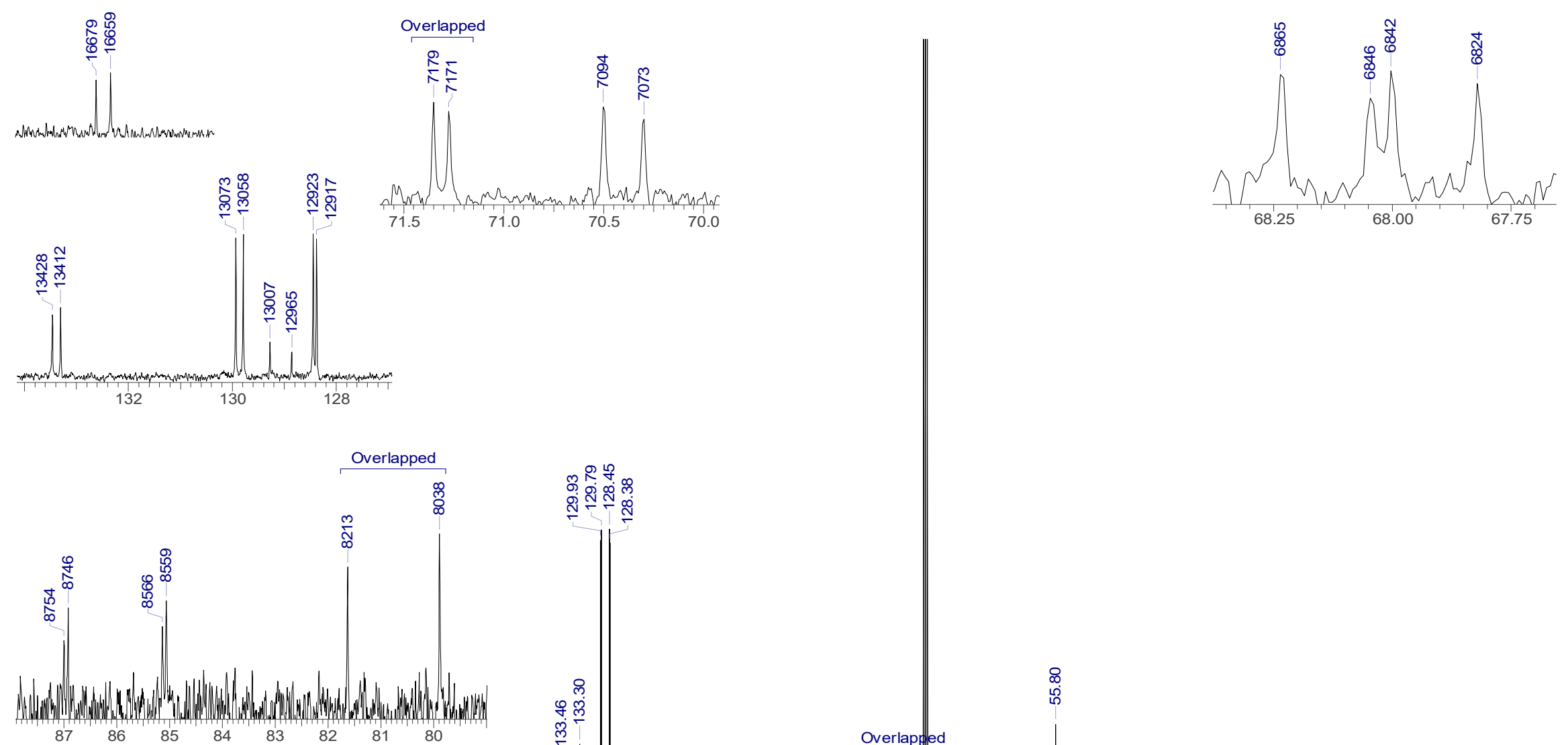

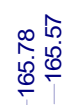

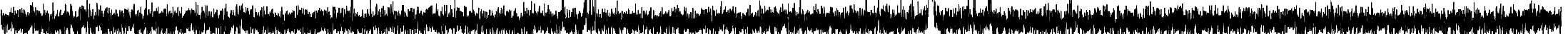

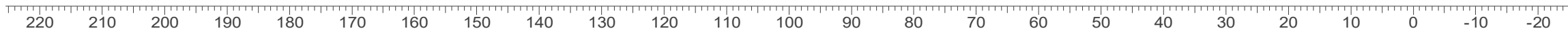


3.20.3. COSY, $\mathrm{HSQC} \& \mathrm{HMBC}\left(\mathrm{CDCl}_{3}\right)$

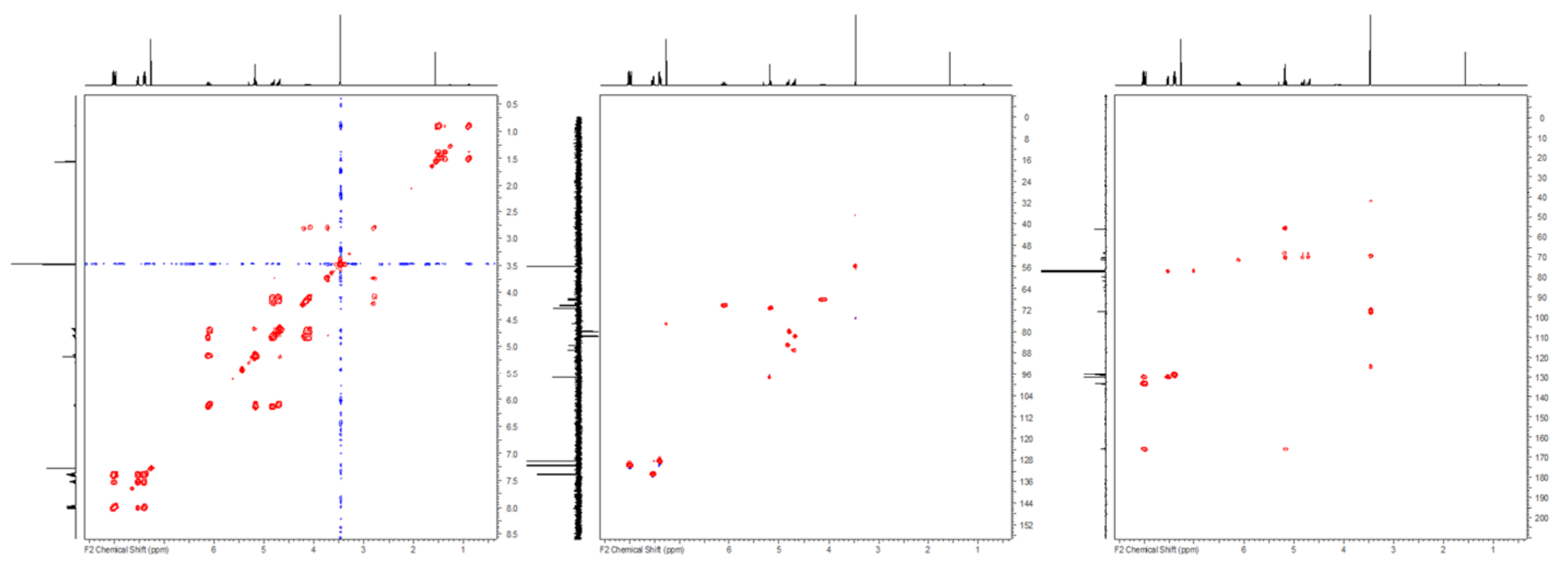


3.20.4. $\left.{ }^{19} \mathrm{~F} \mathrm{NMR} \mathrm{(376} \mathrm{MHz,} \mathrm{CDCl}_{3}\right)$
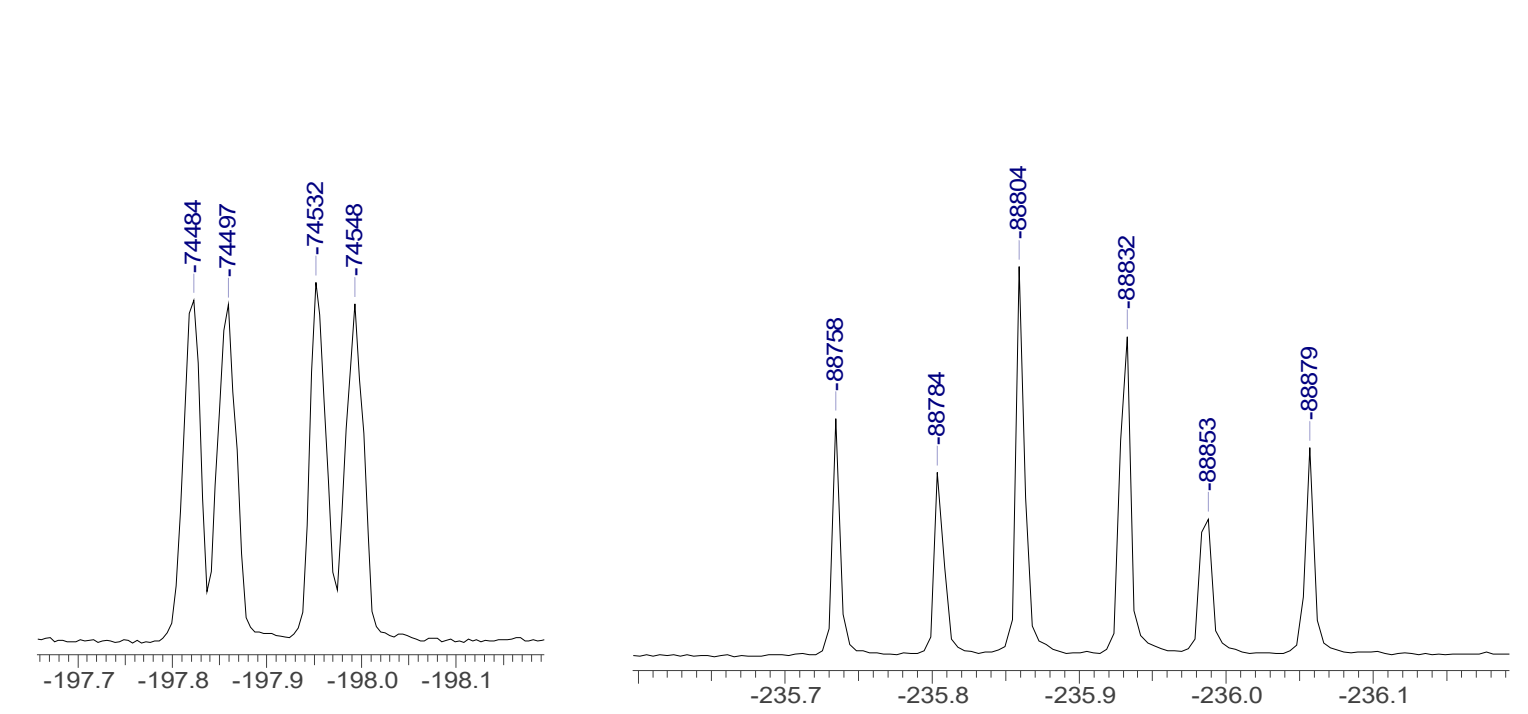

$$
\mathrm{O}_{\mathrm{OBz}}^{\mathrm{O}} \mathrm{. \textrm {OM }}
$$

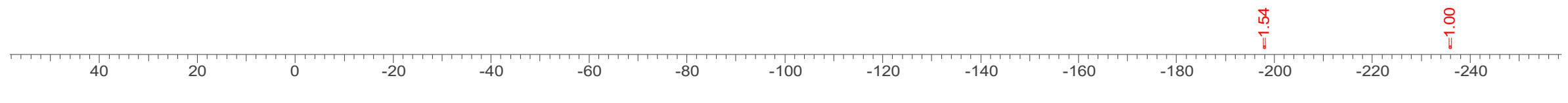


3.20.5. ${ }^{19} \mathrm{~F}\left\{{ }^{1} \mathrm{H}\right\}$ NMR (376 MHz, $\left.\mathrm{CDCl}_{3}\right)$

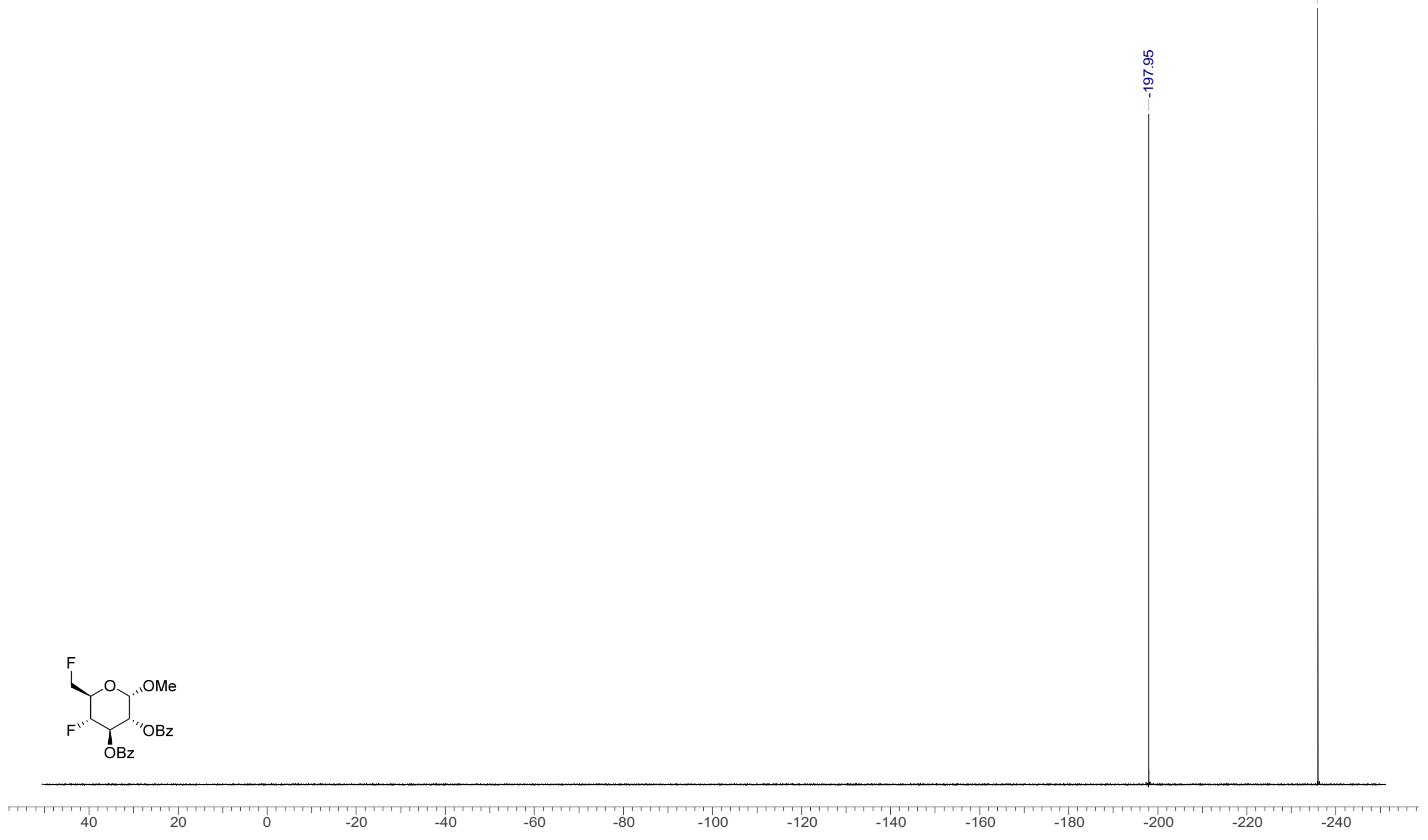


3.21. Methyl 6-O-tert-butyldimethylsilyl-2,3-O-((2'R,3'R)-2',3'-dimethoxybutane-2',3'-diyl)- $\alpha$-D-galactopyranoside (47): 3.21.1. ${ }^{1} \mathrm{H} \mathrm{NMR}\left(400 \mathrm{MHz}, \mathrm{CDCl}_{3}\right)$

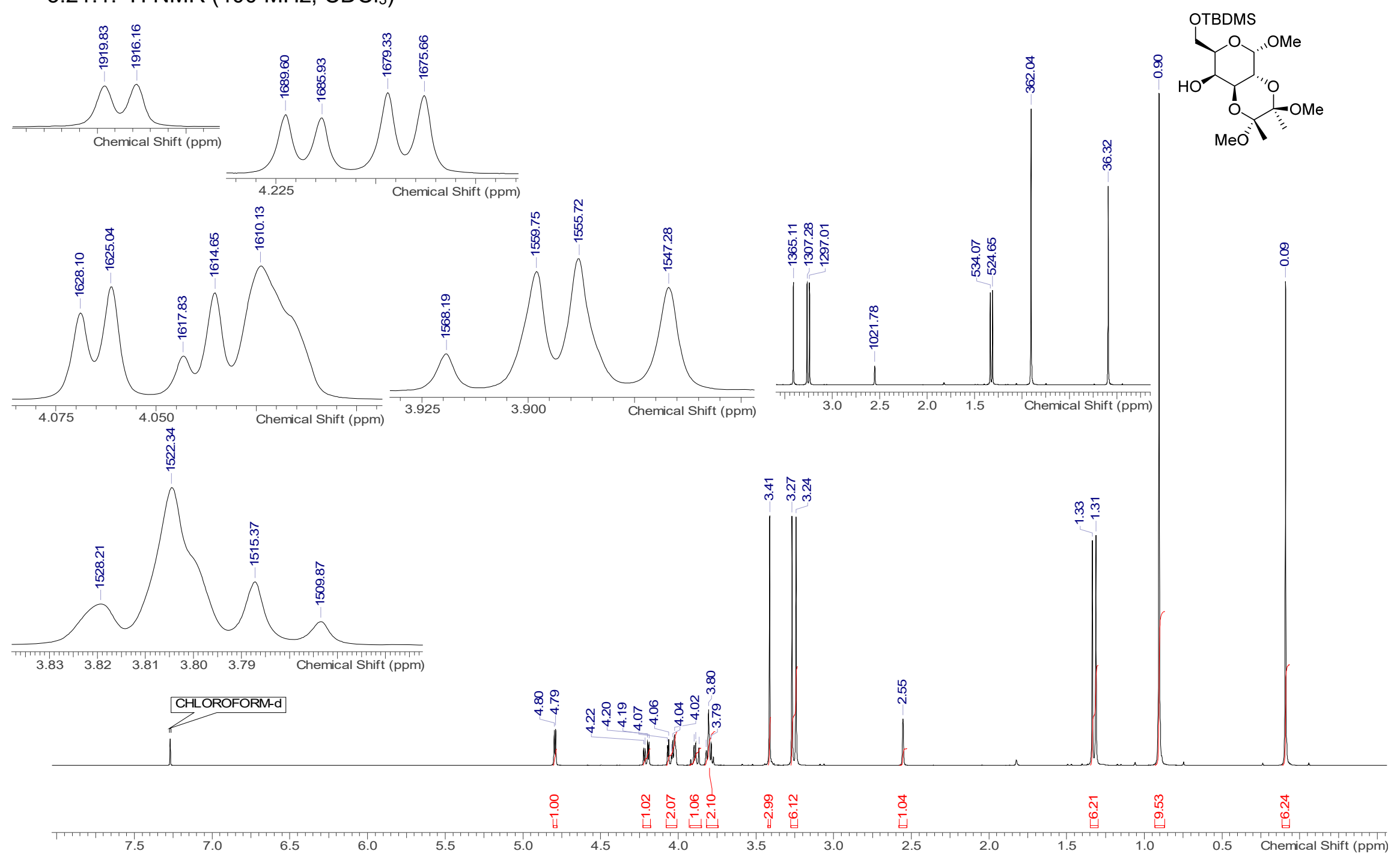


3.21.2. ${ }^{13} \mathrm{C}\left\{{ }^{1} \mathrm{H}\right\}$ NMR $\left(101 \mathrm{MHz}, \mathrm{CDCl}_{3}\right)$

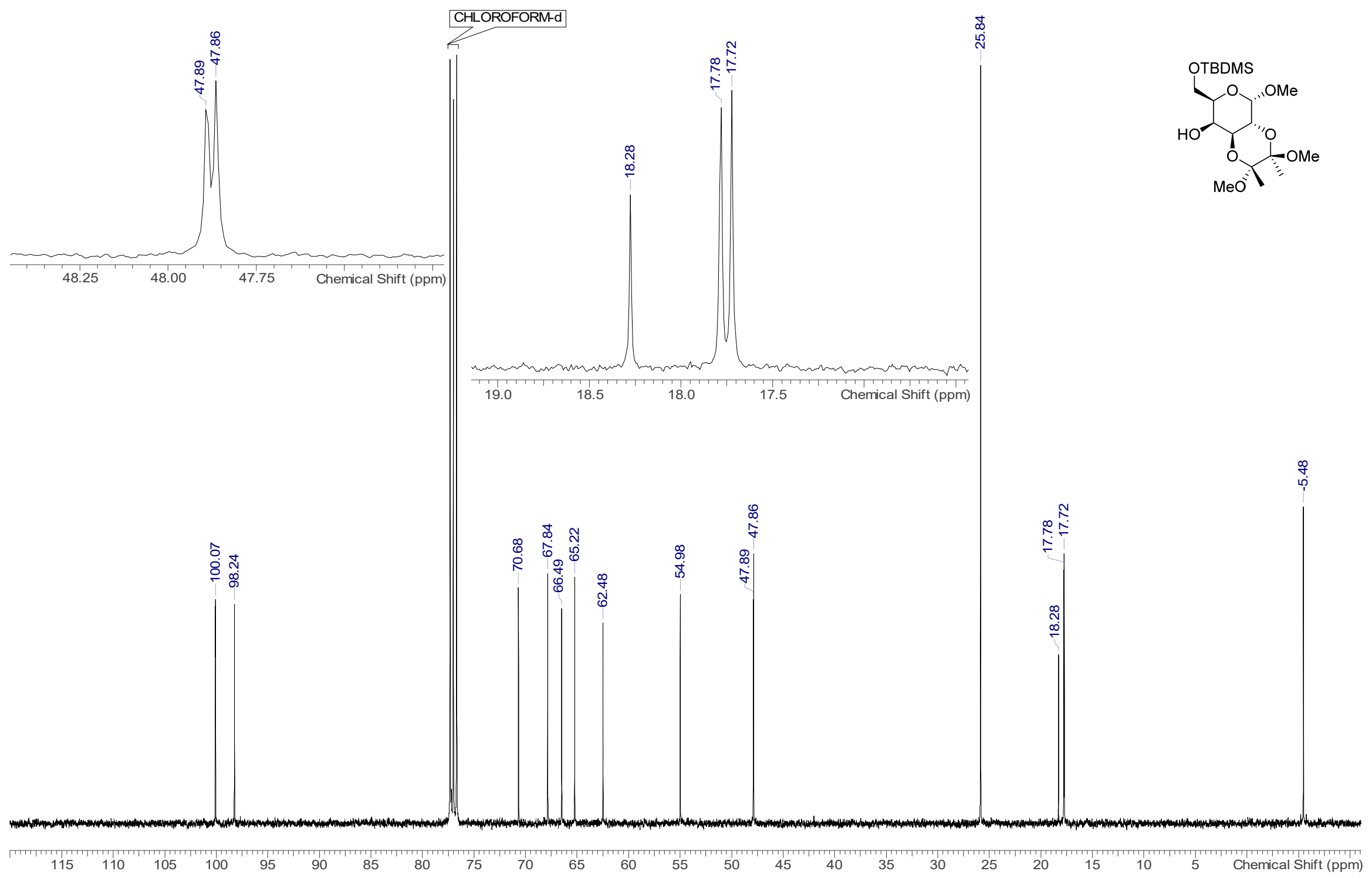


3.21.3. COSY, HSQC \& HMBC $\left(\mathrm{CDCl}_{3}\right)$

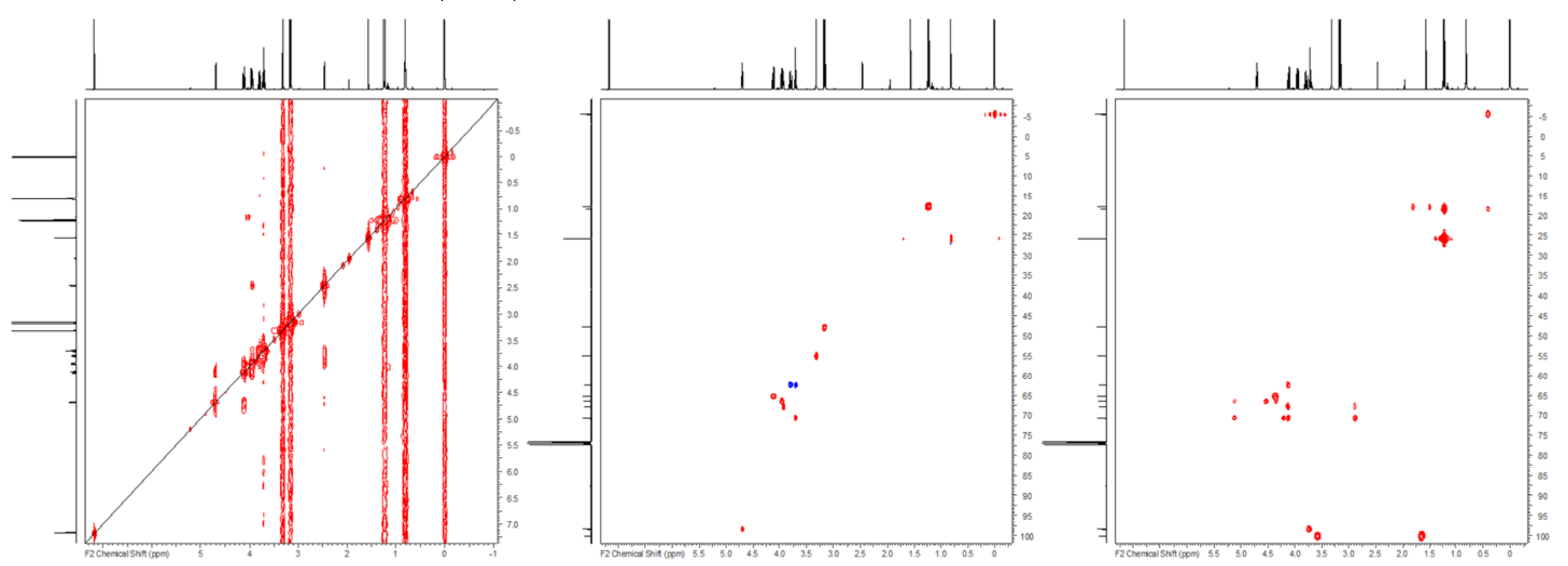


3.22. Methyl 6-O-tert-butyldimethylsilyl-4-O-phenoxythiocarbonyl-2,3-O-((2'R,3'R)-2',3'-dimethoxybutane-2',3'-diyl)- $\alpha-\mathrm{D}-$ galactopyranoside (48):

3.22.1. ${ }^{1} \mathrm{H} \mathrm{NMR}\left(400 \mathrm{MHz}, \mathrm{CDCl}_{3}\right)$

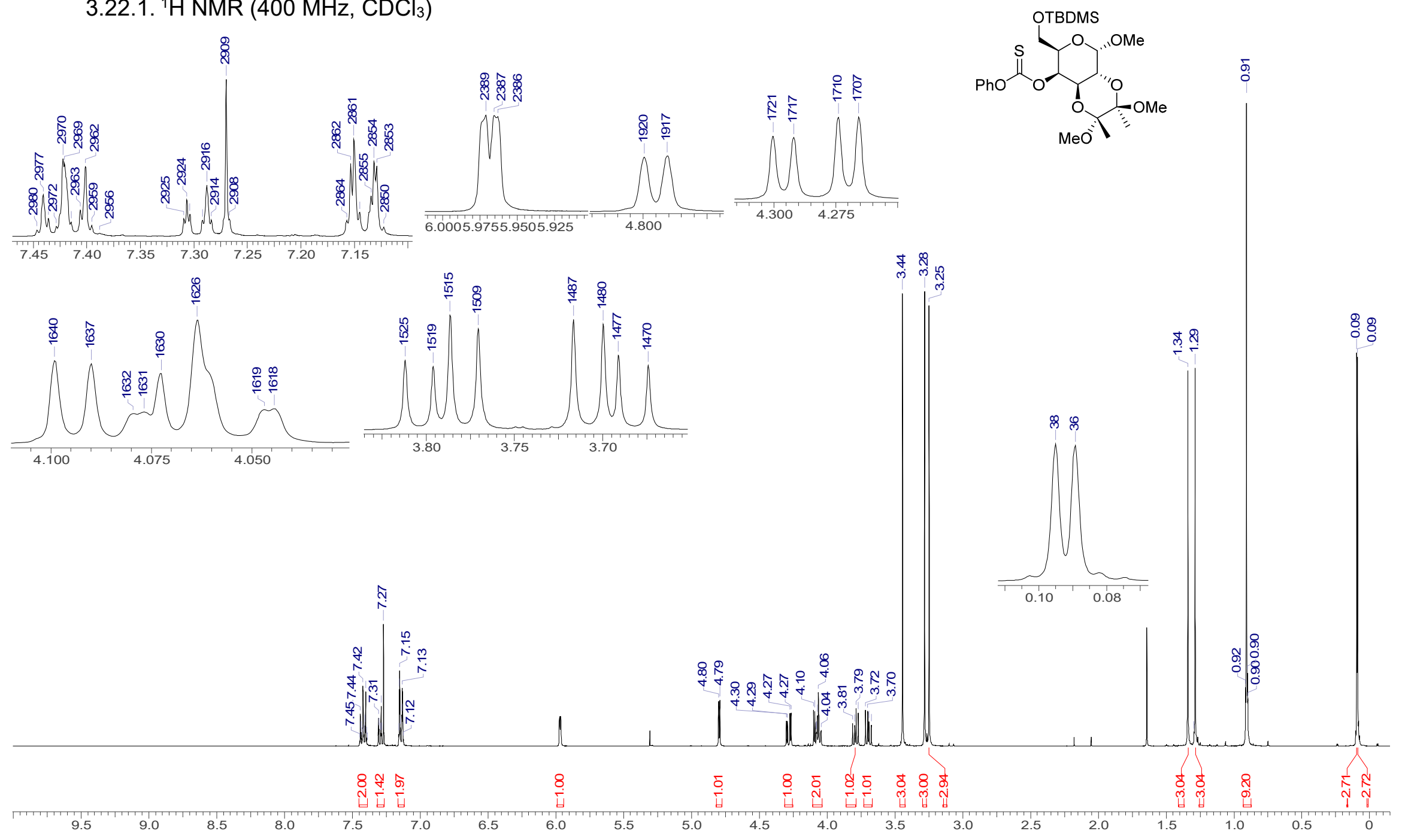




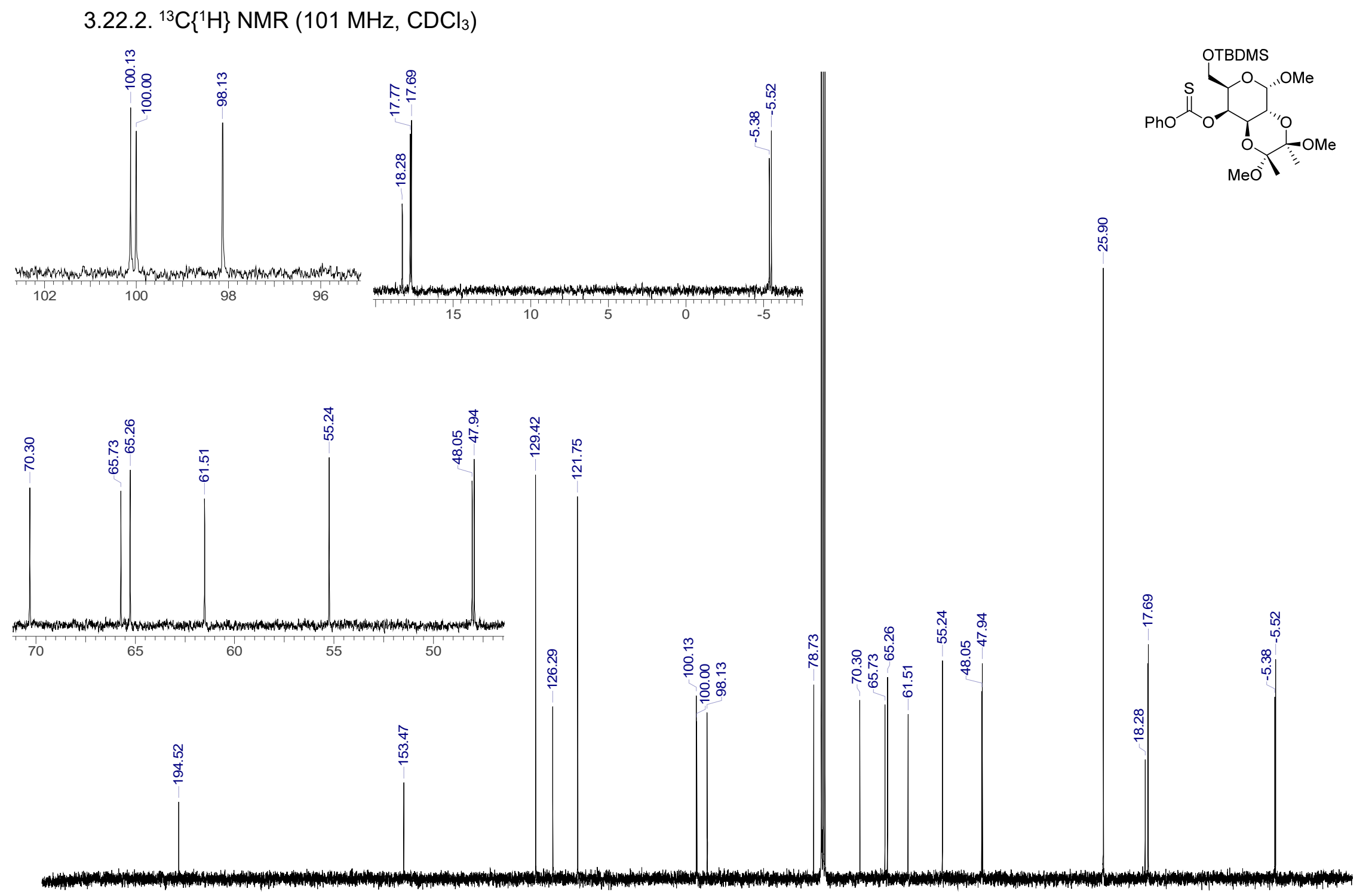

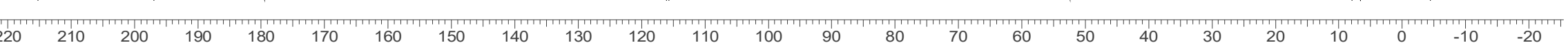


3.22.3. COSY, HSQC-DEPT \& $\mathrm{HMBC}\left(\mathrm{CDCl}_{3}\right)$

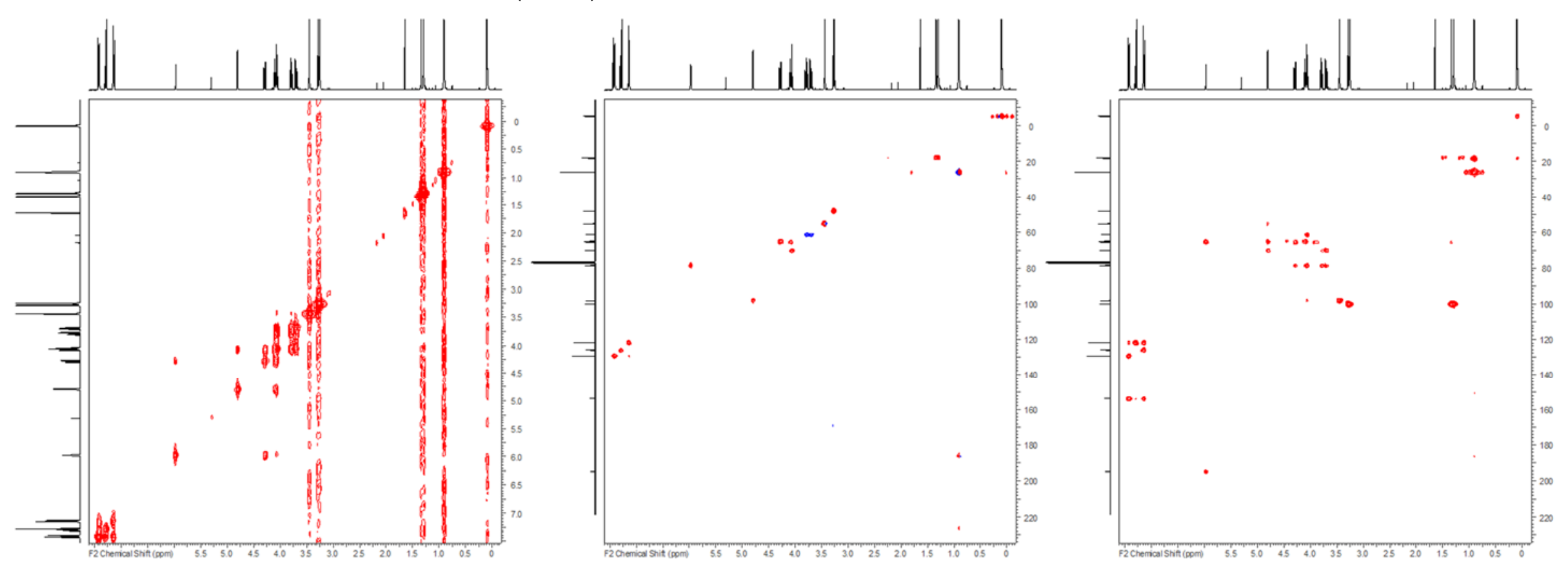


3.23. Methyl 6-O-tert-butyldimethylsilyl-4-deoxy-2,3-O-((2'R,3'R)-2',3'-dimethoxybutane-2', 3'-diyl)- $\alpha$-D-xylo-hexopyranoside (49):

3.23.1. ${ }^{1} \mathrm{H} \mathrm{NMR}\left(400 \mathrm{MHz}, \mathrm{CDCl}_{3}\right)$

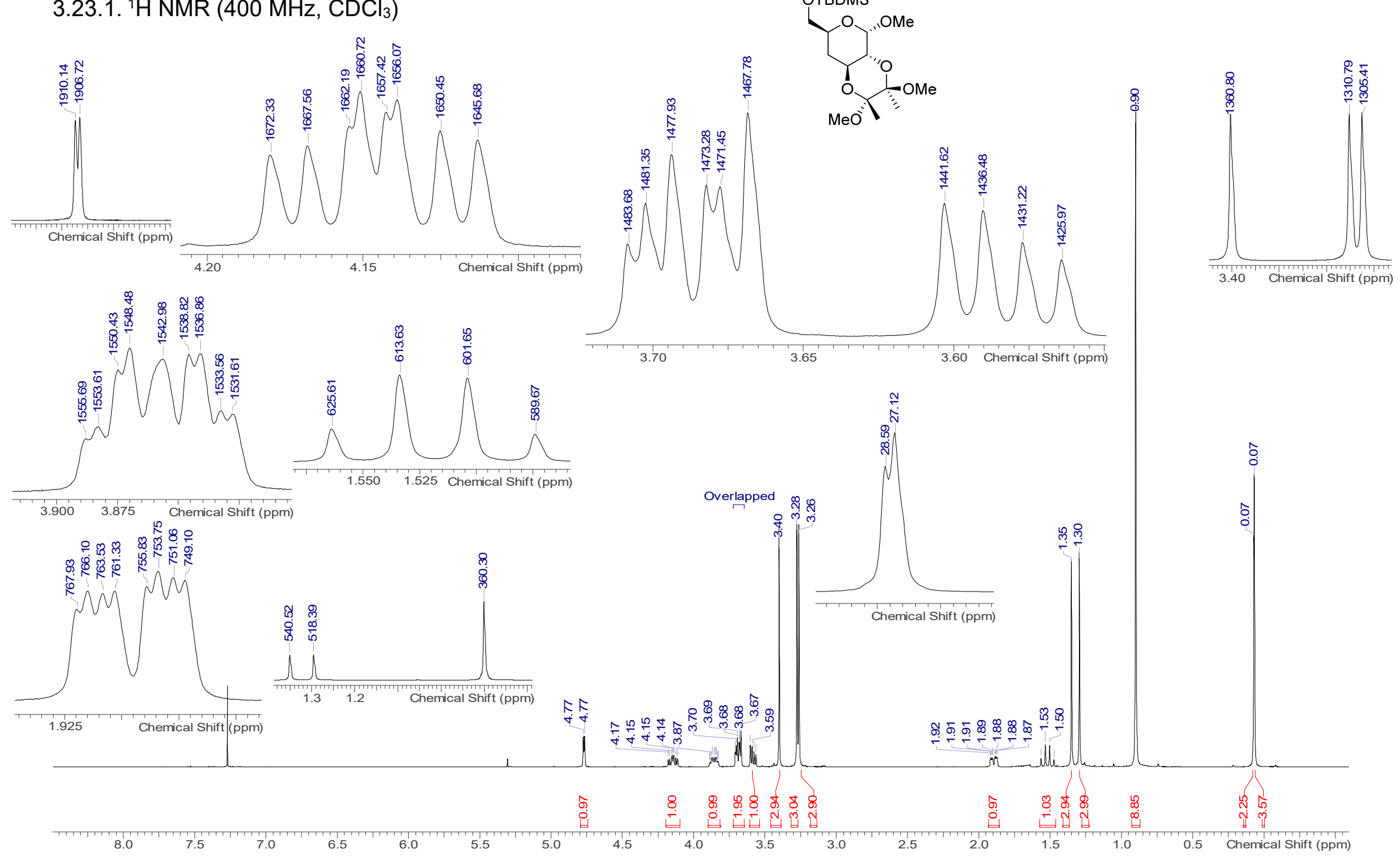


3.23.2. ${ }^{13} \mathrm{C}\left\{{ }^{1} \mathrm{H}\right\}$ NMR (101 MHz, $\left.\mathrm{CDCl}_{3}\right)$

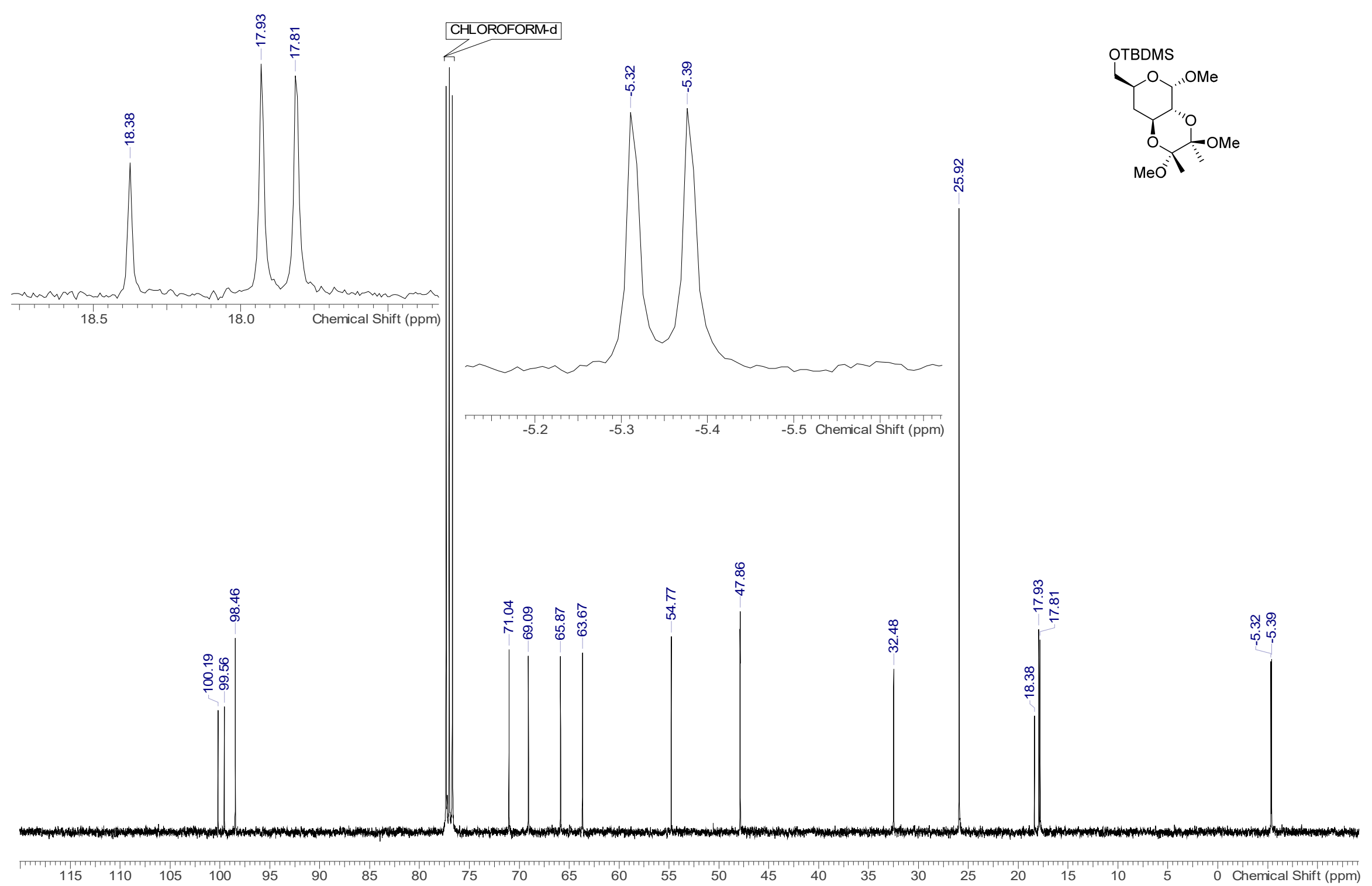


3.23.3. COSY, $\mathrm{HSQC} \& \mathrm{HMBC}\left(\mathrm{CDCl}_{3}\right)$

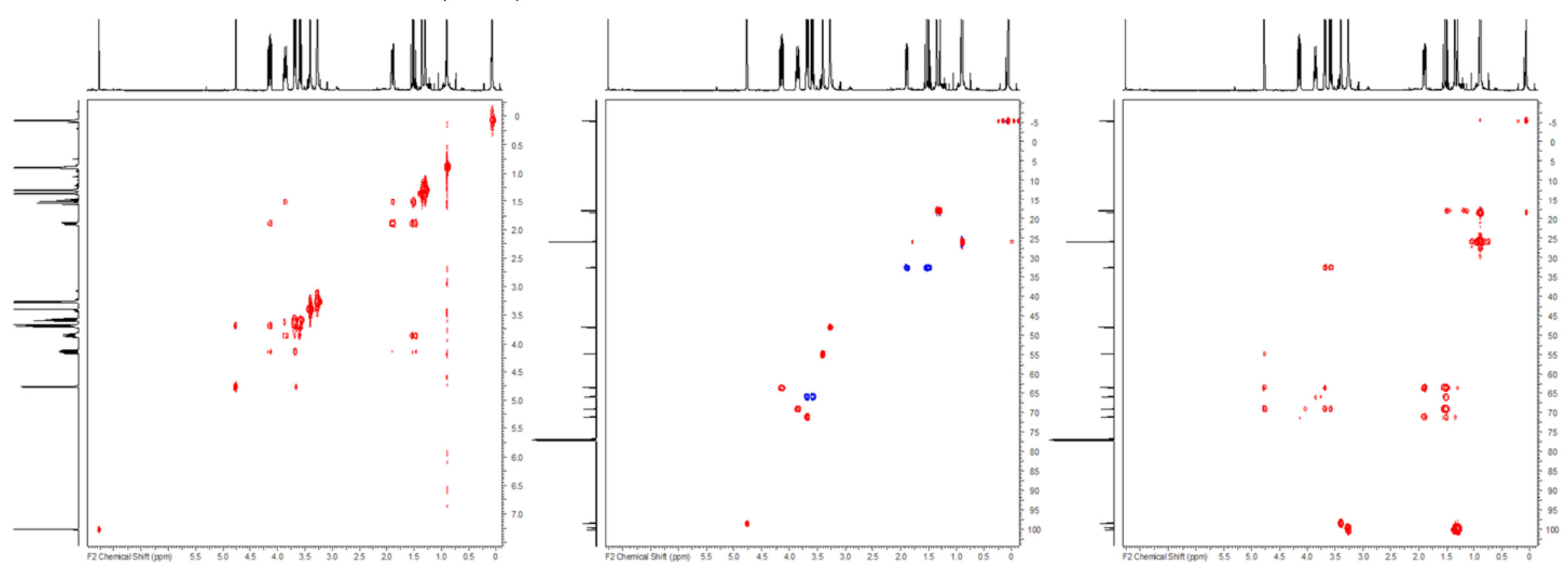


3.24. Mixture of Methyl 4-O-benzoyl-2,3-O-((2'R,3'S)-2',3'-dimethoxybutane-2',3'-diyl)-a-D-fucoopyranoside (52) and Methyl 6O-benzoyl-4-deoxy-2,3-O-((2'S,3'S)-2',3'-dimethoxybutane-2',3'-diyl)-a-D-xylo-hexopyranoside (53):

3.24.1. ${ }^{1} \mathrm{H} \mathrm{NMR}\left(400 \mathrm{MHz}, \mathrm{CDCl}_{3}\right)$

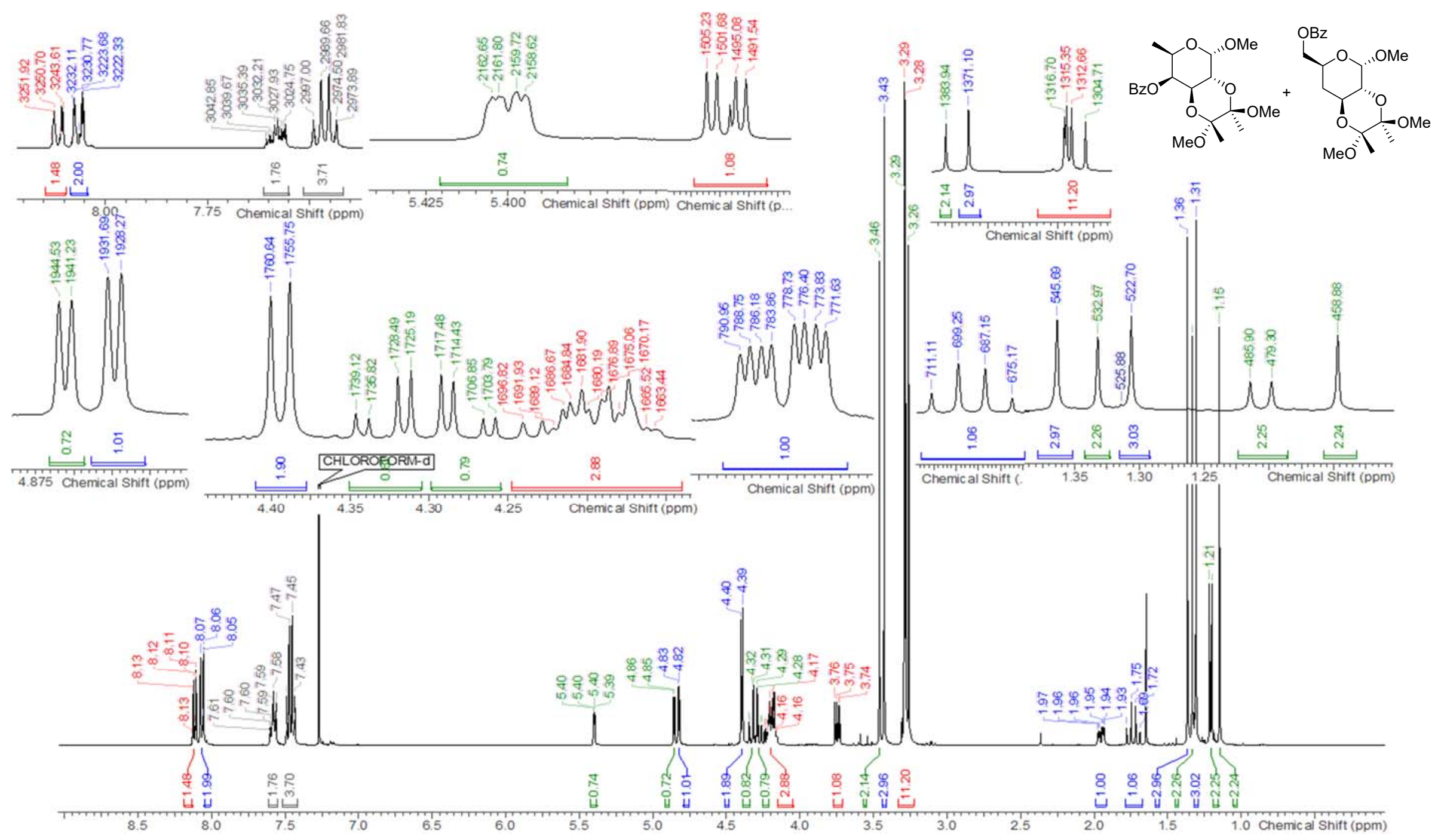


3.24.2. ${ }^{13} \mathrm{C}\left\{{ }^{1} \mathrm{H}\right\}$ NMR $\left(101 \mathrm{MHz}, \mathrm{CDCl}_{3}\right)$
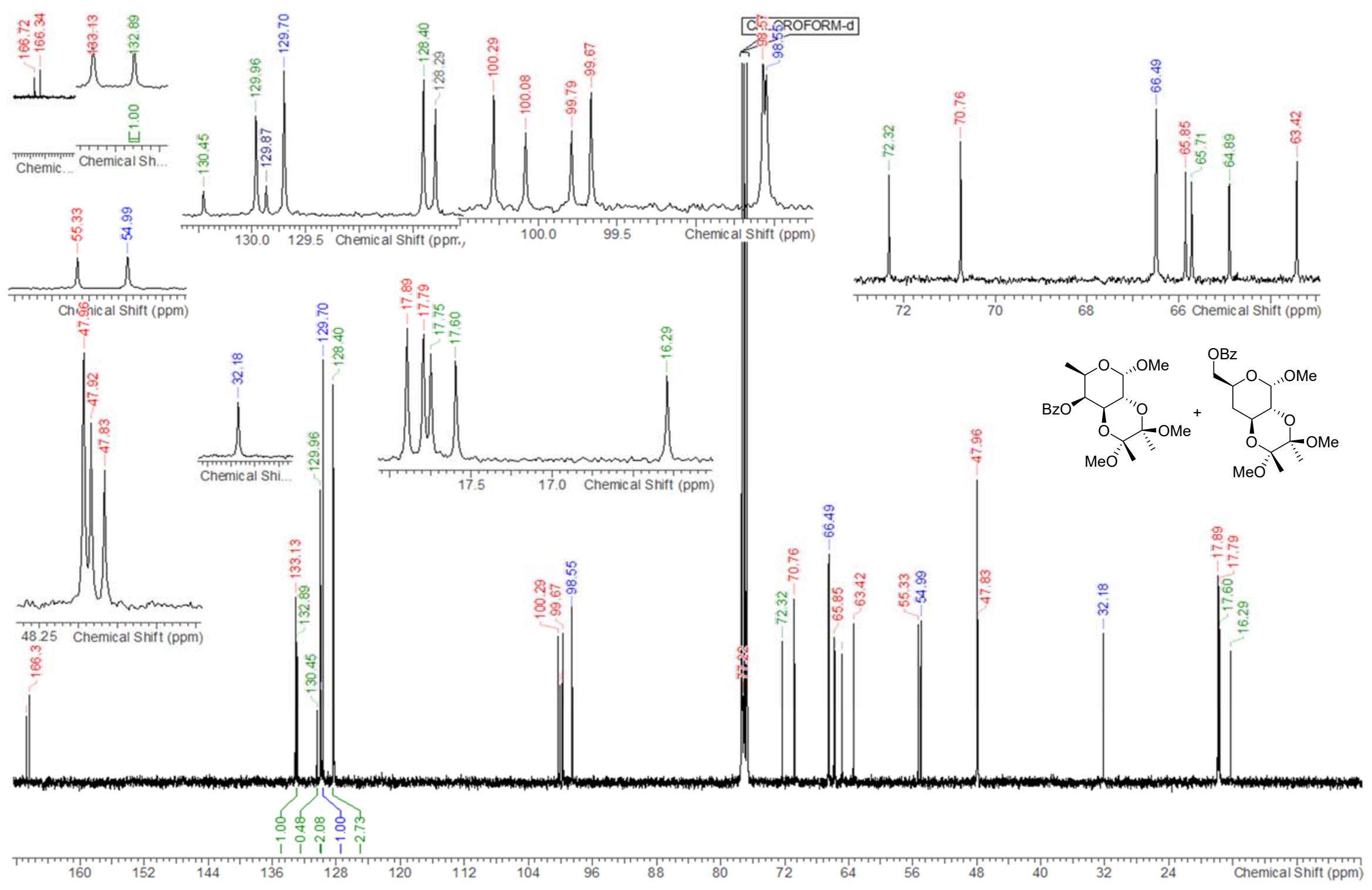
3.24.3. Mixture ratio determination

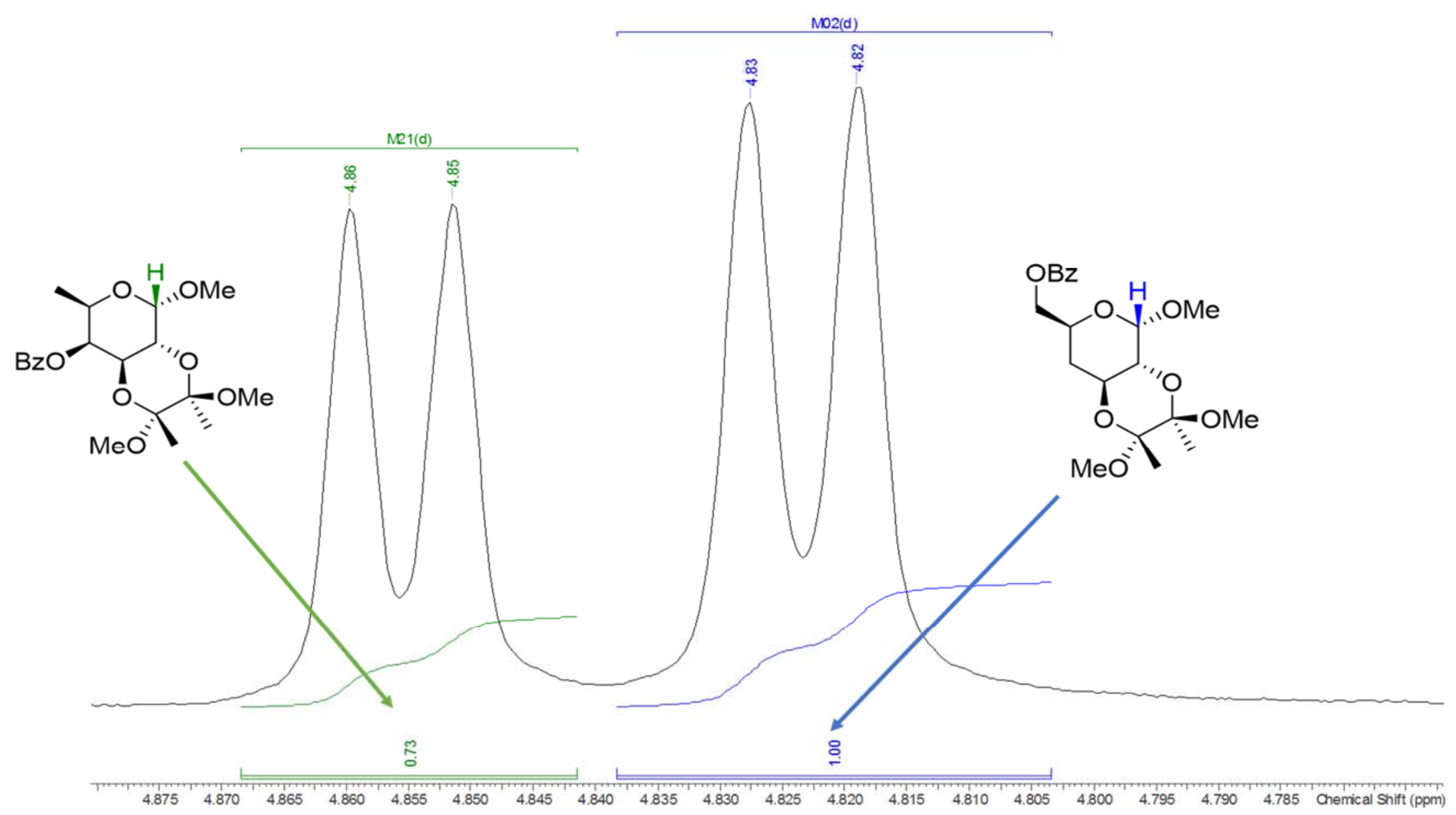

Ratio of $\mathrm{H}_{1}$ intergrals:(52/53) 42:58 


\subsection{Methyl 2,3-O-((2'R,3'R)-2',3'-dimethoxybutane-2',3'-diyl)- $\alpha$-D-fucopyranoside (54):} 3.25.1. ${ }^{1} \mathrm{H}$ NMR $\left(400 \mathrm{MHz}, \mathrm{CDCl}_{3}\right)$

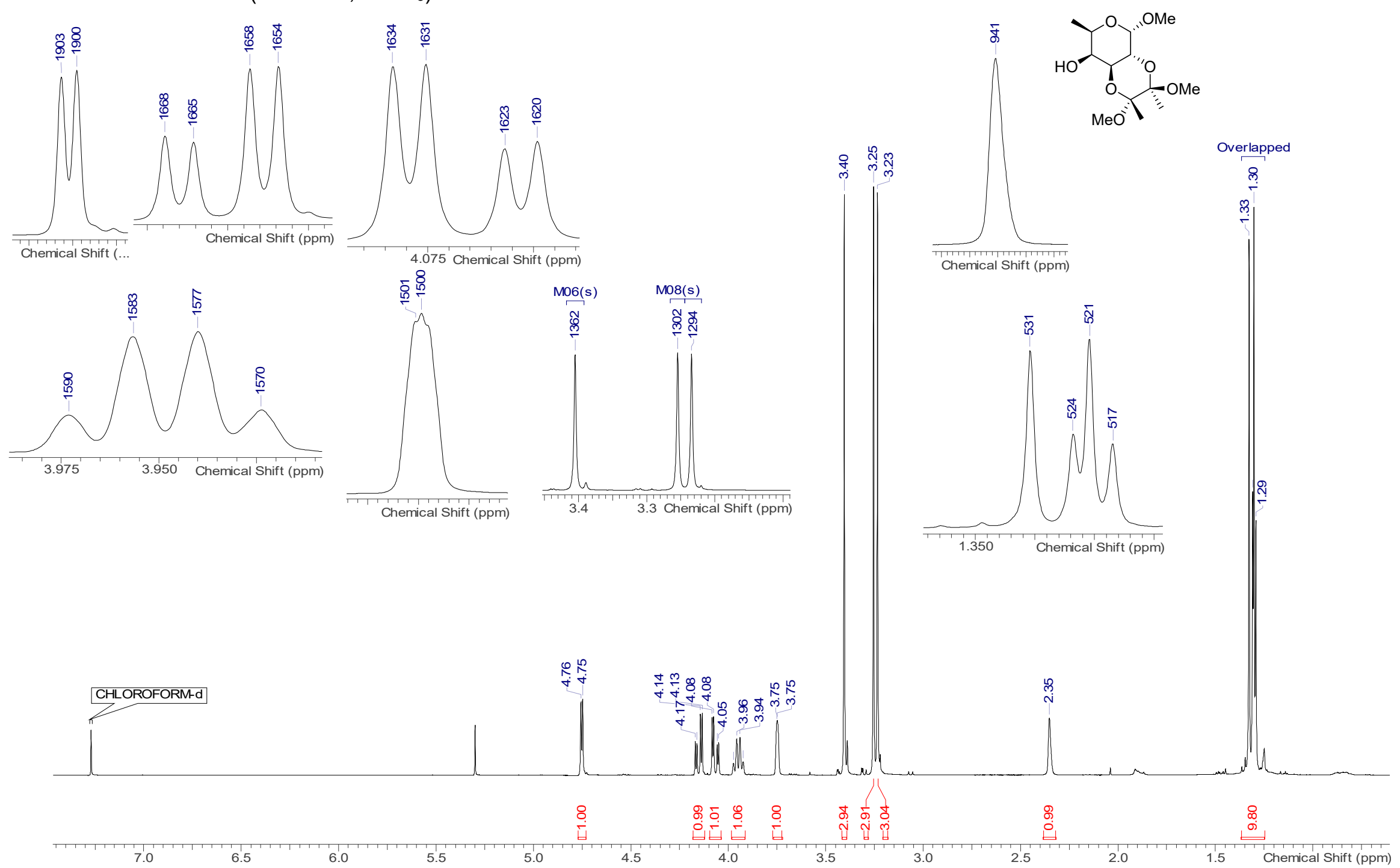


3.25.2. ${ }^{13} \mathrm{C}\left\{{ }^{1} \mathrm{H}\right\}$ NMR (101 MHz, $\left.\mathrm{CDCl}_{3}\right)$
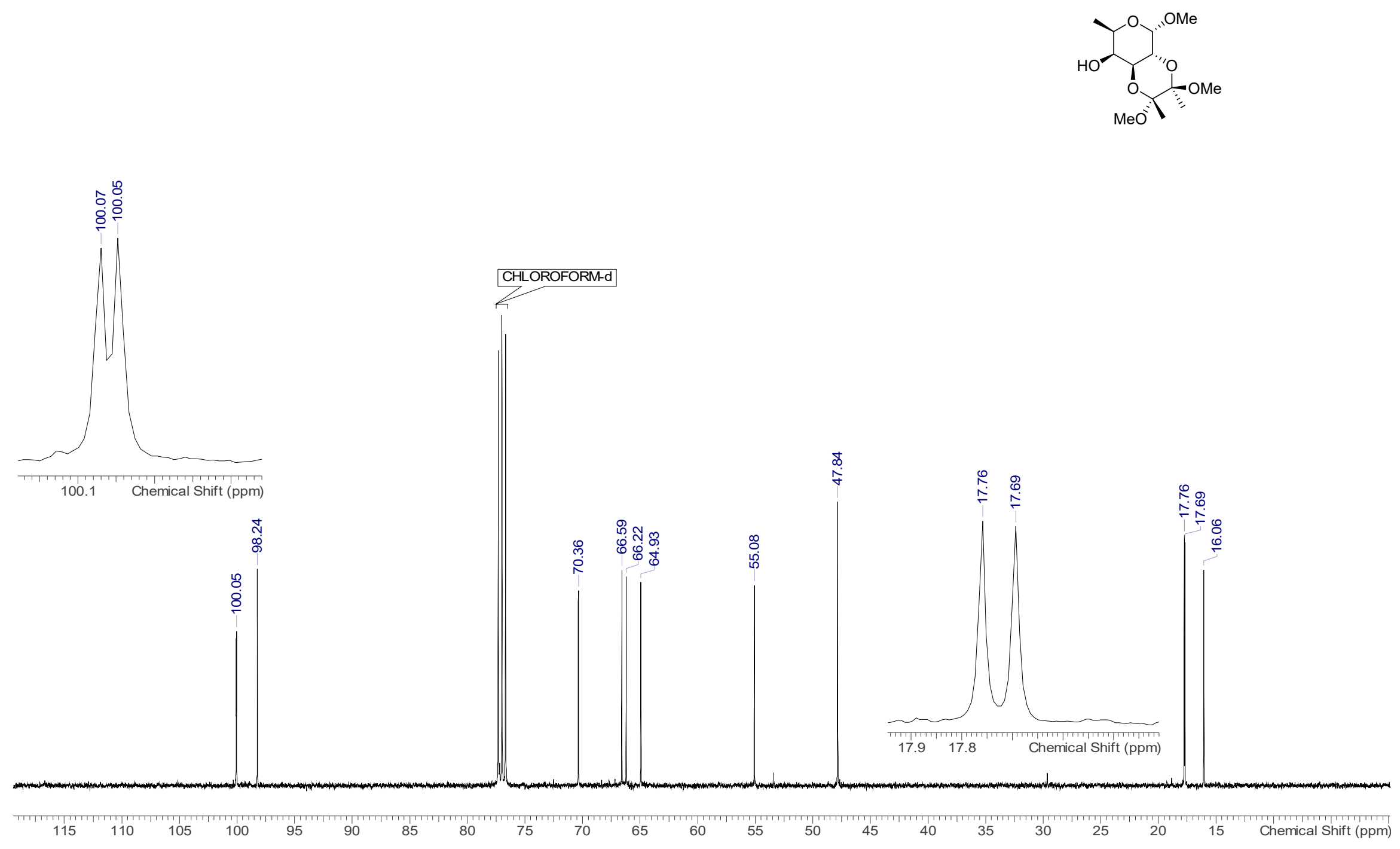
3.25.3. COSY, $\mathrm{HSQC} \& \mathrm{HMBC}\left(\mathrm{CDCl}_{3}\right)$

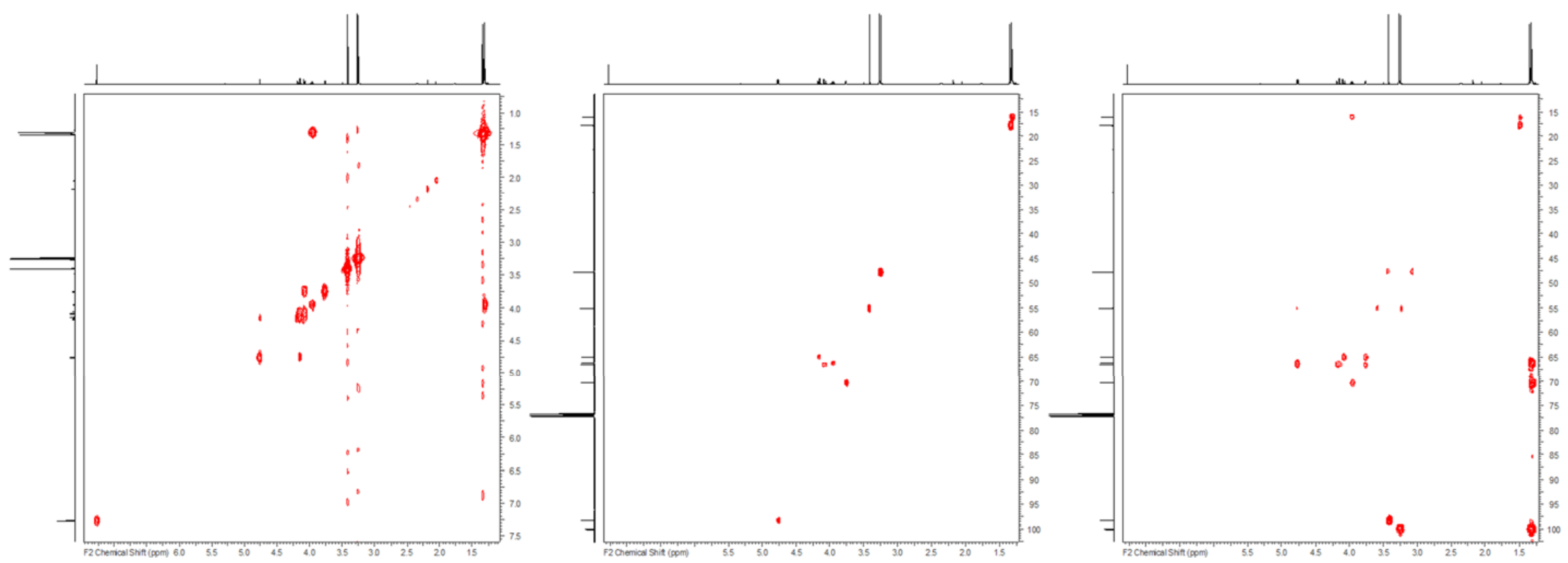




\subsection{Methyl 4-deoxy-2,3-O-((2'R,3'R)-2',3'-dimethoxybutane-2',3'-diyl)- $\alpha-D-x y l o-h e x o p y r a n o s i d e ~(55)$ :} 3.26.1. ${ }^{1} \mathrm{H} \mathrm{NMR}\left(400 \mathrm{MHz}, \mathrm{CDCl}_{3}\right)$

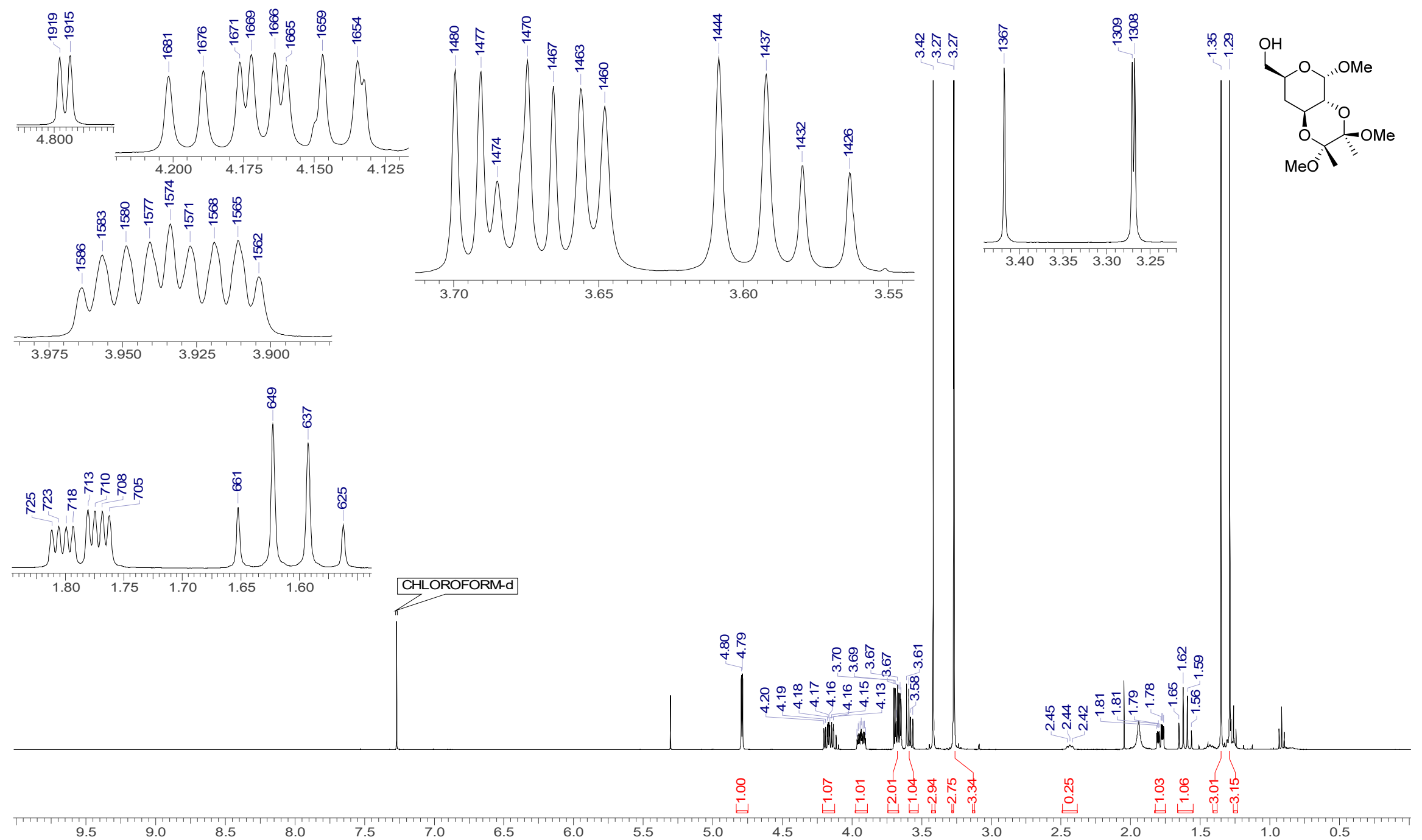


3.26.2. ${ }^{13} \mathrm{C}\left\{{ }^{1} \mathrm{H}\right\} \mathrm{NMR}\left(101 \mathrm{MHz}, \mathrm{CDCl}_{3}\right)$
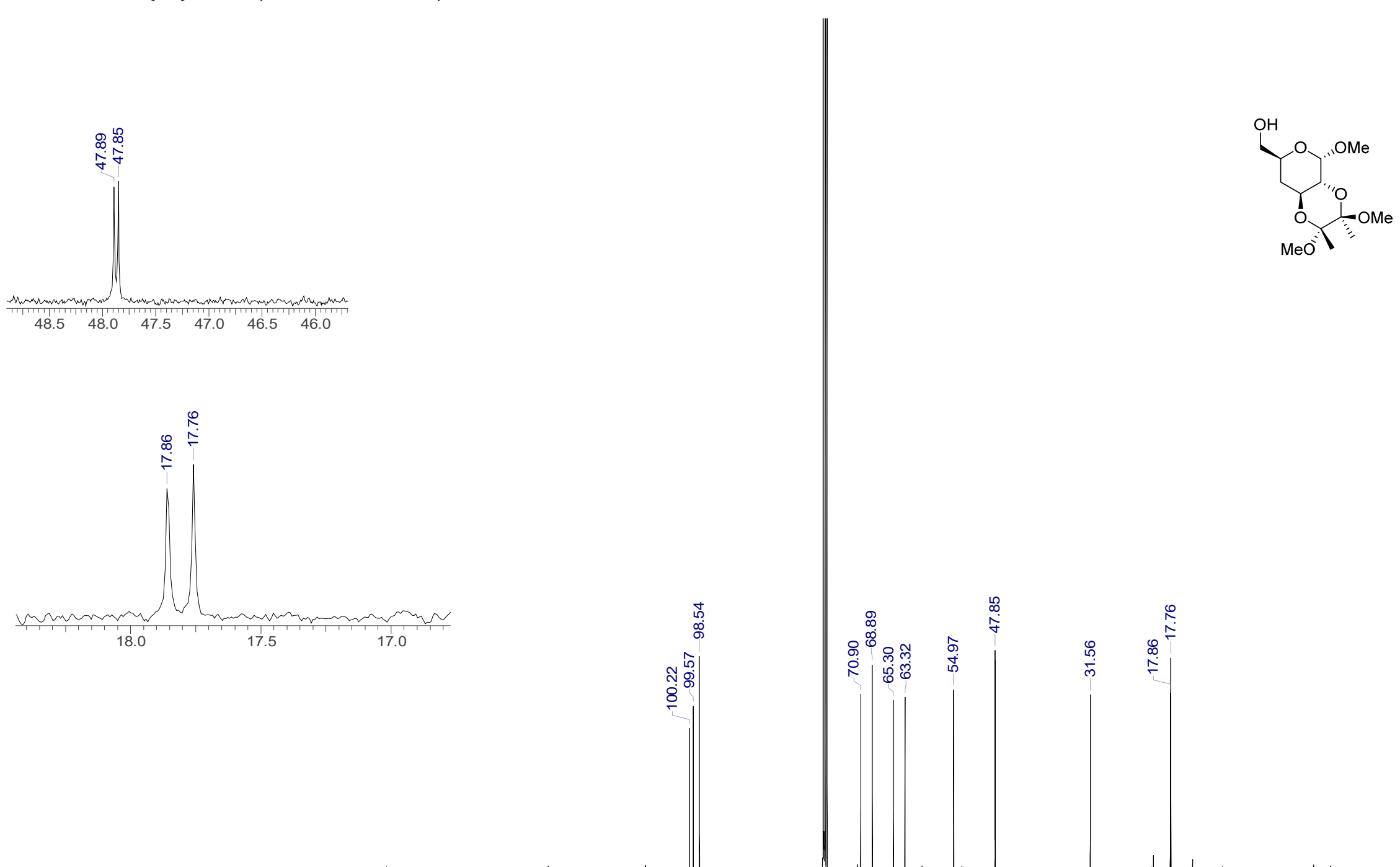

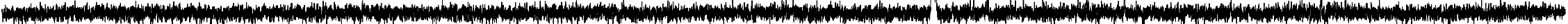

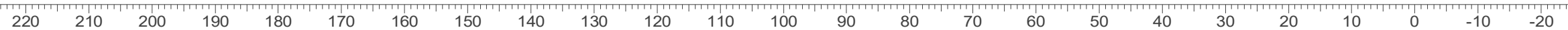


3.26.3. COSY, HSQC-DEPT, $\mathrm{HMBC}\left(\mathrm{CDCl}_{3}\right)$

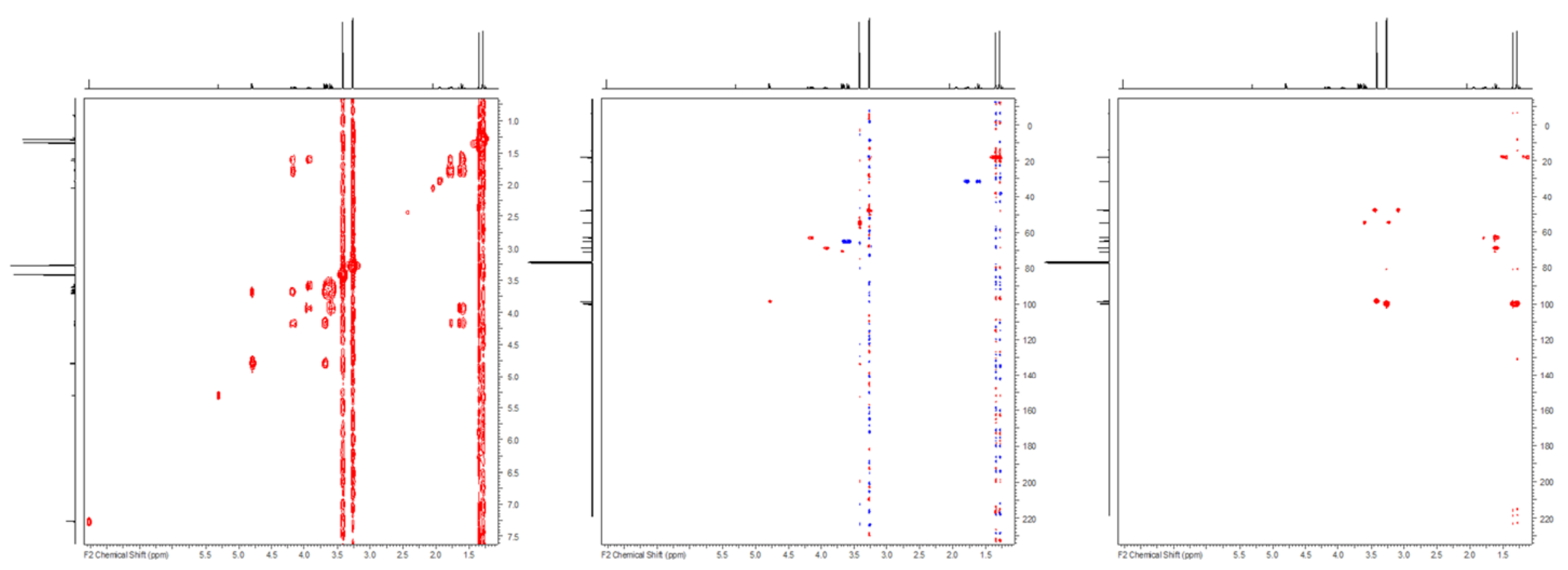


3.27. Methyl 4,6-dideoxy-6-fluoro 2,3-O-((2'R,3'R)-2',3'-dimethoxybutane-2', 3'-diyl)- $\alpha$-D-xylo-hexopyranoside (56): 3.27.1. ${ }^{1} \mathrm{H} \mathrm{NMR}\left(400 \mathrm{MHz}, \mathrm{CDCl}_{3}\right)$

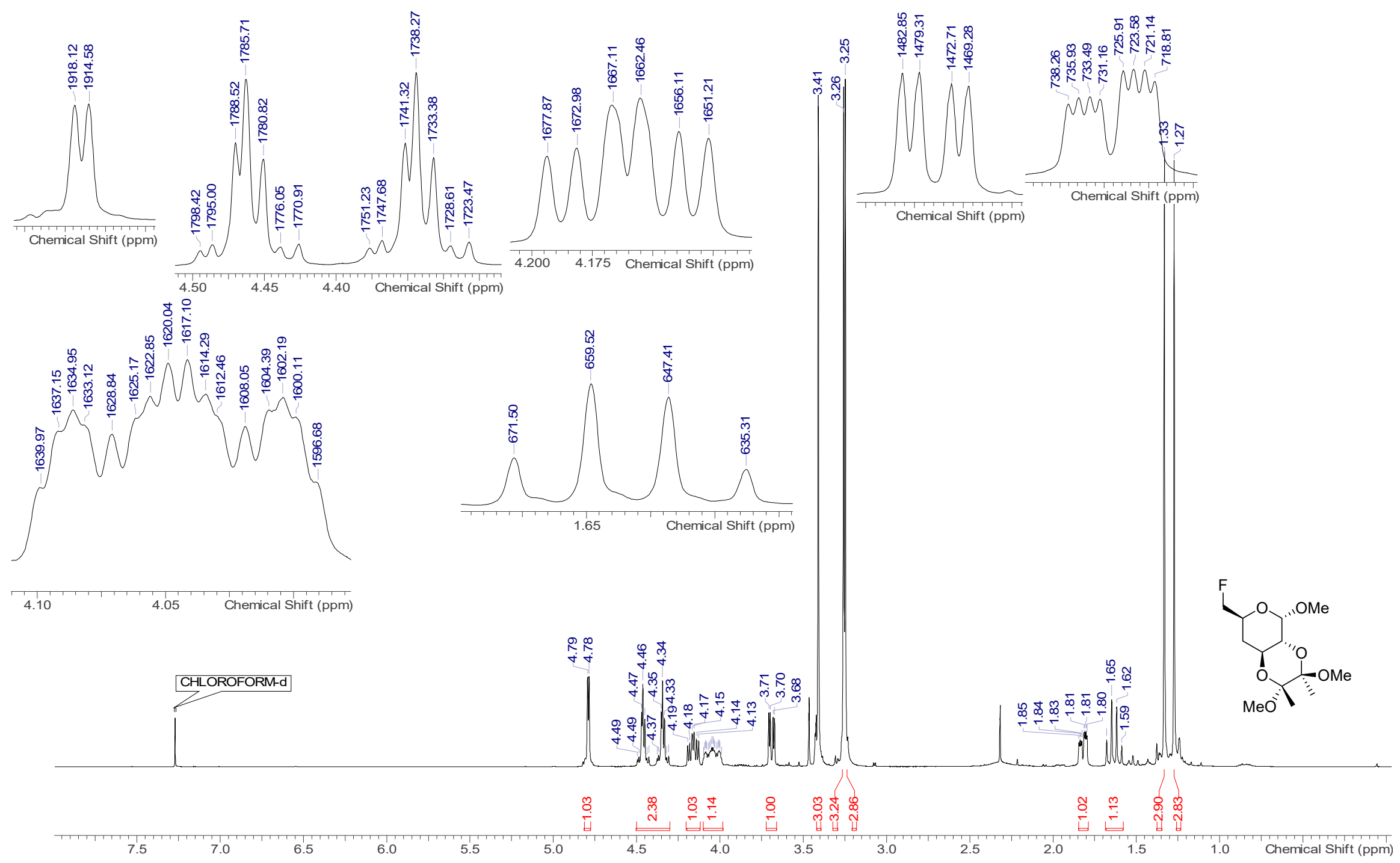




\subsection{2. ${ }^{13} \mathrm{C}\left\{{ }^{1} \mathrm{H}\right\}$ NMR $\left(101 \mathrm{MHz}, \mathrm{CDCl}_{3}\right)$}
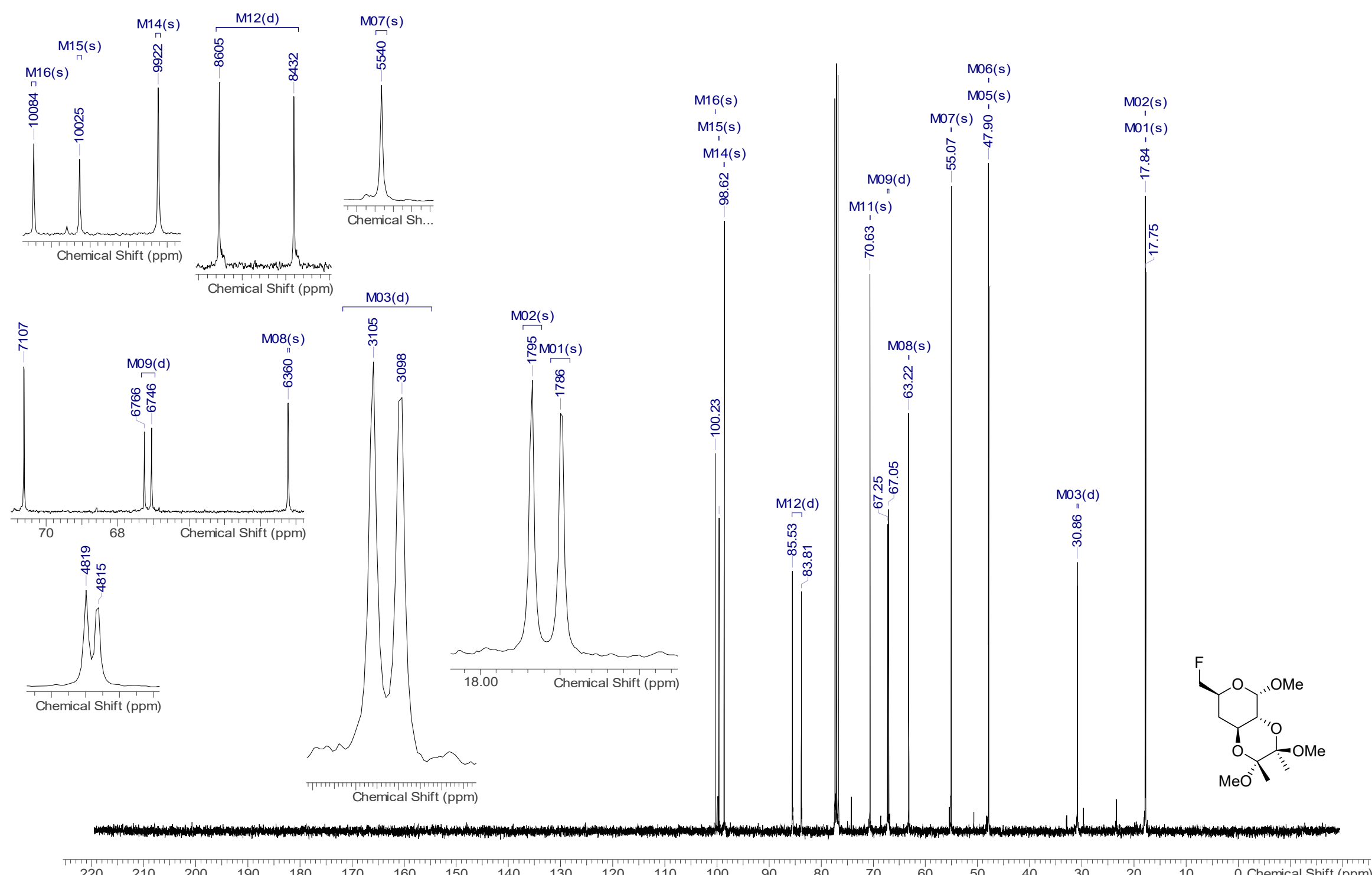
3.27.3. COSY \& HSQC $\left(\mathrm{CDCl}_{3}\right)$

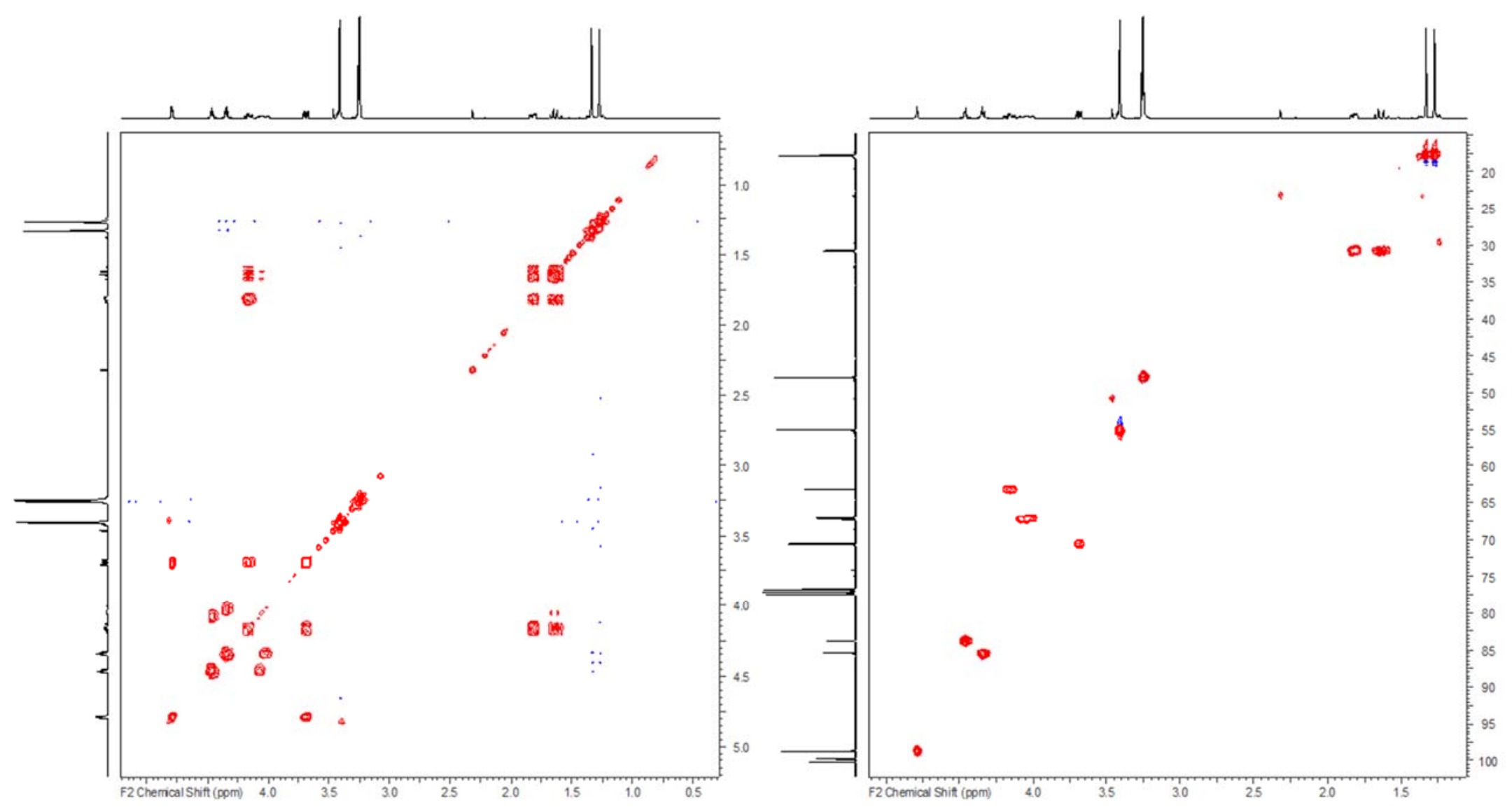


S 158

3.27.4. ${ }^{19} \mathrm{~F} \mathrm{NMR}\left(376 \mathrm{MHz}, \mathrm{CDCl}_{3}\right)$
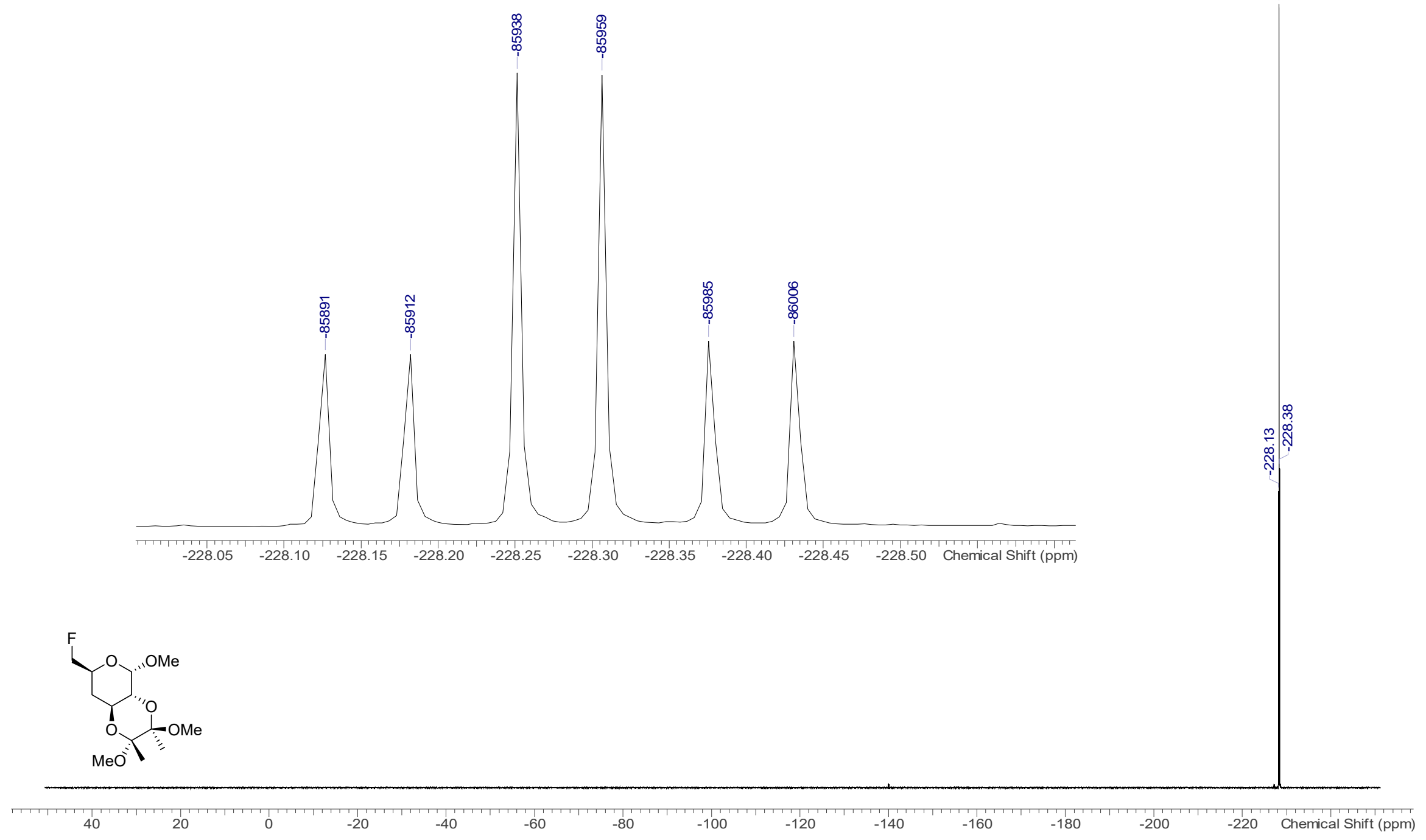
3.27.5. ${ }^{19} \mathrm{~F}\left\{{ }^{1} \mathrm{H}\right\}$ NMR (376 MHz, $\left.\mathrm{CDCl}_{3}\right)$
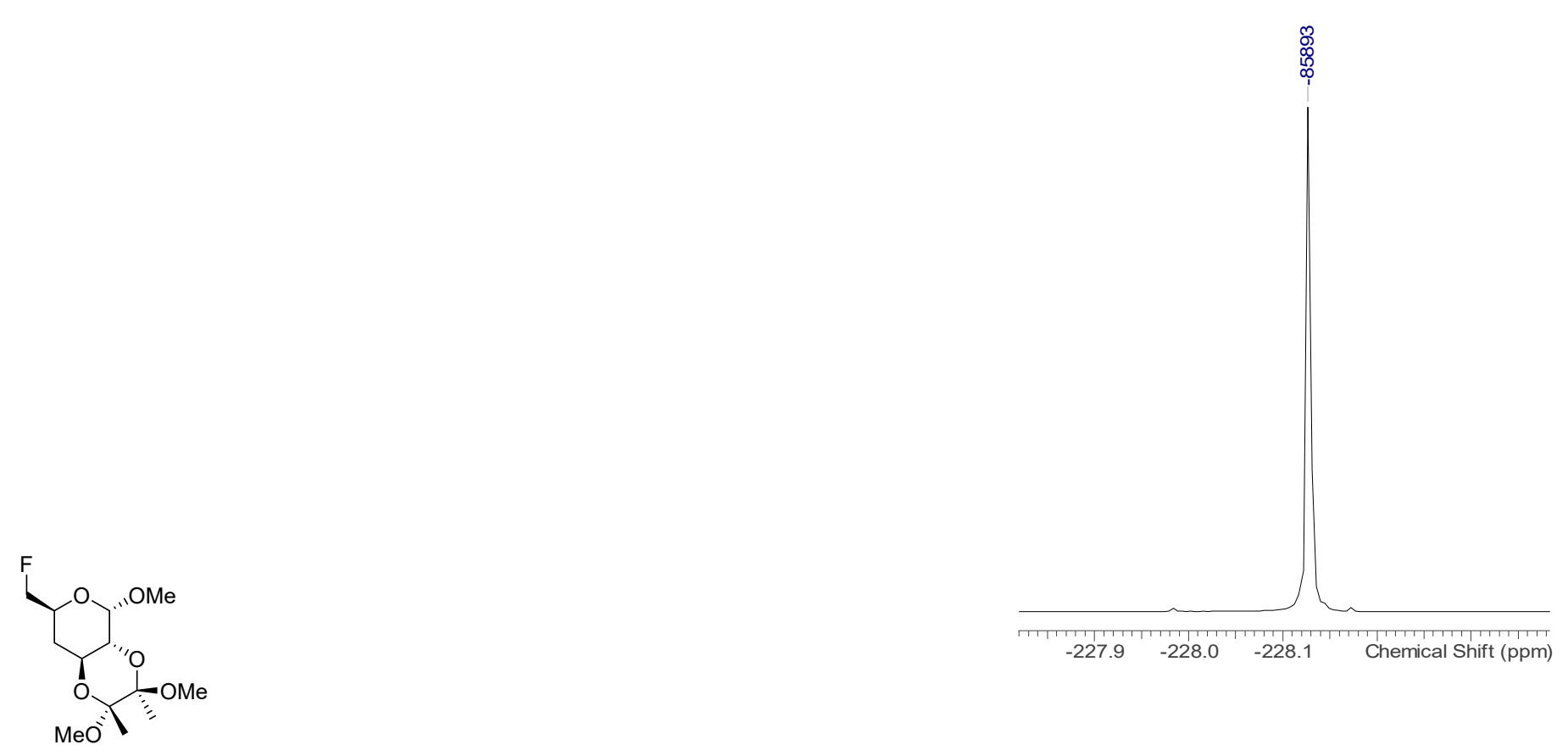
3.28. Mixture of Methyl 4-O-benzoyl-2,3-O-((2'S,3'S)-2',3'-dimethoxybutane-2',3'-diyl)- $\alpha$-D-fucoopyranoside (57) and Methyl 6O-benzoyl-4-deoxy-2,3-O-((2'S,3'S)-2',3'-dimethoxybutane-2',3'-diyl)-a-D-xylo-hexopyranoside (58): 3.28.1. ${ }^{1} \mathrm{H}$ NMR $\left(400 \mathrm{MHz}, \mathrm{CDCl}_{3}\right)$

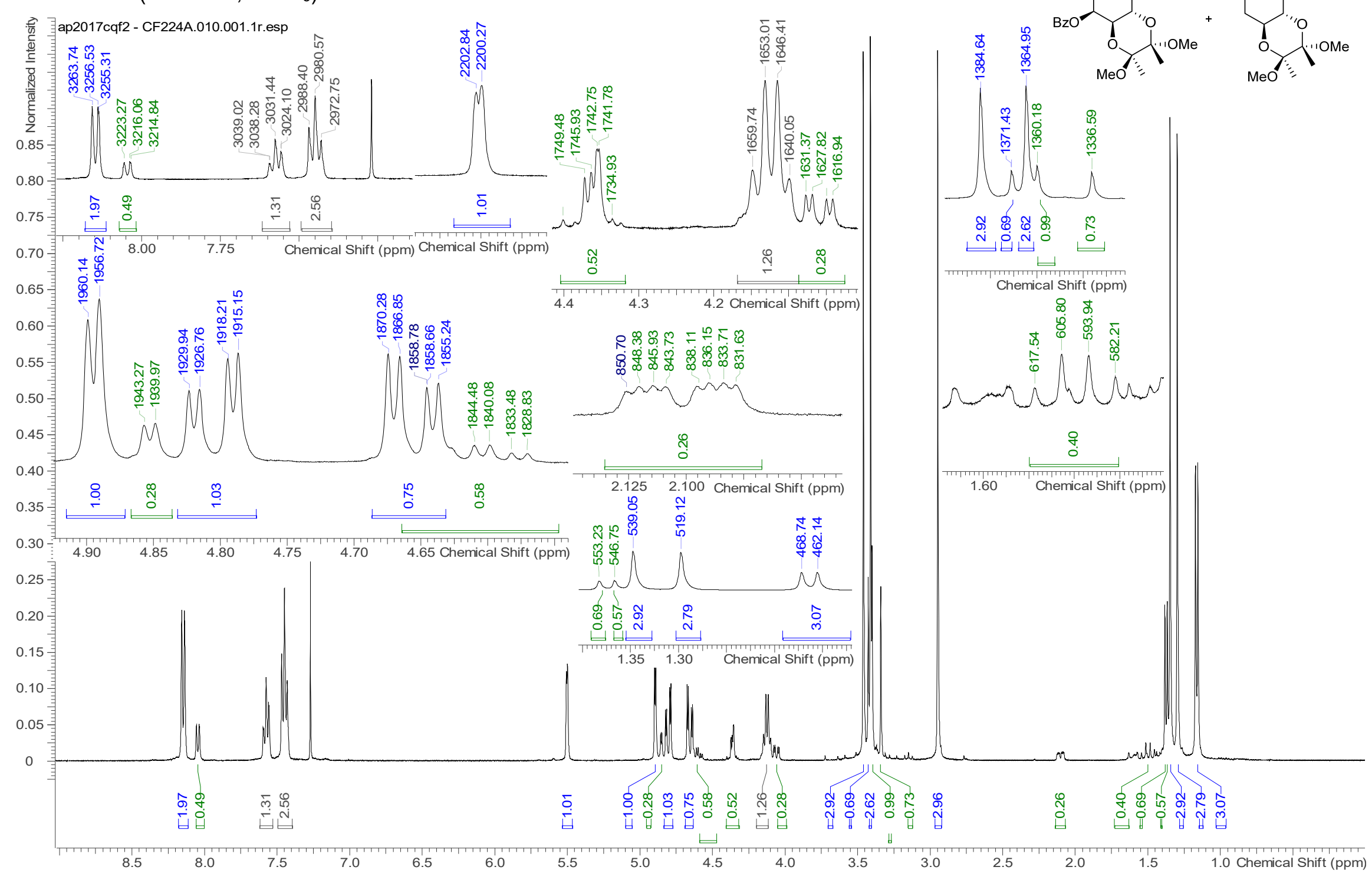


3.28.2. ${ }^{13} \mathrm{C}\left\{{ }^{1} \mathrm{H}\right\}$ NMR $\left(101 \mathrm{MHz}, \mathrm{CDCl}_{3}\right)$

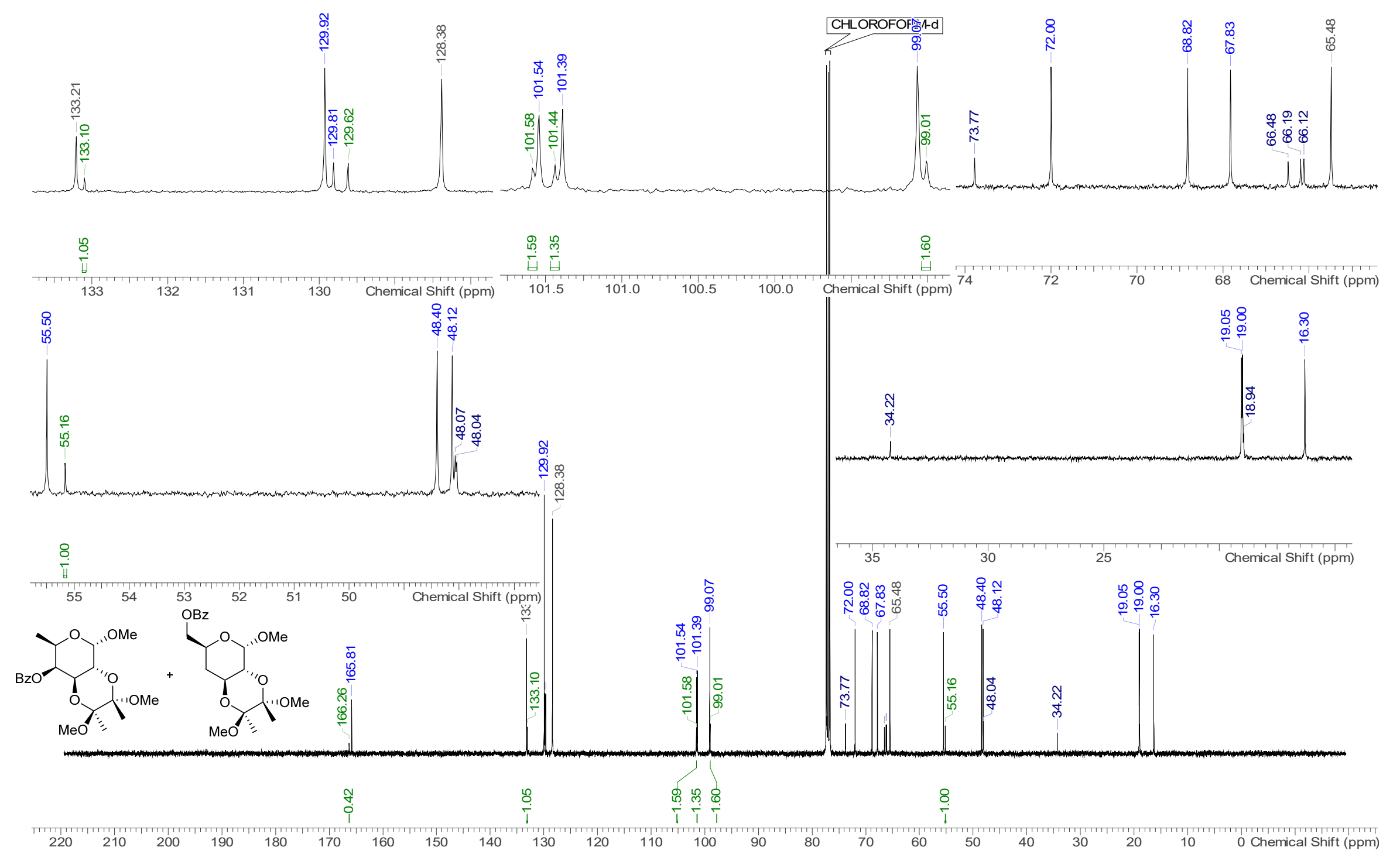


3.28.3. Mixture ratio determination

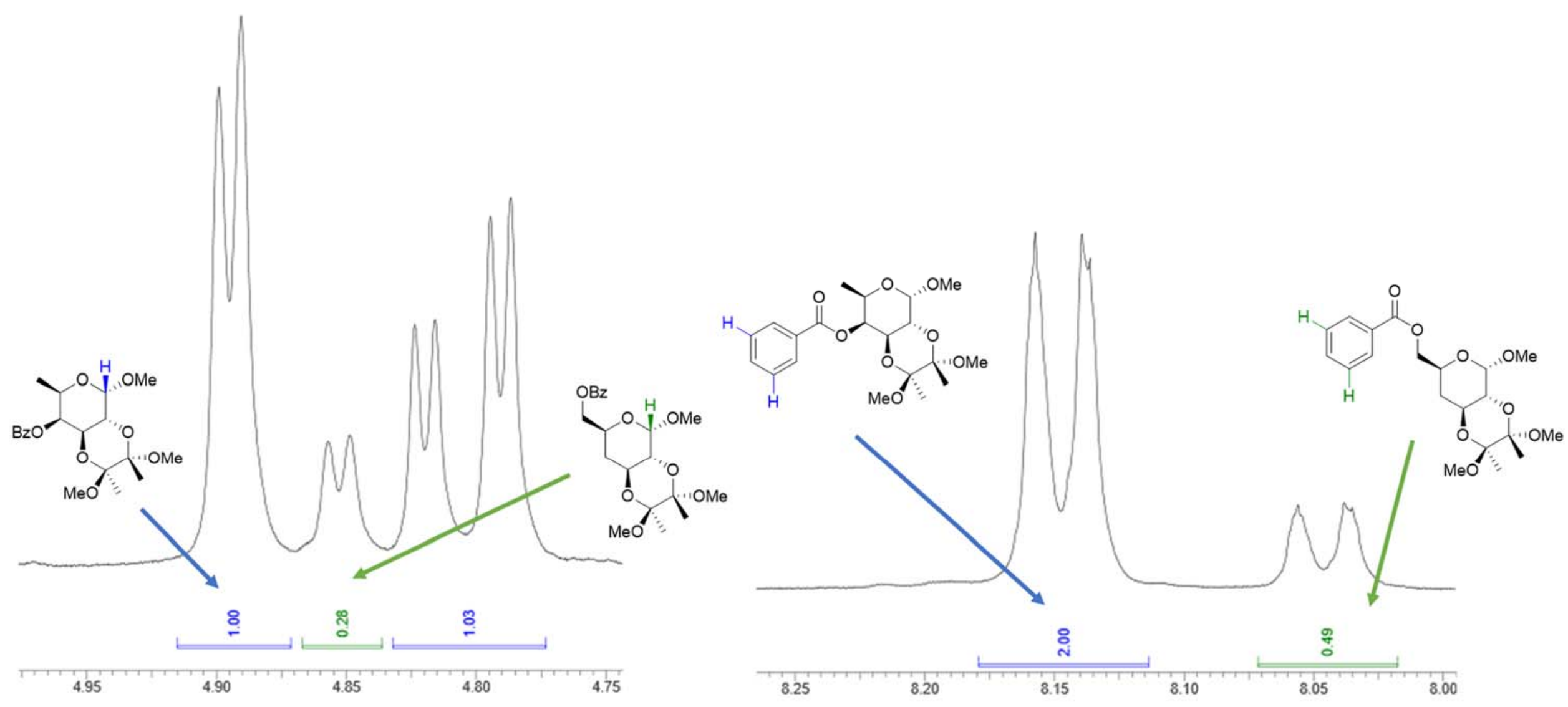

Ratio of $\mathrm{H}_{1}$ intergrals: (57/58) 78/22

Ratio of $\mathrm{Ar}_{H}$ intergrals: (57/58) 80/20 
3.29. Methyl 4,6-dideoxy-4-chloro-6-fluoro- $\alpha$-D-galactopyranoside (60) 3.29.1. ${ }^{1} \mathrm{H} \mathrm{NMR}\left(400 \mathrm{MHz}, \mathrm{CDCl}_{3}\right)$
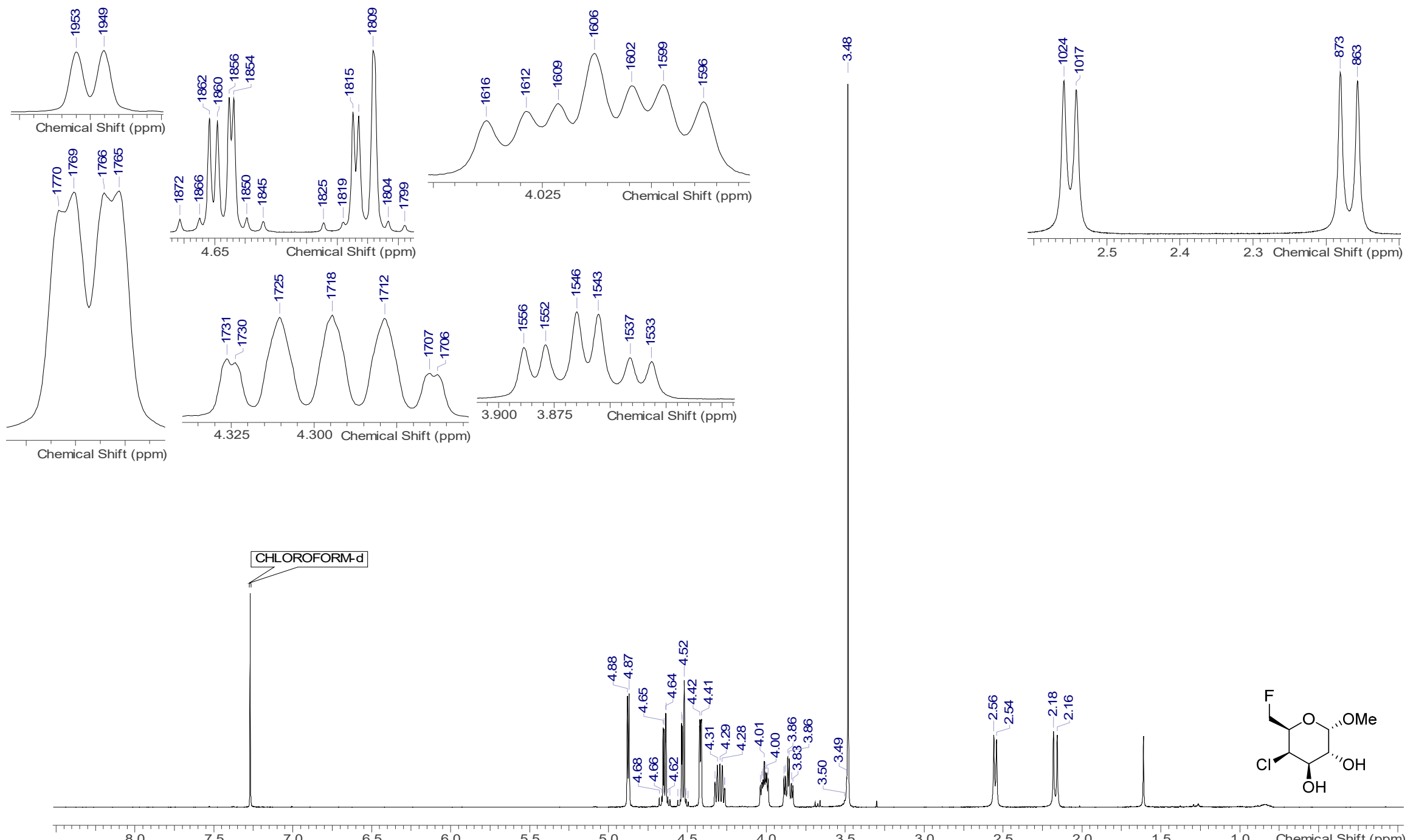

$\begin{array}{lll}1.0 & 1717111.5\end{array}$ 6.5 6.0 5

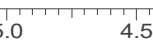

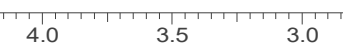
2.5 
3.29.2. ${ }^{13} \mathrm{C}\left\{{ }^{1} \mathrm{H}\right\} \mathrm{NMR}\left(101 \mathrm{MHz}, \mathrm{CDCl}_{3}\right)$
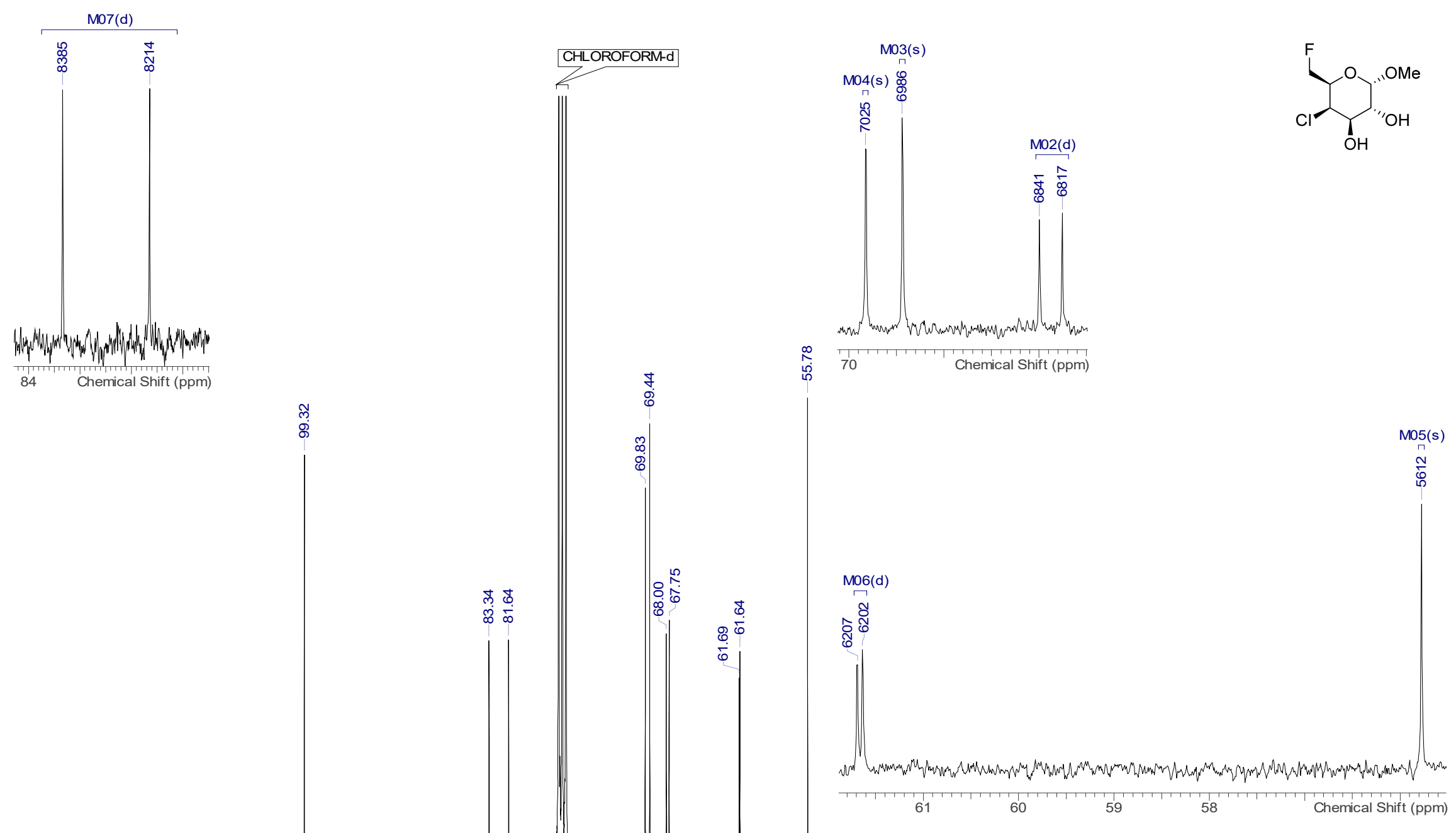

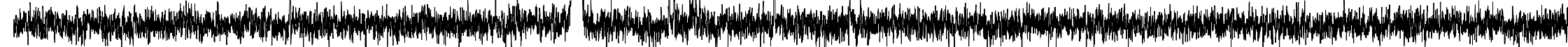

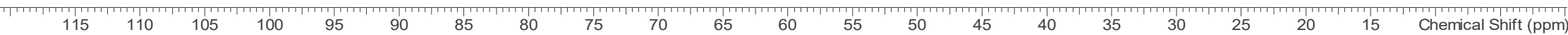


3.29.3. COSY \& HSQC $\left(\mathrm{CDCl}_{3}\right)$

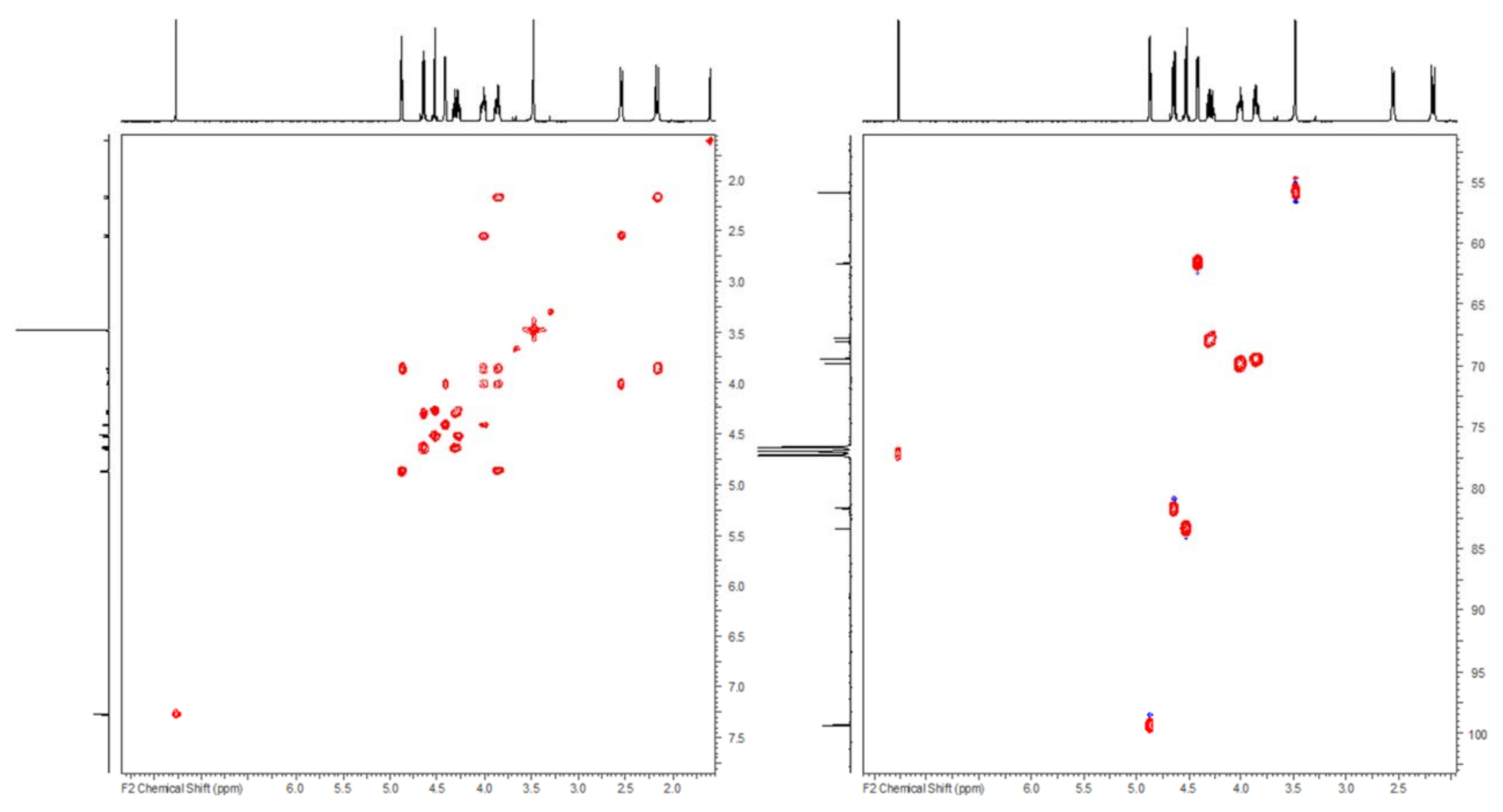


3.29.4. ${ }^{19} \mathrm{~F} \mathrm{NMR}\left(376 \mathrm{MHz}, \mathrm{CDCl}_{3}\right)$

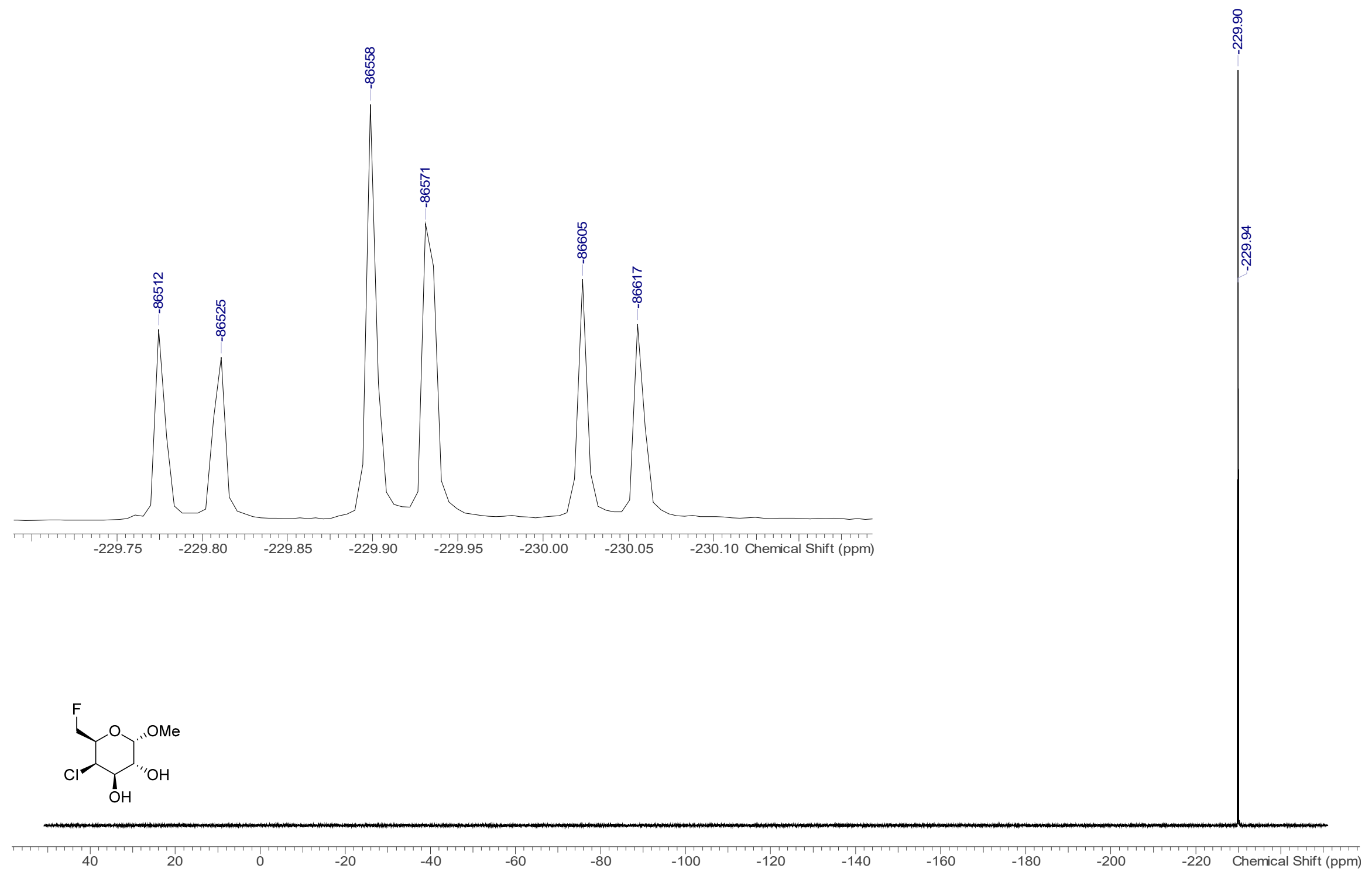


3.29.5. ${ }^{19} \mathrm{~F}\left\{{ }^{1} \mathrm{H}\right\}$ NMR $\left(376 \mathrm{MHz}, \mathrm{CDCl}_{3}\right)$

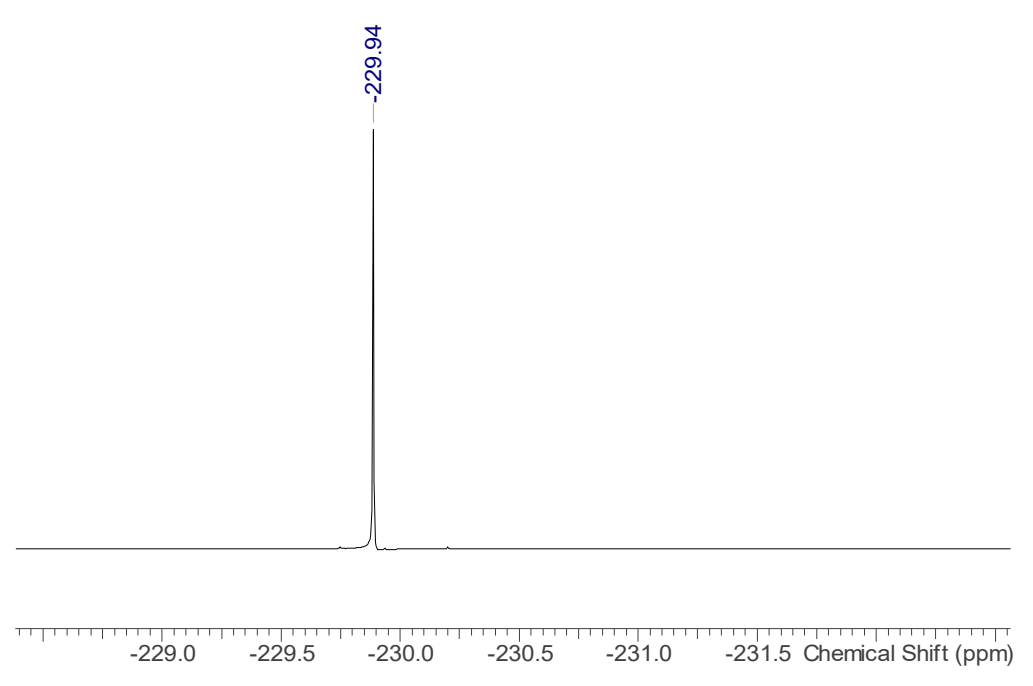

$\underbrace{\mathrm{O}}_{\mathrm{OH}} \mathrm{OH}$ 


\subsection{Methyl 4-deoxy-4-fluoro-6-O-tosyl- $\alpha$-D-galactopyranoside (62)}

3.30.1. ${ }^{1} \mathrm{H} \mathrm{NMR}\left(400 \mathrm{MHz}, \mathrm{CDCl}_{3}\right)$

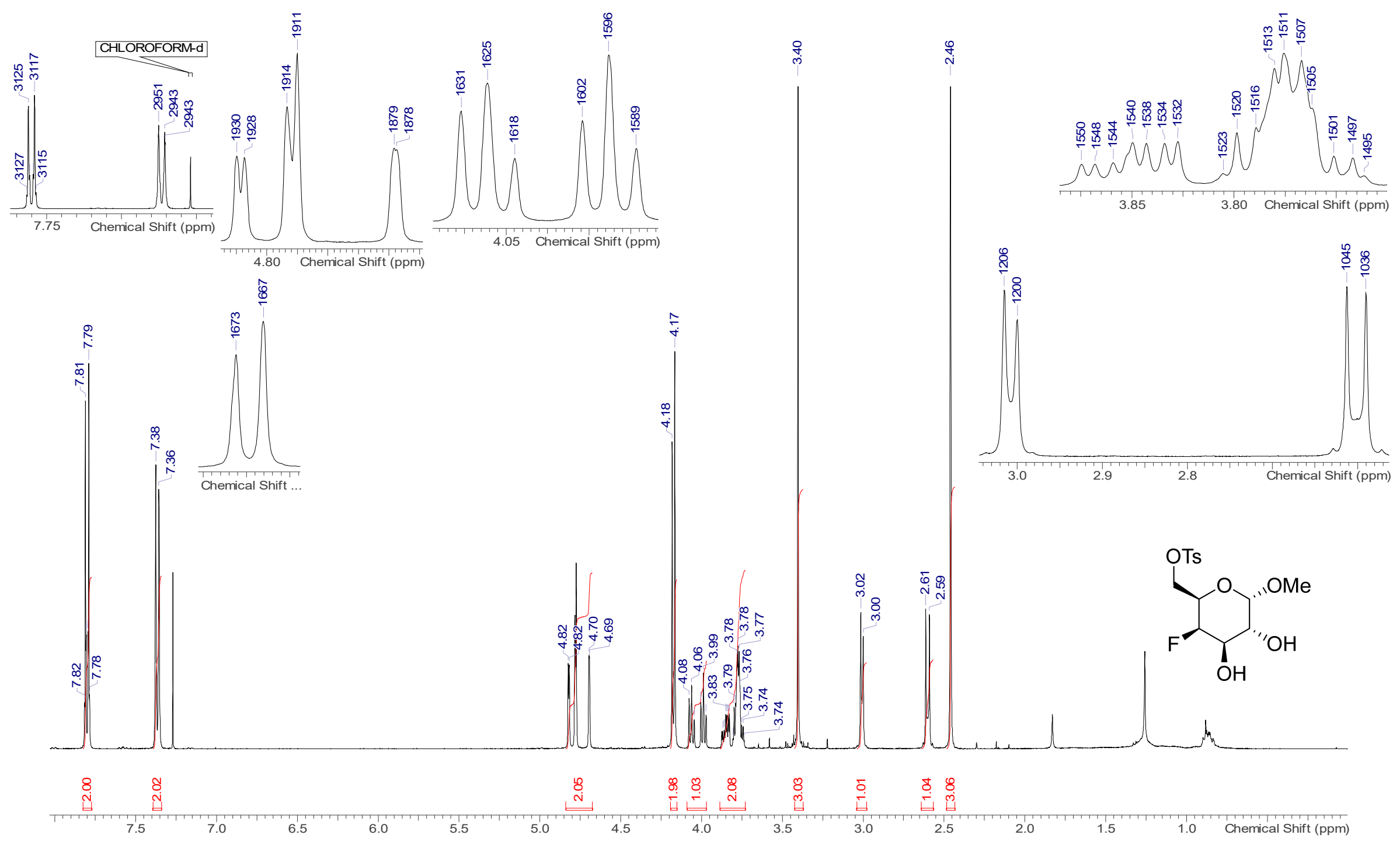


3.30.2. ${ }^{13} \mathrm{C}\left\{{ }^{1} \mathrm{H}\right\}$ NMR $\left(101 \mathrm{MHz}, \mathrm{CDCl}_{3}\right)$

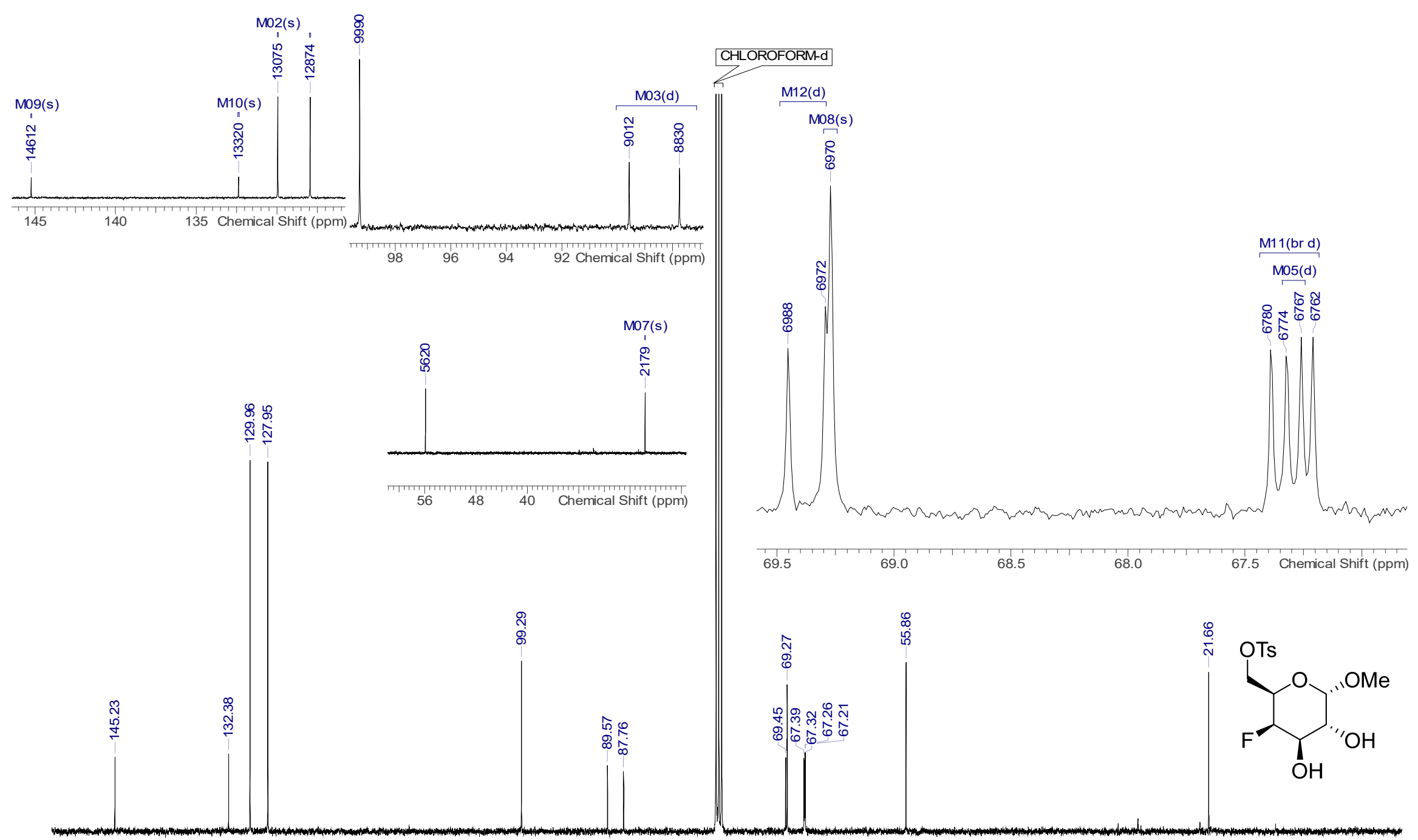

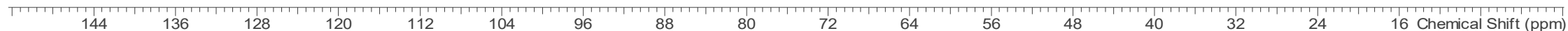


3.30.3. COSY, $\mathrm{HSQC} \& \mathrm{HMBC}\left(\mathrm{CDCl}_{3}\right)$

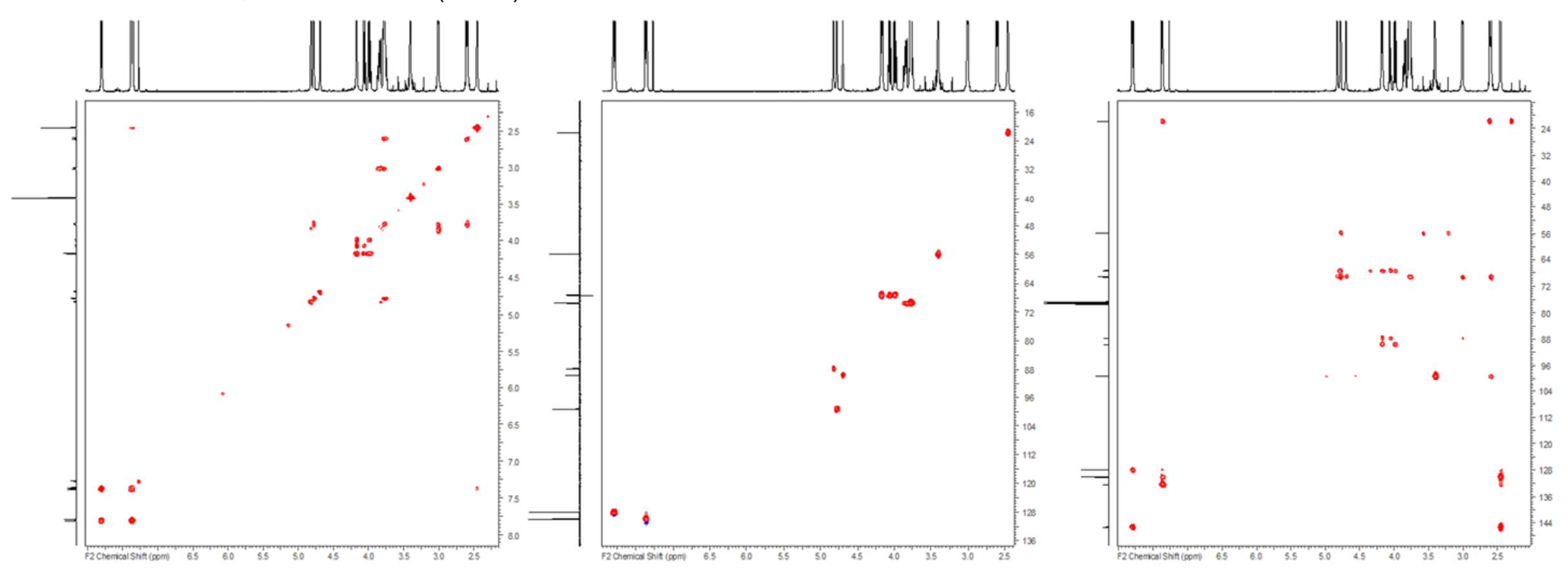


3.30.4. ${ }^{19} \mathrm{~F} \mathrm{NMR}\left(376 \mathrm{MHz}, \mathrm{CDCl}_{3}\right)$

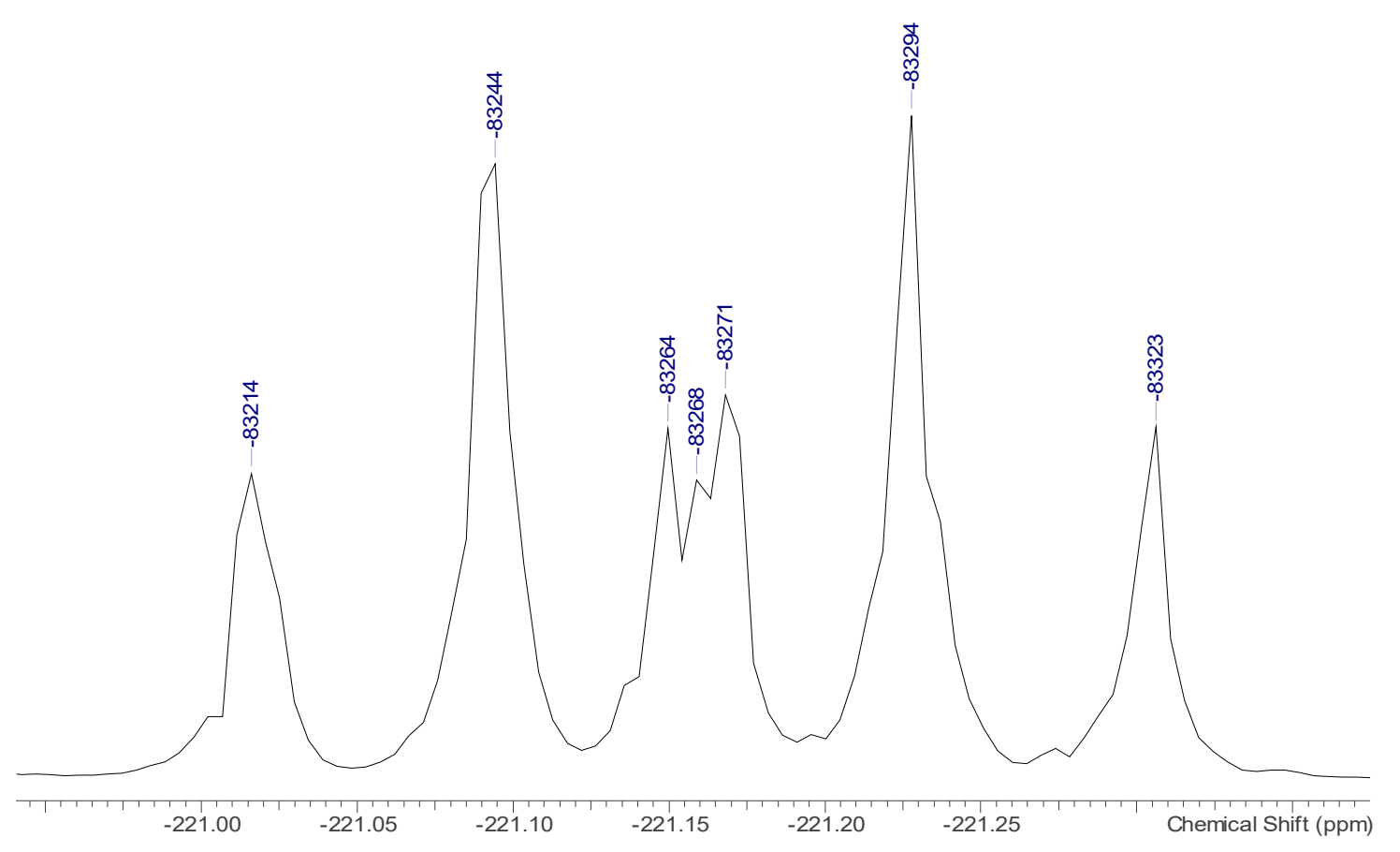

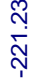

$\bar{m}$

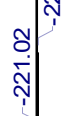

$\underbrace{\mathrm{OTs}}_{\mathrm{OH}} \mathrm{OHOM}$

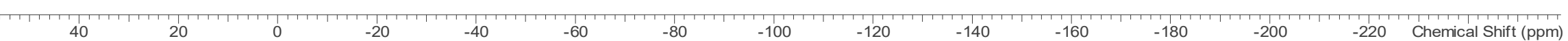


3.30.5. ${ }^{19} \mathrm{~F}\left\{{ }^{1} \mathrm{H}\right\}$ NMR $\left(376 \mathrm{MHz}, \mathrm{CDCl}_{3}\right)$

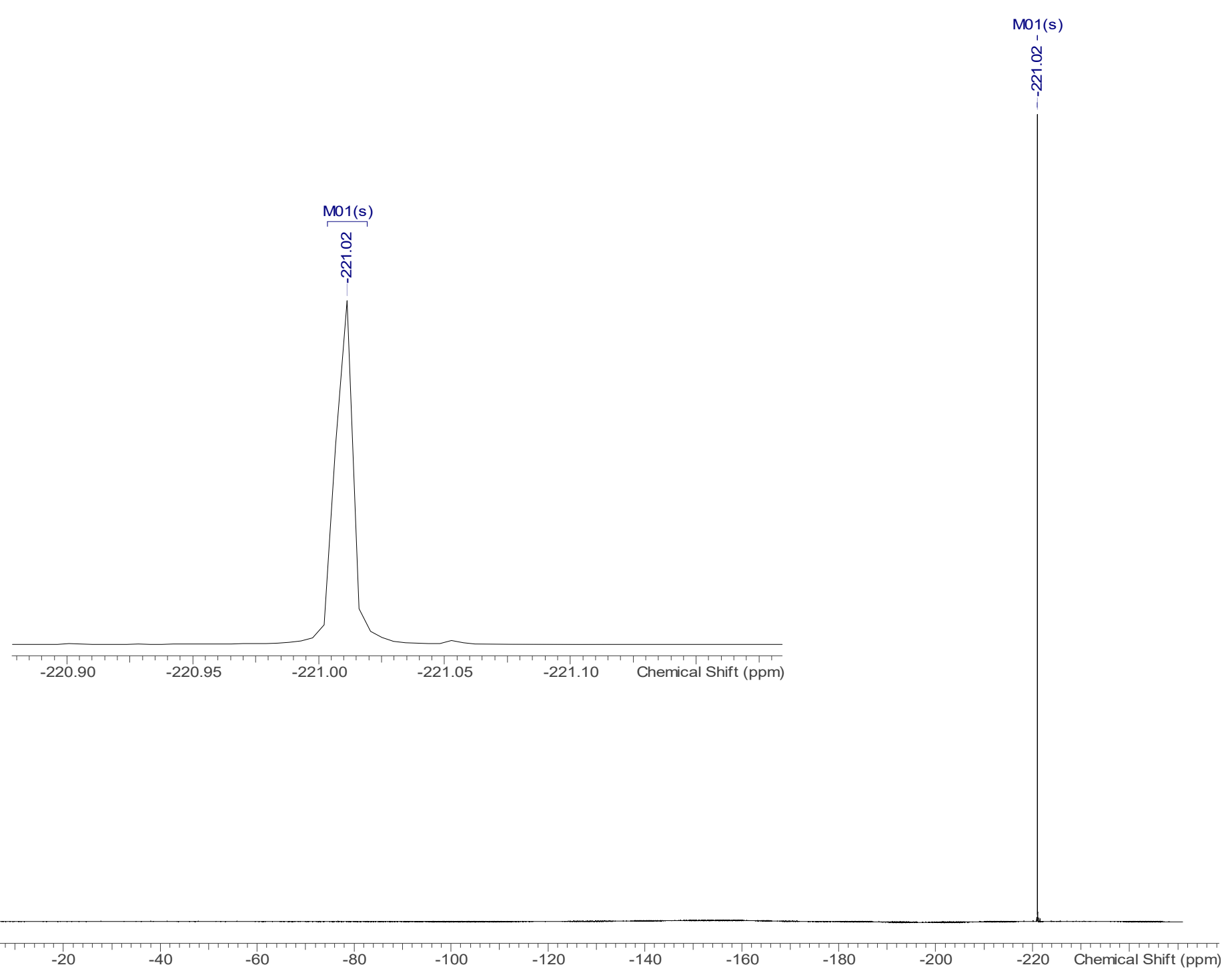


3.31. Methyl-3,6-anhydro-4-deoxy-4-fluoro- $\beta$-D-galactopyranoside (63)

3.31.1. ${ }^{1} \mathrm{H}$ NMR $\left(400 \mathrm{MHz}, \mathrm{CDCl}_{3}\right)$
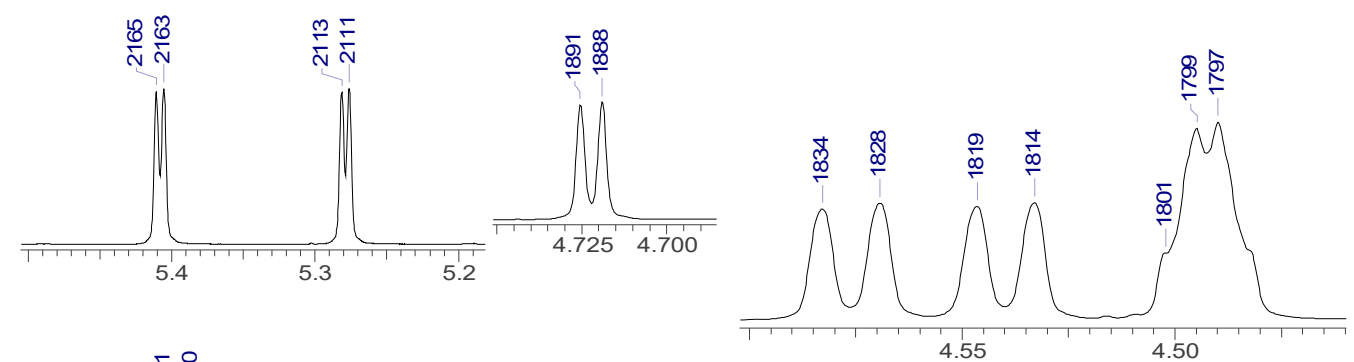

$\stackrel{\leftrightarrow}{\ddot{m}}$
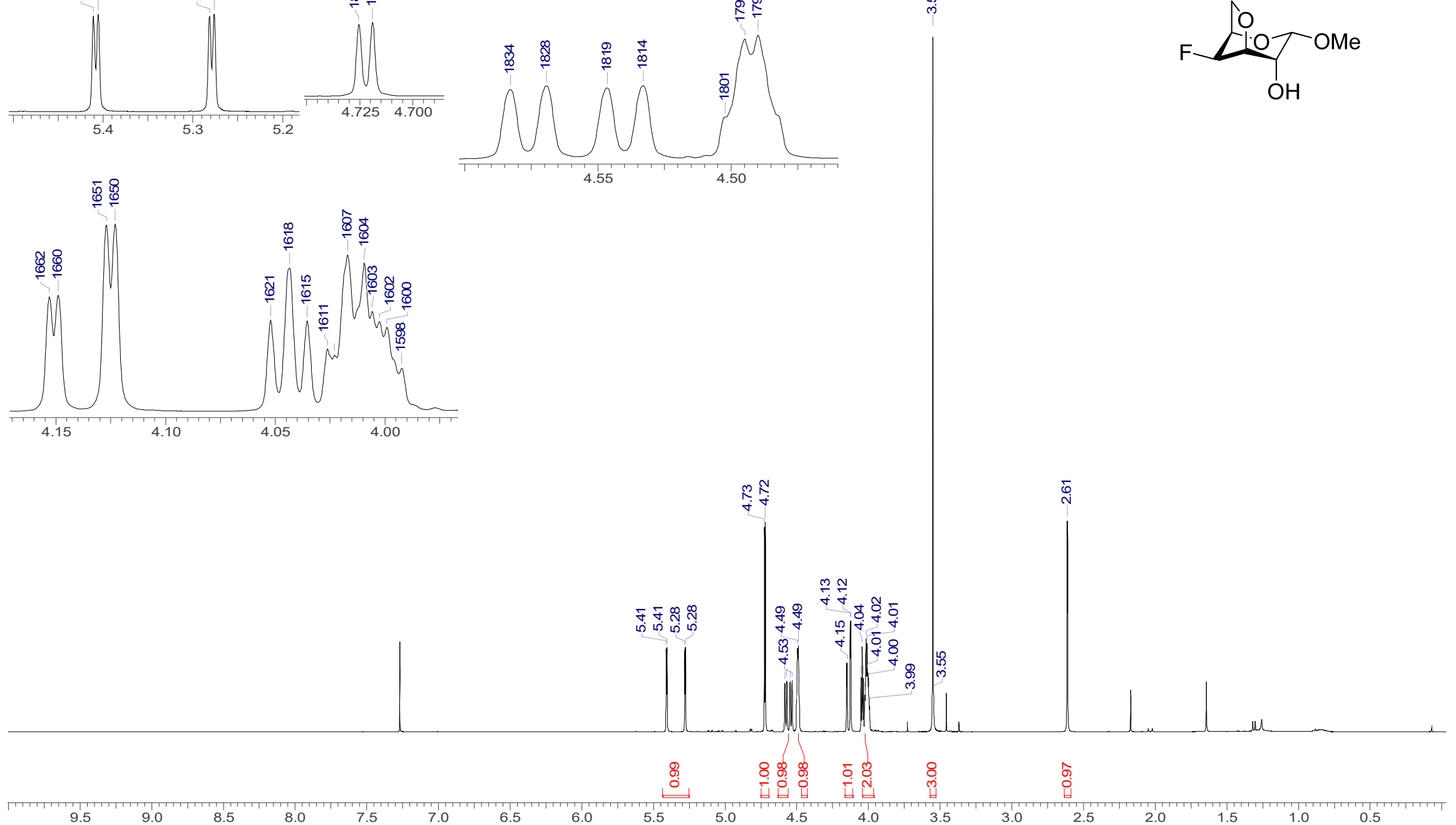
3.31.2. ${ }^{13} \mathrm{C}\left\{{ }^{1} \mathrm{H}\right\}$ NMR (101 MHz, $\left.\mathrm{CDCl}_{3}\right)$
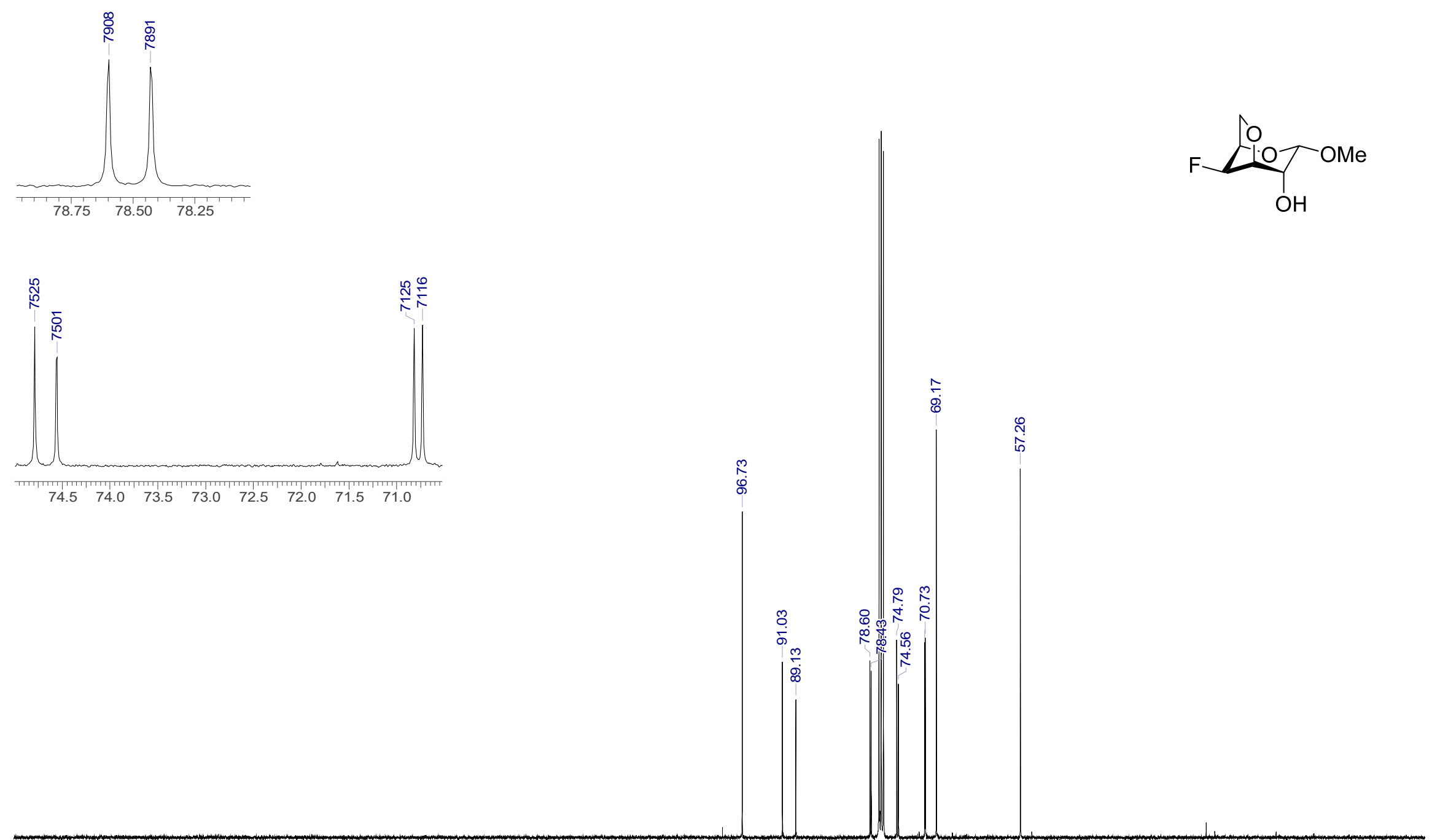


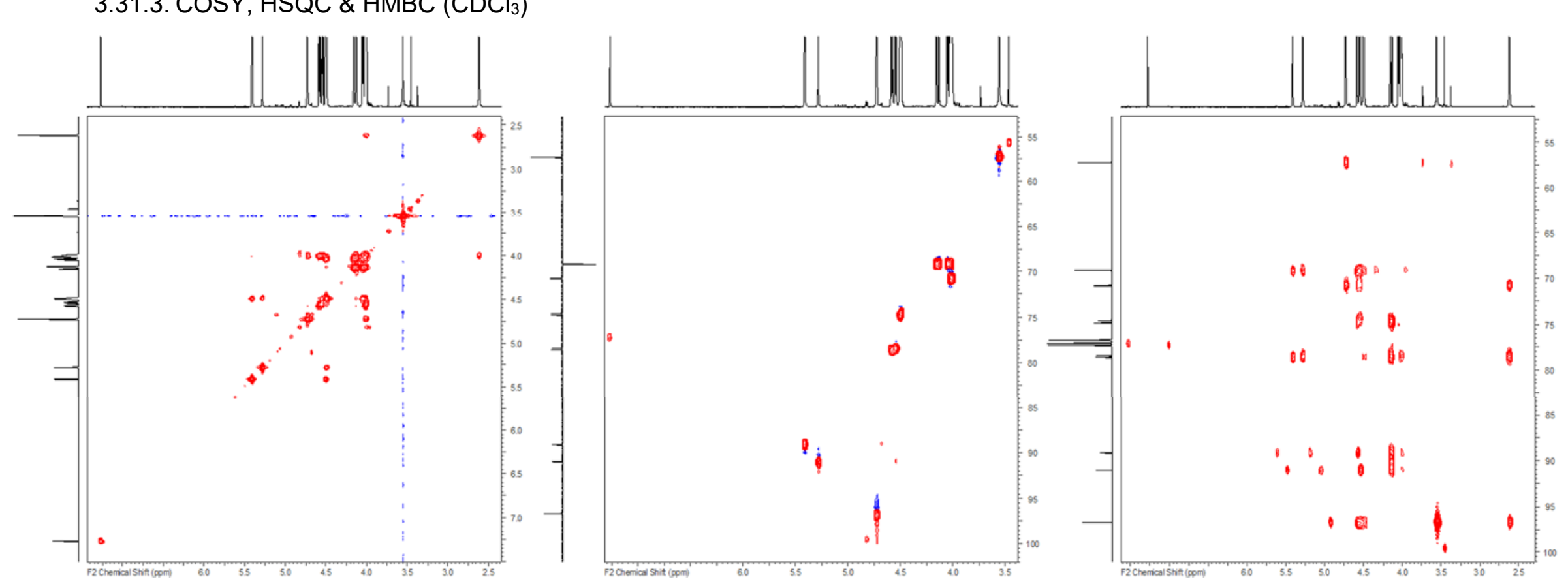


3.31.4. ${ }^{19} \mathrm{~F} \mathrm{NMR}\left(376 \mathrm{MHz}, \mathrm{CDCl}_{3}\right)$

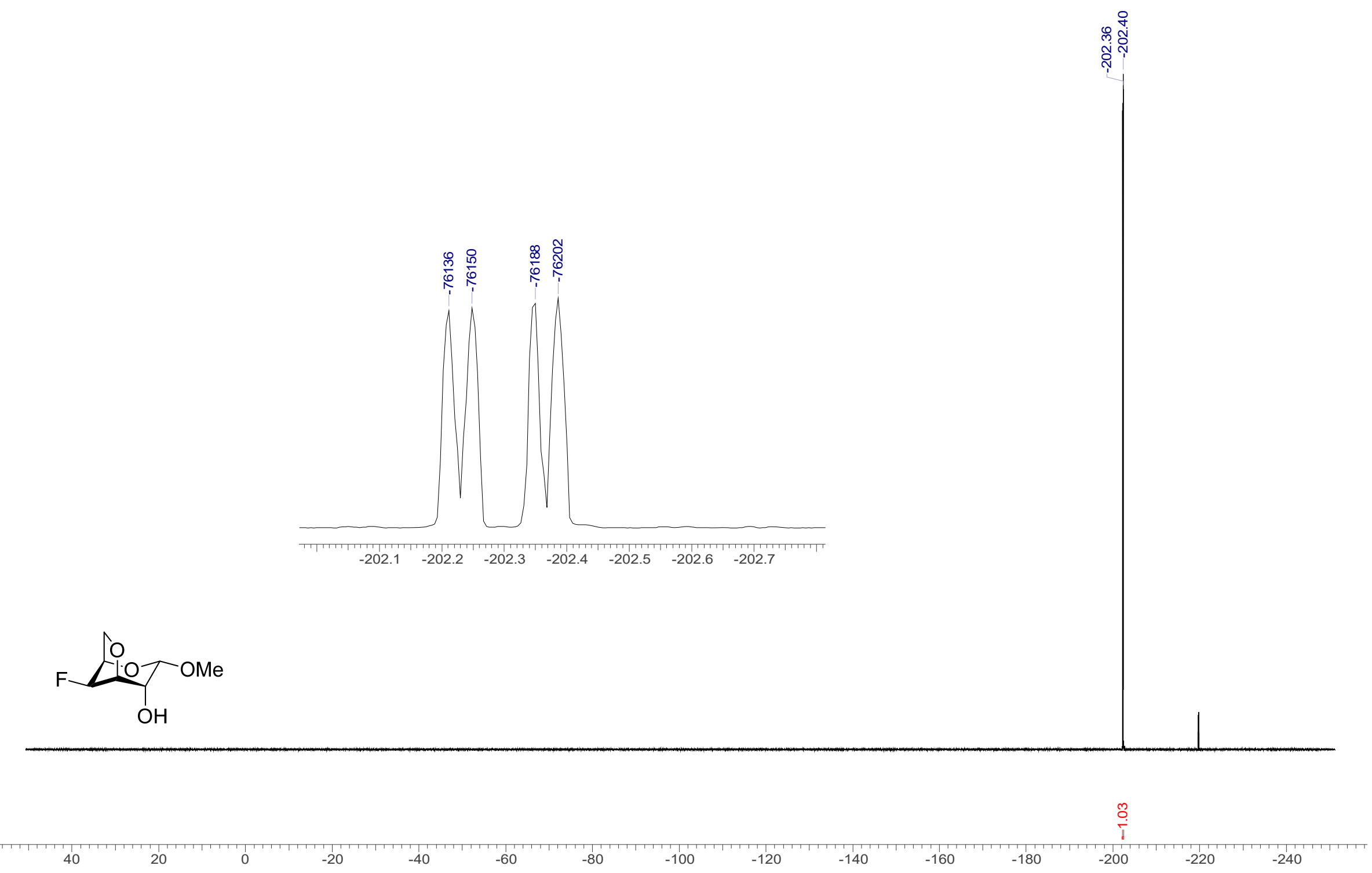


3.31.5. ${ }^{19} \mathrm{~F}\left\{{ }^{1} \mathrm{H}\right\}$ NMR (376 MHz, $\left.\mathrm{CDCl}_{3}\right)$

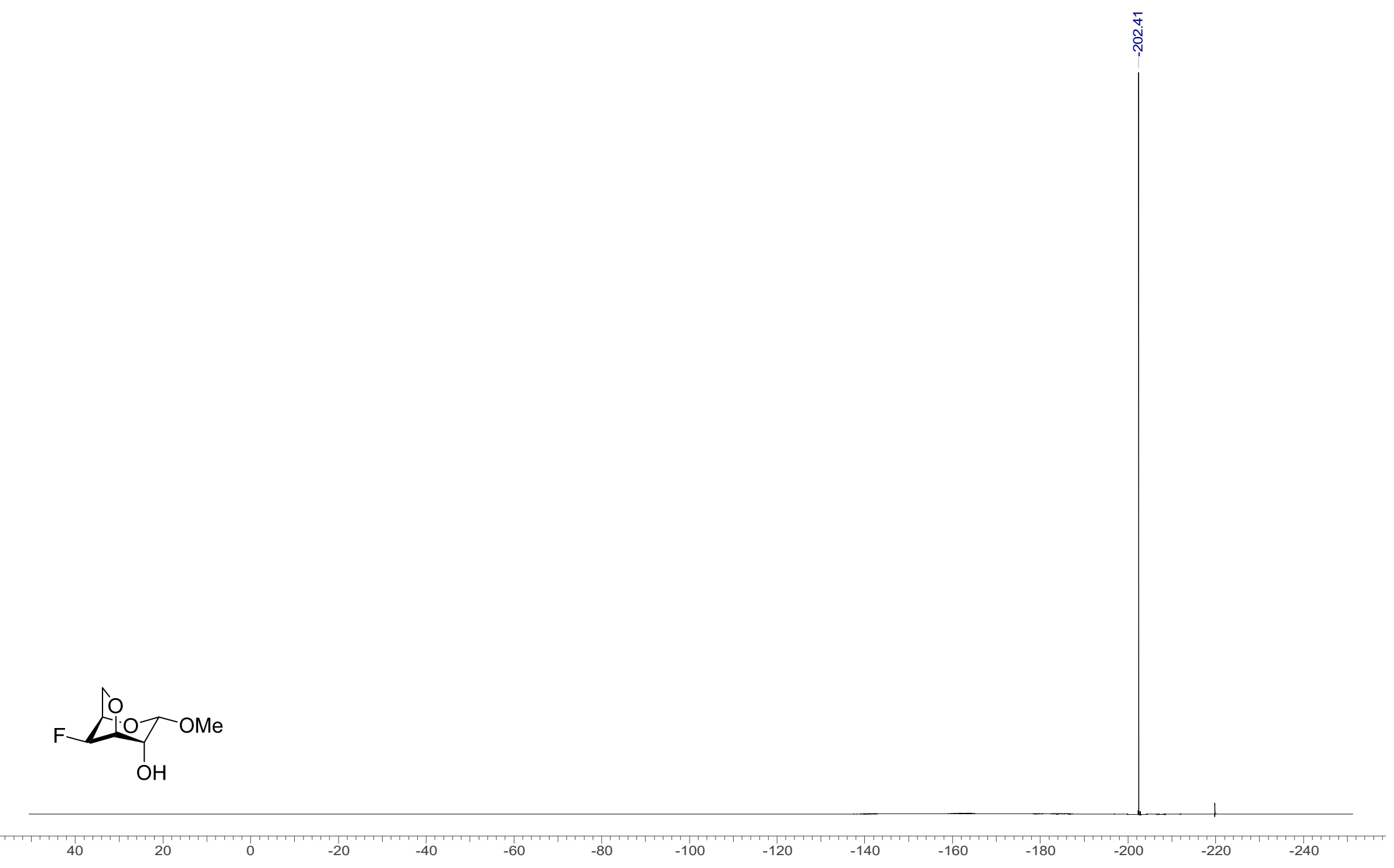




\subsection{Methyl 6-chloro-6-deoxy-a-D-glucopyranoside (64)}

3.32.1. ${ }^{1} \mathrm{H}$ NMR $\left(400 \mathrm{MHz}, \mathrm{DMSO}-\mathrm{d}_{6}\right)$
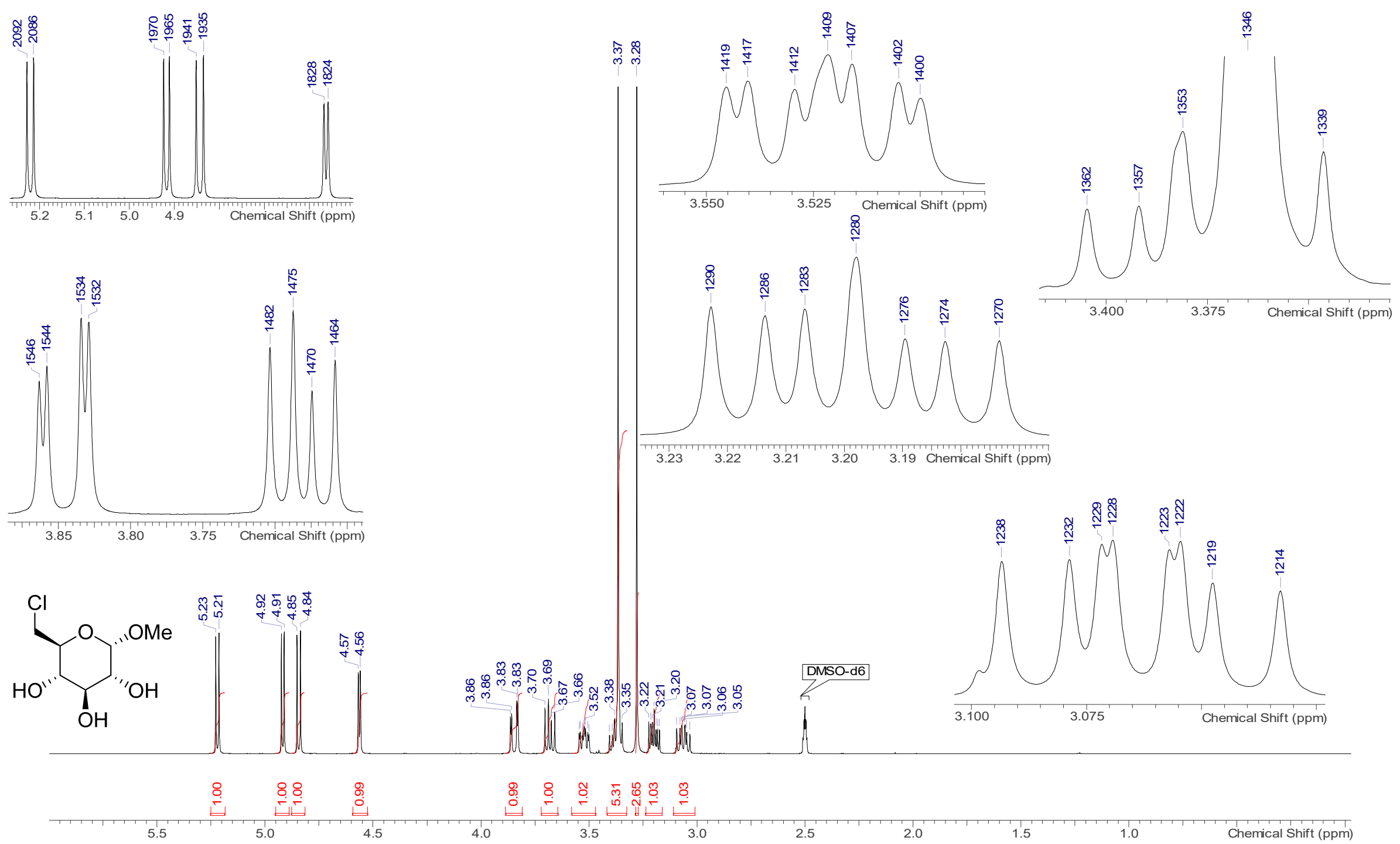

2.5

2.0

1.5

1.0 
3.32.2. ${ }^{13} \mathrm{C}\left\{{ }^{1} \mathrm{H}\right\}$ NMR (101 MHz, DMSO- $\left.\mathrm{d}_{6}\right)$

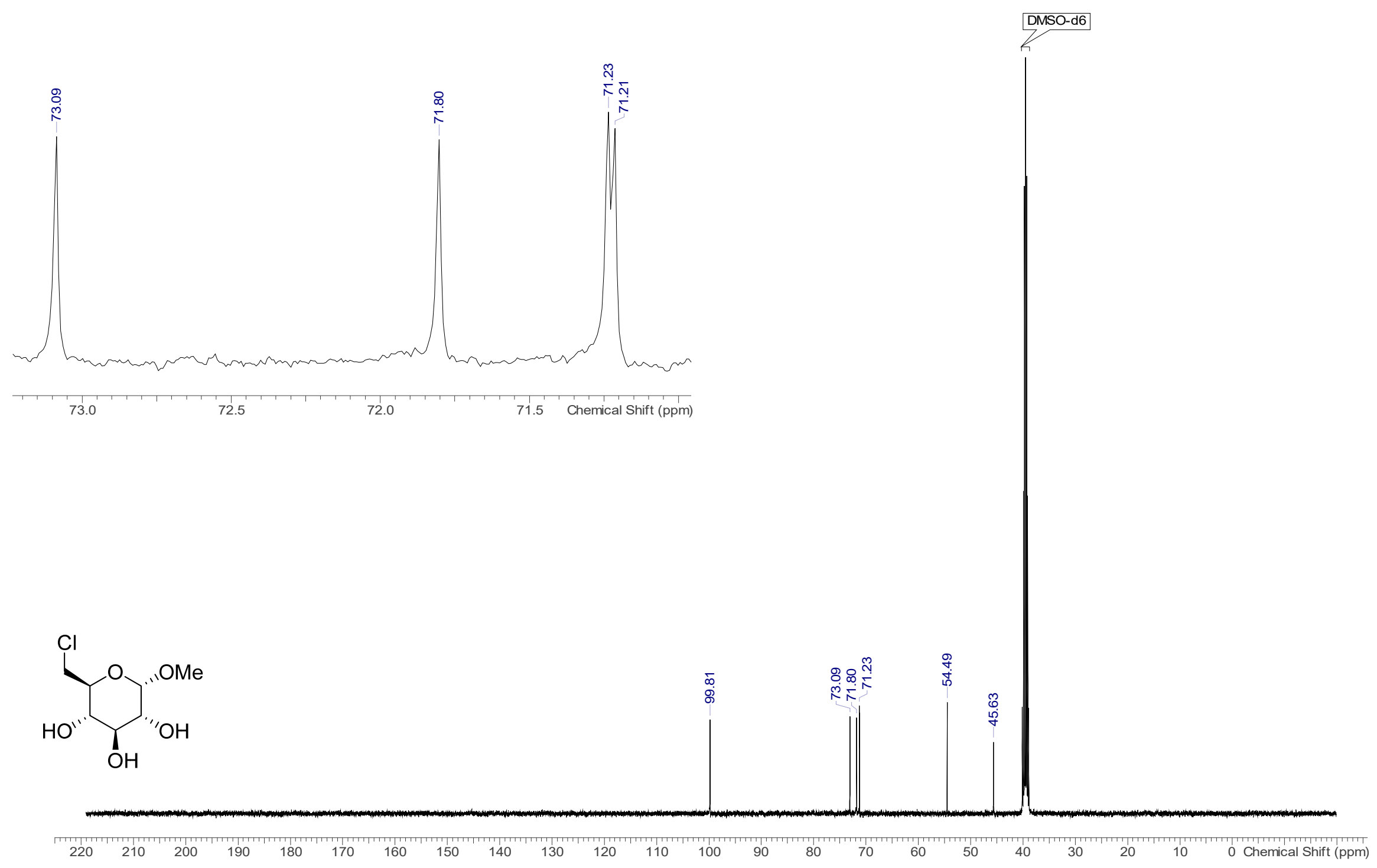


3.32.3. COSY, HSQC-DEPT \& HMBC (DMSO- $\left.\mathrm{d}_{6}\right)$

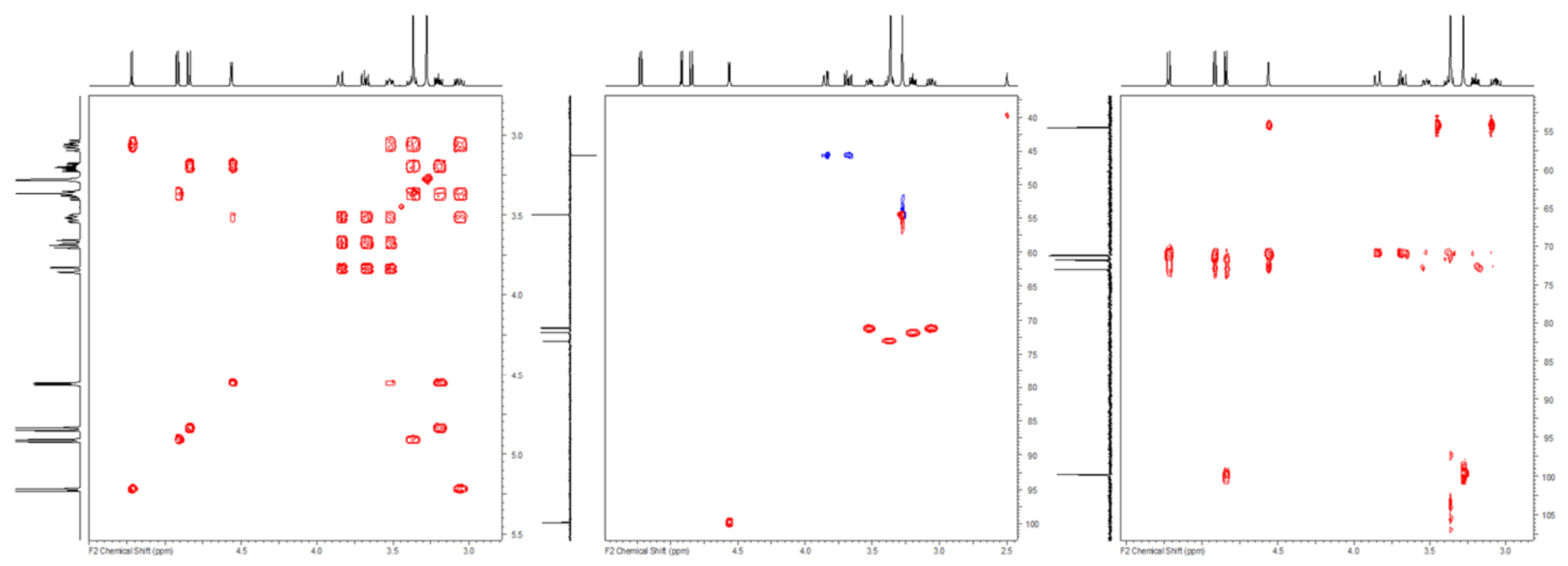


3.33. Methyl 4,6-dideoxy-6-chloro-4-fluoro- $\alpha$-D-galactopyranoside (65)

3.33.1. ${ }^{1} \mathrm{H}$ NMR $\left(500 \mathrm{MHz}, \mathrm{CD}_{3} \mathrm{OD}\right)$

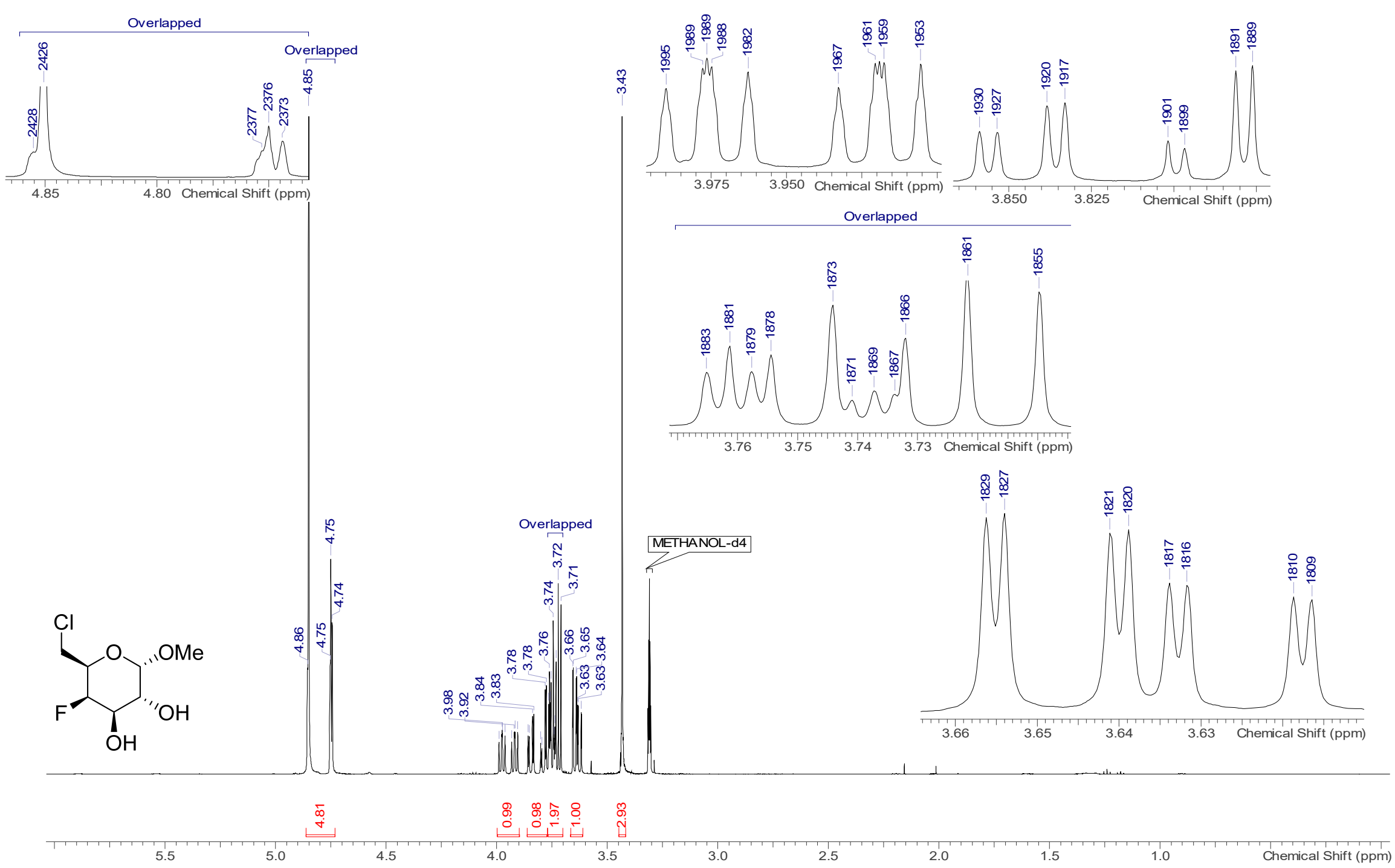




\subsection{2. ${ }^{1} \mathrm{H}\left\{{ }^{19} \mathrm{~F}\right\}$ NMR $\left(500 \mathrm{MHz}, \mathrm{CD}_{3} \mathrm{OD}\right)$}
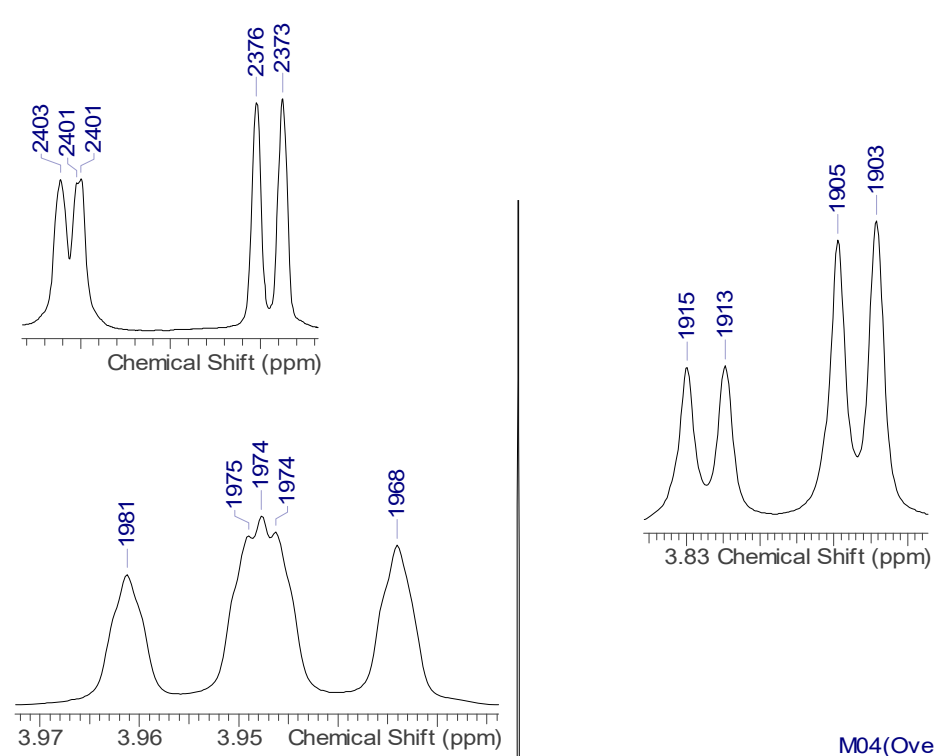

M05(s)

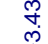

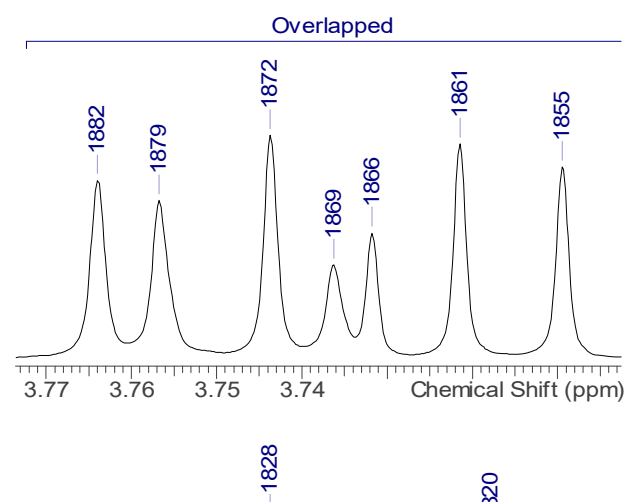

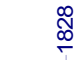

M03 $(\mathrm{m})$

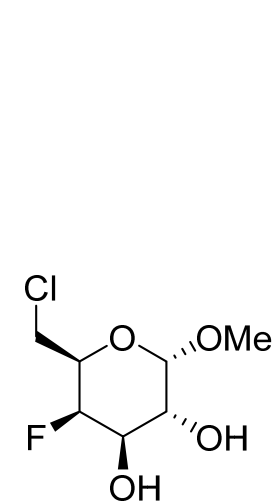

$\mathrm{MO2}(\mathrm{m}) \square \mathrm{MO1}(\mathrm{m})$

M06(d)
$\quad$
M07(d)

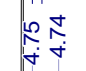

赵雨

METHANOL-d4
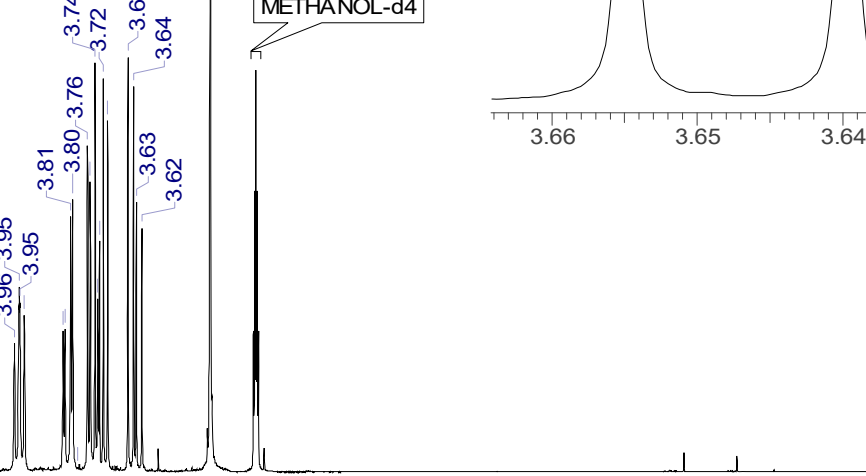

3.63

Chemical Shift (ppm)

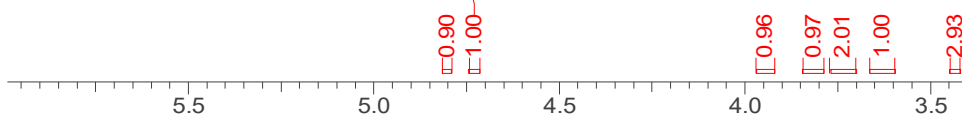

3.0

2.5

2.0

1.5

1.0 
3.33.3. ${ }^{13} \mathrm{C}\left\{{ }^{1} \mathrm{H}\right\}$ NMR $\left(126 \mathrm{MHz}, \mathrm{CD}_{3} \mathrm{OD}\right)$
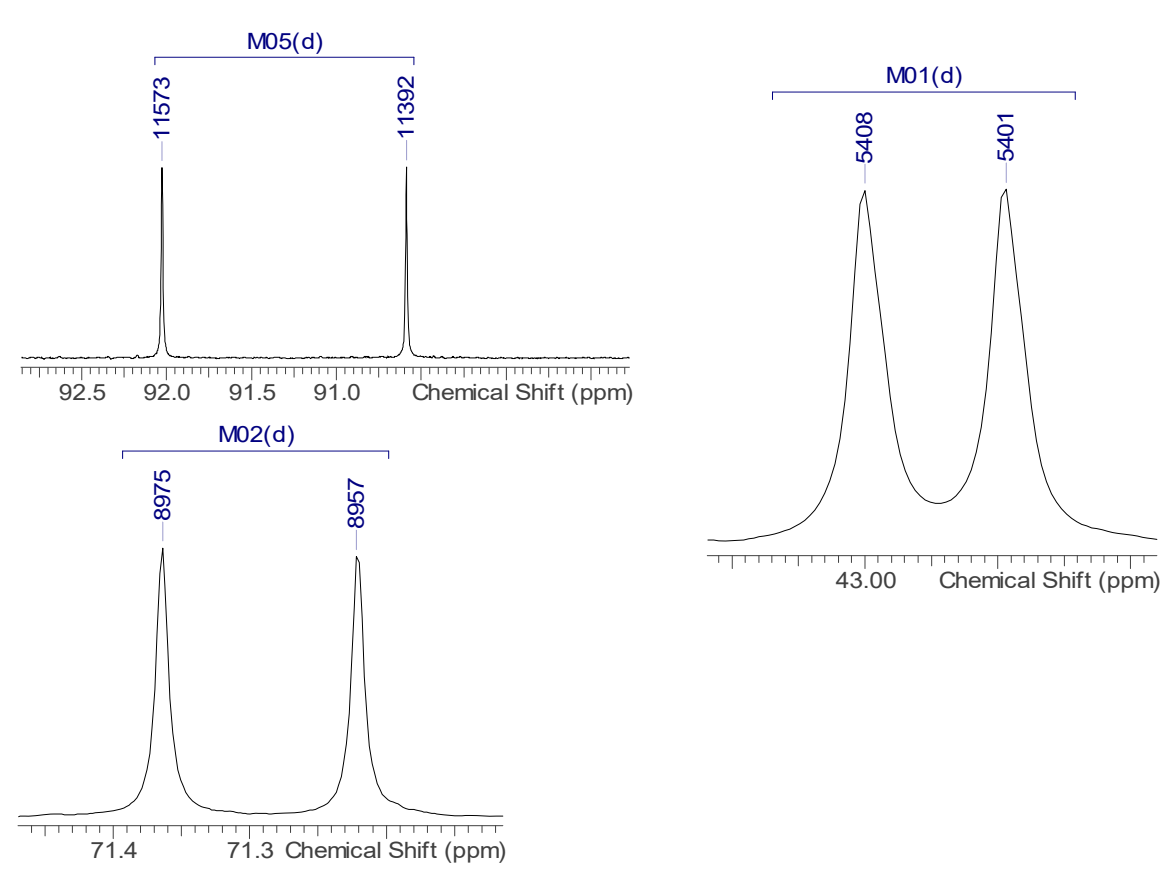

METHANOL-d4
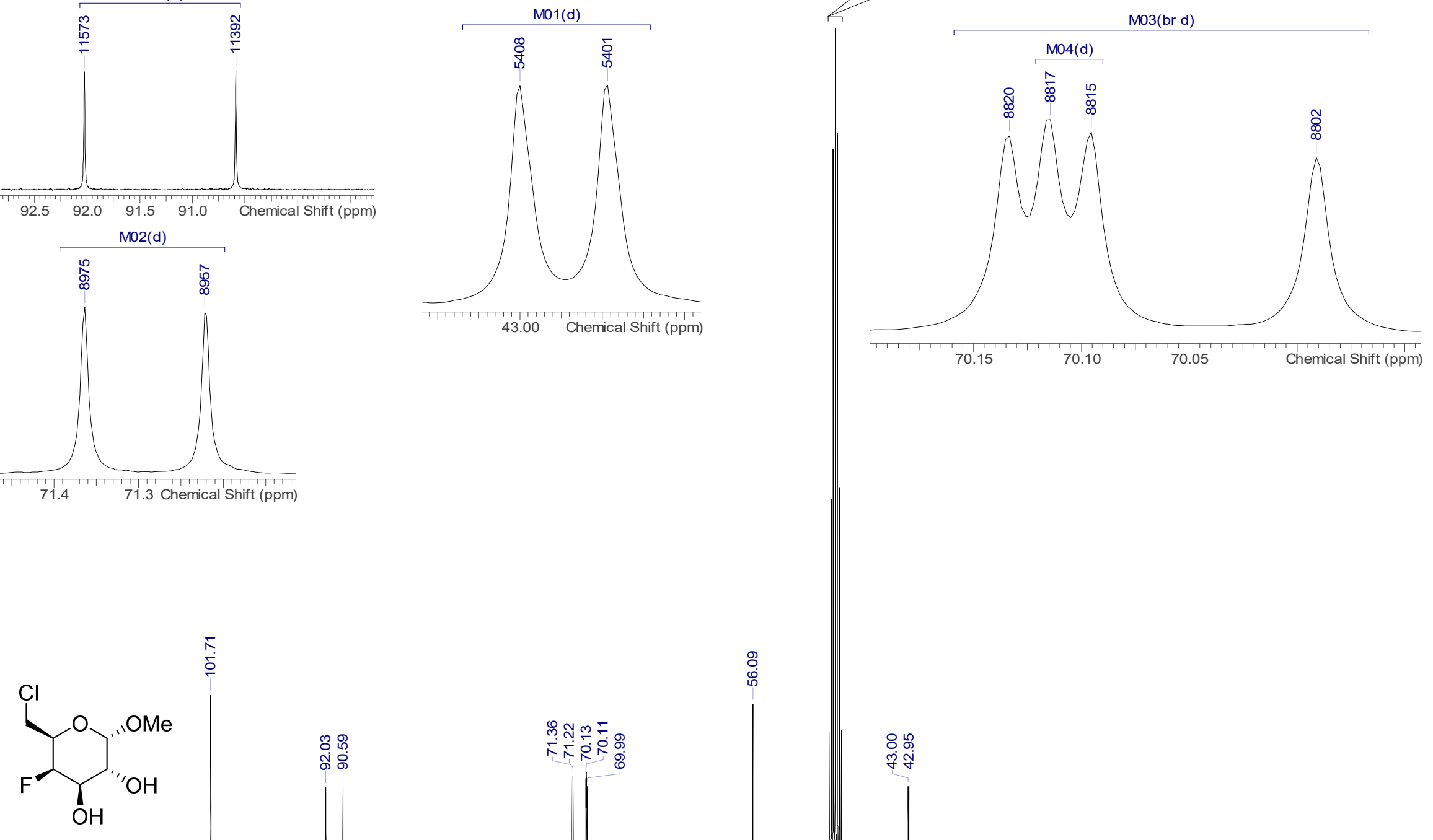

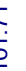

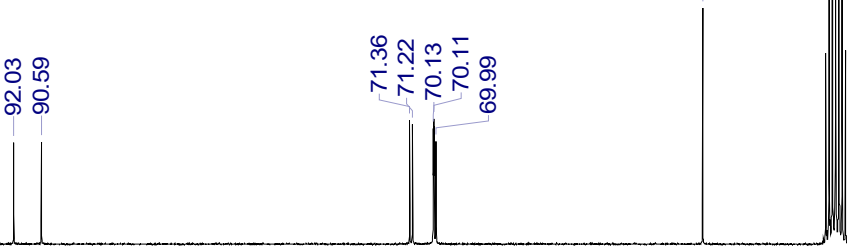

ஓํำ

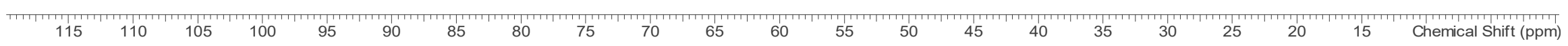




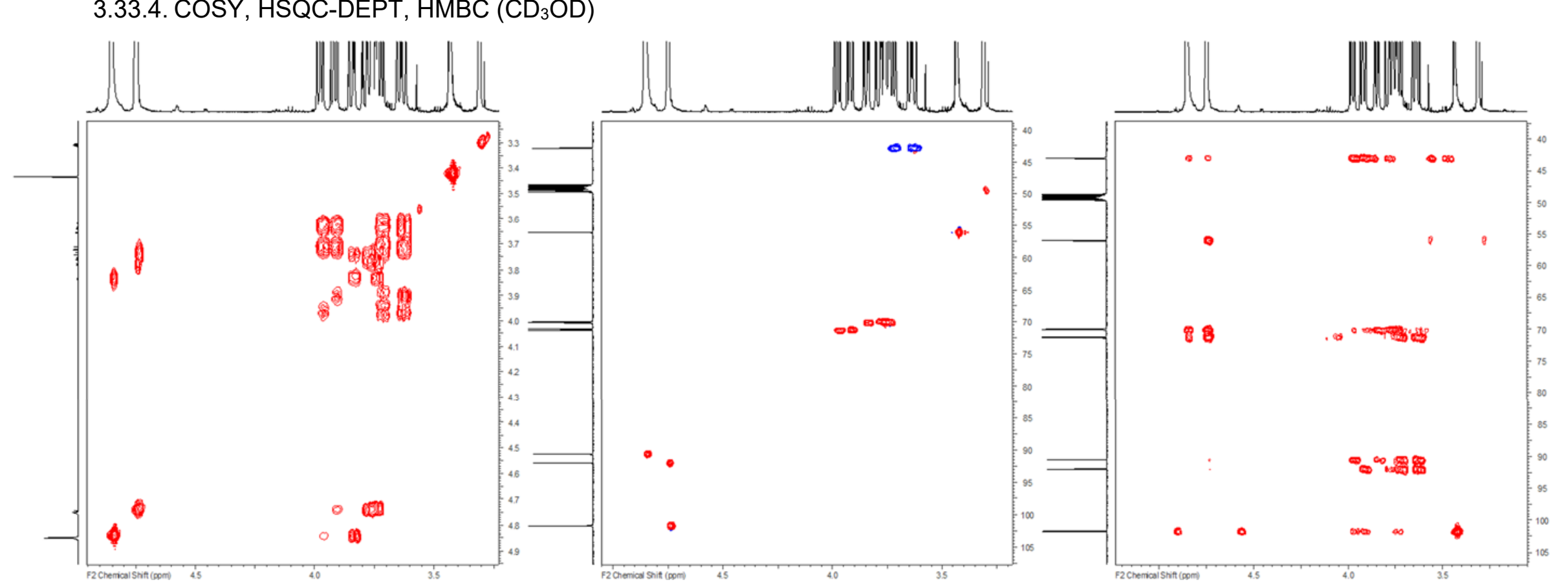


3.33.5. ${ }^{19} \mathrm{~F} \mathrm{NMR}\left(470 \mathrm{MHz}, \mathrm{CD}_{3} \mathrm{OD}\right)$

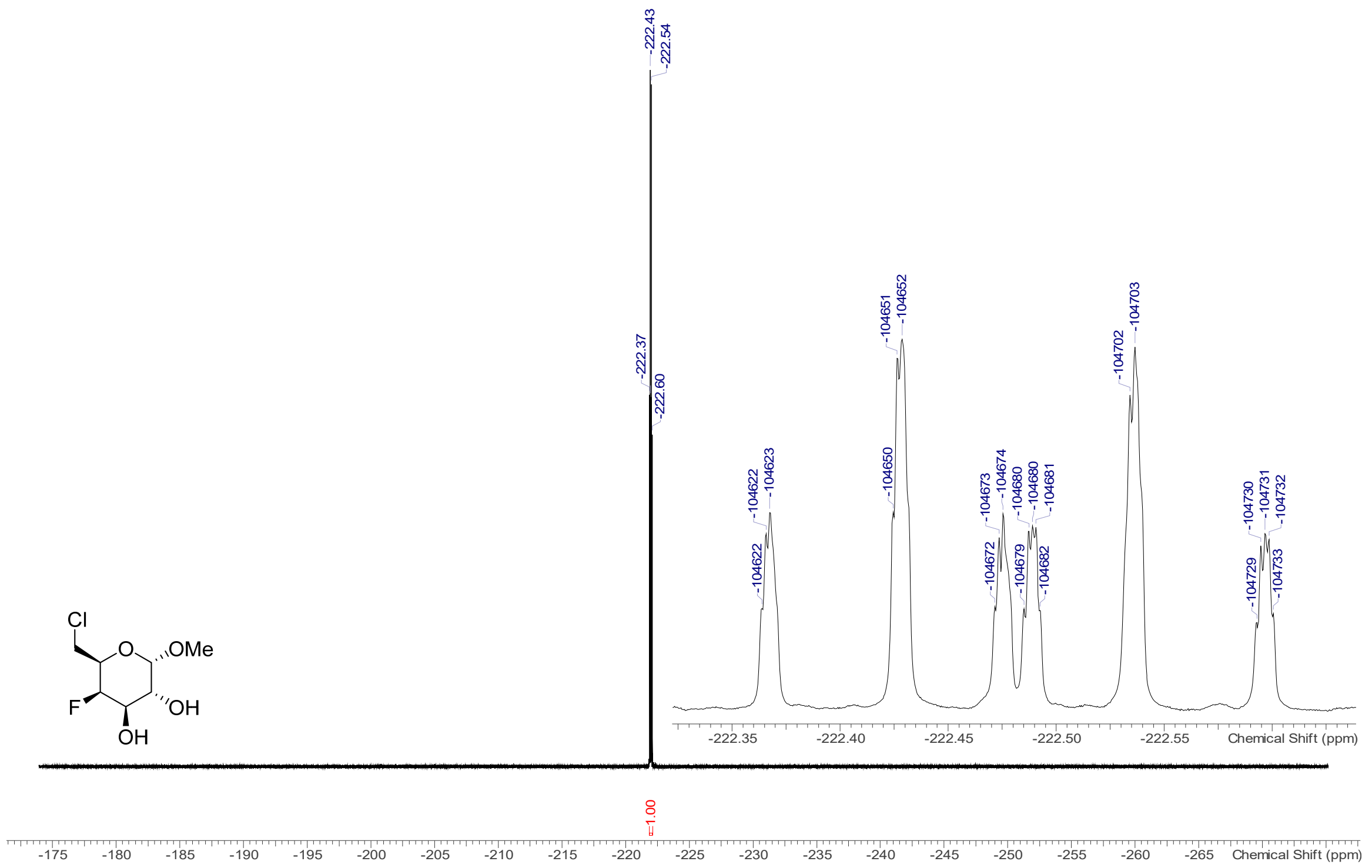


3.33.6. ${ }^{19} \mathrm{~F}\left\{{ }^{1} \mathrm{H}\right\}$ NMR $\left(470 \mathrm{MHz}, \mathrm{CD}_{3} \mathrm{OD}\right)$

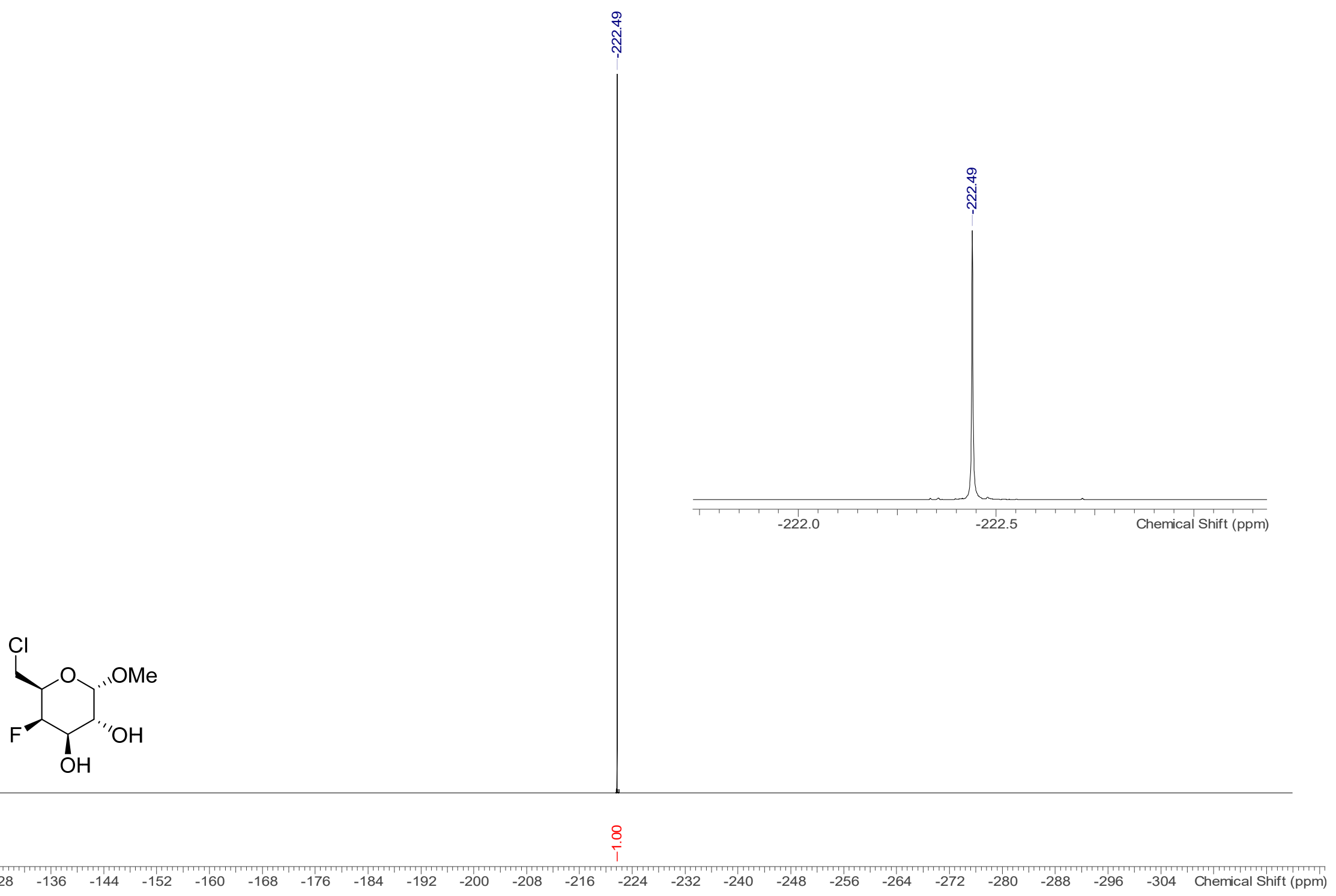


3.34. Methyl 2,3-di-O-benzoyl-6-deoxy-6-bromo-a-D-galactopyranoside (66) 3.34.1. ${ }^{1} \mathrm{H}$ NMR $\left(400 \mathrm{MHz}, \mathrm{CDCl}_{3}\right)$
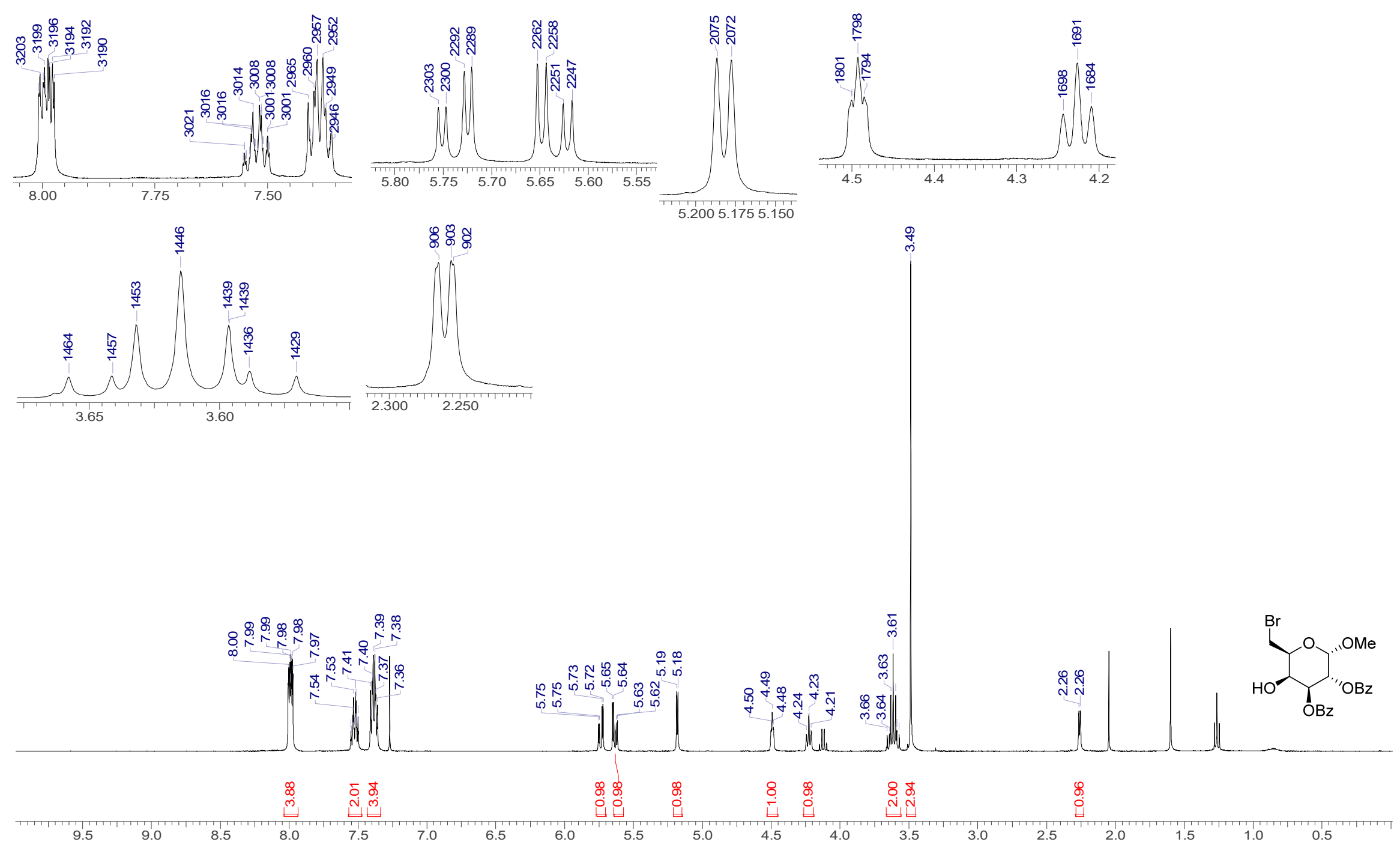
3.34.2. ${ }^{13} \mathrm{C}\left\{{ }^{1} \mathrm{H}\right\}$ NMR $\left(101 \mathrm{MHz}, \mathrm{CDCl}_{3}\right)$

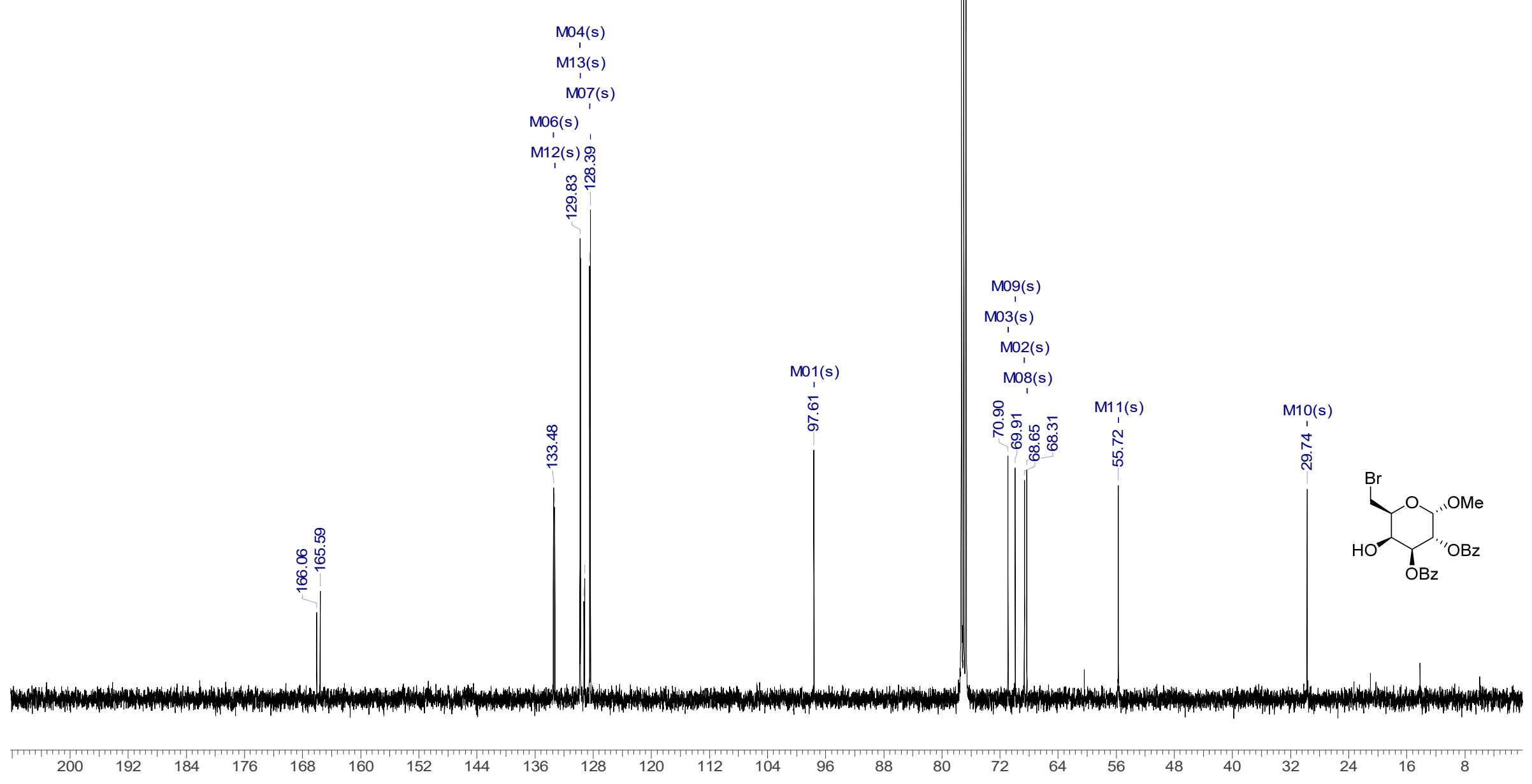


3.34.3. COSY \& HSQC $\left(\mathrm{CDCl}_{3}\right)$

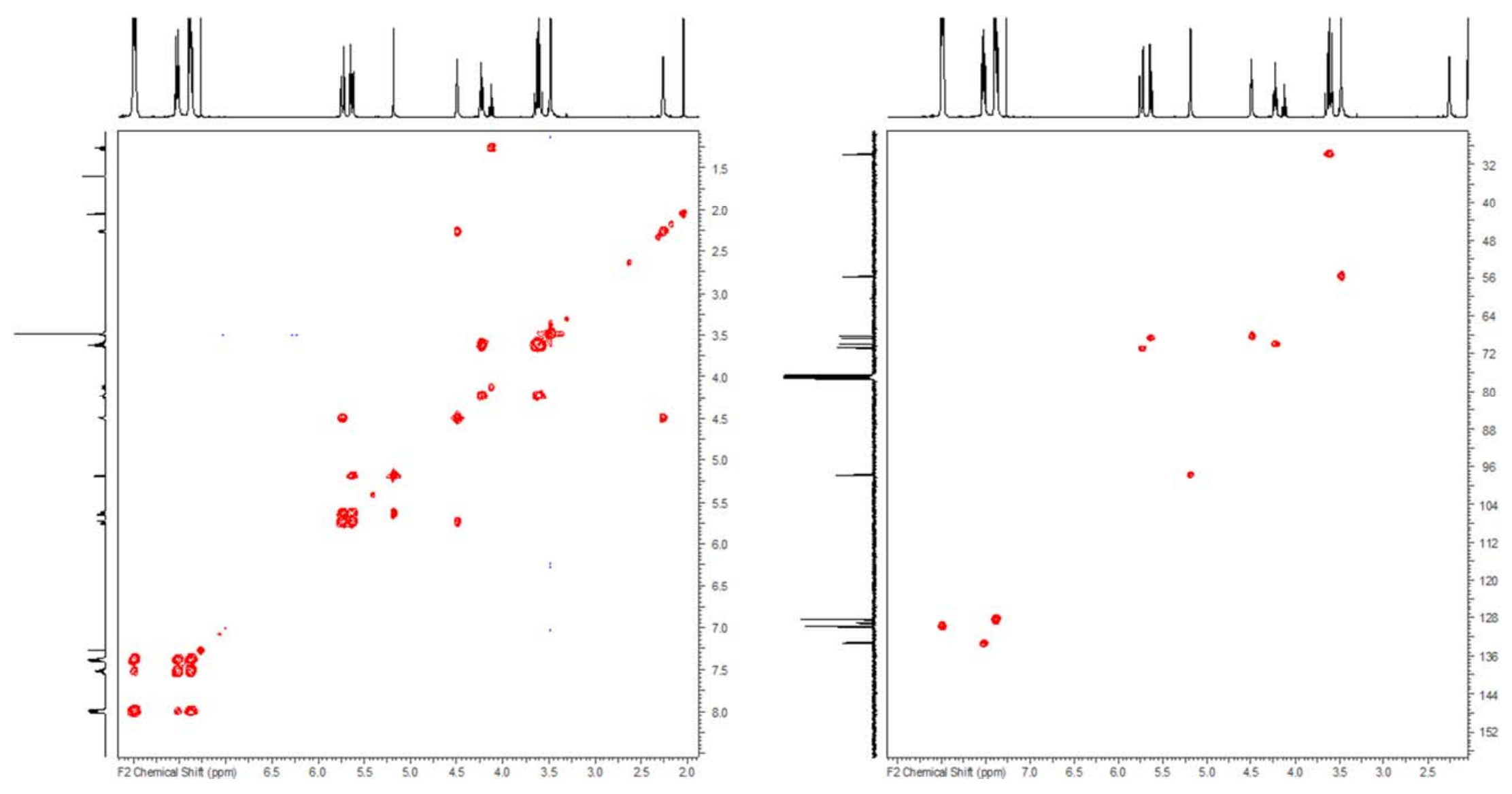




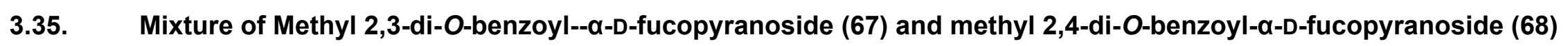
3.35.1. ${ }^{1} \mathrm{H} \mathrm{NMR}\left(400 \mathrm{MHz}, \mathrm{CDCl}_{3}\right)$

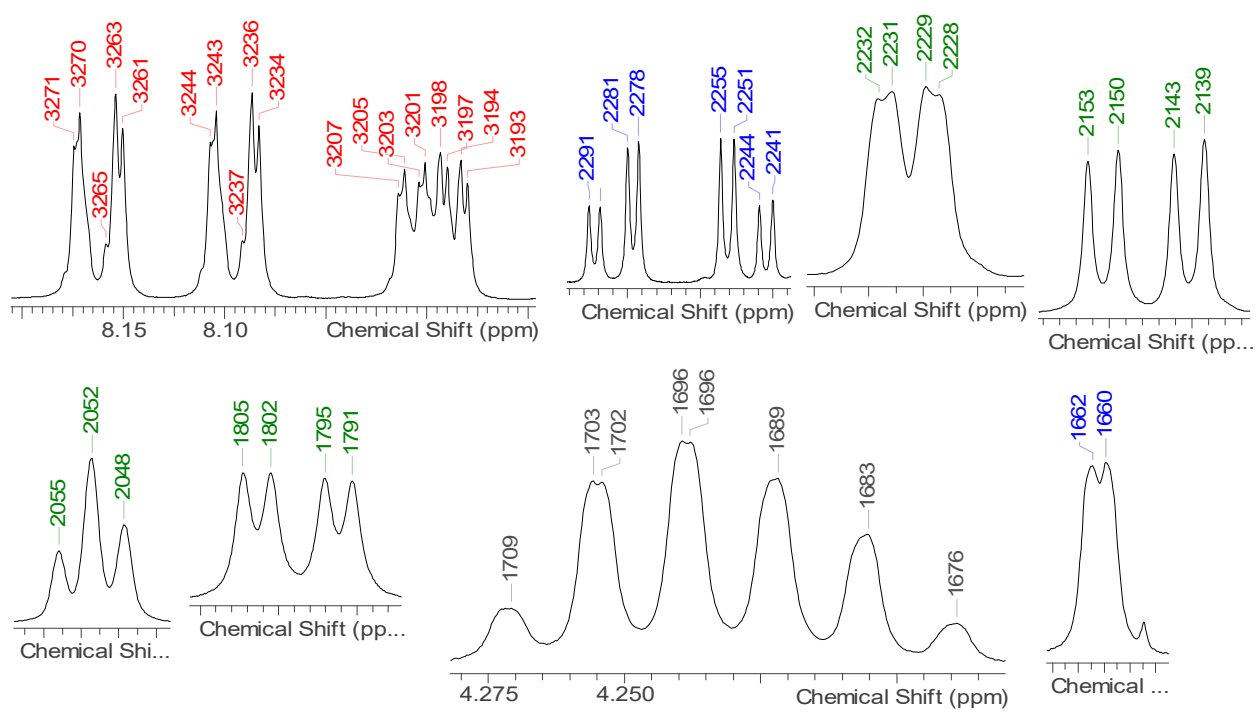

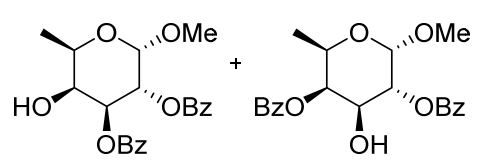
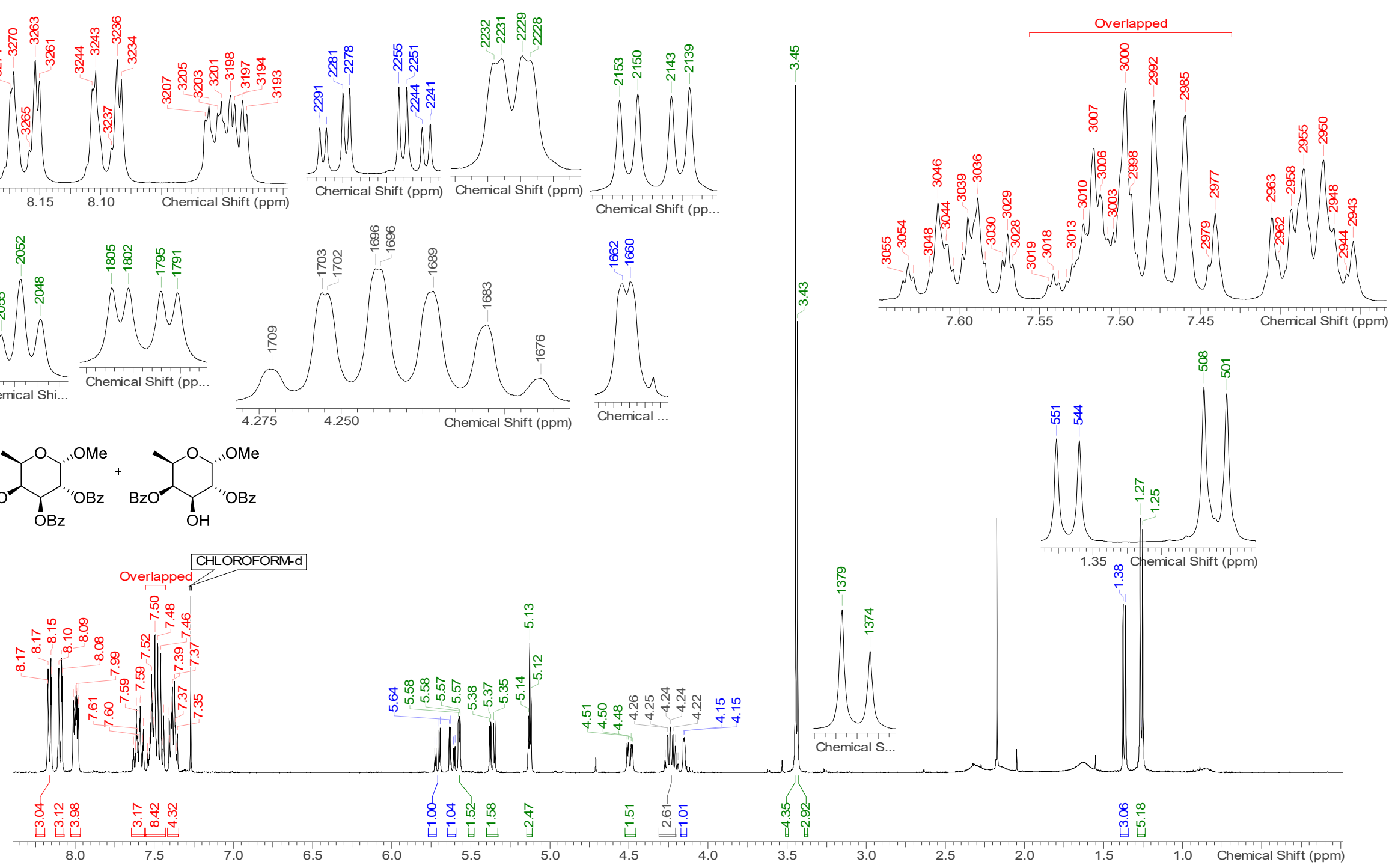
3.35.2. ${ }^{13} \mathrm{C}\left\{{ }^{1} \mathrm{H}\right\}$ NMR $\left(101 \mathrm{MHz}, \mathrm{CDCl}_{3}\right)$
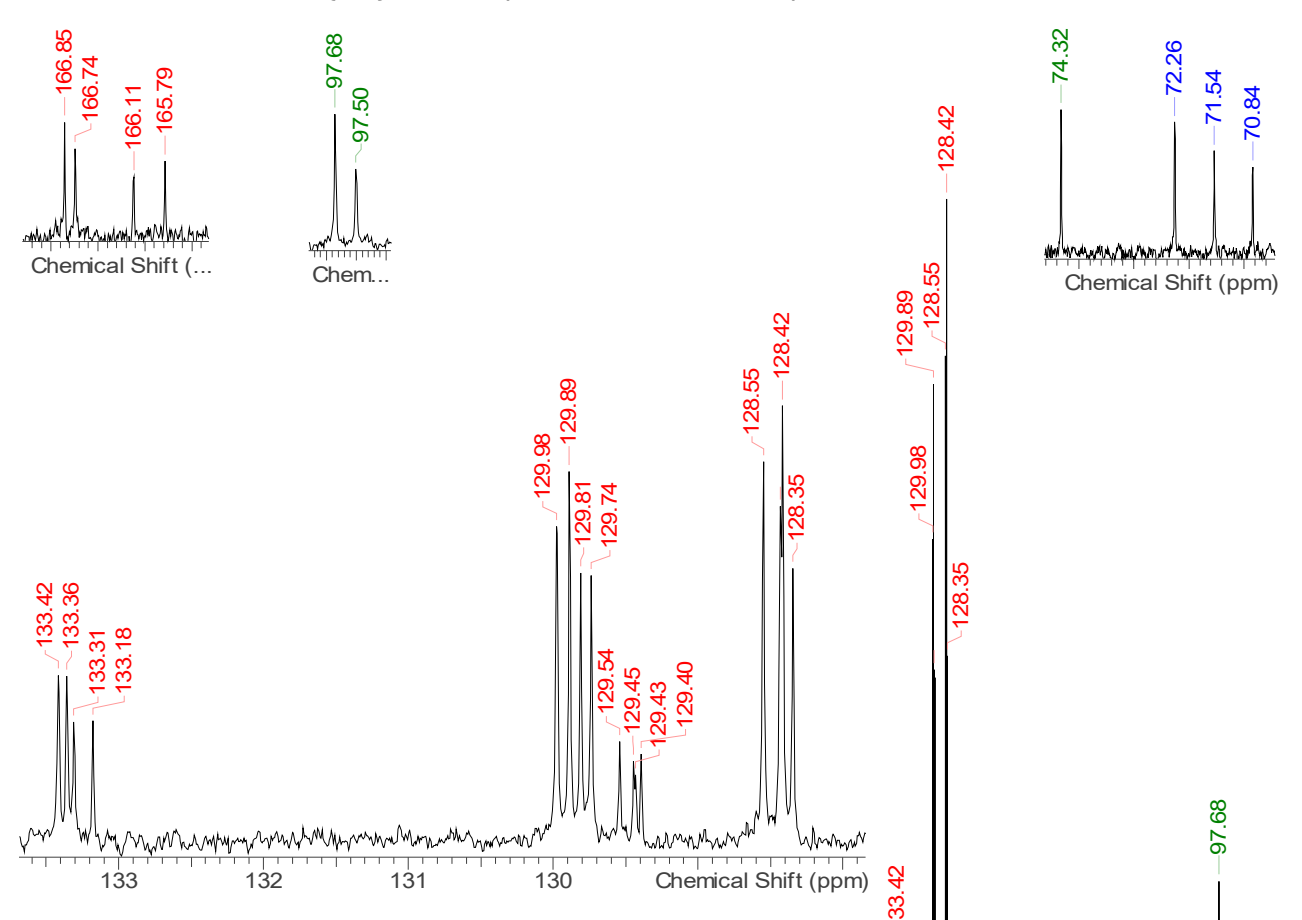

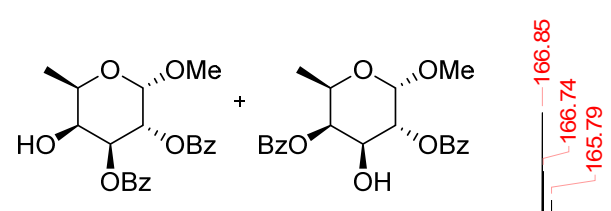
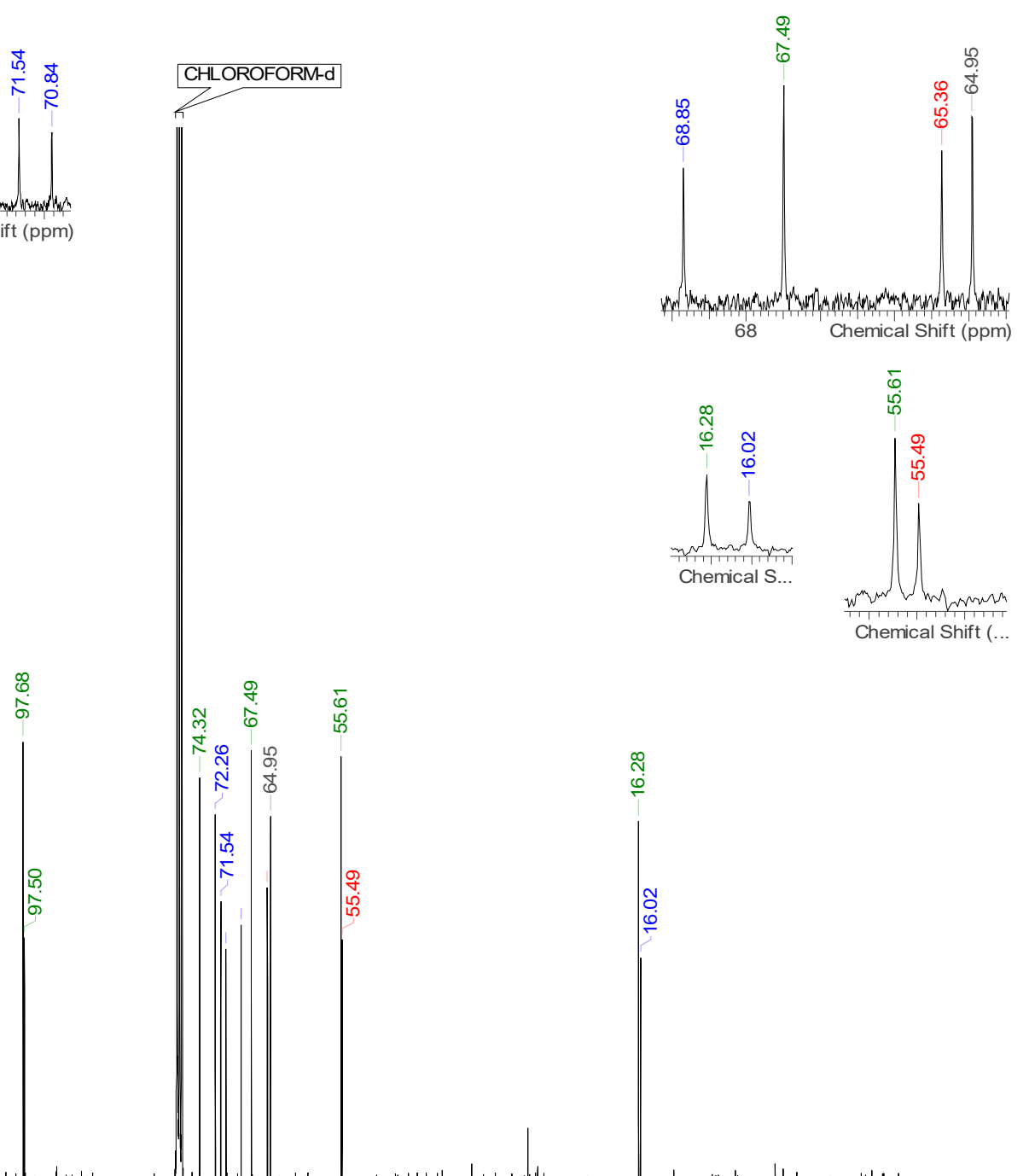

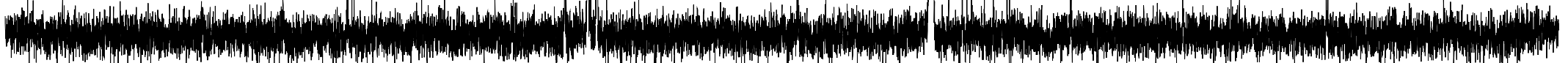

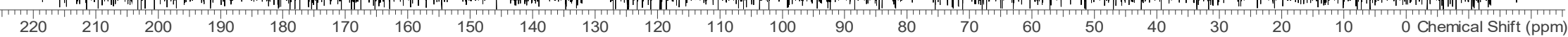




\subsubsection{Mixture ratio determination}

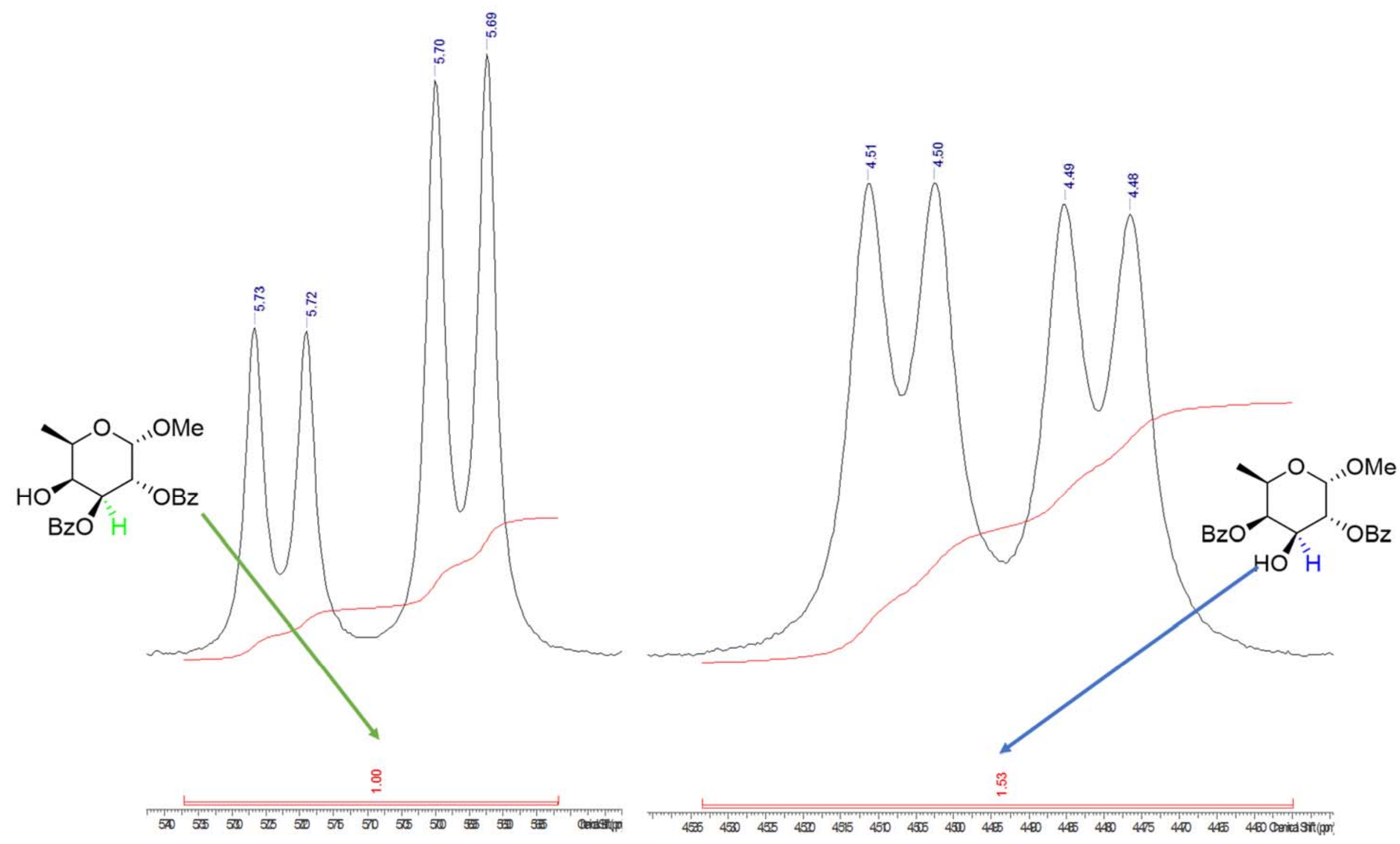




\subsection{Methyl 2,3-di-O-benzoyl-4,6-dideoxy-4-fluoro-6-bromo- $\alpha$-D-glucopyranoside (69)}

3.36.1. ${ }^{1} \mathrm{H} \mathrm{NMR}\left(400 \mathrm{MHz}, \mathrm{CDCl}_{3}\right)$

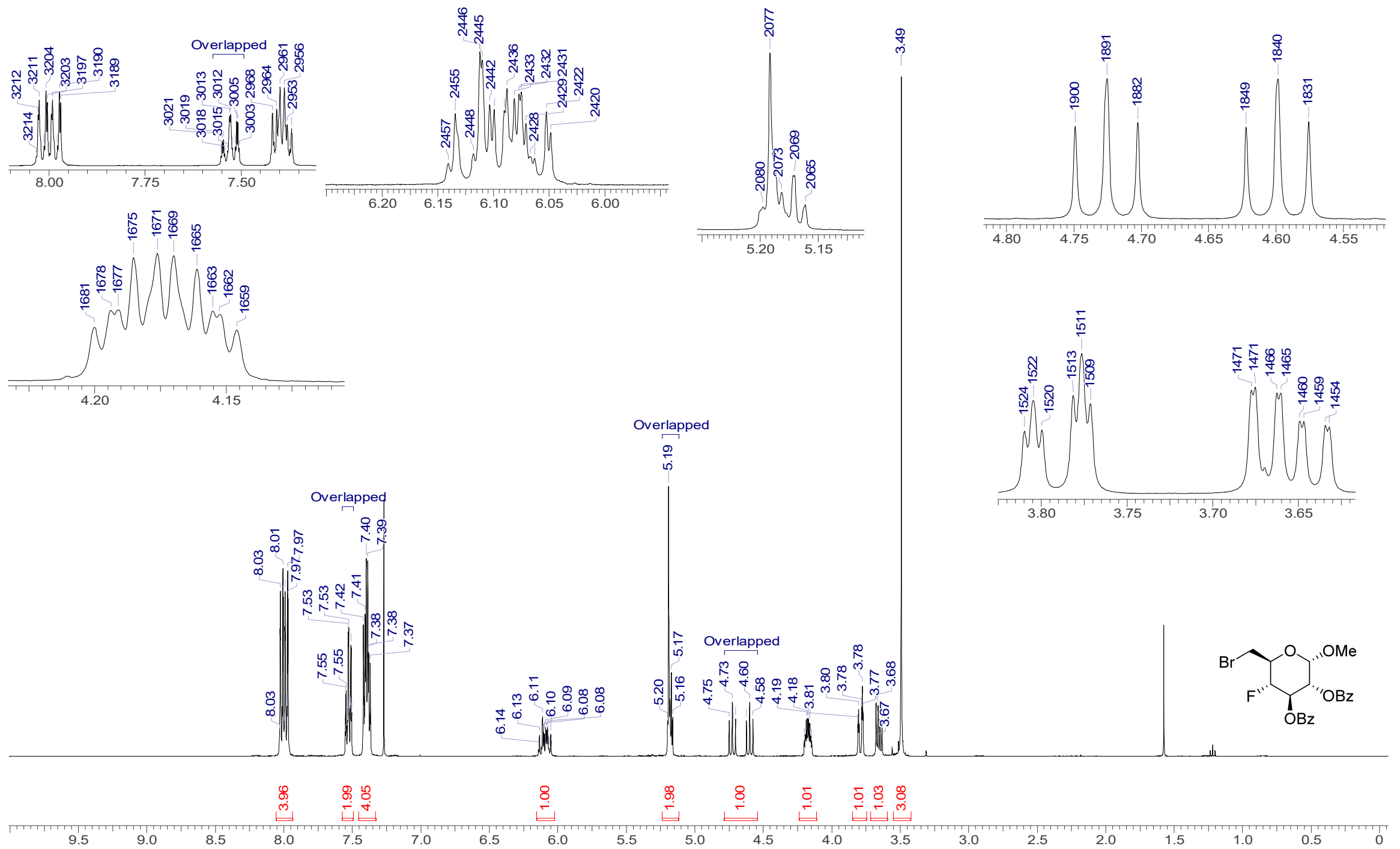


3.36.2. ${ }^{13} \mathrm{C}\left\{{ }^{1} \mathrm{H}\right\}$ NMR $\left(101 \mathrm{MHz}, \mathrm{CDCl}_{3}\right)$

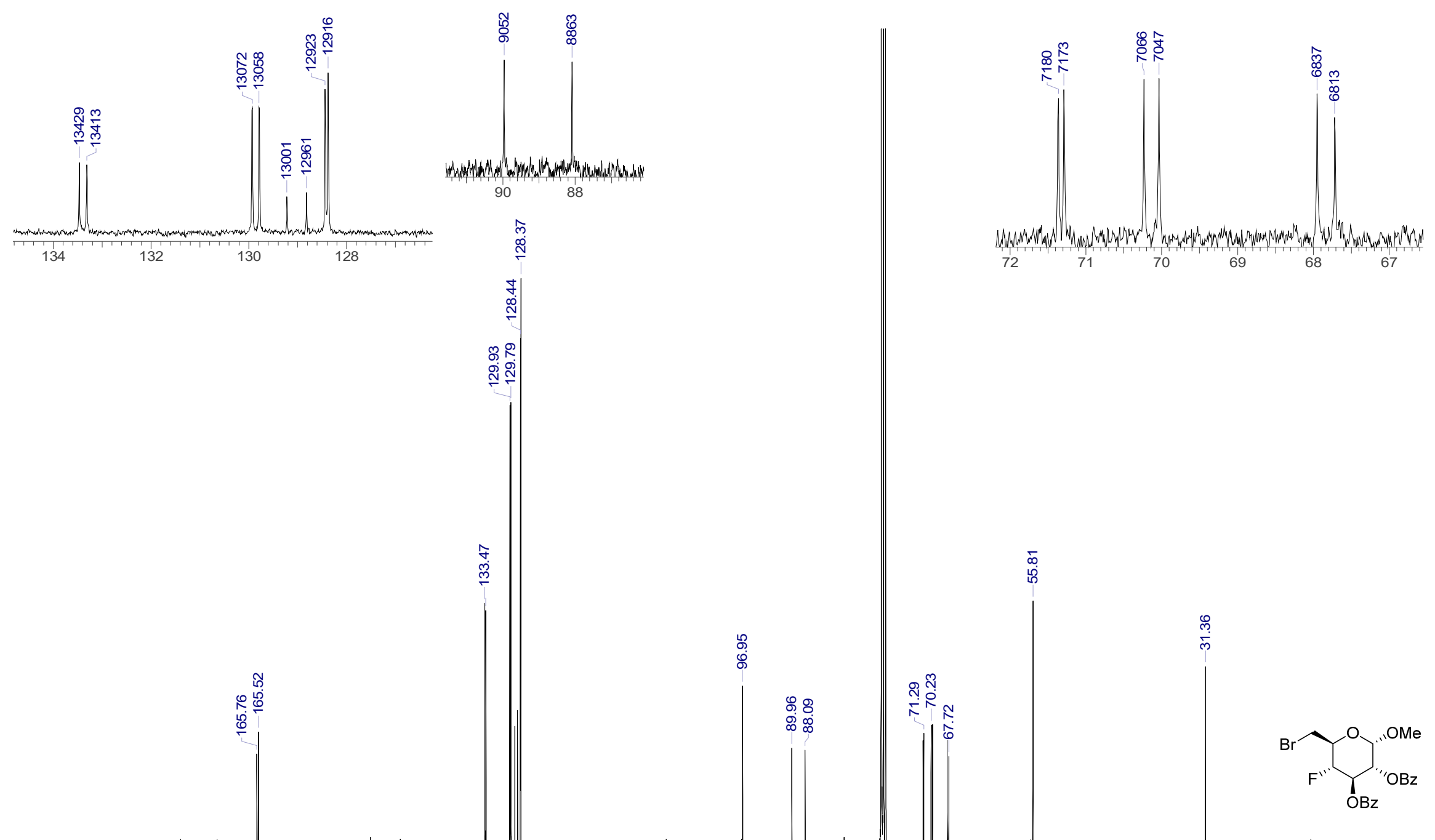

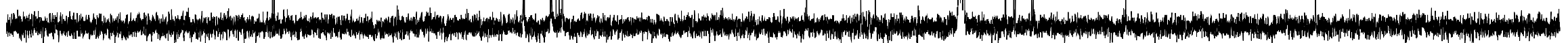

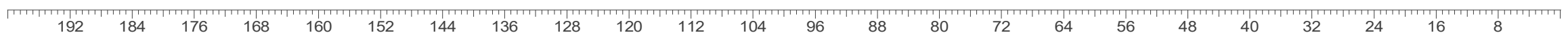


3.36.3. $\mathrm{HSQC}\left(\mathrm{CDCl}_{3}\right)$

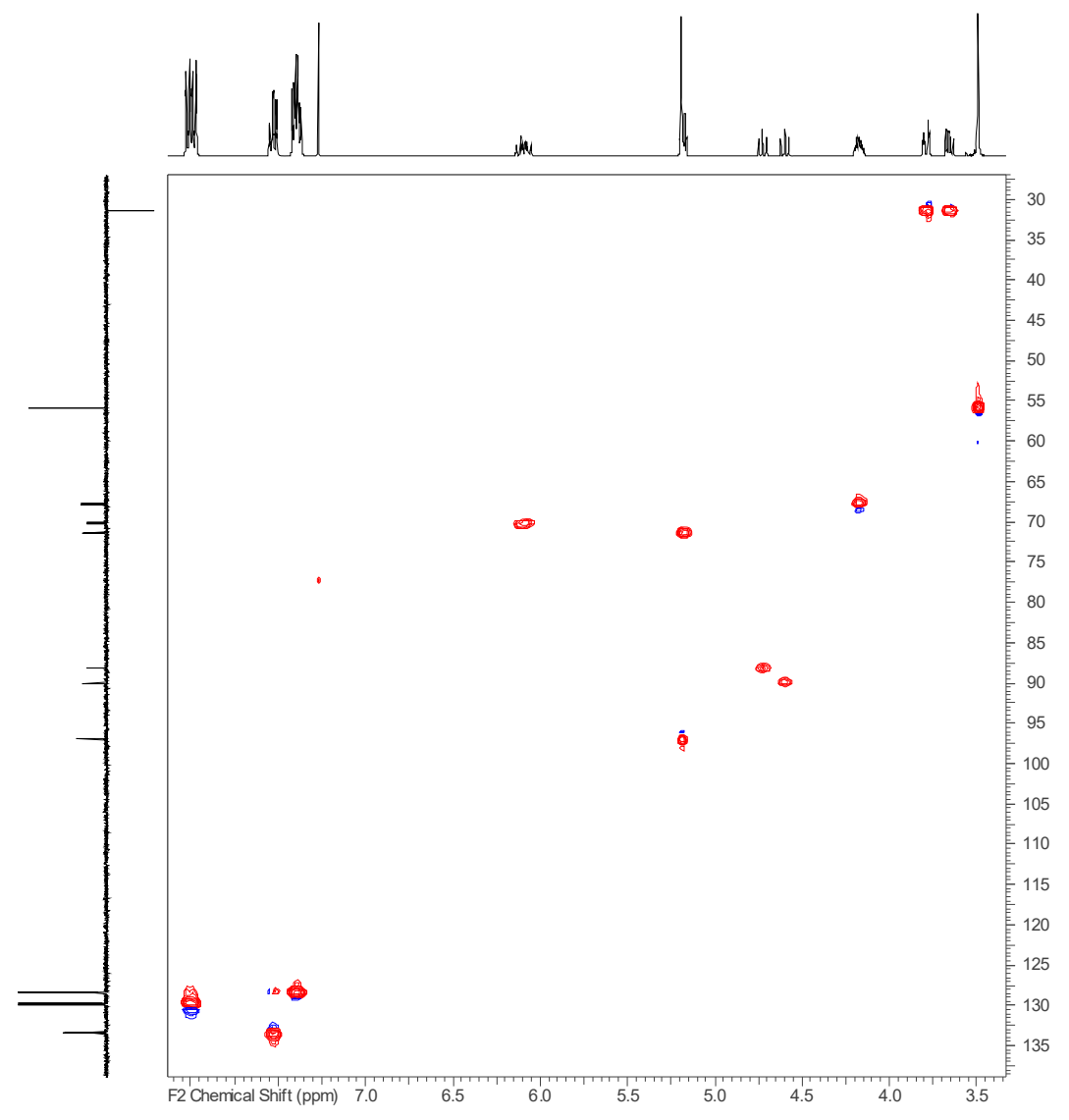


3.36.4. ${ }^{19} \mathrm{~F} \mathrm{NMR}\left(376 \mathrm{MHz}, \mathrm{CDCl}_{3}\right)$

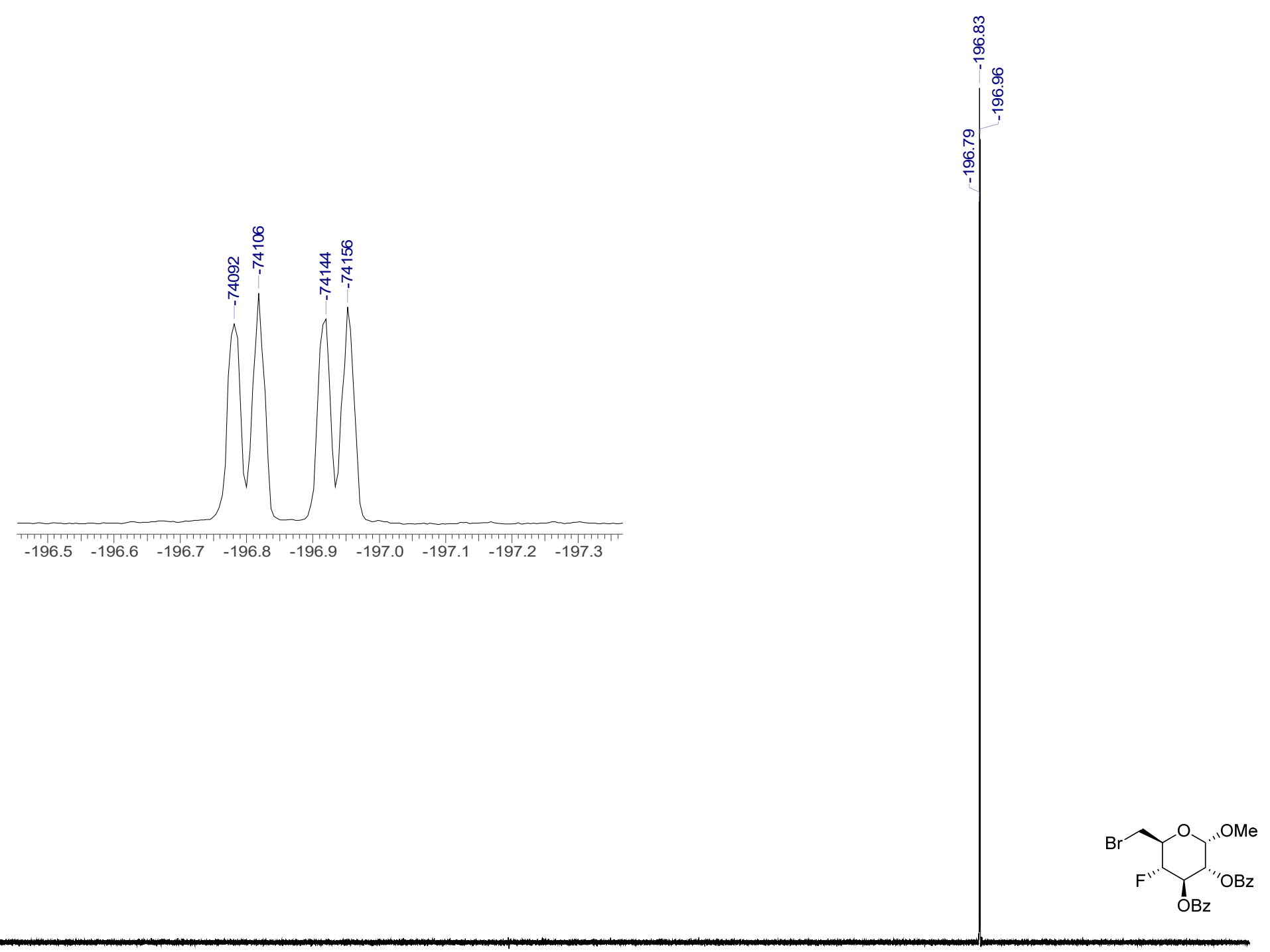


3.36.5. ${ }^{19} \mathrm{~F}\left\{{ }^{1} \mathrm{H}\right\}$ NMR (376 MHz, $\left.\mathrm{CDCl}_{3}\right)$

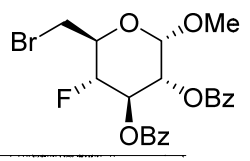




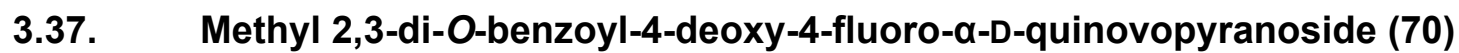

3.37.1. ${ }^{1} \mathrm{H}$ NMR $\left(400 \mathrm{MHz}, \mathrm{CDCl}_{3}\right)$

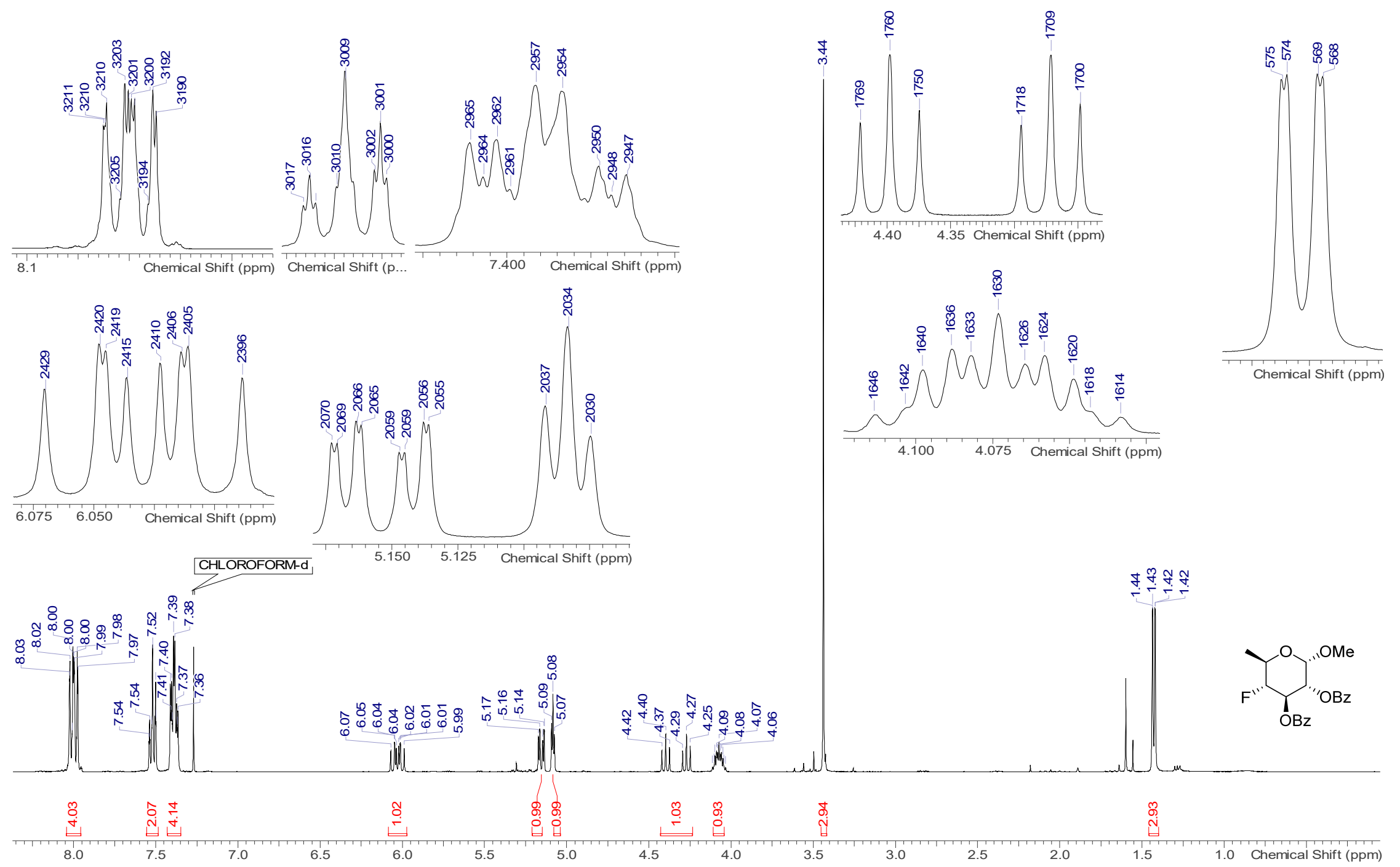


3.37.2. ${ }^{13} \mathrm{C}\left\{{ }^{1} \mathrm{H}\right\}$ NMR $\left(101 \mathrm{MHz}, \mathrm{CDCl}_{3}\right)$

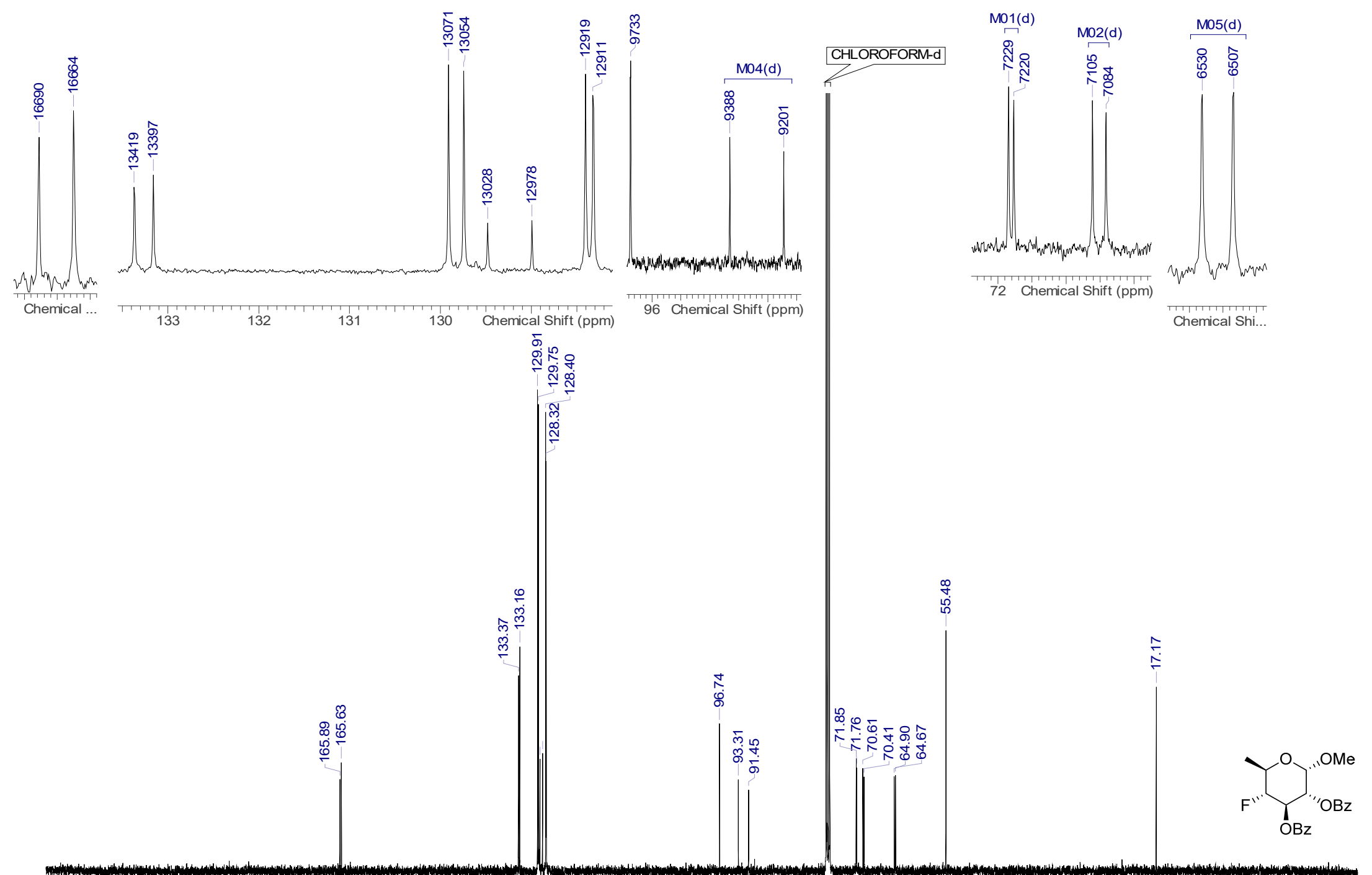

m.

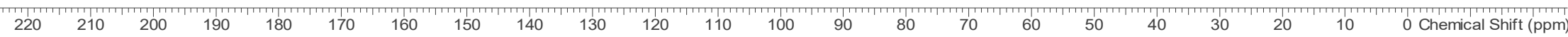


3.37.3. COSY, $\mathrm{HSQC} \& \mathrm{HMBC}\left(\mathrm{CDCl}_{3}\right)$

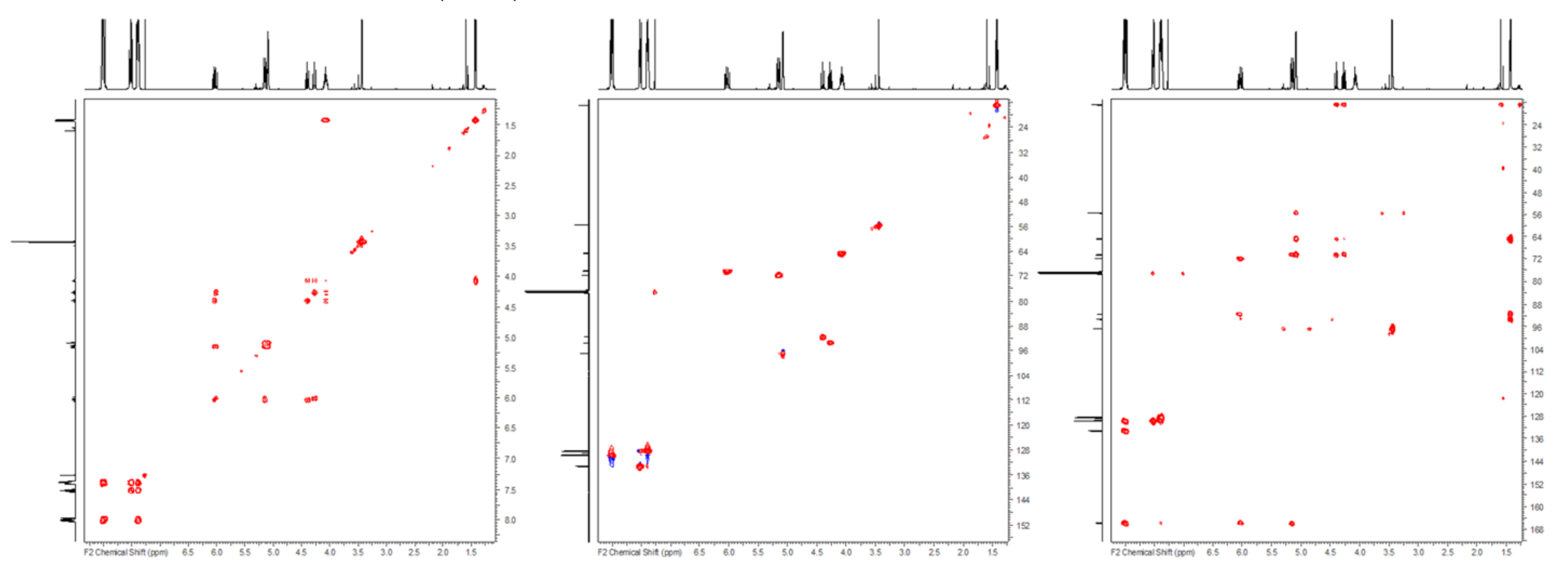


3.37.4. ${ }^{19} \mathrm{~F}$ NMR (376 MHz, $\mathrm{CDCl}_{3}$ )
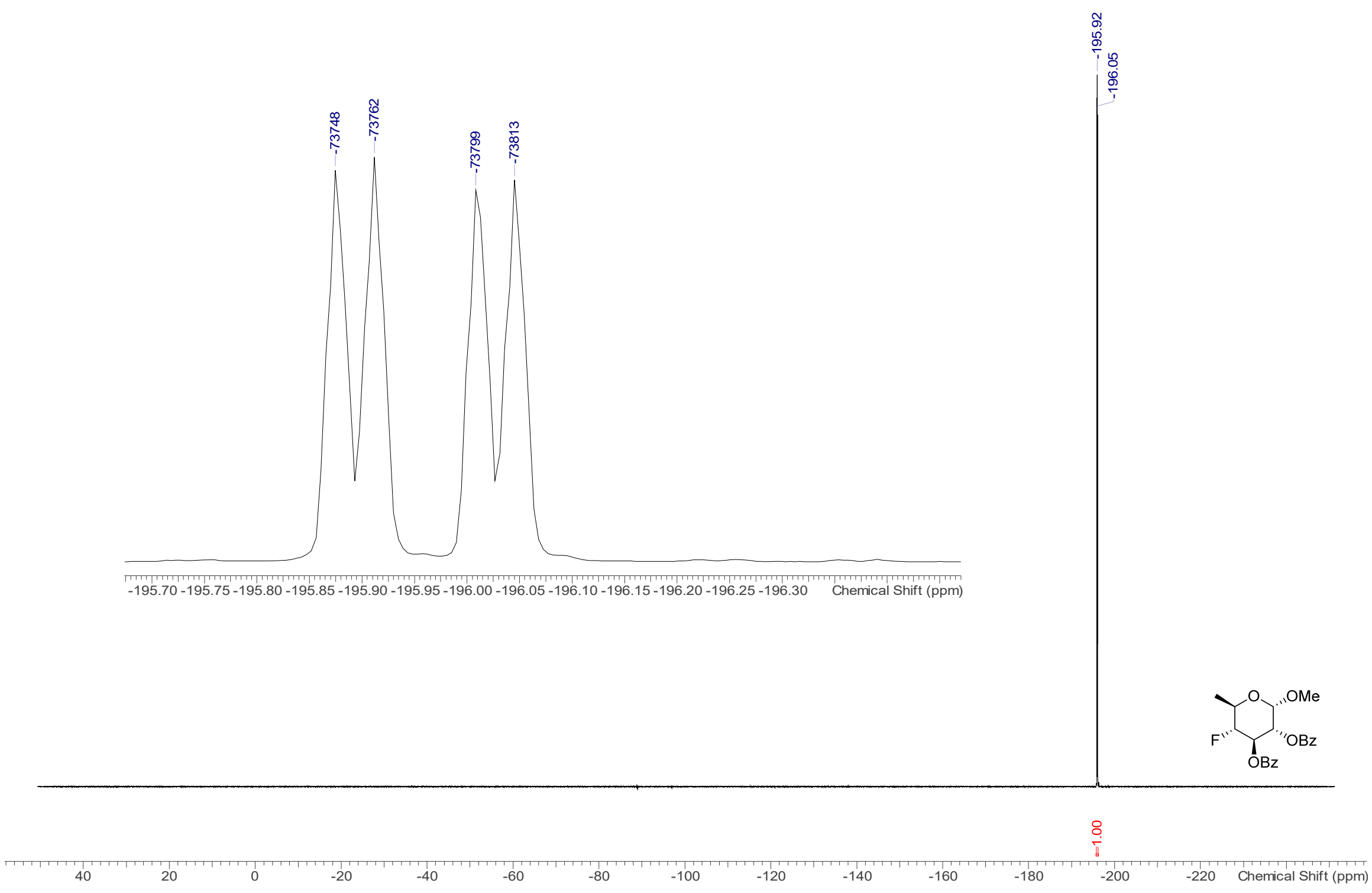
3.37.5. ${ }^{19} \mathrm{~F}\left\{{ }^{1} \mathrm{H}\right\} \mathrm{NMR}\left(376 \mathrm{MHz}, \mathrm{CDCl}_{3}\right)$
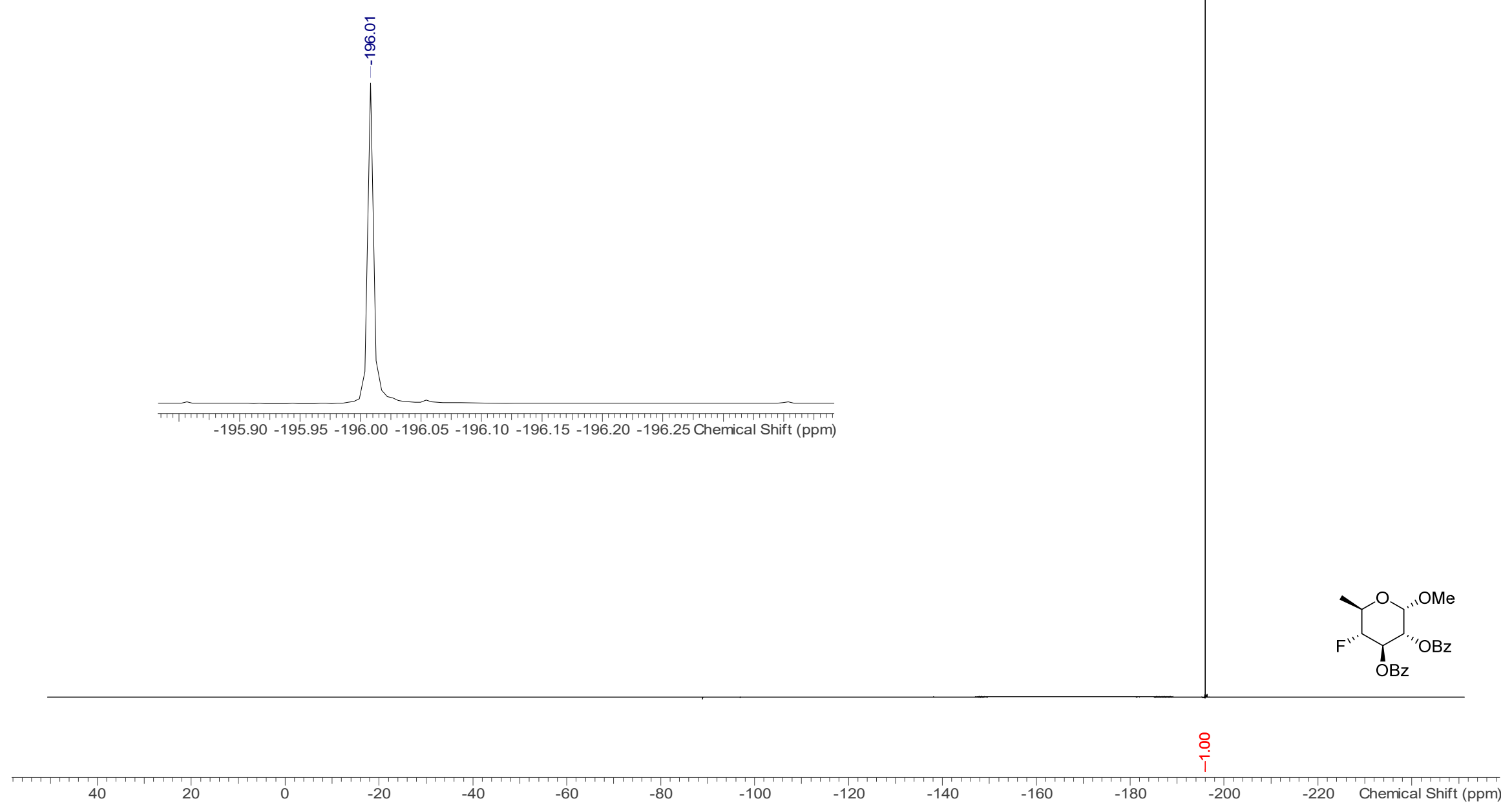


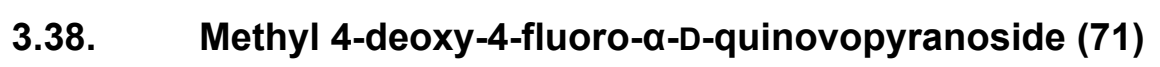

3.38.1. ${ }^{1} \mathrm{H}$ NMR $\left(500 \mathrm{MHz}, \mathrm{CD}_{3} \mathrm{OD}\right)$

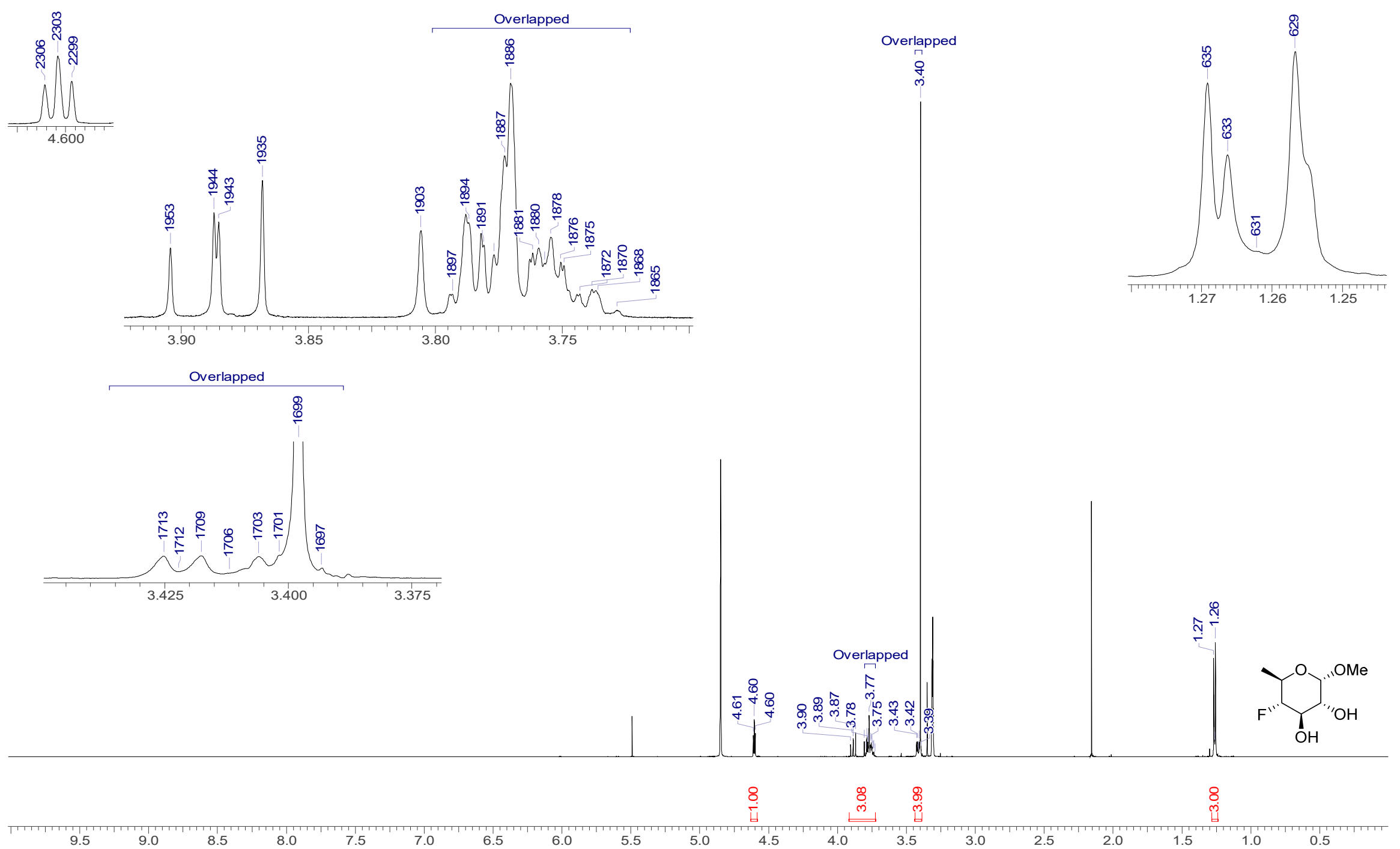


3.38.2. ${ }^{1} \mathrm{H}\left\{{ }^{19} \mathrm{~F}\right\} \mathrm{NMR}\left(500 \mathrm{MHz}, \mathrm{CD}_{3} \mathrm{OD}\right)$

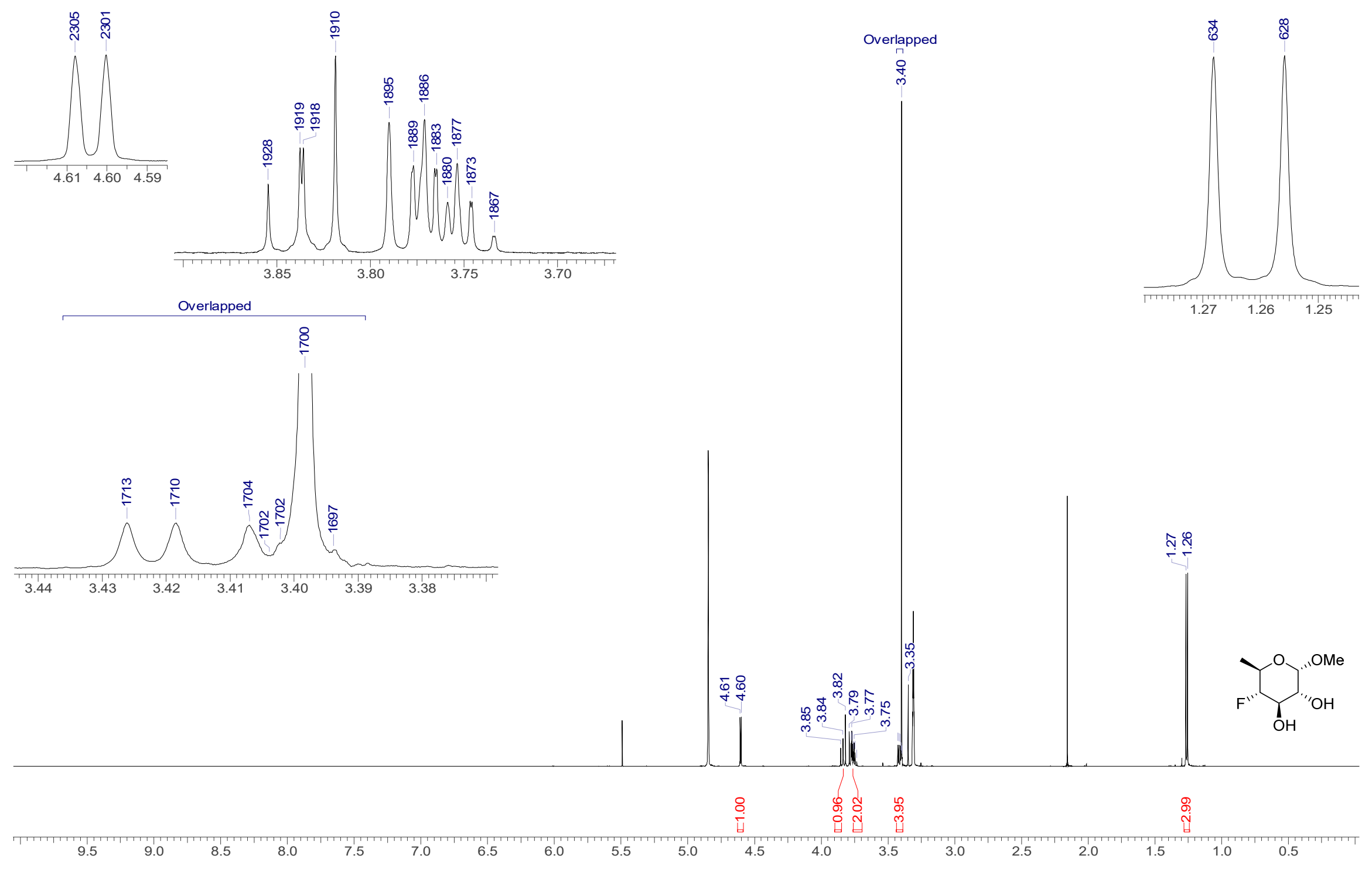


3.38.3. ${ }^{13} \mathrm{C}\left\{{ }^{1} \mathrm{H}\right\}$ NMR $\left(101 \mathrm{MHz}, \mathrm{CD}_{3} \mathrm{OD}\right)$
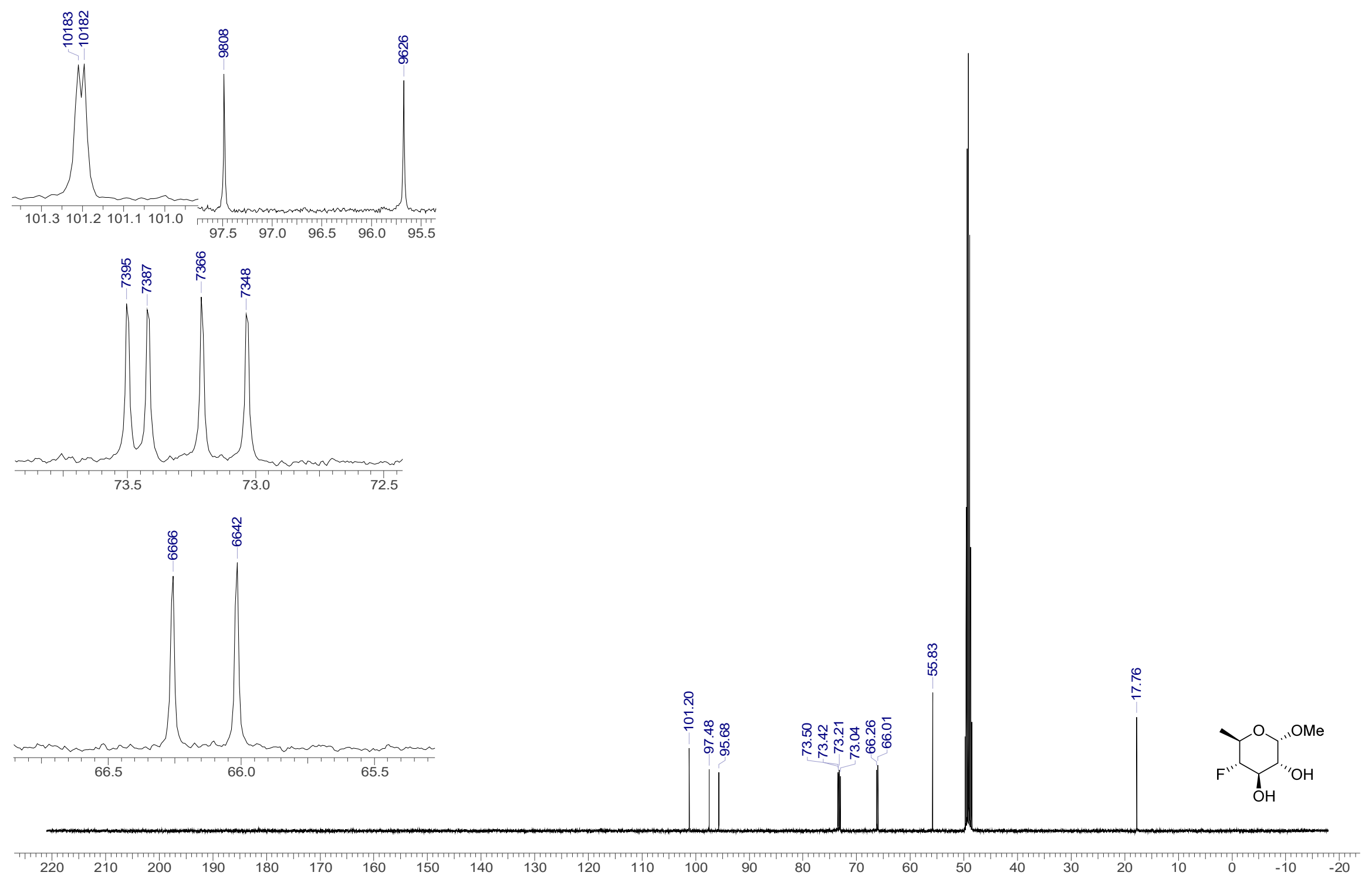
3.38.4. COSY, HSQC-DEPT $\left(\mathrm{CD}_{3} \mathrm{OD}\right)$

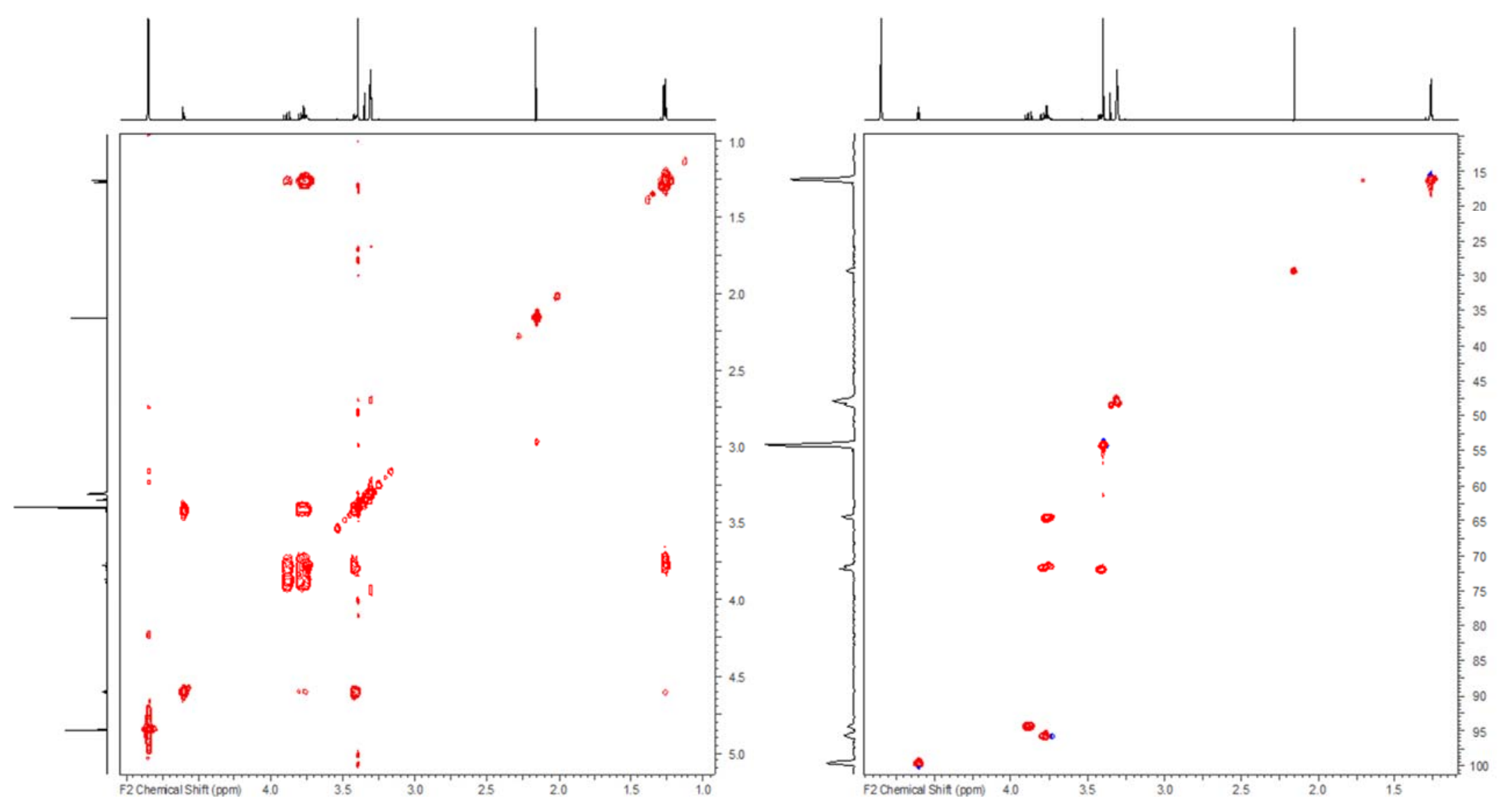


3.38.5. ${ }^{19} \mathrm{~F} \mathrm{NMR}\left(470 \mathrm{MHz}, \mathrm{CD}_{3} \mathrm{OD}\right)$

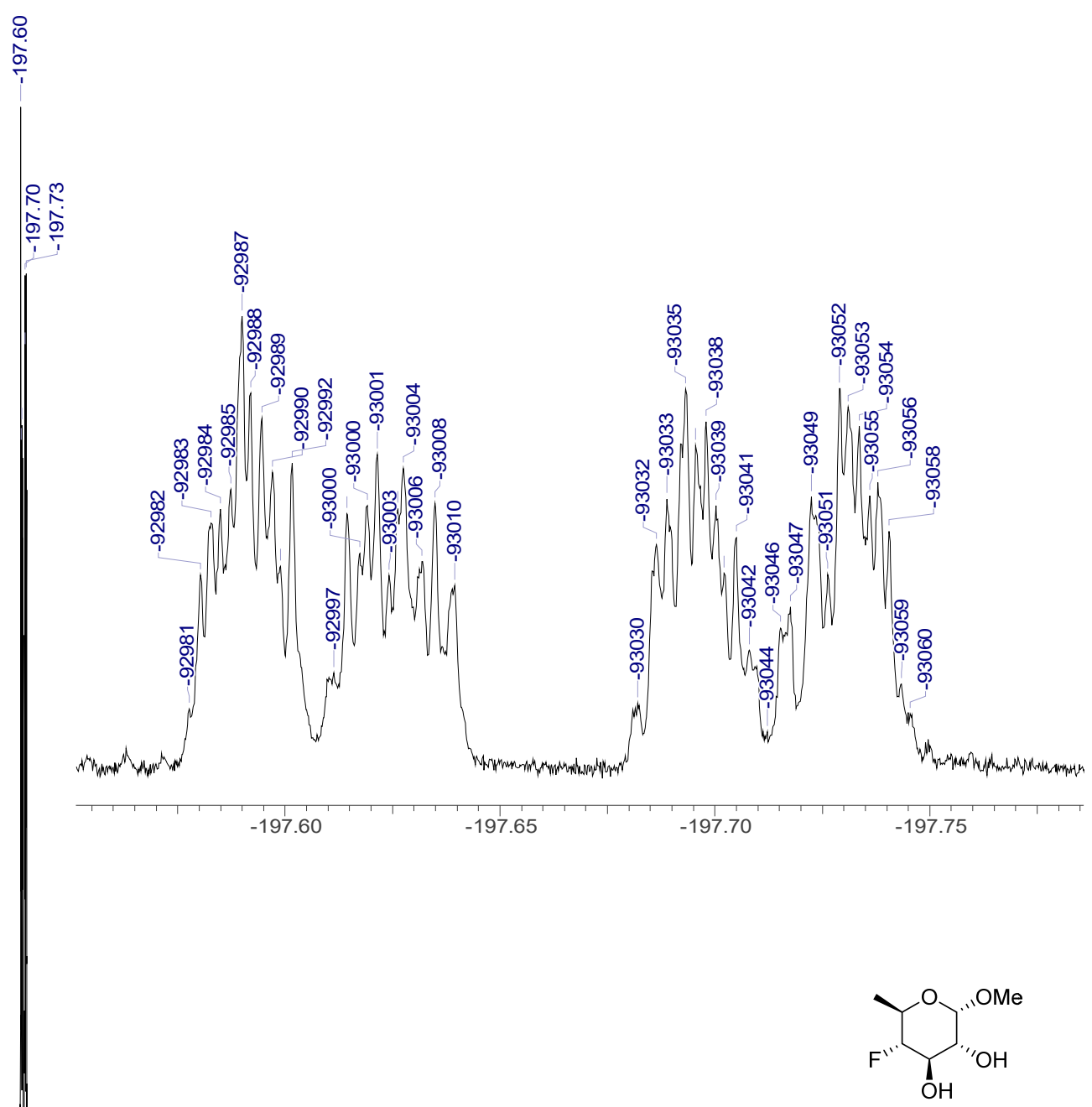

Iow

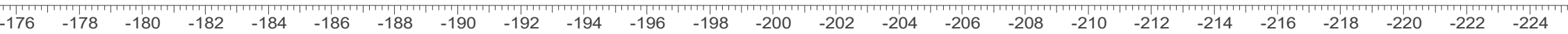


3.38.6. ${ }^{19} \mathrm{~F}\left\{{ }^{1} \mathrm{H}\right\} \mathrm{NMR}\left(470 \mathrm{MHz}, \mathrm{CD}_{3} \mathrm{OD}\right)$

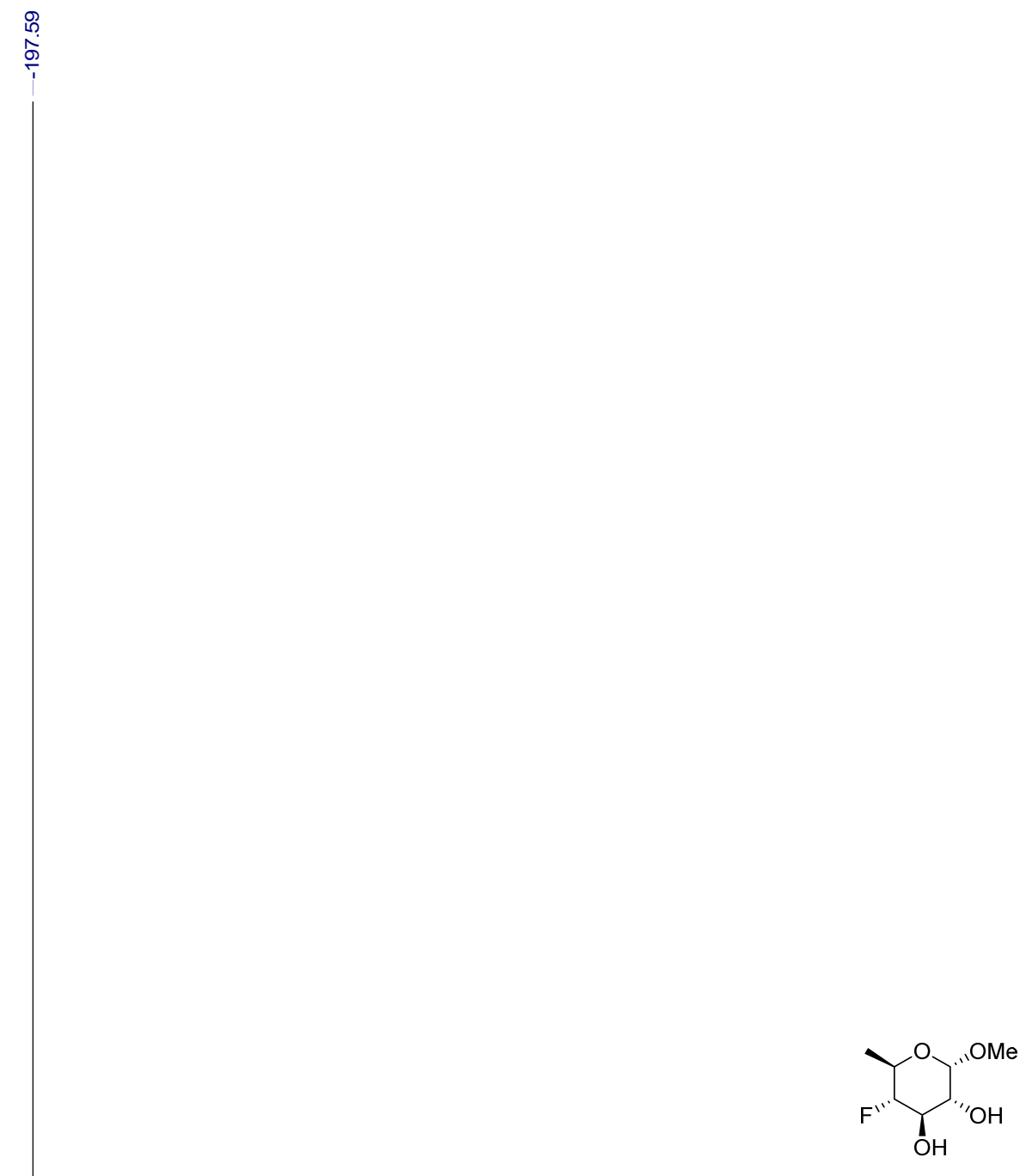




\subsection{Methyl 6-O-p-toluenesufonyl-2,3-O-((2'R,3'R)-2',3'-dimethoxybutane -2',3'-diyl)-a-D-galactopyransoside (72)} 3.39.1. ${ }^{1} \mathrm{H} \mathrm{NMR}\left(400 \mathrm{MHz}, \mathrm{CDCl}_{3}\right)$
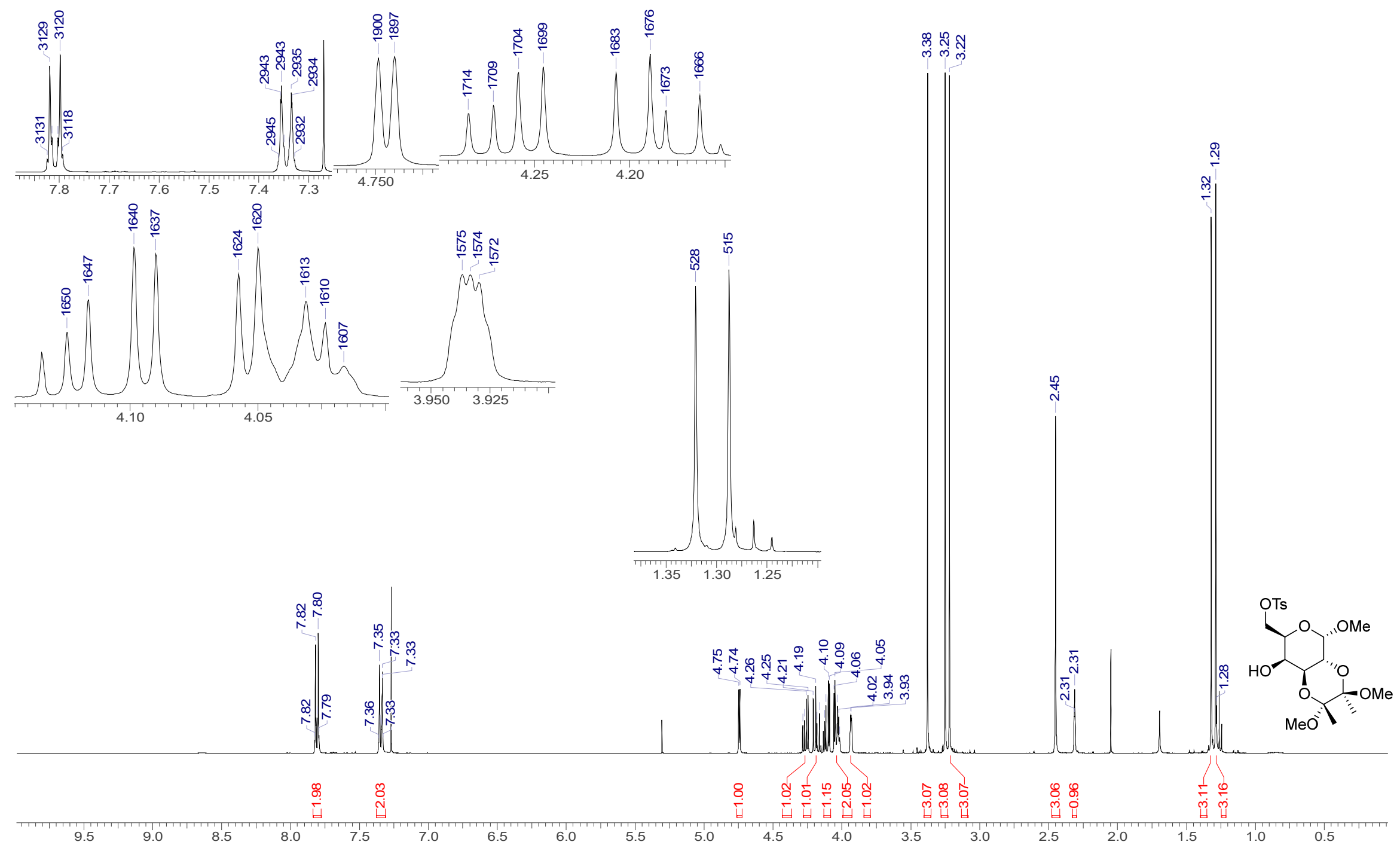
3.39.2. ${ }^{13} \mathrm{C}\left\{{ }^{1} \mathrm{H}\right\}$ NMR $\left(101 \mathrm{MHz}, \mathrm{CDCl}_{3}\right)$

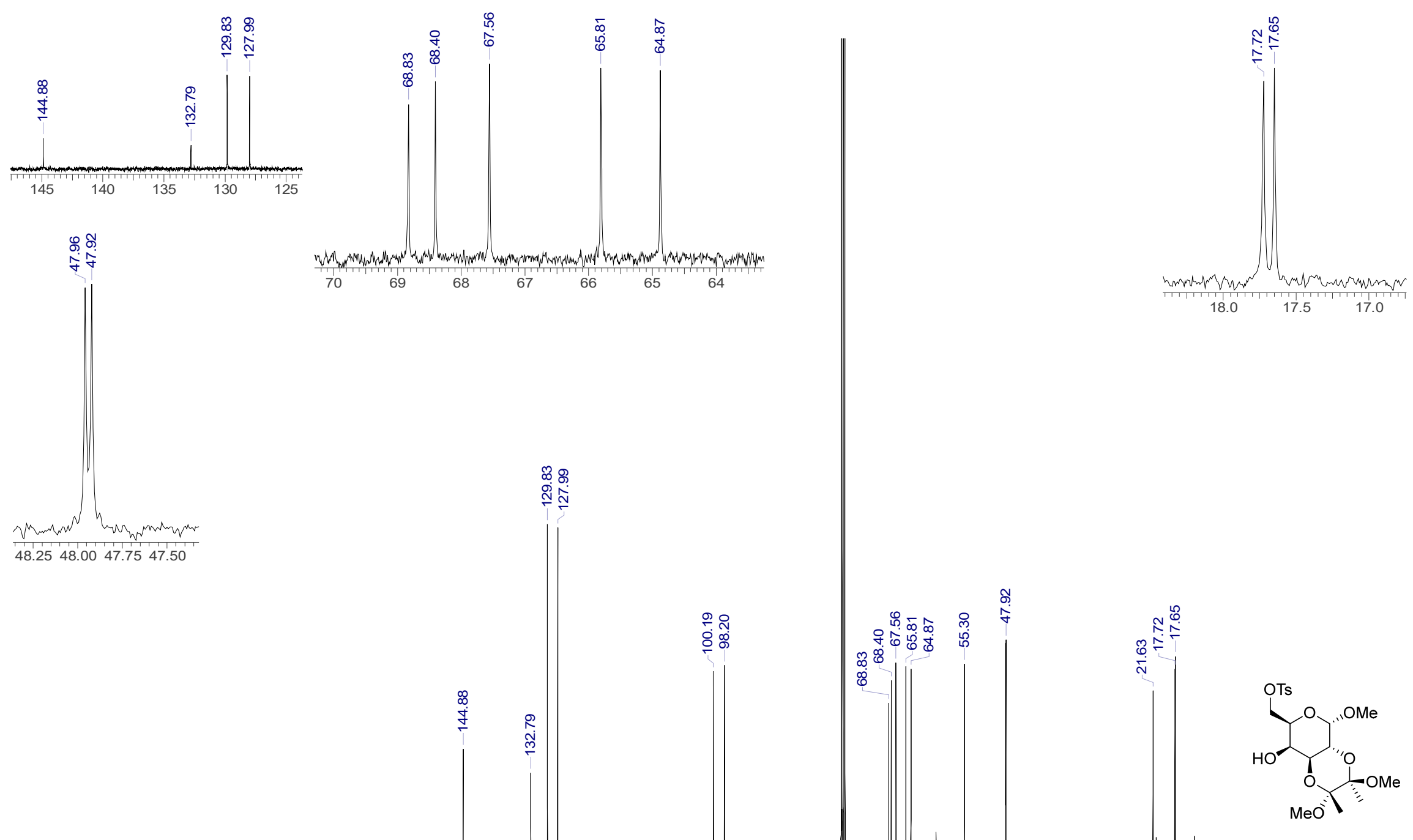

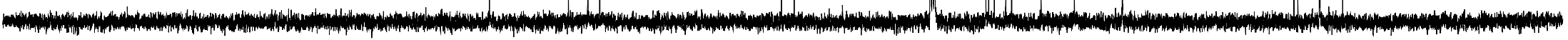

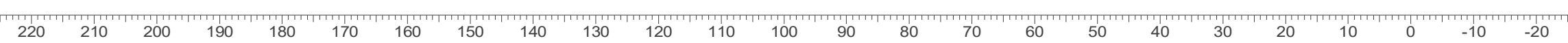


3.39.3. COSY, HSQC-DEPT, $\mathrm{HMBC}\left(\mathrm{CDCl}_{3}\right)$

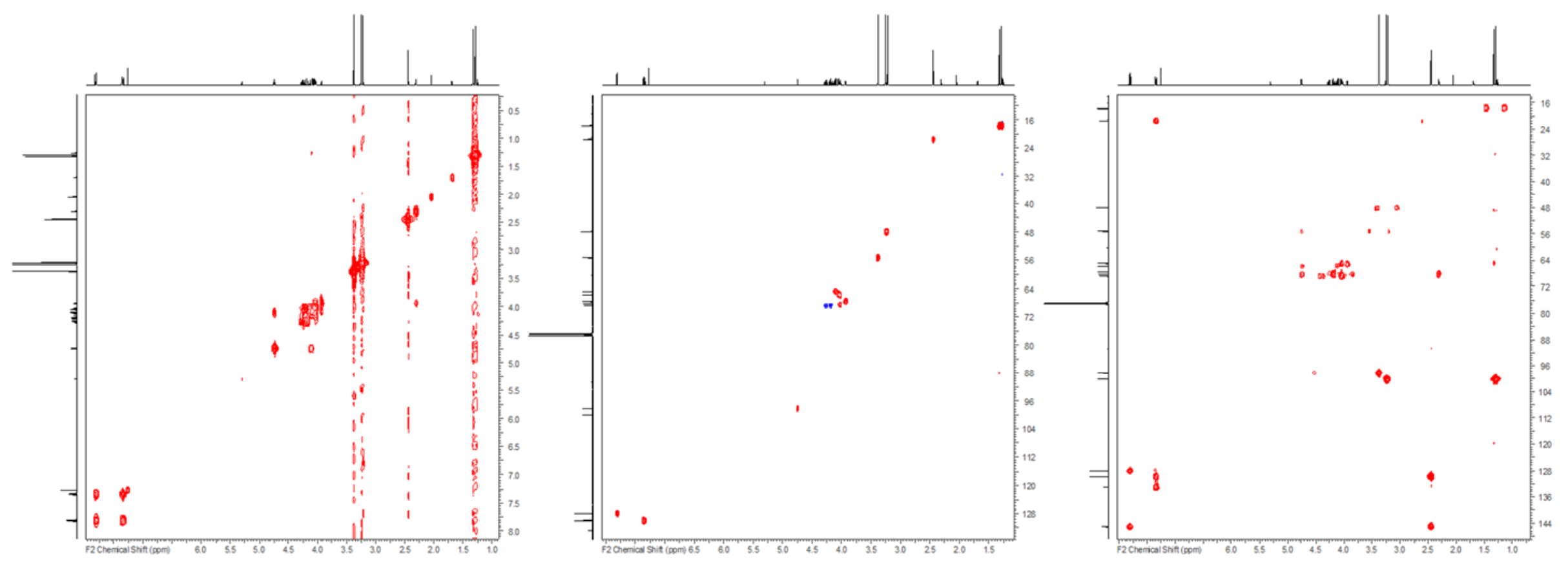




\subsection{Methyl 4-deoxy-4-fluoro-2,3-O-((2'R,3'R)-2',3'-dimethoxybutane-2',3'-diyl)- $\alpha$-D-quinovopyranoside (73)}

3.40.1. ${ }^{1} \mathrm{H} \mathrm{NMR}\left(400 \mathrm{MHz}, \mathrm{CDCl}_{3}\right)$
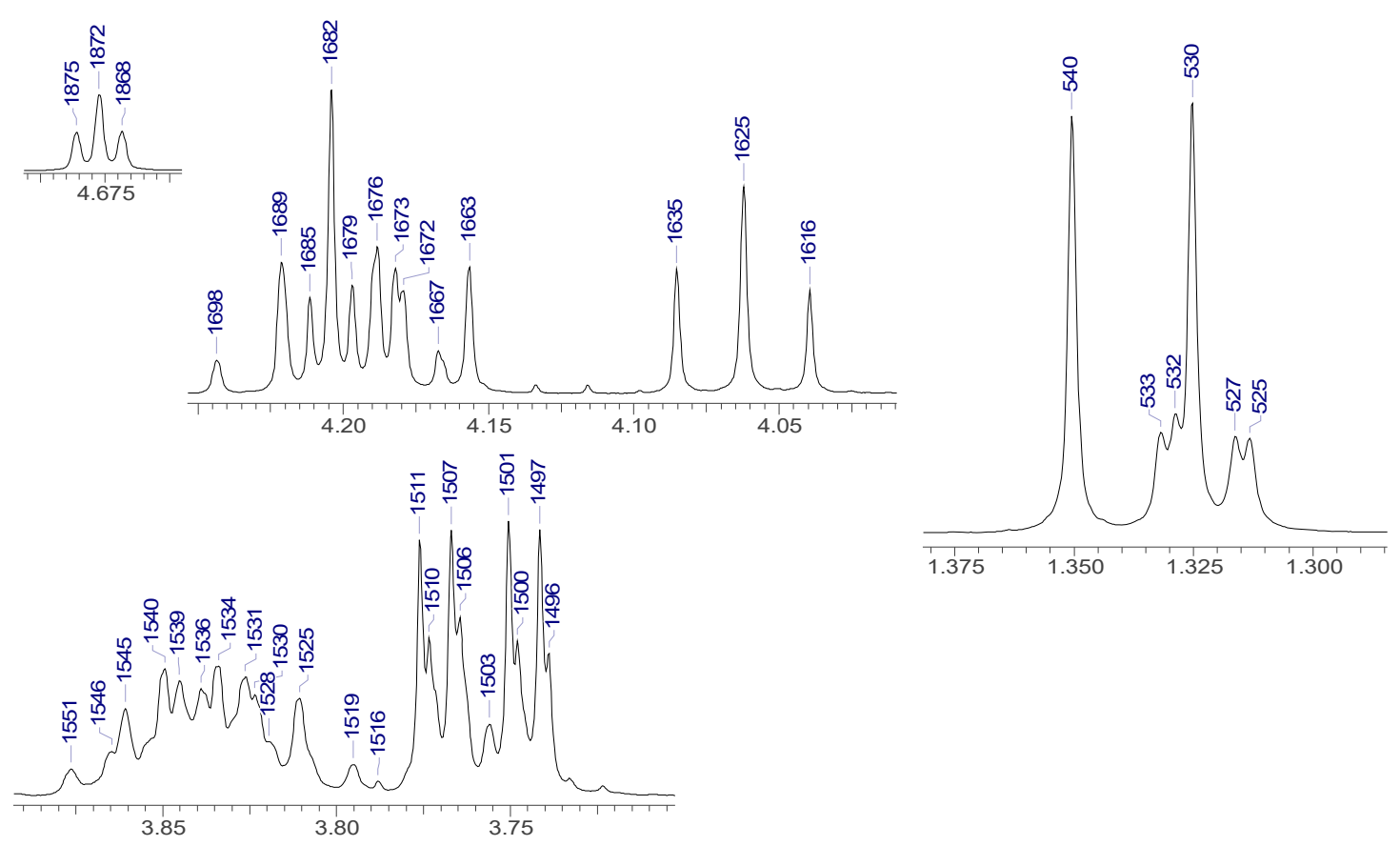

悉总品

을

लำ
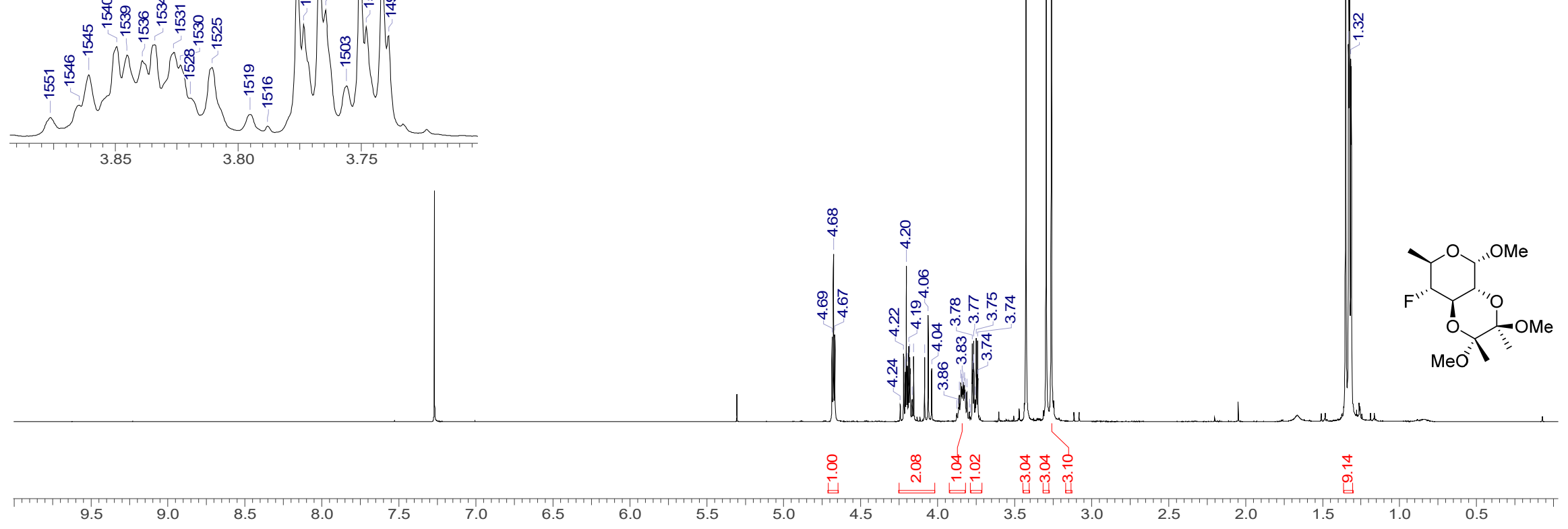
3.40.2. ${ }^{13} \mathrm{C}\left\{{ }^{1} \mathrm{H}\right\}$ NMR $\left(101 \mathrm{MHz}, \mathrm{CDCl}_{3}\right)$
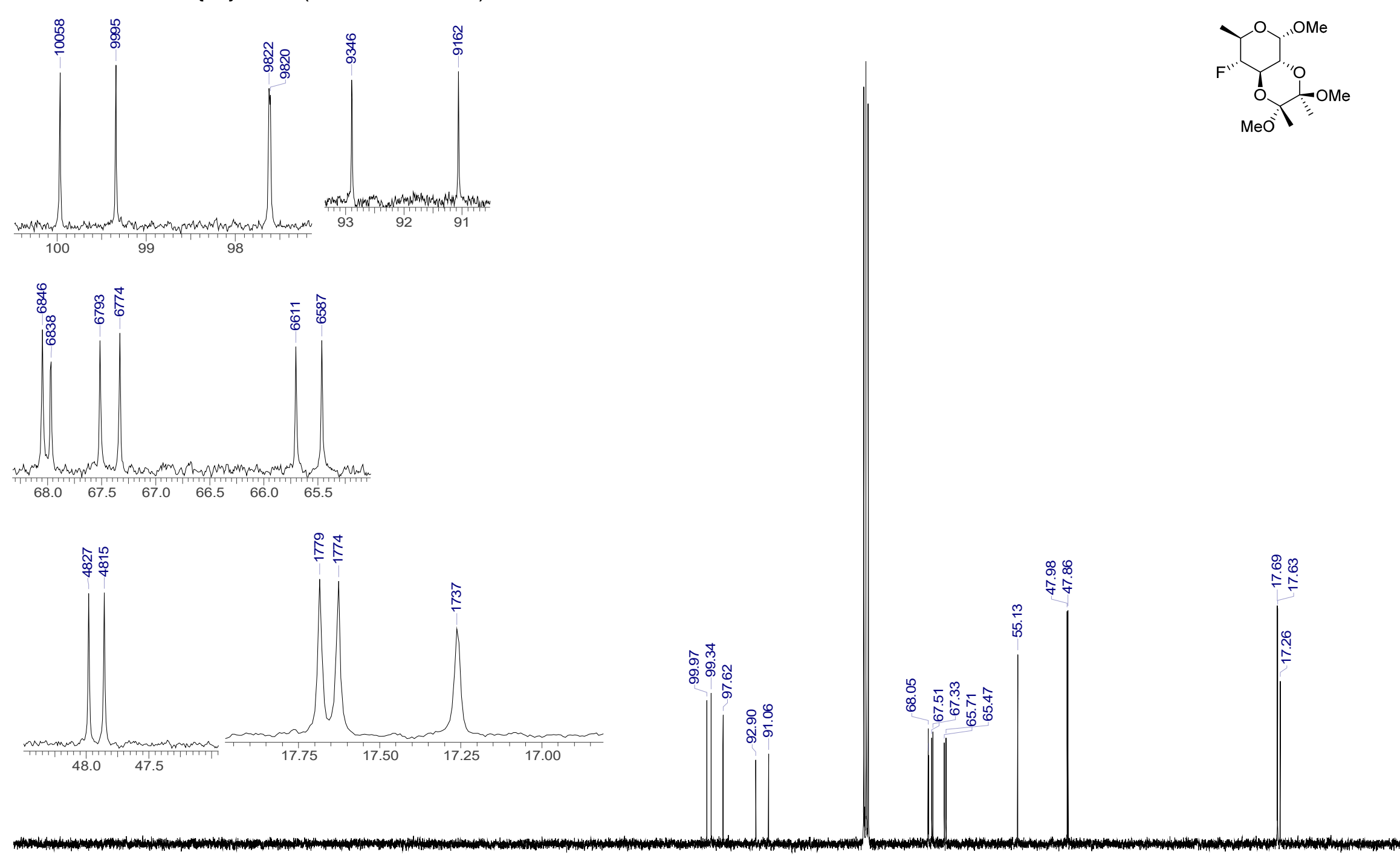

mow

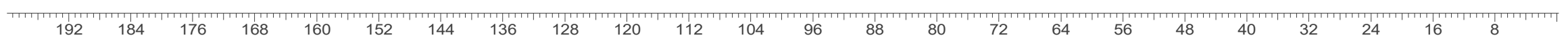


3.40.3. COSY, $\mathrm{HSQC} \& \mathrm{HMBC}\left(\mathrm{CDCl}_{3}\right)$

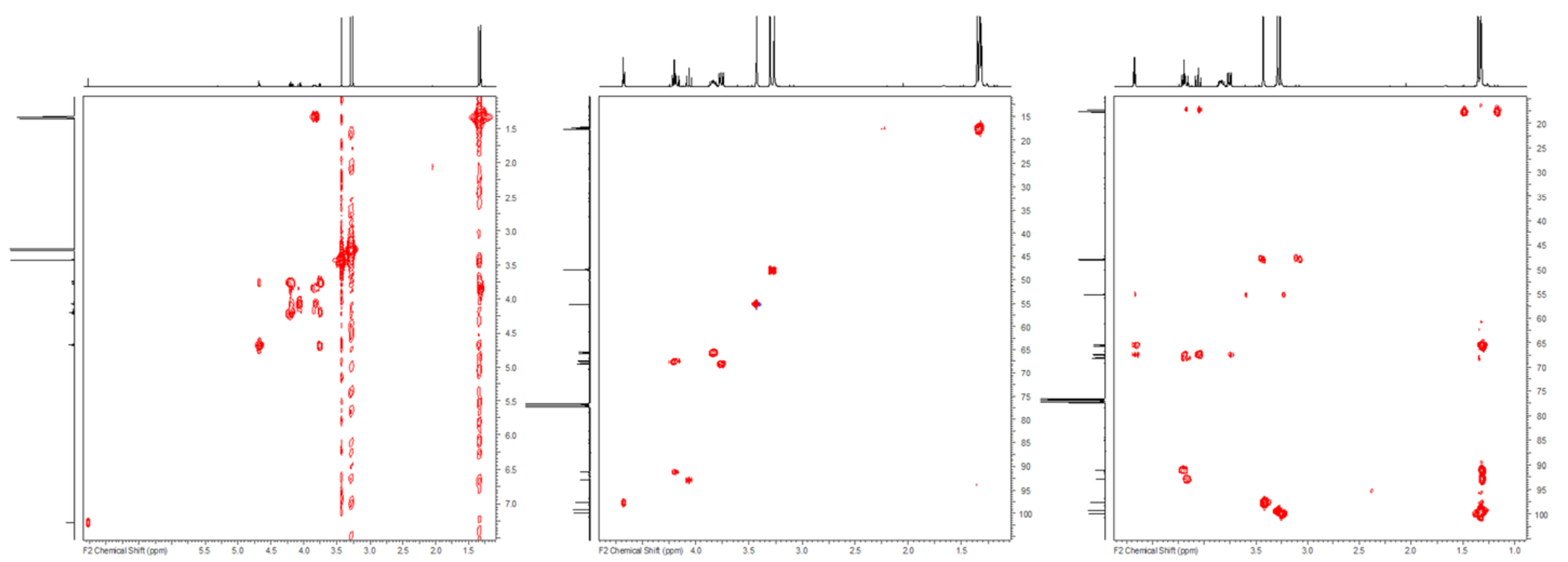


3.40.4. ${ }^{19} \mathrm{~F} \mathrm{NMR}\left(376 \mathrm{MHz}, \mathrm{CDCl}_{3}\right)$
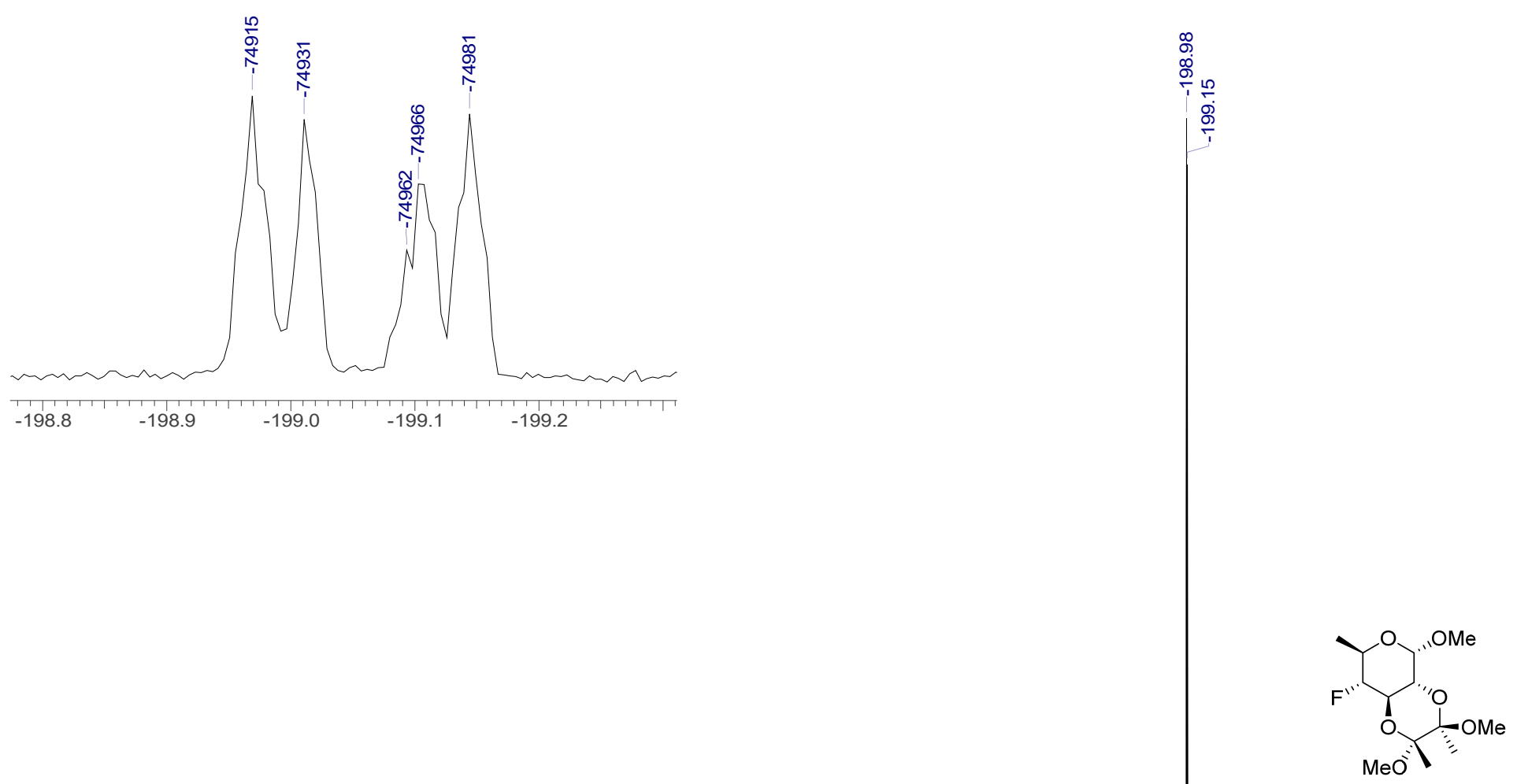

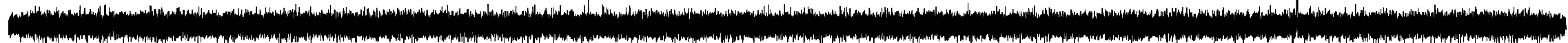


3.40.5. ${ }^{19} \mathrm{~F}\left\{{ }^{1} \mathrm{H}\right\} \mathrm{NMR}\left(376 \mathrm{MHz}, \mathrm{CDCl}_{3}\right)$

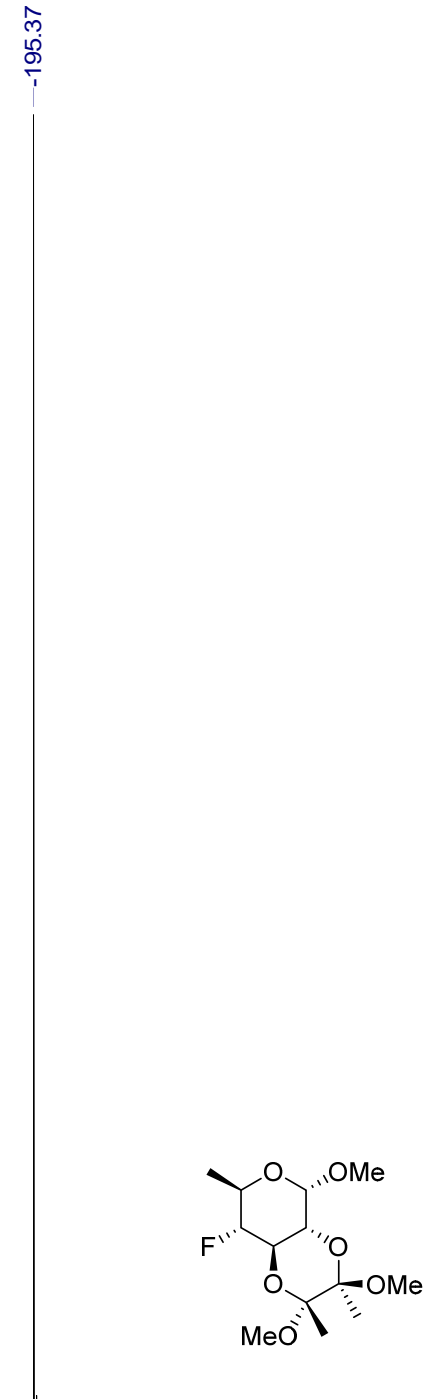




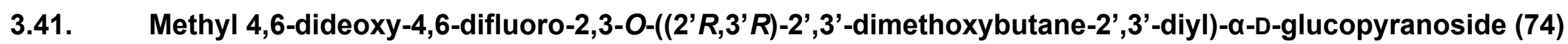
3.41.1. ${ }^{1} \mathrm{H} \mathrm{NMR}\left(400 \mathrm{MHz}, \mathrm{CDCl}_{3}\right)$
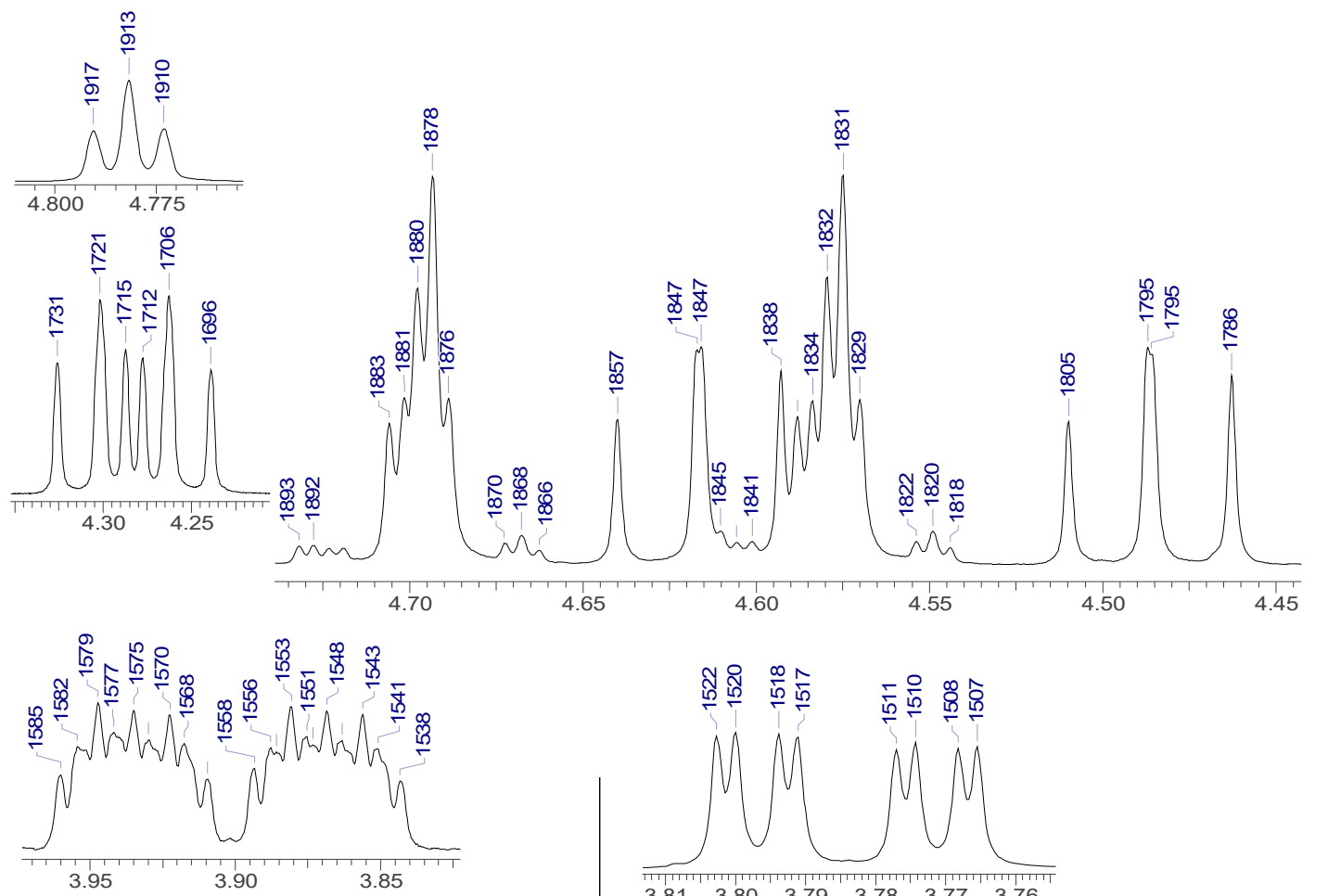

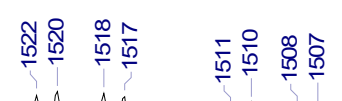
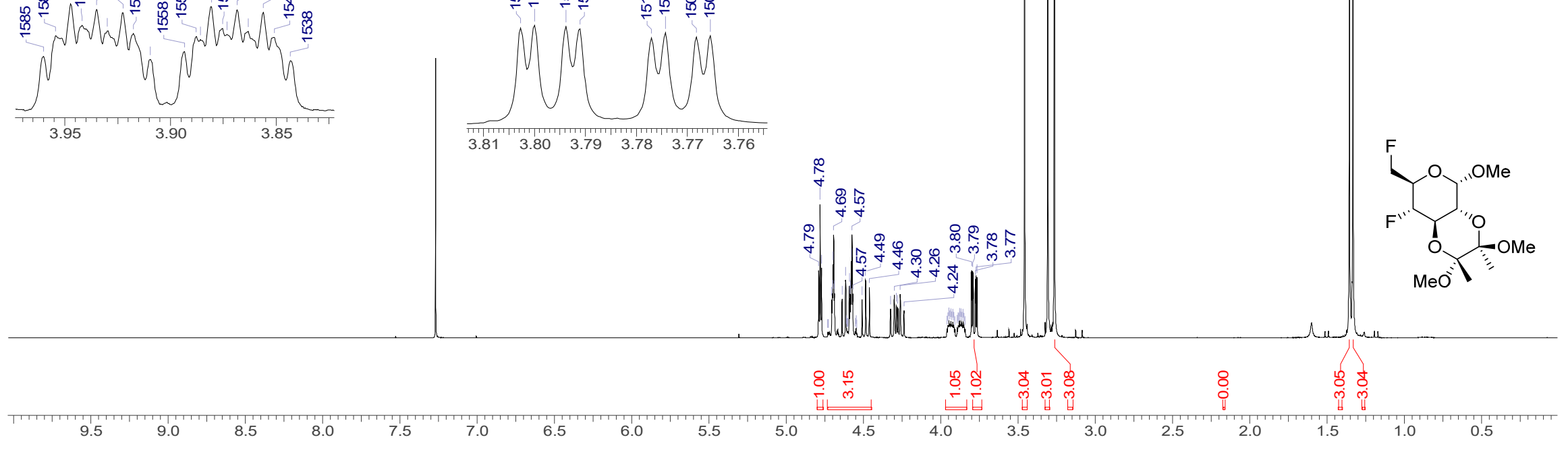


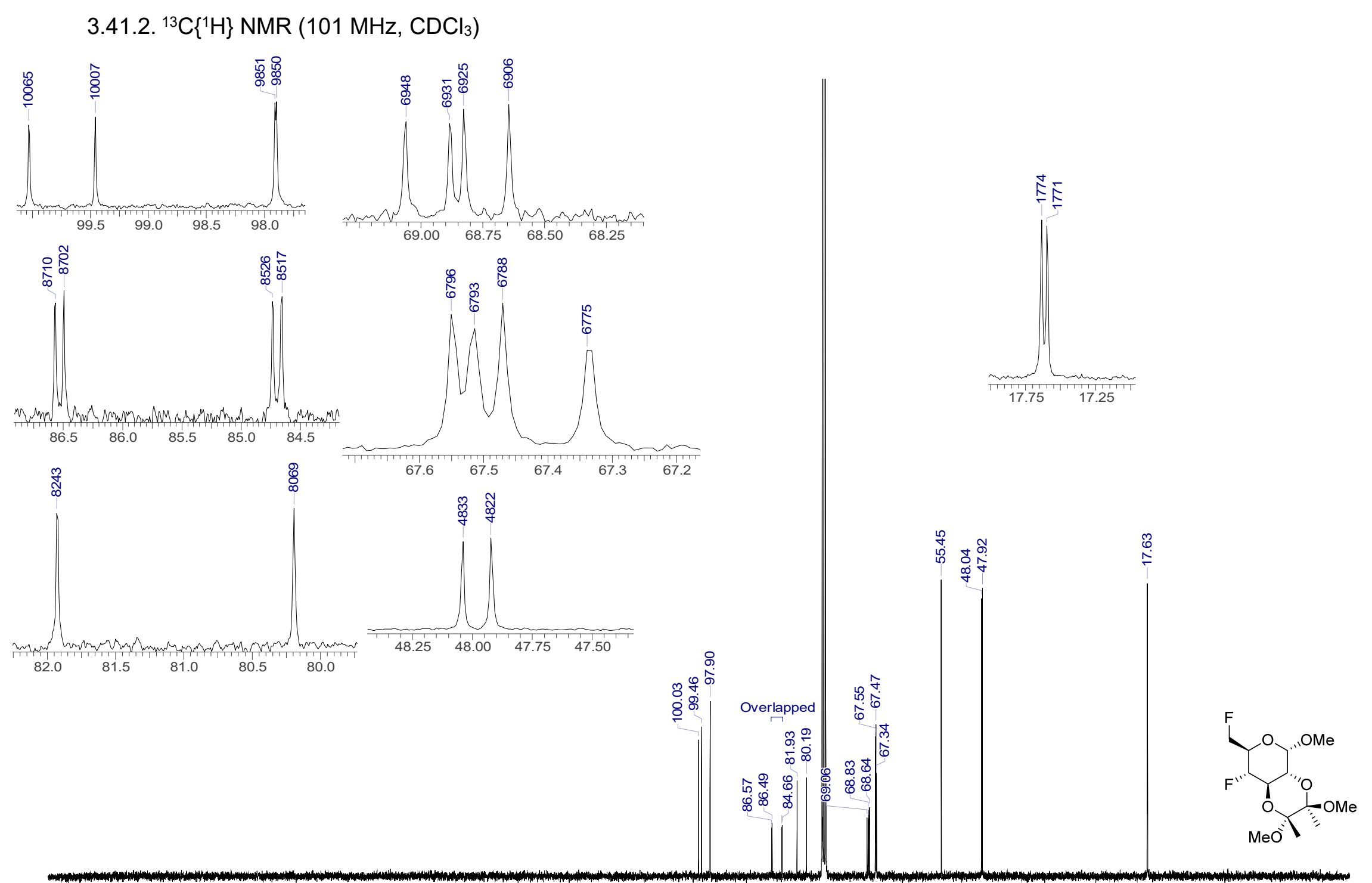

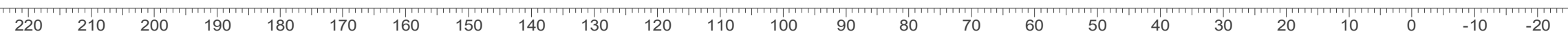


3.41.3. COSY, $\mathrm{HSQC} \& \mathrm{HMBC}\left(\mathrm{CDCl}_{3}\right)$

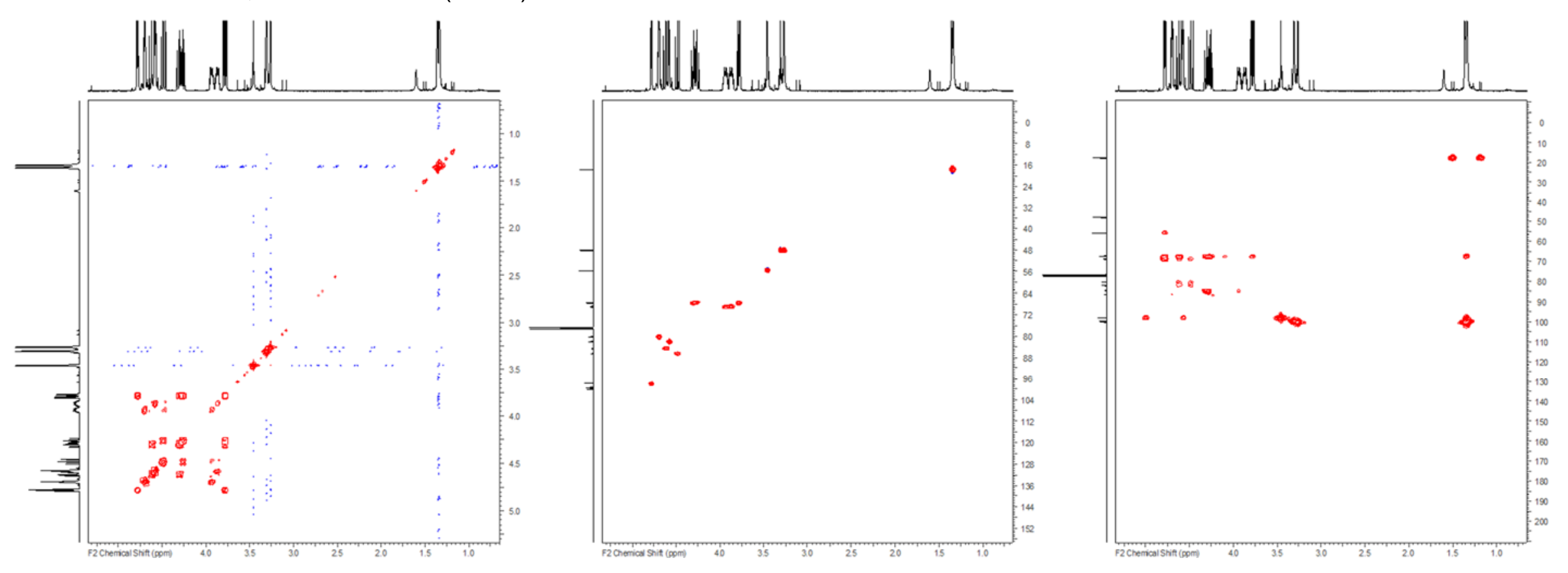


3.41.4. ${ }^{19} \mathrm{~F} \mathrm{NMR}\left(376 \mathrm{MHz}, \mathrm{CDCl}_{3}\right)$
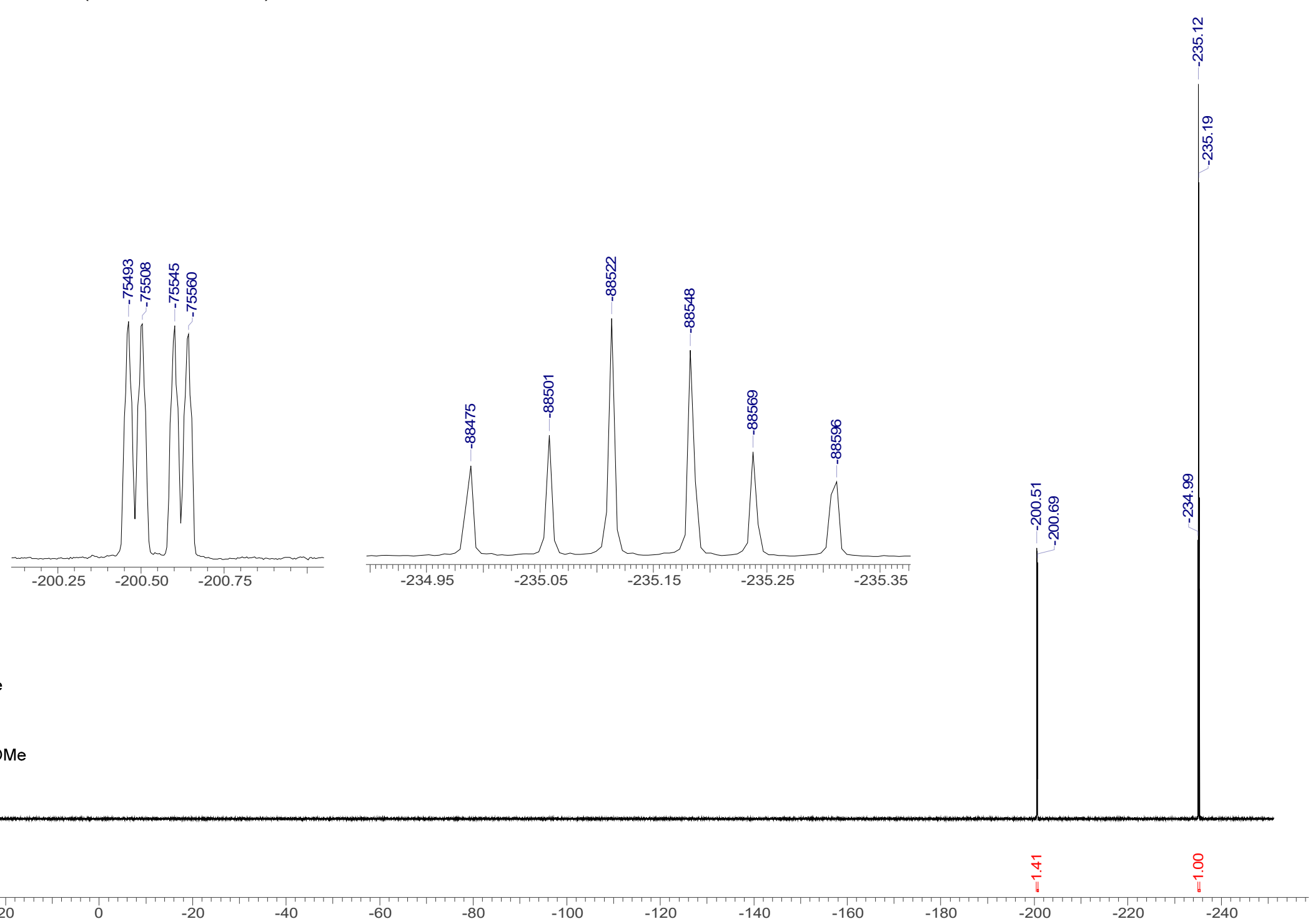

$$
\text { OOOMe }
$$


3.41.5. ${ }^{19} \mathrm{~F}\left\{{ }^{1} \mathrm{H}\right\} \mathrm{NMR}\left(376 \mathrm{MHz}, \mathrm{CDCl}_{3}\right)$

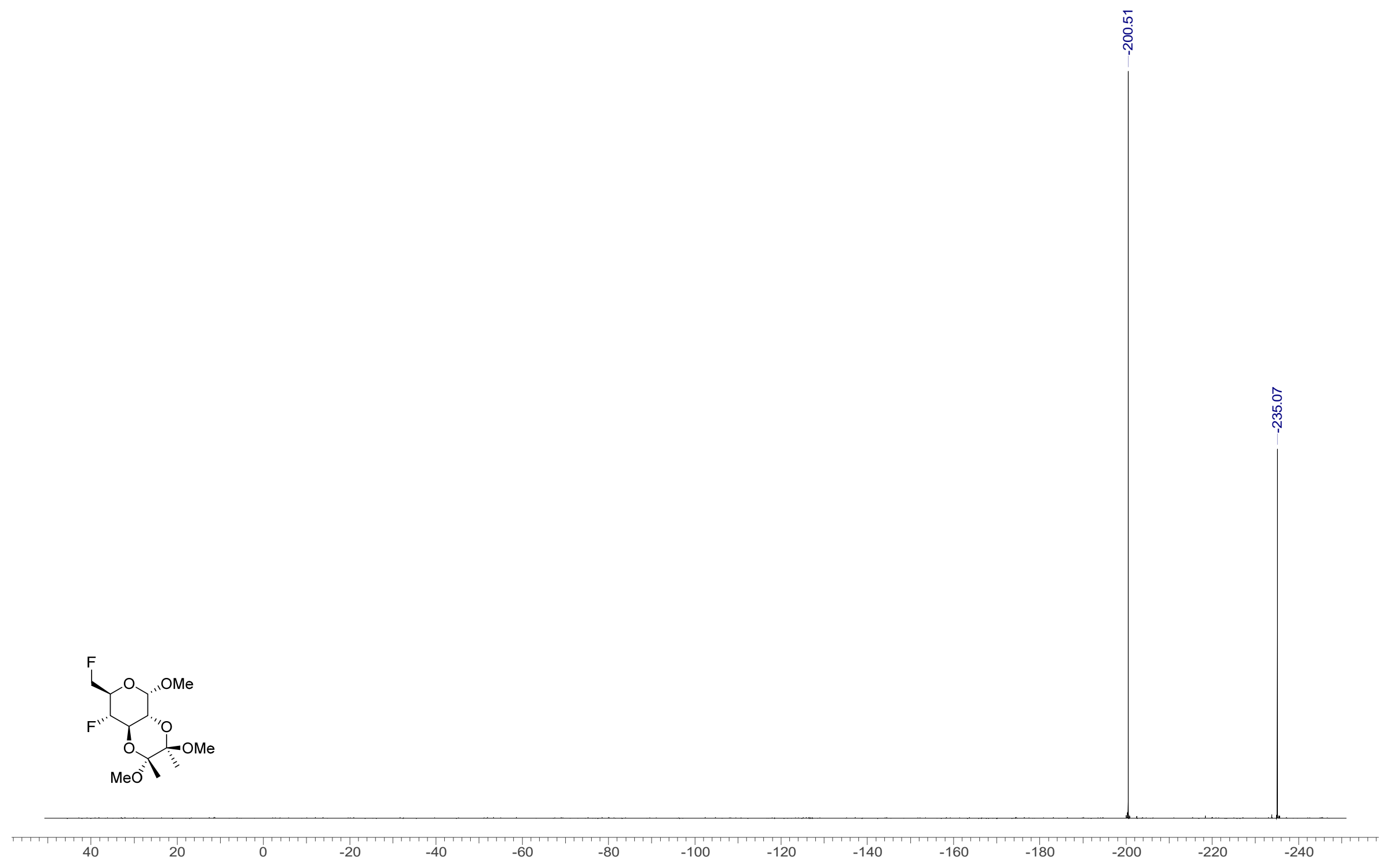


S 222

3.42. Methyl 4,6-dideoxy-4,4-difluoro-2,3-O-((2'R,3'R)-2',3'-dimethoxybutane-2',3'-diyl)- $\alpha-\mathrm{D}-x y l o-h e x o p y r a n o s i d e ~(76)$ 3.42.1. ${ }^{1} \mathrm{H} \mathrm{NMR}\left(400 \mathrm{MHz}, \mathrm{CDCl}_{3}\right)$
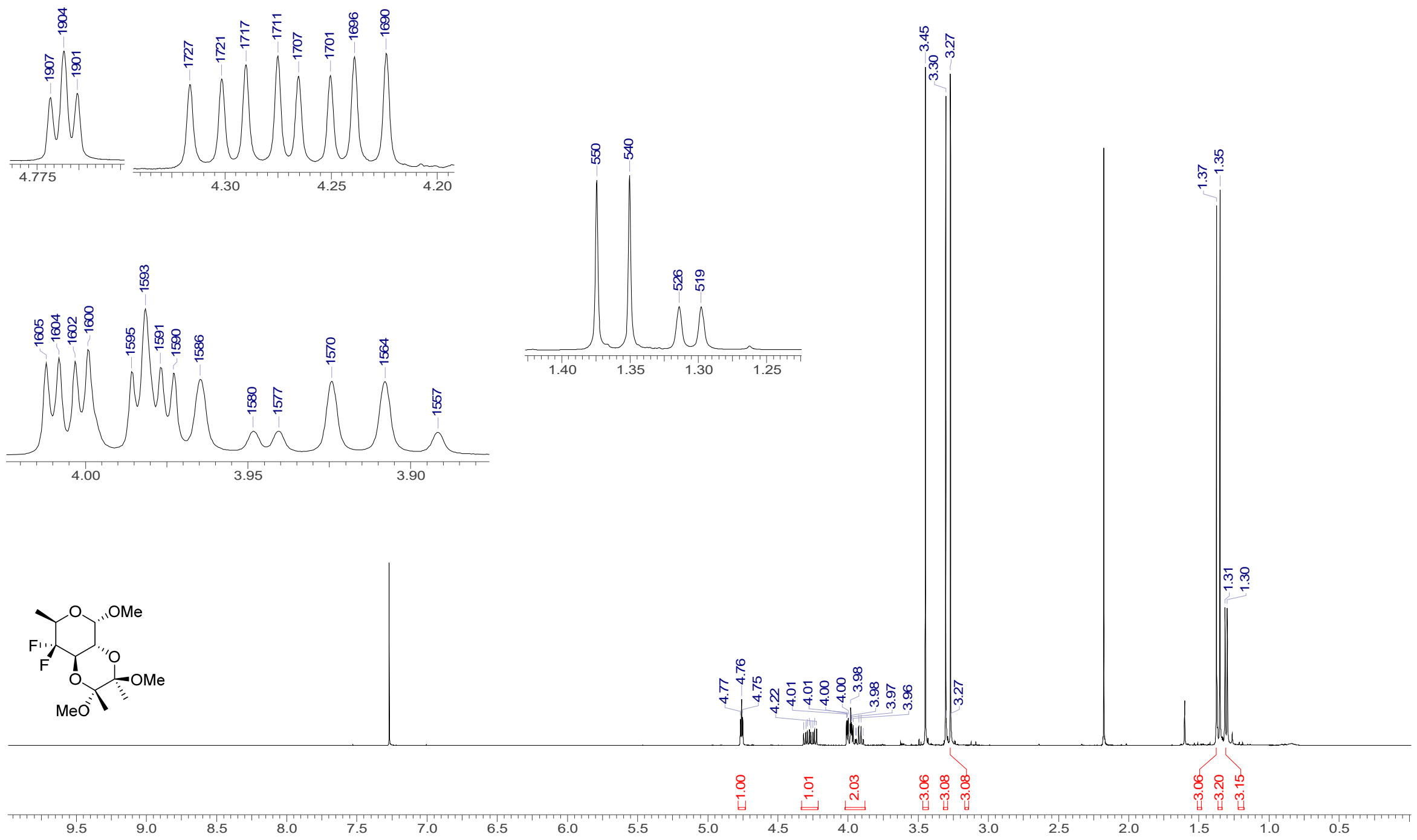


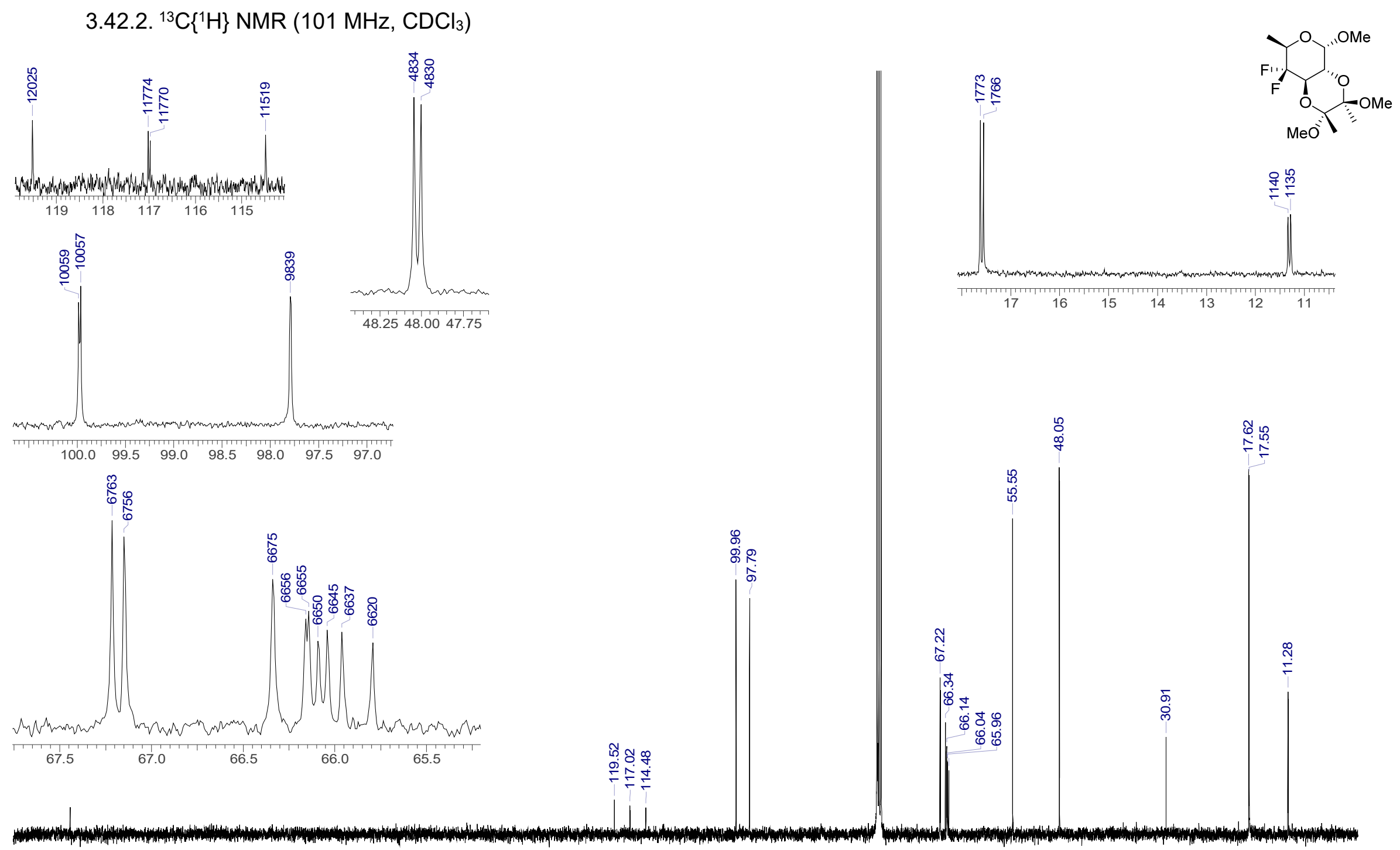

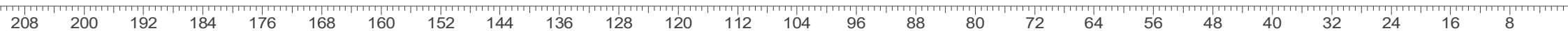


3.42.3. COSY, $\mathrm{HSQC} \& \mathrm{HMBC}\left(\mathrm{CDCl}_{3}\right)$

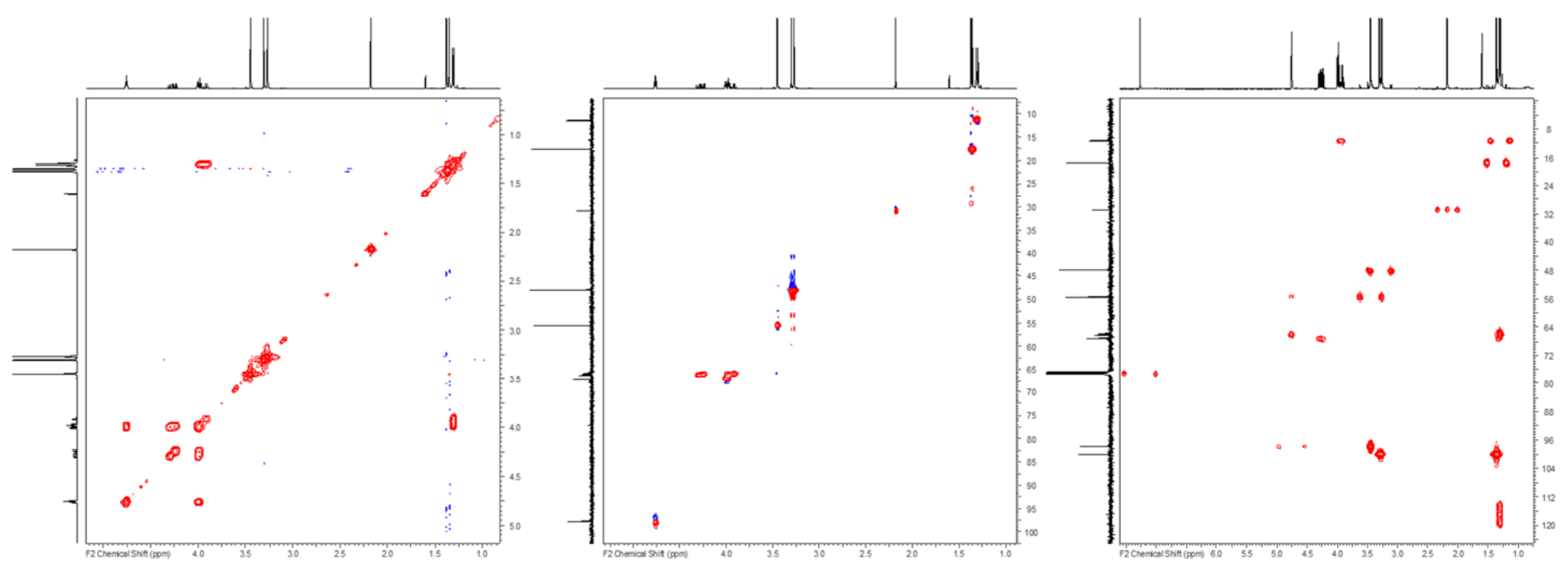


3.42.4. ${ }^{19} \mathrm{~F} \mathrm{NMR}\left(376 \mathrm{MHz}, \mathrm{CDCl}_{3}\right)$
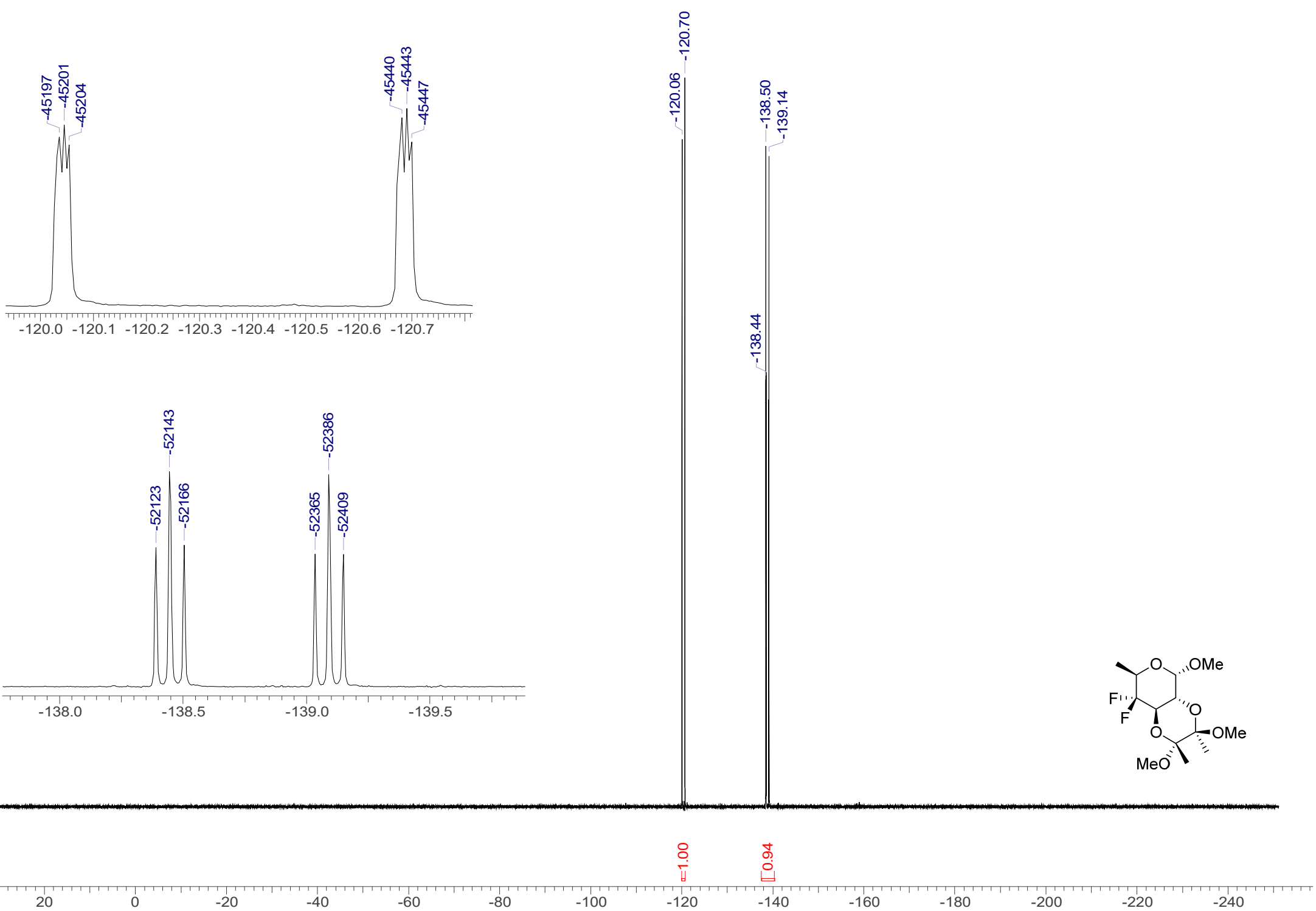
3.42.5. ${ }^{19} \mathrm{~F}\left\{{ }^{1} \mathrm{H}\right\}$ NMR (376 MHz, $\left.\mathrm{CDCl}_{3}\right)$

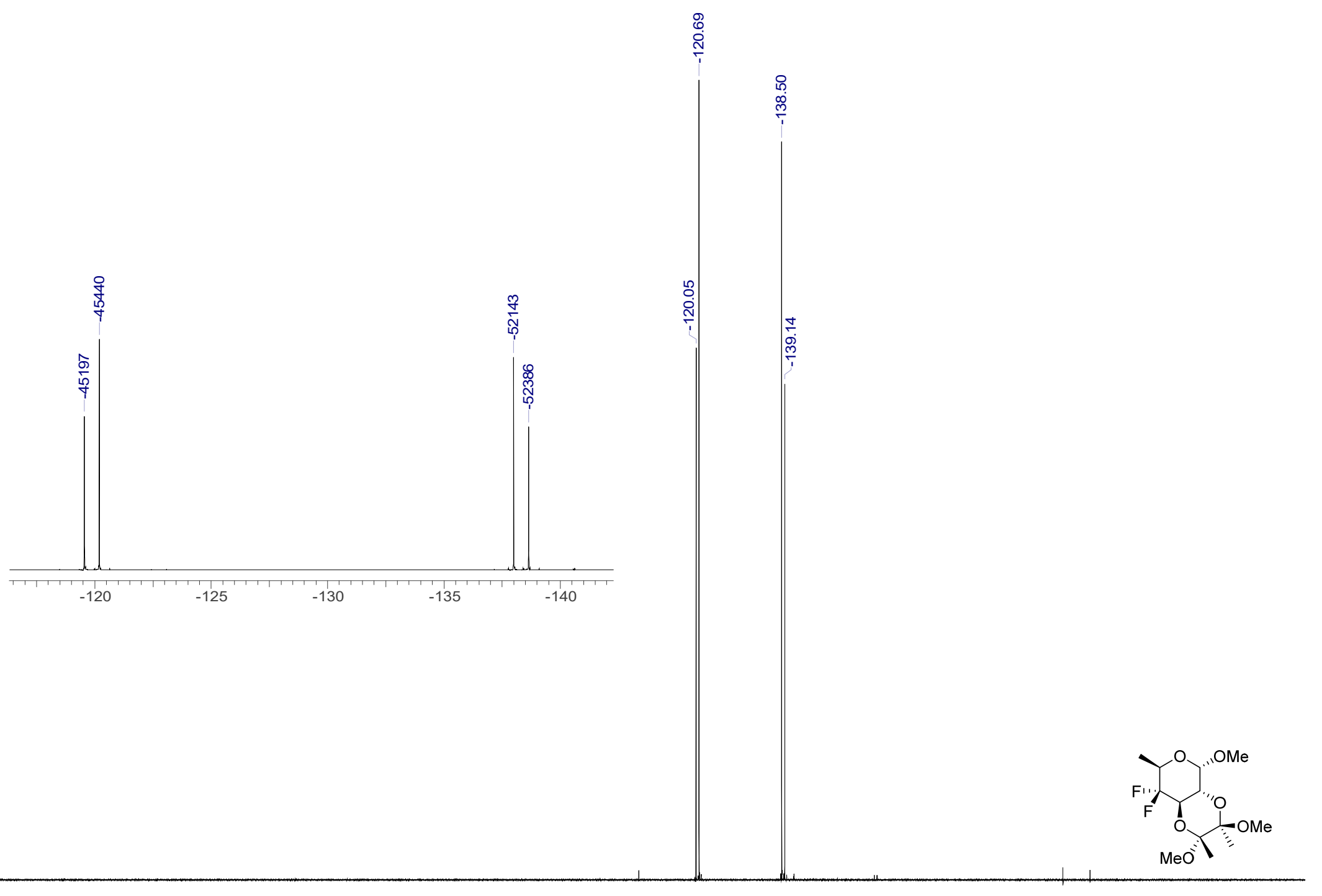

$40 \quad 20$

$20-20$

$-40$

$-60$

$-80 \quad-100$

$-120$

$-160$

$-180$ 
3.43. Methyl 4,6-dideoxy-4-fluoro-2,3-O-((2' $\left.R, 3^{\prime} R\right)-2^{\prime}, 3^{\prime}$-dimethoxybutane-2', 3'-diyl)-a-D-erythro-hex-3-enopyranoside (77) 3.43.1. ${ }^{1} \mathrm{H}$ NMR $\left(400 \mathrm{MHz}, \mathrm{CDCl}_{3}\right)$
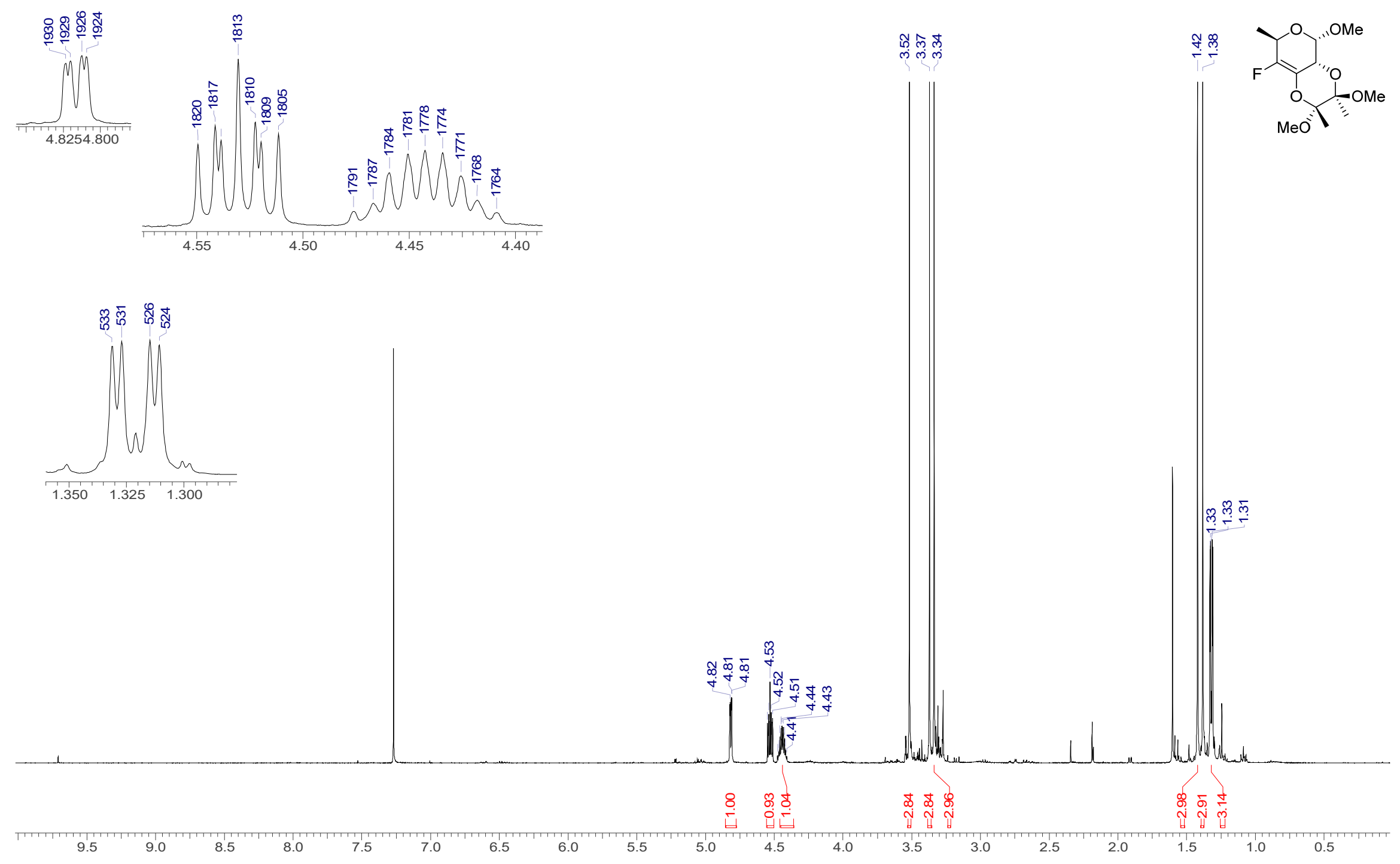
3.43.2. ${ }^{13} \mathrm{C}\left\{{ }^{1} \mathrm{H}\right\} \mathrm{NMR}\left(101 \mathrm{MHz}, \mathrm{CDCl}_{3}\right)$

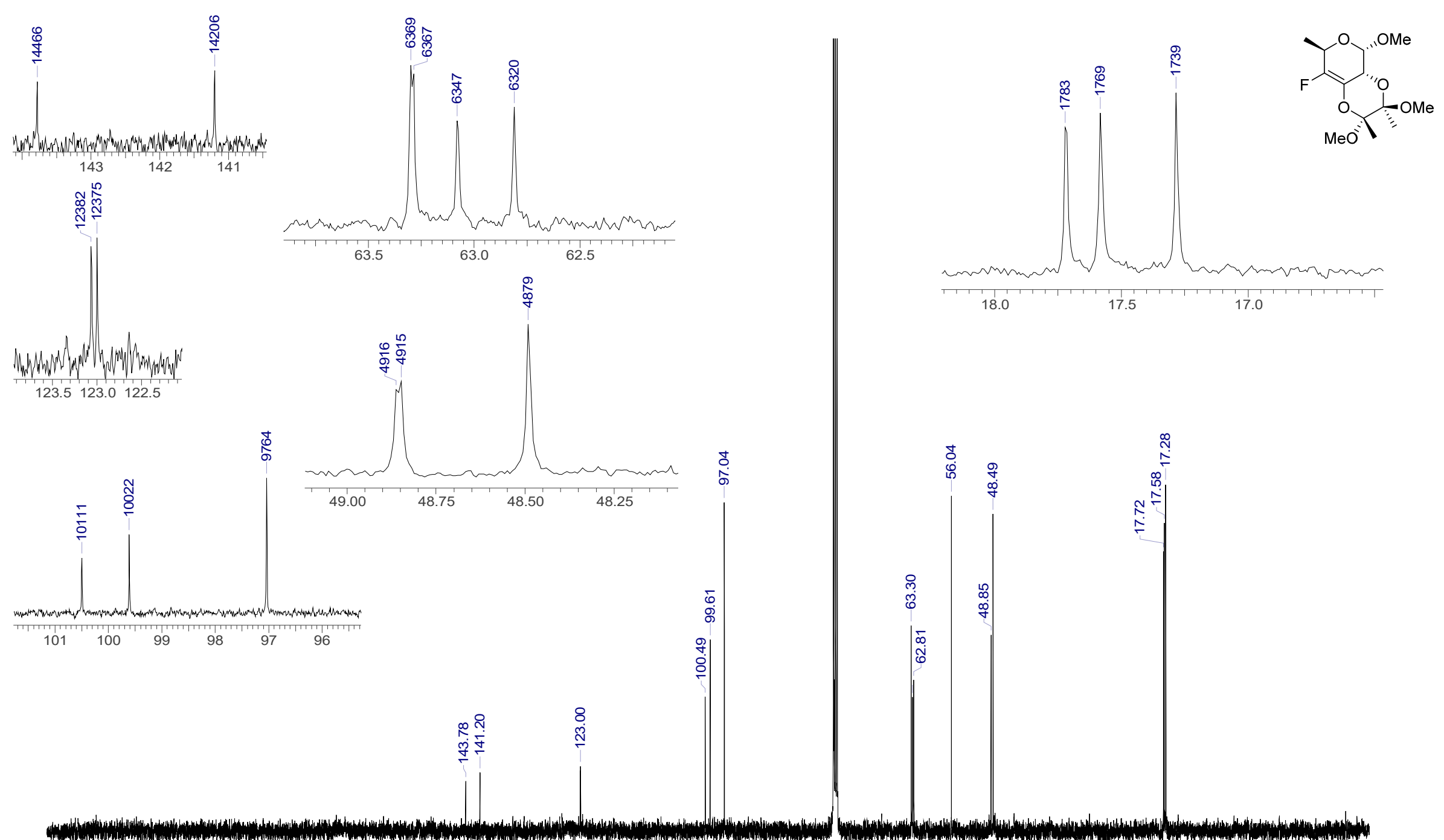

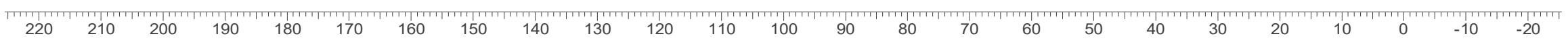


3.43.3. COSY, $\mathrm{HSQC}, \mathrm{HMBC}\left(\mathrm{CDCl}_{3}\right)$

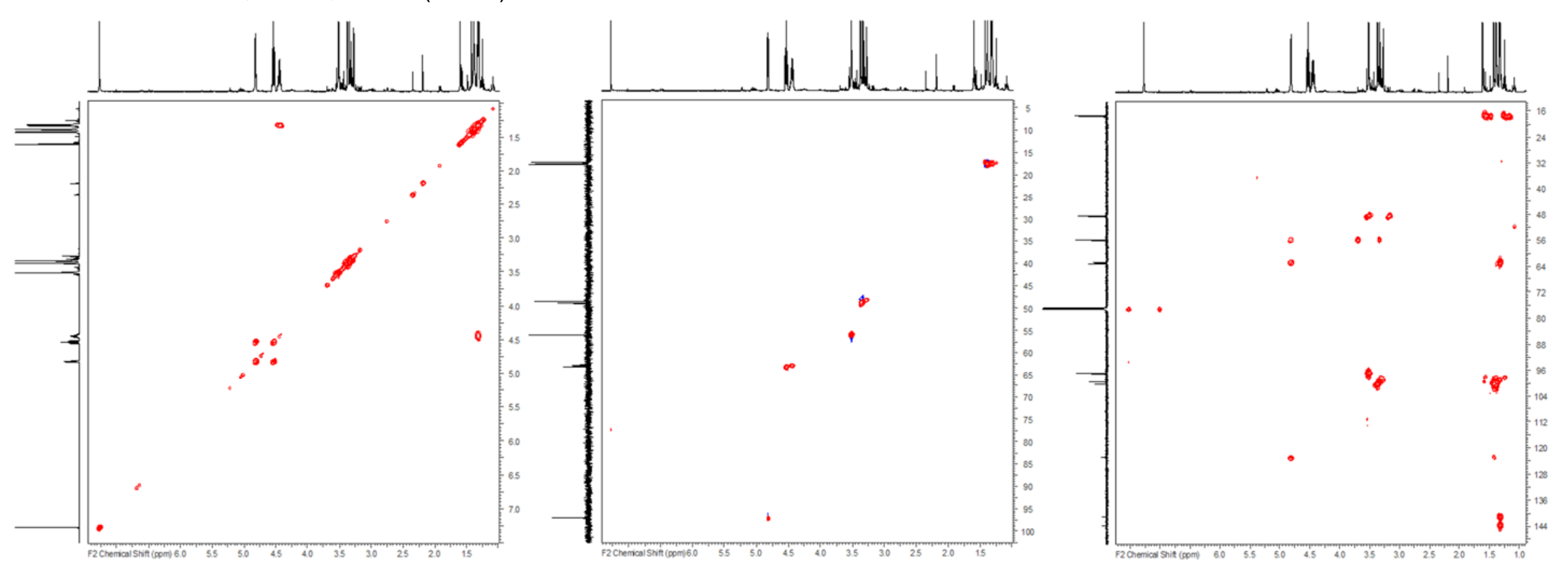


3.43.4. $\left.{ }^{19} \mathrm{~F} \mathrm{NMR} \mathrm{(376} \mathrm{MHz,} \mathrm{CDCl}_{3}\right)$
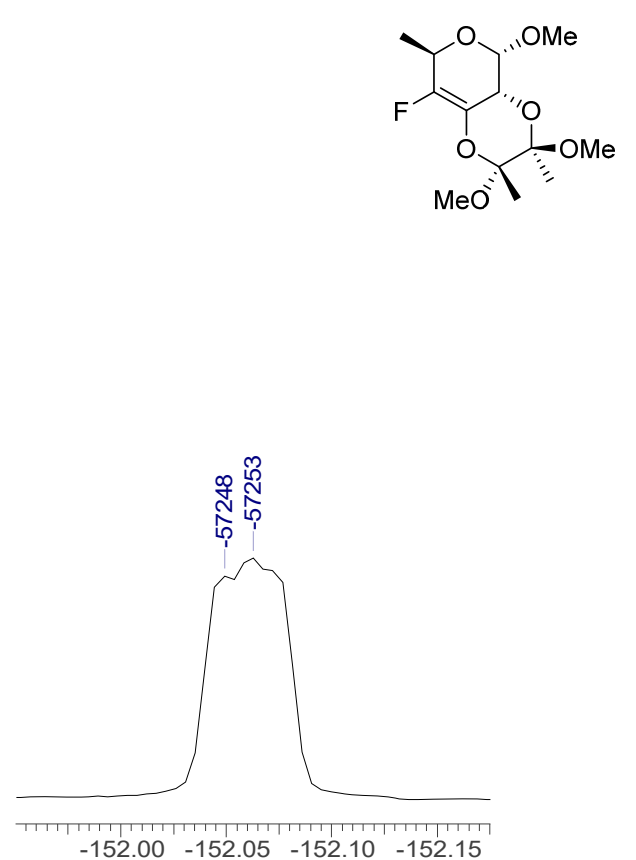
3.43.5. ${ }^{19} \mathrm{~F}\left\{{ }^{1} \mathrm{H}\right\}$ NMR (376 MHz, $\left.\mathrm{CDCl}_{3}\right)$

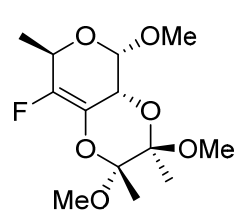


3.44. Methyl 4,6-dideoxy-4-fluoro-2,3-O-((2' $\left.R, 3^{\prime} R\right)-2^{\prime}, 3^{\prime}$-dimethoxybutane-2', 3'-diyl)- $\alpha$-L-threo-hex-4-enopyranoside (78) 3.44.1. ${ }^{1} \mathrm{H} \mathrm{NMR}\left(400 \mathrm{MHz}, \mathrm{CDCl}_{3}\right)$
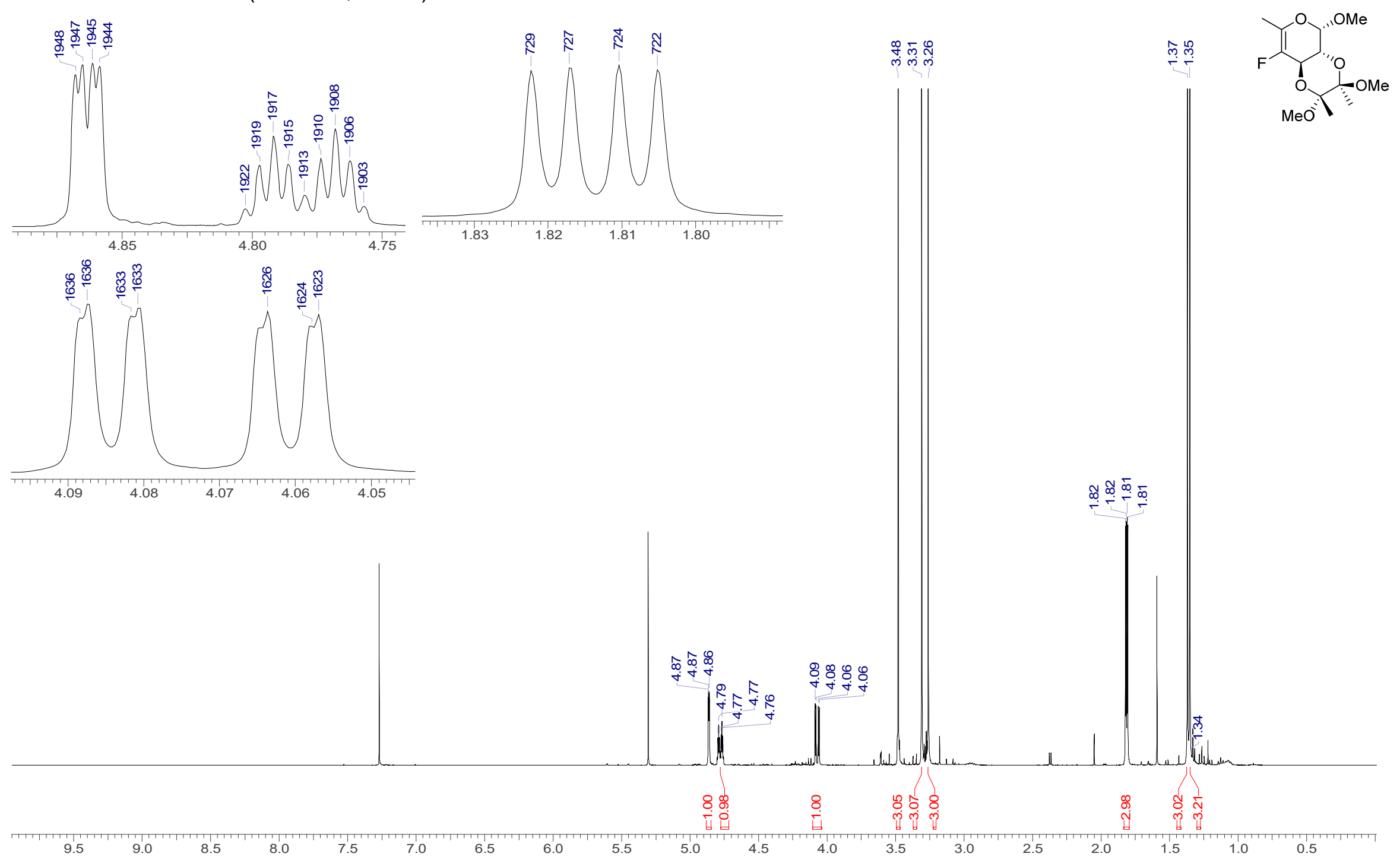
3.44.2. ${ }^{13} \mathrm{C}\left\{{ }^{1} \mathrm{H}\right\}$ NMR $\left(101 \mathrm{MHz}, \mathrm{CDCl}_{3}\right)$
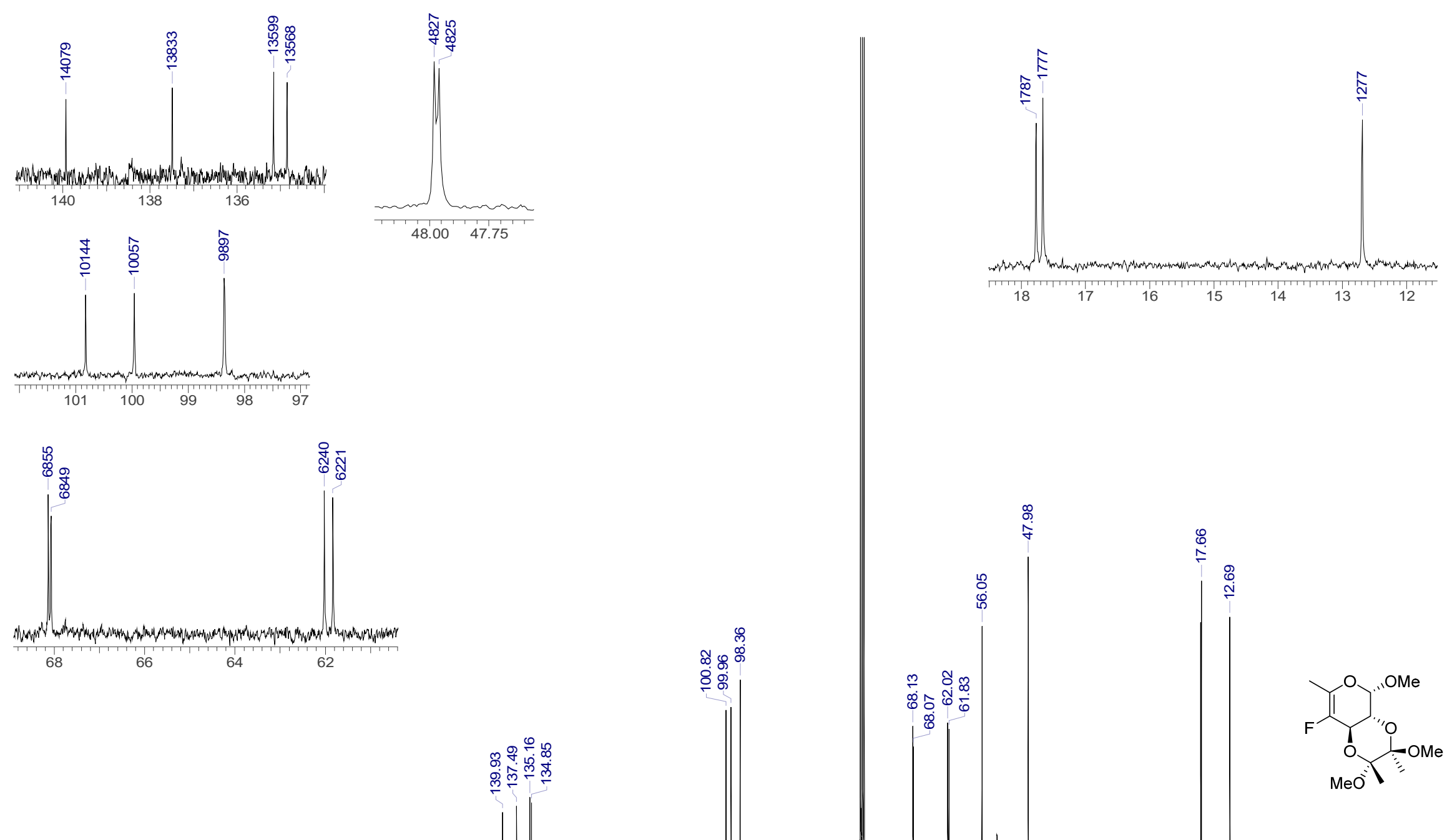

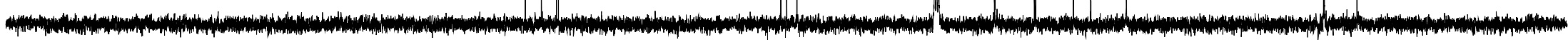

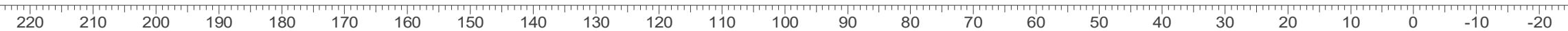


3.44.3. COSY \& HSQC $\left(\mathrm{CDCl}_{3}\right)$

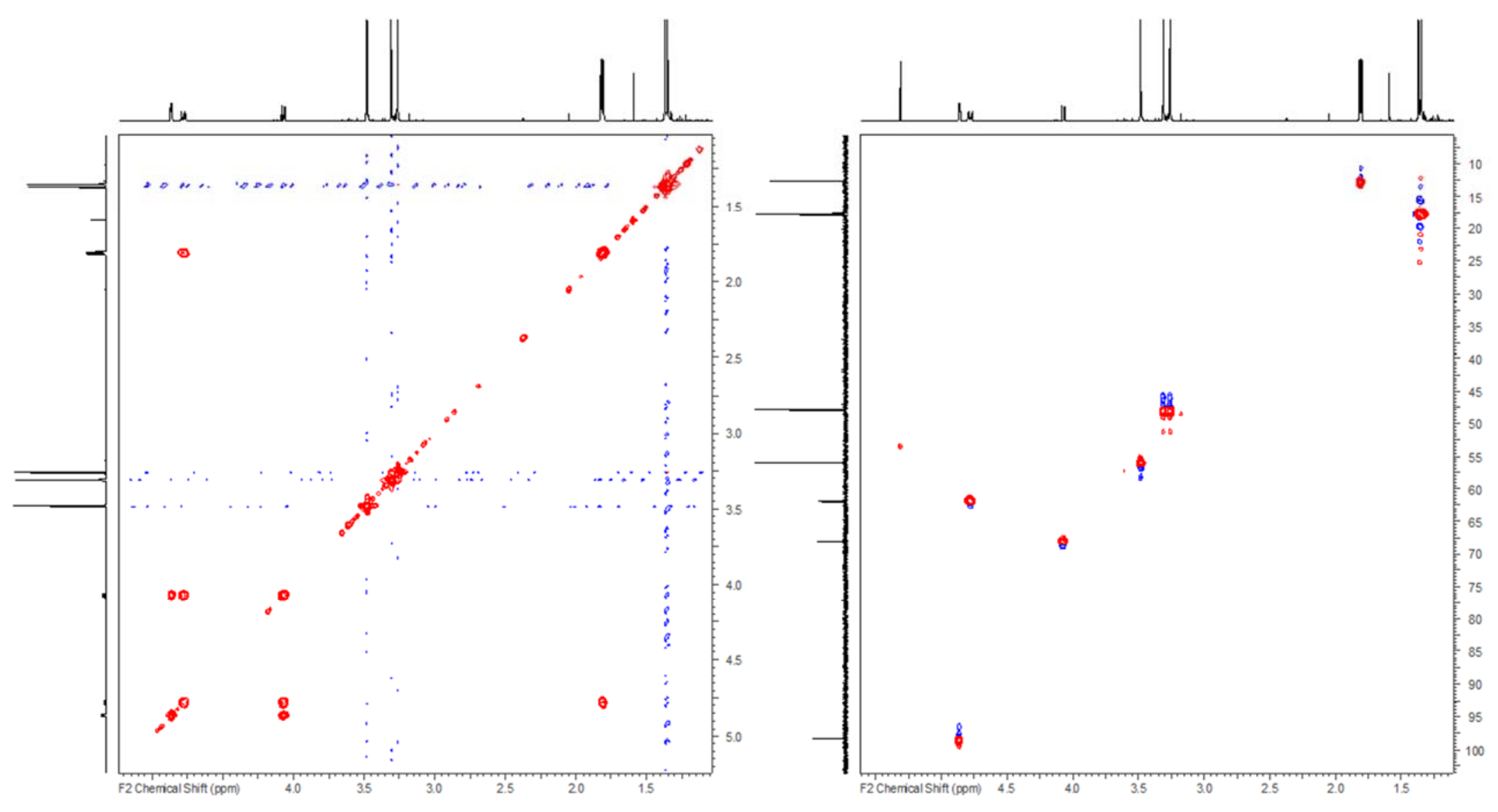


3.44.4. ${ }^{19} \mathrm{~F} \mathrm{NMR}\left(376 \mathrm{MHz}, \mathrm{CDCl}_{3}\right)$

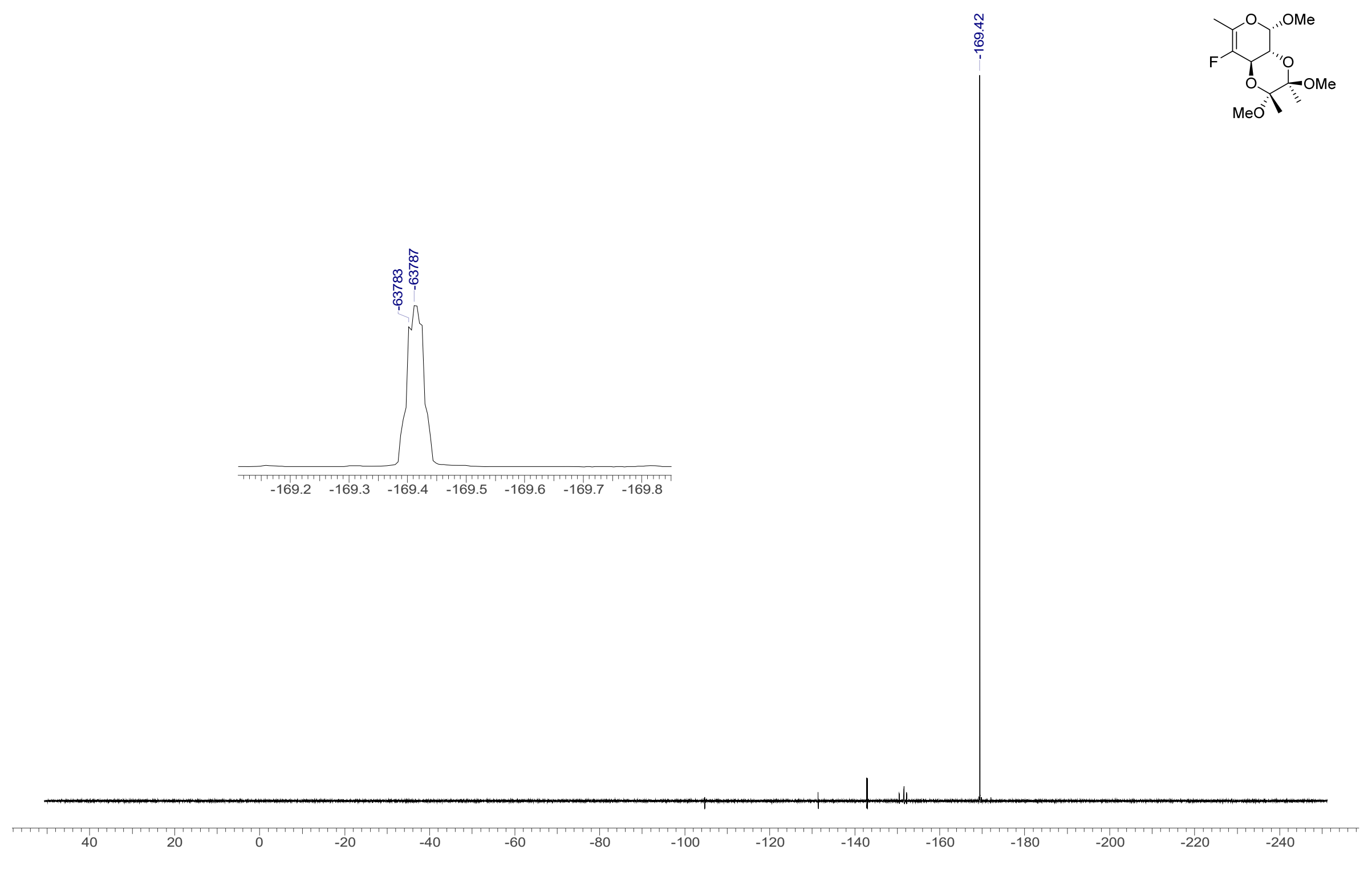


3.44.5. ${ }^{19} \mathrm{~F}\left\{{ }^{1} \mathrm{H}\right\} \mathrm{NMR}\left(376 \mathrm{MHz}, \mathrm{CDCl}_{3}\right)$

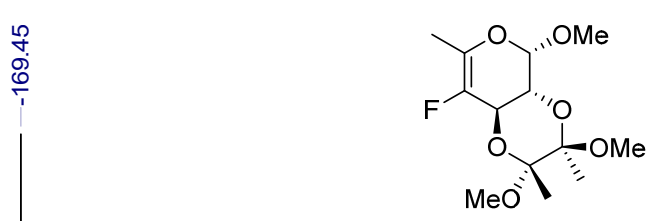


3.45. Methyl 4,6-dideoxy-6,6-difluoro-2,3-O-((2'R,3'R)-2',3'-dimethoxybutane-2',3'-diyl)- $\alpha$-D-xylo-hexopyranoside (80) 3.45.1. ${ }^{1} \mathrm{H} \mathrm{NMR}\left(400 \mathrm{MHz}, \mathrm{CDCl}_{3}\right)$

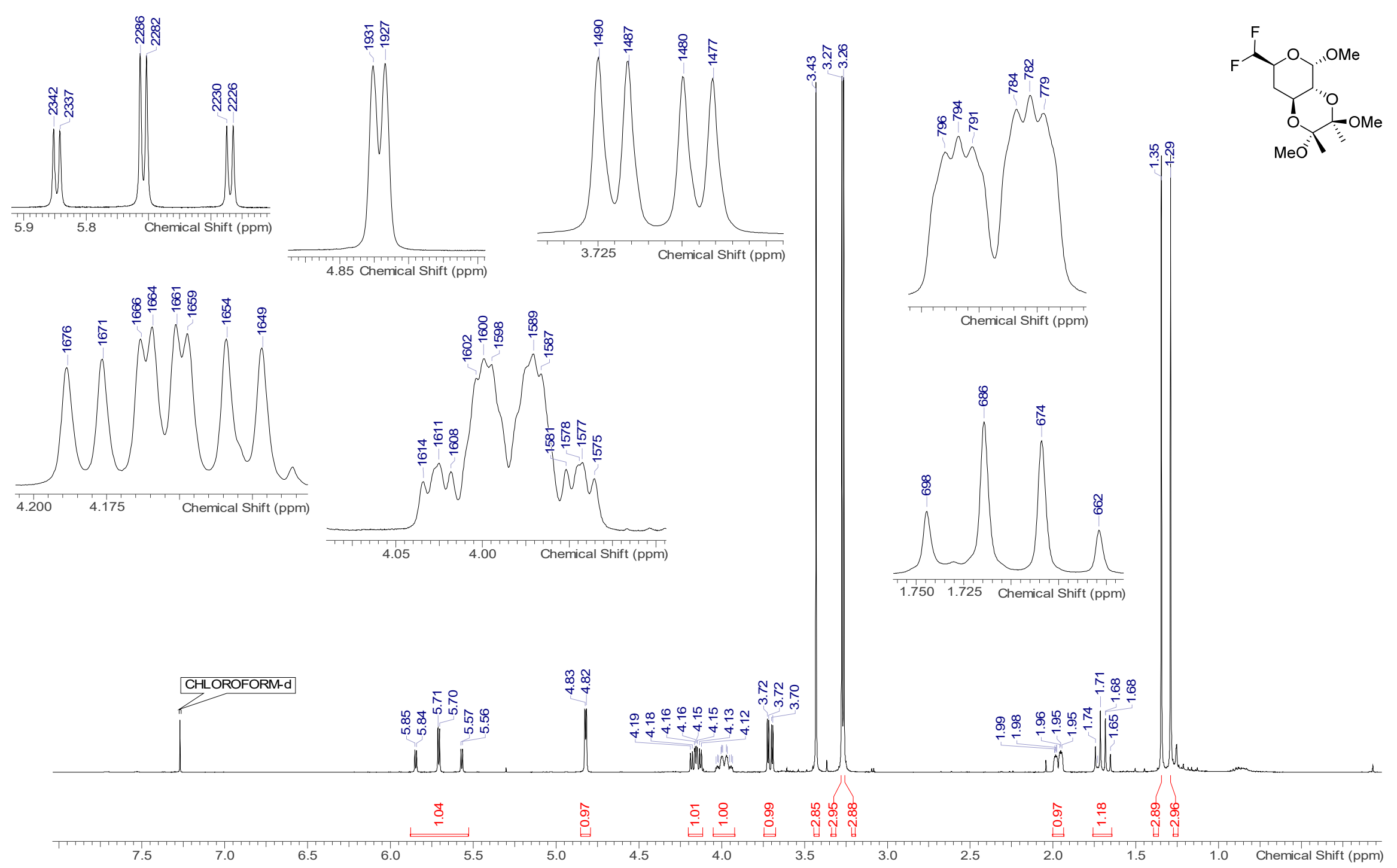


3.45.2. ${ }^{13} \mathrm{C}\left\{{ }^{1} \mathrm{H}\right\}$ NMR $\left(101 \mathrm{MHz}, \mathrm{CDCl}_{3}\right)$
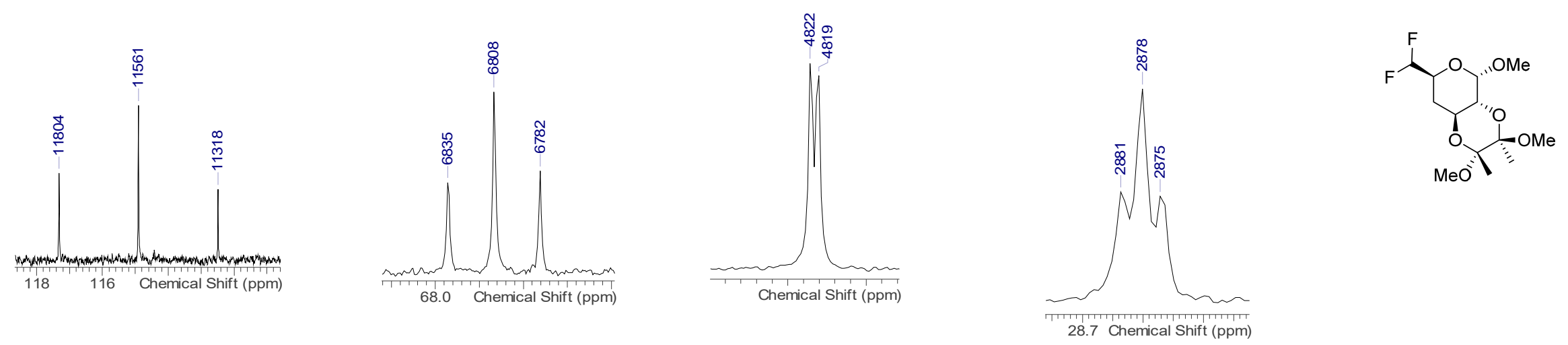

CHLOROFORM-d
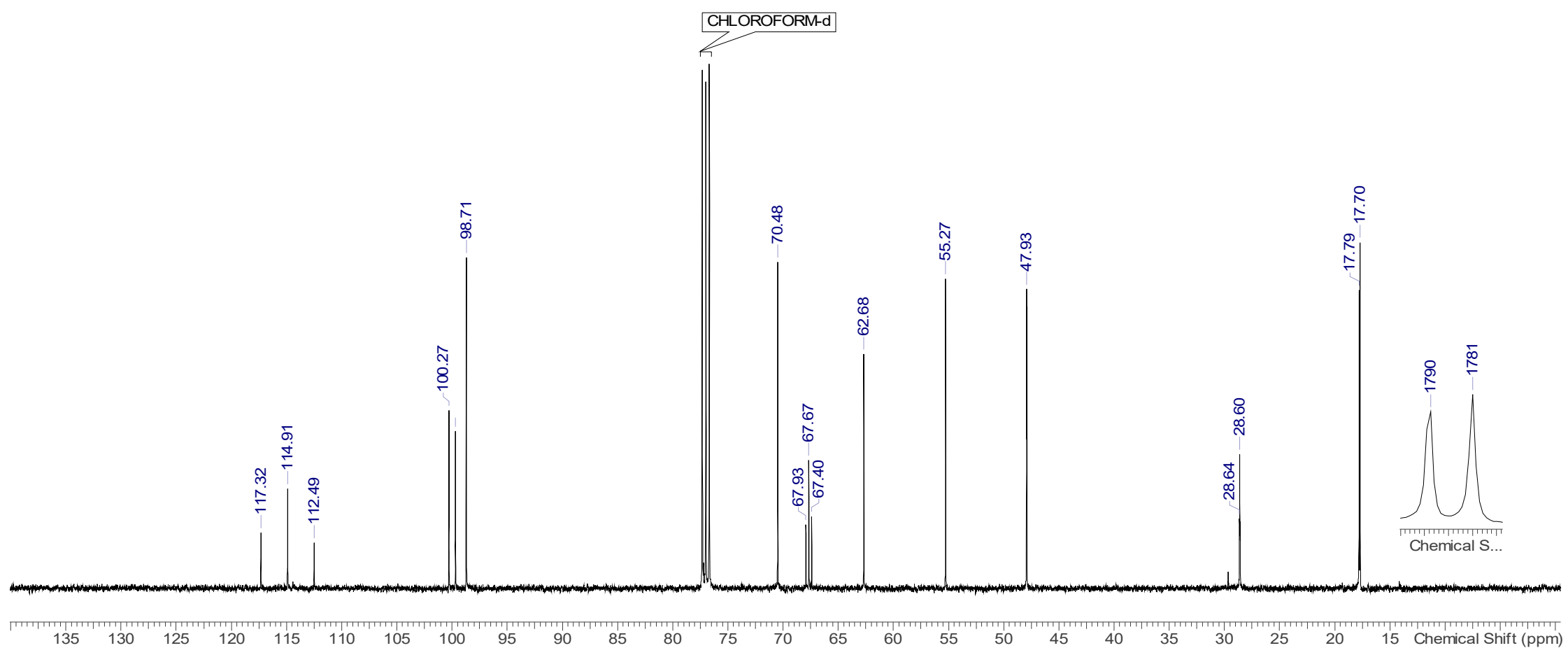
3.45.3. COSY, $\mathrm{HSQC} \& \mathrm{HMBC}\left(\mathrm{CDCl}_{3}\right)$

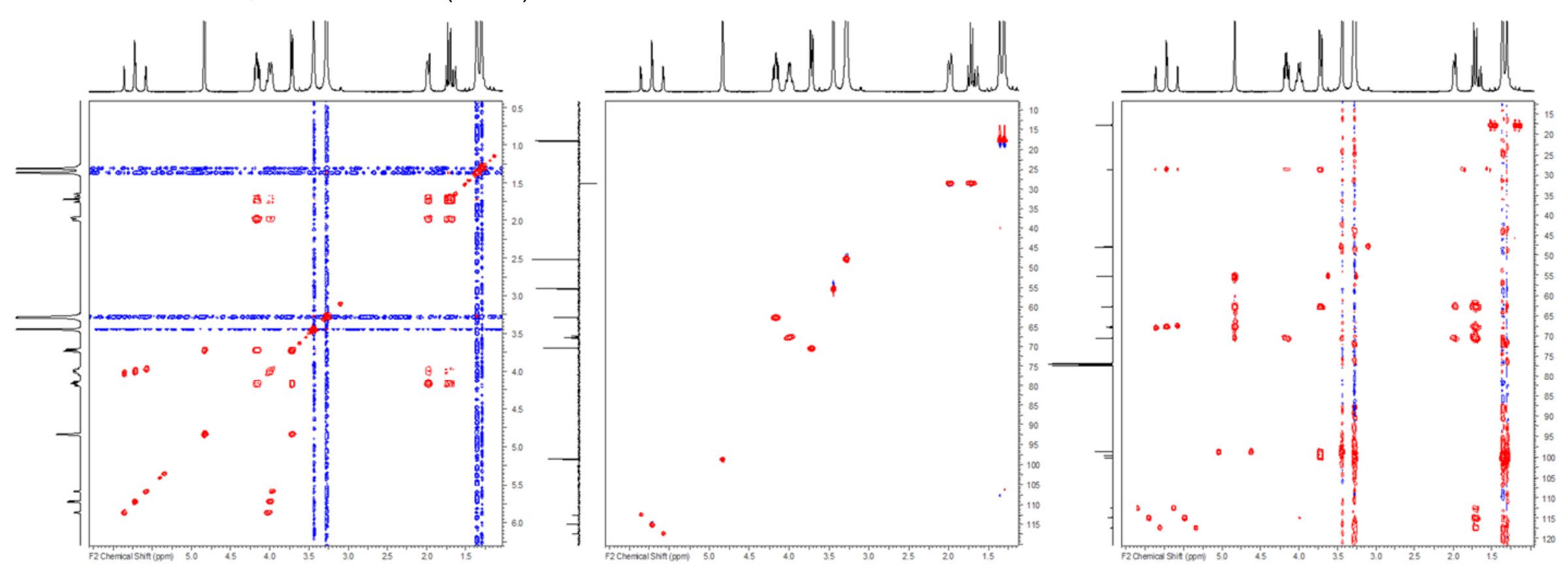


3.45.4. ${ }^{19} \mathrm{~F}$ NMR (376 MHz, $\mathrm{CDCl}_{3}$ )

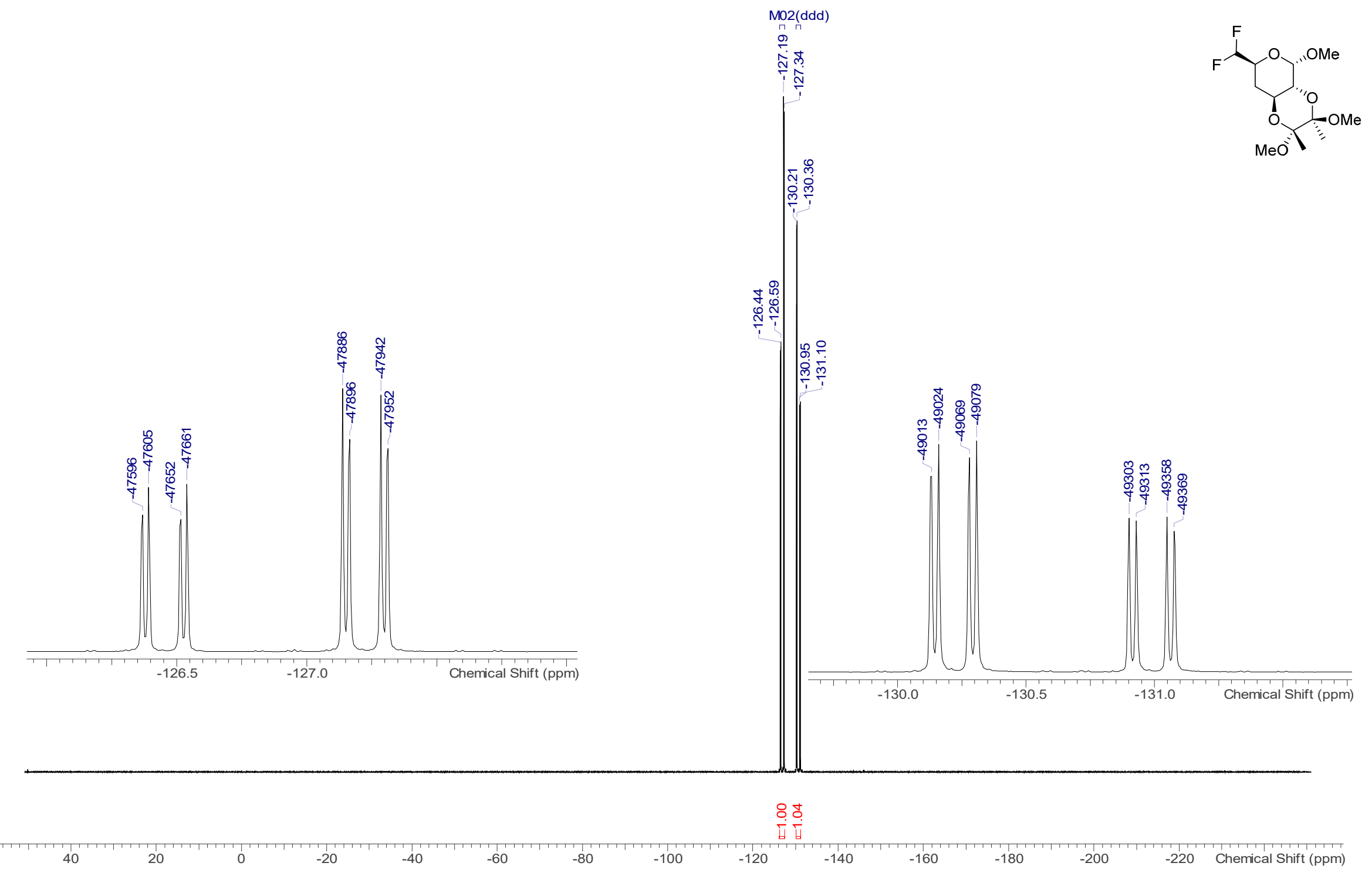


3.45.5. ${ }^{19} \mathrm{~F}\left\{{ }^{1} \mathrm{H}\right\}$ NMR (376 MHz, $\left.\mathrm{CDCl}_{3}\right)$

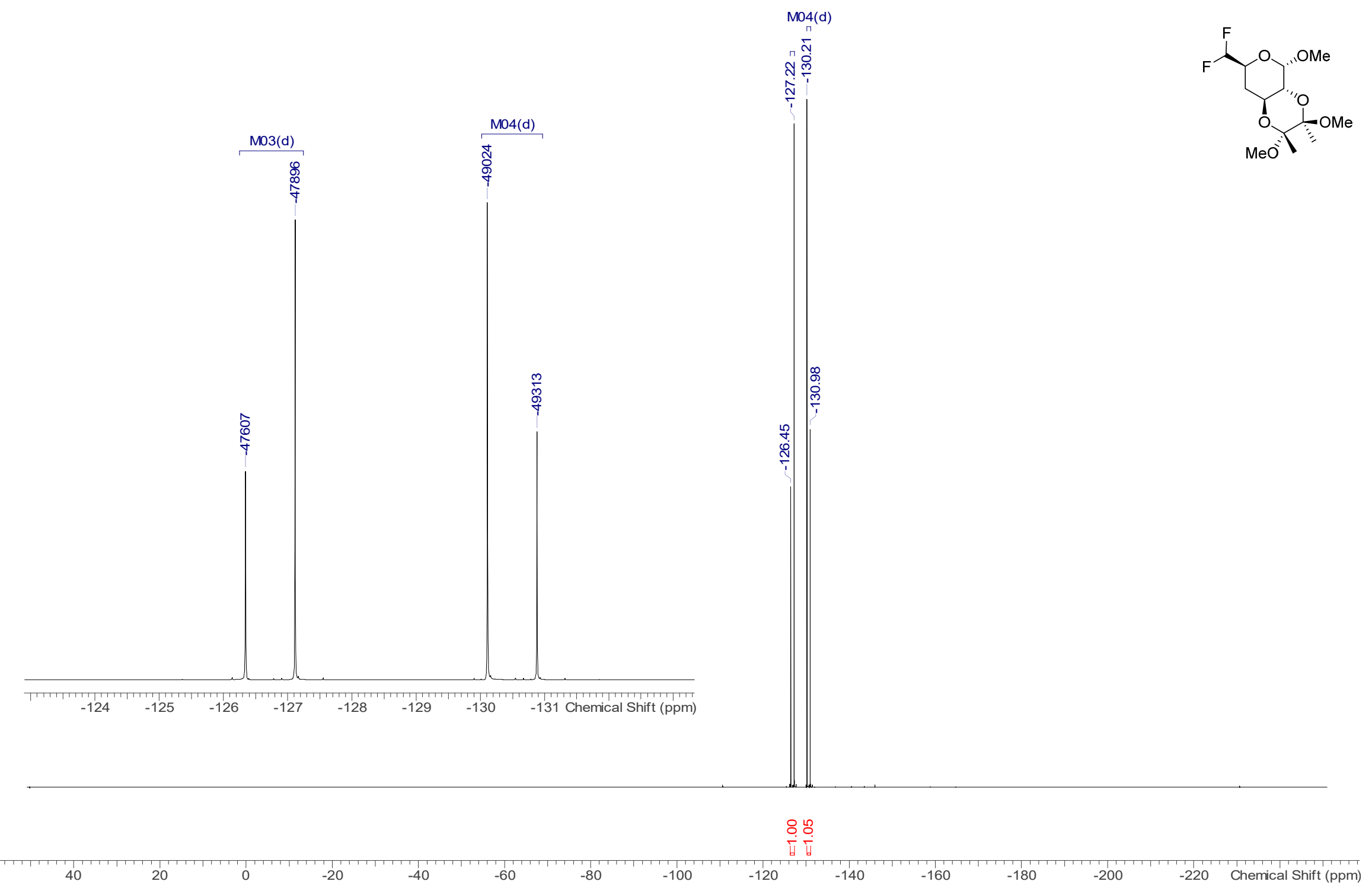




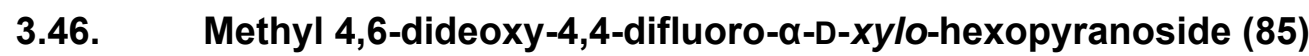

3.46.1. ${ }^{1} \mathrm{H}$ NMR $\left(500 \mathrm{MHz}, \mathrm{CDCl}_{3}\right)$
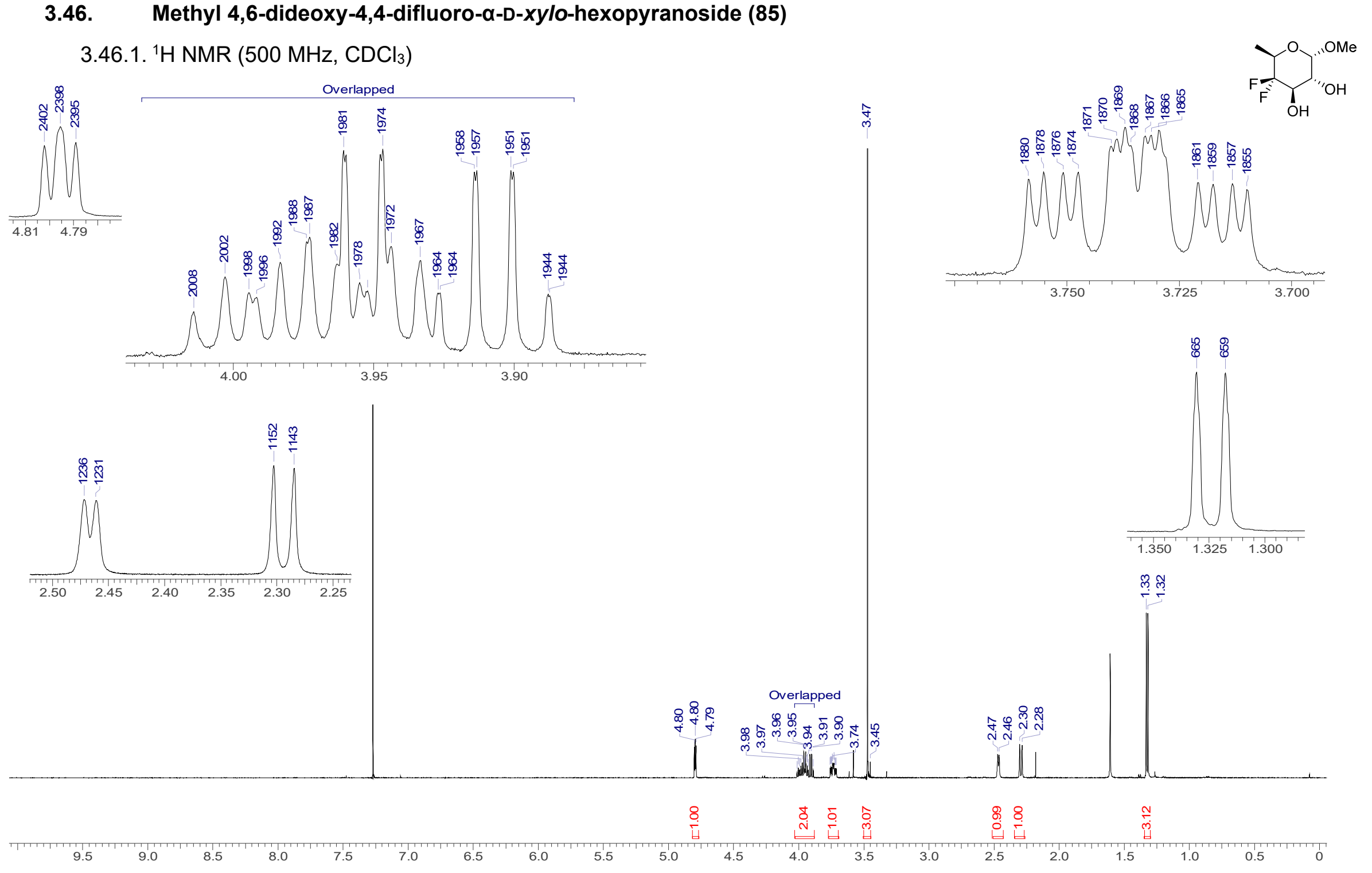
3.46.2. ${ }^{1} \mathrm{H}\left\{{ }^{19} \mathrm{~F}\right\}$ NMR (500 MHz, $\left.\mathrm{CDCl}_{3}\right)$

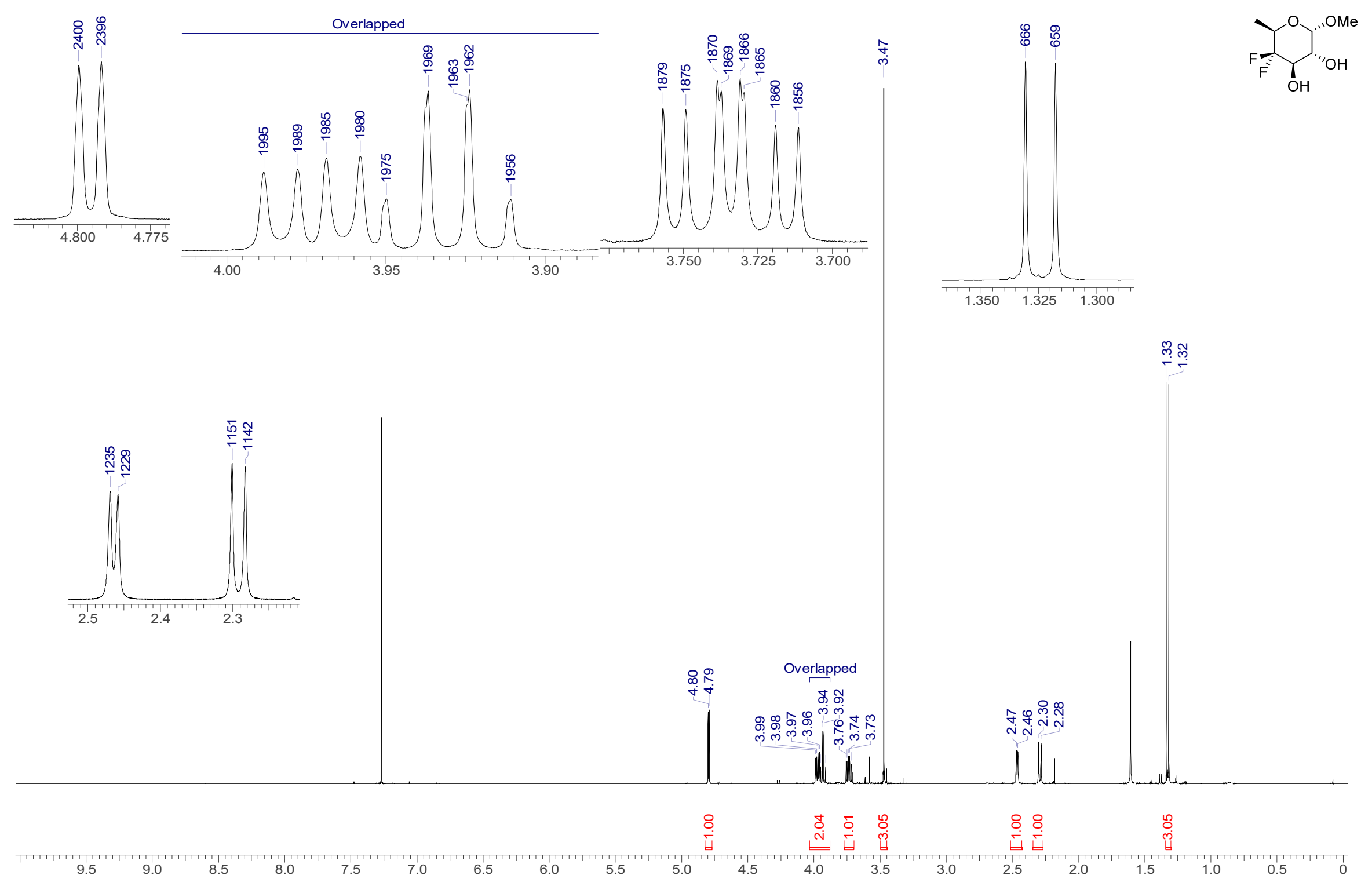


3.46.3. ${ }^{13} \mathrm{C}\left\{{ }^{1} \mathrm{H}\right\} \mathrm{NMR}\left(126 \mathrm{MHz}, \mathrm{CDCl}_{3}\right)$

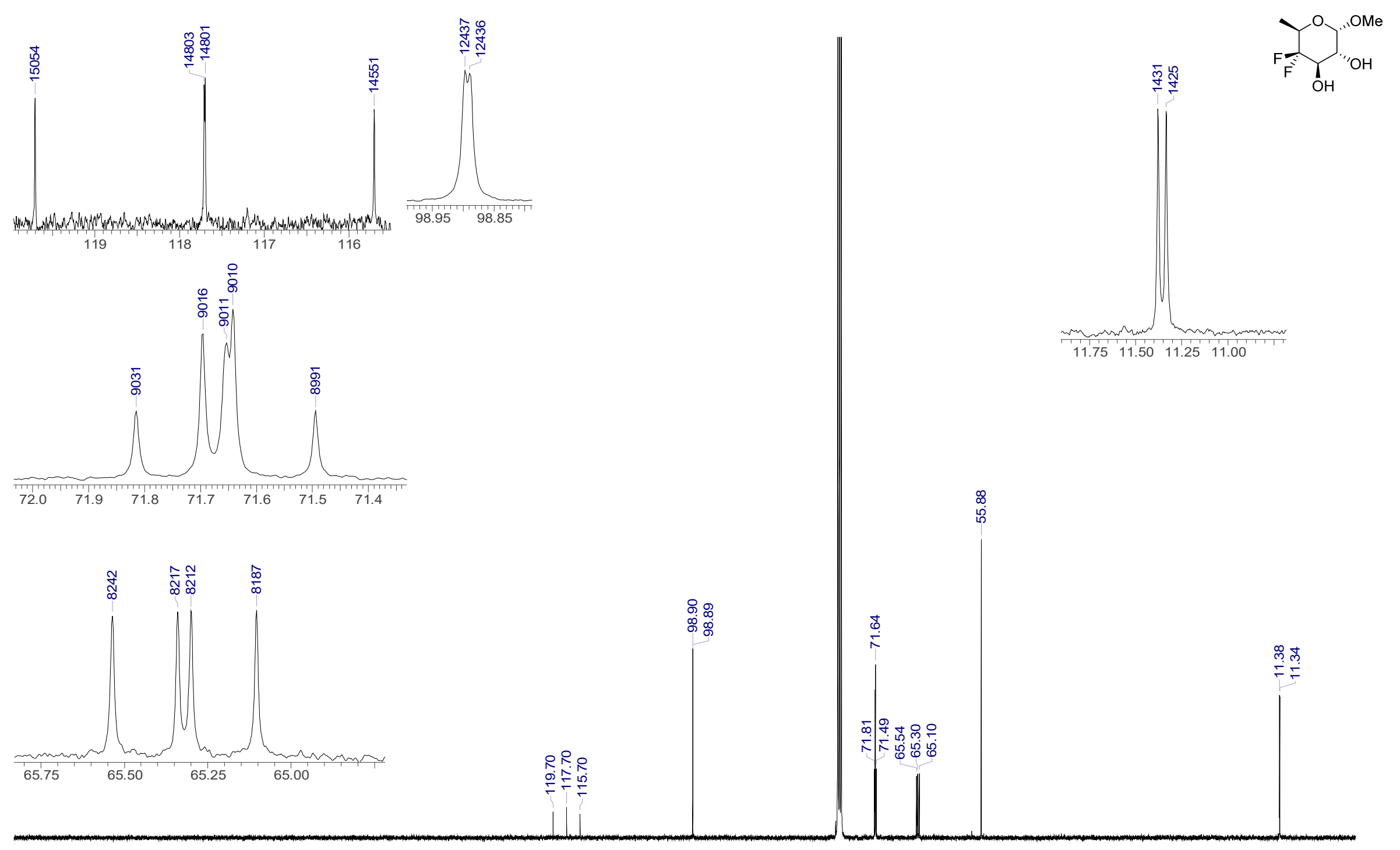

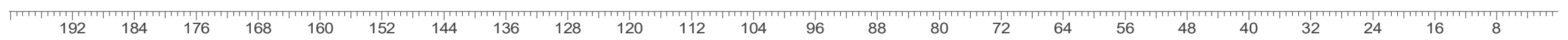


3.46.4. COSY, HSQC-DEPT, $\mathrm{HMBC}\left(\mathrm{CDCl}_{3}\right)$

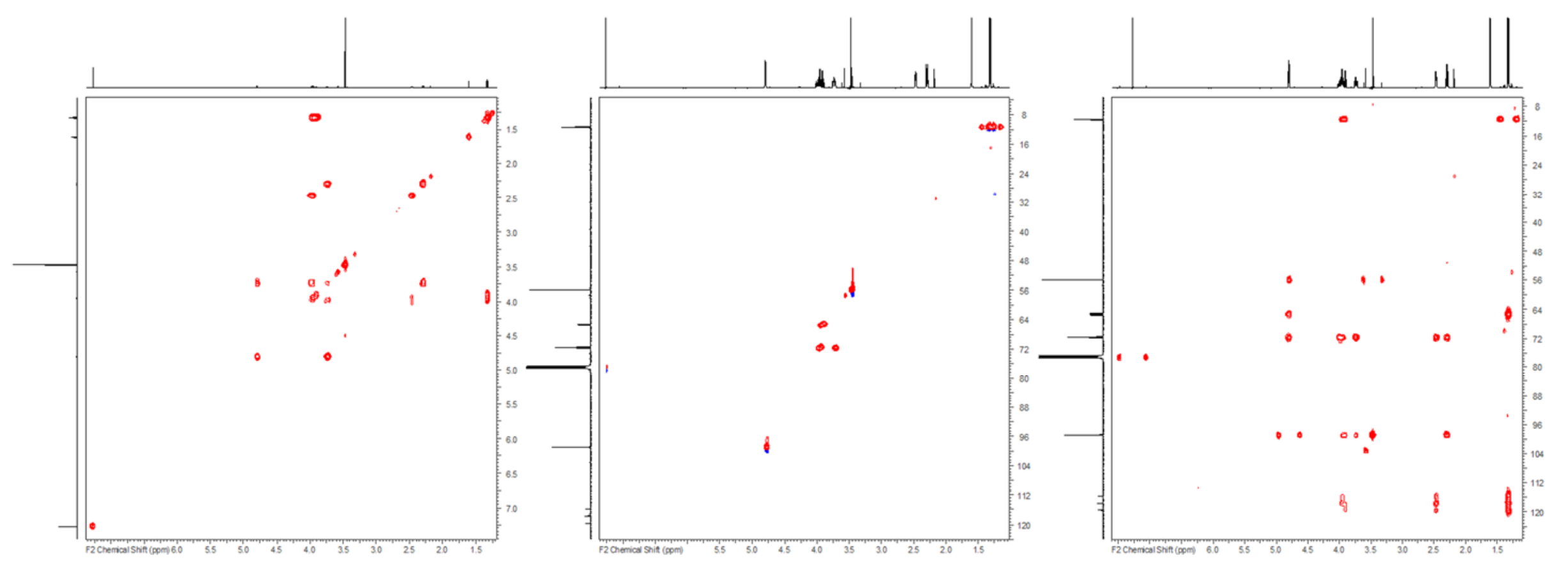


3.46.5. ${ }^{19} \mathrm{~F} \mathrm{NMR}\left(470 \mathrm{MHz}, \mathrm{CDCl}_{3}\right)$

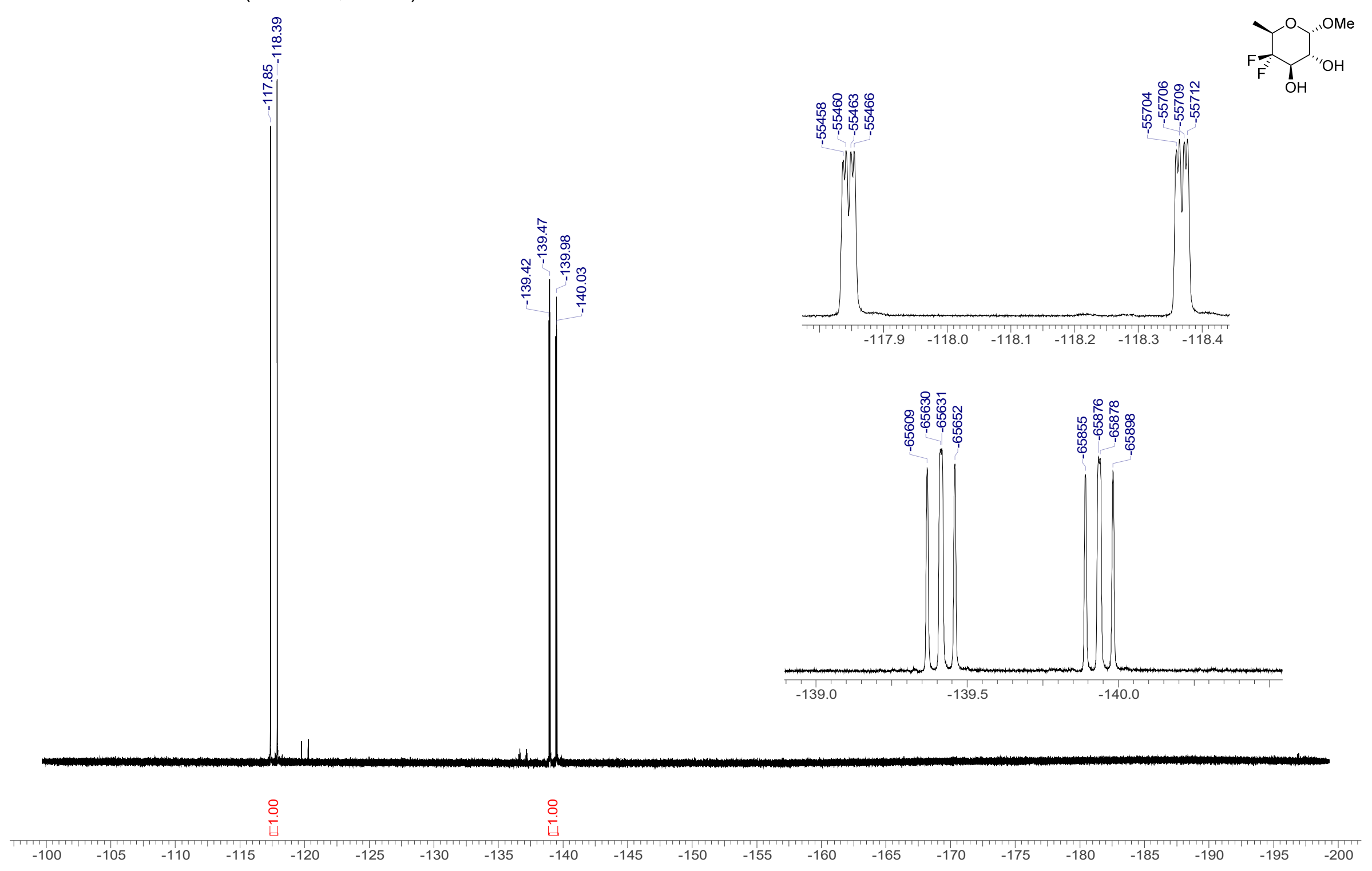


3.46.6. ${ }^{19} \mathrm{~F}\left({ }^{1} \mathrm{H}\right) \mathrm{NMR}\left(470 \mathrm{MHz}, \mathrm{CDCl}_{3}\right)$

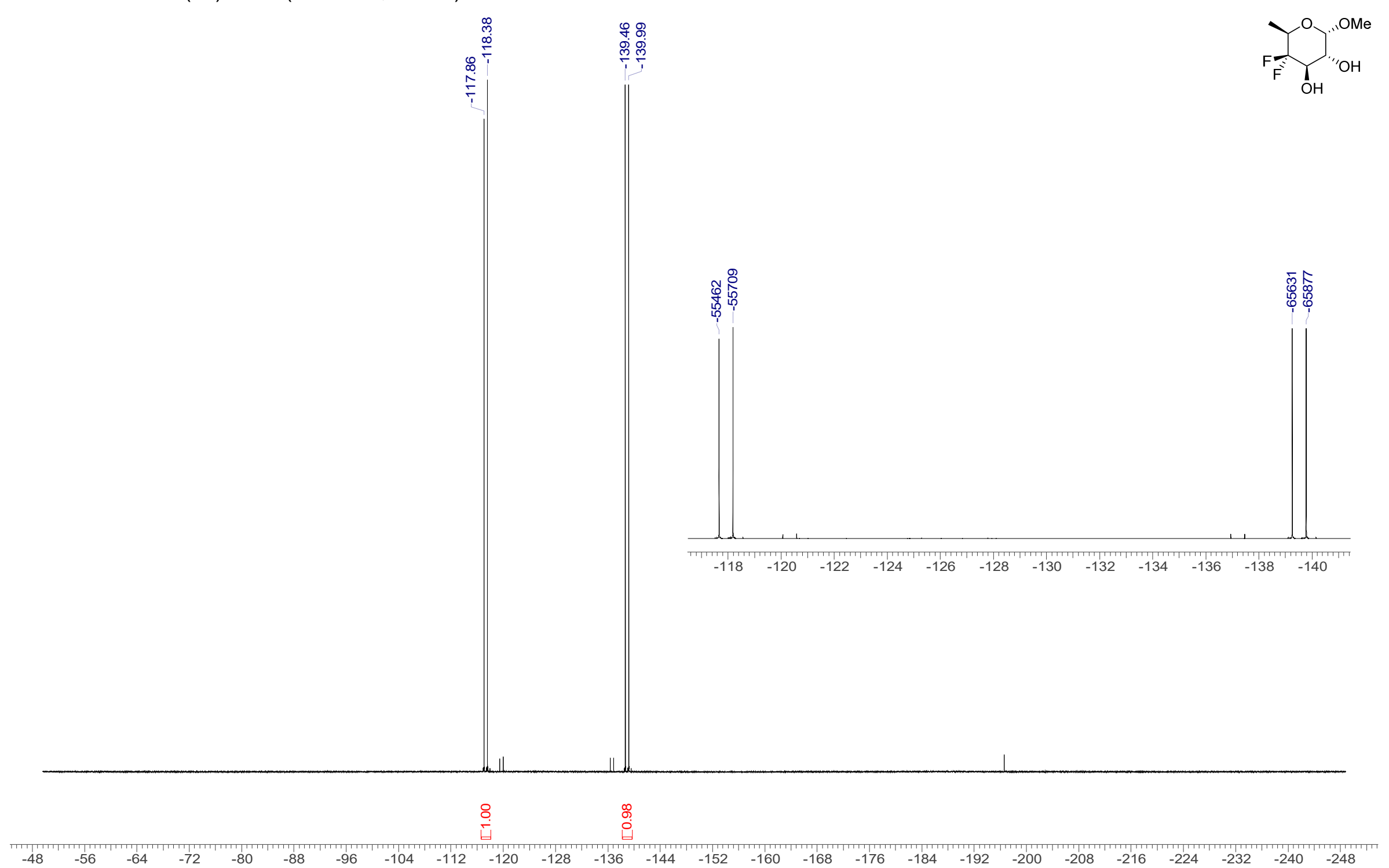




\subsection{4,6-Dideoxy-D-xylo-hexopyranose (86)}

3.47.1. ${ }^{1} \mathrm{H}$ NMR $\left(500 \mathrm{MHz}, \mathrm{CD}_{3} \mathrm{OD}\right)$

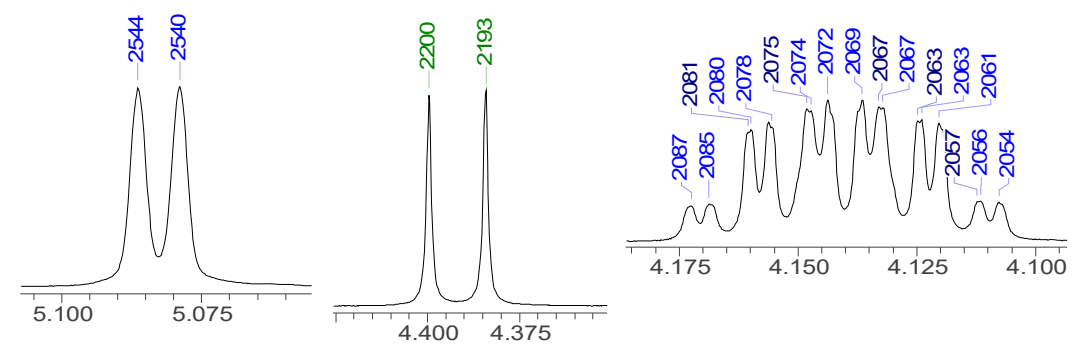

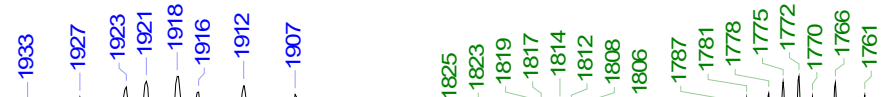
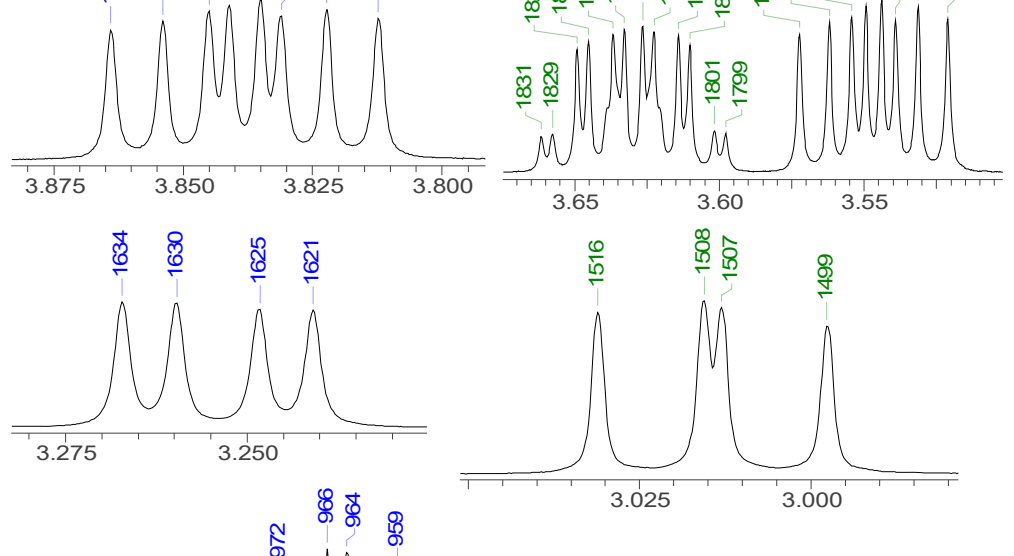

象草 8

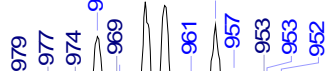

1.95

1.90

1.85
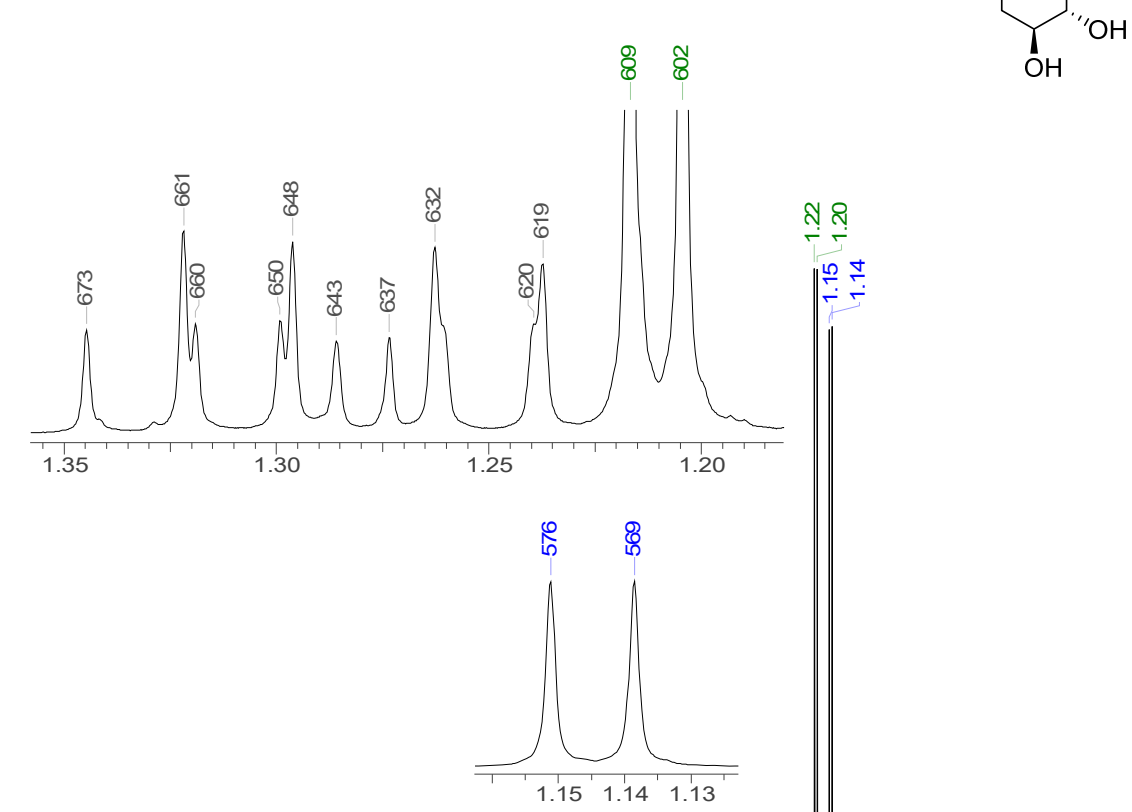

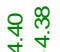

is
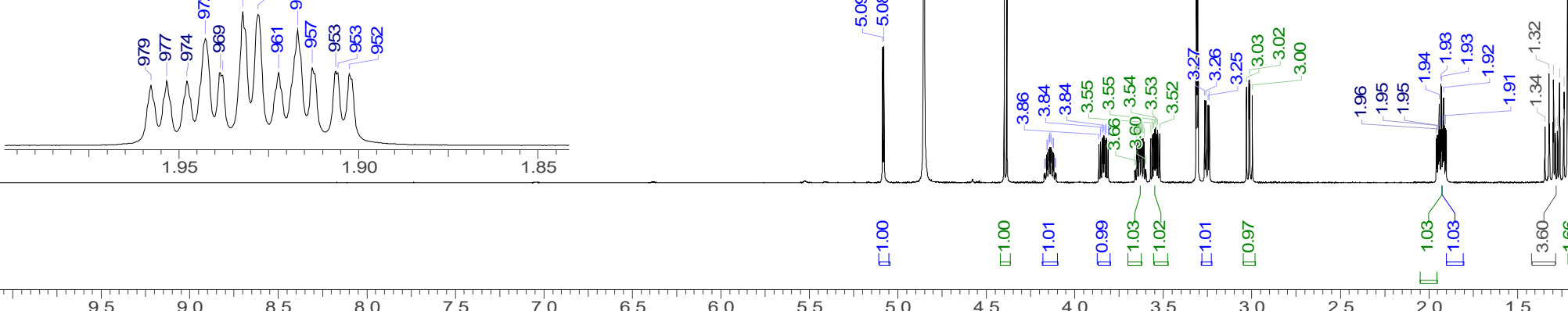

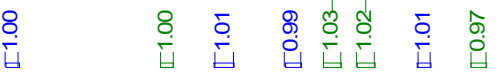

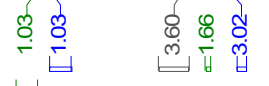

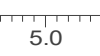

4.5

0
$11+11111111$

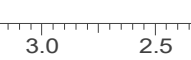

\begin{tabular}{llllll}
\hline & \\
2.0 & 1.5 & 1.0 & 0.5 & 0
\end{tabular} 
3.47.2. ${ }^{13} \mathrm{C}\left\{{ }^{1} \mathrm{H}\right\}$ NMR $\left(101 \mathrm{MHz}, \mathrm{CD}_{3} \mathrm{OD}\right)$
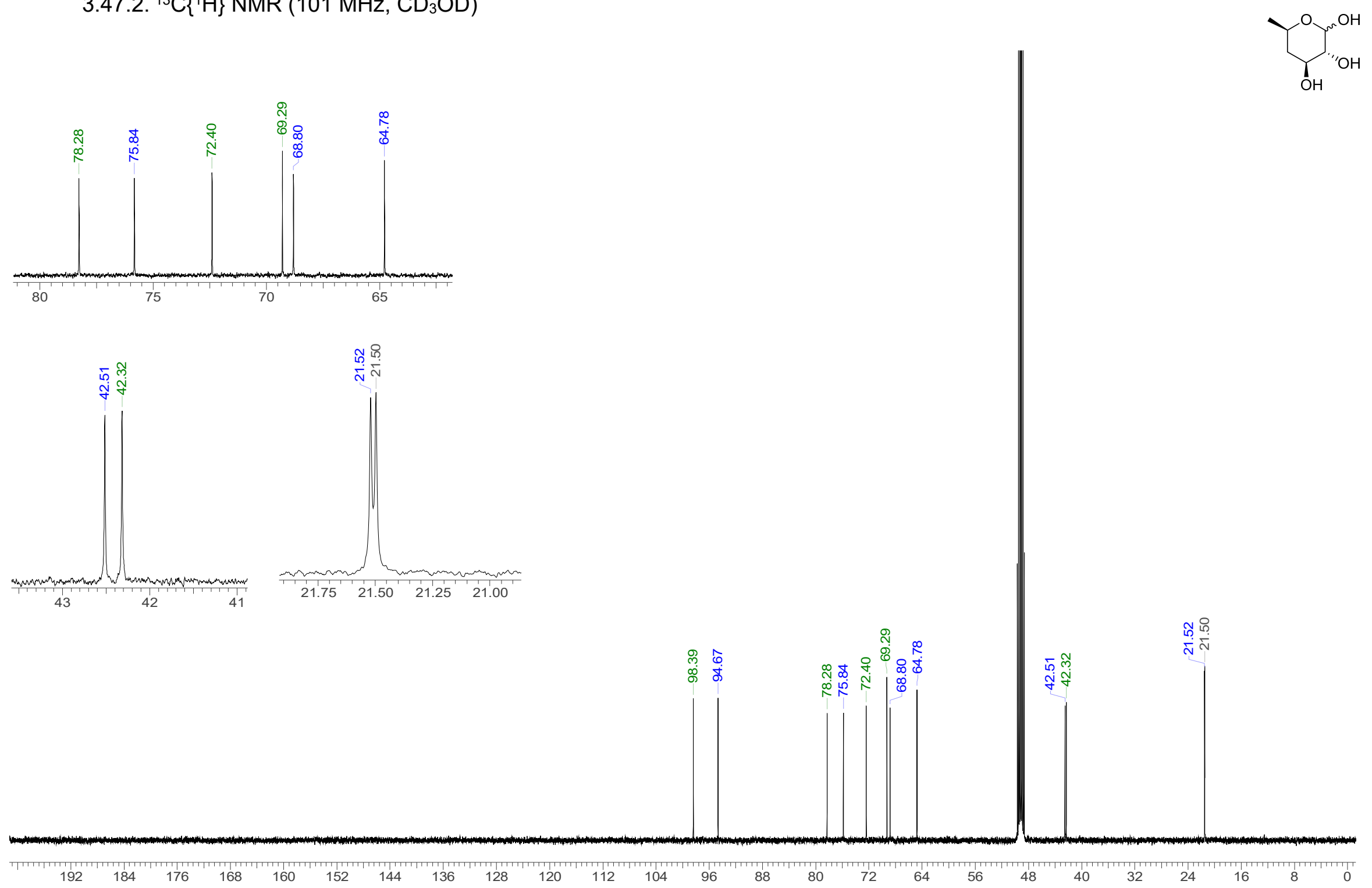


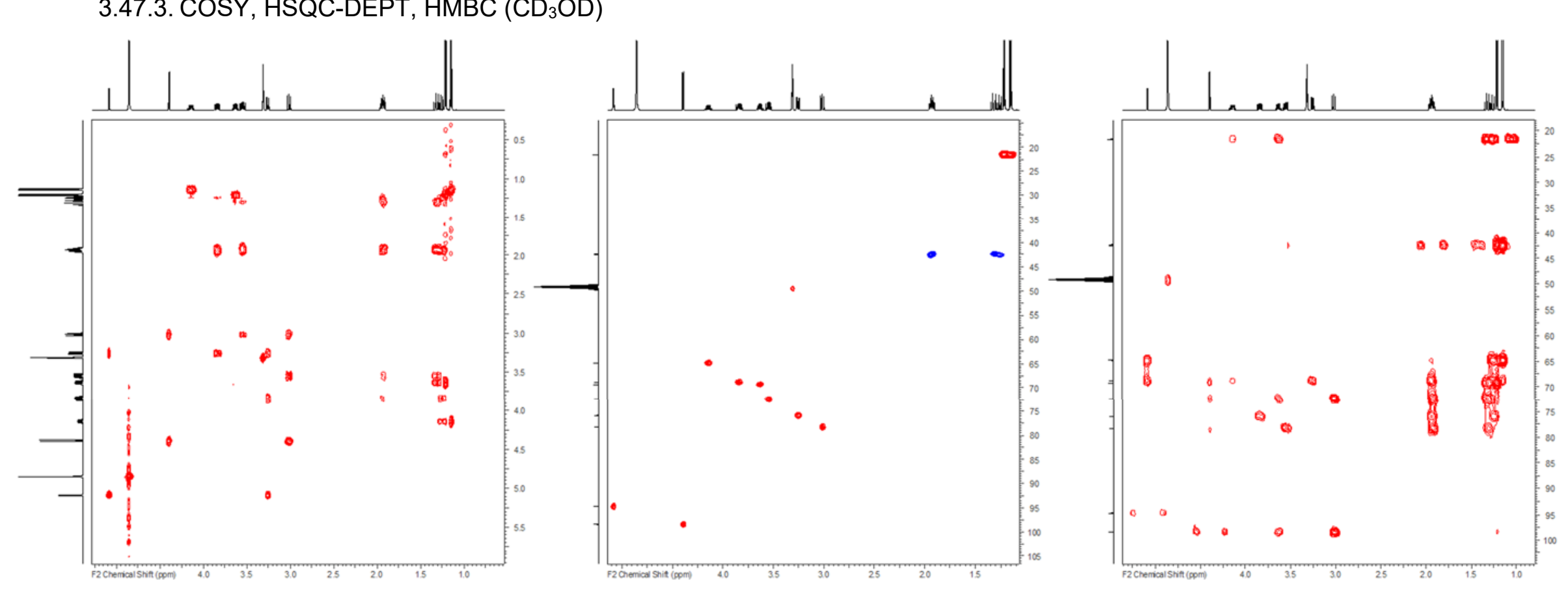


3.48. 1,2,3-Tri-O-acetyl-4,6-dideoxy-D-xylo-hexopyranoside (94)

3.48.1. ${ }^{1} \mathrm{H}$ NMR $\left(400 \mathrm{MHz}, \mathrm{CDCl}_{3}\right)$
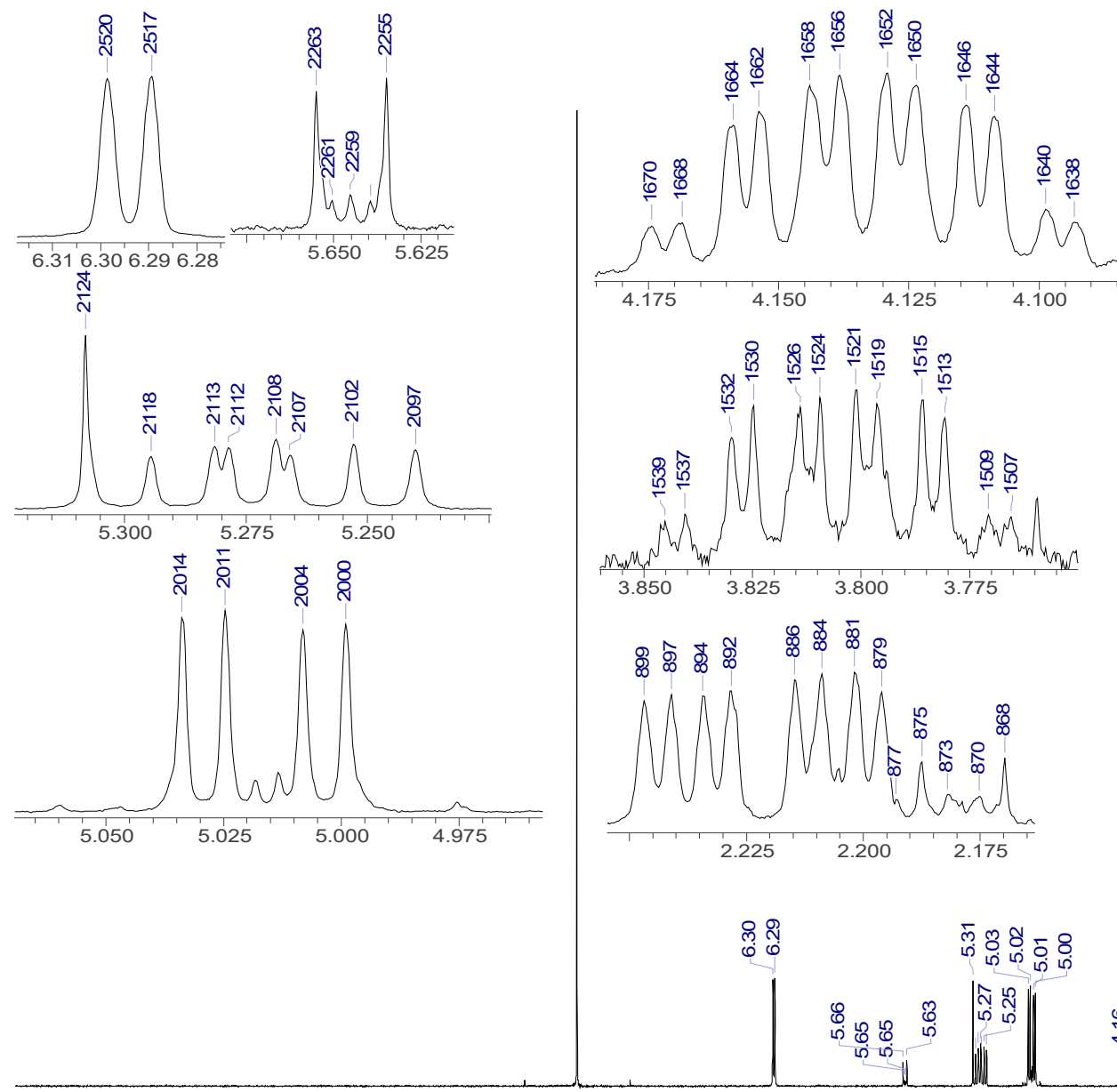
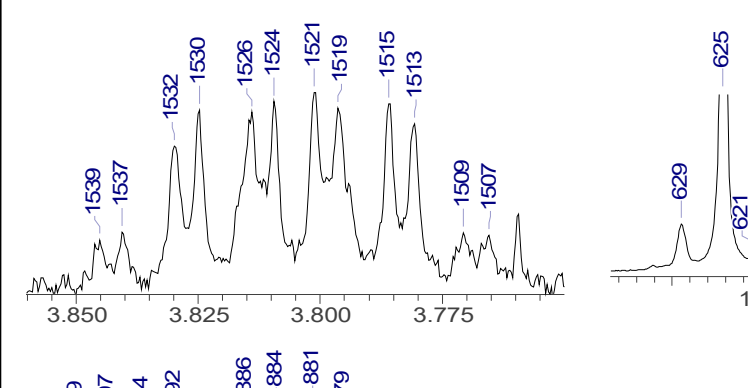

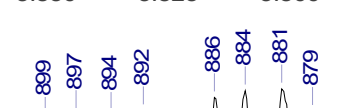
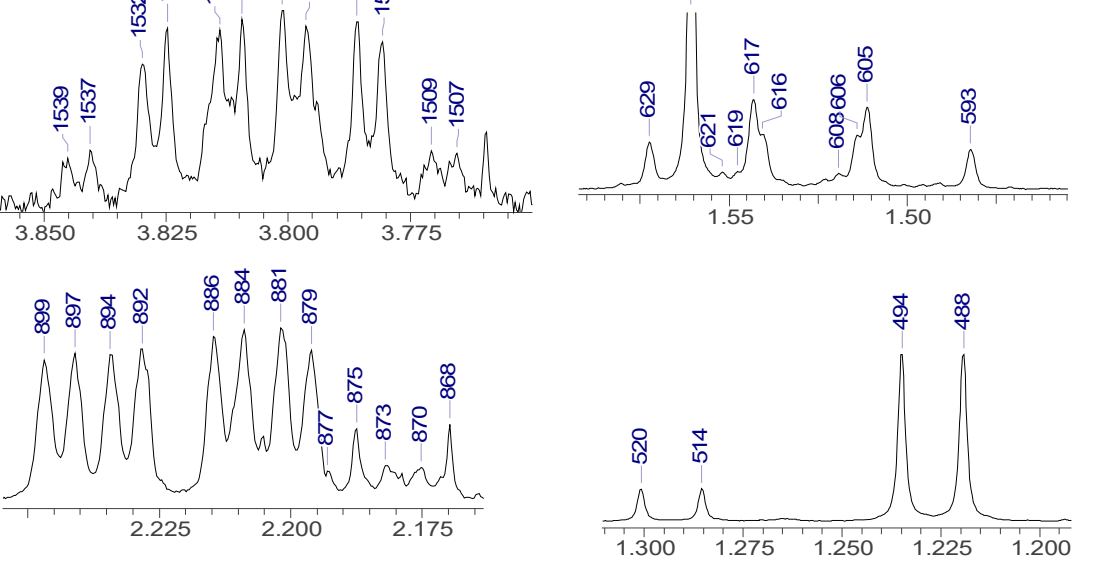

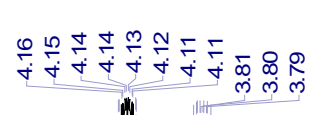

ชูสสรส

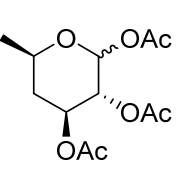

ำํำ

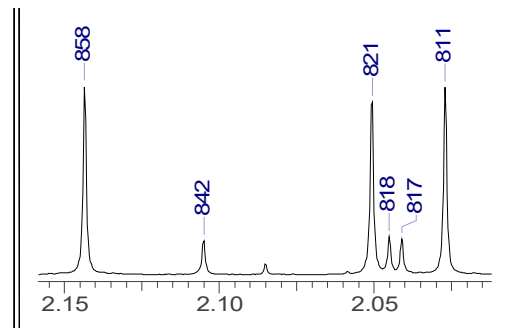

$\stackrel{0}{\mathscr{2}}$

$56 \frac{9}{4}$
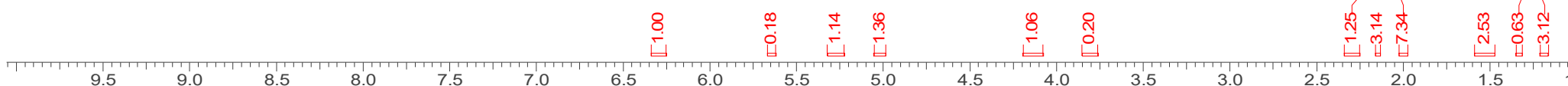
3.48.2. ${ }^{13} \mathrm{C}\left\{{ }^{1} \mathrm{H}\right\}$ NMR $\left(101 \mathrm{MHz}, \mathrm{CDCl}_{3}\right)$
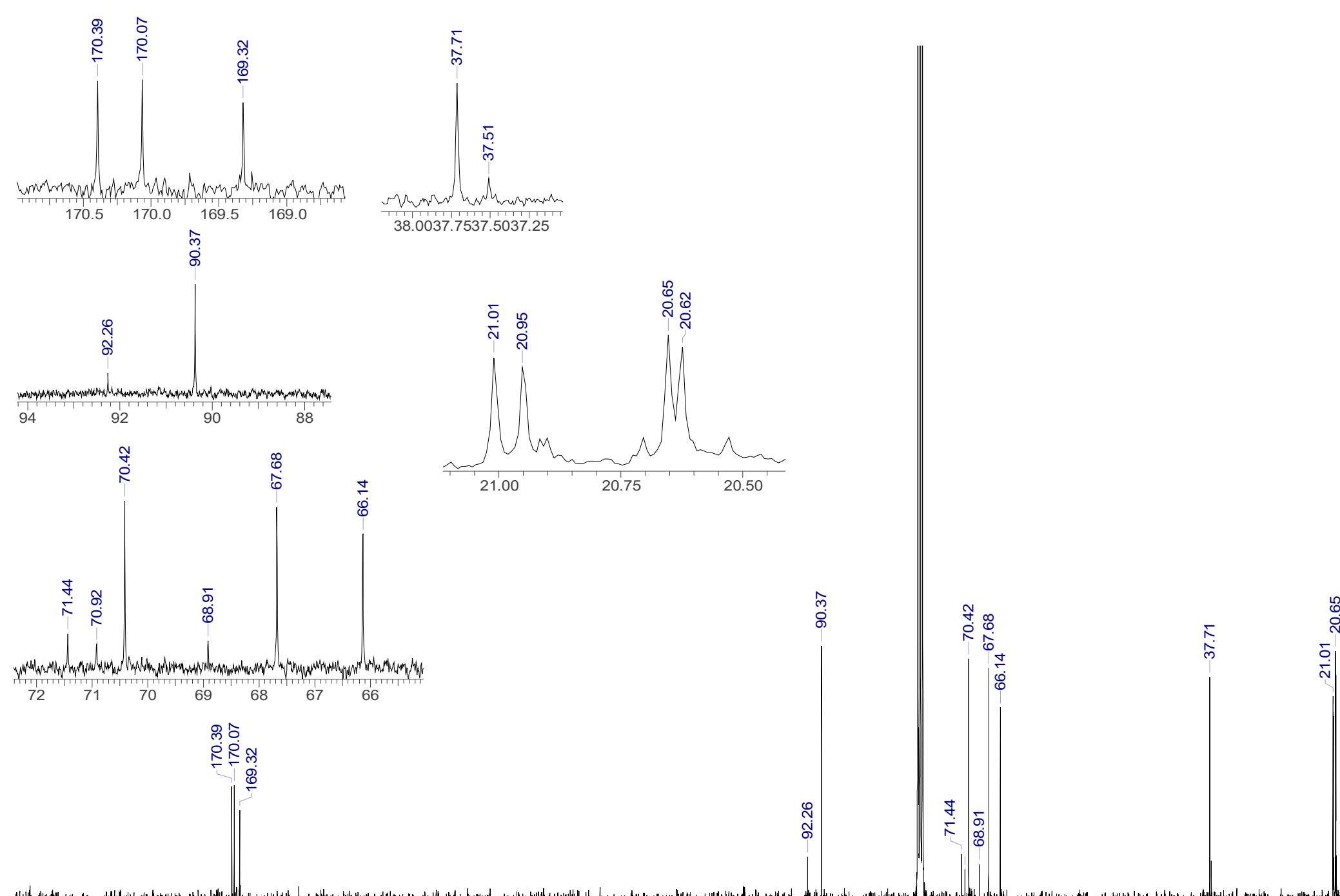

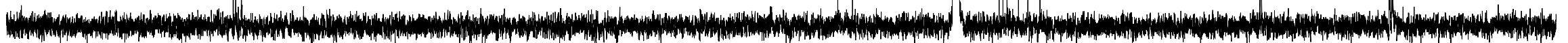

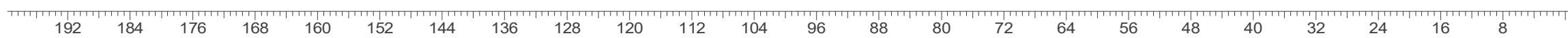




$$
A \square
$$




\section{4. $D_{2} O$ NMR spectra of compounds $1 \mathrm{a}-7 \mathrm{a}$ and $83,84,86,87,89,92 \& 93$ including $q N M R$ experiments}

\subsection{Procedure}

qNMR experiments were conducted adapting a previously published method. ${ }^{2,3}$

Sample Preparation

NMR samples of $\mathbf{1 a - 7 a}$ and $\mathbf{8 3}, \mathbf{8 4}, \mathbf{8 6}, \mathbf{8 7}, \mathbf{8 9}, \mathbf{9 2}$ \& 93 were prepared using $64 \mu$ mol of substrate in $\mathrm{D}_{2} \mathrm{O}(0.75 \mathrm{~mL})$ in flame-sealed NMR tubes. The samples were left to equilibrate, which was determined by comparison of ${ }^{1} \mathrm{H}$ NMR spectra over several days. Most samples reached equilibration within 24 hours, however all samples were left at least an additional 2 days before analysis.

\section{NMR Experiments}

Quantification of fluorinated pyranoses was achieved by integration of ${ }^{19} \mathrm{~F}\left\{{ }^{1} \mathrm{H}\right\} \mathrm{NMR}$ resonances, obtained at $376 \mathrm{MHz}$. Non-fluorinated sugars were determined by ${ }^{1} \mathrm{H}$ NMR obtained at $500 \mathrm{MHz}$. In a typical experiment for fluorinated pyranoses, the delay time (D1) was set to $30 \mathrm{~s}$ for mono-fluorinated substrates, and $60 \mathrm{~s}$ for di-fluorinated substrates, in order to achieve an appropriate pulse delay time of $\geq 5 \times \mathrm{T}_{1}$. The frequency offset point (O1P) was set to the midpoint of all fluorine signals of interest, to allow all nuclei to be equally excited. The spectral width (SW) was set to 300 ppm, and the number of transients (NS) set to 64 . Inverse-gated decoupling was used to obtain a decoupled spectrum without nOe (nuclear Overhauser effect) enhancements. ${ }^{4}$

In a typical experiment for non-fluorinated sugars, the longest $T_{1}$ of each sample was determined by using an inversion-recovery sequence, ${ }^{5}$ and $D 1$ set to an appropriate amount for sufficient pulse delay times (noted on spectrum). O1P was set to 6.175 ppm, and the spectral width set to 30 ppm, with 16 transients.

For quantitative integration, a signal-to-noise (SNR) ratio of $\geq 300$ is desired. ${ }^{6}$

\section{Data Processing}

Obtained spectral data was processed using ACD/Labs 2020.1.2 (File Version S15S41, Build 117479). For ${ }^{19} \mathrm{~F}$ qNMR, all FIDs were processed with zero-filling equal to 4 times the original value, and apodization (exponential) equivalent to $2 \mathrm{~Hz}$ Lorentzian line broadening, before undergoing Fourier transformation. ${ }^{1} \mathrm{H}$ qNMR was analysed directly. If needed, manual phase correction was applied, and then baseline corrected (spectrum averaging, Box Half Width $=500$ points). Fully resolved signals from each anomer were then integrated, and values compared to determine the ratio of each anomer. 


\subsection{4,6-dideoxy-6-fluoro-D-xylo-hexopyranose (1a)}

4.2.1. ${ }^{1} \mathrm{H}$ NMR $\left(500 \mathrm{MHz}, \mathrm{D}_{2} \mathrm{O}\right)$

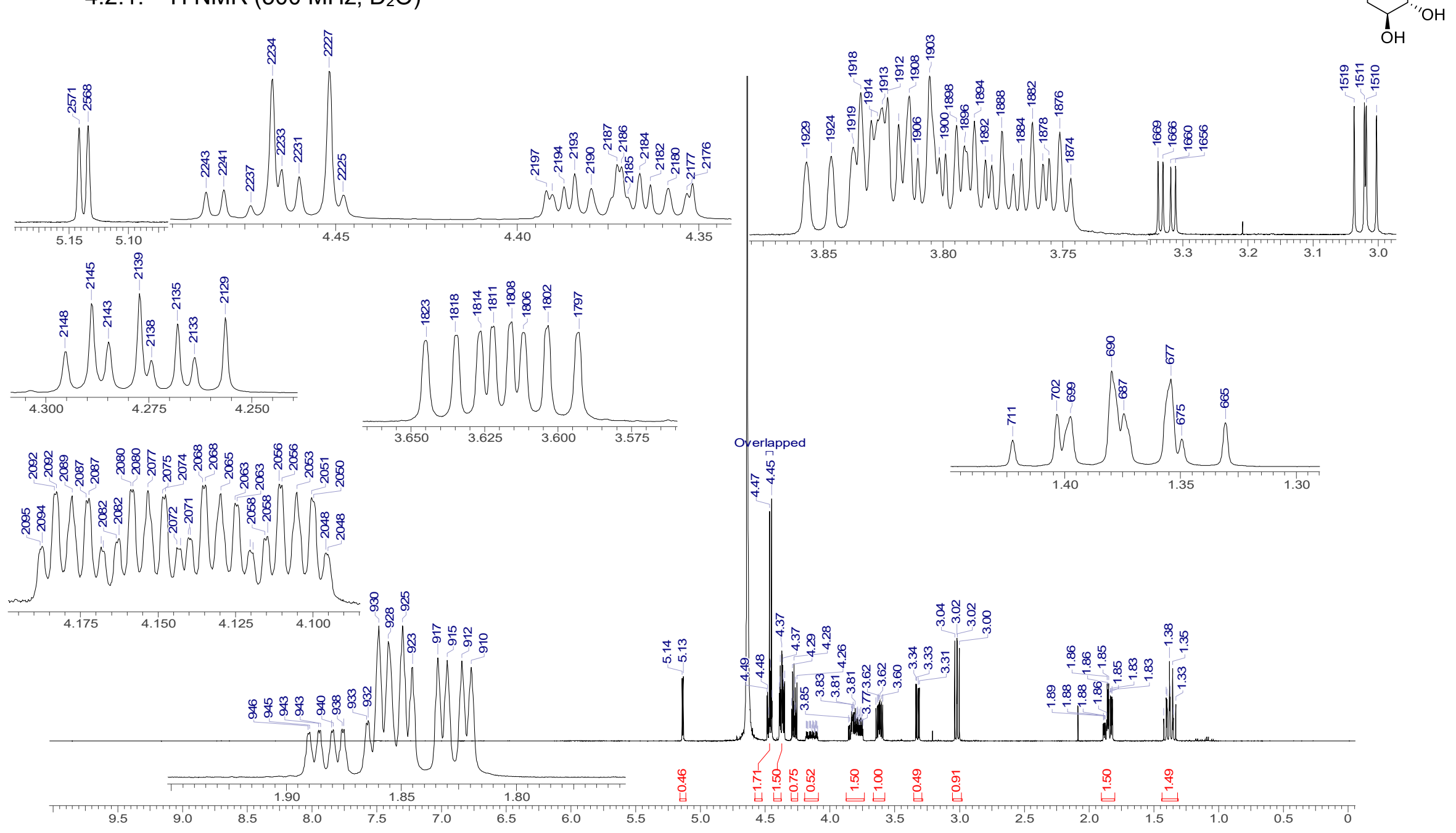


4.2.2. ${ }^{1} \mathrm{H}\left\{{ }^{19} \mathrm{~F}\right\} \mathrm{NMR}\left(500 \mathrm{MHz}, \mathrm{D}_{2} \mathrm{O}\right)$

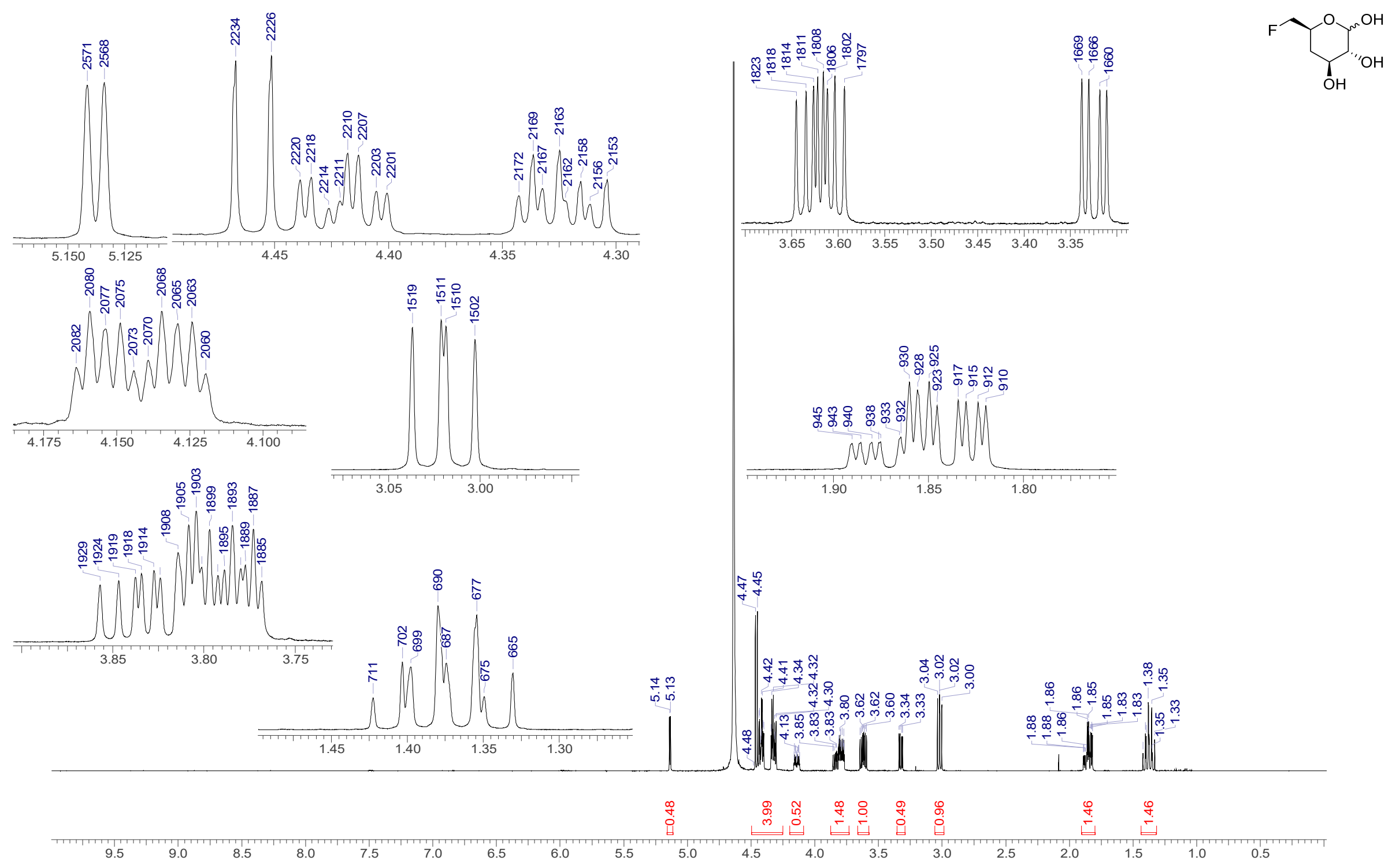


4.2.3. ${ }^{13} \mathrm{C}\left\{{ }^{1} \mathrm{H}\right\} \operatorname{NMR}\left(126 \mathrm{MHz}, \mathrm{D}_{2} \mathrm{O}\right)$

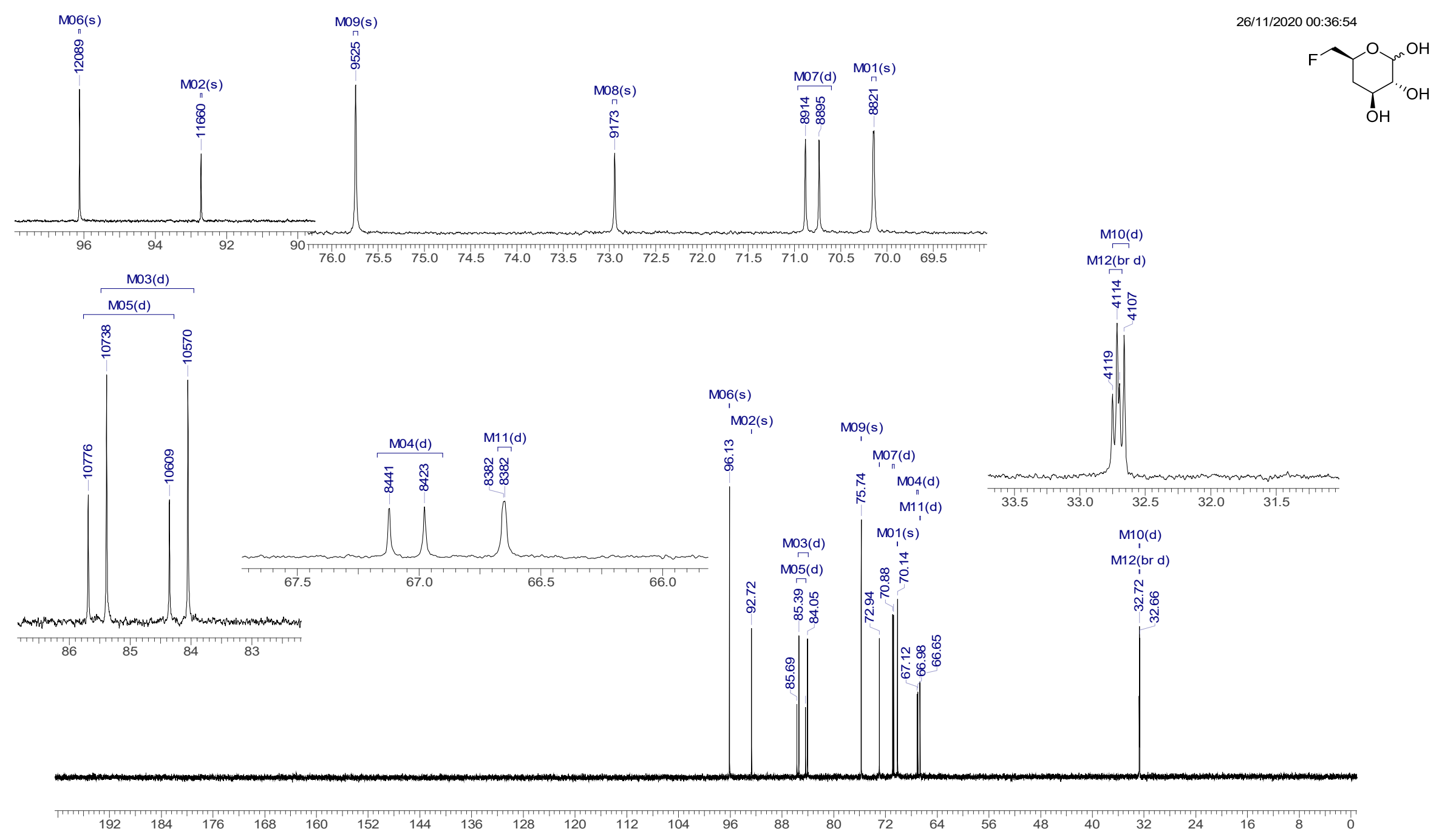


4.2.4. COSY, HSQC-DEPT, $\mathrm{HMBC}\left(\mathrm{D}_{2} \mathrm{O}\right)$

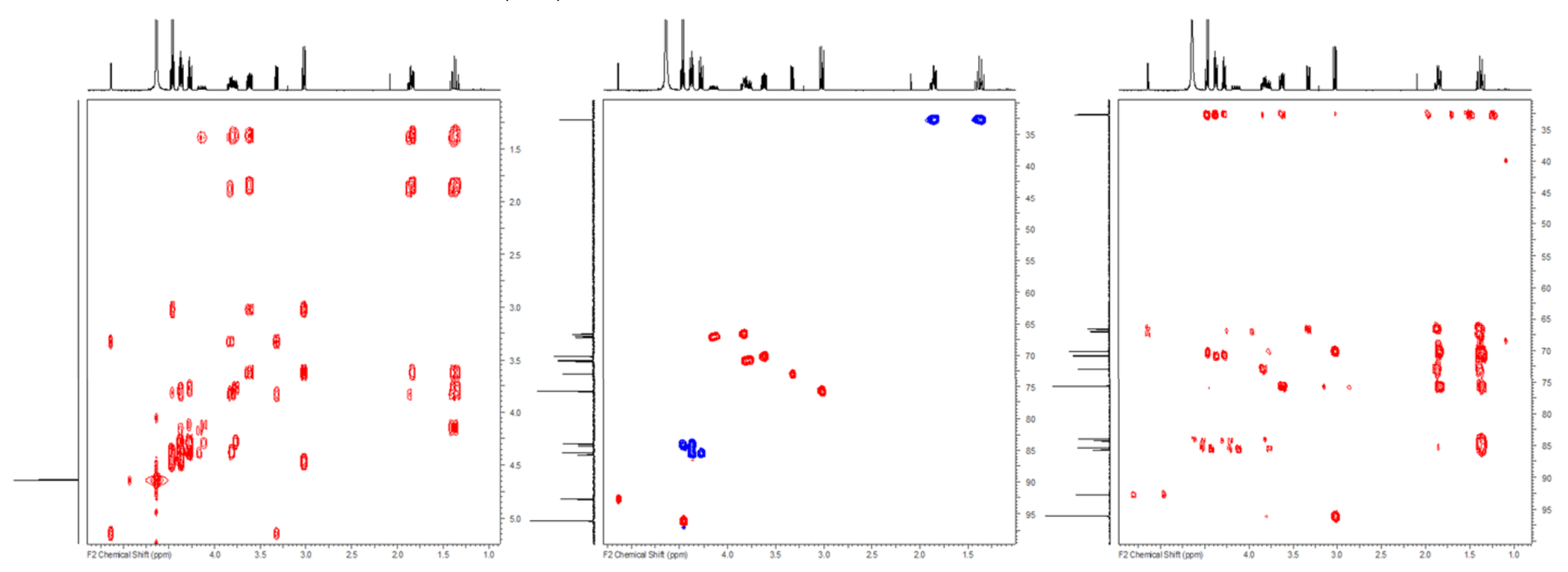


4.2.5. ${ }^{19} \mathrm{~F} \mathrm{NMR}\left(470 \mathrm{MHz}, \mathrm{D}_{2} \mathrm{O}\right)$

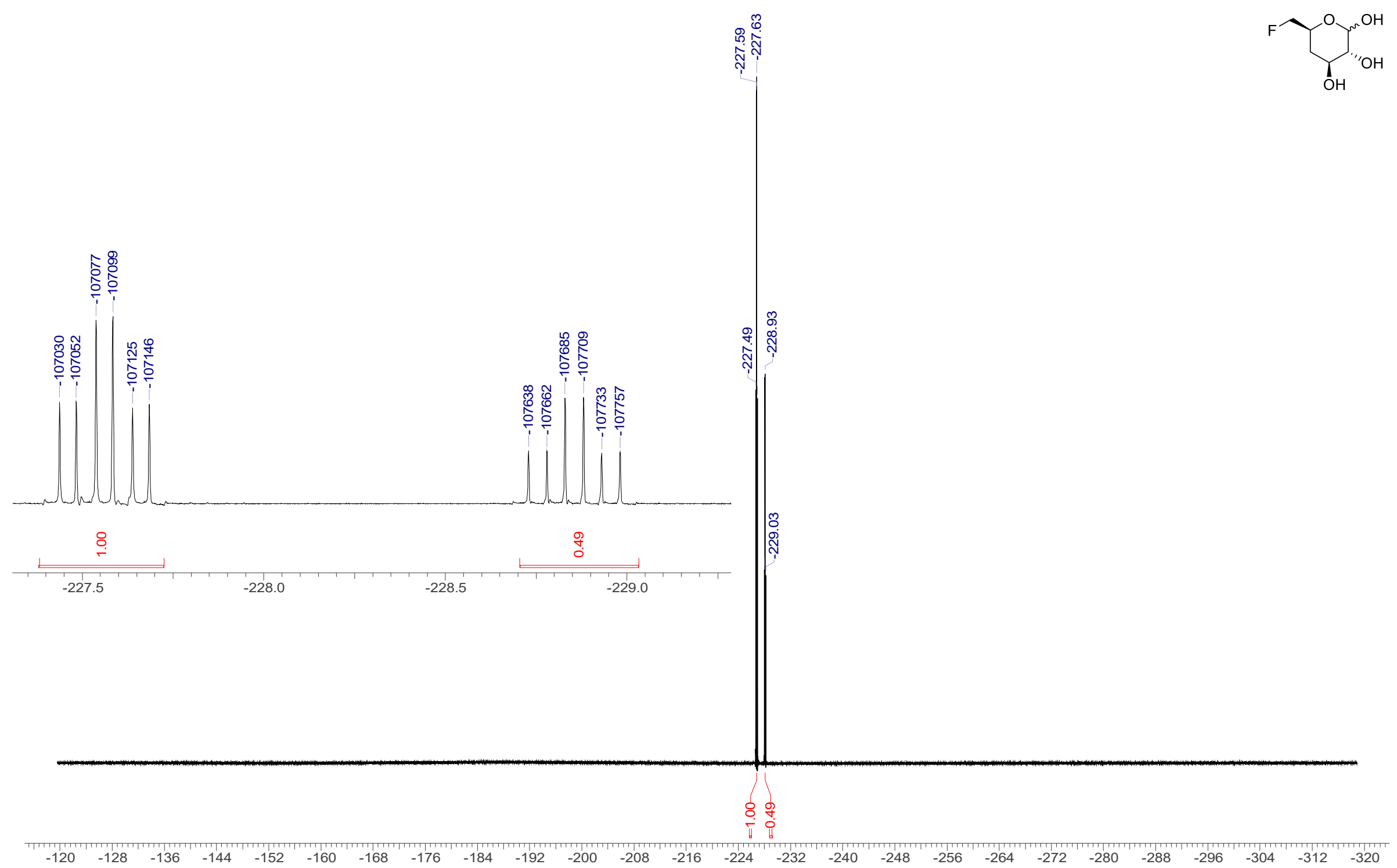


4.2.6. ${ }^{19} \mathrm{~F}\left\{{ }^{1} \mathrm{H}\right\} \mathrm{NMR}\left(470 \mathrm{MHz}, \mathrm{D}_{2} \mathrm{O}\right)$ 
4.2.7. ${ }^{19} \mathrm{~F}\left\{{ }^{1} \mathrm{H}\right\}$ qNMR $\left(376 \mathrm{MHz}, \mathrm{D}_{2} \mathrm{O}\right)$

Duplicate \#1

DW/4,6 V4 D2O An1 qNMR

SW: 300 ppm
O1P: $-228.18 \mathrm{ppm}$
D1: 30 s sec
Proton-decoupled ${ }^{19} \mathrm{~F}$
NMR
$\alpha / \beta=33: 67$
(see next page for detailed integration
information after data processing)

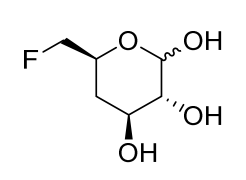

Current Data Parameters
NAME se1820dw3

EXPNO

12

F2 - Acquisition Parameters

$\begin{array}{ll}\text { Date } & 20200920 \\ \text { Time } & 11.07 \mathrm{~h}\end{array}$

INSTRUM $11.07 \mathrm{~h}$

PROBHD Z116098_0218

PULPROG zgfhigqn.2

$\begin{array}{ll}\text { TD } & 131072 \\ \text { SOLVENT } & \text { D2O }\end{array}$

$\begin{array}{lc}\text { NS } & 64 \\ \text { DS } & 4\end{array}$

SWH $\quad 113636.367 \mathrm{~Hz}$

FIDRES $\quad 1.733953 \mathrm{~Hz}$

RG $\quad 212.69$

DW $\quad 4.400$ usec

TE $298.0 \mathrm{~K}$ ec

D1 $\quad 30.00000000 \mathrm{sec}$

D12 $0.00002000 \mathrm{sec}$

$\begin{array}{ll}\text { TDO } & 1 \\ \text { SFO1 } & 376.4124567 \mathrm{MHz}\end{array}$

NUC1 $19 \mathrm{~F}$

$\begin{array}{ll}\text { P1 } & 14.00 \mathrm{usec} \\ \text { PLW1 } & 22.69199944\end{array}$

SFO2 $\quad 400.1316005 \mathrm{MHz}$

NUC2 $1 \mathrm{H}$

$\begin{array}{ll}\text { CPDPRG } & \text { waltz16 } \\ \text { PCPD2 } & 90.00 \text { usec }\end{array}$

$\begin{array}{lr}\text { PLW2 } & 90.00 \mathrm{usec} \\ \text { PLW12 } & 0.38100052 \mathrm{~W}\end{array}$

$\begin{array}{lr}\text { PLW12 } & 0.20223001 \mathrm{~W}\end{array}$

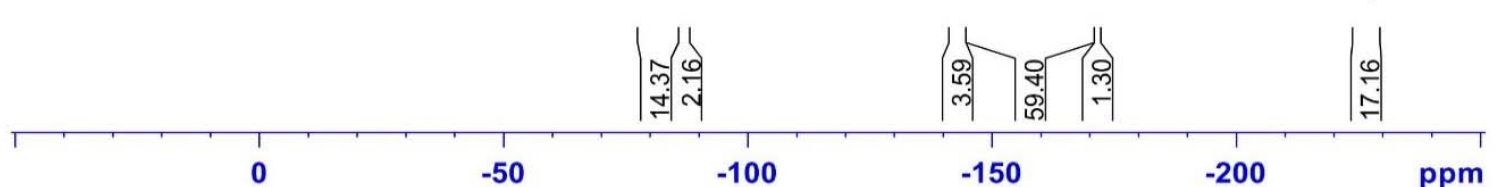

F2 - Processing parameters

$\begin{array}{ll}\text { SF } & 65536 \\ \text { S76.4984132 MHz }\end{array}$

$\begin{array}{lc}\text { WDW } & 376.4984132 \mathrm{MHz} \\ \mathrm{SSB} & \mathrm{EM}\end{array}$

SSB

$0.30 \mathrm{~Hz}$

$\begin{array}{lc}\mathrm{GB} & 0 \\ \mathrm{PC} & 1.00\end{array}$ 
Duplicate \#1

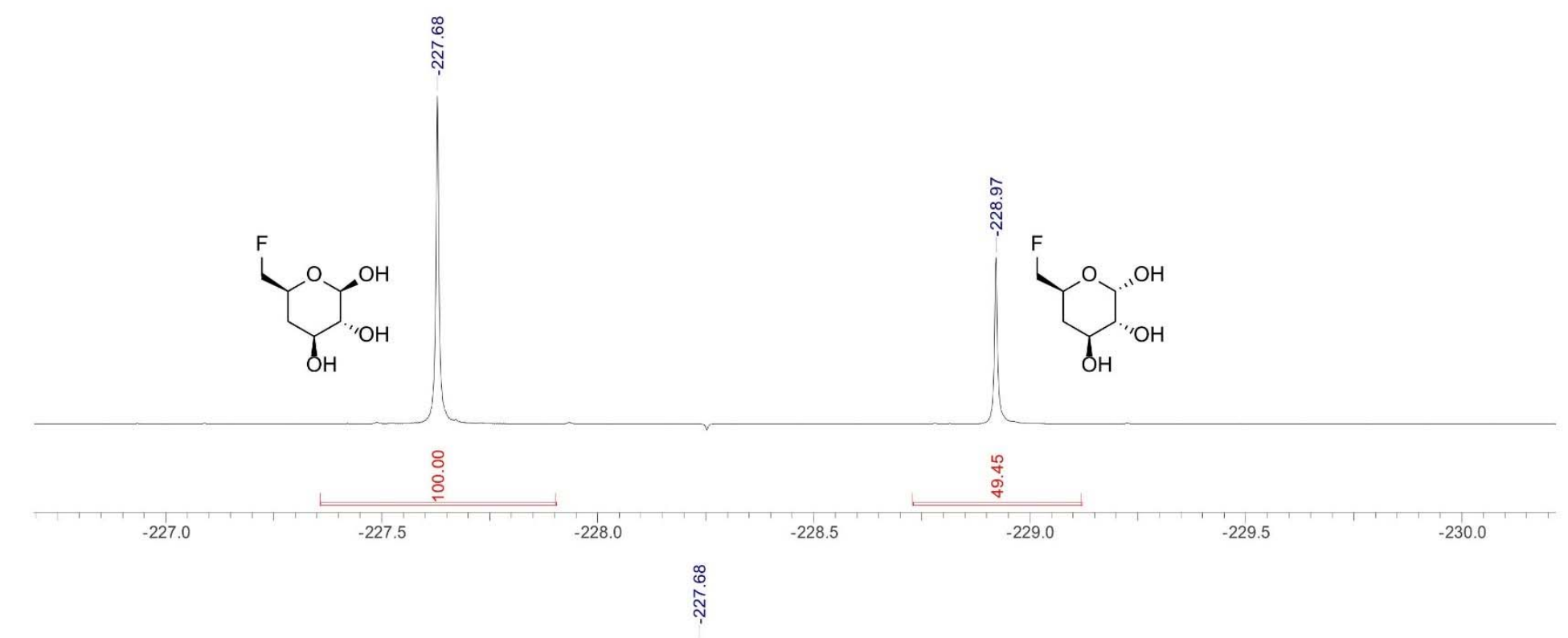

SW: $300 \mathrm{ppm}$

O1P: -228.18 ppm

D1: 30 s sec

Proton-decoupled ${ }^{19} \mathrm{~F}$

NMR

$\alpha / \beta 33: 67$

ำ

$\begin{array}{lllllllllllllllllllll}-195 & -200 & -205 & -210 & -215 & -220 & -225 & -230 & -235 & -240 & -245 & -250 & -255 & -260 & -265\end{array}$




\section{Duplicate \#2}

DW/4,6 V4 D2O An2 qNMR

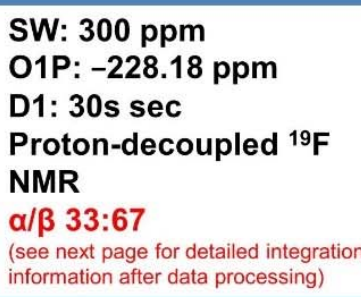

D1: $30 \mathrm{~s} \mathrm{sec}$

Proton-decoupled ${ }^{19} \mathrm{~F}$

NMR

$\alpha / \beta 33: 67$

(see next page for detailed integration

information after data processing)
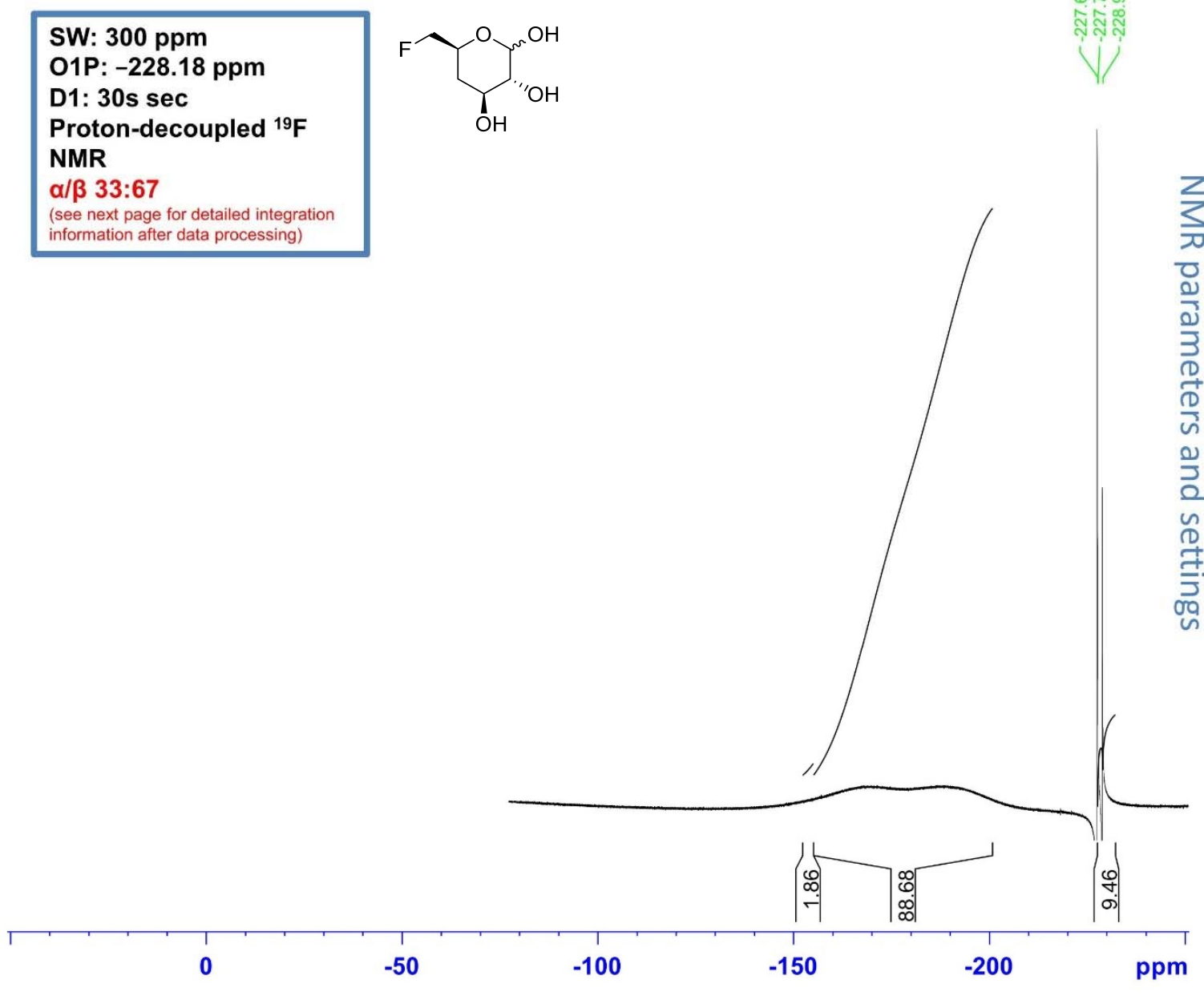

AVIIIHD400 [3]

Current Data Parameters
NAME se1820dw4

EXPNO $\quad 12$

12

F2 - Acquisition Parameters

$\begin{array}{lc}\text { Date__ } & 20200920 \\ \text { Time } & 11.51 \mathrm{~h}\end{array}$

PROBHD spect

PULPROG Z116098_0218

TD zgfhiggn.2

SOLVENT 131072 D2O

NS

DS

64
4

SWH $\quad 113636.367 \mathrm{~Hz}$

AQ $\quad 1.733953 \mathrm{~Hz}$

RG $\quad 212.69$

DW $\quad 4.400$ usec

DE $\quad 6.50$ usec

$\begin{array}{ll}\text { TE } & 298.0 \mathrm{~K} \\ \text { D1 } & 30.00000000 \mathrm{sec}\end{array}$

D11 $0.03000000 \mathrm{sec}$

D12 $0.0002000 \mathrm{sec}$

TD0 1

$\begin{array}{lc}\text { SFO1 } & 376.4124567 \\ \text { NUC1 } & 19 \mathrm{~F}\end{array}$

P1 14.00 usec

PLW1 22.69199944 W

$\begin{array}{lc}\text { SFO2 } & 400.1316005 \mathrm{MHz} \\ \text { NUC2 } & 1 \mathrm{H}\end{array}$

CPDPRG[2 waltz16

$90.00 \mathrm{usec}$
$16.38100052 \mathrm{~W}$

PLW12 $0.20223001 \mathrm{~W}$

F2 - Processing parameters

$\begin{array}{ll}\text { SI } & 65536 \\ \text { SF } & 376.4984132 \mathrm{MHz}\end{array}$

WDW $\begin{aligned} & 376.4984132 \\ & \text { EM }\end{aligned}$

$\begin{array}{ll}\text { SSB } & 0 \\ \text { LB } & 0.30 \mathrm{~Hz}\end{array}$

$\begin{array}{lc}\text { GB } & 0.30 \mathrm{~Hz} \\ \text { PC } & 0\end{array}$ 
Duplicate \#2

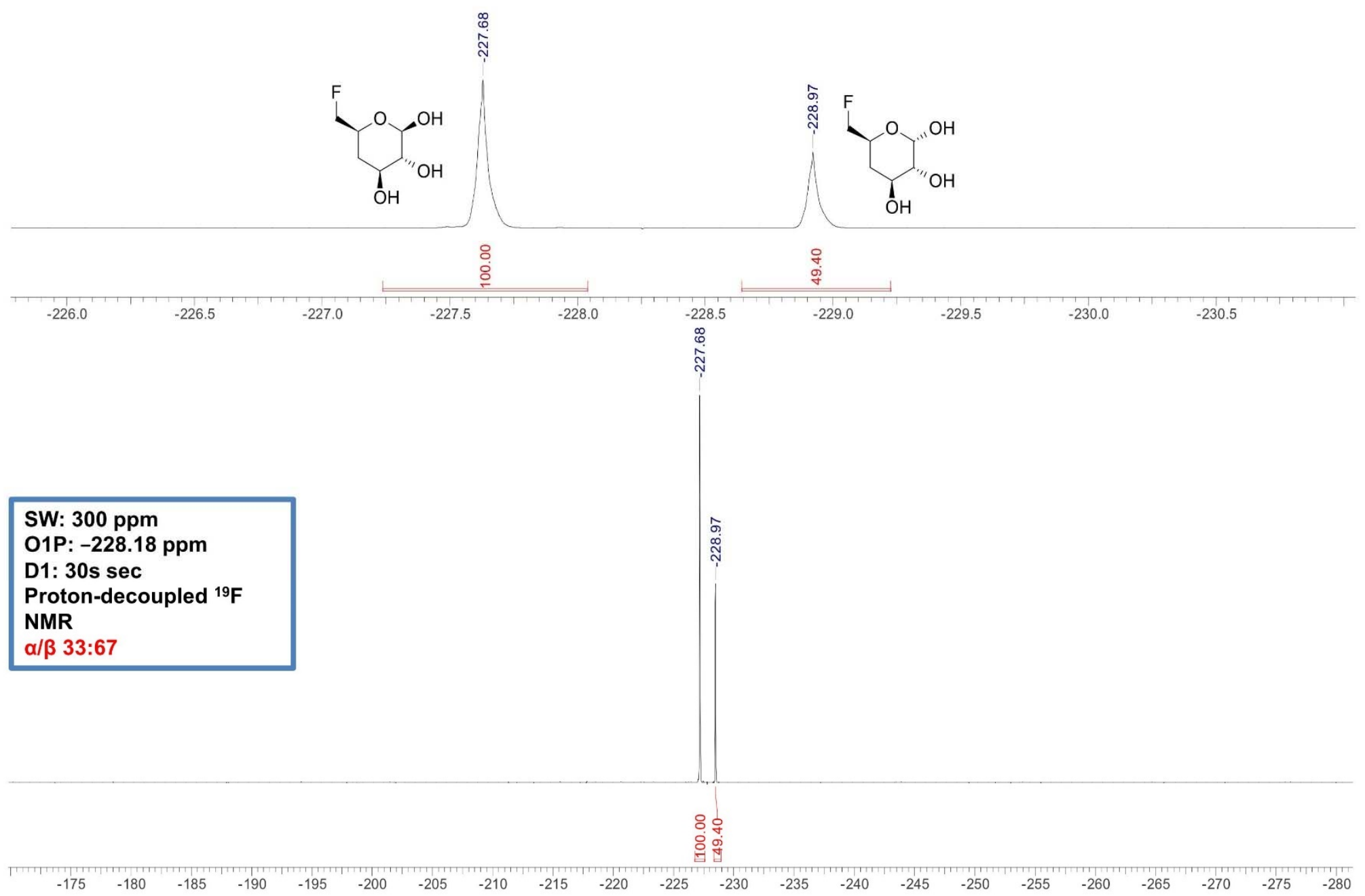




\subsection{4-deoxy-4-fluoro-D-fucose (2a)}

4.3.1. ${ }^{1} \mathrm{H}$ NMR $\left(500 \mathrm{MHz}, \mathrm{D}_{2} \mathrm{O}\right)$
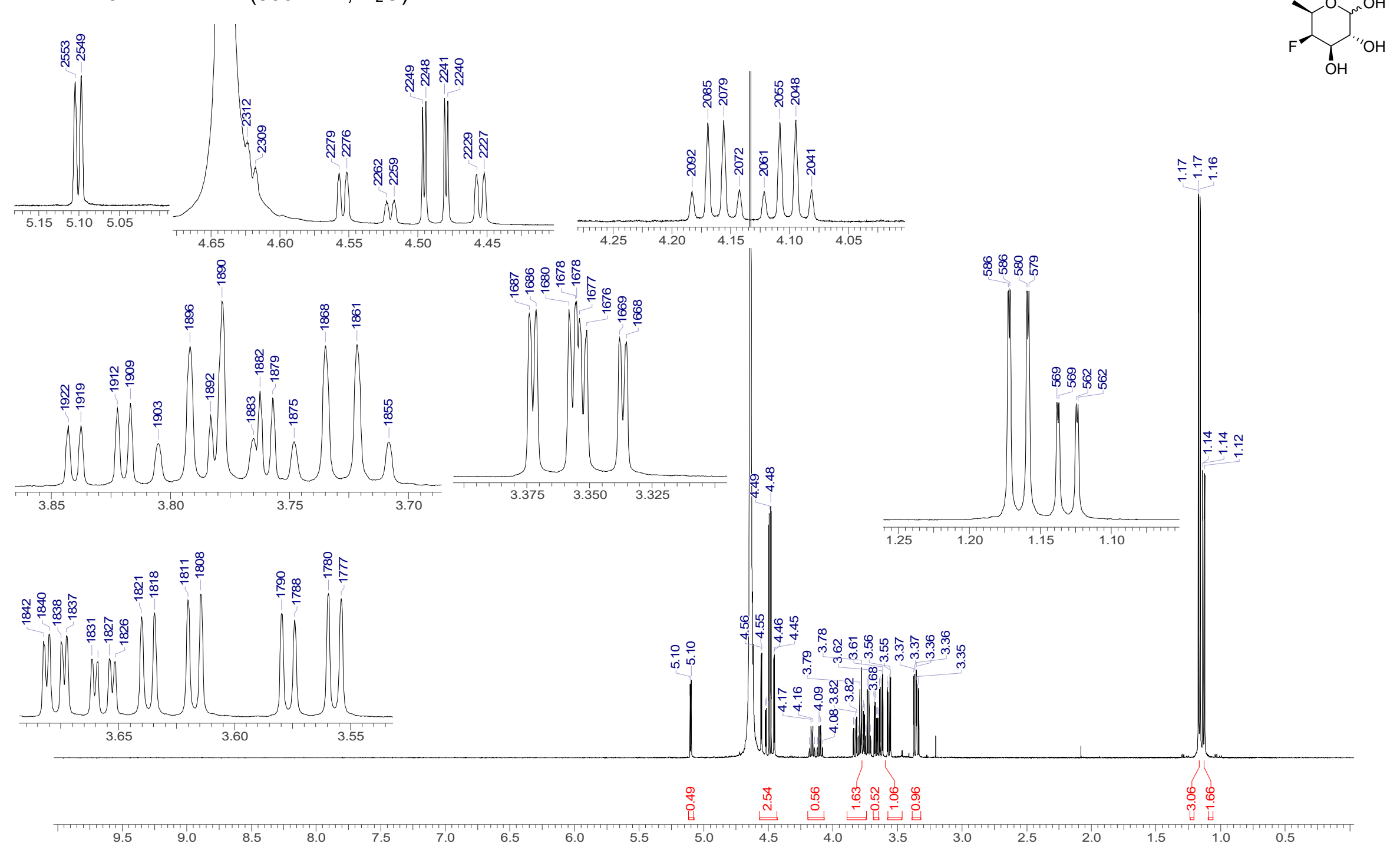
4.3.2. ${ }^{1} \mathrm{H}\left\{{ }^{19} \mathrm{~F}\right\} \mathrm{NMR}\left(500 \mathrm{MHz}, \mathrm{D}_{2} \mathrm{O}\right)$
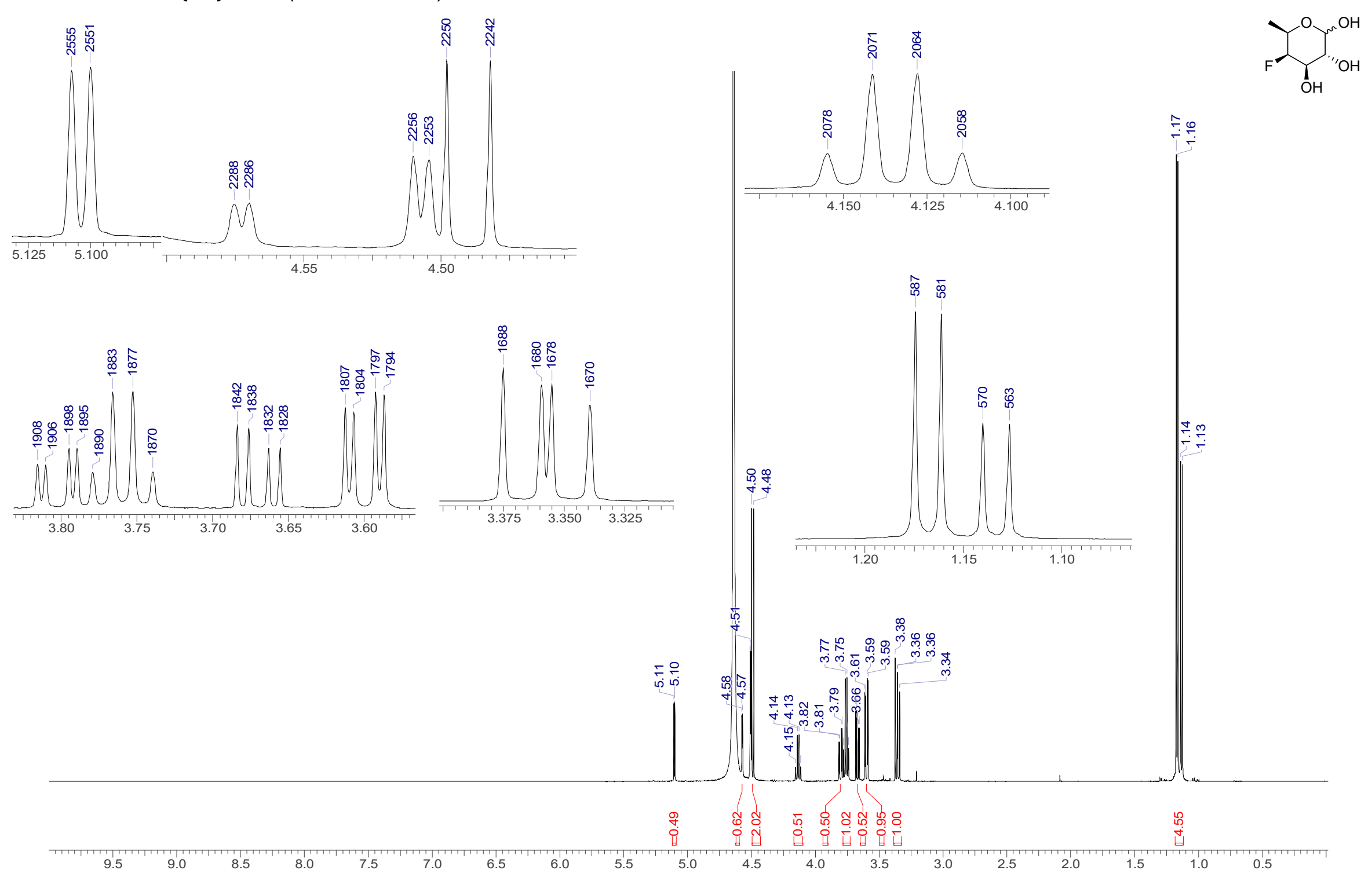
4.3.3. ${ }^{13} \mathrm{C}\left\{{ }^{1} \mathrm{H}\right\} \operatorname{NMR}\left(126 \mathrm{MHz}, \mathrm{D}_{2} \mathrm{O}\right)$

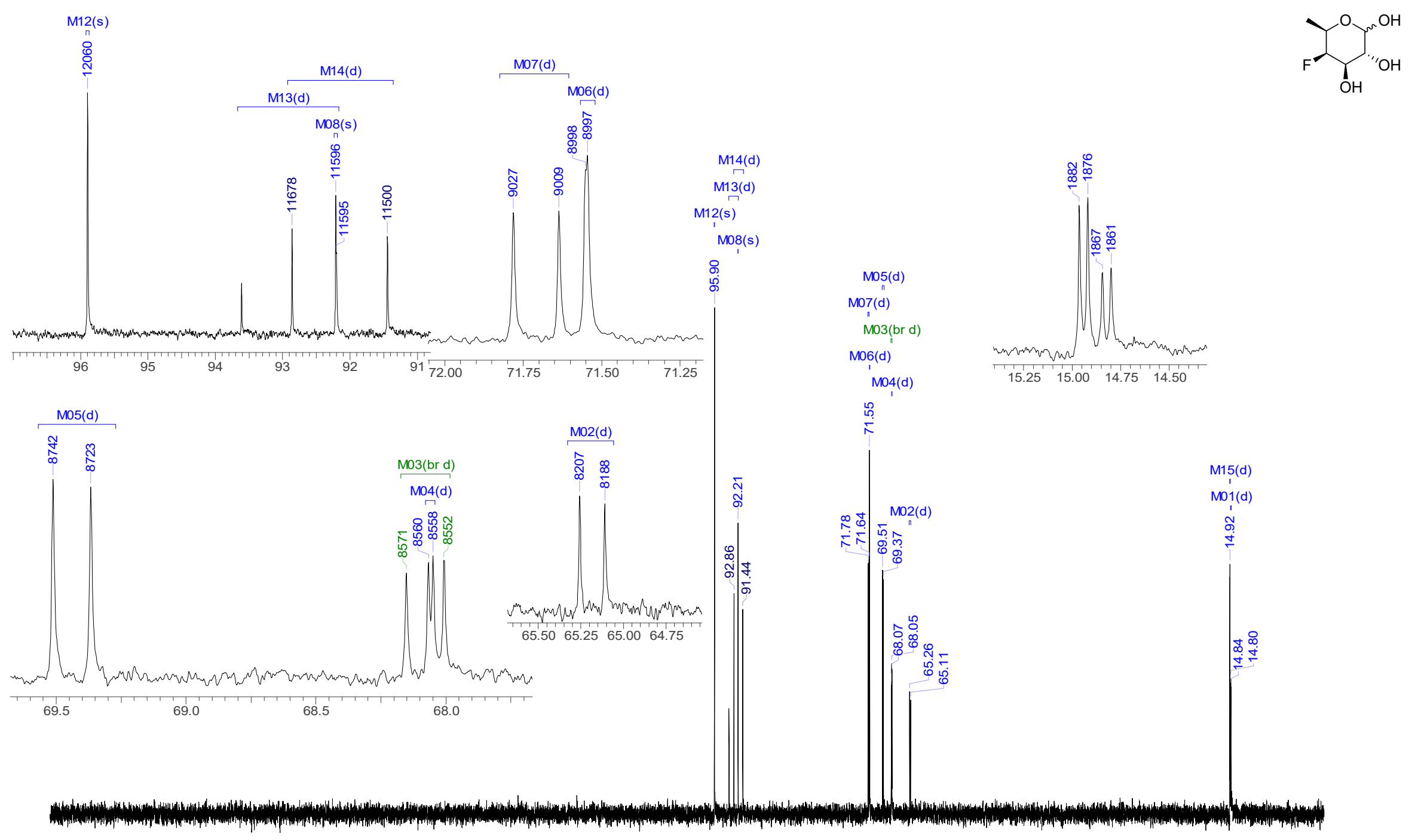

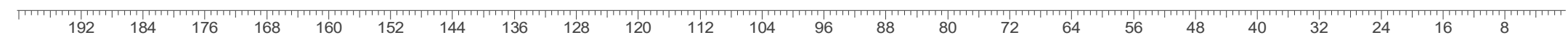




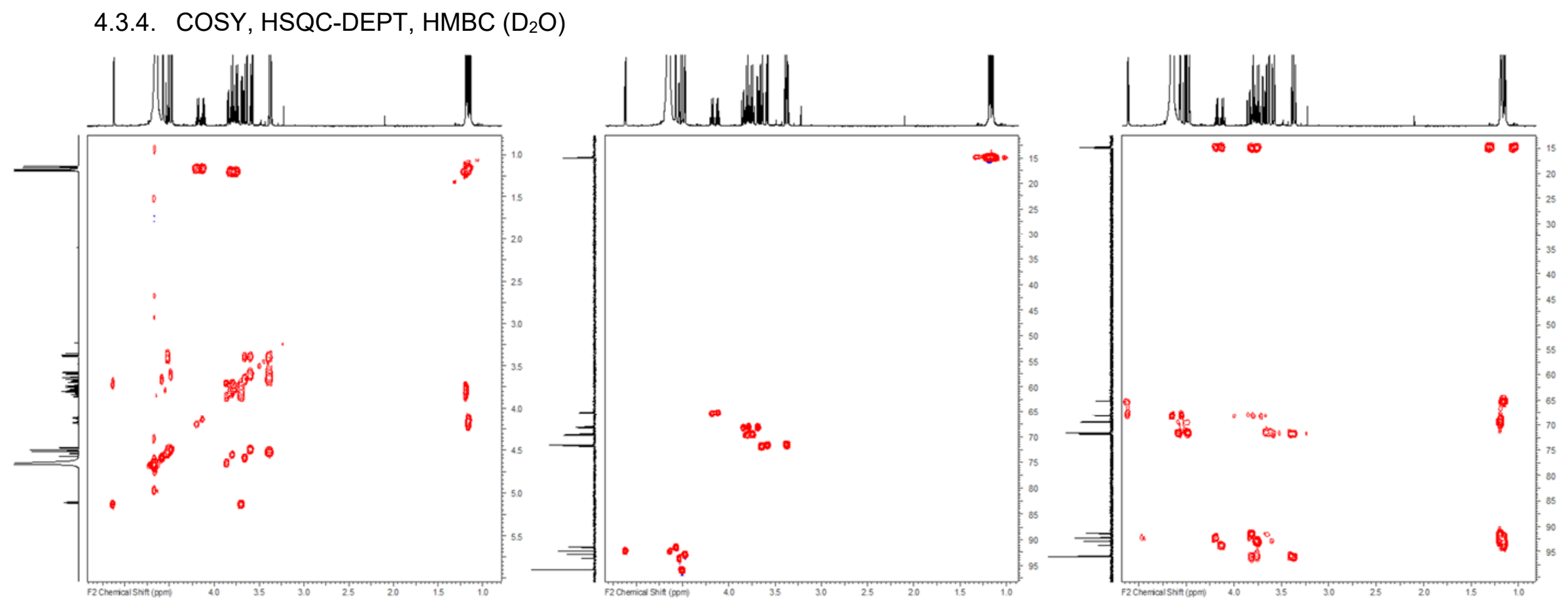


4.3.5. ${ }^{19} \mathrm{~F} \mathrm{NMR}\left(470 \mathrm{MHz}, \mathrm{D}_{2} \mathrm{O}\right)$

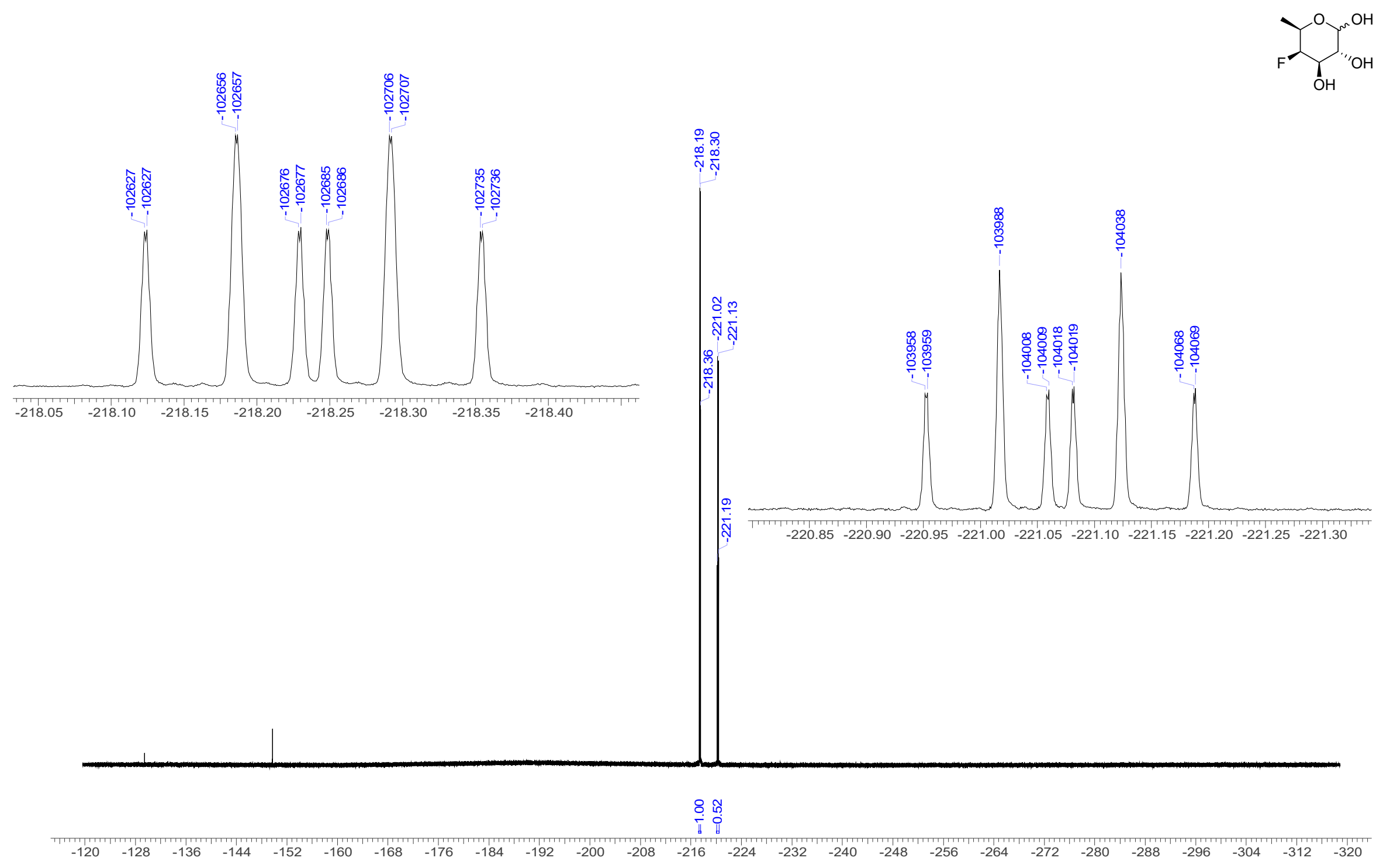


4.3.6. ${ }^{19} \mathrm{~F}\left\{{ }^{1} \mathrm{H}\right\} \mathrm{NMR}\left(470 \mathrm{MHz}, \mathrm{D}_{2} \mathrm{O}\right)$ 
4.3.7. ${ }^{19} \mathrm{~F}\left\{{ }^{1} \mathrm{H}\right\}$ qNMR $\left(376 \mathrm{MHz}, \mathrm{D}_{2} \mathrm{O}\right)$

Duplicate \#1

DW/4,6 V3 D2O An1 qNMR

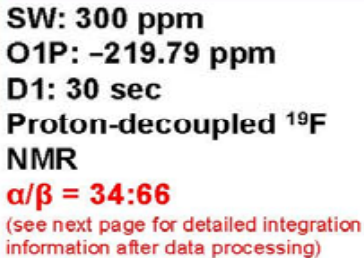

W: 300 ppm

$\alpha / \beta=34: 66$

(see next page for detailed integration
information after data processing)
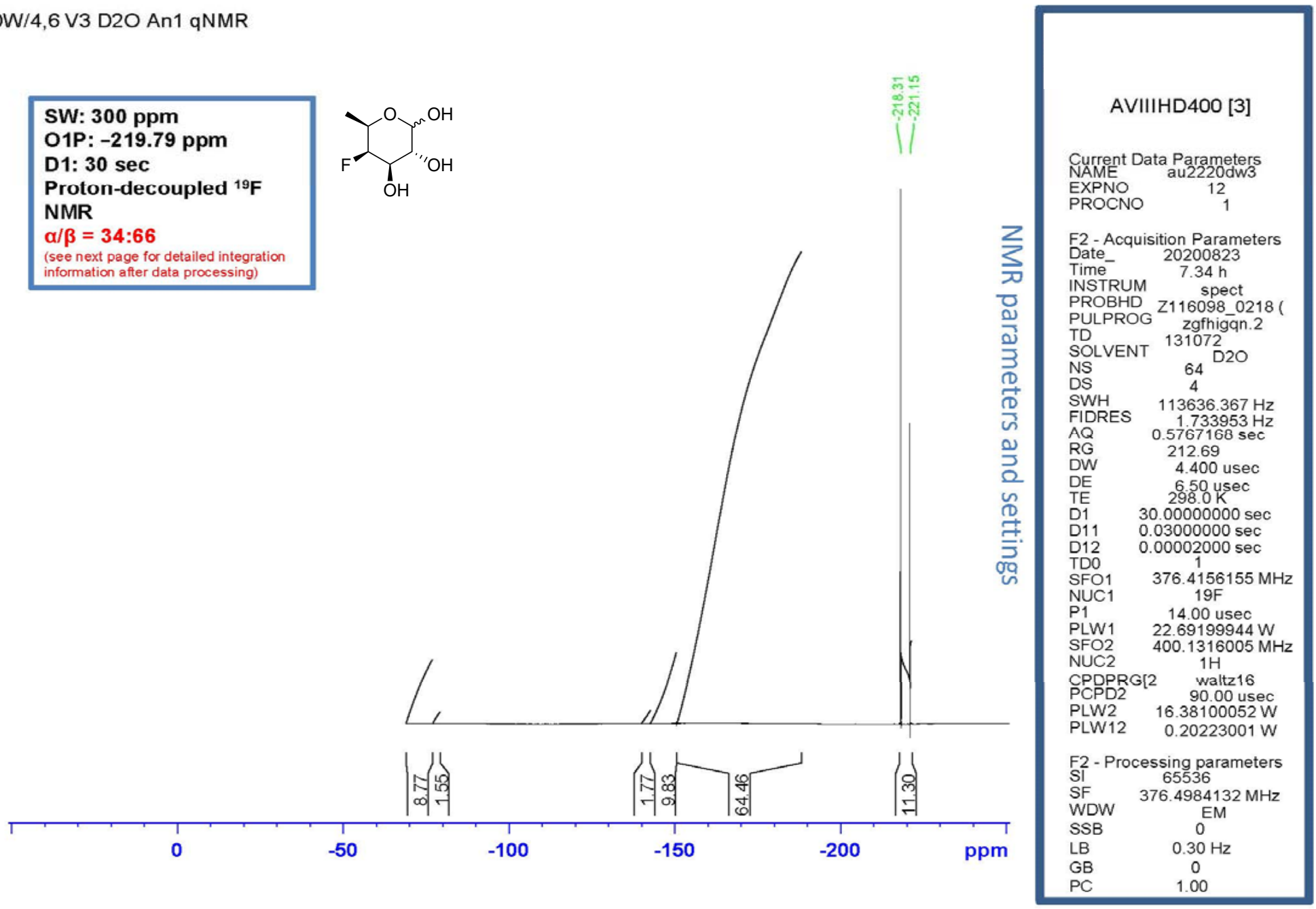
Duplicate \#1

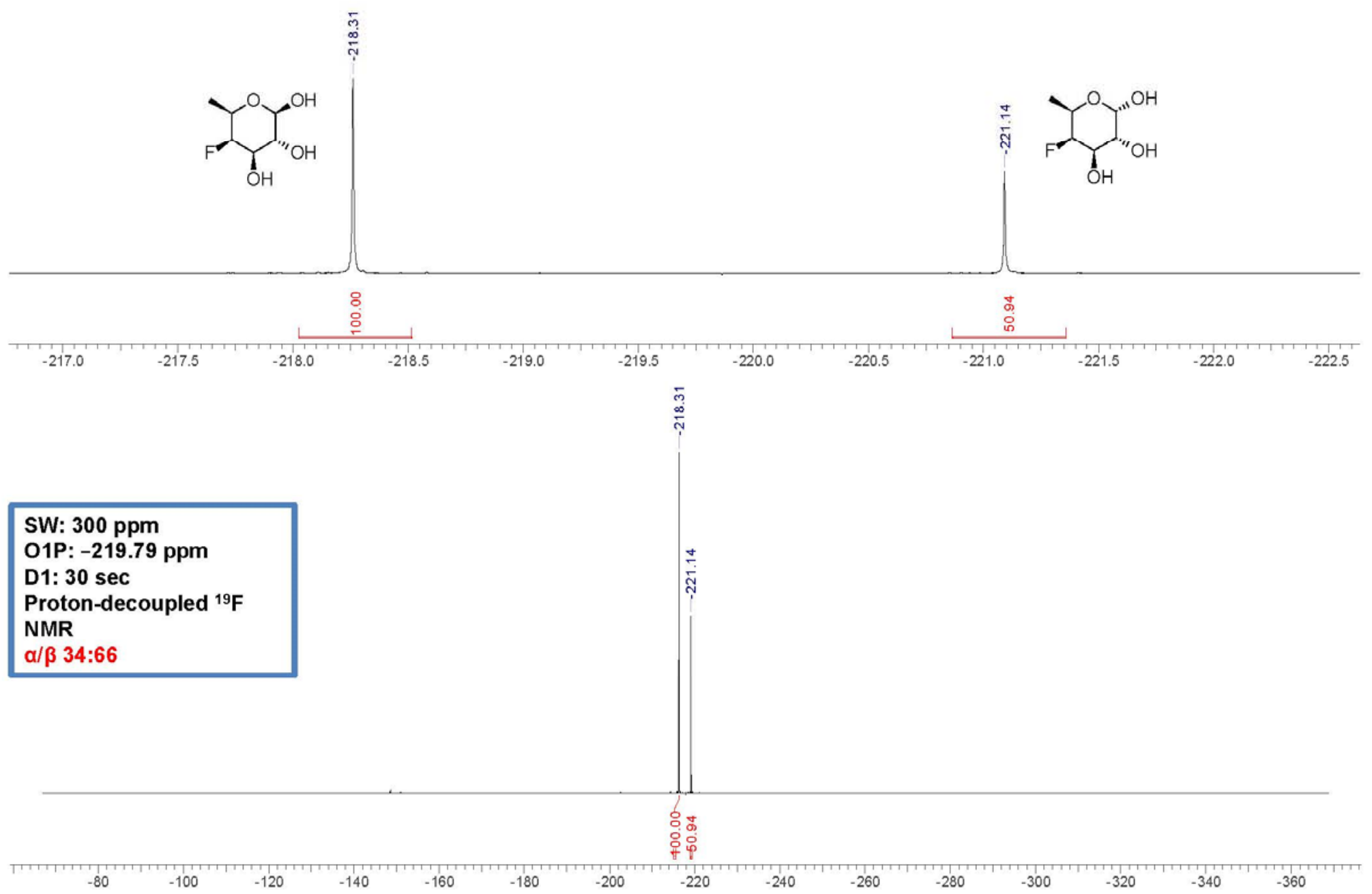




\section{Duplicate \#2}

DW/4,6 V3 D2O An2 qNMR

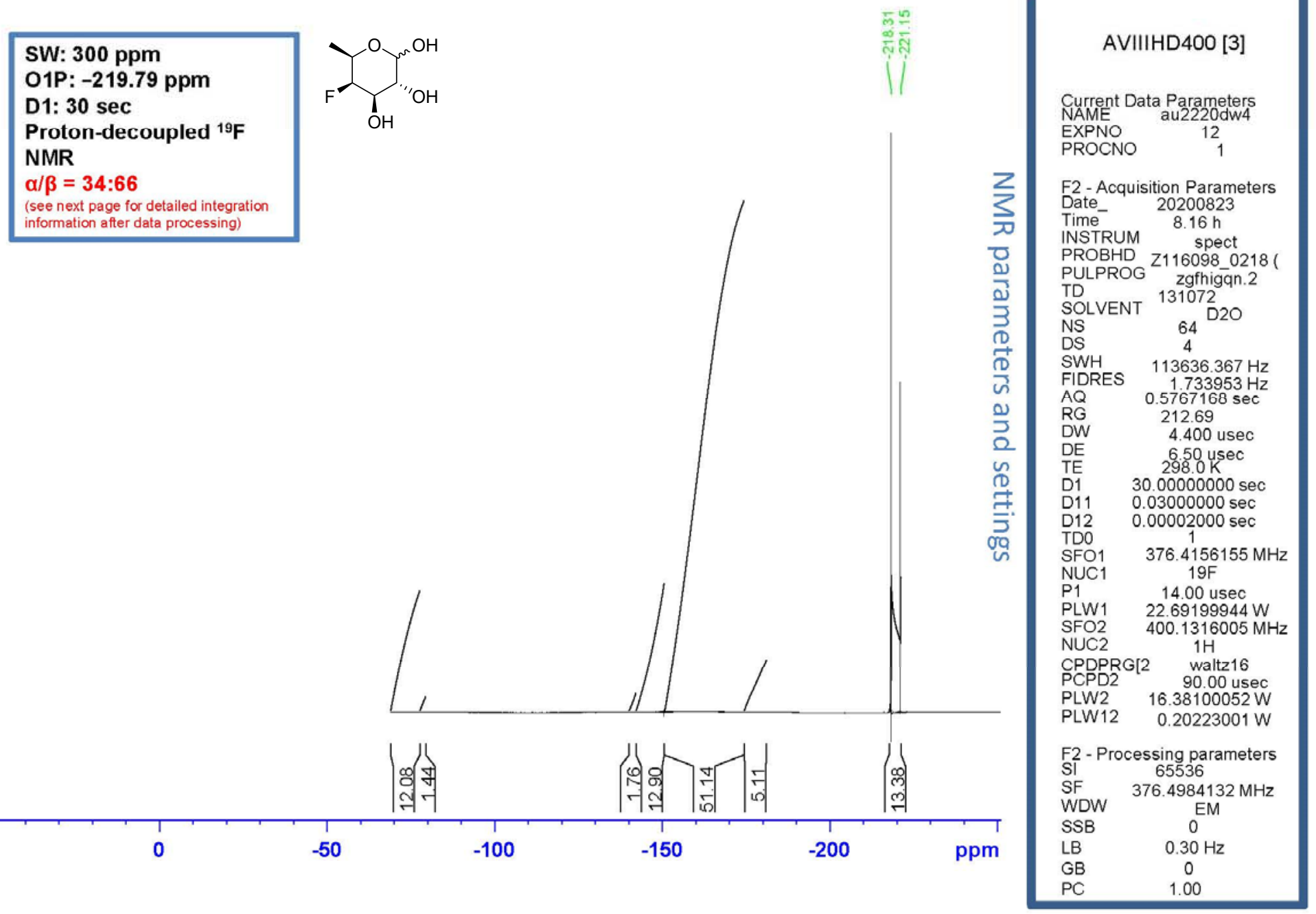


Duplicate \#2

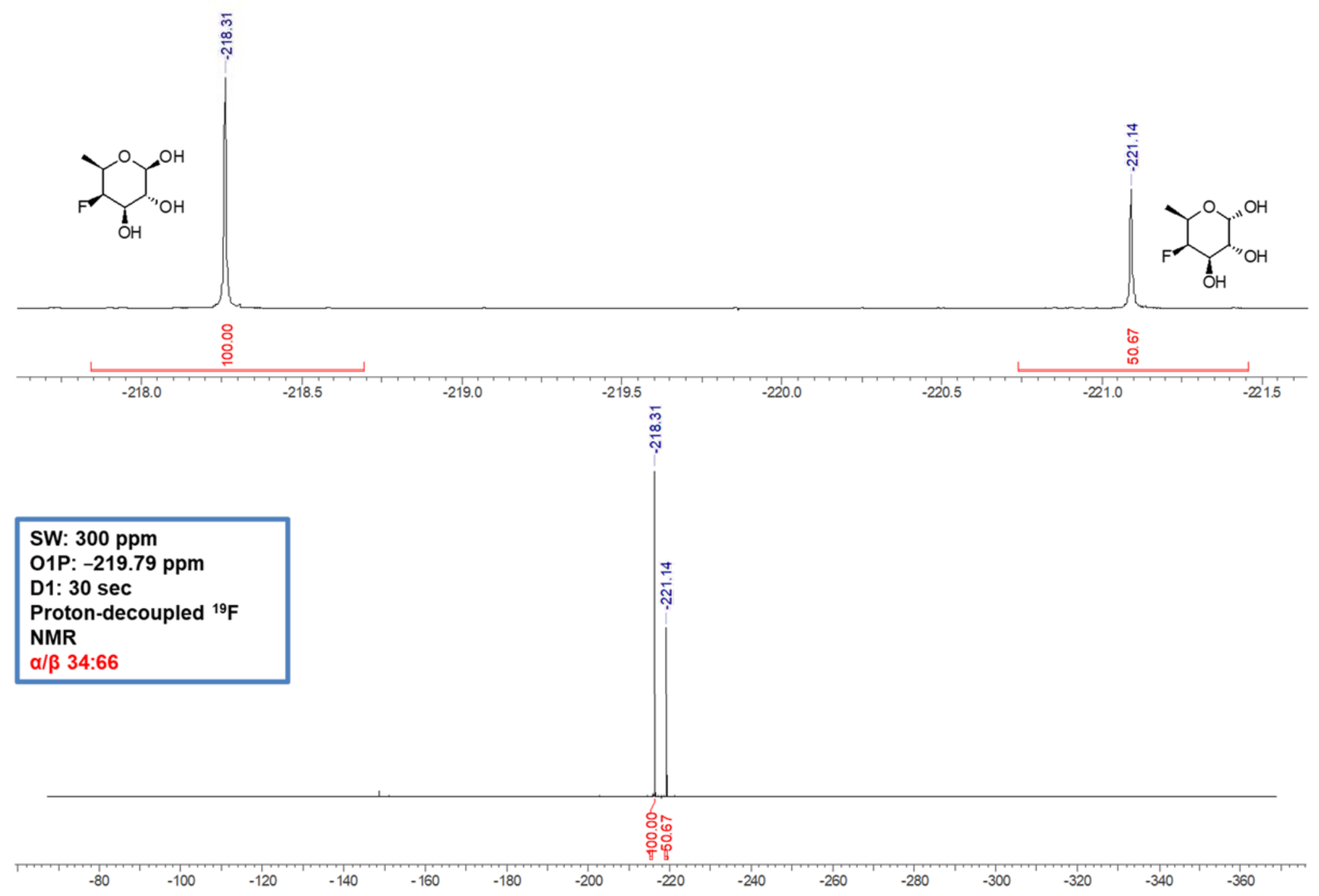




\subsection{4-Deoxy-4-fluoro-D-quinovose (3a)}

4.4.1. ${ }^{1} \mathrm{H}$ NMR $\left(500 \mathrm{MHz}, \mathrm{D}_{2} \mathrm{O}\right)$
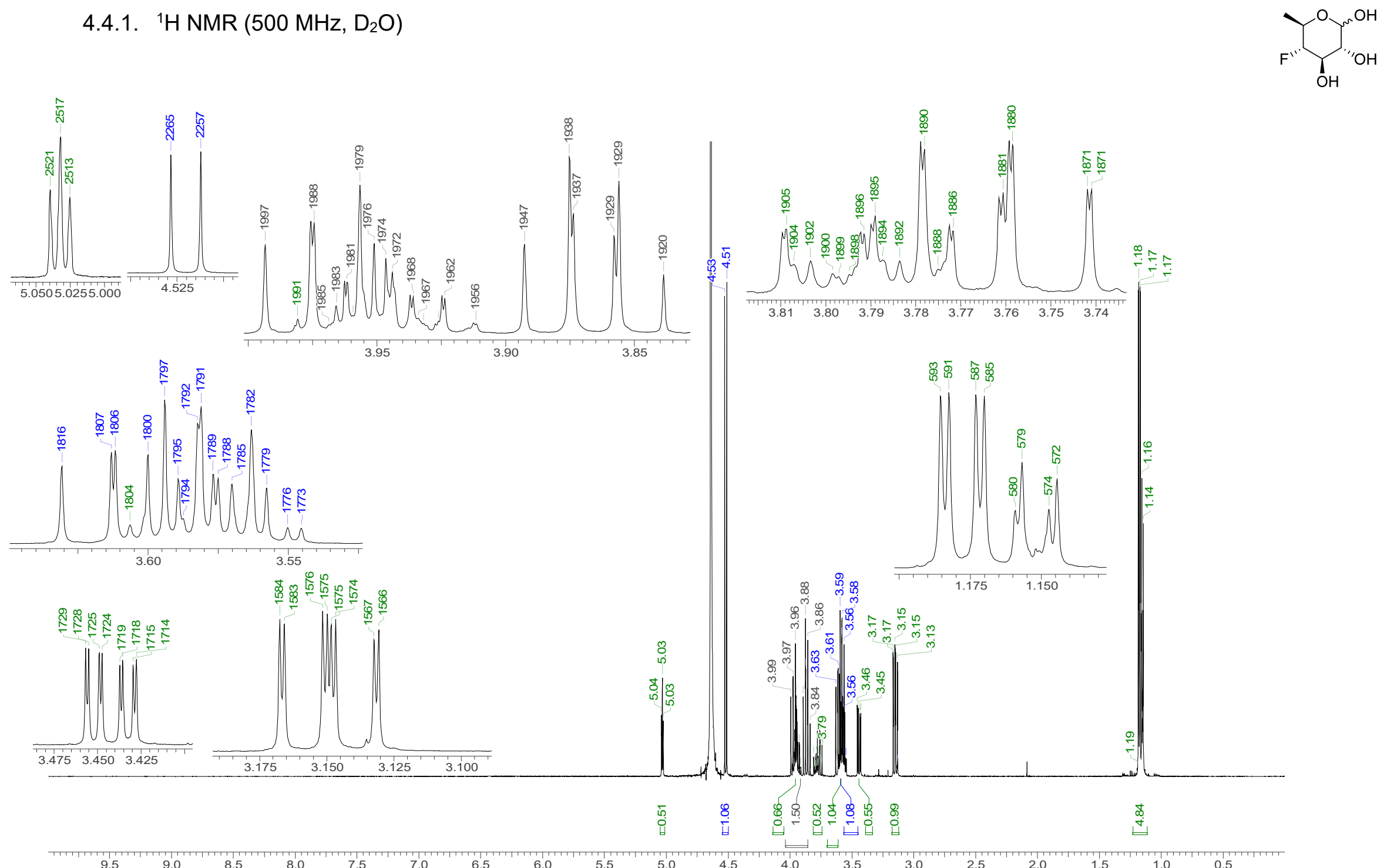
4.4.2. ${ }^{1} \mathrm{H}\left\{{ }^{19} \mathrm{~F}\right\} \operatorname{NMR}\left(500 \mathrm{MHz}, \mathrm{D}_{2} \mathrm{O}\right)$
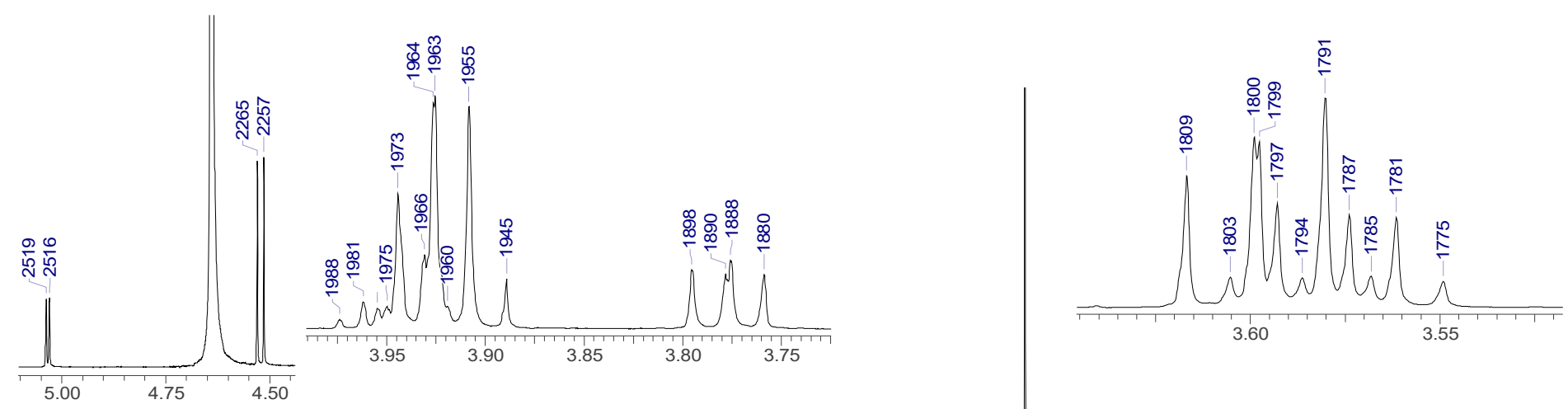

$\mathrm{OH}_{\mathrm{OH}}^{\mathrm{O} O \mathrm{OH}}$
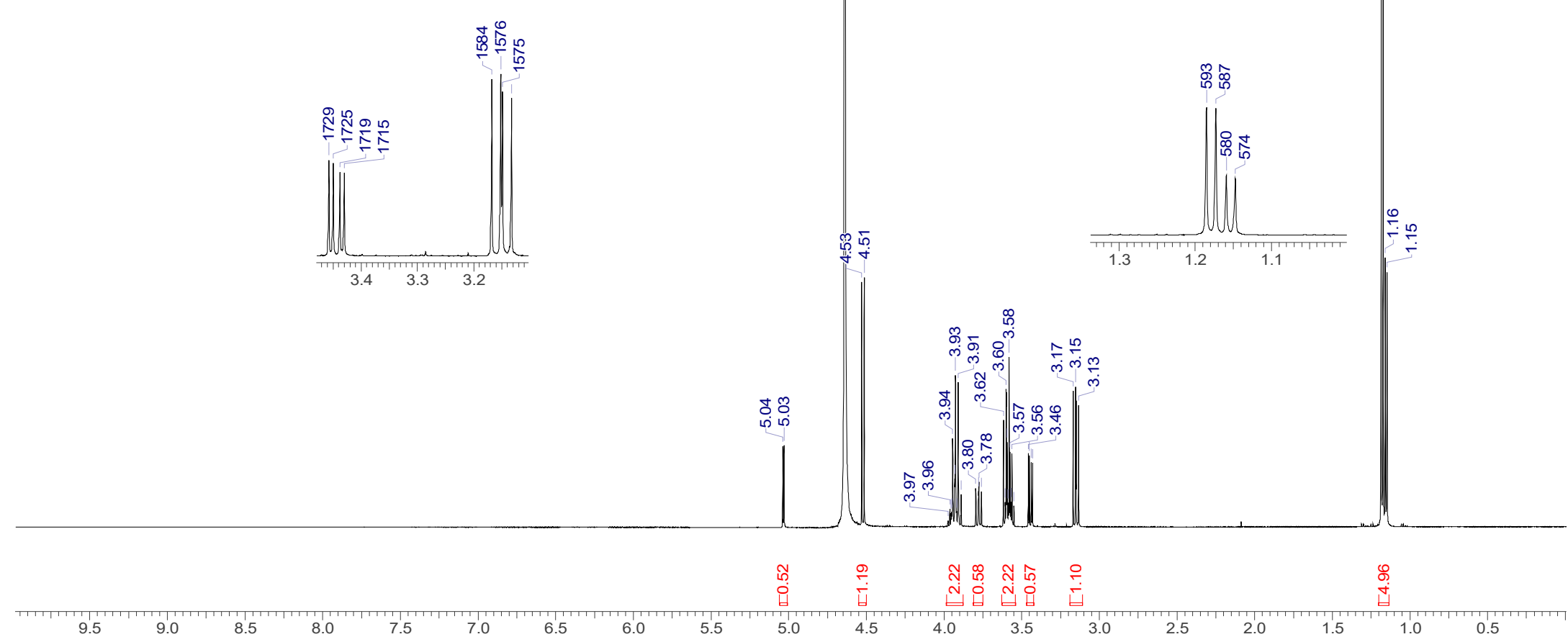
4.4.3. ${ }^{13} \mathrm{C}\left\{{ }^{1} \mathrm{H}\right\} \operatorname{NMR}\left(126 \mathrm{MHz}, \mathrm{D}_{2} \mathrm{O}\right)$
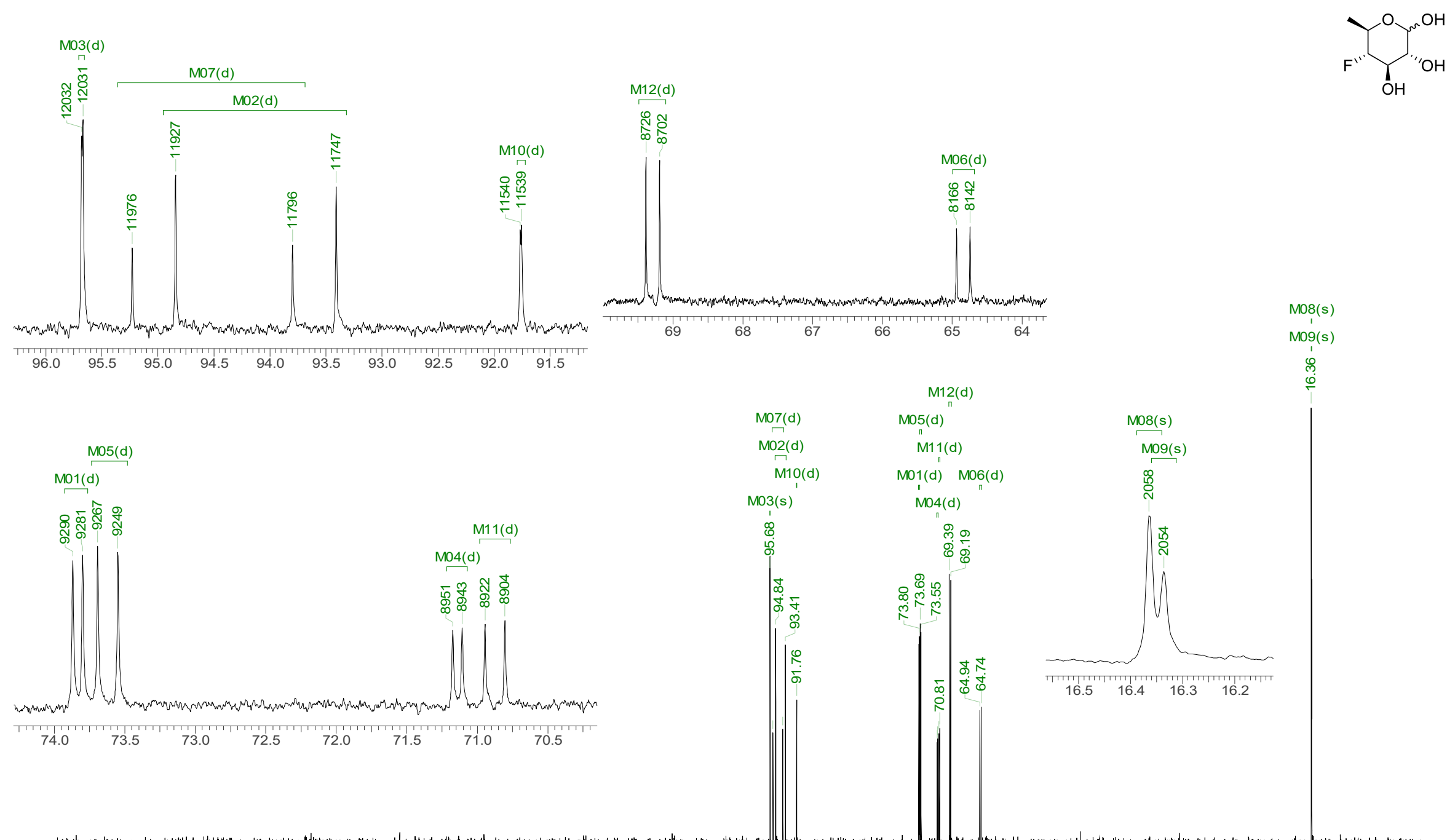

M08(s)

M09(s)

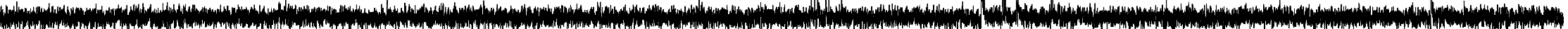




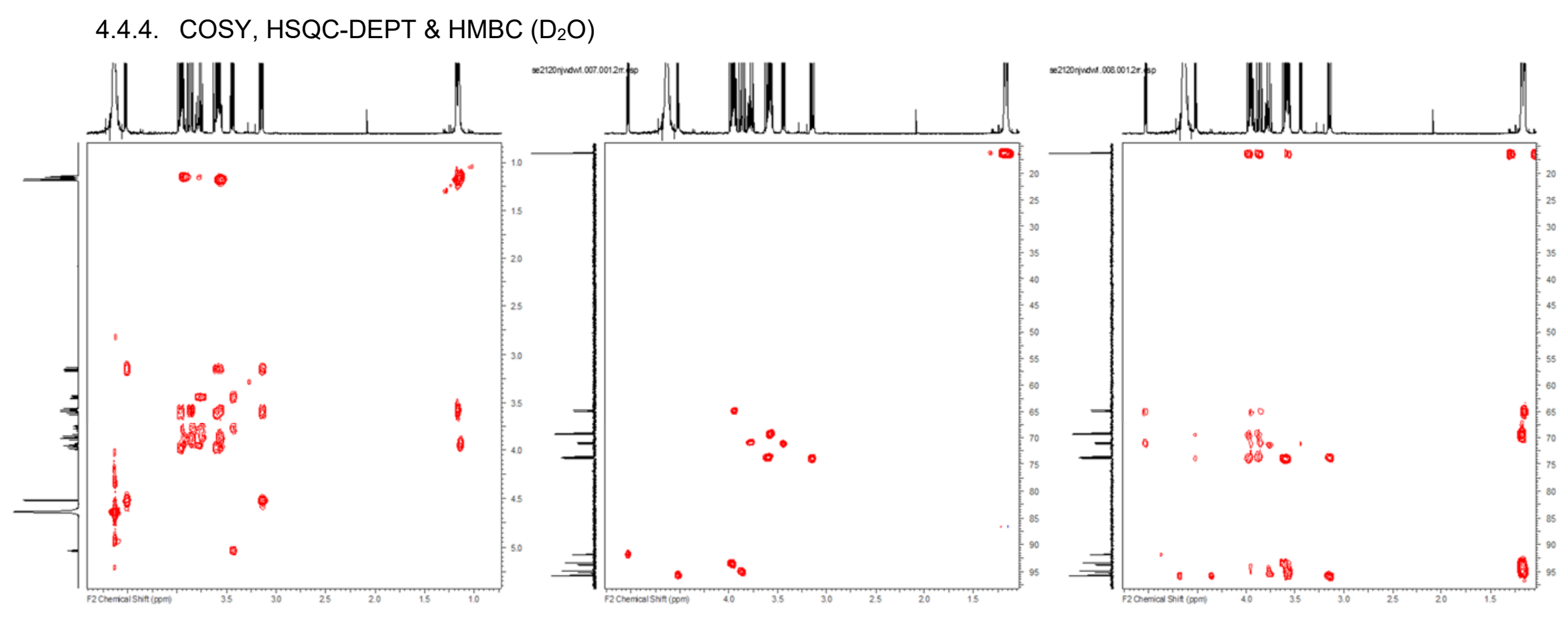


4.4.5. ${ }^{19} \mathrm{~F} \mathrm{NMR}\left(470 \mathrm{MHz}, \mathrm{D}_{2} \mathrm{O}\right)$

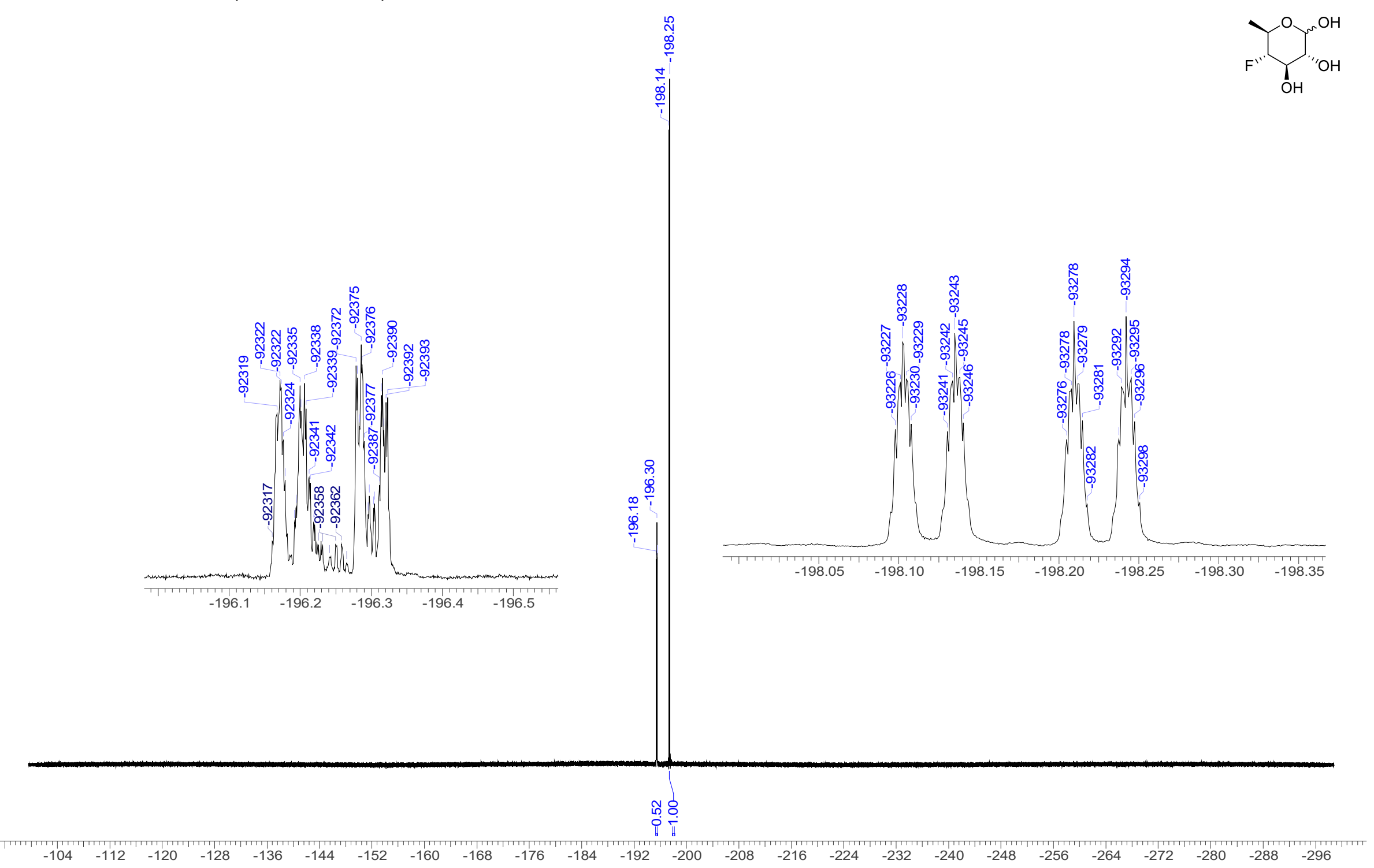


4.4.6. ${ }^{19} \mathrm{~F}\left\{{ }^{1} \mathrm{H}\right\} \mathrm{NMR}\left(470 \mathrm{MHz}, \mathrm{D}_{2} \mathrm{O}\right)$

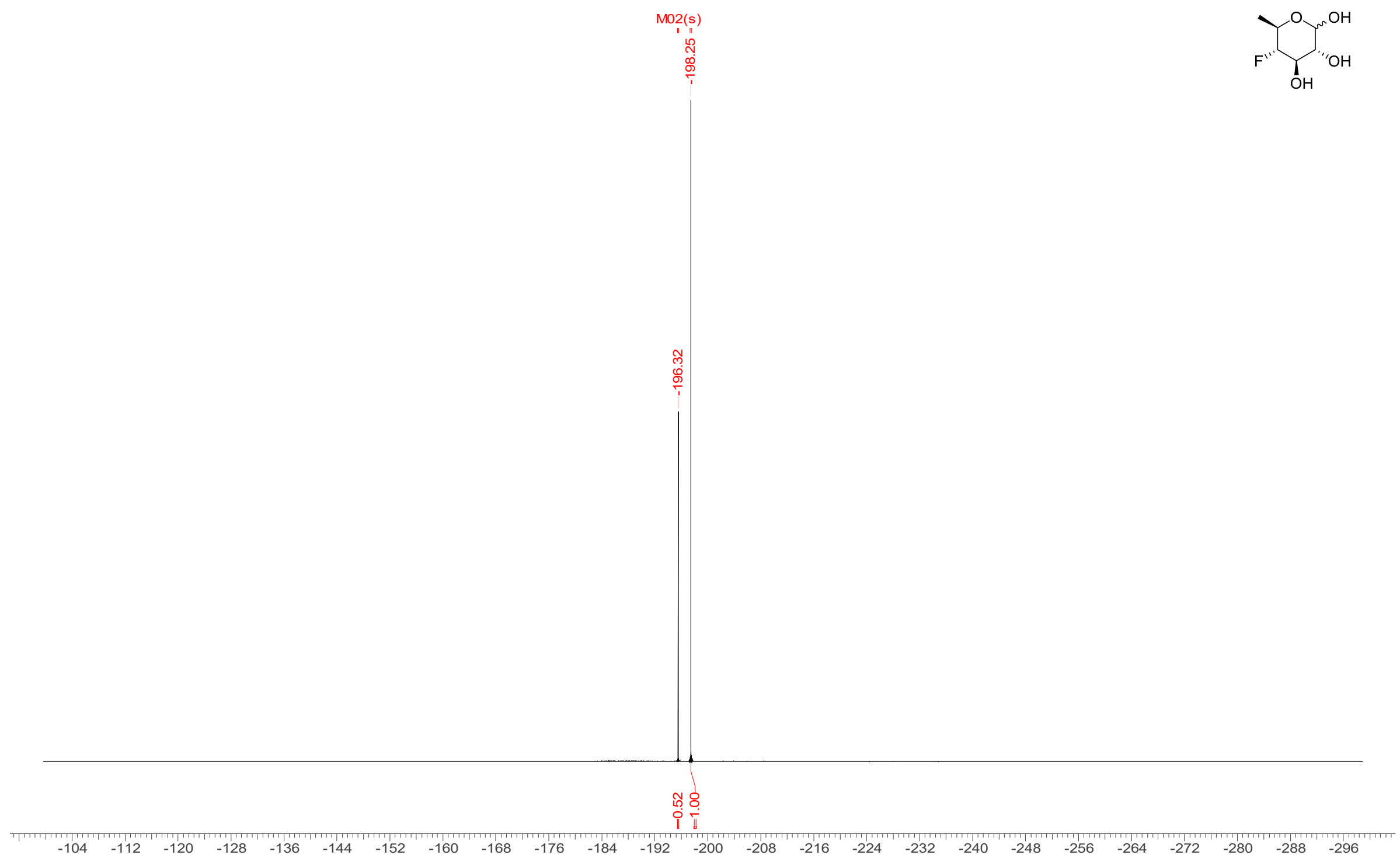


4.4.7. ${ }^{19} \mathrm{~F}\left\{{ }^{1} \mathrm{H}\right\}$ qNMR $\left(376 \mathrm{MHz}, \mathrm{D}_{2} \mathrm{O}\right)$

Duplicate \#1

DW/4,6 V2 D2O An1 qNMR

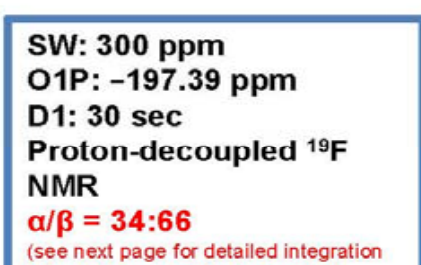

$\alpha / \beta=34: 66$
(see next page for detailed integration
information after data processing)

$$
\mathrm{OH}_{\mathrm{OH}}^{\mathrm{O}} \mathrm{OH}
$$

(8) 9

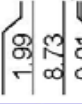

$0-50$

$-100$

$-150$

$-200$

ppm

\section{AVIIIHD400 [3]}

Current Data Parameters
NAME au2520dw4

EXPNO

F2 - Acquisition Parameters

Date_ 20200826

INSTRUM $8.49 \mathrm{~h}$

PROBHD Z116098_0218(

PULPROG zgfhiggn.

$\begin{array}{ll}\text { SOLVENT } & 131072 \\ \text { NS } & 640\end{array}$

NS 64

SWH

FIDRES $\quad 113636.367 \mathrm{~Hz}$

AQ $\quad 0.5767168 \mathrm{sec}$

RG 212.69

DE $\quad 6.400$ usec

6.50
$298.0 \mathrm{~K}$

$30.00000000 \mathrm{sec}$

$0.00002000 \mathrm{sec}$

$\begin{array}{lc}\text { SFO1 } & 376.4240491 \mathrm{MHz} \\ \text { NUC1 } & 19 \mathrm{~F}\end{array}$

$\begin{array}{ll}\text { P1 } & 14.00 \text { usec } \\ \text { PLW1 } & 22.69199944 \mathrm{~W}\end{array}$

SFO2 $400.1316005 \mathrm{MHz}$

CPDPRG[2 waltz16

PCPD2 90.00 usec

PLW2 16.38100052 W

F2 - Processing parameters

$\begin{array}{ll}\text { SF } & 376.4984132 \mathrm{MHz}\end{array}$

WDW $\quad$ EM

$\begin{array}{lc}\text { SSB } & 0 \\ \text { LB } & 0.30 \mathrm{~Hz}\end{array}$

$\begin{array}{ll}\text { GB } & 0 \\ \text { PC } & 100\end{array}$ 
Duplicate \#1

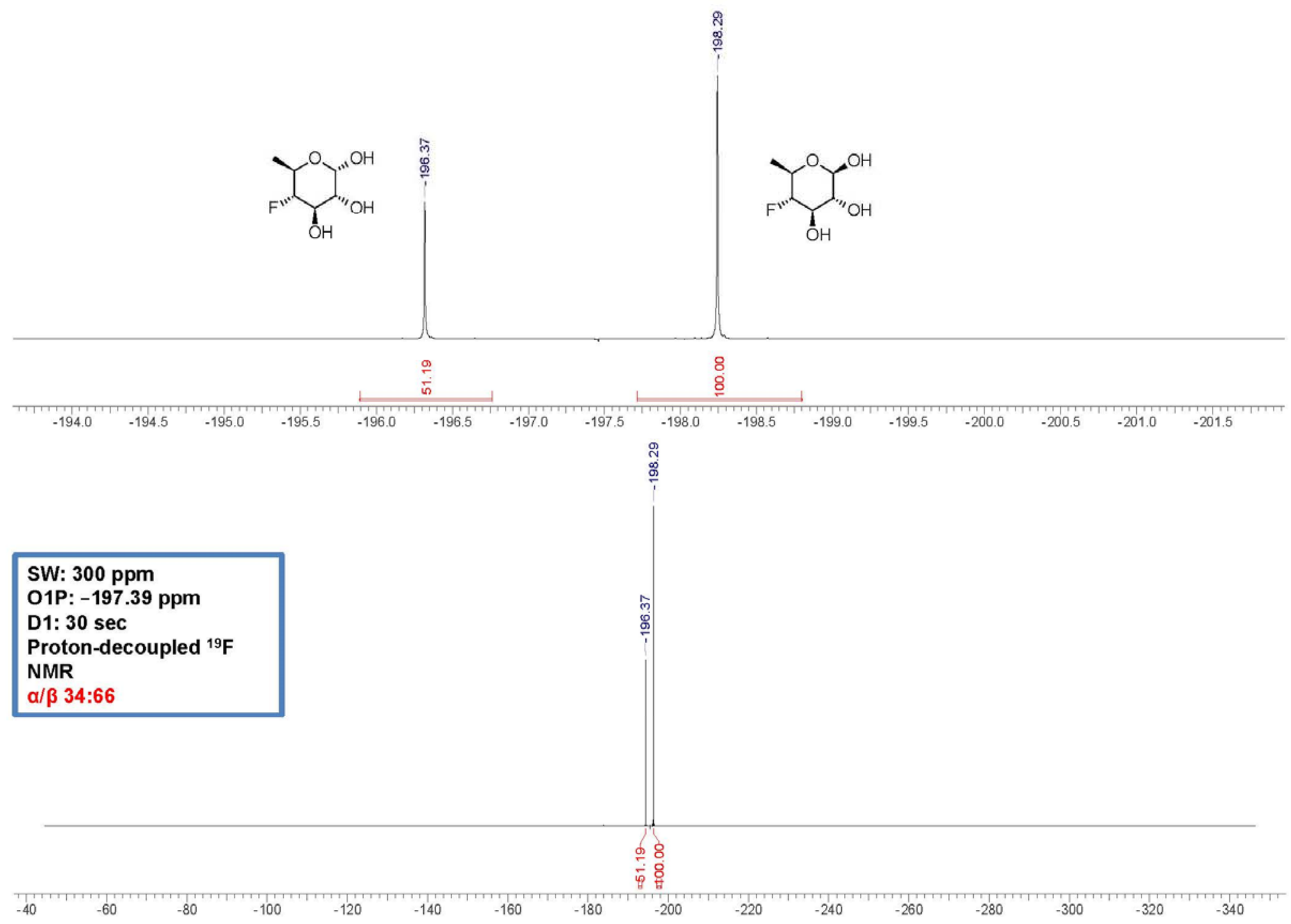




\section{Duplicate \#2}

DW/4,6 V2 D2O An2 qNMR

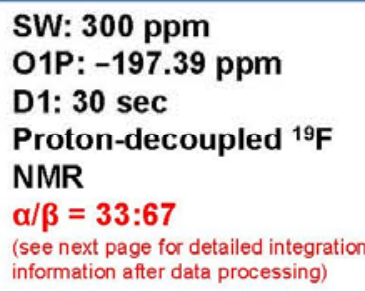

D1: 30 sec

Proton-decoupled ${ }^{19} \mathrm{~F}$

NMR

$\alpha / \beta=33: 67$

(see next page for detailed integration

information after data processing)
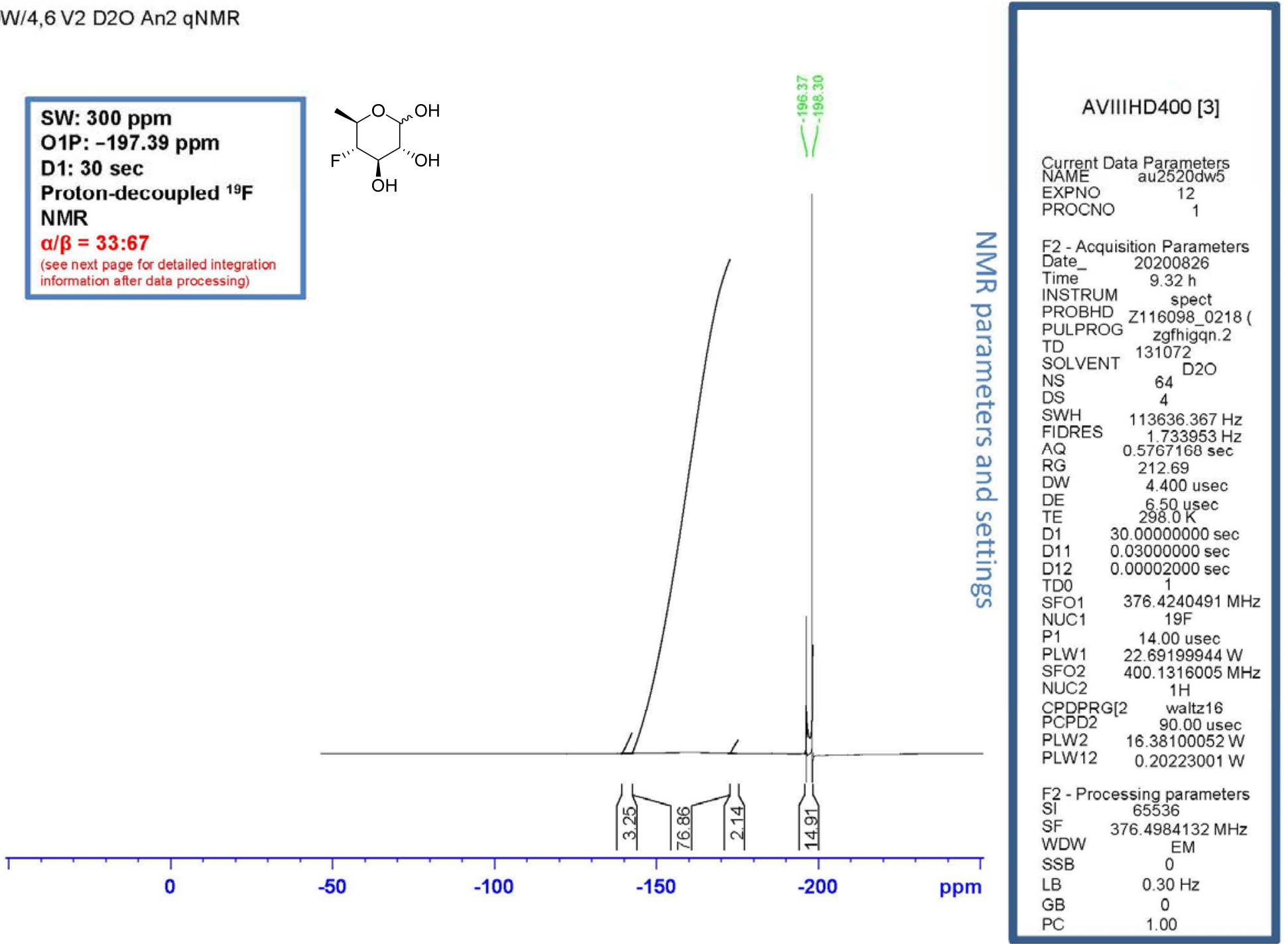
Duplicate \#2

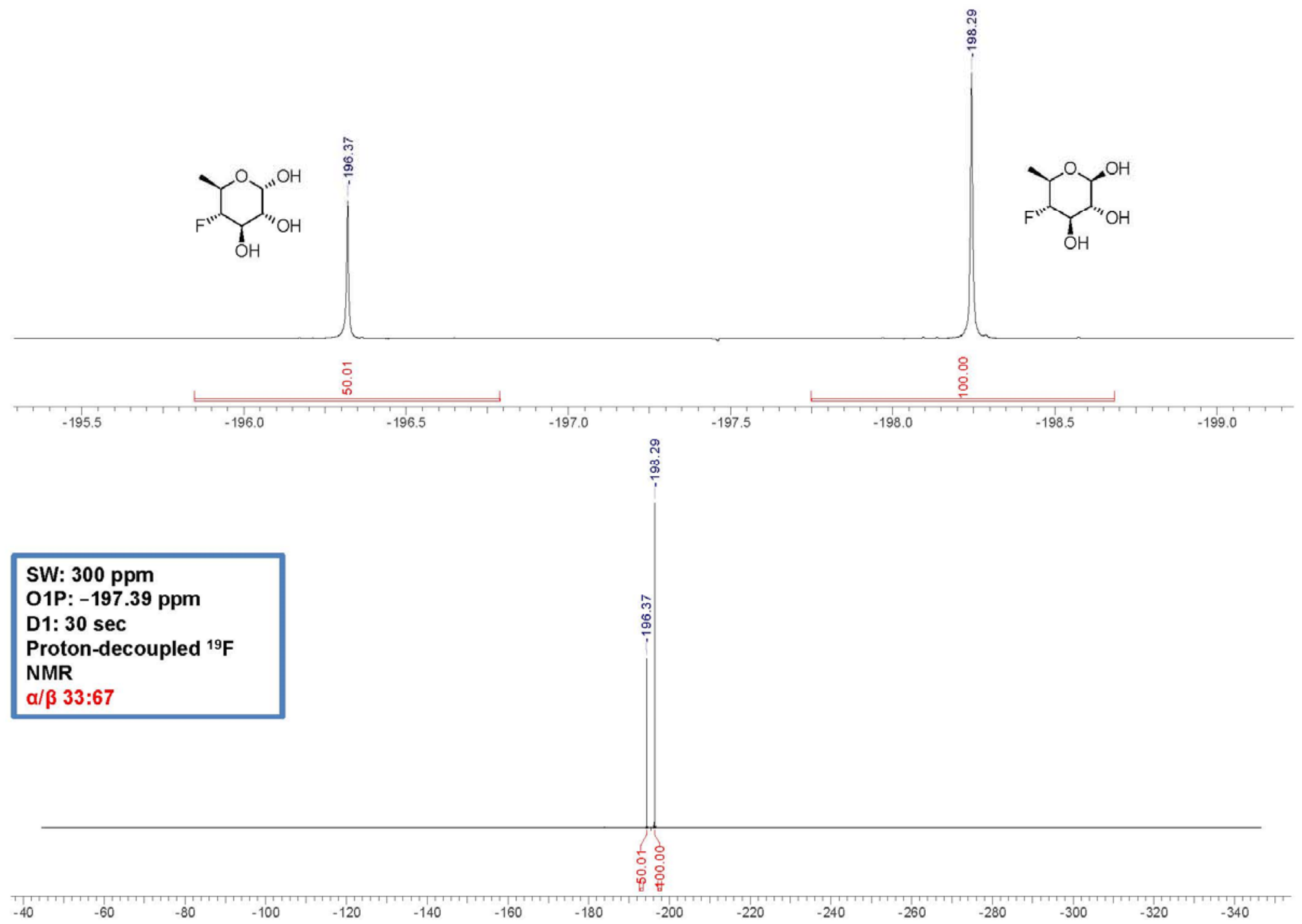




\subsection{4,6-Dideoxy-4,6-difluoro-D-galactose (4a)}

4.5.1. ${ }^{1} \mathrm{H}$ NMR $\left(500 \mathrm{MHz}, \mathrm{D}_{2} \mathrm{O}\right)$
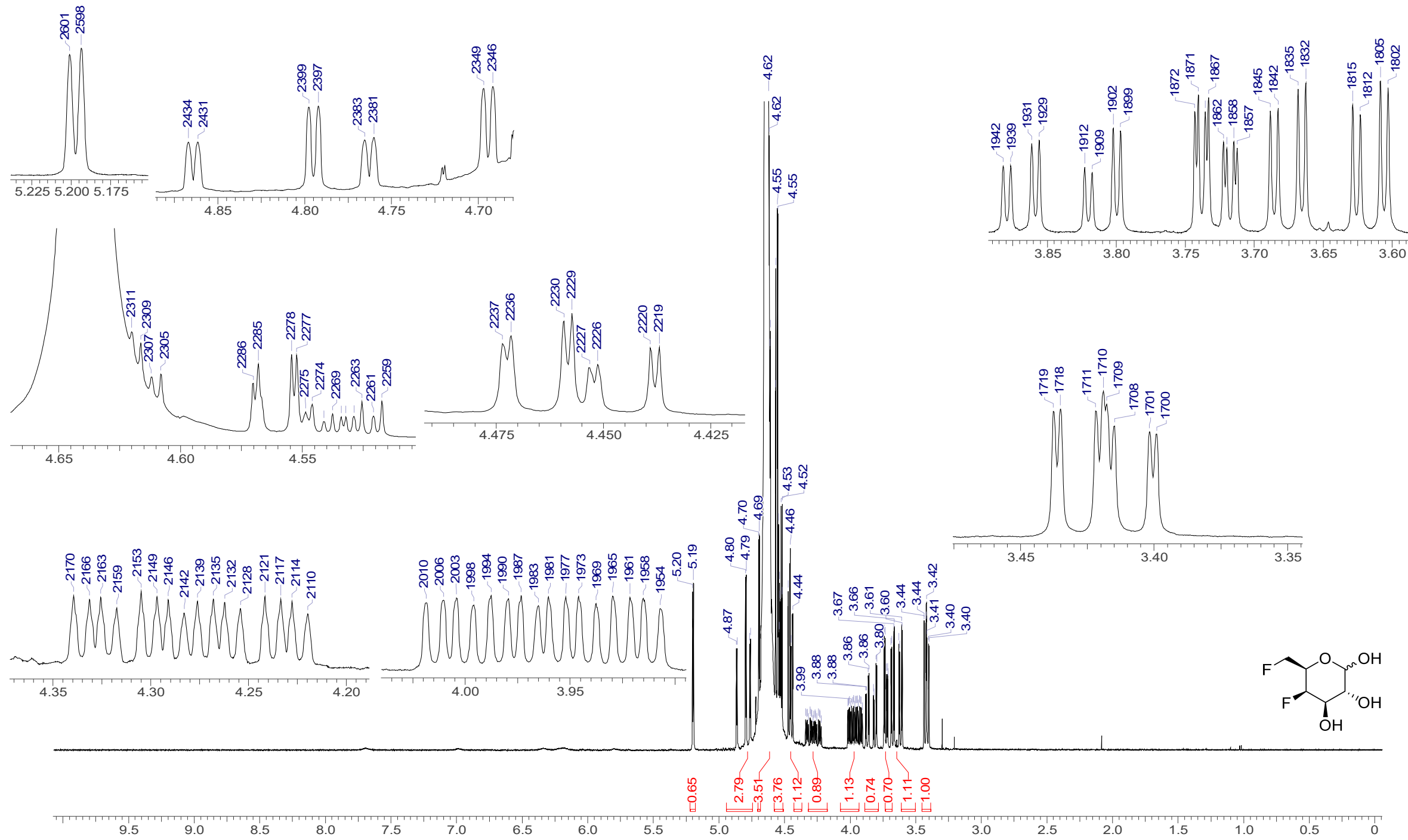
4.5.2. ${ }^{1} \mathrm{H}\left\{{ }^{19} \mathrm{~F}\right\} \mathrm{NMR}\left(500 \mathrm{MHz}, \mathrm{D}_{2} \mathrm{O}\right)$
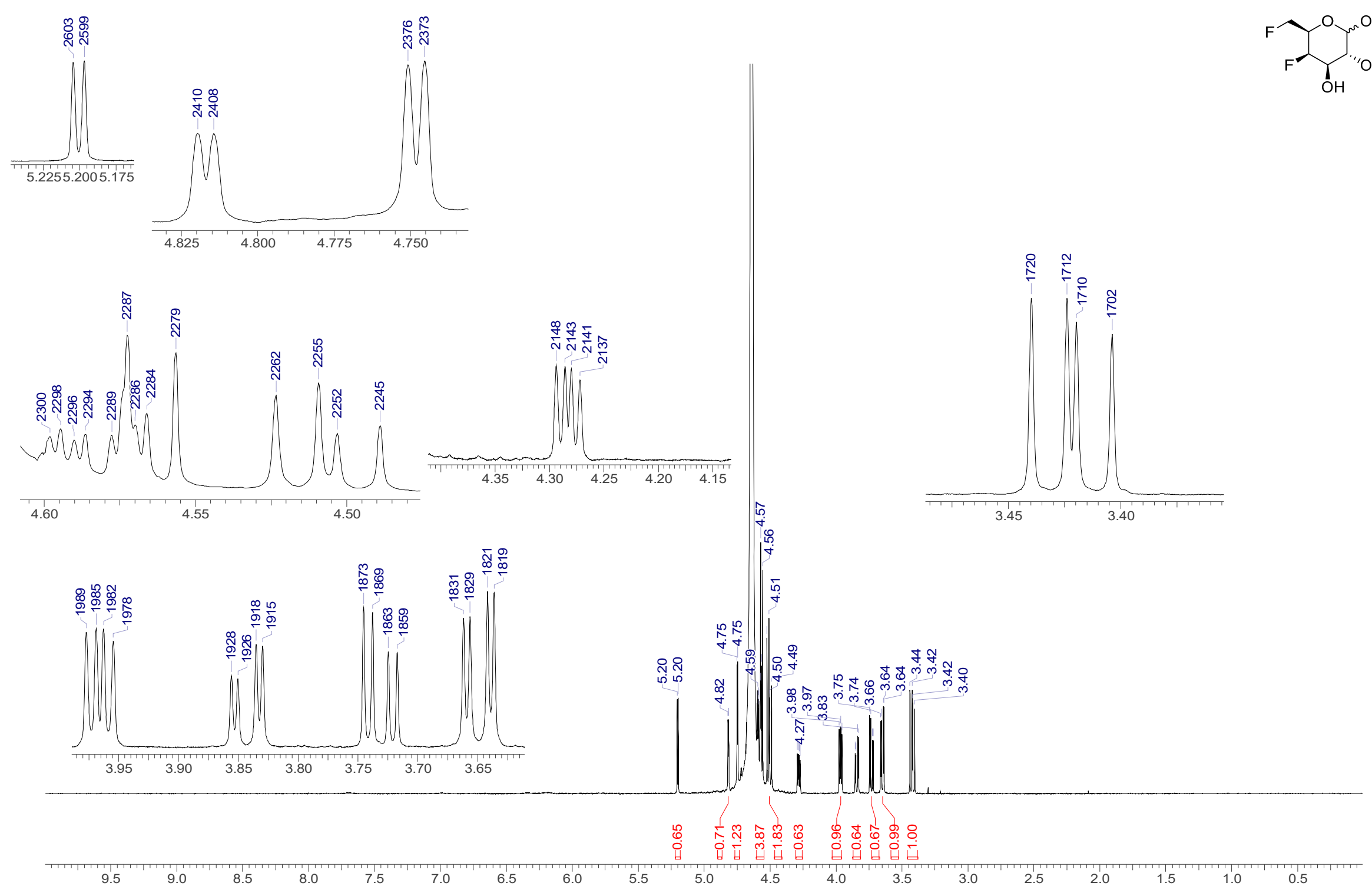
4.5.3. ${ }^{13} \mathrm{C}\left\{{ }^{1} \mathrm{H}\right\}$ NMR $\left(126 \mathrm{MHz}, \mathrm{D}_{2} \mathrm{O}\right)$
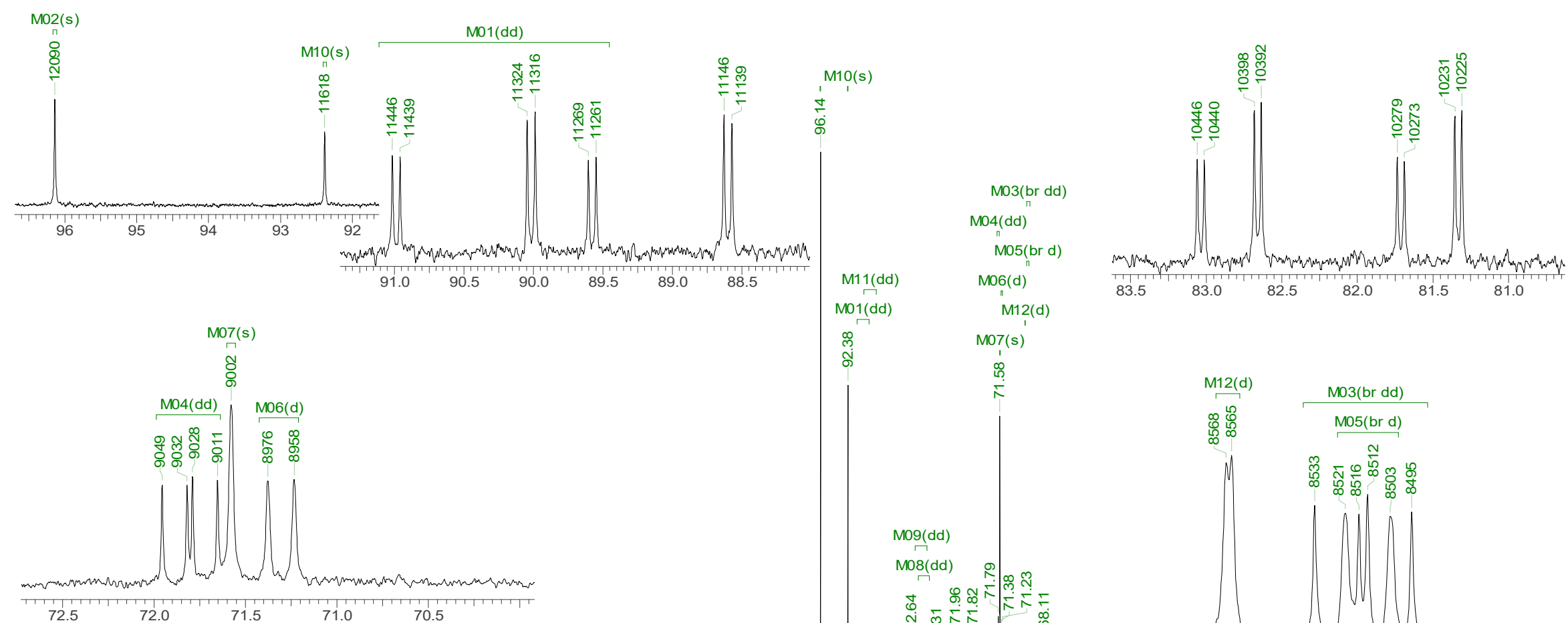

ले

$\mathrm{MO7}(\mathrm{s})$

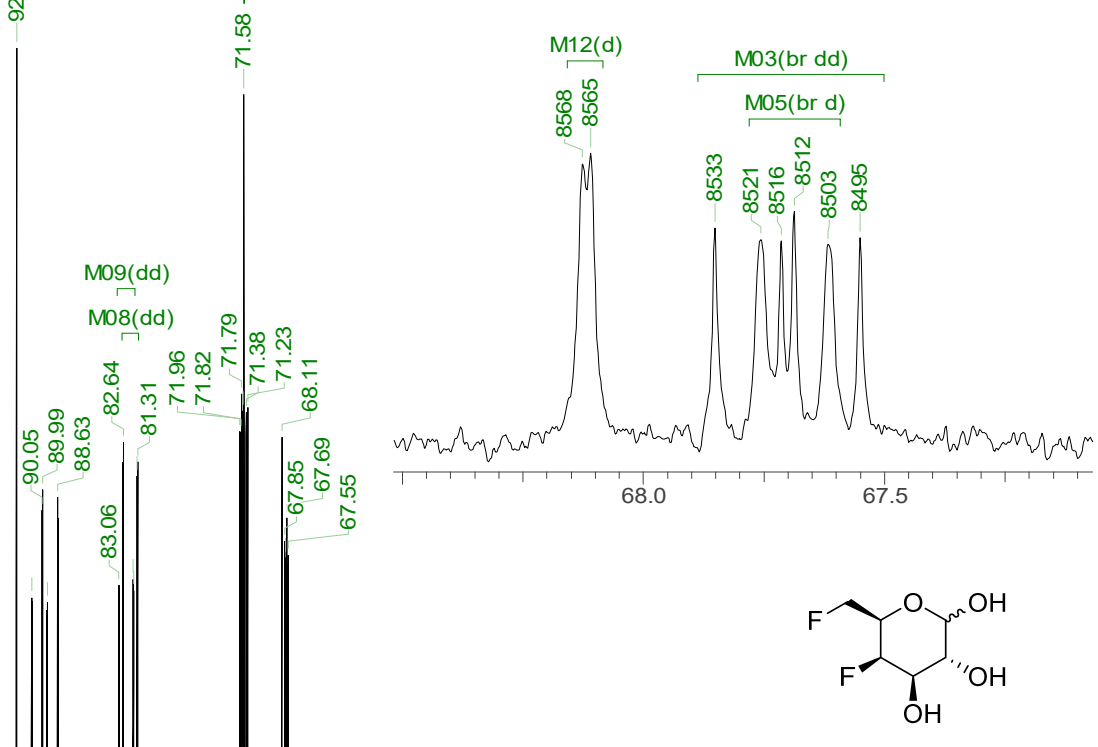

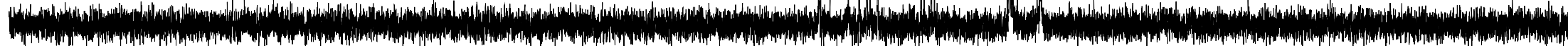

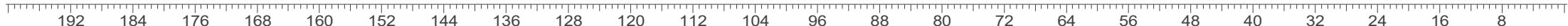


4.5.4. COSY, HSQC-DEPT \& HMBC $\left(D_{2} O\right)$
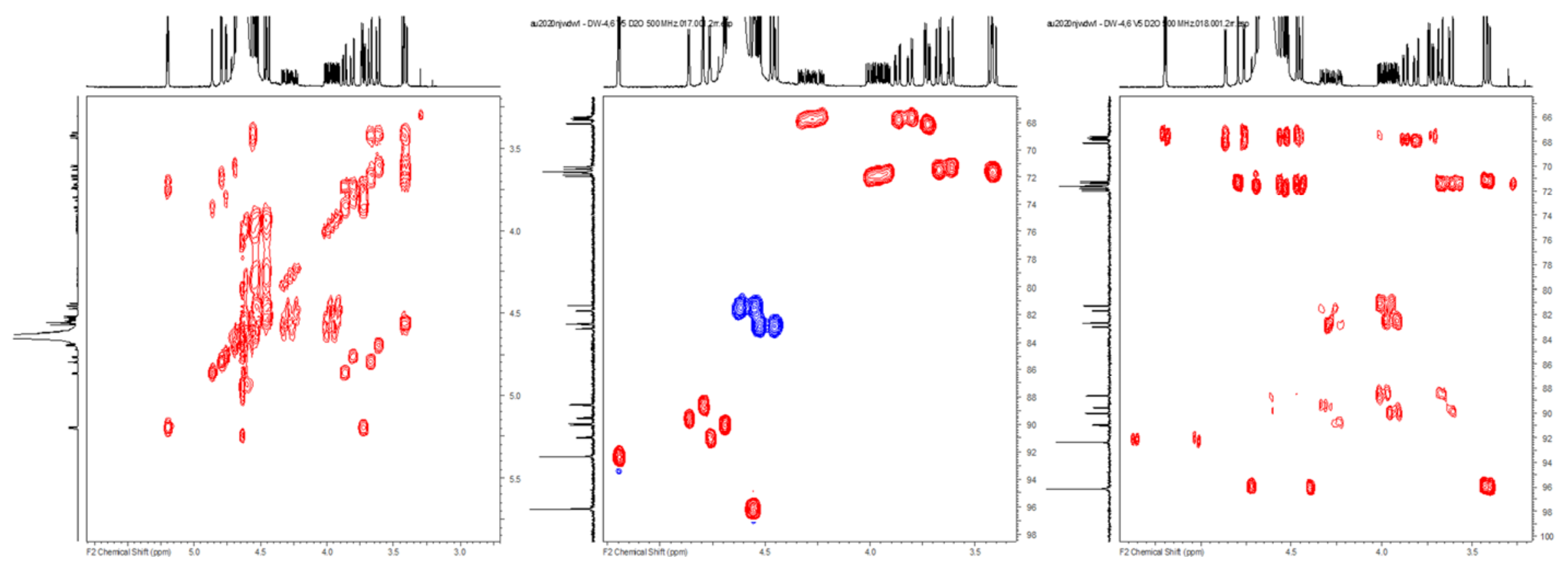
S 289

4.5.5. ${ }^{19} \mathrm{~F} \mathrm{NMR}\left(470 \mathrm{MHz}, \mathrm{D}_{2} \mathrm{O}\right)$
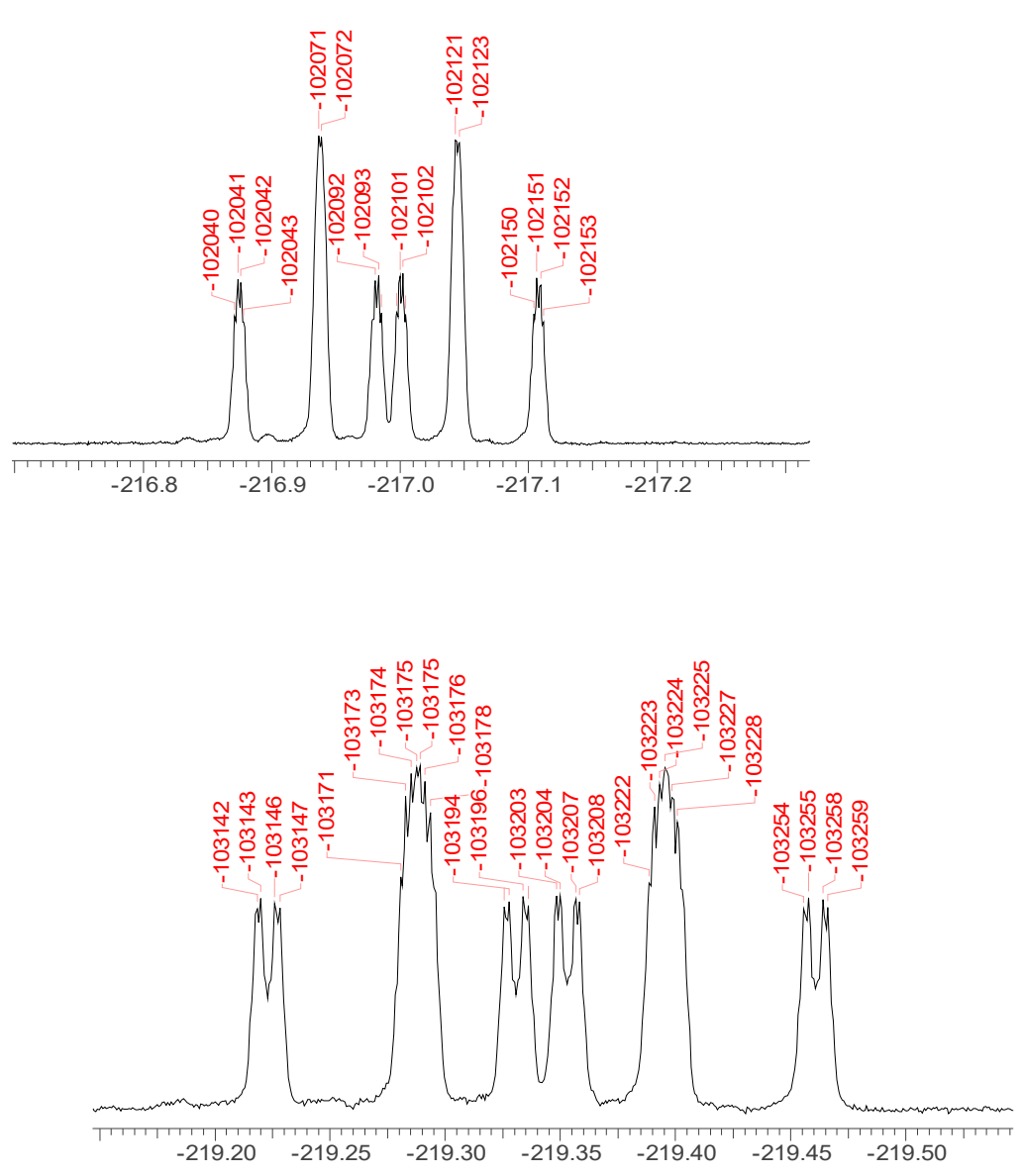<smiles>OC1O[C@H](O)[C@H](O)C(O)C1F</smiles>

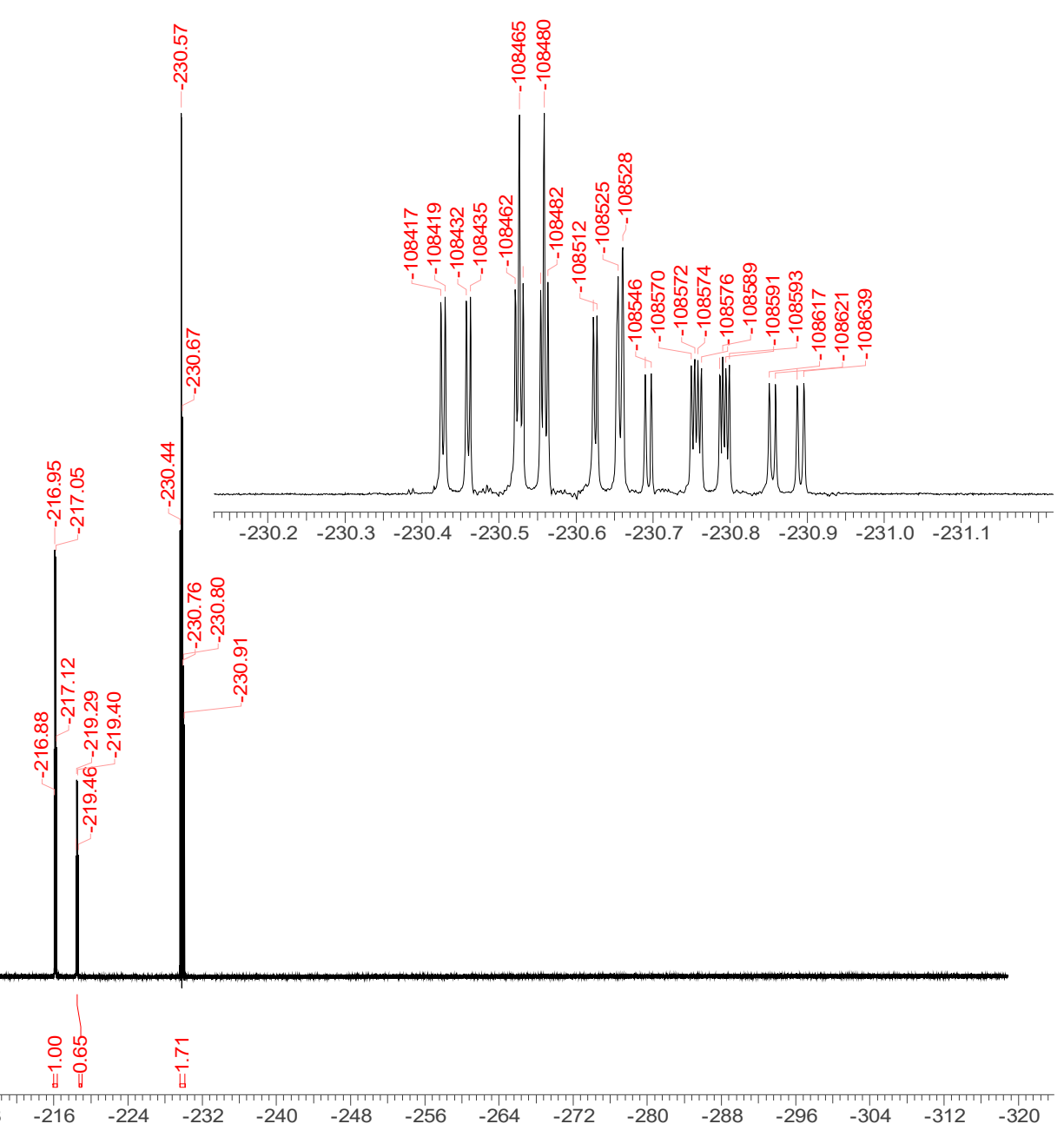


4.5.6. ${ }^{19} \mathrm{~F}\left\{{ }^{1} \mathrm{H}\right\} \mathrm{NMR}\left(470 \mathrm{MHz}, \mathrm{D}_{2} \mathrm{O}\right)$

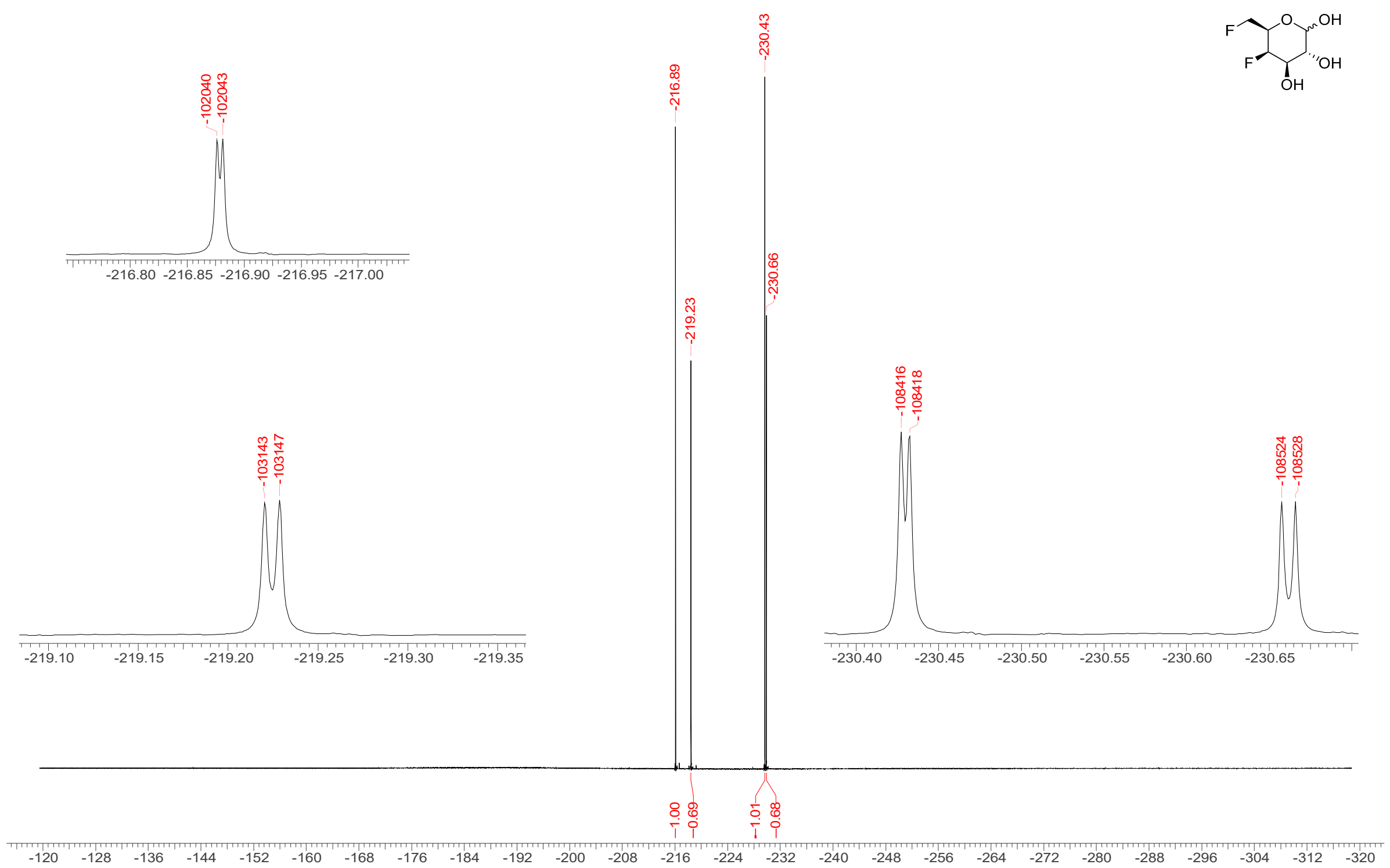




\subsection{7. ${ }^{1} \mathrm{H}$ NMR $\left(500 \mathrm{MHz}, \mathrm{D}_{2} \mathrm{O}\right.$, Spectral Width $\left.=\mathbf{6 . 0} \mathbf{p p m}\right)$}

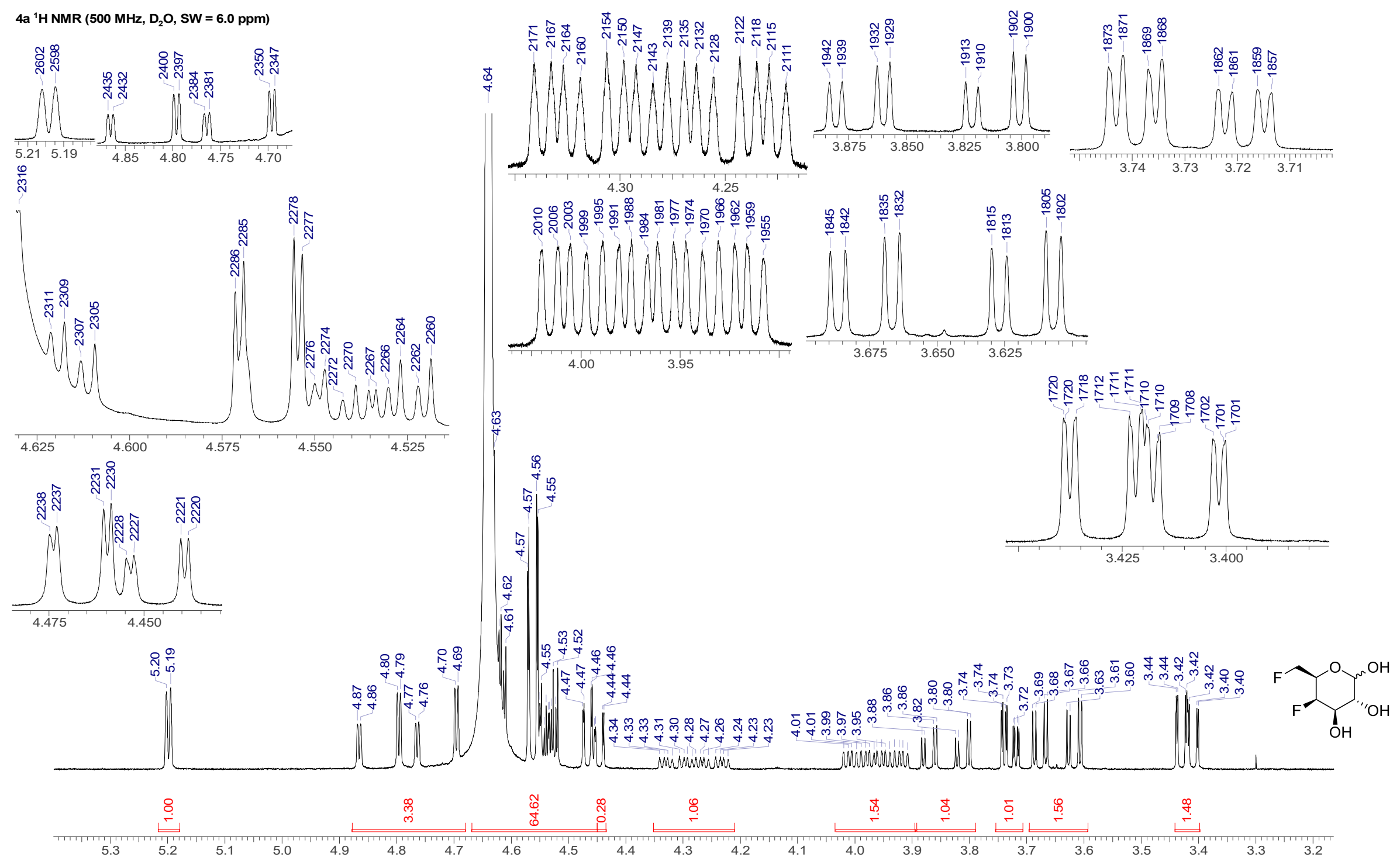


4.5.8. ${ }^{1} \mathrm{H}\left\{{ }^{19} \mathrm{~F}\right\} \mathrm{NMR}\left(500 \mathrm{MHz}, \mathrm{D}_{2} \mathrm{O}\right.$, Spectral Width $\left.=\mathbf{6 . 0} \mathbf{p p m}\right)$

$4 a^{1} \mathrm{H}^{19}{ }^{19} \mathrm{~F}$ NMR $\left(500 \mathrm{MHz}, \mathrm{D}_{2} \mathrm{O}, \mathrm{SW}=6.0 \mathrm{ppm}\right)$

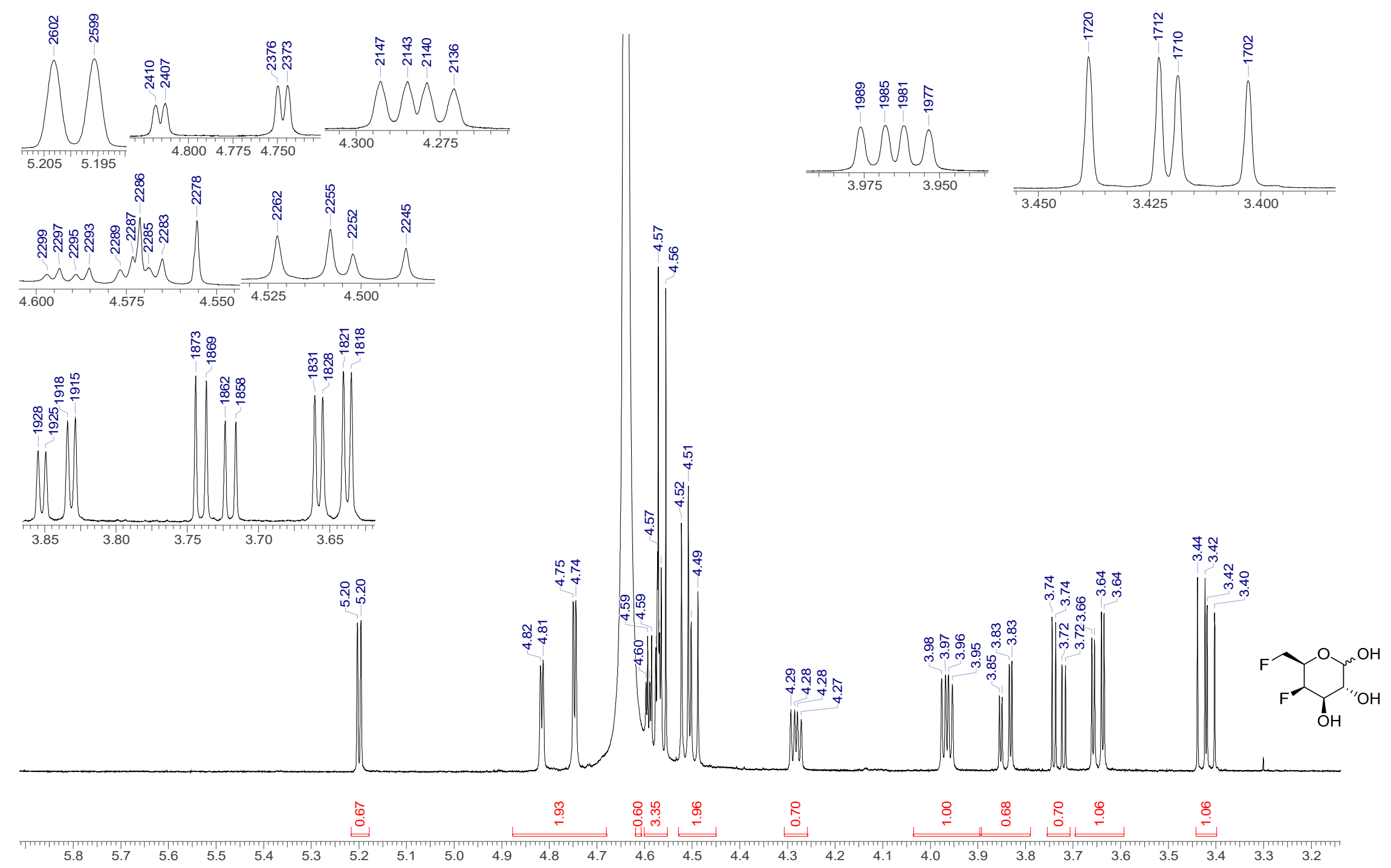


4.5.9. ${ }^{19} \mathrm{~F}$ NMR $\left(470 \mathrm{MHz}, \mathrm{D}_{2} \mathrm{O}\right.$, Spectral width $\left.=\mathbf{3 0 . 0} \mathbf{p p m}\right)$

4a ${ }^{19} \mathrm{~F}$ NMR $\left(470 \mathrm{MHz}, \mathrm{D}_{2} \mathrm{O}, \mathrm{SW}=30.0 \mathrm{ppm}\right)$

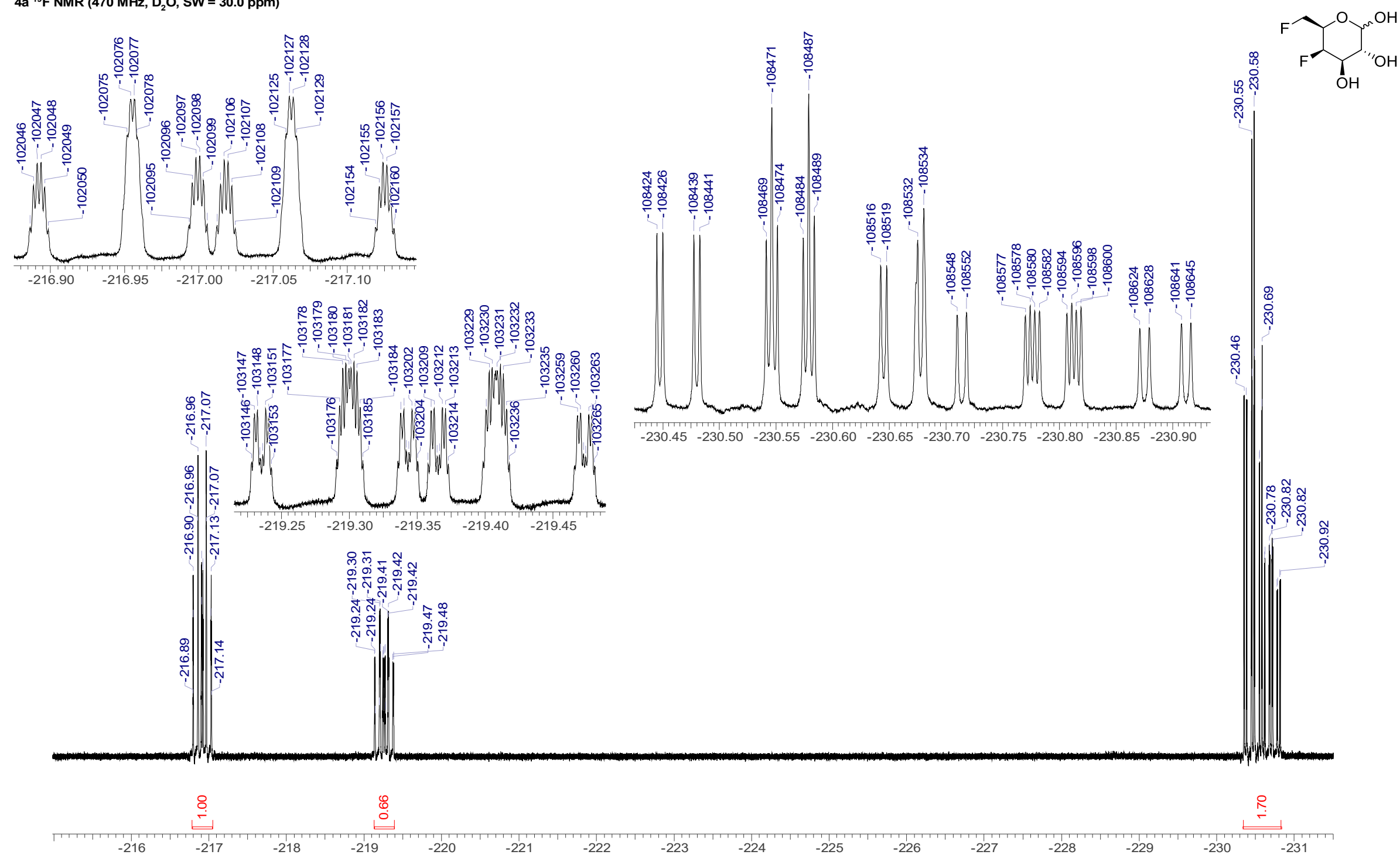




\subsubsection{0. $\operatorname{COSY}\left(D_{2} O\right.$, Optimized for long-range couplings)}

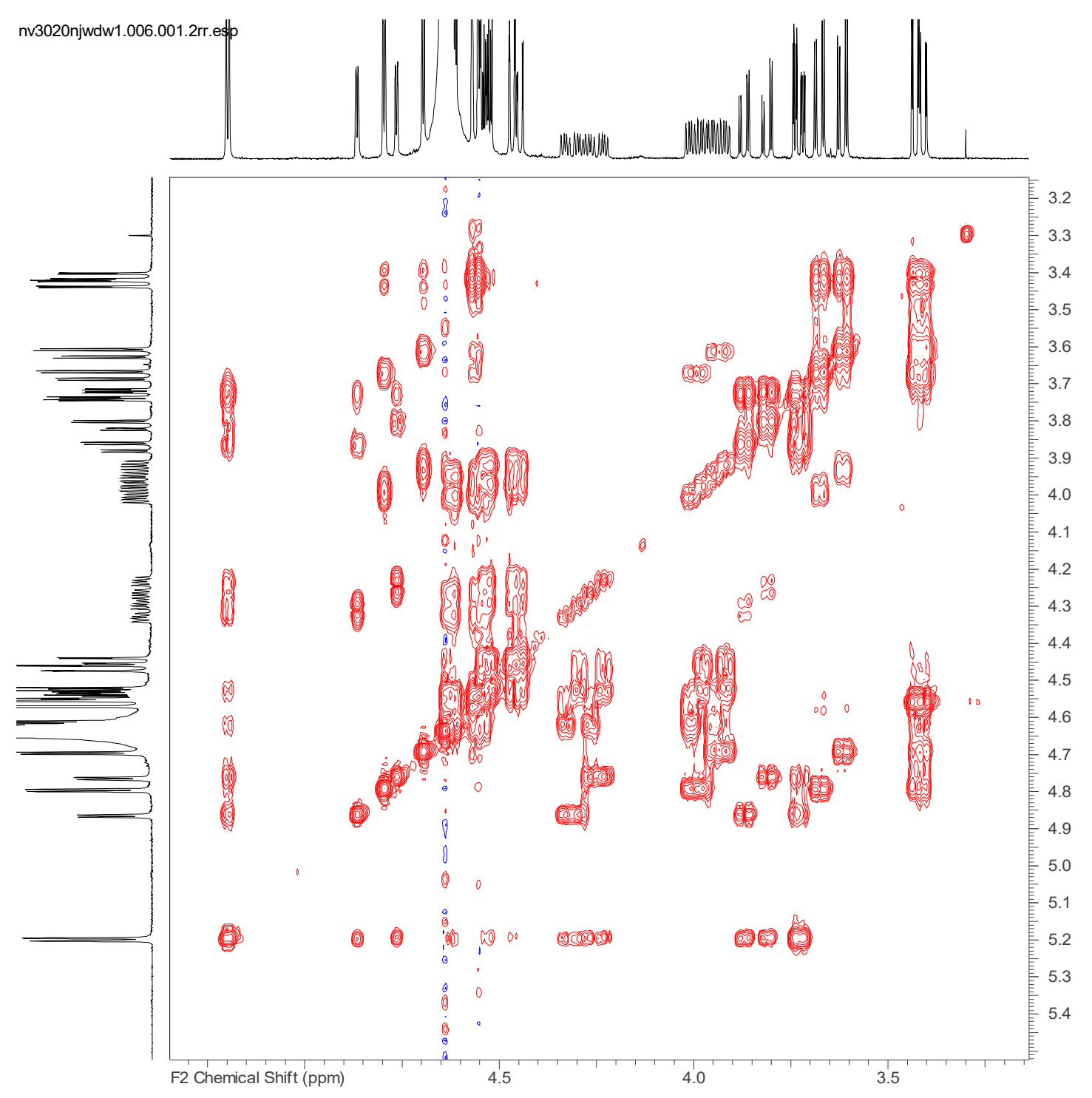


4.5.11. ${ }^{19} \mathrm{~F}\left\{{ }^{1} \mathrm{H}\right\}$ qNMR $\left(376 \mathrm{MHz}, \mathrm{D}_{2} \mathrm{O}\right)$

Duplicate \#1

DW/4,6 V5 D2O Equilibrated qNMR 19F $\{1 \mathrm{H}\}$ An1

\section{SW: 300 ppm}

O1P: $-223.97 \mathrm{ppm}$

D1: 60 sec

Proton-decoupled ${ }^{19} \mathrm{~F}$

NMR

$\alpha / \beta$ 40:60

(see next page for detailed integration

information after data processing)

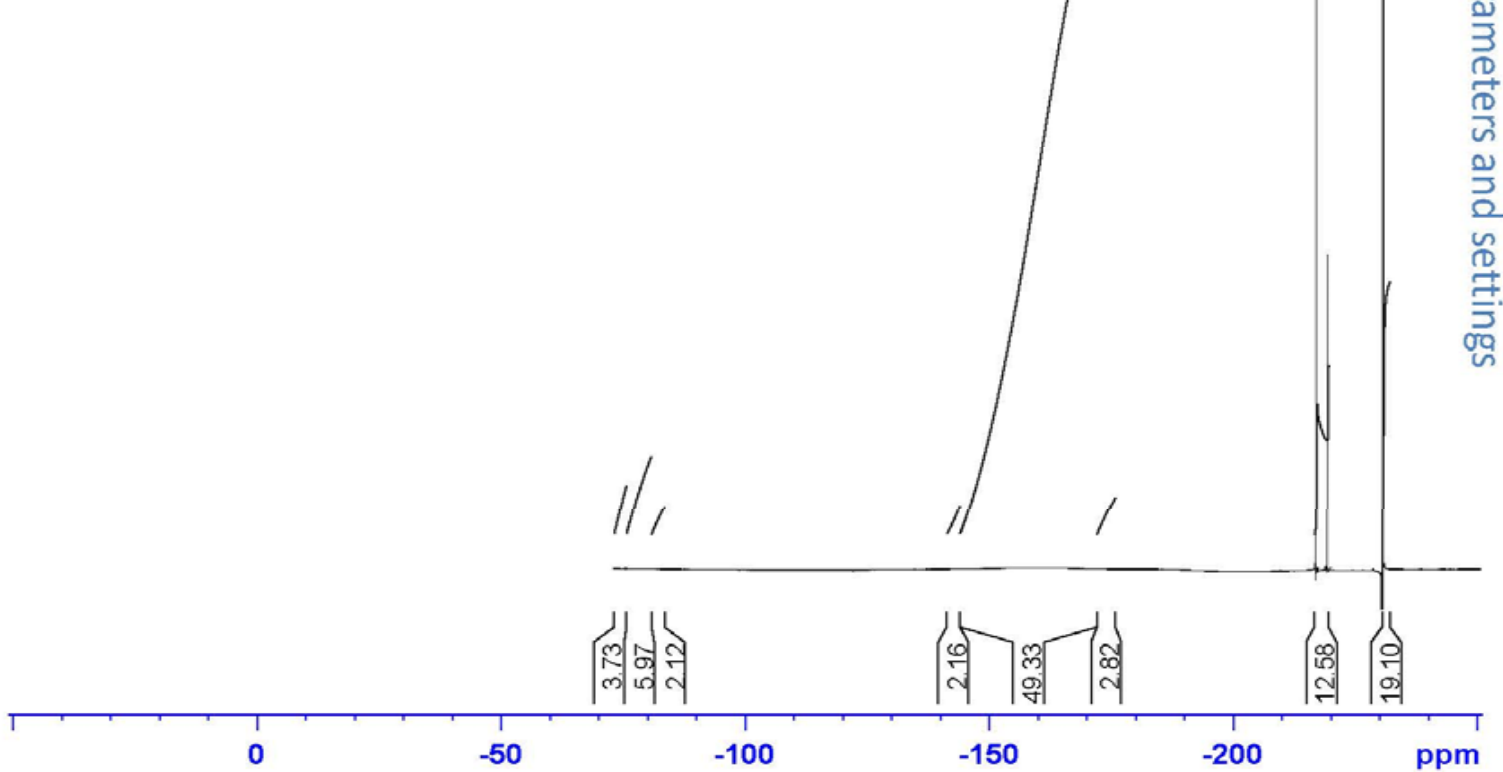

AVIIIHD400 [3]

Current Data Parameters

EXPNO

F2 - Acquisition Parameters

Date_ 20200730

Time $-\quad 5.55 \mathrm{~h}$

INSTRUM spect
PROBHD Z116098_0218
PULPROG

$\begin{array}{ll}\text { TD } & \text { zgfhigan.2 } \\ \text { SOLVNT } & 131072\end{array}$

NS $\quad 64$ D2

SWH

SWH $113636367 \mathrm{~Hz}$

FIDRES

$113636.367 \mathrm{~Hz}$
$1.733953 \mathrm{~Hz}$

$0.5767168 \mathrm{se}$

212.69
$4.400 \mathrm{usec}$

650 usec

$298.0 \mathrm{~K}$

$60.00000000 \mathrm{sec}$

$0.00002000 \mathrm{sec}$

376. $4140417 \mathrm{MHz}$$$
19 \mathrm{~F}
$$

14.00 usec $400.1316005 \mathrm{MHz}$

NUC2 $1 \mathrm{H}$

CPDPRG[2 waltz16

PLW2 $1638100052 \mathrm{~W}$

PLW12 $\quad 0.20223001 \mathrm{~W}$

F2 - Processing parameters

SF $\quad 376.4984132 \mathrm{MHz}$

WDW

SSB

SSB $\quad 0$

$\begin{array}{lc}\text { LB } & 0.30 \\ \text { GB } & 0\end{array}$ 
Duplicate \#1
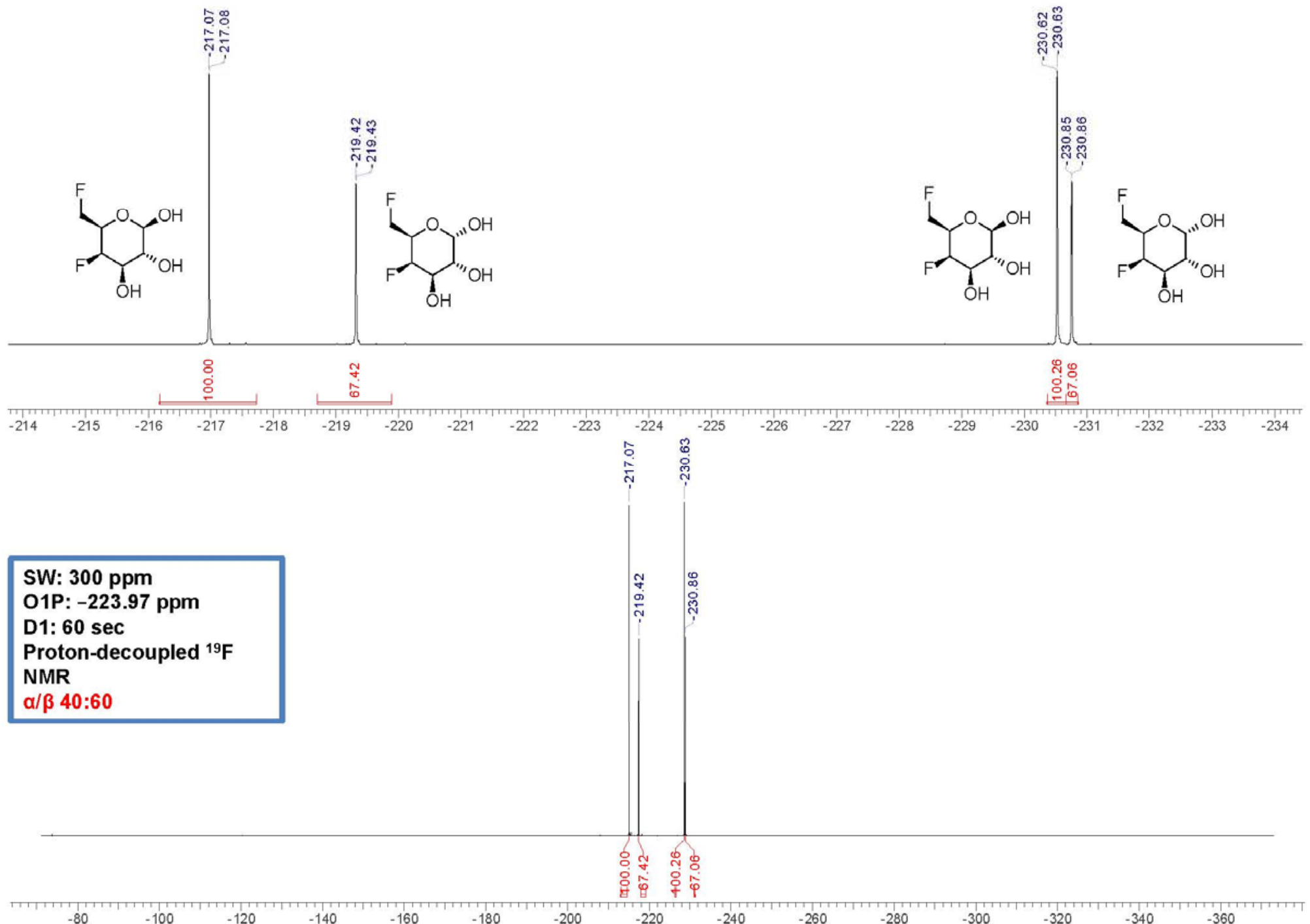


\section{Duplicate \#2}

DW/4,6 V5 D2O Equilibrated qNMR 19F $\{1 \mathrm{H}\}$ An2

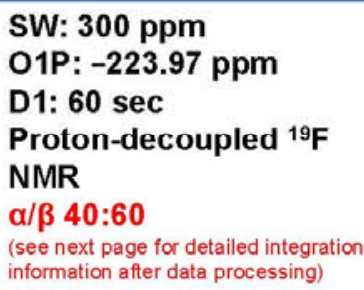

D1: $60 \mathrm{sec}$

Proton-decoupled ${ }^{19} \mathrm{~F}$

NMR

$\alpha / \beta$ 40:60

see next page for detailed integration

information after data processing)
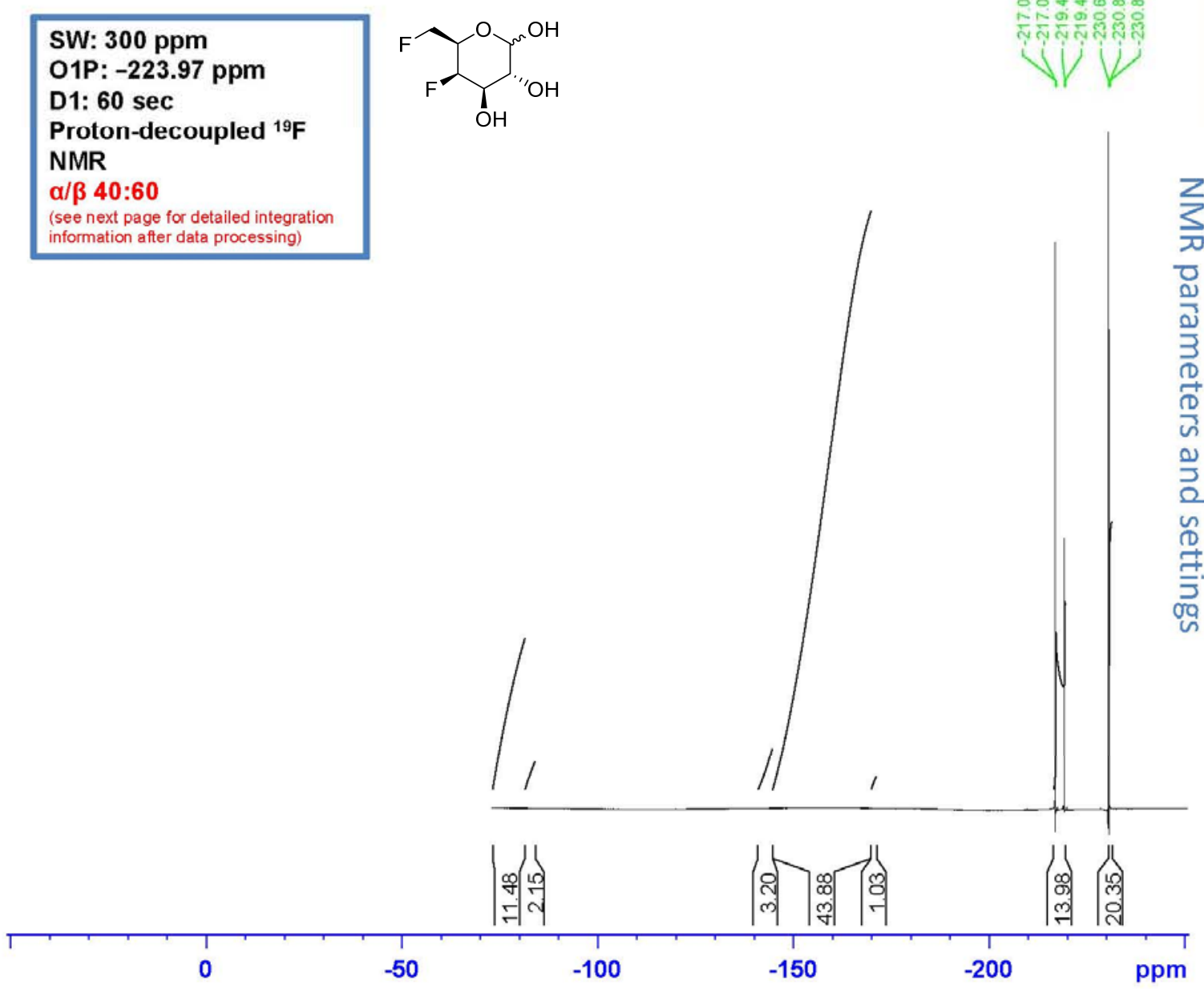

AVIIIHD400 [3]

Current Data Parameters
NAME

$\begin{array}{ll}\text { EXPNO } & 10 \\ \text { PROCNO } & 1\end{array}$

10

F2 - Acquisition Parameters

Date_ 20200730

$7.12 \mathrm{~h}$

spect

PULPROG Z116098_0218

TD 131072 zgn.

SOLVENT 131072

$\begin{array}{lc}\text { NS } & 64 \\ \text { DS } & 4\end{array}$

$\begin{array}{ll}\text { SWH } & 4 \\ \text { SW } & 113636.367 \mathrm{~Hz}\end{array}$

$1.733953 \mathrm{~Hz}$

DW 4.400 usec

DE $\quad 6.50$ usec

D1 $\quad 60.00000000 \mathrm{sec}$

D11 $0.03000000 \mathrm{sec}$

D12 $0.00002000 \mathrm{sec}$

TD0 $\quad 376.4140417 \mathrm{MH}$

NUC1 $19 \mathrm{~F}$

P1 14.00 usec

PLW1 22.69199944 W

SF2 $400.1316005 \mathrm{MHz}$

CPDPRG[2 waltz16

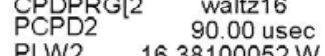

$\begin{array}{lr}\text { PLW2 } & 16.38100052 \mathrm{~W} \\ \text { PLW12 } & 0.20223001 \mathrm{~W}\end{array}$

F2 - Processing parameters

SF $376.4984132 \mathrm{MHz}$

SF $376.4984132 \mathrm{MHz}$

SSB EM

LB $\quad 0.30 \mathrm{~Hz}$

GB $\quad 1.00$ 


\section{Duplicate \#2}

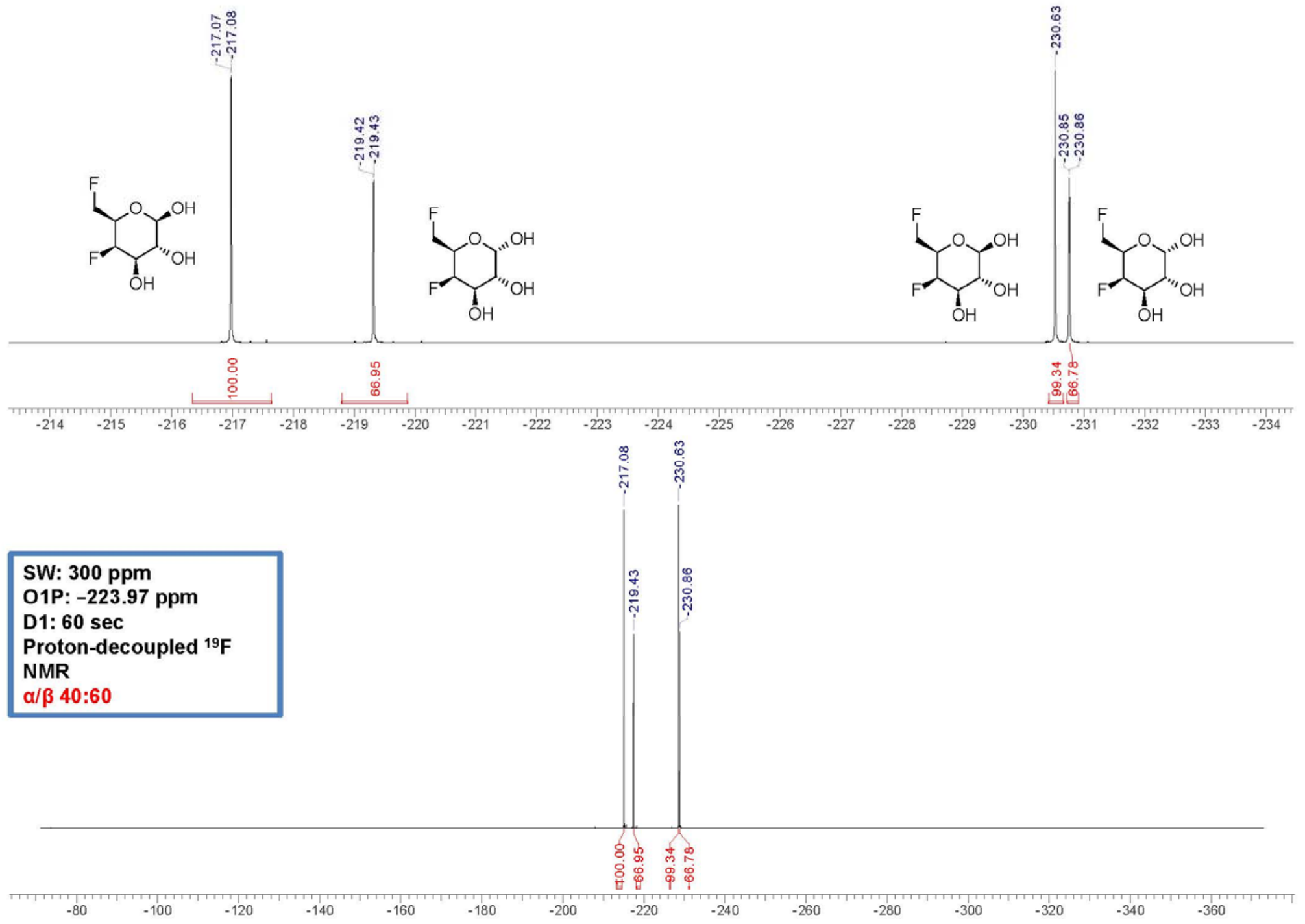




\subsection{4,6-Dideoxy-4,6-difluoro-D-glucose (5a)}

4.6.1. ${ }^{1} \mathrm{H}$ NMR $\left(500 \mathrm{MHz}, \mathrm{D}_{2} \mathrm{O}\right)$
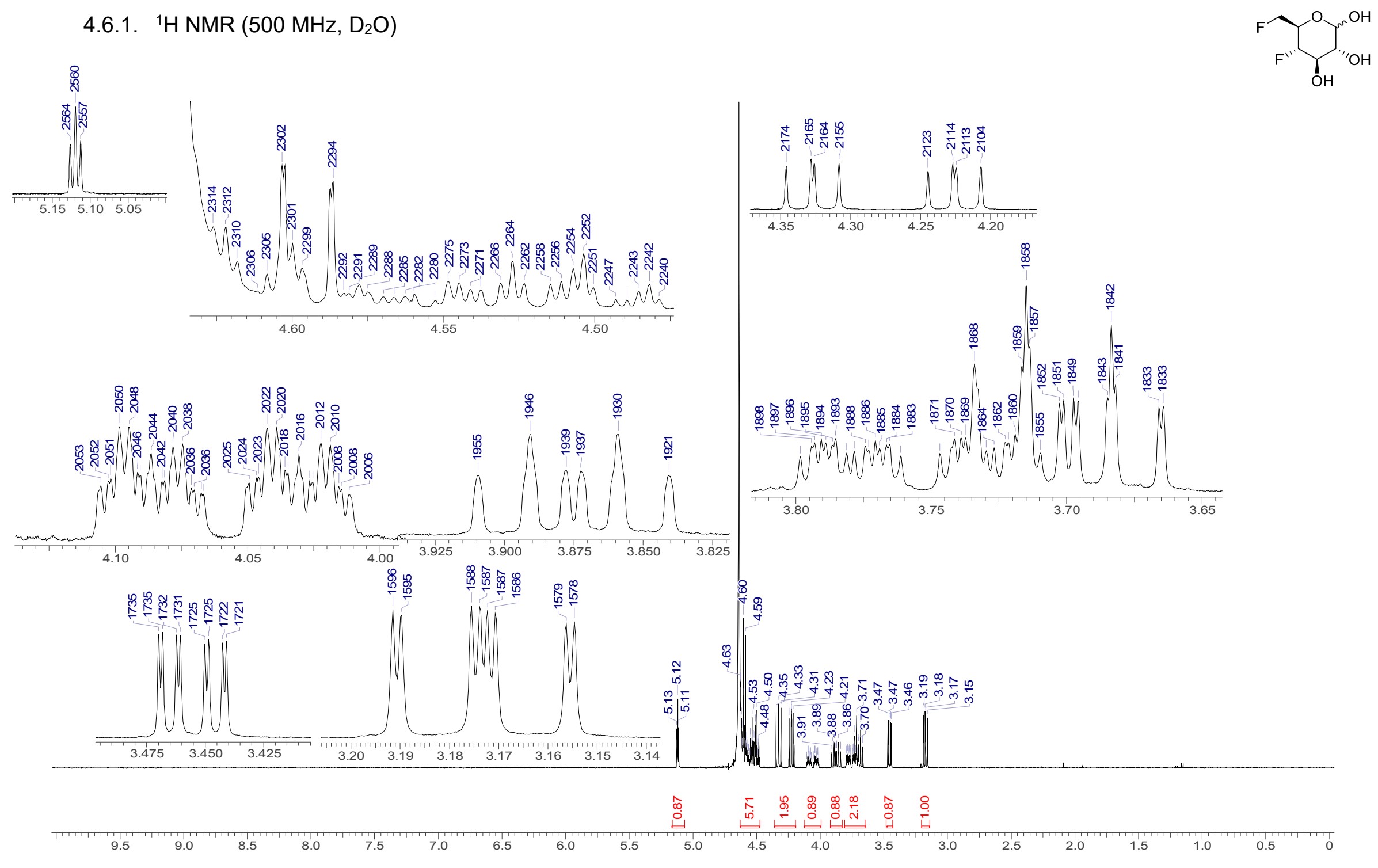
4.6.2. ${ }^{1} \mathrm{H}\left\{{ }^{19} \mathrm{~F}\right\} \mathrm{NMR}\left(500 \mathrm{MHz}, \mathrm{D}_{2} \mathrm{O}\right)$
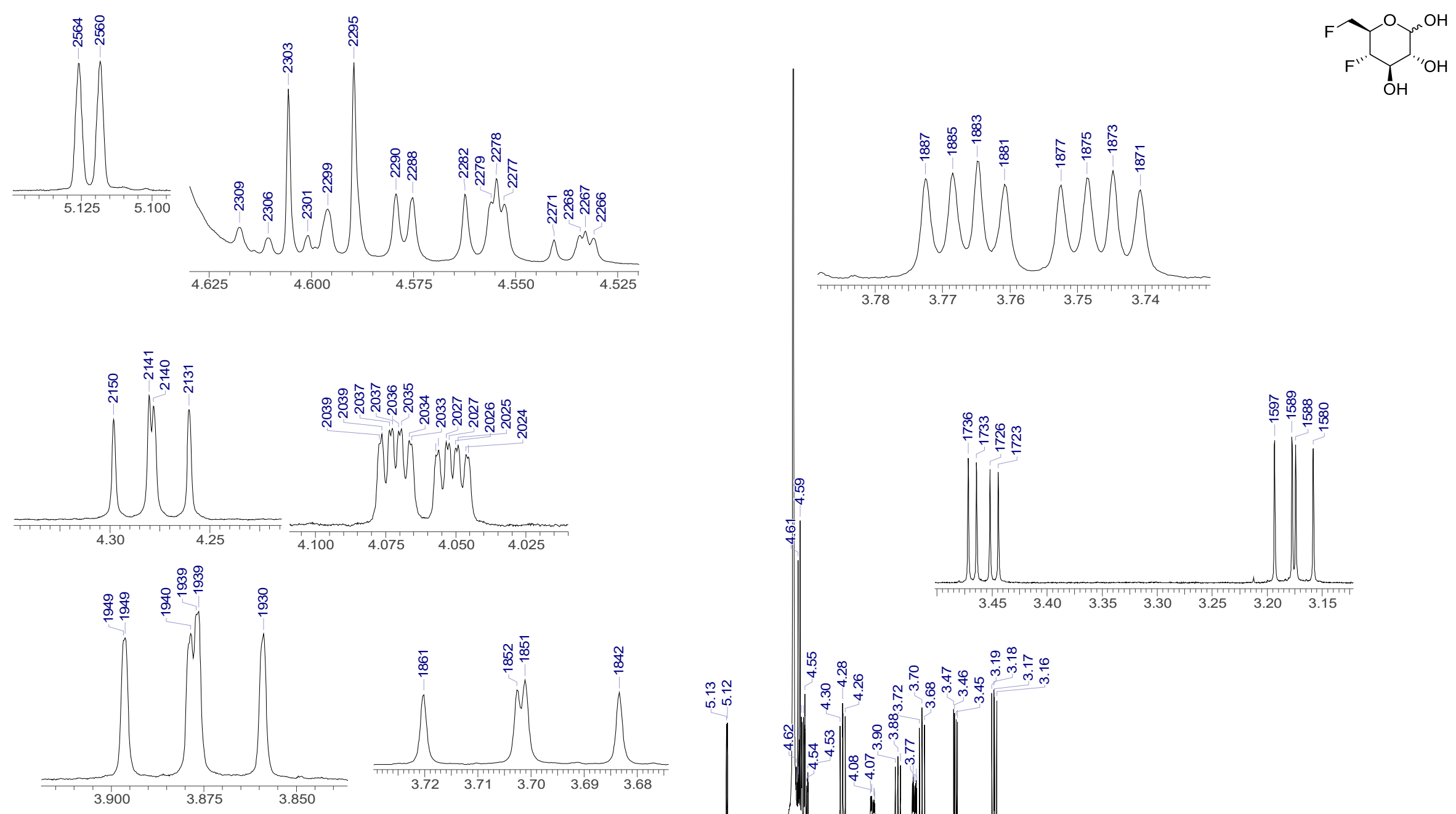

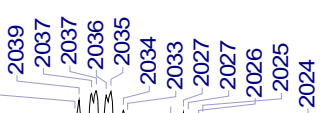

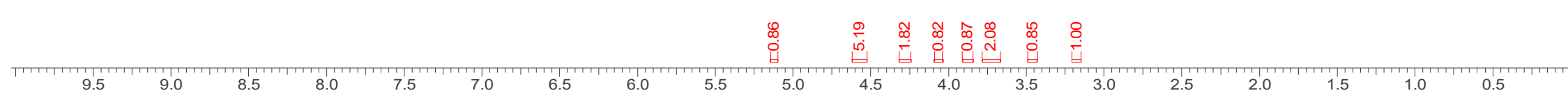


4.6.3. ${ }^{13} \mathrm{C}\left\{{ }^{1} \mathrm{H}\right\}$ NMR $\left(126 \mathrm{MHz}, \mathrm{D}_{2} \mathrm{O}\right)$

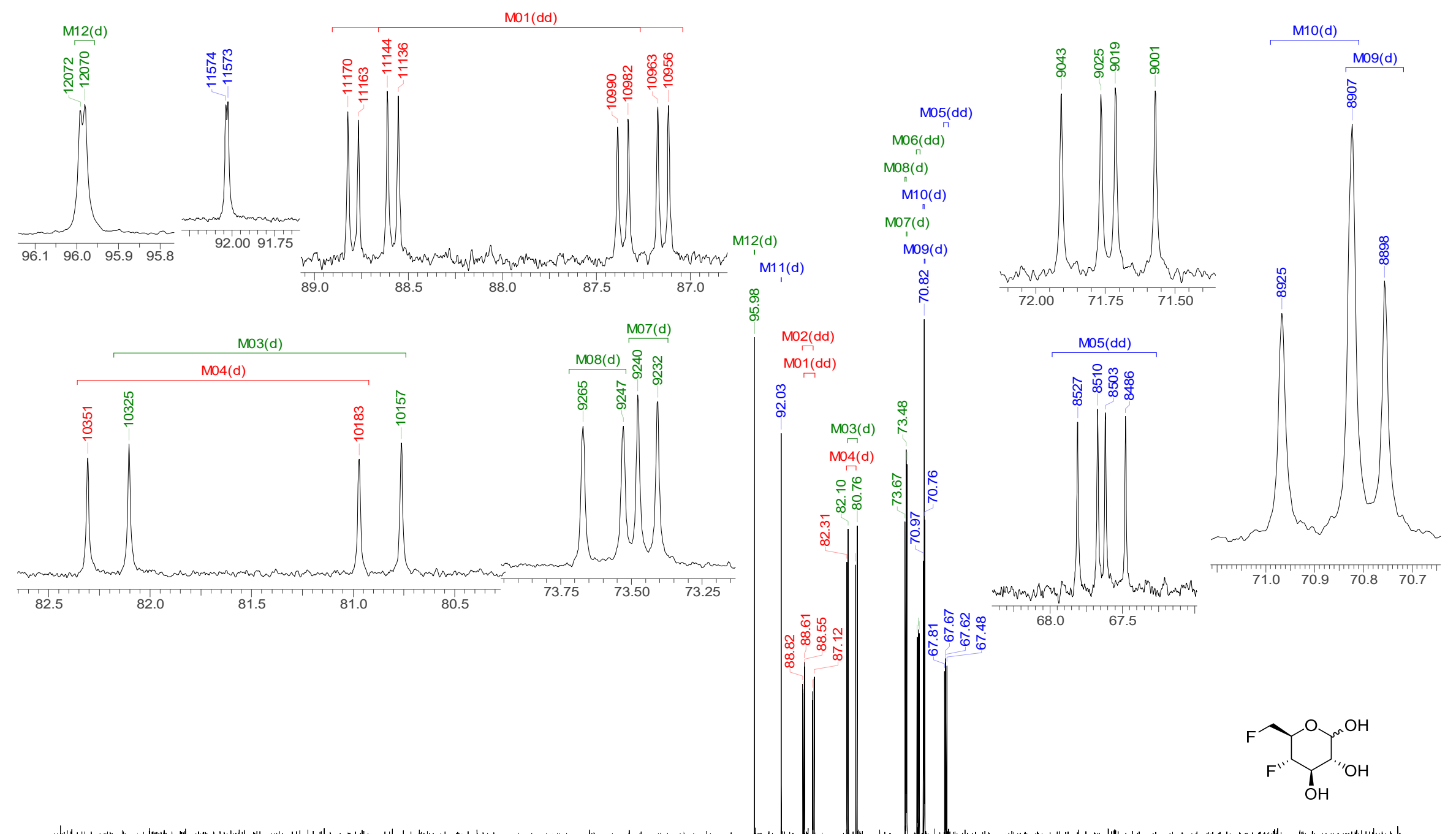

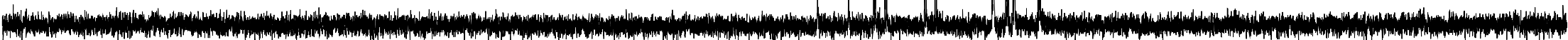


4.6.4. COSY, HSQC-DEPT \& HMBC $\left(D_{2} \mathrm{O}\right)$

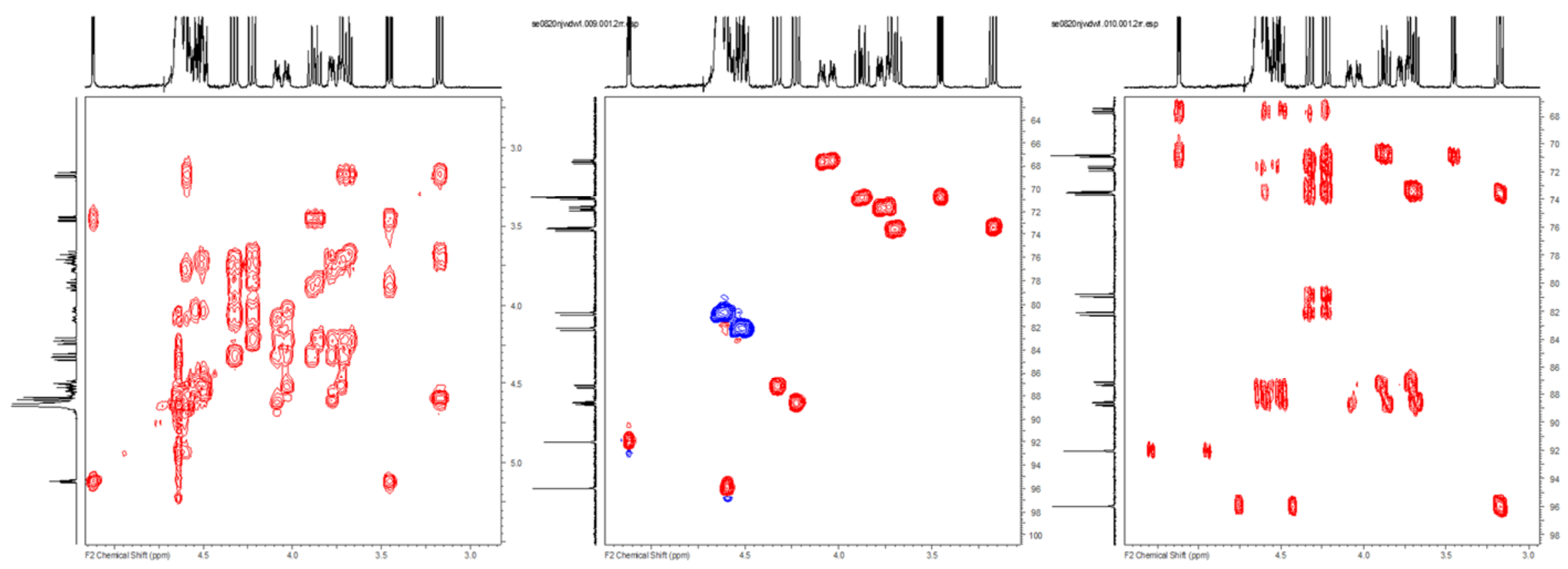


4.6.5. ${ }^{19} \mathrm{~F} \mathrm{NMR}\left(470 \mathrm{MHz}, \mathrm{D}_{2} \mathrm{O}\right)$
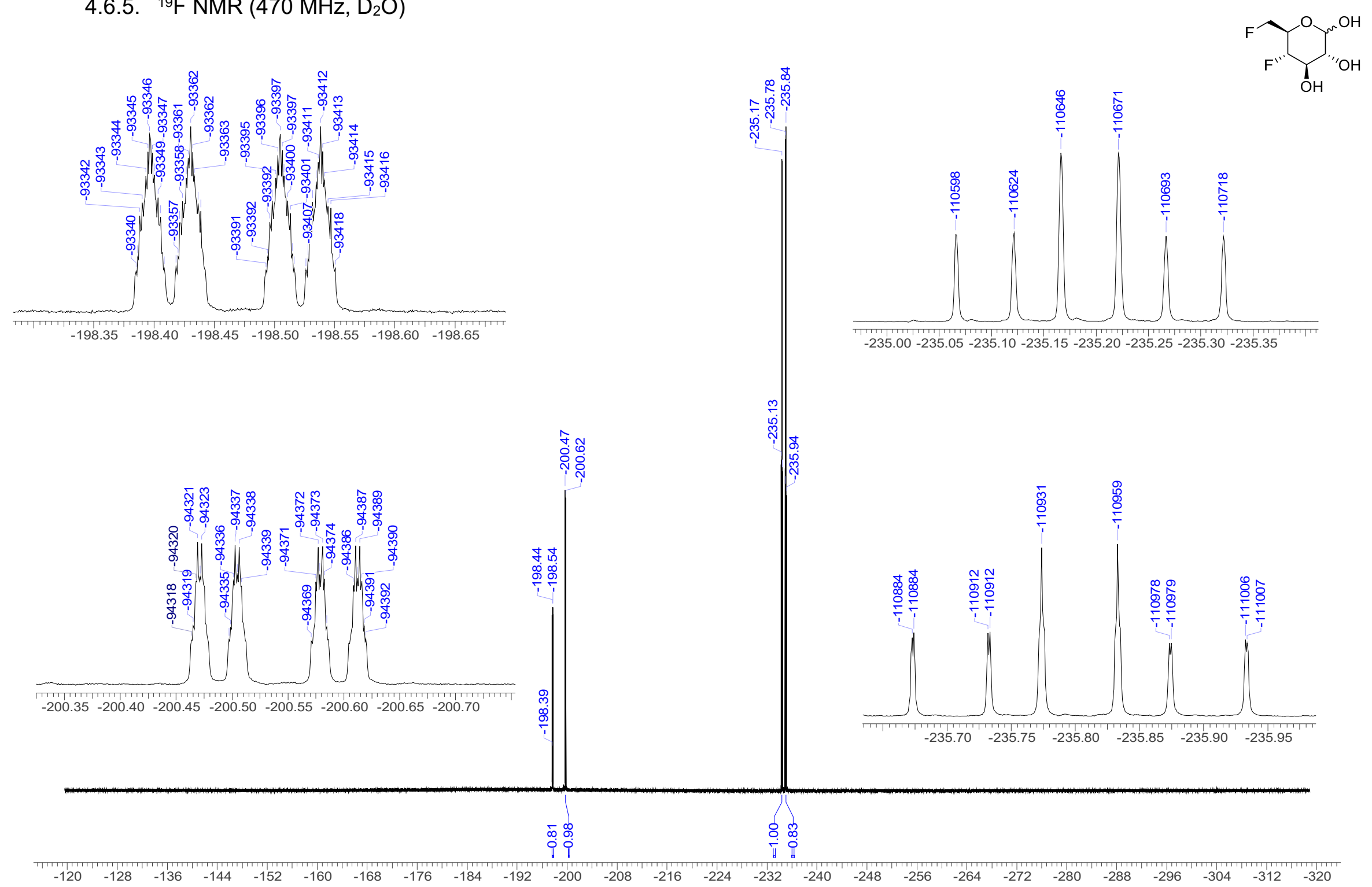
4.6.6. ${ }^{19} \mathrm{~F}\left\{{ }^{1} \mathrm{H}\right\} \mathrm{NMR}\left(470 \mathrm{MHz}, \mathrm{D}_{2} \mathrm{O}\right)$

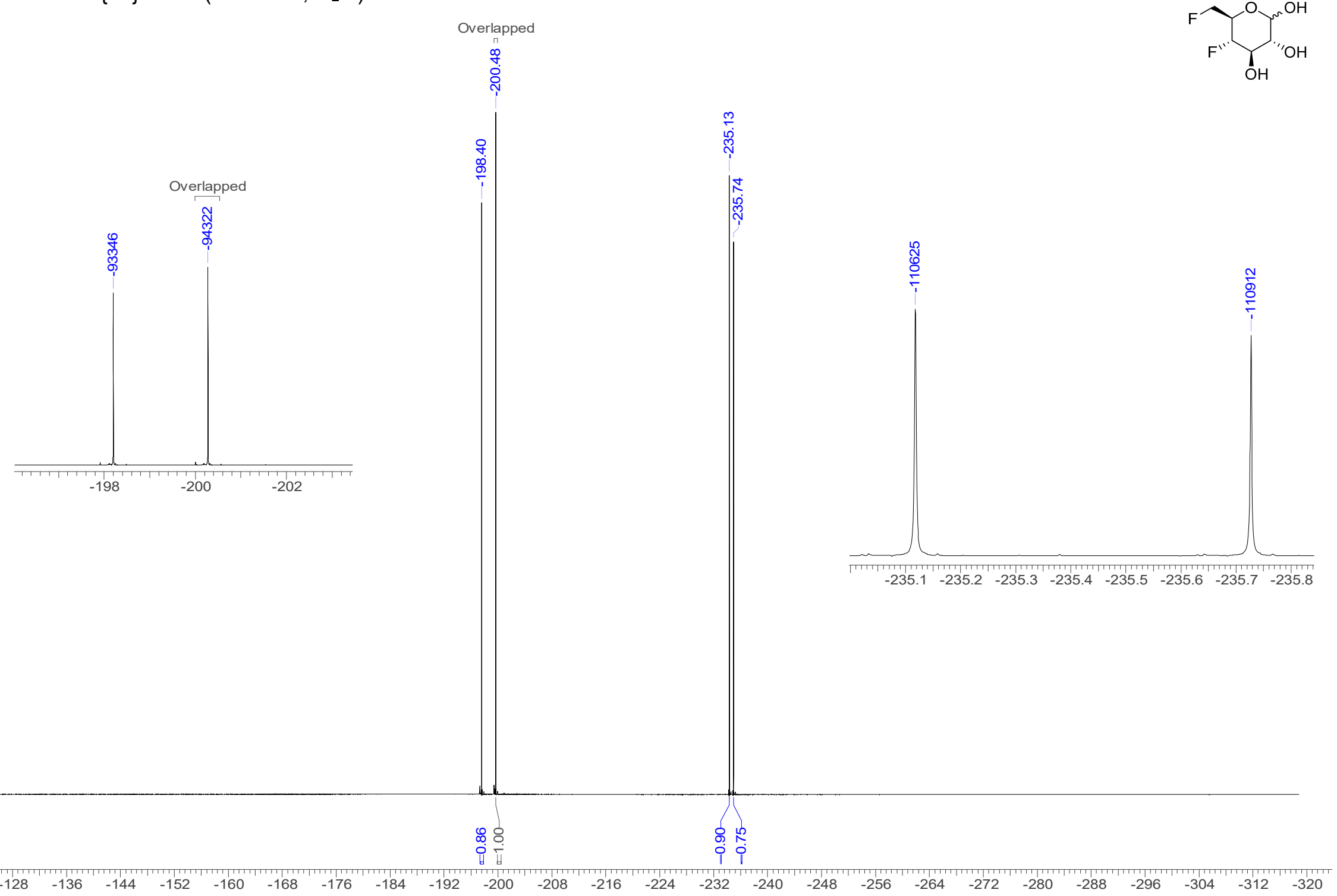




\subsection{7. ${ }^{1} \mathrm{H}$ NMR $\left(500 \mathrm{MHz}, \mathrm{D}_{2} \mathrm{O}\right.$, Spectral Width $=6.0$ ppm $)$}

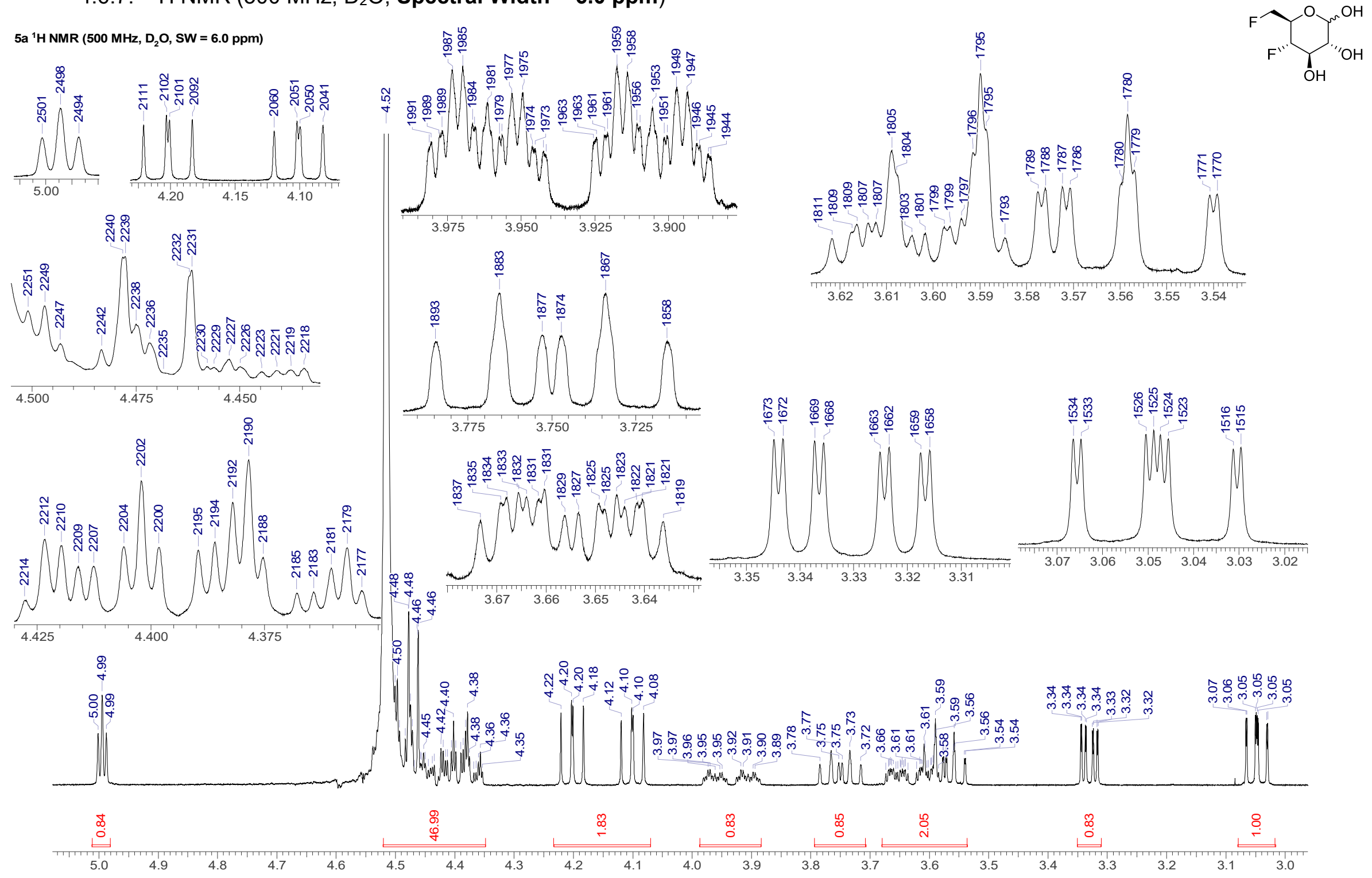


4.6.8. ${ }^{1} \mathrm{H}\left\{{ }^{19} \mathrm{~F}\right\} \mathrm{NMR}\left(500 \mathrm{MHz}, \mathrm{D}_{2} \mathrm{O}\right.$, Spectral Width $\left.=\mathbf{6 . 0} \mathbf{p p m}\right)$

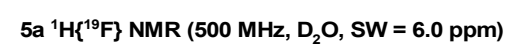

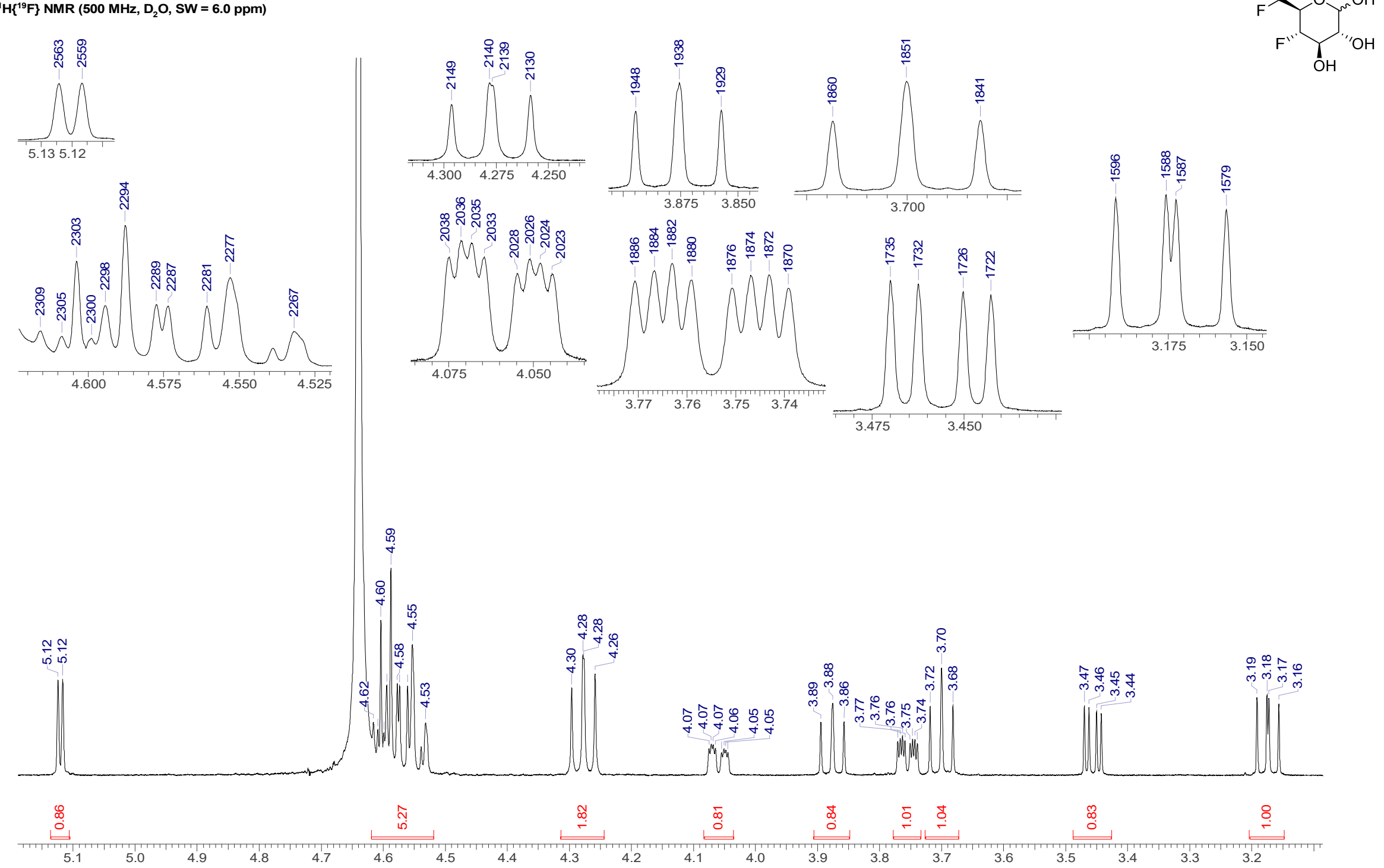


4.6.9. $\operatorname{COSY}\left(D_{2} O\right.$, Optimized for long-range couplings $)$

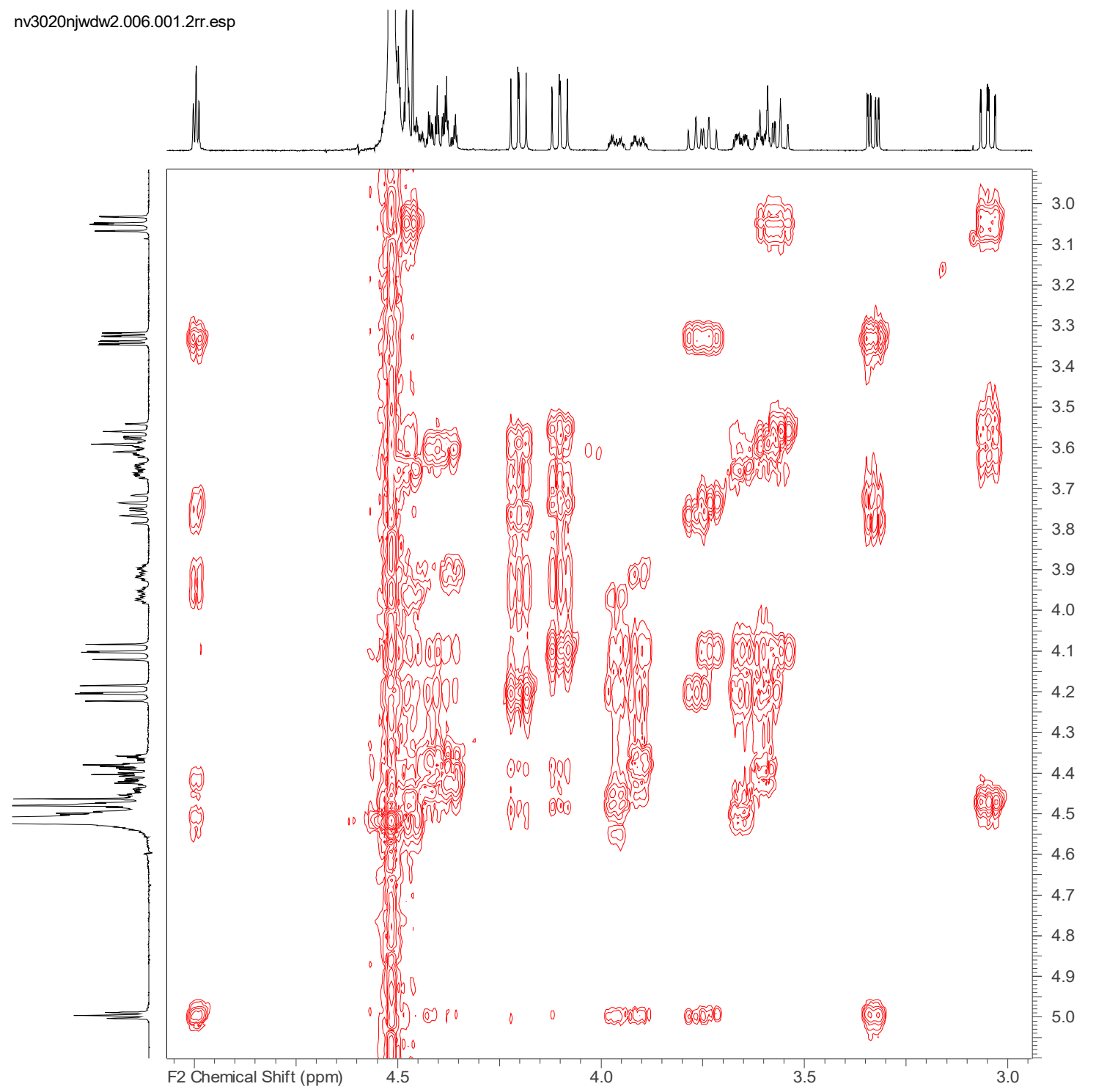


4.6.10. ${ }^{19} \mathrm{~F}$ NMR $\left(470 \mathrm{MHz}, \mathrm{D}_{2} \mathrm{O}\right.$, Spectral width $\left.=\mathbf{3 0 . 0} \mathbf{p p m}\right)$

$5 a{ }^{19} \mathrm{~F} \mathrm{NMR}\left(470 \mathrm{MHz}, \mathrm{D}_{2} \mathrm{O}, \mathrm{SW}=30.0 \mathrm{ppm}\right)$
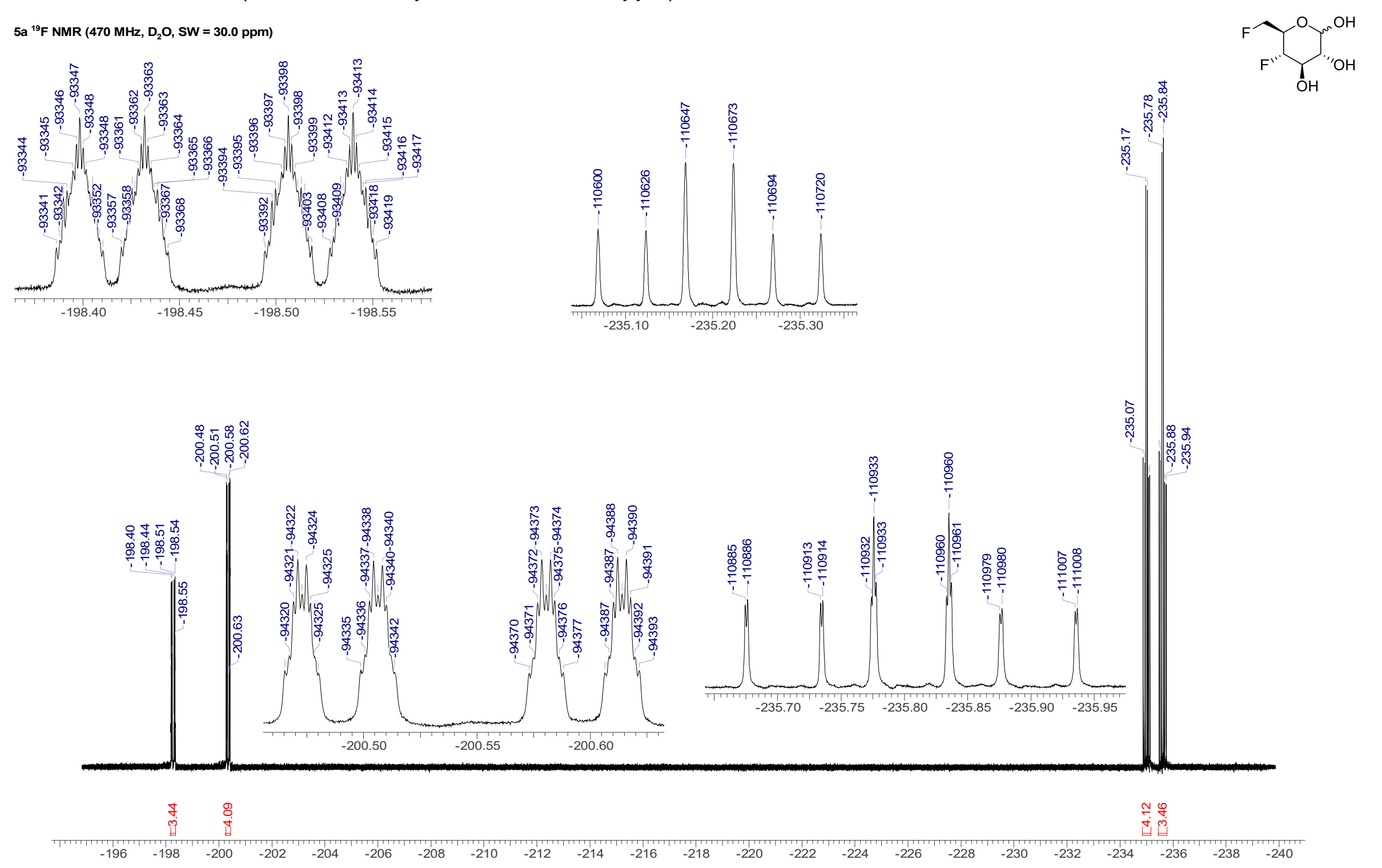
4.6.11. ${ }^{19} \mathrm{~F}\left\{{ }^{1} \mathrm{H}\right\}$ qNMR $\left(376 \mathrm{MHz}, \mathrm{D}_{2} \mathrm{O}\right)$

Duplicate \#1

DW/4,6 V6 D2O qNMR F An1

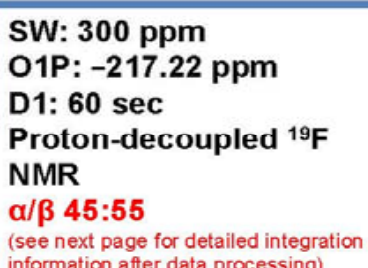

Proton-decoupled ${ }^{19} \mathrm{~F}$

NMR

$\alpha / \beta$ 45:55

see next page for detailed integration

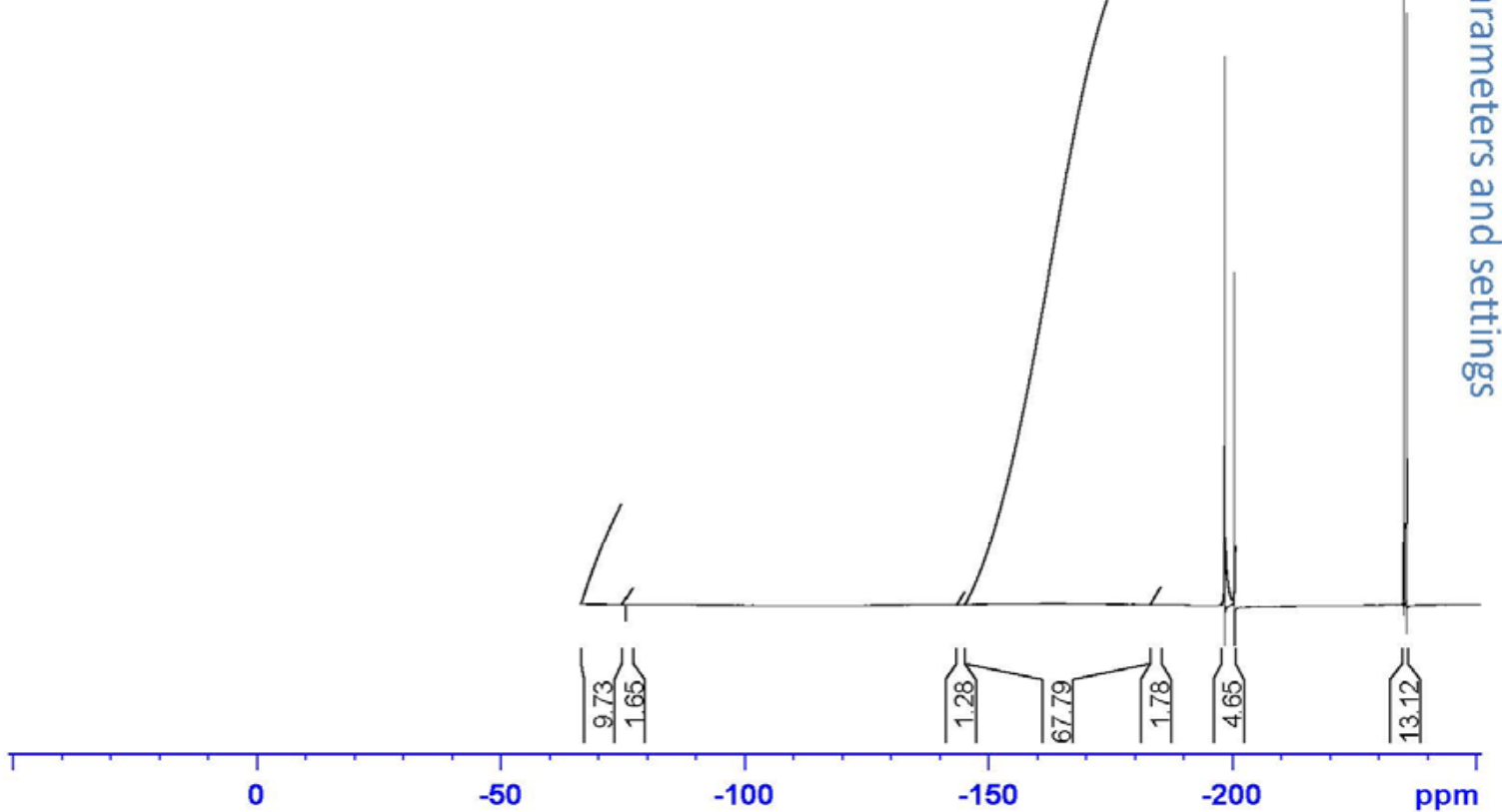

AVIIIHD400 [3]

Current Data Parameters
NAME au1420dw4

EXPNO

12

F2 - Acquisition Parameters

Time- $202008 \mathrm{~h}$

INSTRUM $212 \mathrm{~h}$

PROBHD Z116098 0218

PULPROG zgfhigqn.2

TDOLVENT 131072

$\begin{array}{lc}\text { NS } & 64 \\ \text { DS } & 4\end{array}$

SWH $\quad 113636.367 \mathrm{~Hz}$

$0.57671953 \mathrm{~Hz}$

$\begin{array}{ll}\text { RG } & 0.5767168 \\ \text { RW } & 212.69\end{array}$

DW $\quad 4.400$ usec $6.50 \mathrm{usec}$
$298.0 \mathrm{~K}$

0

$0.030000000 \mathrm{sec}$

$0.00002000 \mathrm{sec}$

TDO $120.4105831 \mathrm{MH}$

SFO1 $\quad 376.4165831 \mathrm{MHz}$

14 19F

$\begin{array}{ll}\text { P1 } & 14.00 \text { usec } \\ \text { PLW1 } & 22.69199944 \mathrm{~W}\end{array}$

$\mathrm{SFO} 2 \quad 400.1316005 \mathrm{MHz}$

$\begin{array}{ll}\text { NUC2 } & 1 \mathrm{H} \\ \text { CPDPRG } & \text { waltz16 }\end{array}$

90.00 usec

\begin{tabular}{lr} 
PLW12 & $16.38100052 \mathrm{~W}$ \\
\hline
\end{tabular}

F2 - Processing parameters

SF $\quad 376.4984132 \mathrm{MHz}$

WDW
SSB

SSB

GB

EM

$0.30 \mathrm{~Hz}$

0
1.00 
Duplicate \#1

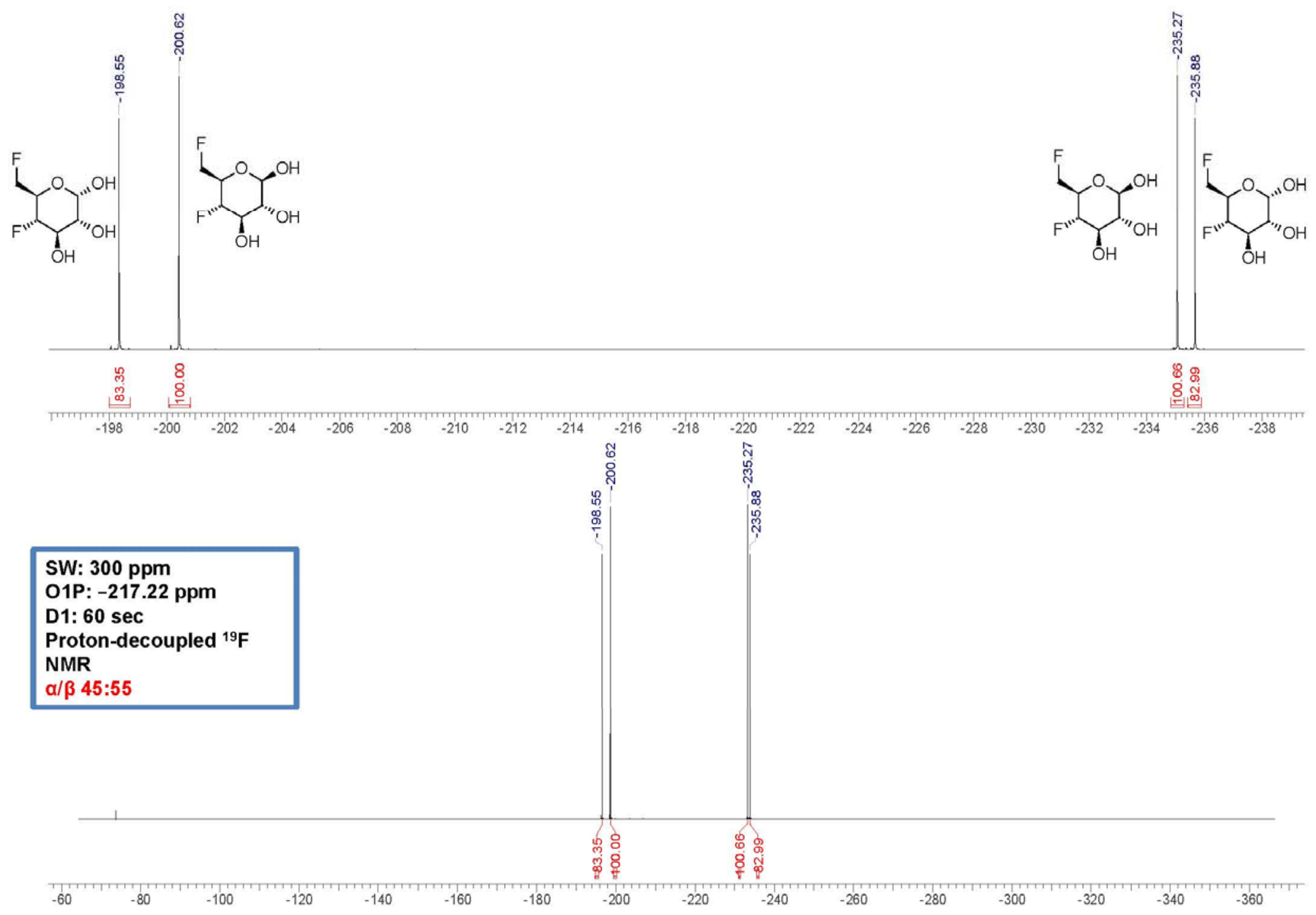




\section{Duplicate \#2}

DW/4,6 V6 D2O qNMR F An2

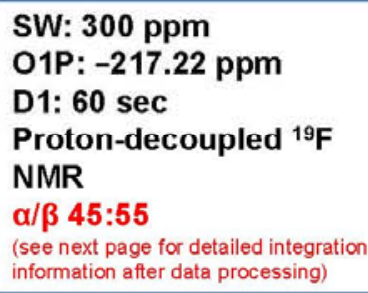

D1: $60 \mathrm{sec}$

Proton-decoupled ${ }^{19} \mathrm{~F}$

NMR

$\alpha / \beta$ 45:55

see next page for detailed integration

information after data processing)
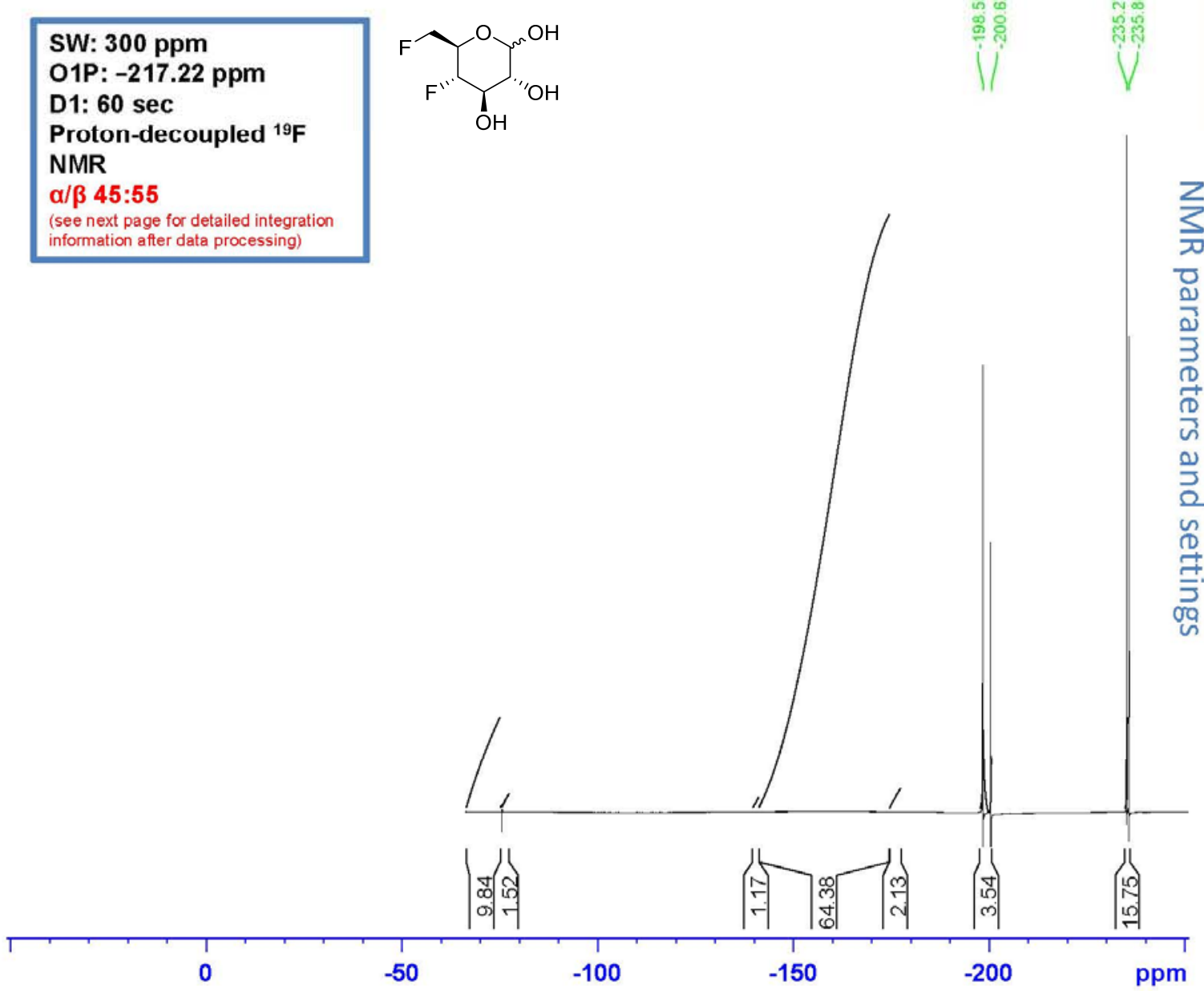

AVIIIHD400 [3]

Current Data Parameters
NAME au1420dw5

EXPNO 12

F2 - Acquisition Parameters

Date_ 20200816

$3.28 \mathrm{~h}$

spect

PULPROG Z116098_0218

TD zgfhigqn 2

SOLVENT 131072 D2O

$\begin{array}{ll}N S & 64 \\ \text { DS } & 4\end{array}$

SWH $\quad 113636.367 \mathrm{~Hz}$

$0.57673953 \mathrm{~Hz}$

RG $\quad 21269$

DW $\quad 4.400$ usec

DE $\quad 6.50$ usec

D1 $60.00000000 \mathrm{sec}$

D11 $\quad 0.00000000 \mathrm{sec}$

D12 $0.00002000 \mathrm{sec}$

TDO

SFO1 $\quad 376.4165831 \mathrm{MHz}$

NUC1 $19 \mathrm{~F}$

14.00 usec

PLW1 22.69199944 W

SFO2 $400.1316005 \mathrm{MHz}$

CPDPRG[2 waltz16

$\begin{array}{ll}\text { CPDPRG } & \text { waltz16 } \\ \text { PCPD2 } & 90.00 \text { usec }\end{array}$

PLW2 16.38100052 W

F2 - Processing parameters

Sl 65536

SF $\quad 376.4984132 \mathrm{MHz}$

SSB EM

LB $\quad 0.30 \mathrm{~Hz}$

GB $\quad 1.00$ 
Duplicate \#2

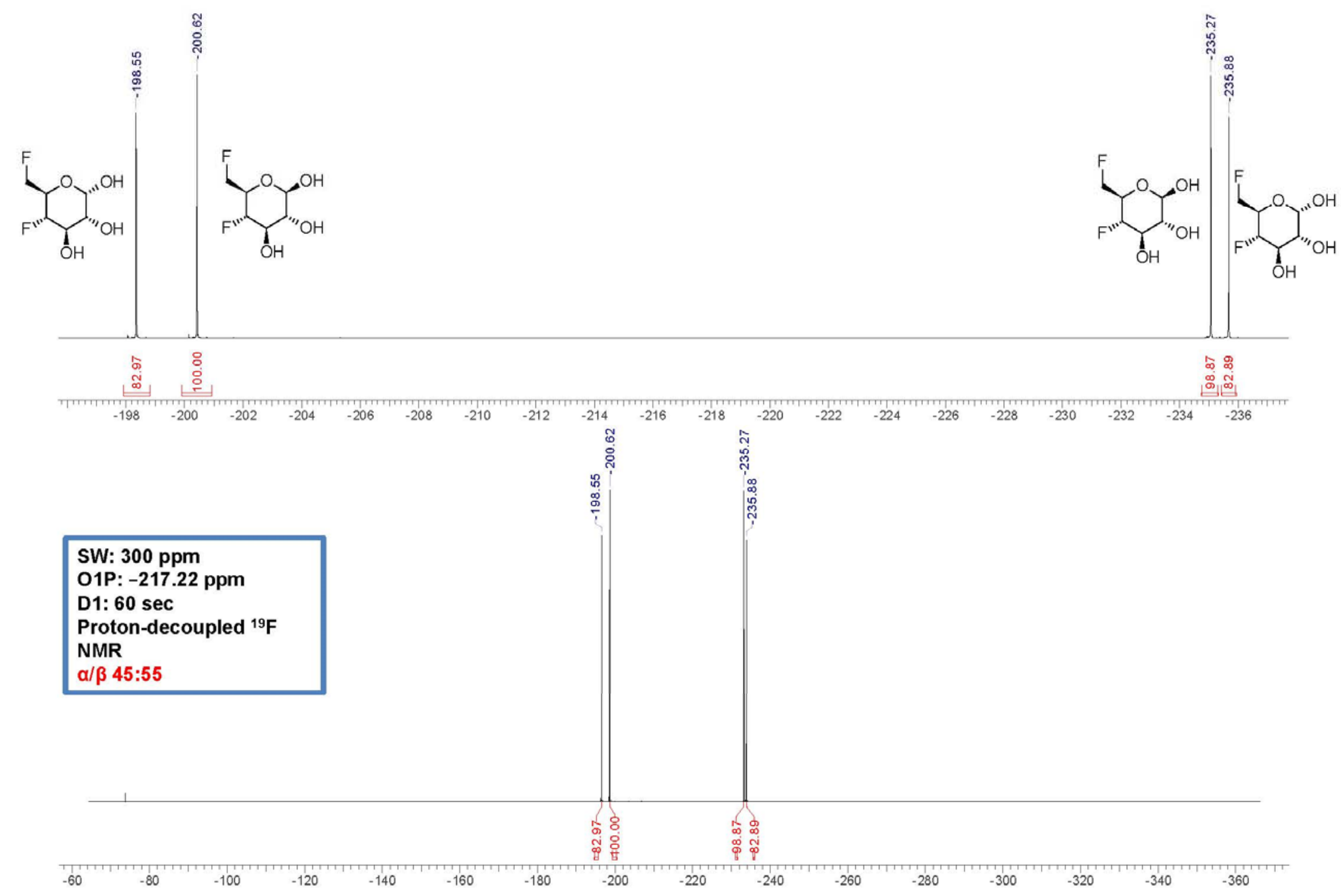




\subsection{4,6-Dideoxy-4,4-difluoro-D-xylo-hexopyranose (6a)}

4.7.1. ${ }^{1} \mathrm{H}$ NMR $\left(500 \mathrm{MHz}, \mathrm{D}_{2} \mathrm{O}\right)$
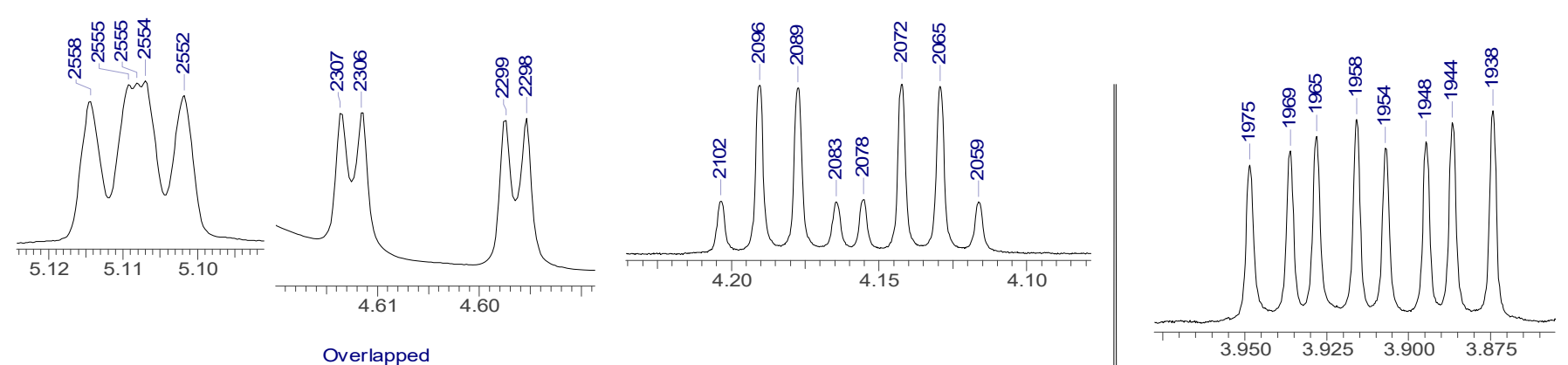

$$
\text { (OH }
$$
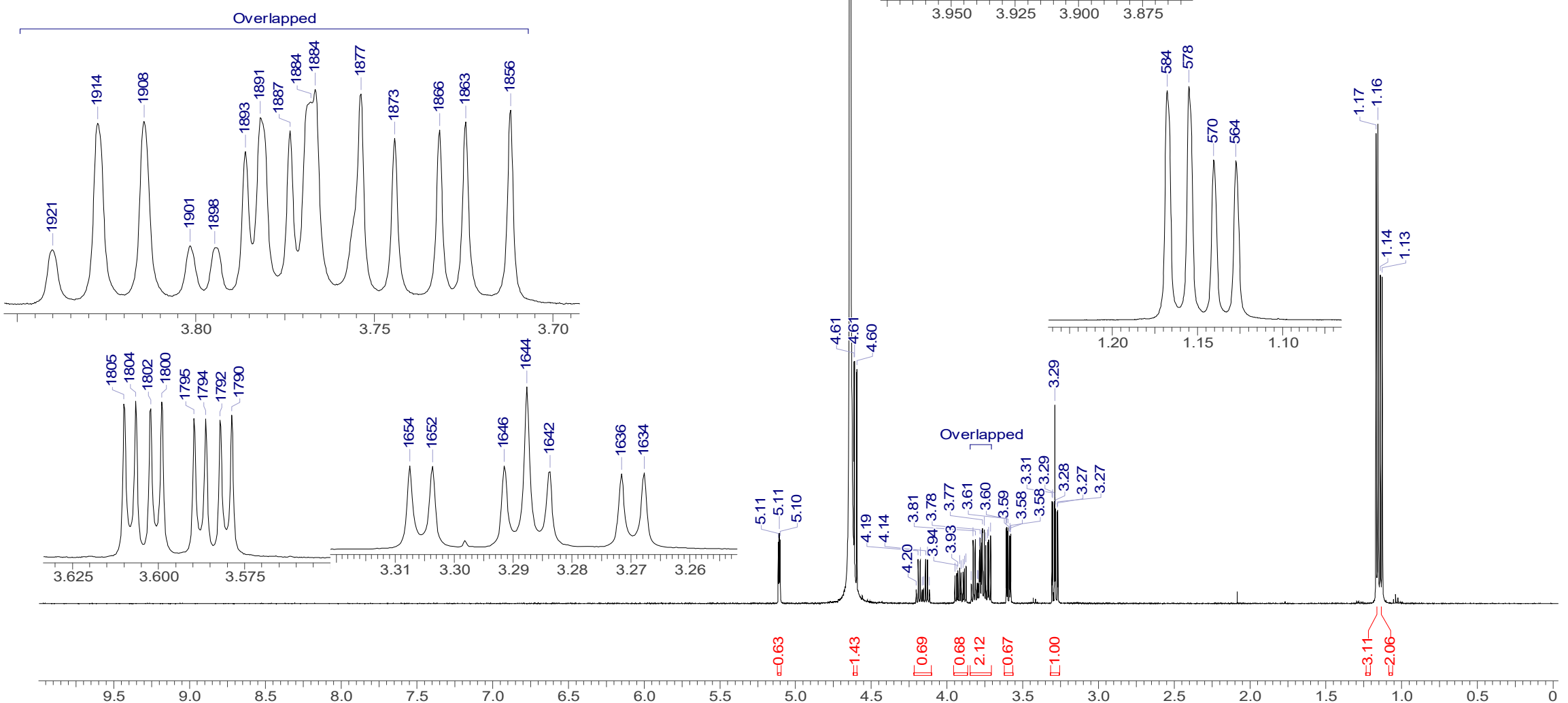
4.7.2. ${ }^{1} \mathrm{H}\left\{{ }^{19} \mathrm{~F}\right\} \mathrm{NMR}\left(500 \mathrm{MHz}, \mathrm{D}_{2} \mathrm{O}\right)$

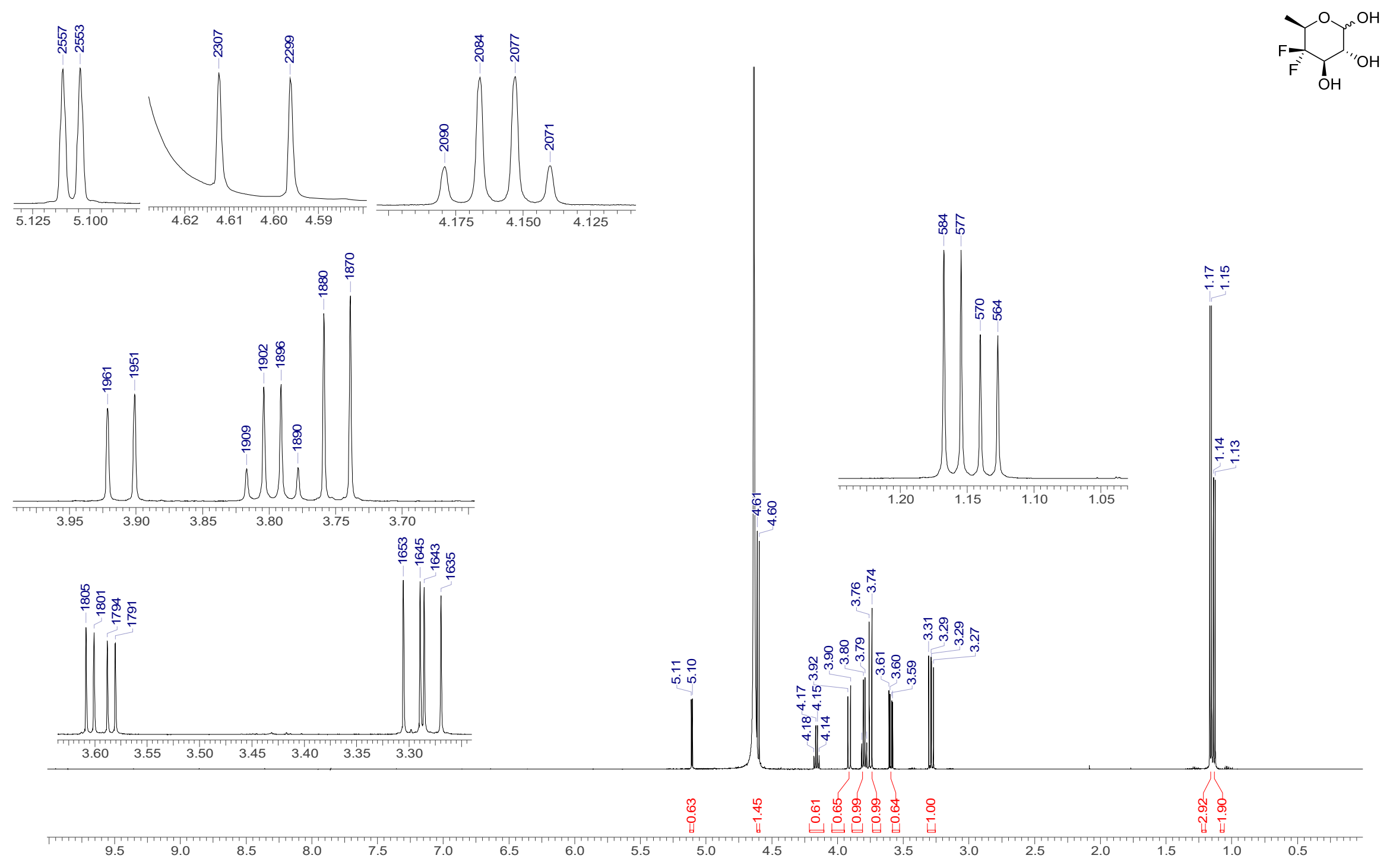


4.7.3. ${ }^{13} \mathrm{C}\left\{{ }^{1} \mathrm{H}\right\} \operatorname{NMR}\left(126 \mathrm{MHz}, \mathrm{D}_{2} \mathrm{O}\right)$
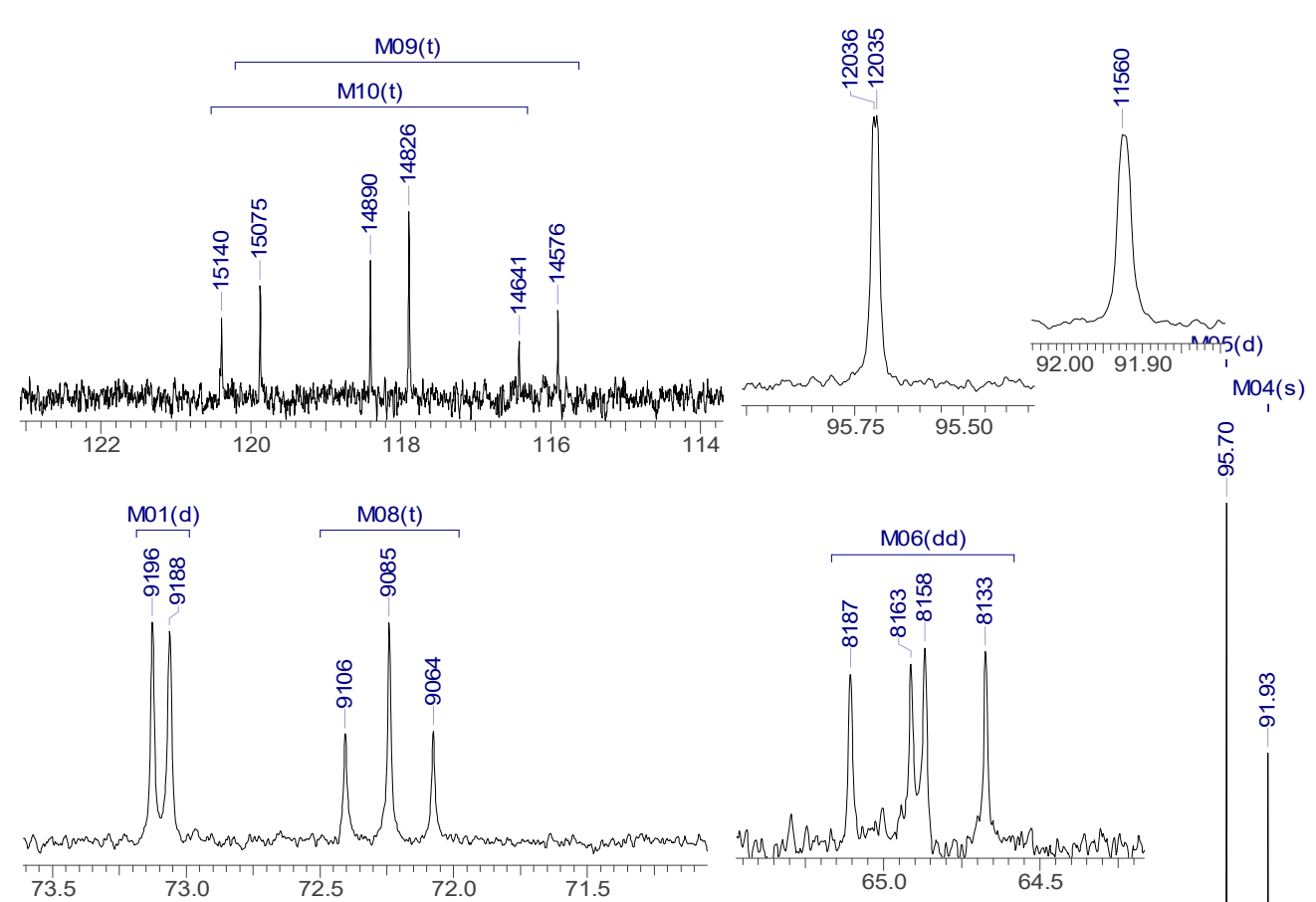

$$
\text { (m09(t) }
$$

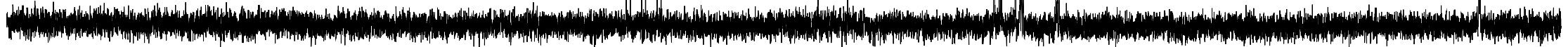
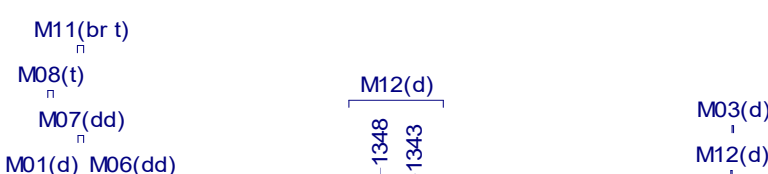

M01(d) MO6(dd)

MO2(d)

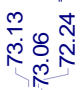

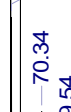

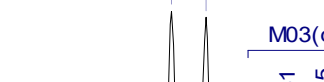

12(d)

(1)

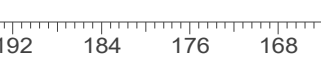

$160+152$

$120 \quad 112 \quad 104$

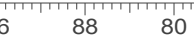

64

$48 \quad 40$

${ }_{32}{ }_{24} 166_{10}$ 
4.7.4. COSY, HSQC-DEPT \& $\operatorname{HMBC}\left(\mathrm{D}_{2} \mathrm{O}\right)$

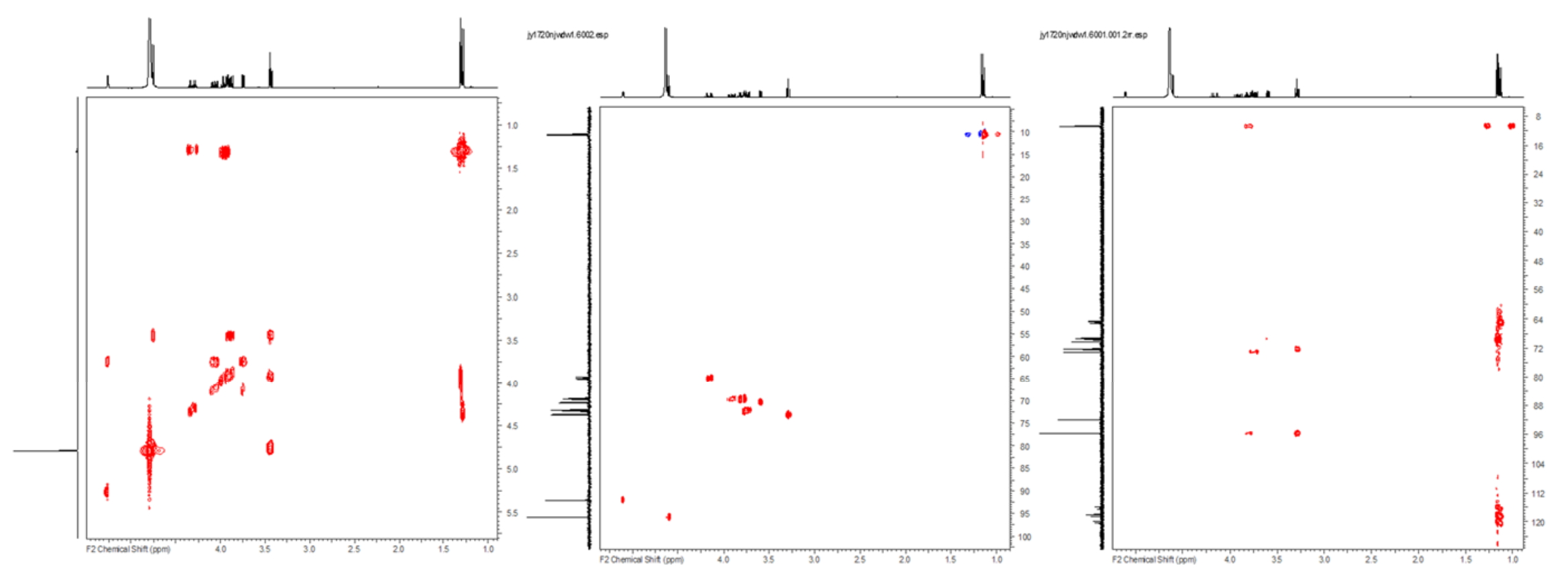




\subsection{5. ${ }^{19} \mathrm{~F}$ NMR $\left(470 \mathrm{MHz}, \mathrm{D}_{2} \mathrm{O}\right)$}

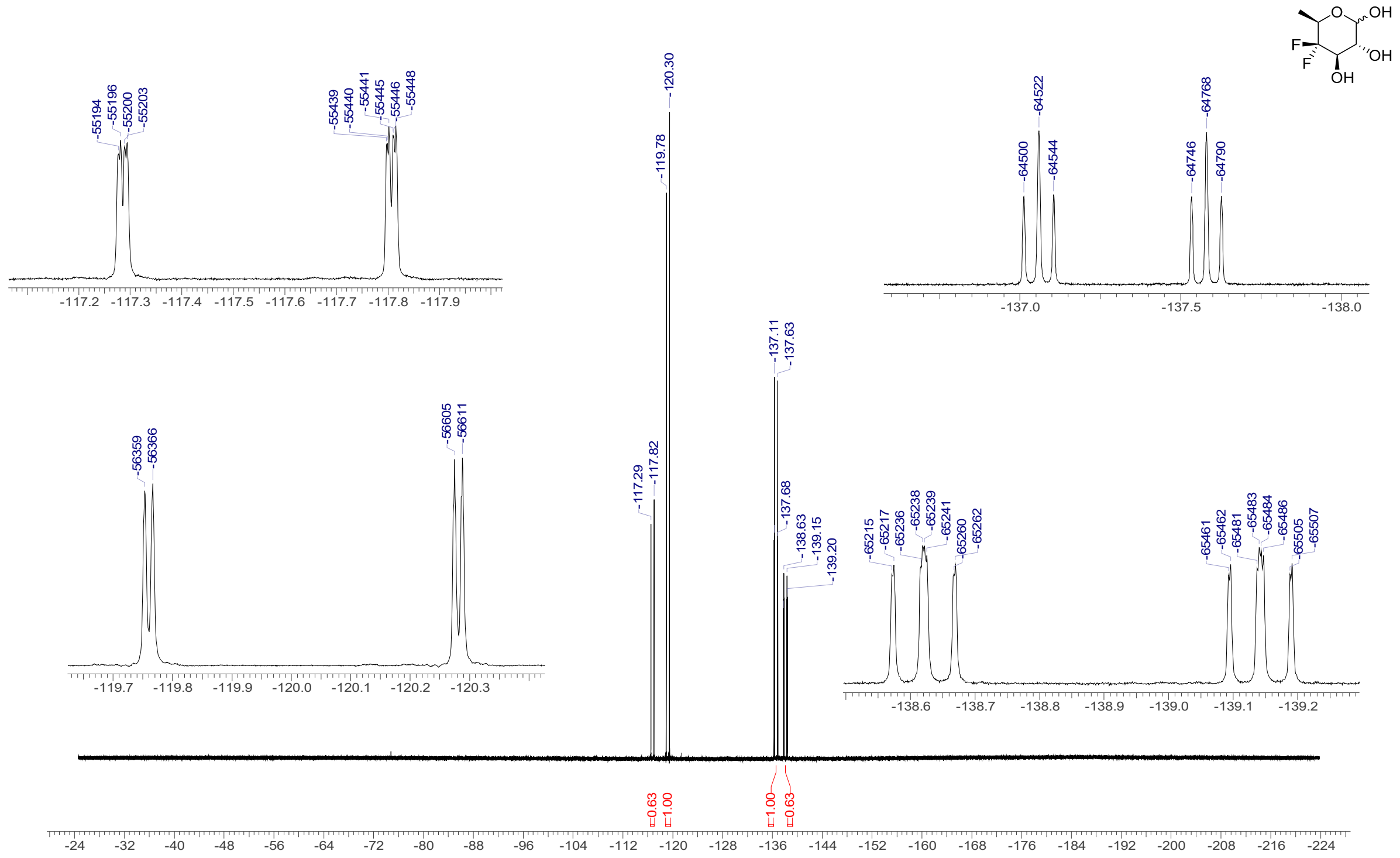


4.7.6. ${ }^{19} \mathrm{~F}\left\{{ }^{1} \mathrm{H}\right\} \mathrm{NMR}\left(470 \mathrm{MHz}, \mathrm{D}_{2} \mathrm{O}\right)$

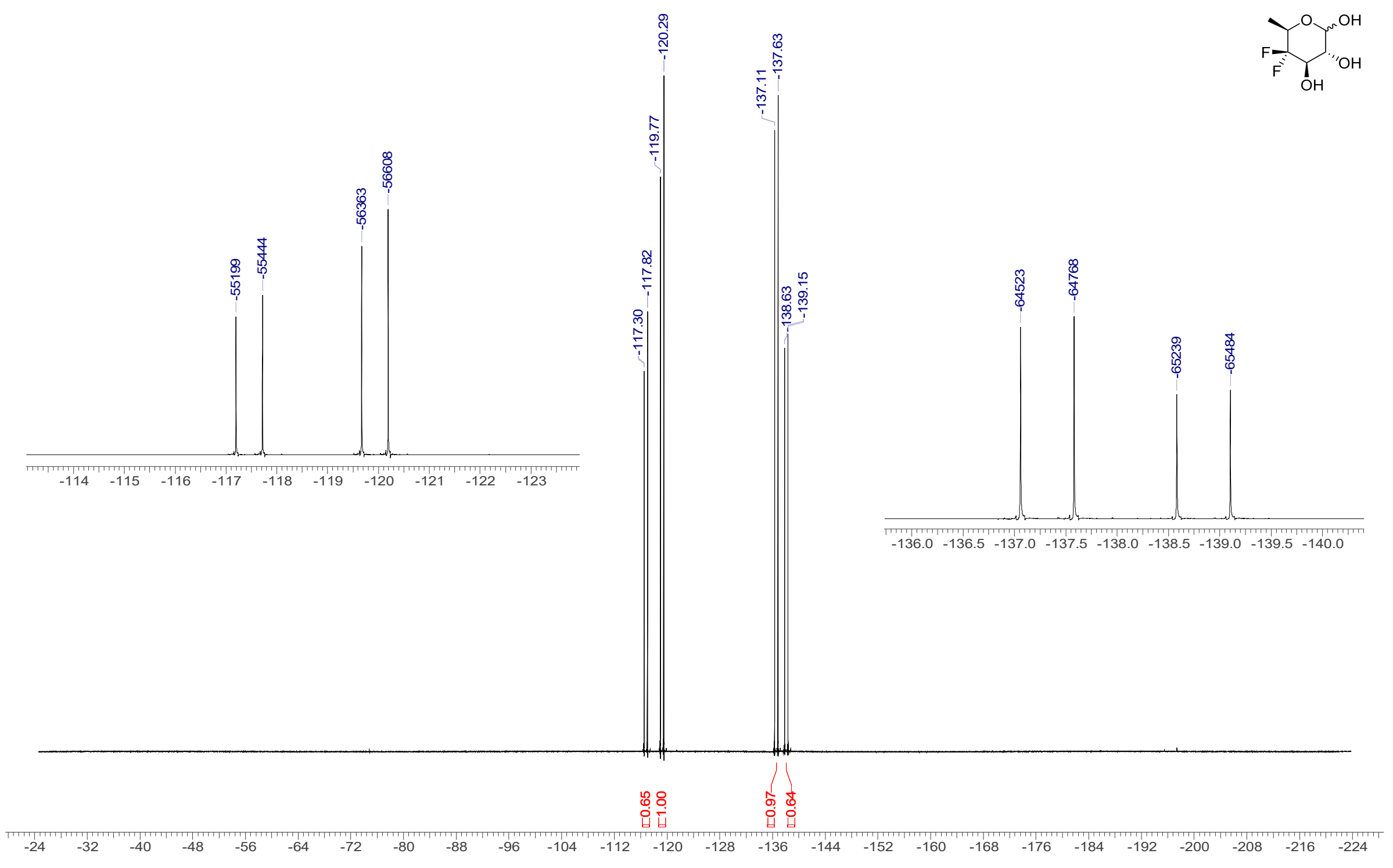


4.7.7. ${ }^{1} \mathrm{H}$ NMR $\left(500 \mathrm{MHz}, \mathrm{D}_{2} \mathrm{O}\right.$, Spectral Width $\left.=\mathbf{6 . 0} \mathbf{p p m}\right)$

$6 a^{1} \mathrm{H}$ NMR (500 MHz, $\left.\mathrm{D}_{2} \mathrm{O}, \mathrm{SW}=6.0 \mathrm{ppm}\right)$

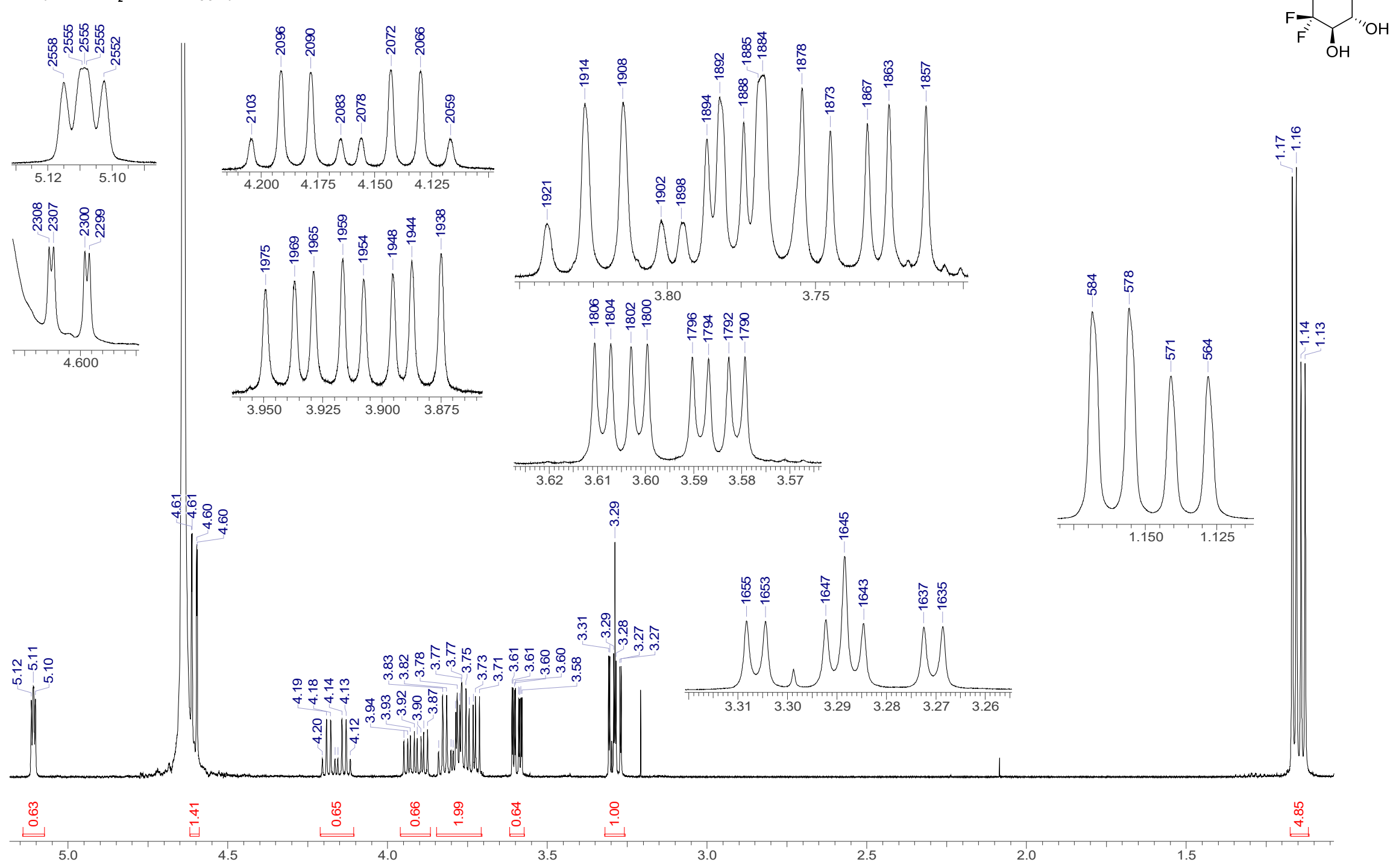


4.7.8. ${ }^{1} \mathrm{H}\left\{{ }^{19} \mathrm{~F}\right\} \mathrm{NMR}\left(500 \mathrm{MHz}, \mathrm{D}_{2} \mathrm{O}\right.$, Spectral Width $\left.=\mathbf{6 . 0} \mathbf{p p m}\right)$

$\left.6 a^{1} \mathrm{H}^{19}{ }^{19} \mathrm{~F}\right\} \mathrm{NMR}\left(500 \mathrm{MHz}, \mathrm{D}_{2} \mathrm{O}, \mathrm{SW}=6.0 \mathrm{ppm}\right)$
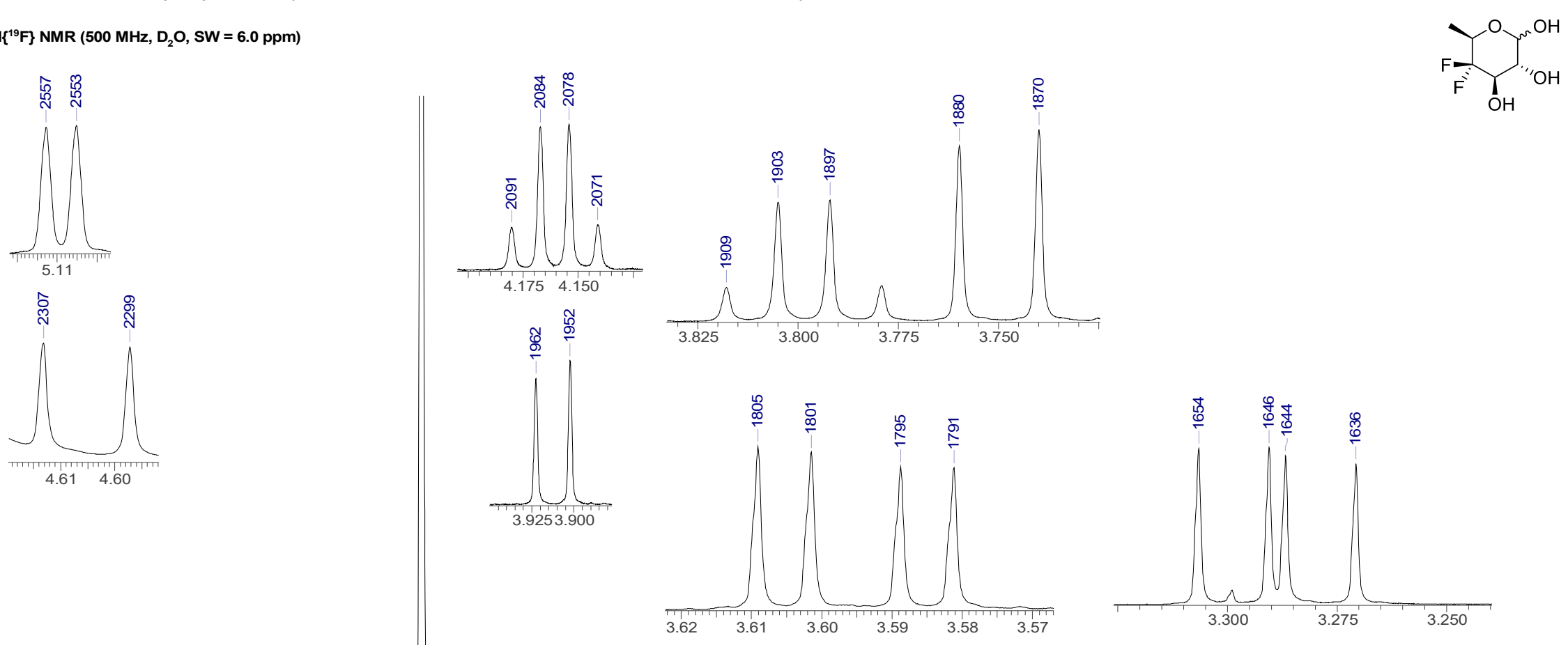

$4.61 \quad 4.60$

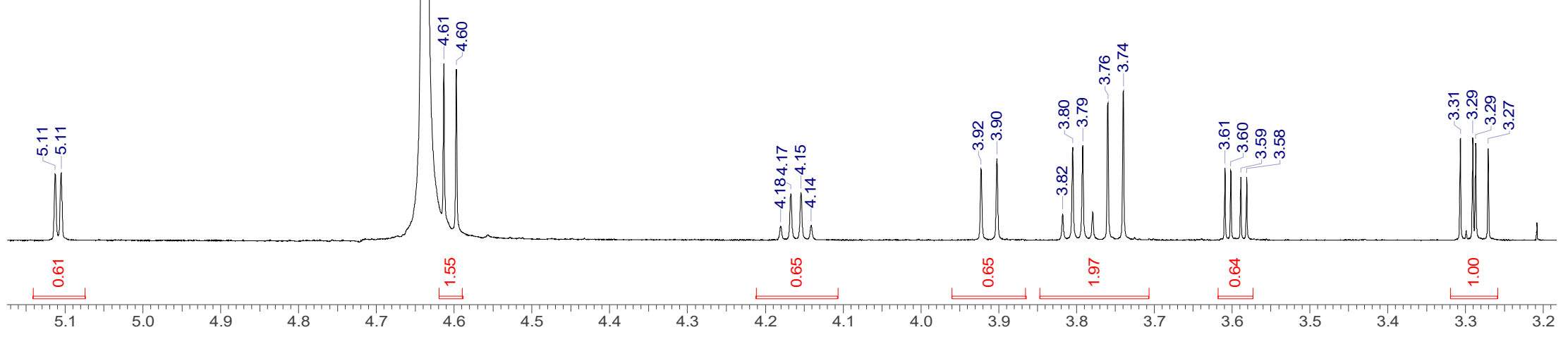




\subsection{9. $\operatorname{COSY}\left(D_{2} \mathrm{O}\right.$, Optimized for long-range couplings $)$}

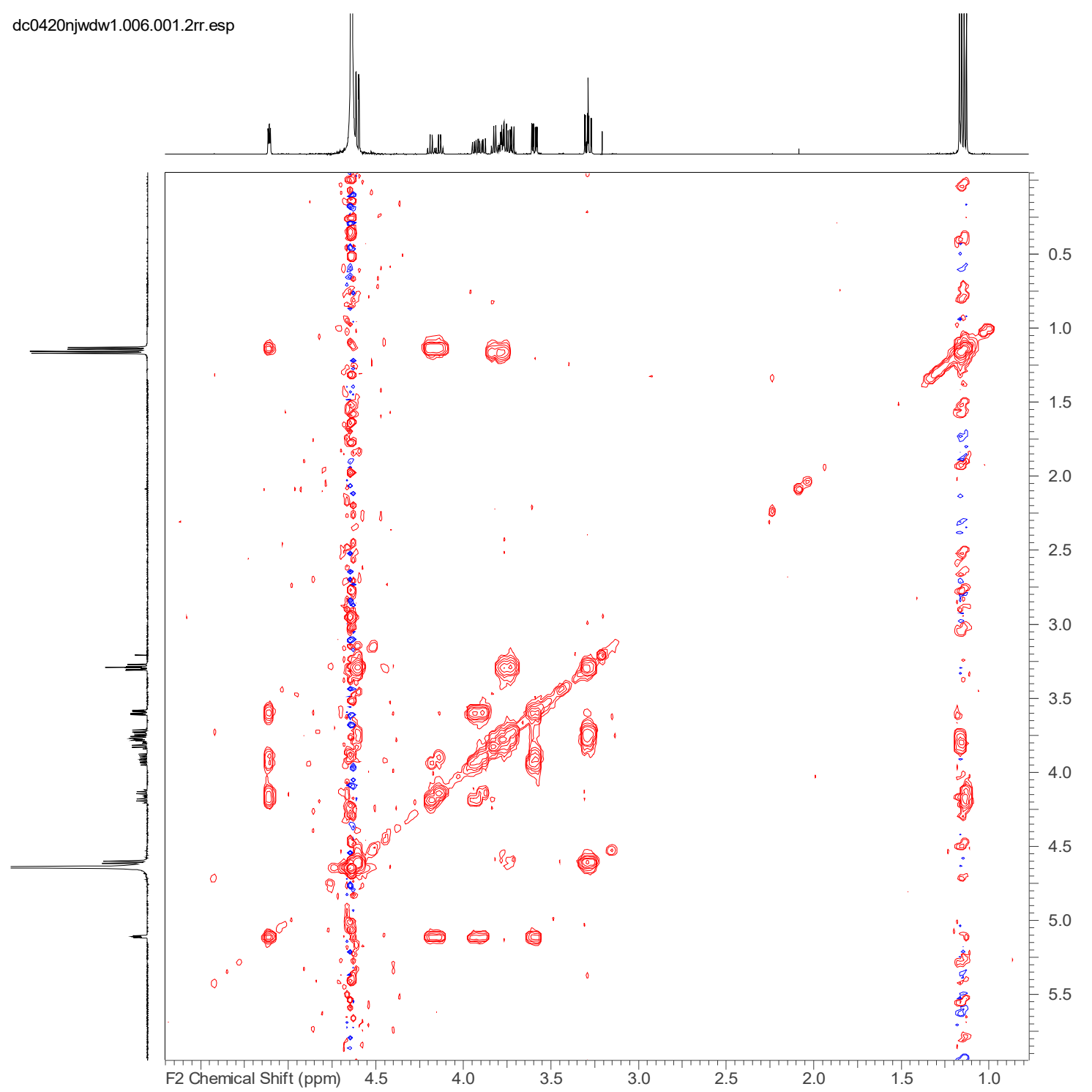


4.7.10. ${ }^{19} \mathrm{~F}$ NMR $\left(470 \mathrm{MHz}, \mathrm{D}_{2} \mathrm{O}\right.$, Spectral Width = $\mathbf{3 0 . 0}$ ppm $)$

$6{ }^{19} \mathrm{~F} \mathrm{NMR}\left(470 \mathrm{MHz}, \mathrm{D}_{2} \mathrm{O}, \mathrm{SW}=30.0 \mathrm{ppm}\right)$

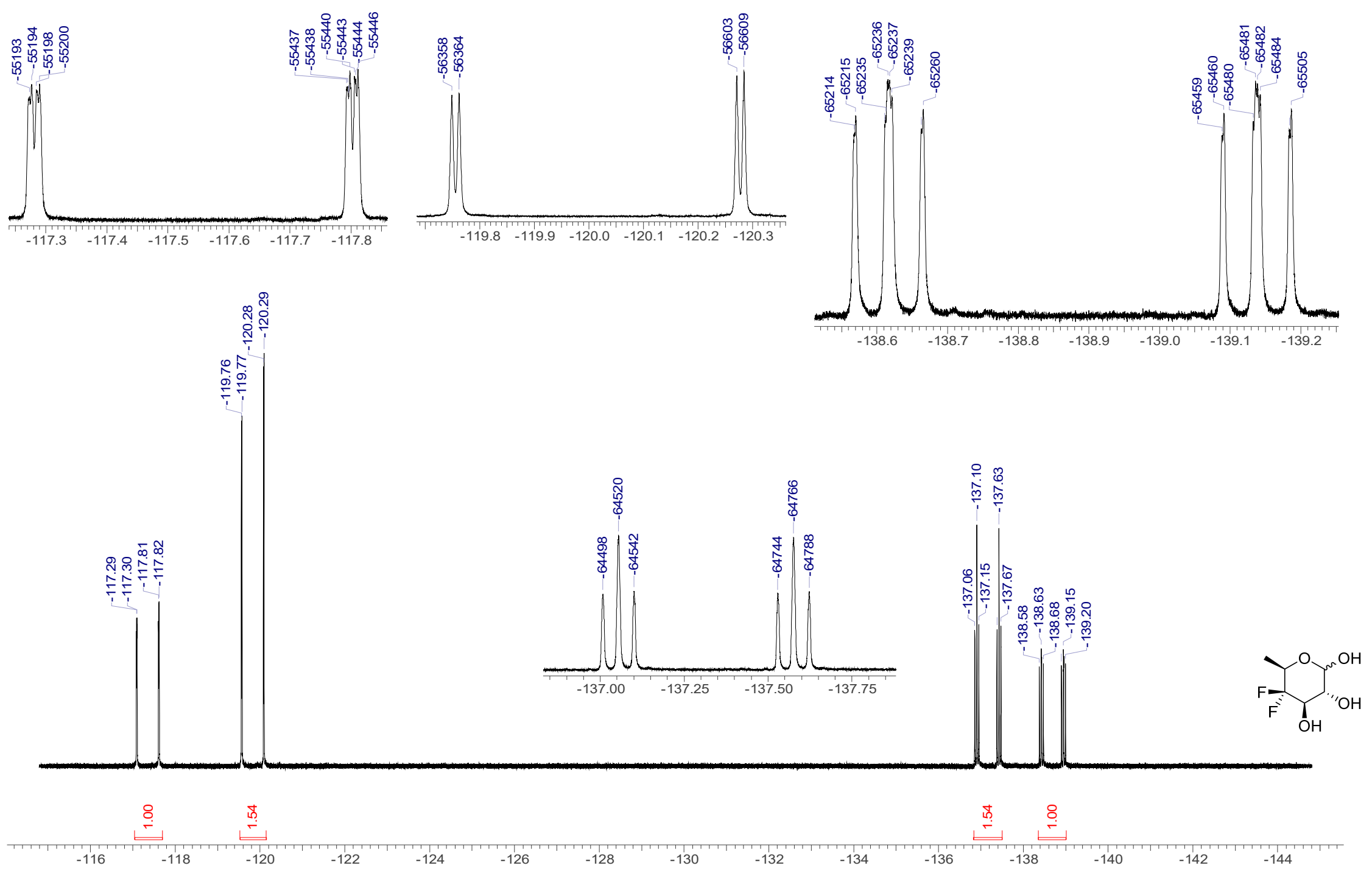


4.7.11. ${ }^{19} \mathrm{~F}\left\{{ }^{1} \mathrm{H}\right\}$ qNMR $\left(376 \mathrm{MHz}, \mathrm{D}_{2} \mathrm{O}\right)$

Duplicate \#1

DW/4,6 V8 qNMR D2O An1 240920 2pm

\section{sW: 300 ppm}

O1P: $\mathbf{- 1 2 8 . 3 4} \mathrm{ppm}$

D1: $60 \mathrm{sec}$

Proton-decoupled ${ }^{19} \mathrm{~F}$

NMR

$\alpha / \beta 39: 61$

(see next page for detailed integration

information after data processing)
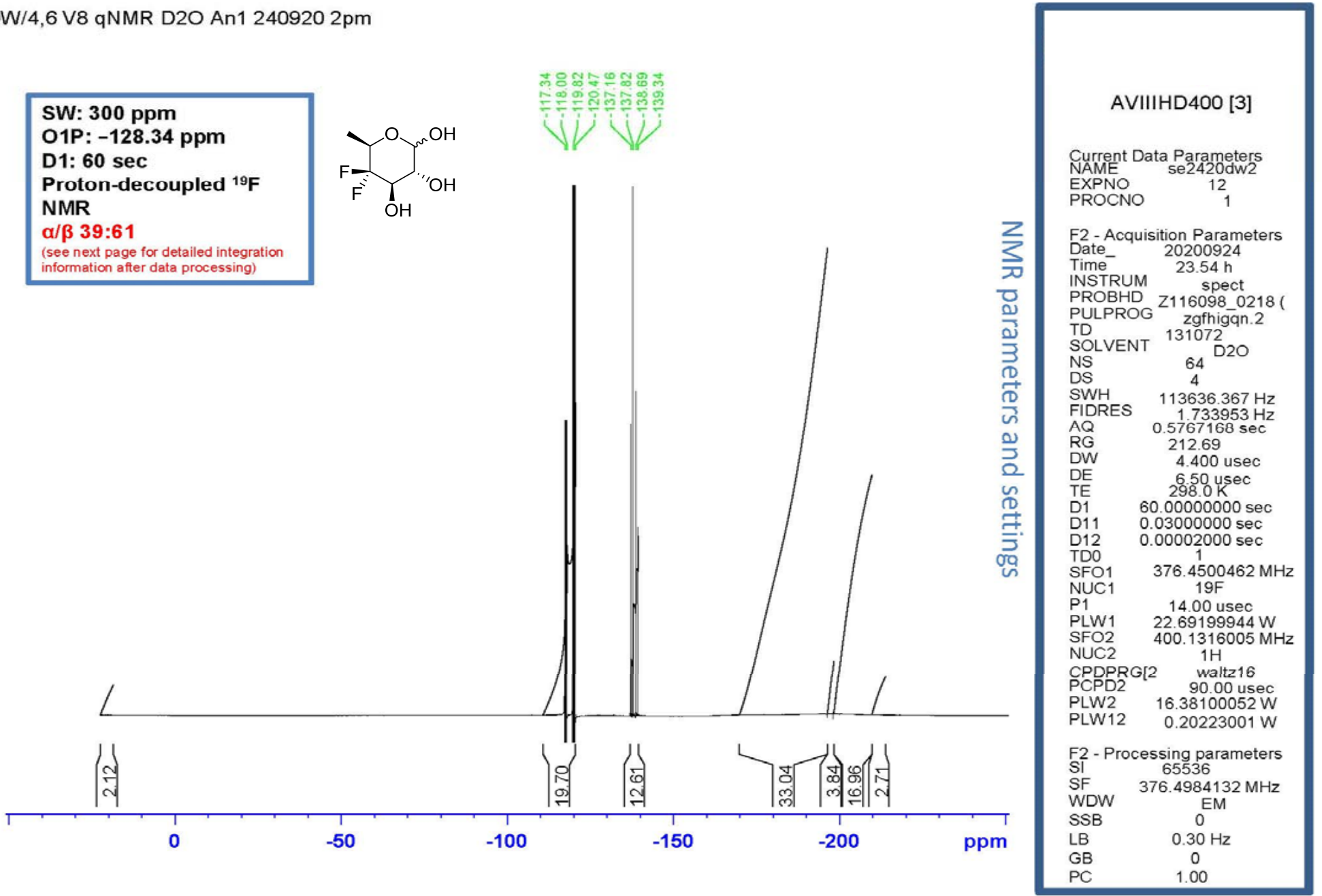


\section{Duplicate \#1}

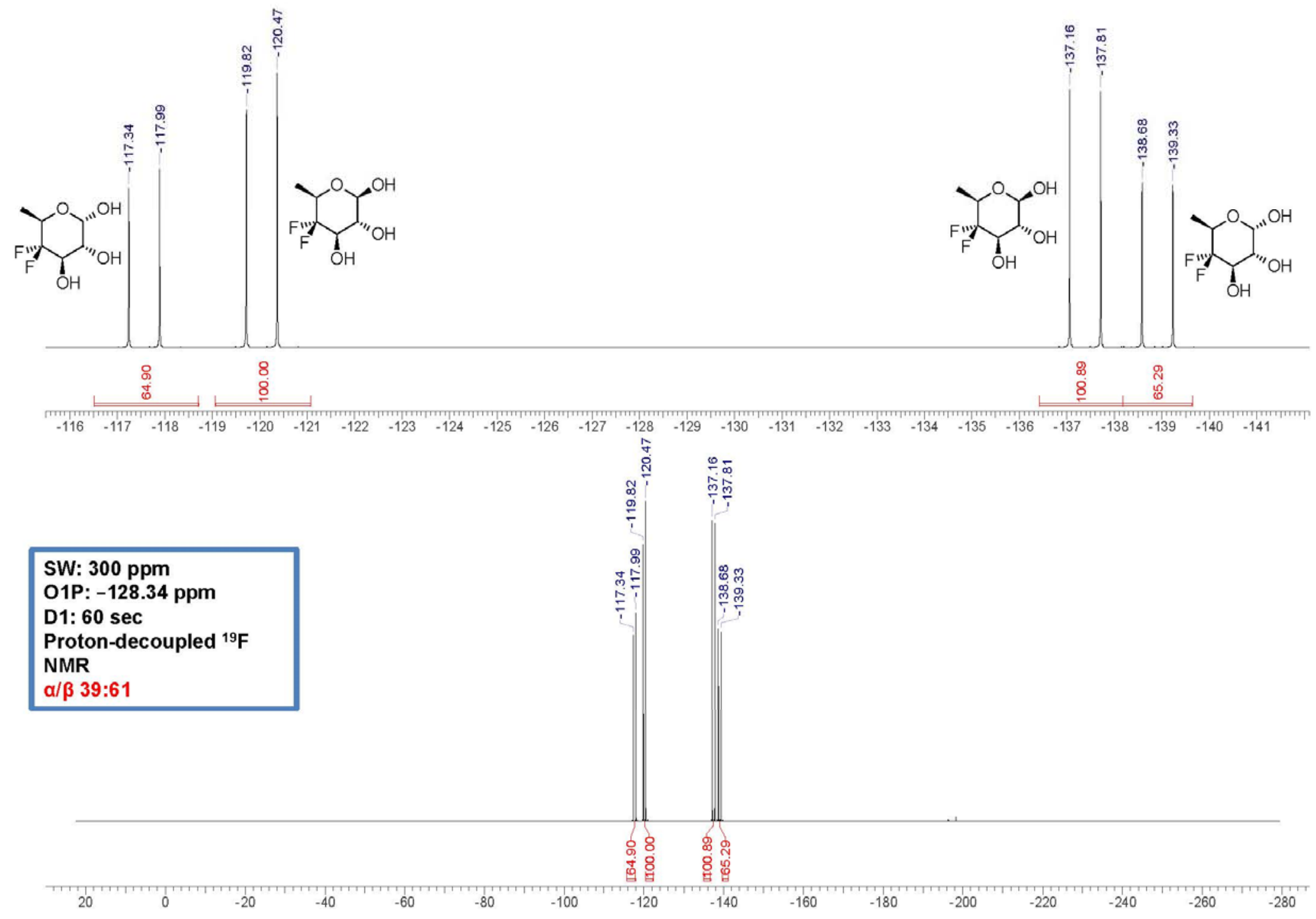




\section{Duplicate \#2}

DW/4,6 V8 qNMR D2O An2 240920 2pm

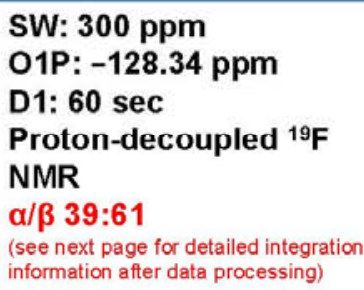

Proton-decoupled ${ }^{19} \mathrm{~F}$

NMR

$\alpha / \beta 39: 61$

see next page for detailed integration

information after data processing)
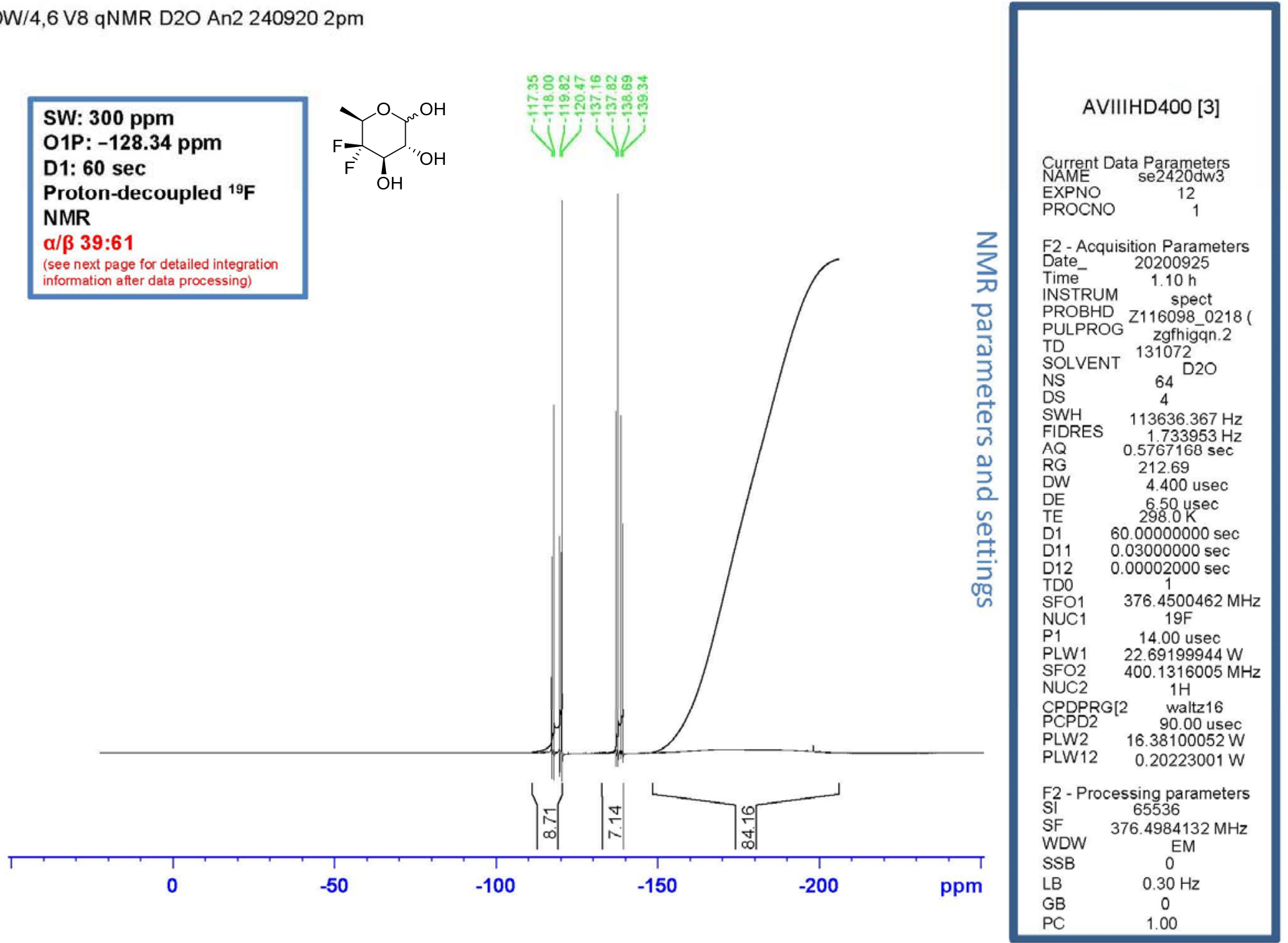
Duplicate \#2

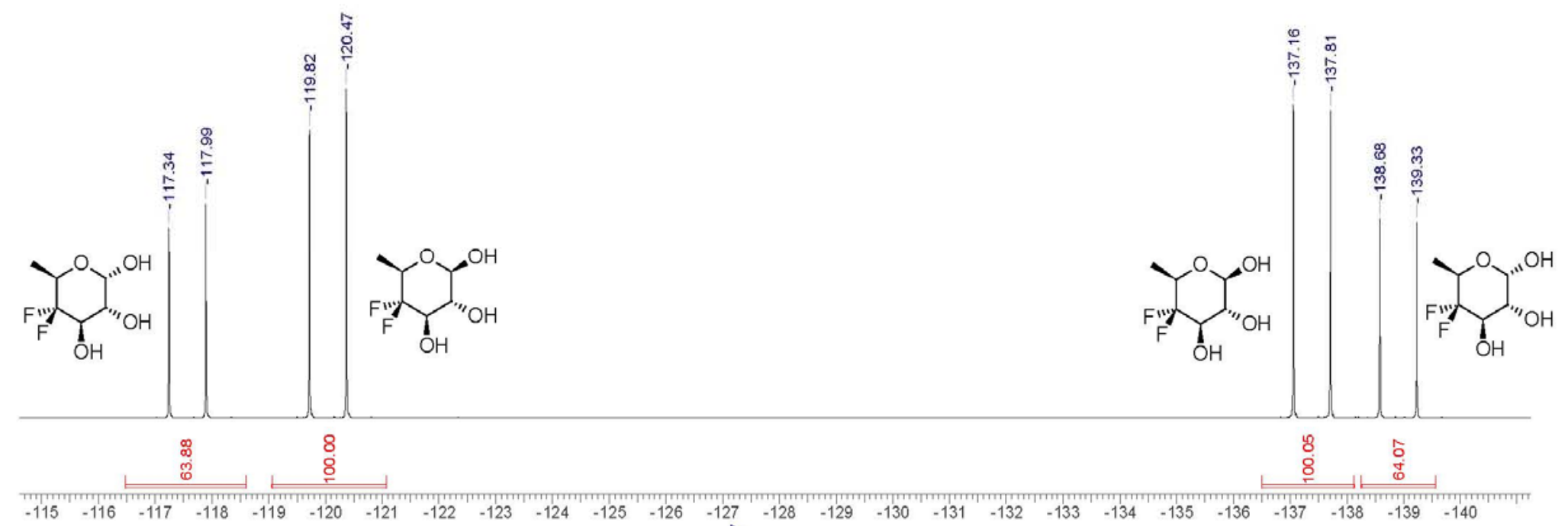

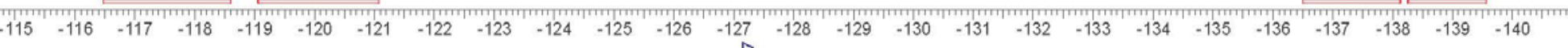

SW: $300 \mathrm{ppm}$
O1P: $-128.34 \mathrm{ppm}$
D1: $60 \mathrm{sec}$
Proton-decoupled ${ }^{19} \mathrm{~F}$
NMR
$\alpha / \beta 39: 61$

$\alpha / \beta 39: 61$

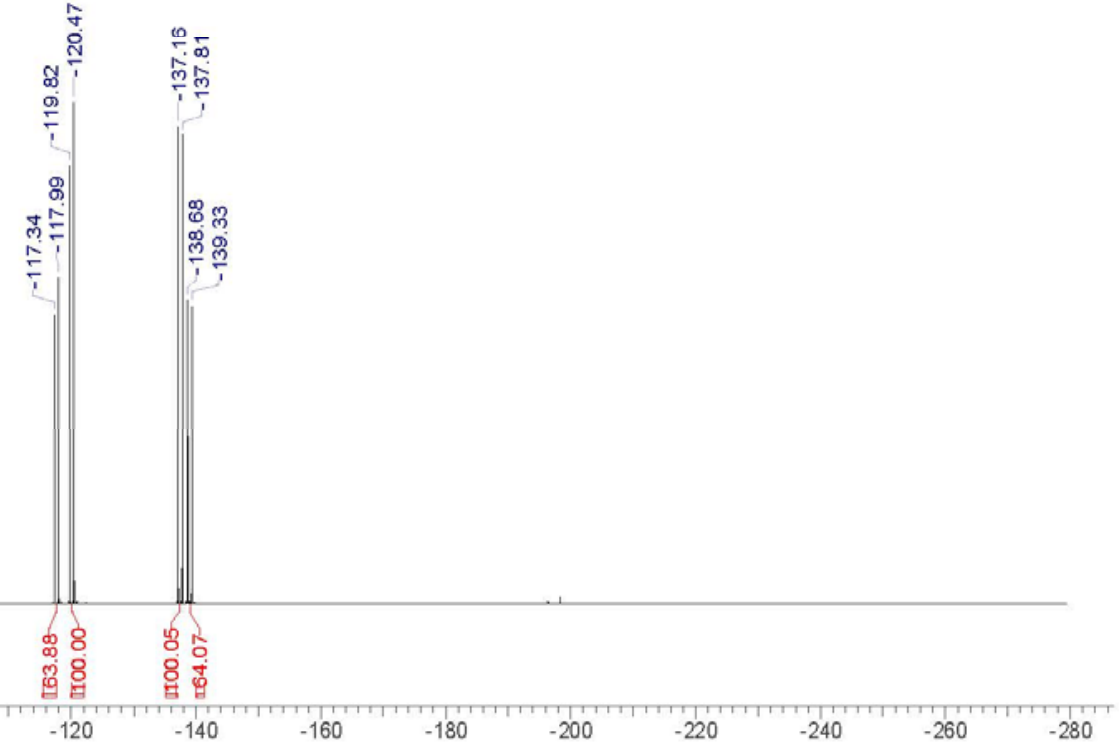




\subsection{4,6-Dideoxy-6,6-difluoro-D-xylo-hexopyranoside (7a)}

4.8.1. ${ }^{1} \mathrm{H}$ NMR $\left(500 \mathrm{MHz}, \mathrm{D}_{2} \mathrm{O}\right)$
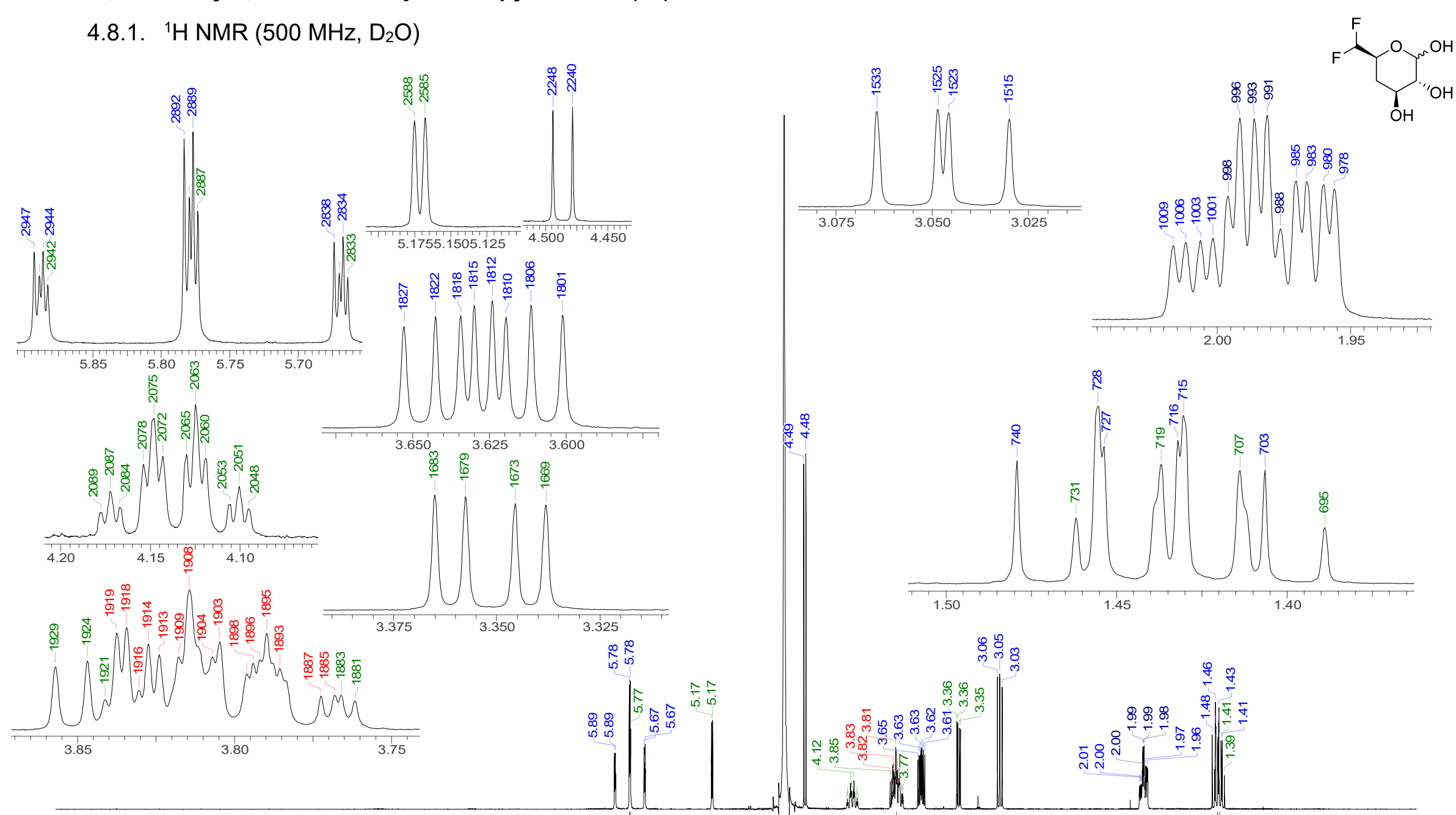

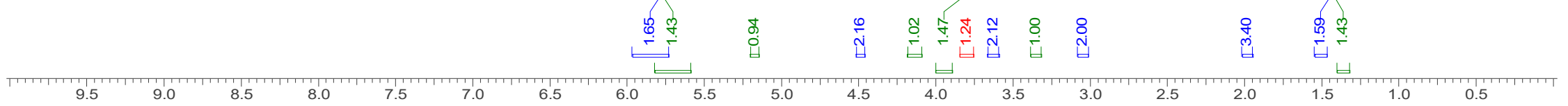


4.8.2. ${ }^{1} \mathrm{H}\left\{{ }^{19} \mathrm{~F}\right\} \mathrm{NMR}\left(500 \mathrm{MHz}, \mathrm{D}_{2} \mathrm{O}\right)$

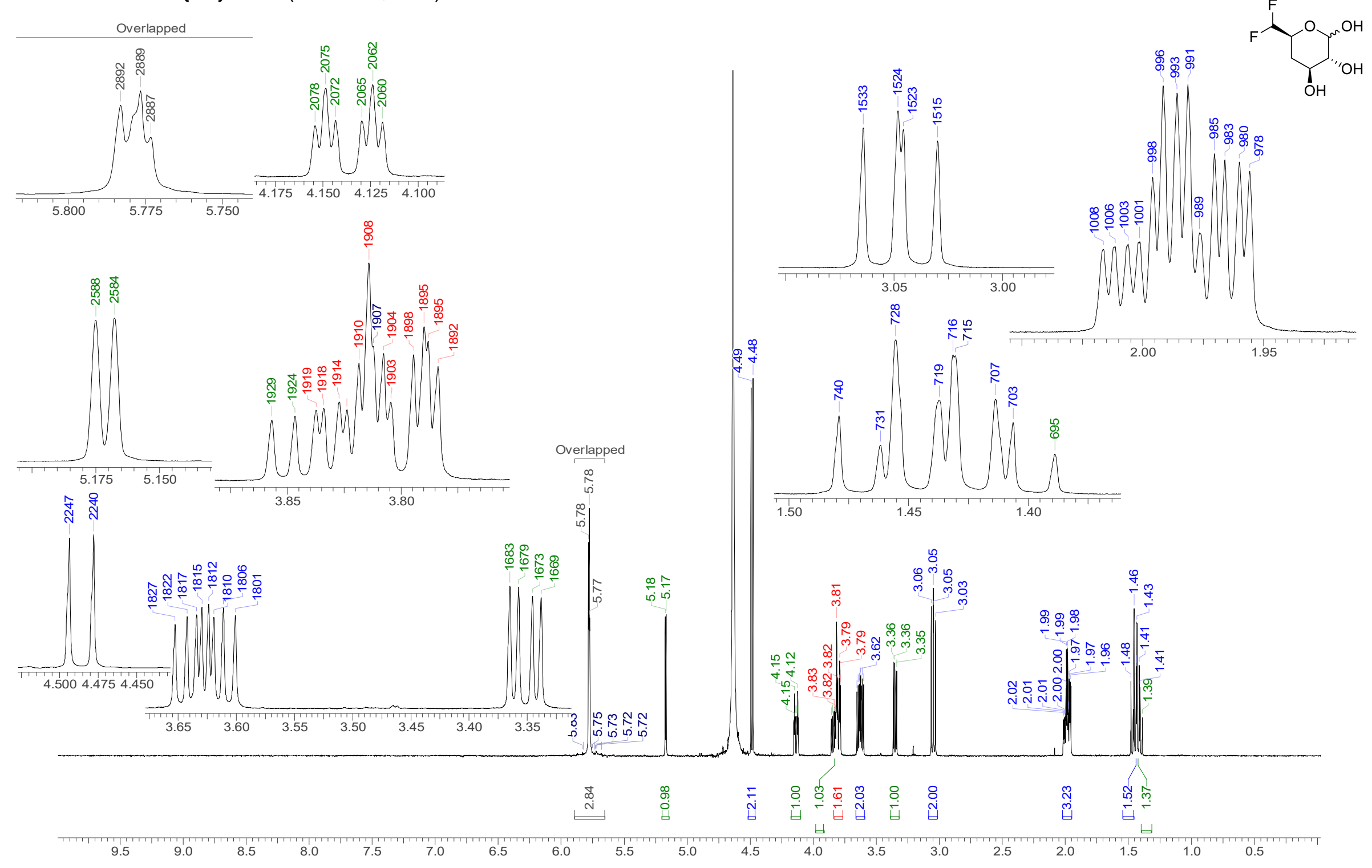


4.8.3. ${ }^{13} \mathrm{C}\left\{{ }^{1} \mathrm{H}\right\} \operatorname{NMR}\left(126 \mathrm{MHz}, \mathrm{D}_{2} \mathrm{O}\right)$

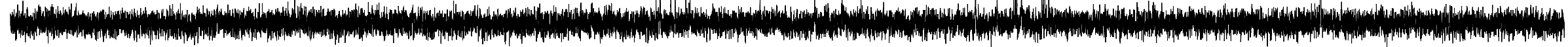

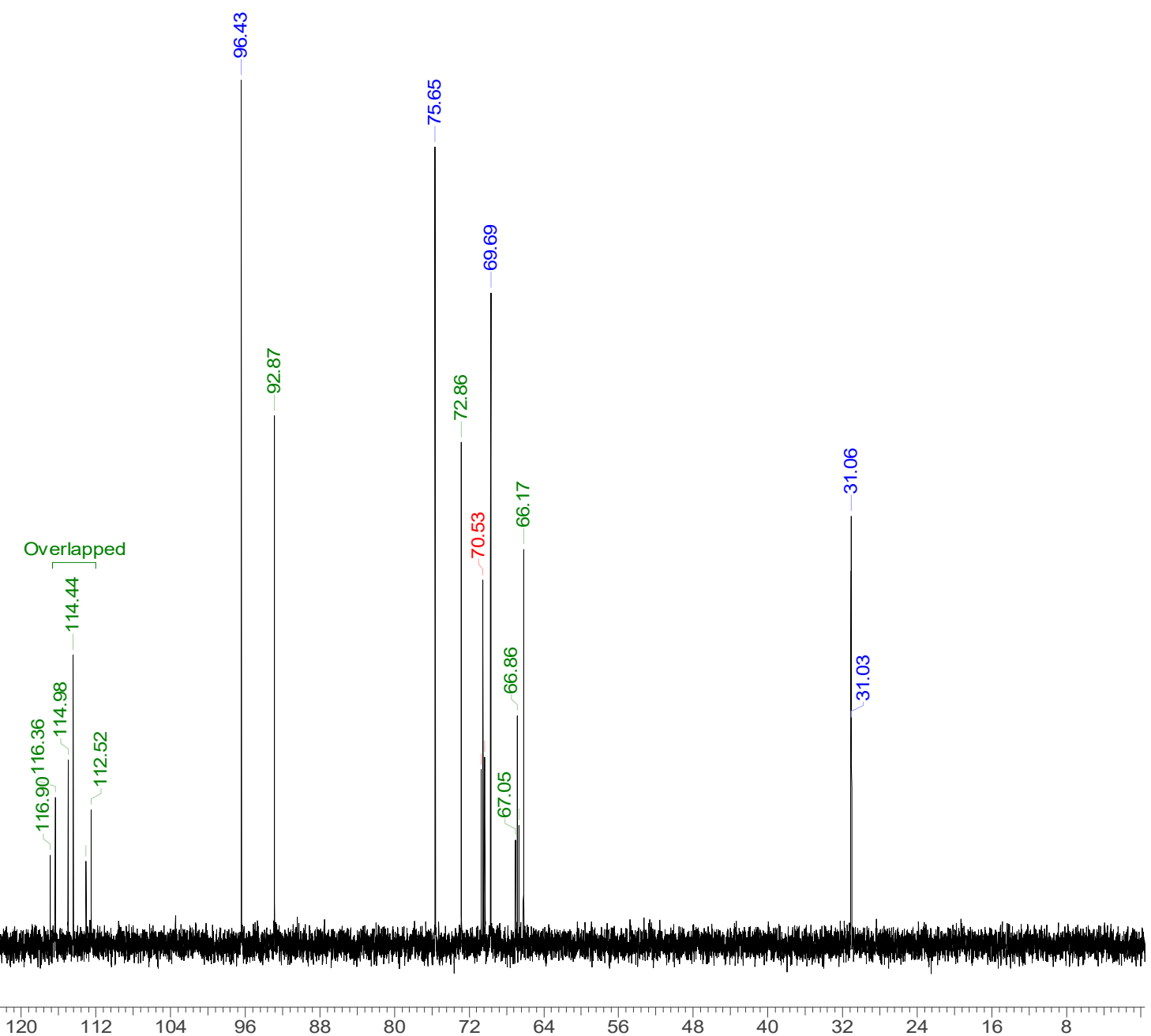


4.8.4. COSY, HSQC-DEPT \& HMBC $\left(D_{2} \mathrm{O}\right)$

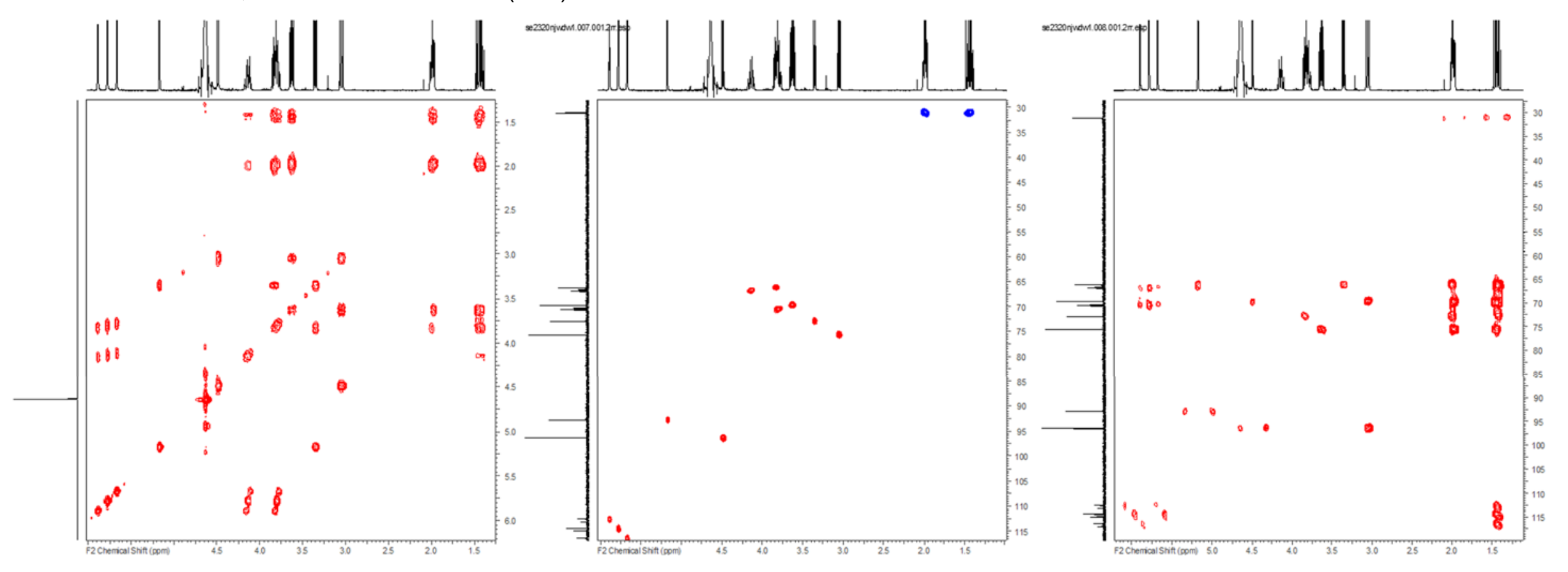


4.8.5. ${ }^{19} \mathrm{~F}$ NMR $\left(470 \mathrm{MHz}, \mathrm{D}_{2} \mathrm{O}\right)$

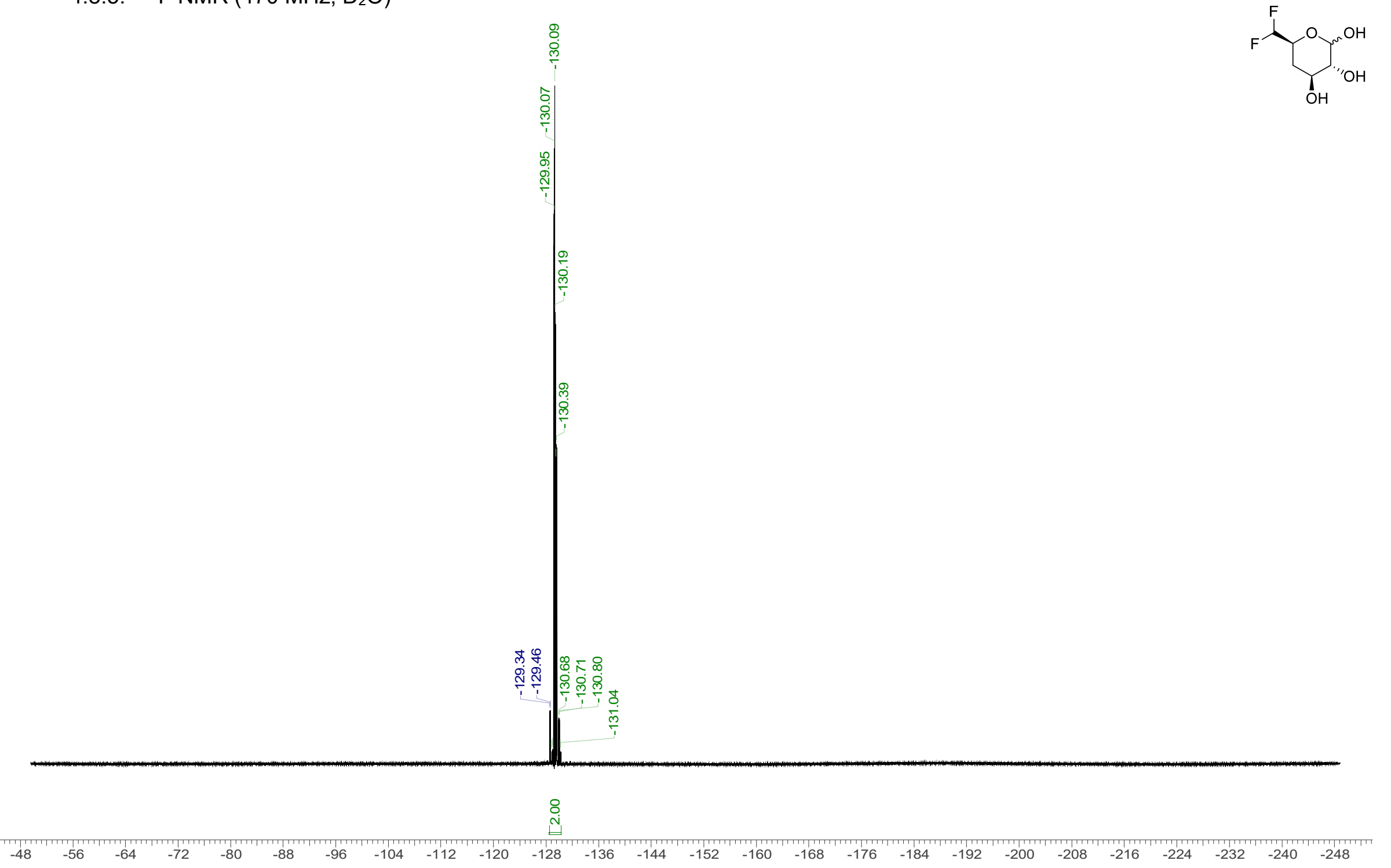




\section{${ }^{19} \mathrm{~F}$ expansion}

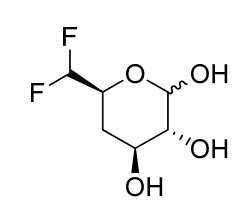

M02(ddd)

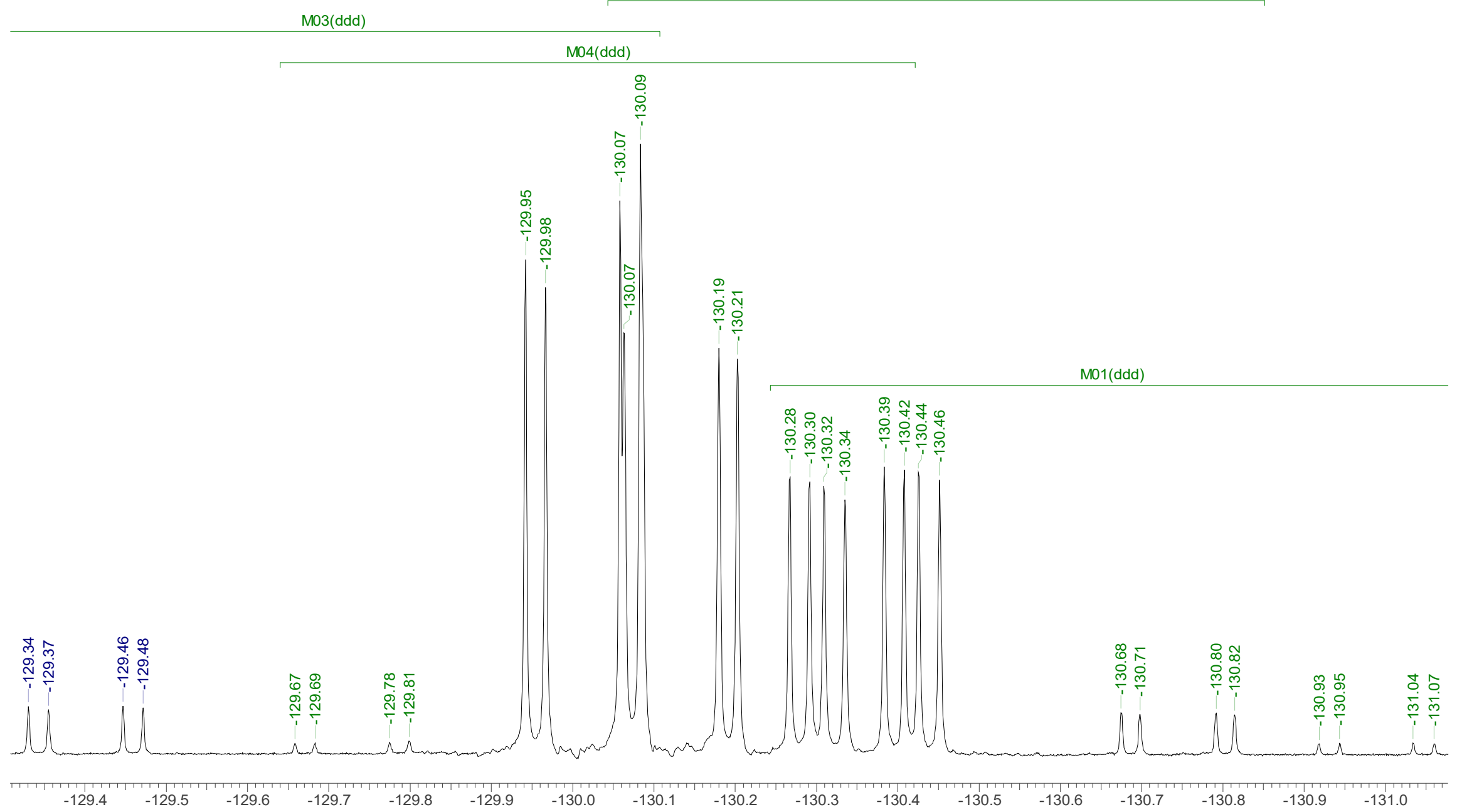


4.8.6. ${ }^{19} \mathrm{~F}\left\{{ }^{1} \mathrm{H}\right\} \mathrm{NMR}\left(470 \mathrm{MHz}, \mathrm{D}_{2} \mathrm{O}\right)$

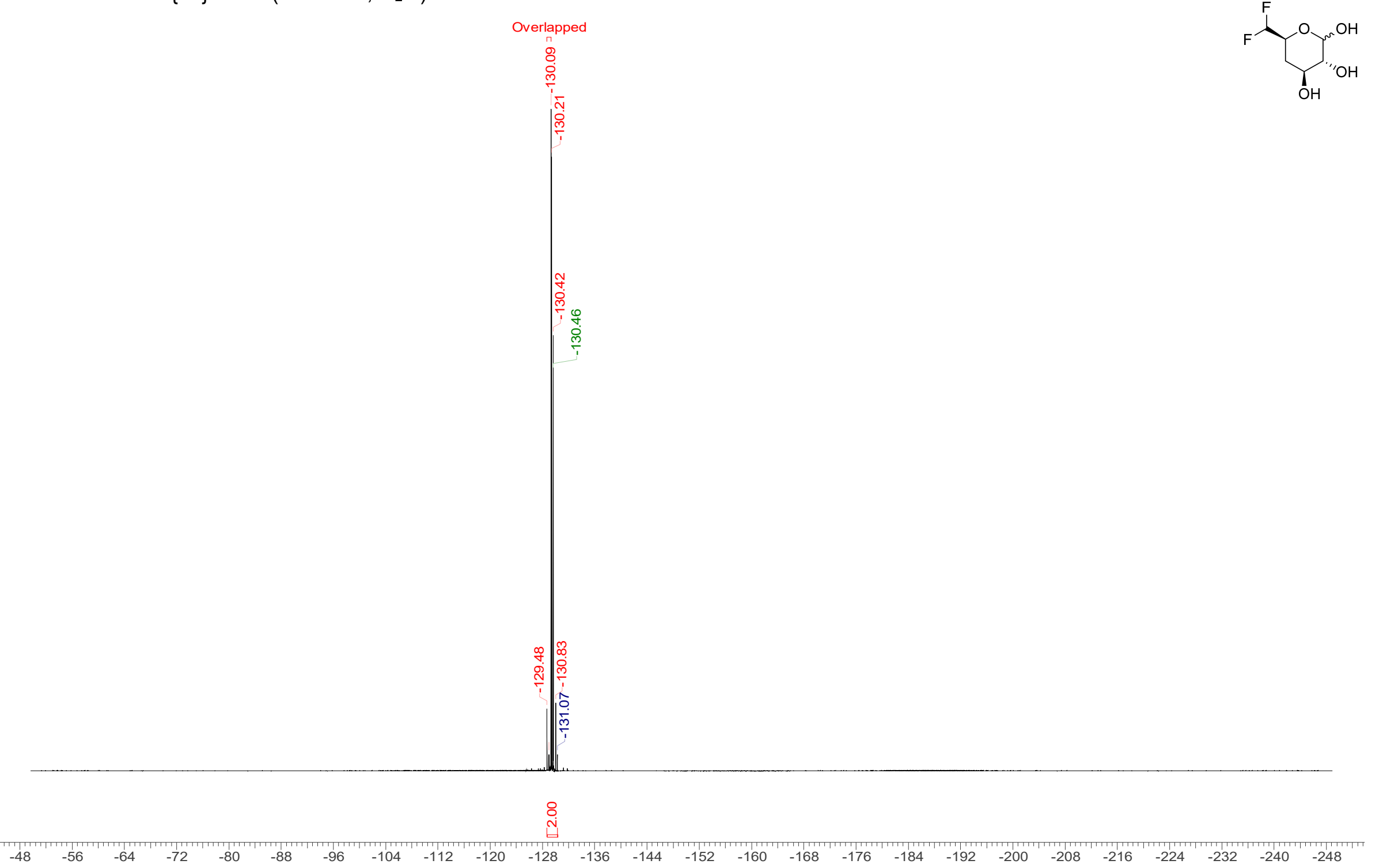




\section{${ }^{19} \mathrm{~F}\left\{{ }^{1} \mathrm{H}\right\}$ expansion}

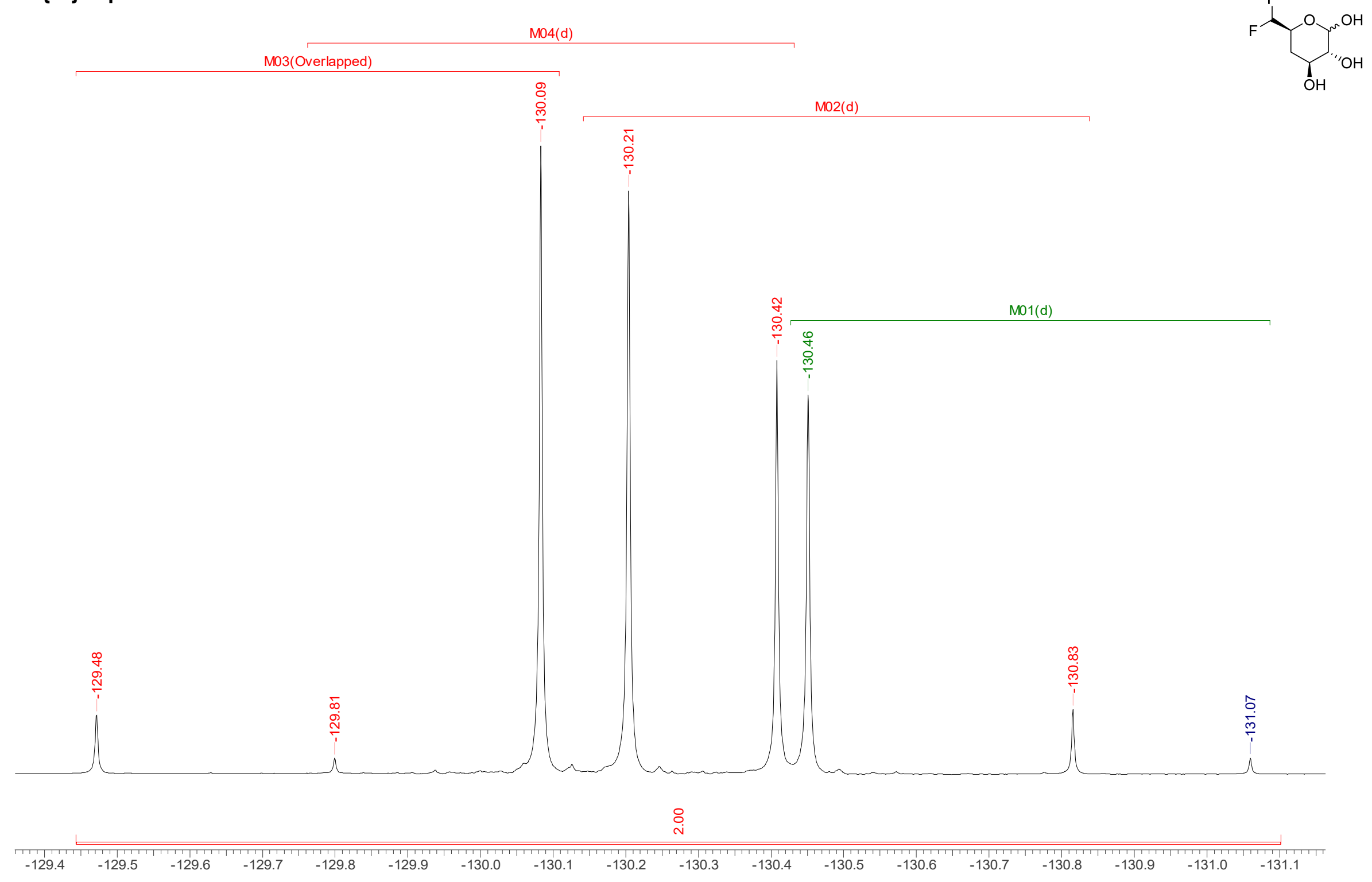


4.8.7. ${ }^{1} \mathrm{H}$ qNMR $\left(500 \mathrm{MHz}, \mathrm{D}_{2} \mathrm{O}\right)$

Duplicate \#1

DW/4,6/V7 1H qNMR (1)

Quant. proton

Longest T1 $3.7 \mathrm{~s}$ (delay set to $30 \mathrm{~s}$ for full relaxation)

\section{SW: $\mathbf{3 0}$ ppm}

O1P: $6.175 \mathrm{ppm}$

D1: 30 sec

${ }^{1} \mathrm{H}$ NMR

$\alpha / \beta 38: 62$
(see next page for detailed integration

information after data processing)
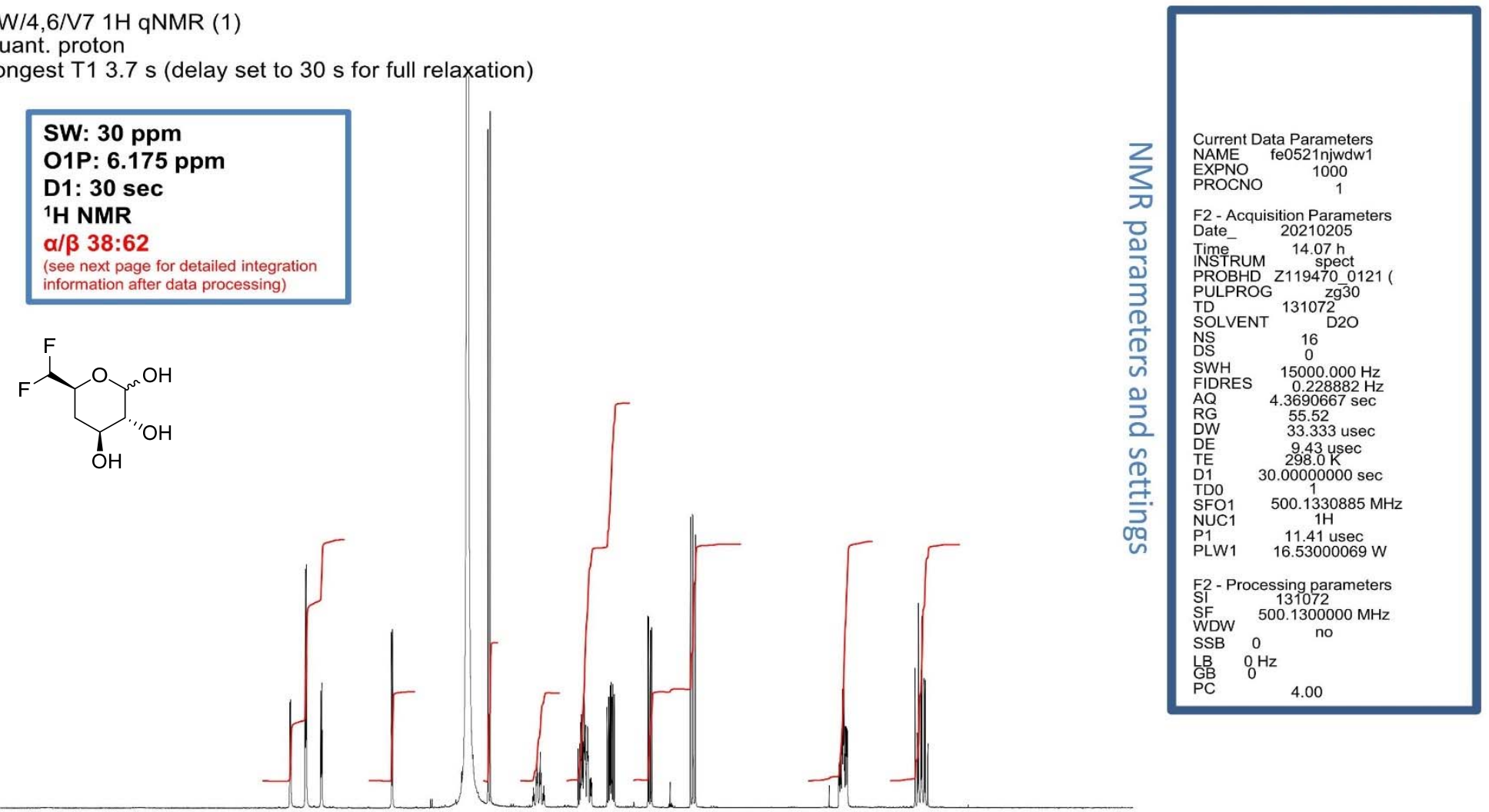

$\begin{array}{lll}7.5 & 7.0 & 6.5\end{array}$
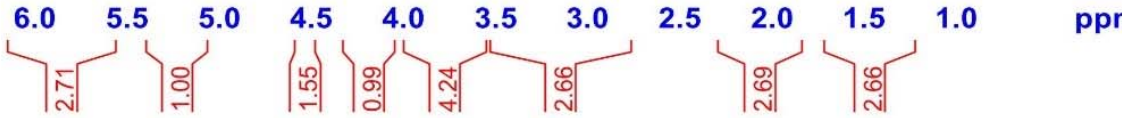

ppm 
Duplicate \#1

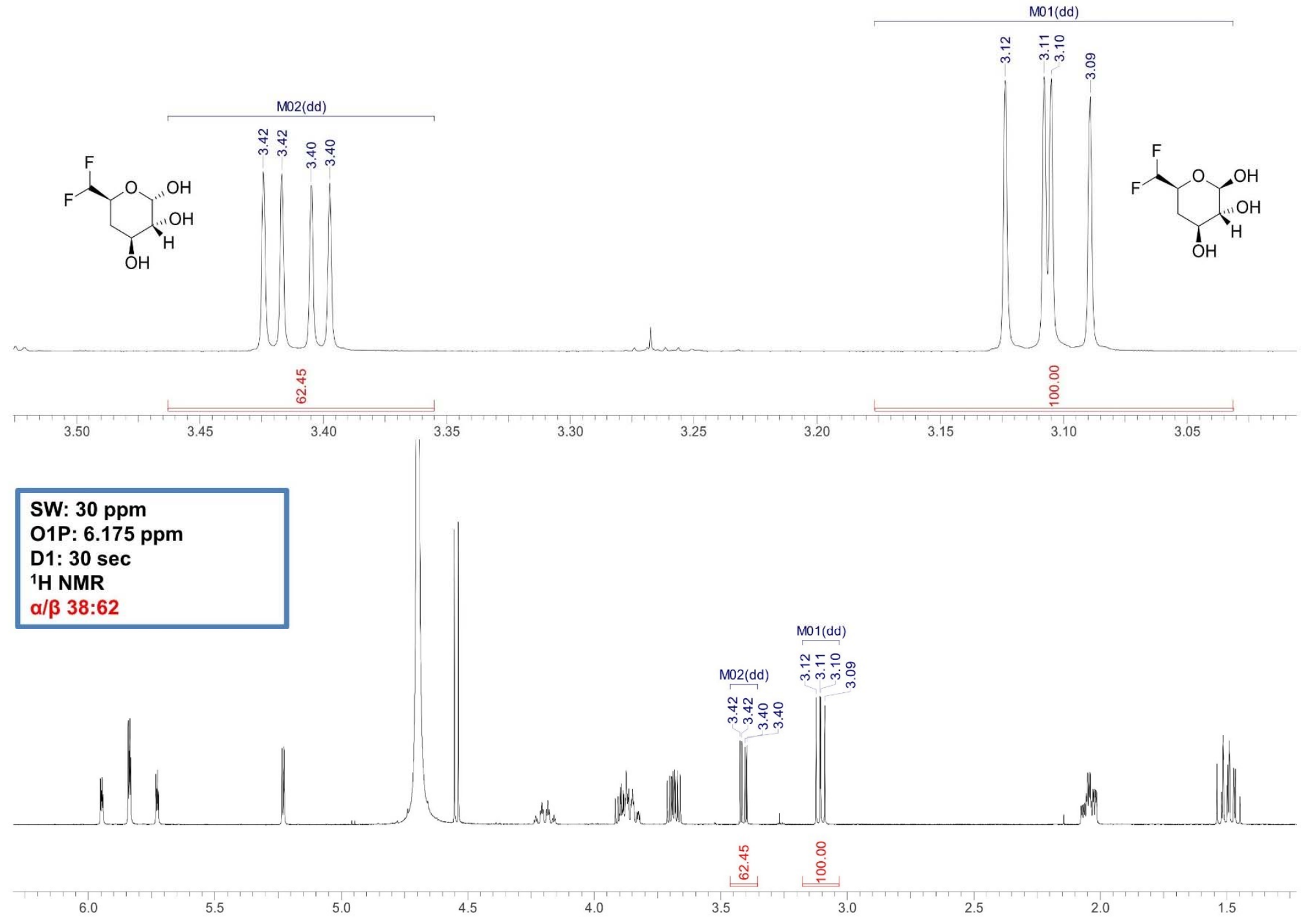




\section{Duplicate \#2}

DW/4,6/V7 1H qNMR (2)

Quant. proton

Longest T1 $3.5 \mathrm{~s}$ (delay set to $30 \mathrm{~s}$ for full relaxation)

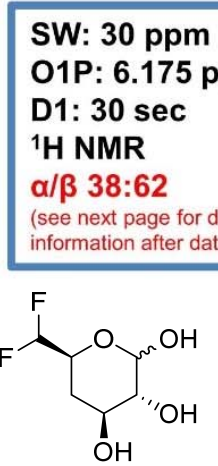

SW: $30 \mathrm{ppm}$

O1P: $6.175 \mathrm{ppm}$

D1: 30 sec

age for detailed integration

${ }_{\mathrm{OH}}^{\mathrm{O}}$
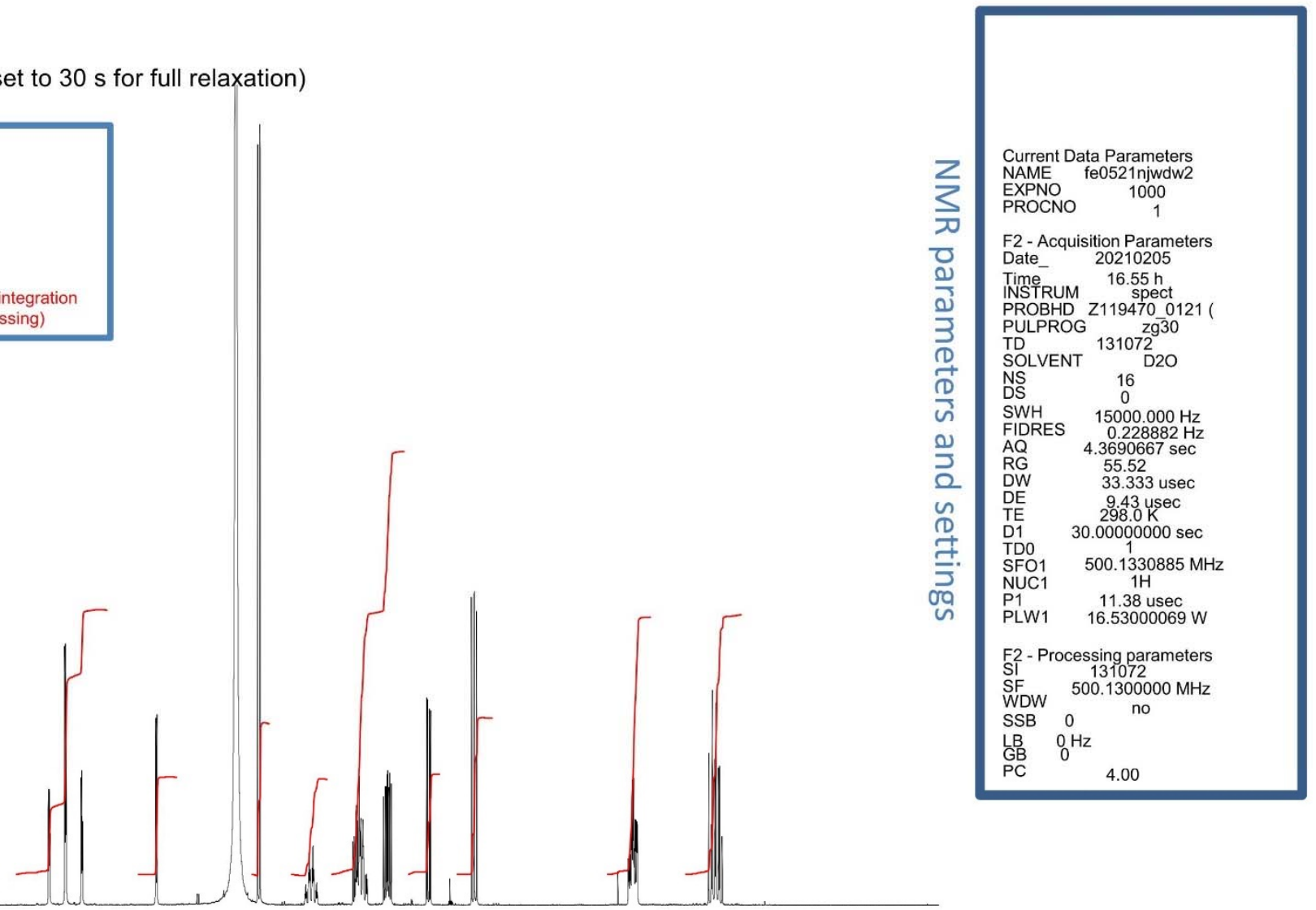

\begin{tabular}{ll}
$7.5 \quad 7.0$ \\
\hline
\end{tabular}

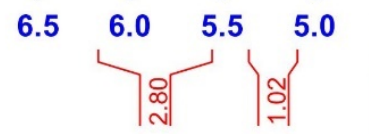

$4.5 \quad 4.03 .5 \quad 3.0$

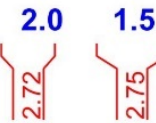

ppm 


\section{Duplicate \#2}

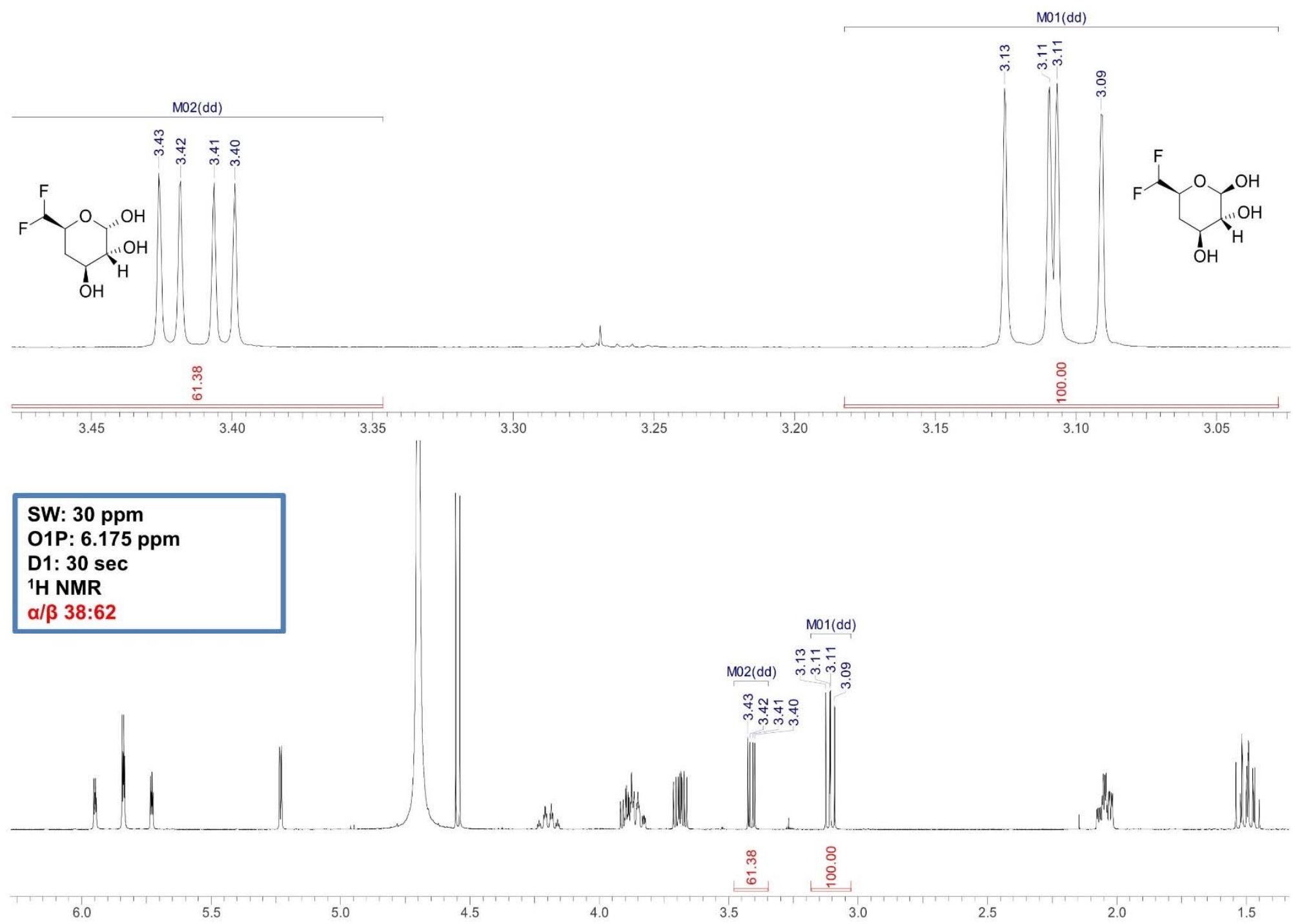




\subsection{D-Glucose (83)}

4.9.1. ${ }^{1} \mathrm{H}$ NMR $\left(500 \mathrm{MHz}, \mathrm{D}_{2} \mathrm{O}\right)$
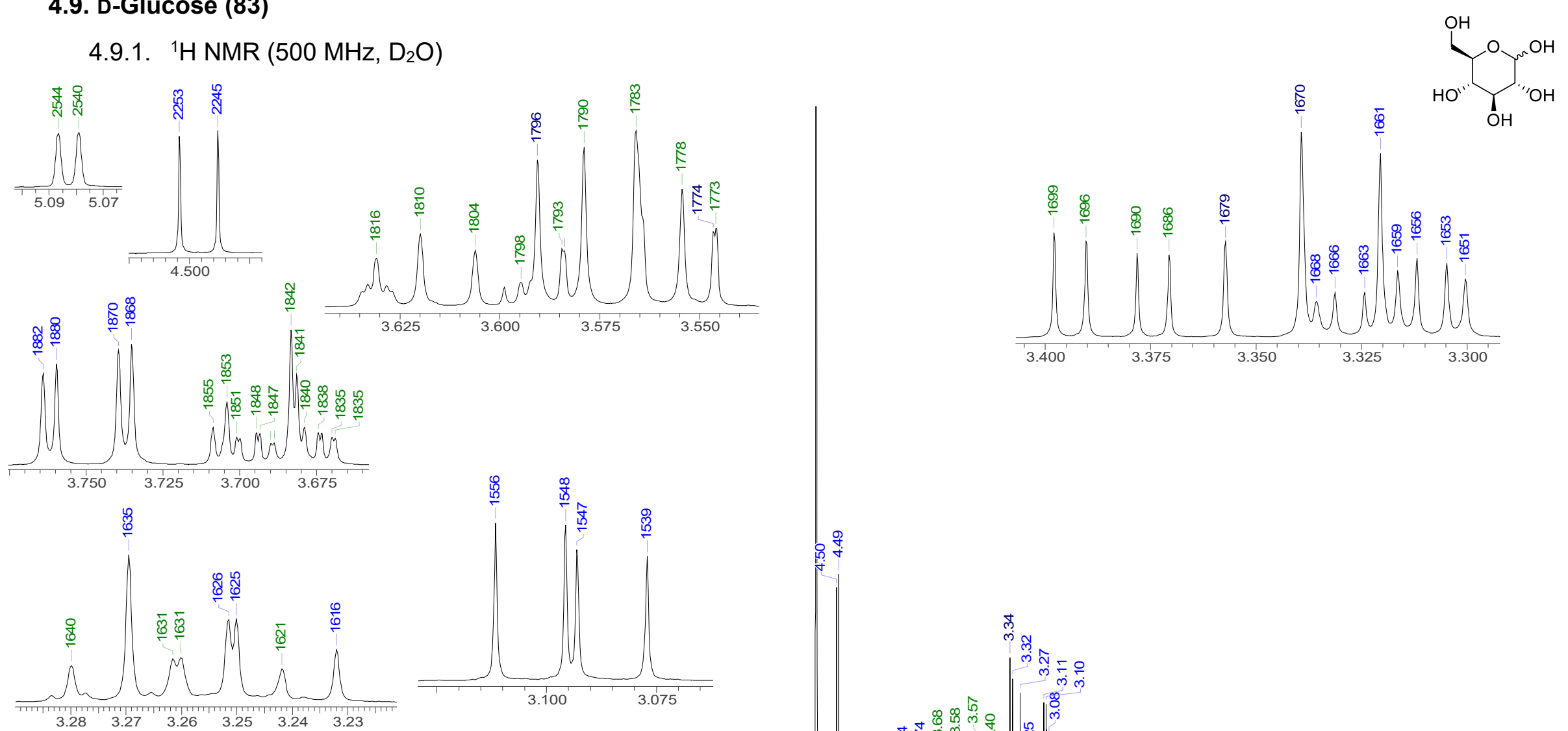

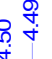

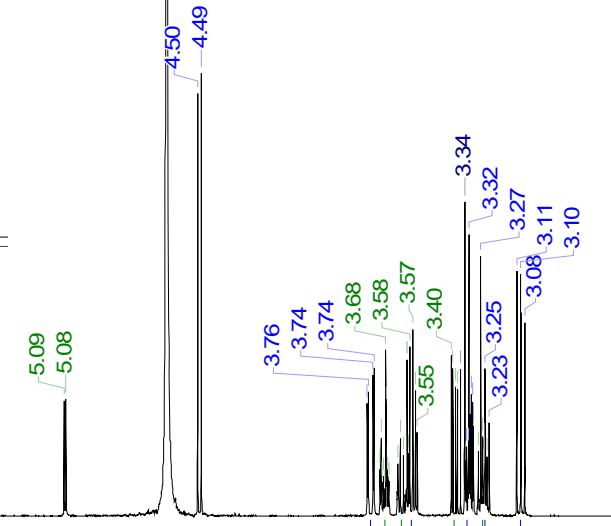

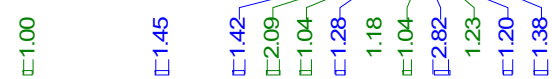

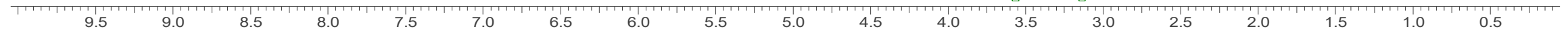


4.9.2. ${ }^{13} \mathrm{C}\left\{{ }^{1} \mathrm{H}\right\} \operatorname{NMR}\left(126 \mathrm{MHz}, \mathrm{D}_{2} \mathrm{O}\right)$
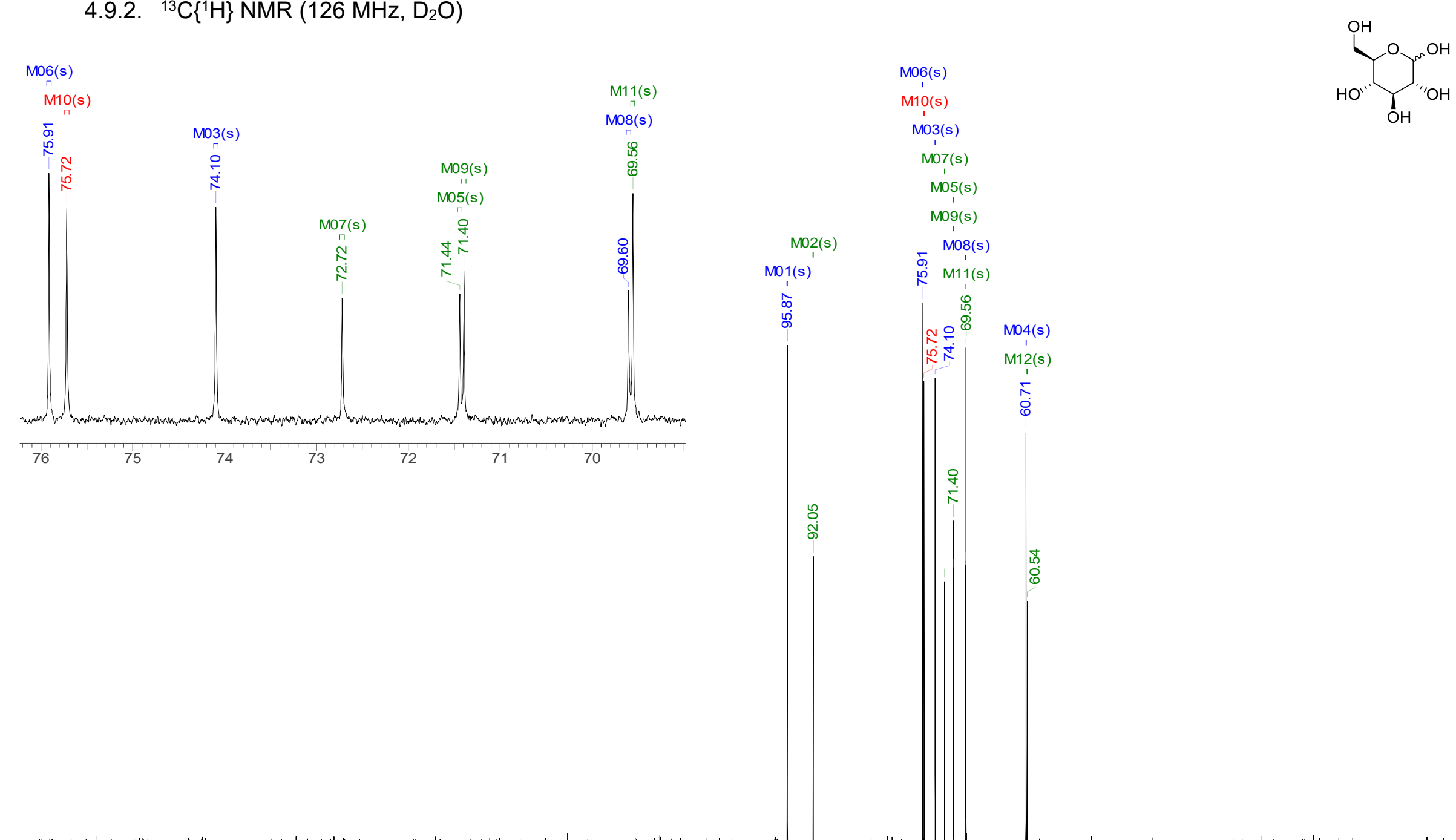

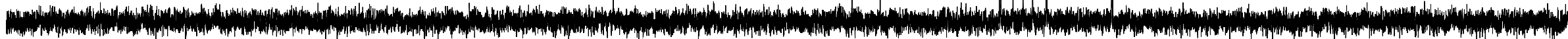

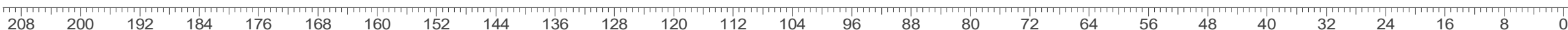


4.9.3. COSY, HSQC-DEPT \& HMBC $\left(\mathrm{D}_{2} \mathrm{O}\right)$

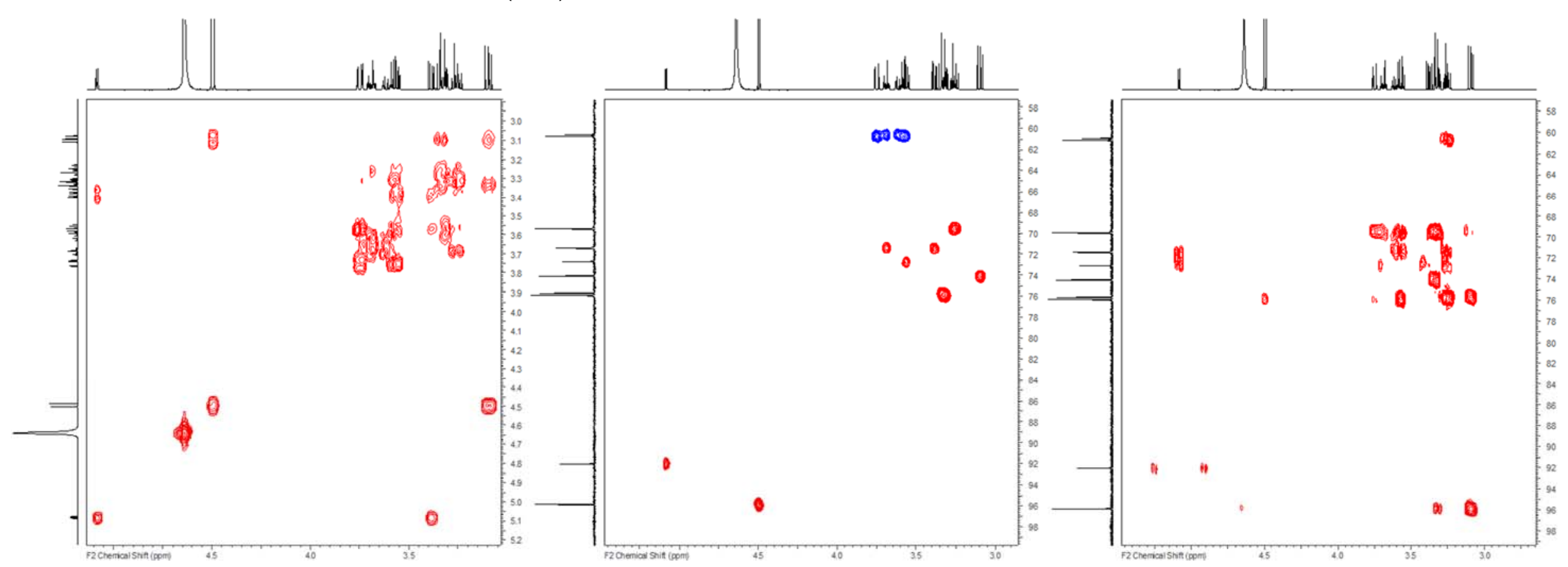


4.9.4. ${ }^{1} \mathrm{H}$ qNMR $\left(500 \mathrm{MHz}, \mathrm{D}_{2} \mathrm{O}\right)$

Duplicate \#1

DW/4,6 Glu D2O $500 \mathrm{MHz}$

Quant. proton

Longest T1 $2.8 \mathrm{~s}$ (delay set to $30 \mathrm{~s}$ for full relaxation)

"OH
SW: 30 ppm

O1P: $6.175 \mathrm{ppm}$

D1: $30 \mathrm{sec}$

1H NMR

$\alpha / \beta=38: 62$

for detailed integration

information after data processing)

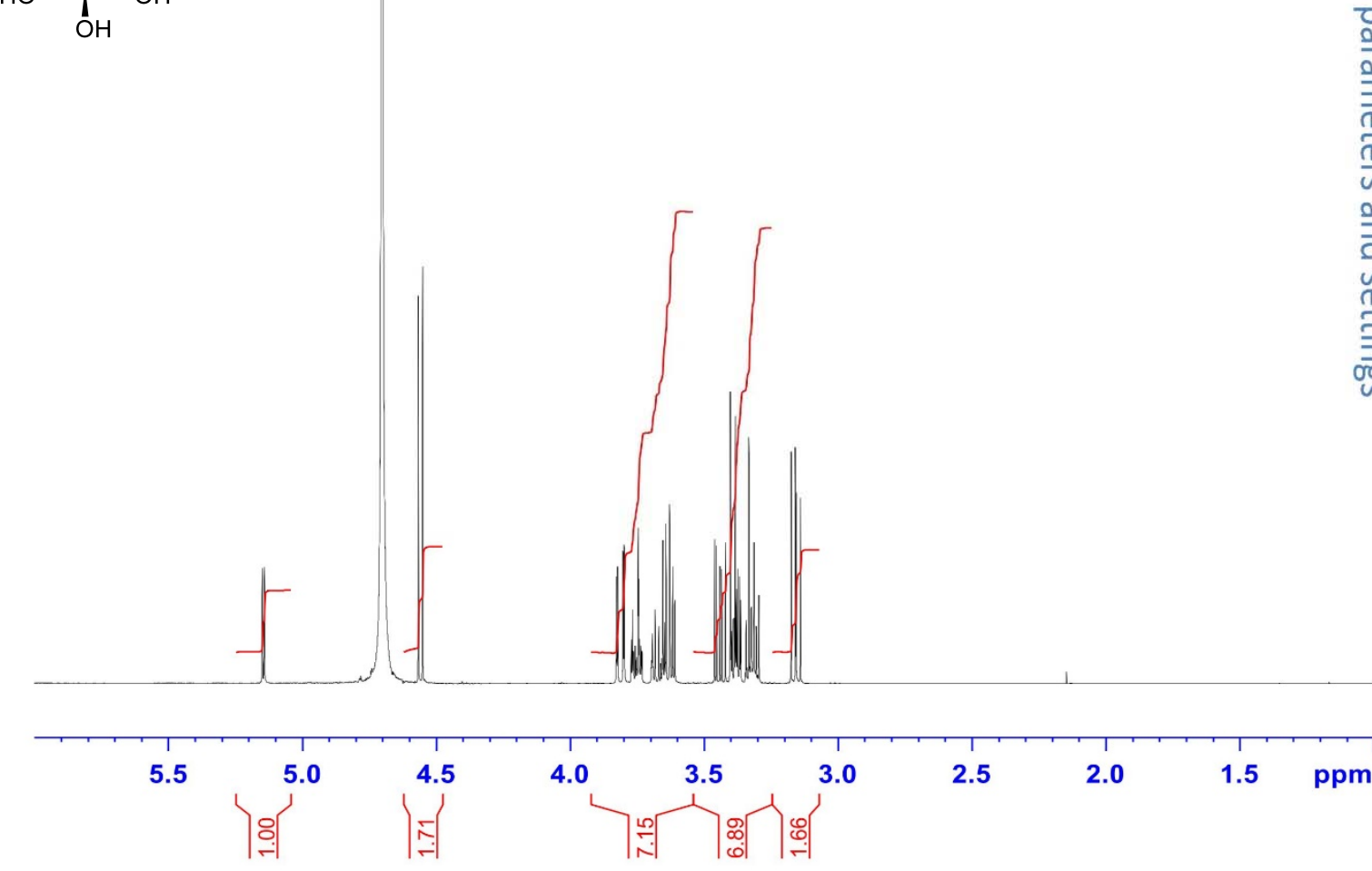


Duplicate \#1

SW: 30 ppm

01P: $6.175 \mathrm{ppm}$

D1: $30 \mathrm{sec}$

H NMR

(1"

$\alpha / \beta=38: 62$

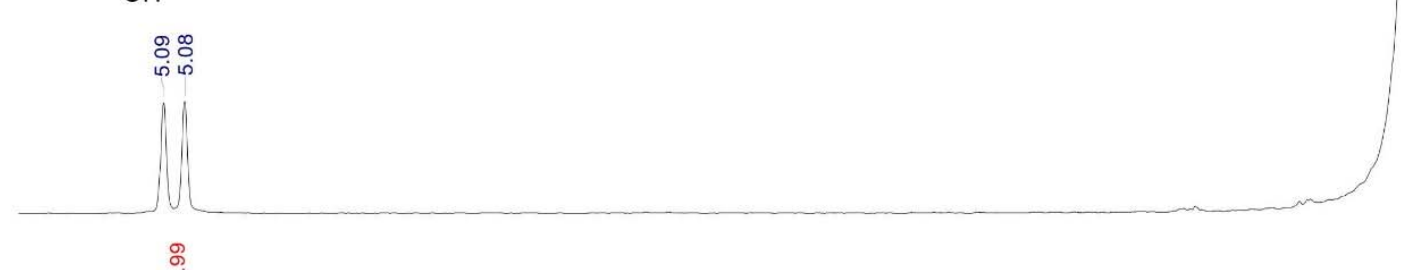

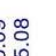
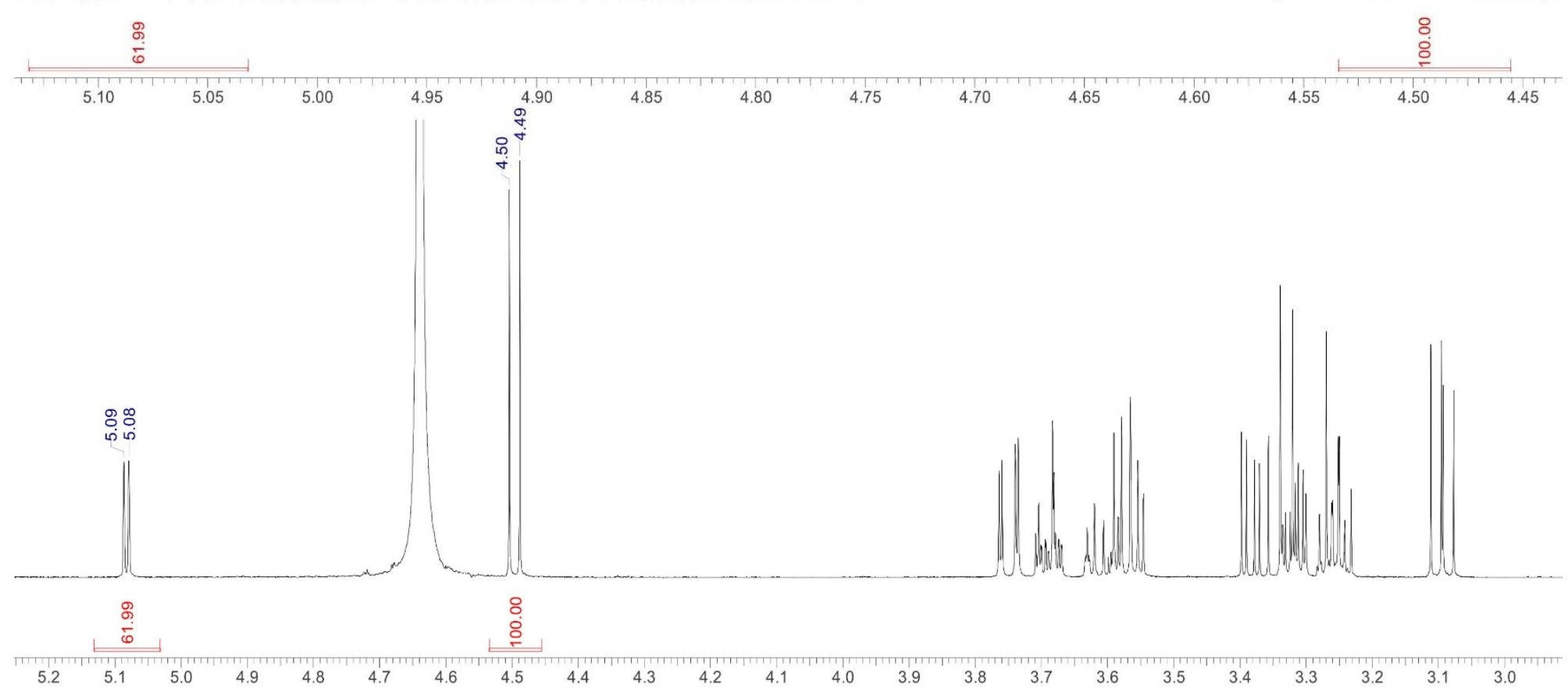


\section{Duplicate \#2}

DW/4,6 Glu $500 \mathrm{MHz}$

Quant. proton (qNMR 2)

Longest T1 $2.8 \mathrm{~s}$ (delay set to $30 \mathrm{~s}$ for full relaxation)

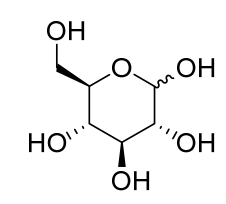

$\mathrm{O}_{\mathrm{OH}}^{\mathrm{OH}}$

SW: $30 \mathrm{ppm}$

01P: $6.175 \mathrm{ppm}$

D1: 30 sec

${ }^{1} \mathrm{H}$ NMR

$\alpha / \beta=38: 62$

iled integratio

information after data processing)

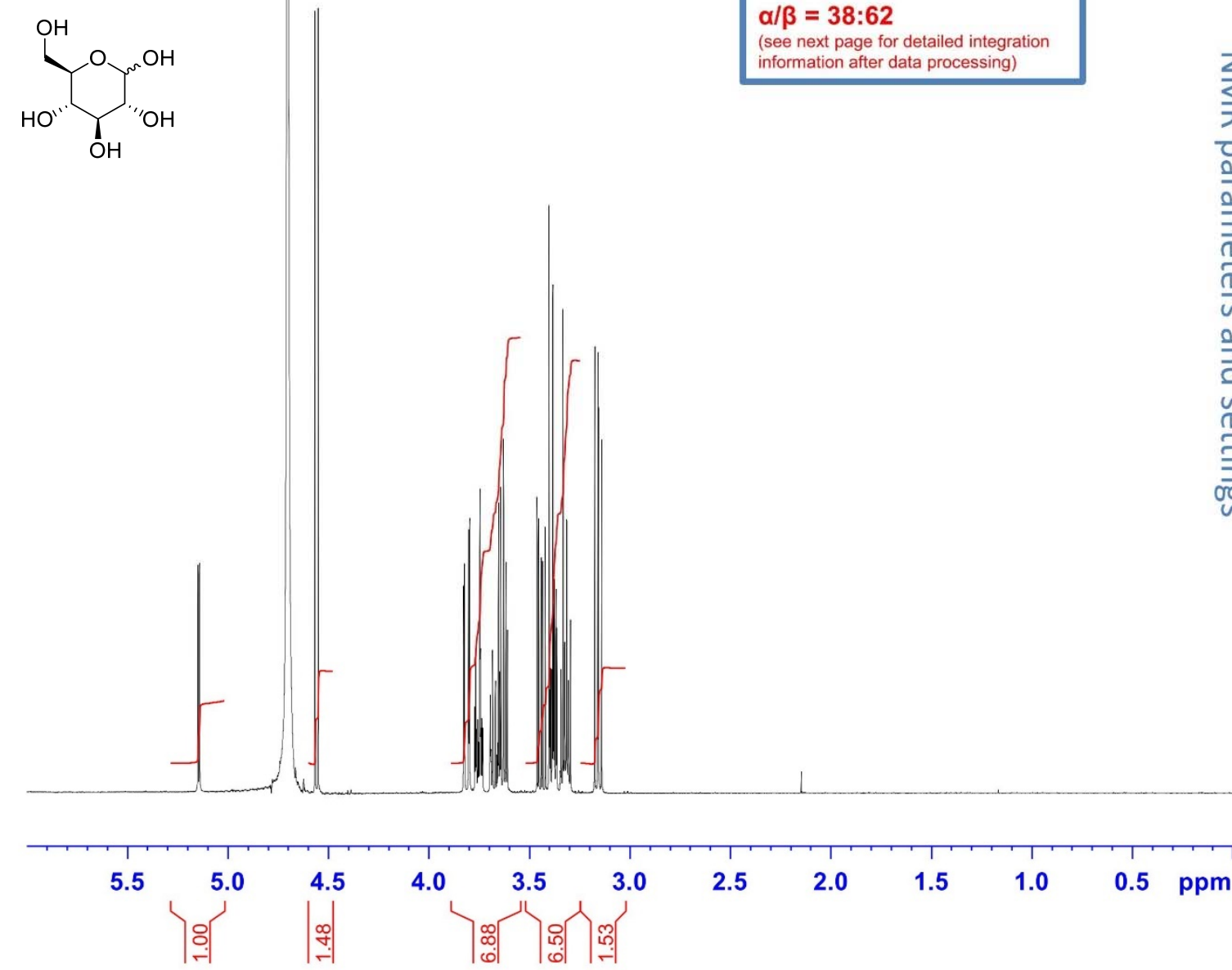

Current Data Parameters

$\begin{array}{ll}\text { EXPNO } & 2 \\ \text { PROCNO } & 1\end{array}$

F2 - Acquisition Parameters

Time ${ }_{\text {INSTRM }} 15.22 \mathrm{~h}$

PROBHD Z119470 0121

PULPROG $\mathrm{zg}^{3} 30$

SOLVENT ${ }^{131072}$ D2O

$\begin{array}{lc}\text { NS } & 16 \\ \text { DS } & 0 \\ \text { SWH } & 15000.000 \mathrm{~Hz}\end{array}$

AQ $\quad 4.3690667 \mathrm{sec}$

$\begin{array}{ll}\text { RG } & 55.52 \\ \text { DW } & 33.333 \text { usec }\end{array}$

$\begin{array}{ll}\text { DE } & 9.29 \text { usec } \\ \text { TE } & 298.0 \mathrm{~K}\end{array}$

$\begin{array}{ll}\text { TE } & 298.0 \mathrm{~K} \\ \text { D1 } & 30.0000000 \mathrm{sec}\end{array}$

TD0 1

$\begin{array}{lc}\text { SFO1 } & 500.1330885 \mathrm{MHz} \\ \text { NUC1 } & 1 \mathrm{H}\end{array}$

$\begin{array}{ll}\text { P1 } & 12.06 \text { usec } \\ \text { PLW1 } & 15.15600014 \mathrm{~W}\end{array}$

F2 - Processing parameters

$\begin{array}{ll} & \\ \text { SI } & \text { - Processing parameters } \\ \text { SF } & 131072 \\ \text { SF } & 500.1300000 \mathrm{MHz}\end{array}$

NSW 0

$\begin{array}{lll}L B & 0 \\ G B & 0 & 1 \\ S & 0\end{array}$

$\begin{array}{lll}\mathrm{CB} & \mathrm{O} & \\ \mathrm{PC} & 0 & 4.00\end{array}$ 
Duplicate \#2

SW: 30 ppm

01P: $6.175 \mathrm{ppm}$

D1: 30 sec

${ }^{1} \mathrm{H}$ NMR

$\alpha / \beta=38: 62$

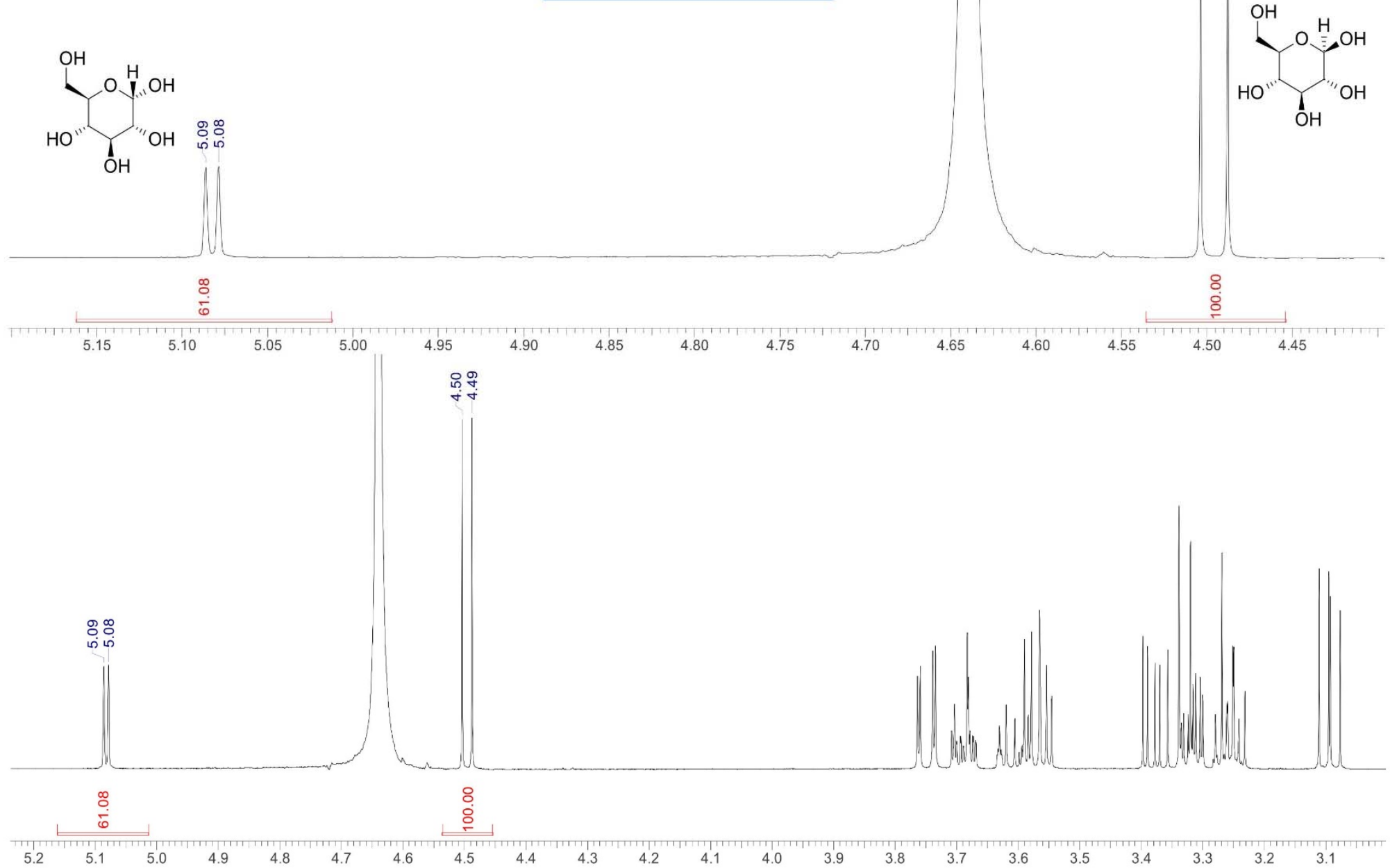




\subsection{L-Fucose (84)}

4.10.1. ${ }^{1} \mathrm{H}$ NMR $\left(500 \mathrm{MHz}, \mathrm{D}_{2} \mathrm{O}\right)$
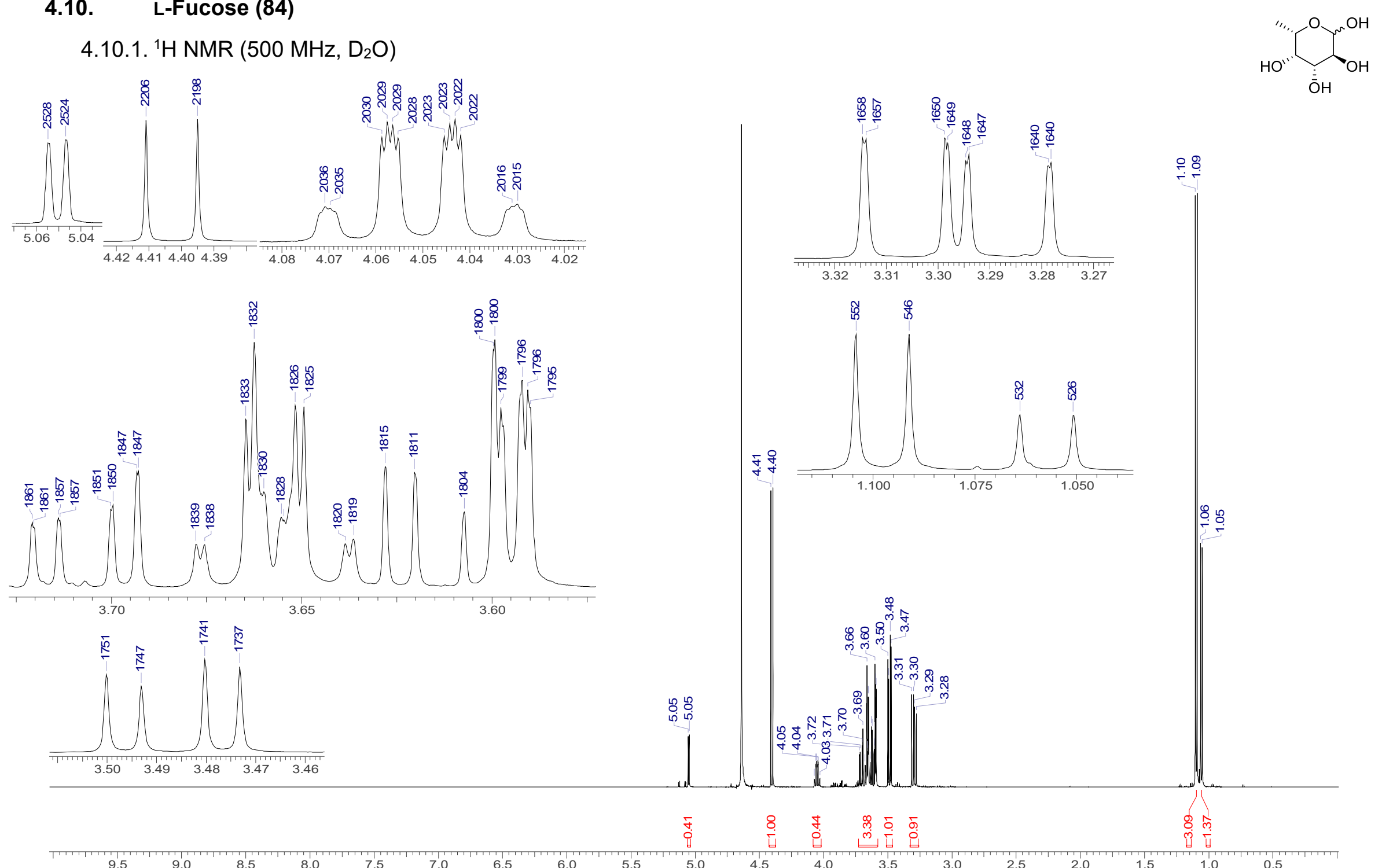
4.10.2. ${ }^{13} \mathrm{C}\left\{{ }^{1} \mathrm{H}\right\}$ NMR $\left(126 \mathrm{MHz}, \mathrm{D}_{2} \mathrm{O}\right)$

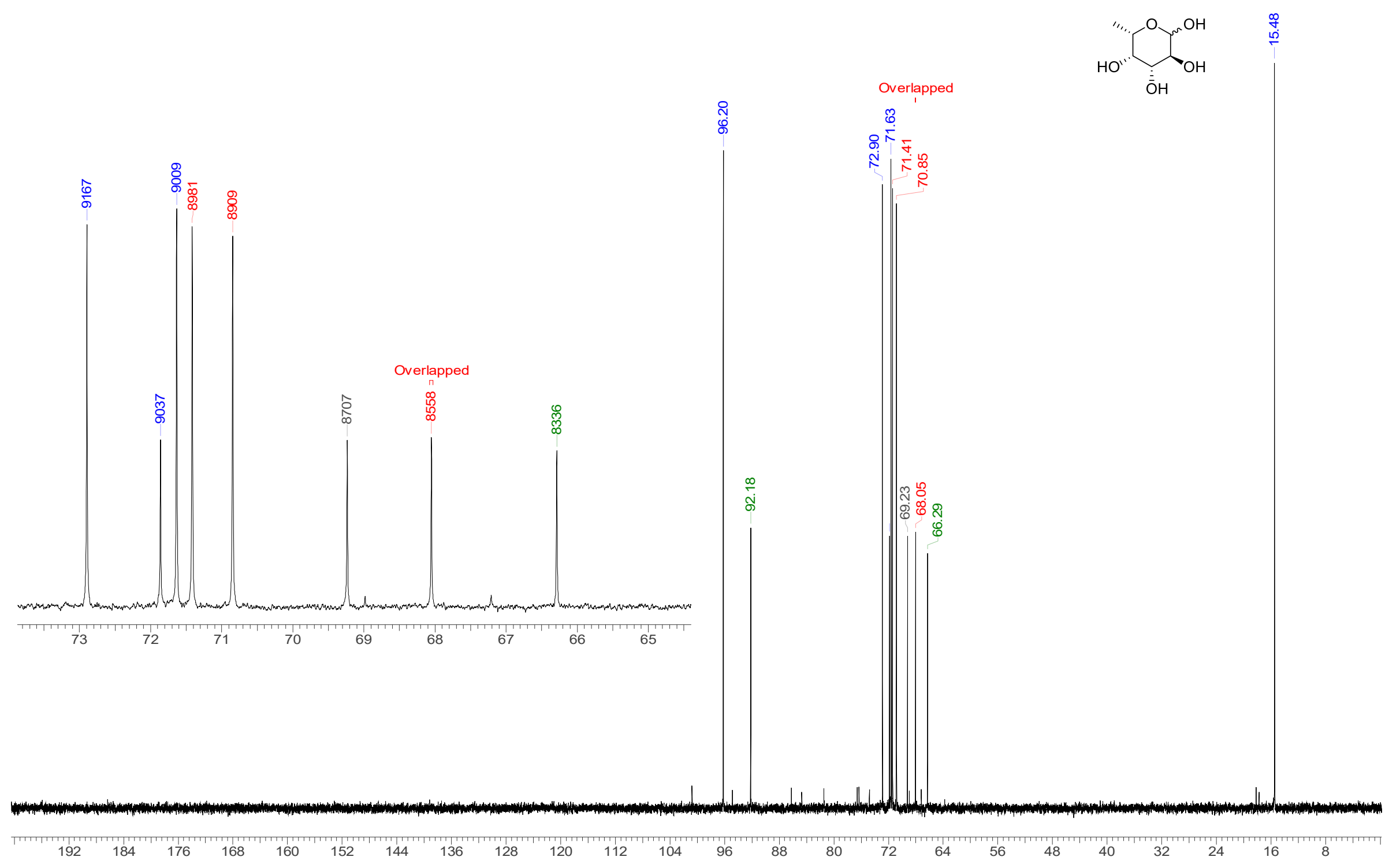




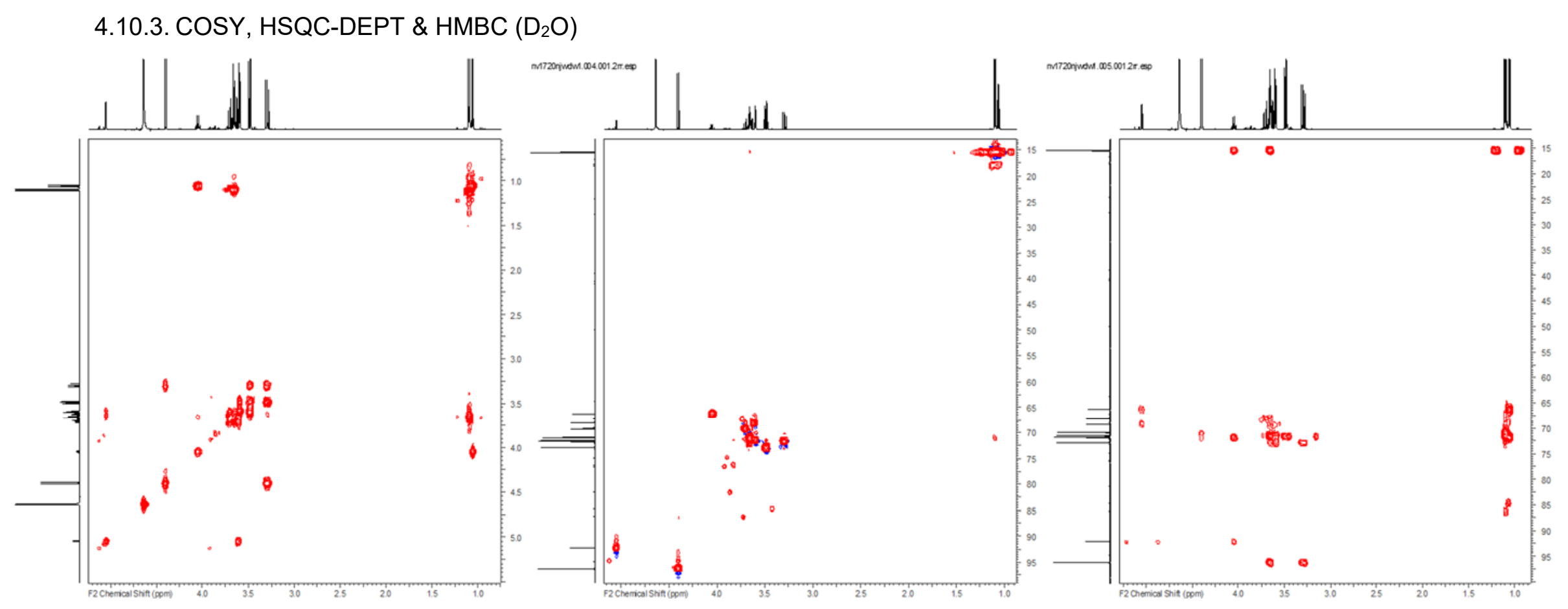


4.10.4. ${ }^{1} \mathrm{H}$ qNMR $\left(500 \mathrm{MHz}, \mathrm{D}_{2} \mathrm{O}\right)$

Duplicate \#1

DW/4,6/LFuc D2O $500 \mathrm{MHz}$ (1)

Quant. proton

Longest T1 $4.6 \mathrm{~s}$ (delay set to $45 \mathrm{~s}$ for full relaxation)

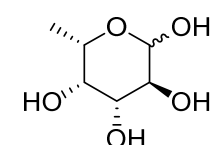

SW: $30 \mathrm{ppm}$

O1P: $6.175 \mathrm{ppm}$

D1: $45 \mathrm{sec}$

${ }^{1} \mathrm{H}$ NMR

$\alpha / \beta=31: 69$

(see next page for detailed integration

Current Data Parameters

EXPNO 1000

F2 - Acquisition Parameters
Date 20201117

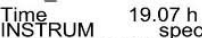

Spect

PULPROG $2 \bar{g} 30$

SOLVENT ${ }^{131072}$

$\begin{array}{lc}\text { SWH } & 0 \\ \text { SWH } & 15000.000 \mathrm{~Hz}\end{array}$

FIDRES $\quad 0.228882 \mathrm{H}$

$\begin{array}{lc}\text { RG } & 4.3690667 \mathrm{sec} \\ \mathrm{RW} & 55.52 \\ \mathrm{DW} & 33.333 \text { usec }\end{array}$

$\begin{array}{ll}\text { DW } & 33.333 \text { usec } \\ \text { DE } & 9.31 \text { usec }\end{array}$

$\begin{array}{ll}9.31 & \text { usec } \\ \text { TE } & 298.0 \mathrm{~K} \\ \text { D1 } & 45.00000000 \mathrm{sec}\end{array}$

$\begin{array}{ll}\text { TD0 } & 1 \\ \text { SFO1 } & 500.1330885 \mathrm{MHz}\end{array}$

$\begin{array}{ll}\text { P1 } 11.95 \text { usec } \\ \text { PLW1 } & 15.15600014 \mathrm{~W}\end{array}$

F2 - Processing parameters

$\begin{array}{lr}\text { FI - Processing parameters } \\ \text { SF } & 131072 \\ \text { SF } & 500.1300000 \mathrm{MHz}\end{array}$

$\begin{array}{ll}\mathrm{SSB} & 0 \\ \mathrm{LB} & 0 \\ \mathrm{~GB} & 0\end{array}$

$\begin{array}{ll}\mathrm{LB} & 0 \mathrm{~Hz} \\ \mathrm{GC} & 0\end{array}$

$$
4.00
$$


Duplicate \#1

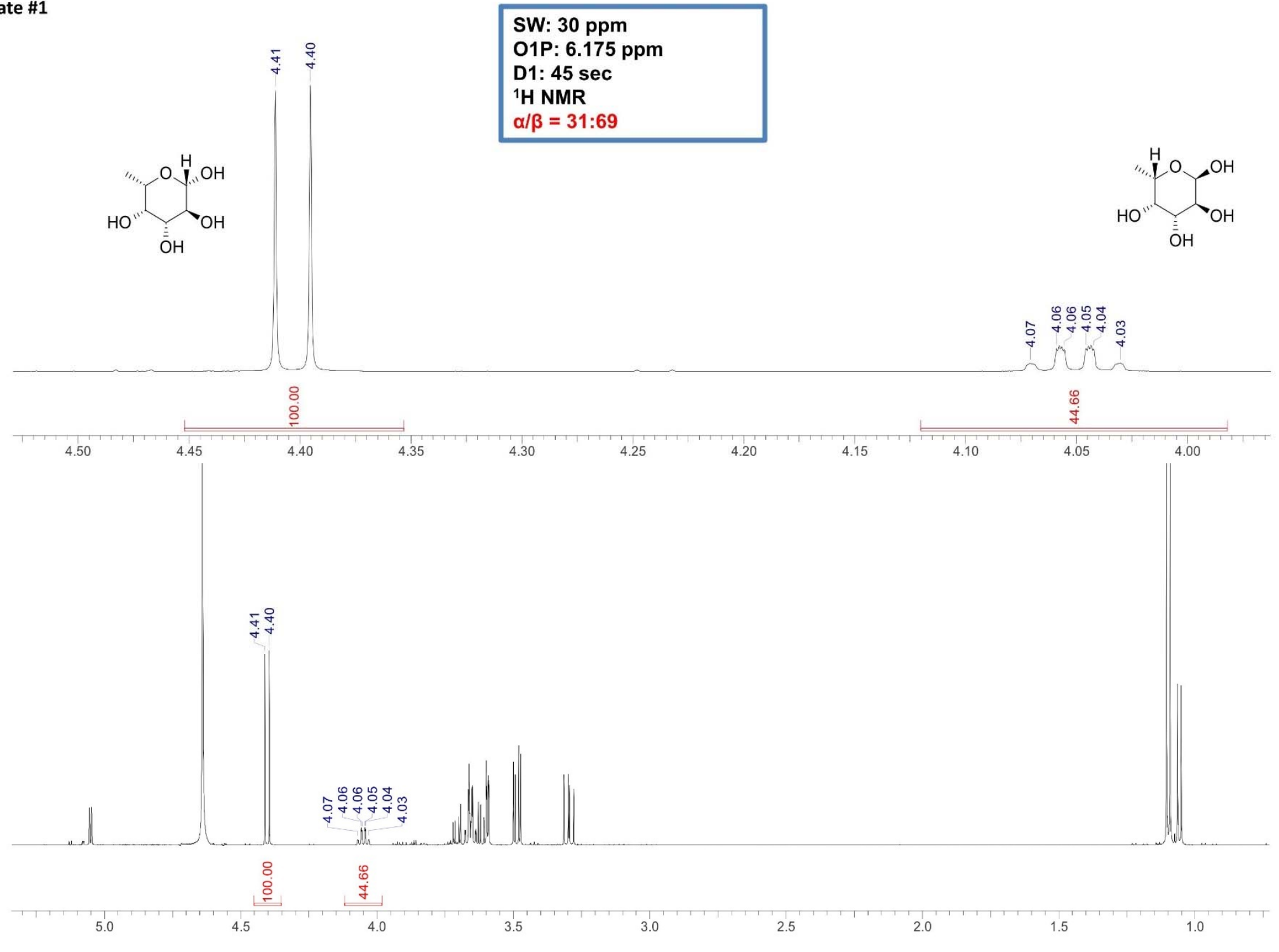




\section{Duplicate \#2}

DW/4,6/LFuc D2O 500 MHz (2)

Quant. proton

Longest T1 $4.6 \mathrm{~s}$ (delay set to $45 \mathrm{~s}$ for full relaxation)

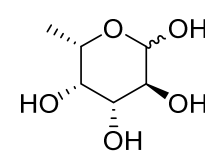

SW: $30 \mathrm{ppm}$

O1P: 6.175 ppm

D1: 45 sec

${ }^{1} \mathrm{H}$ NMR

$\alpha / \beta=31: 69$

for detailed integratio

formation after data processing)

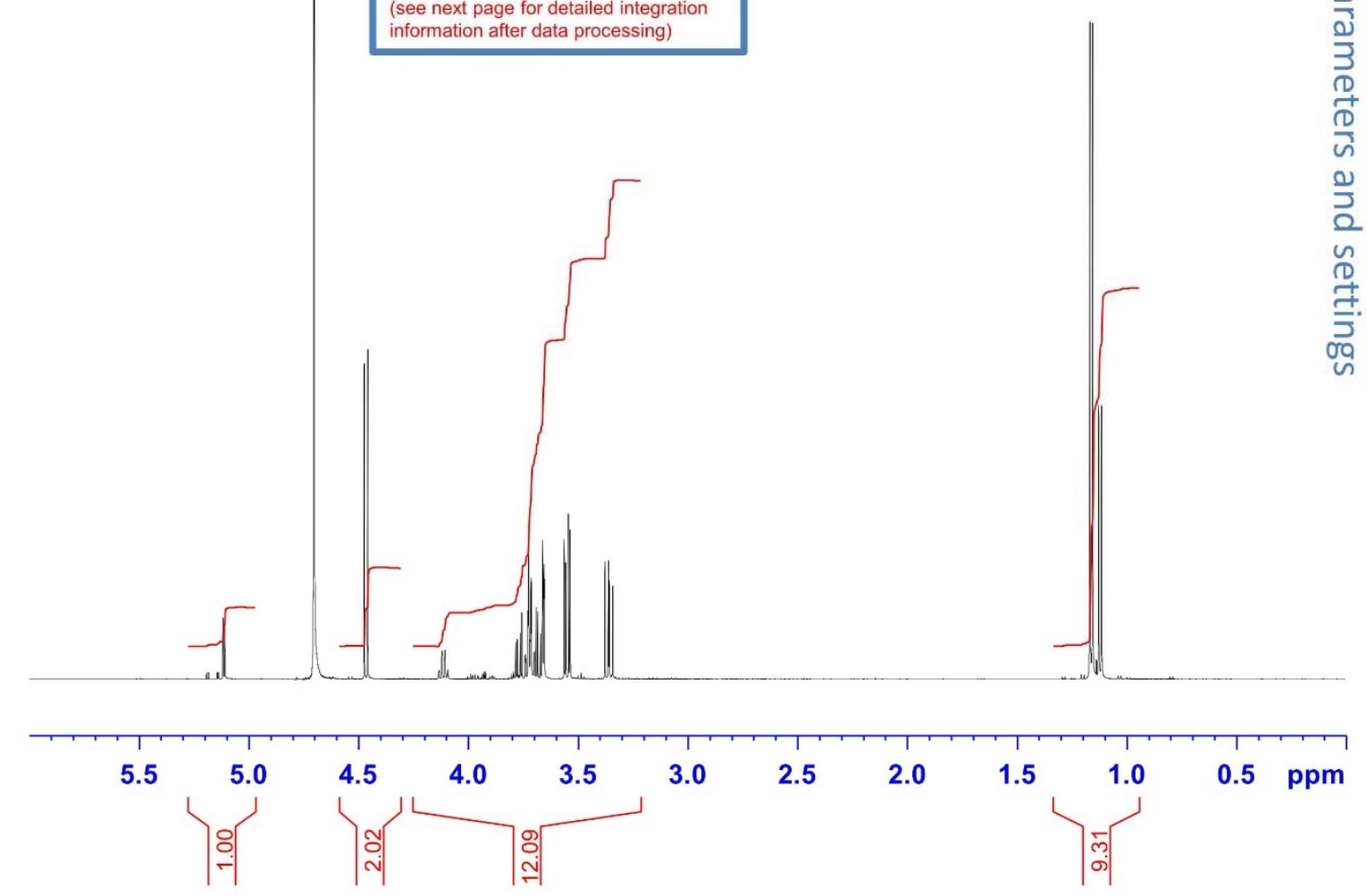


Duplicate \#2

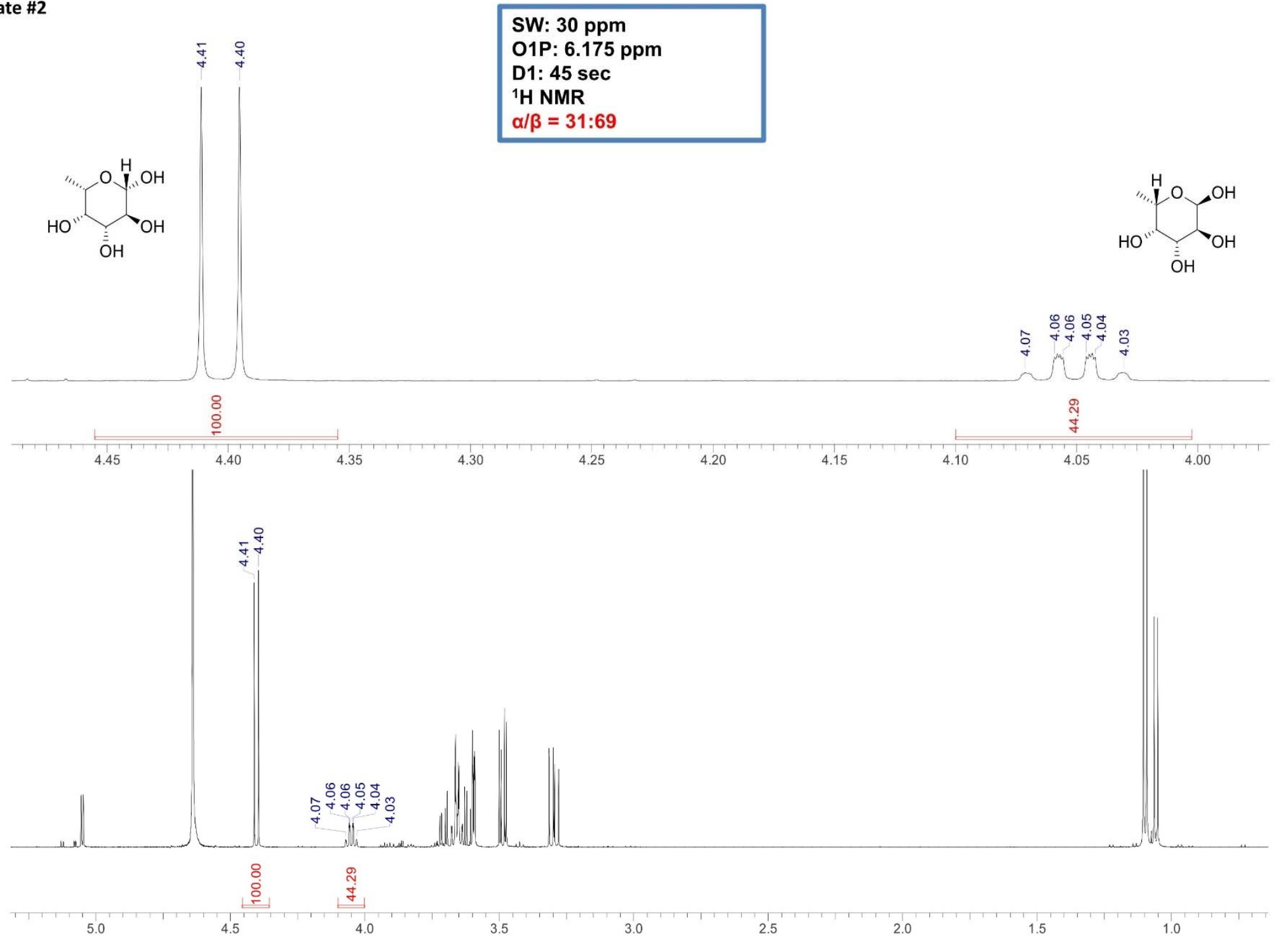




\subsection{4,6-Dideoxy-D-xylo-hexopyranose (86)}

4.11.1. ${ }^{1} \mathrm{H}$ NMR $\left(500 \mathrm{MHz}, \mathrm{D}_{2} \mathrm{O}\right)$
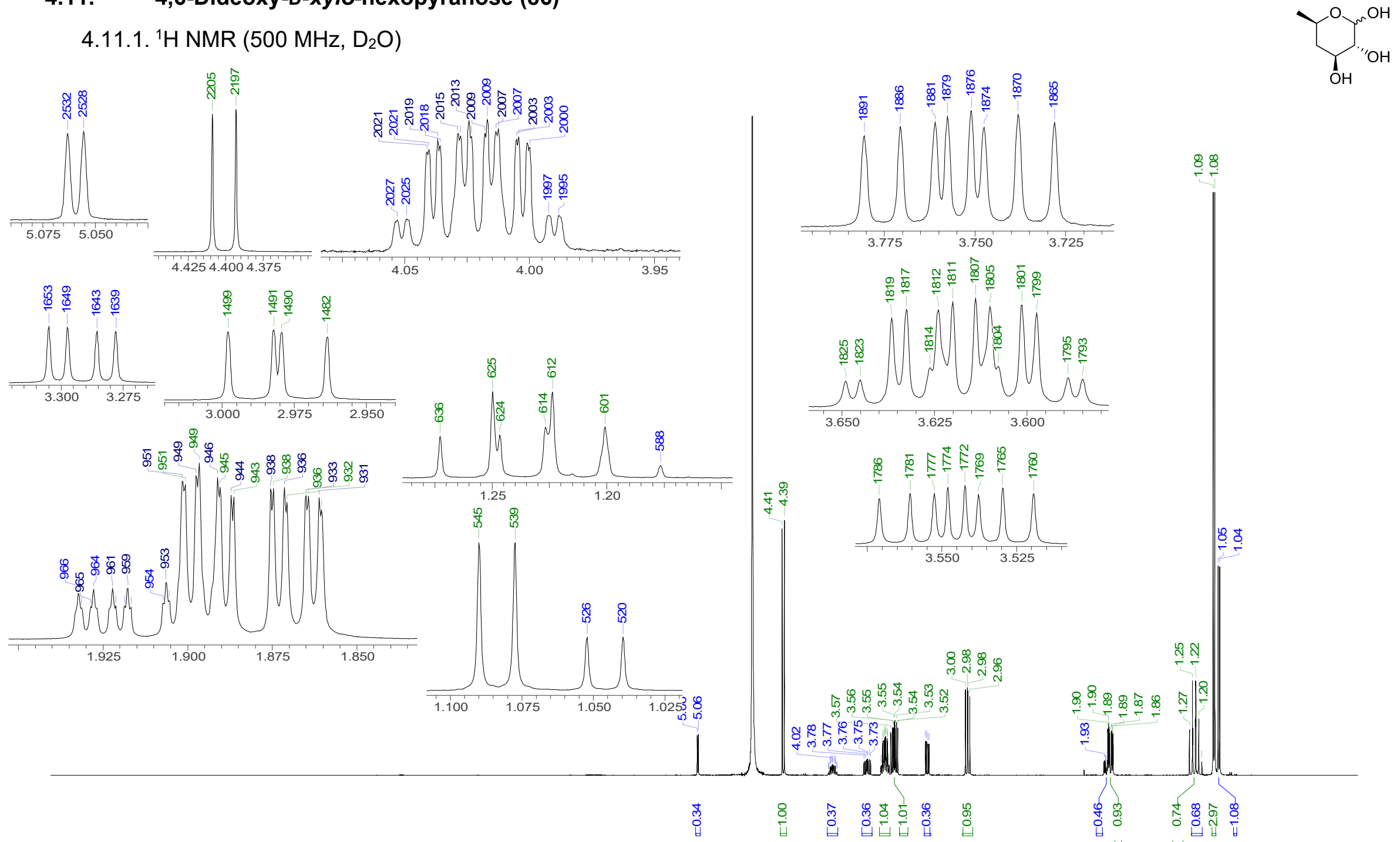

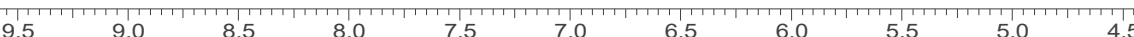

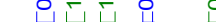

2.5


4.11.2. ${ }^{13} \mathrm{C}\left\{{ }^{1} \mathrm{H}\right\} \operatorname{NMR}\left(126 \mathrm{MHz}, \mathrm{D}_{2} \mathrm{O}\right)$

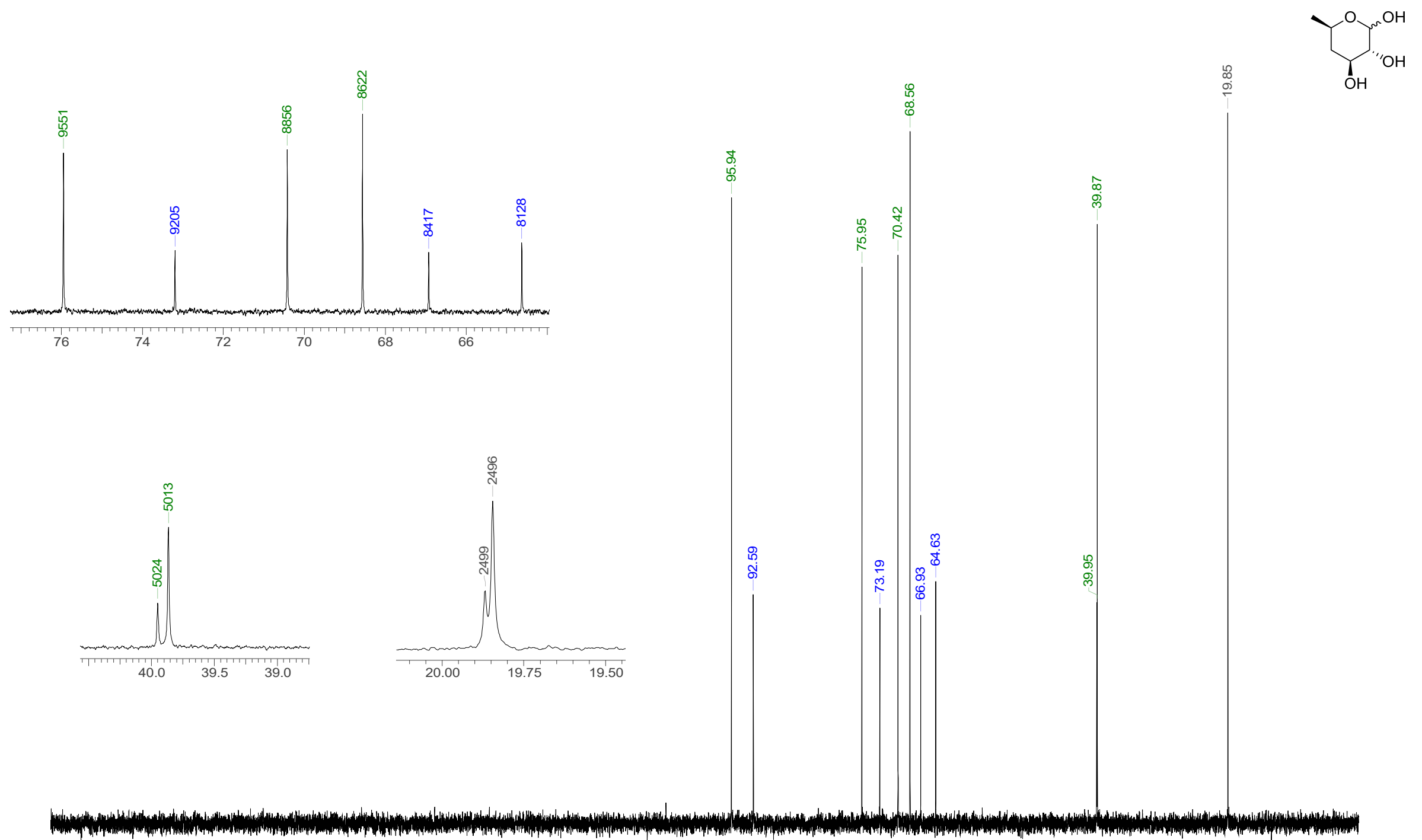


4.11.3. COSY, HSQC-DEPT \& HMBC $\left(D_{2} O\right)$

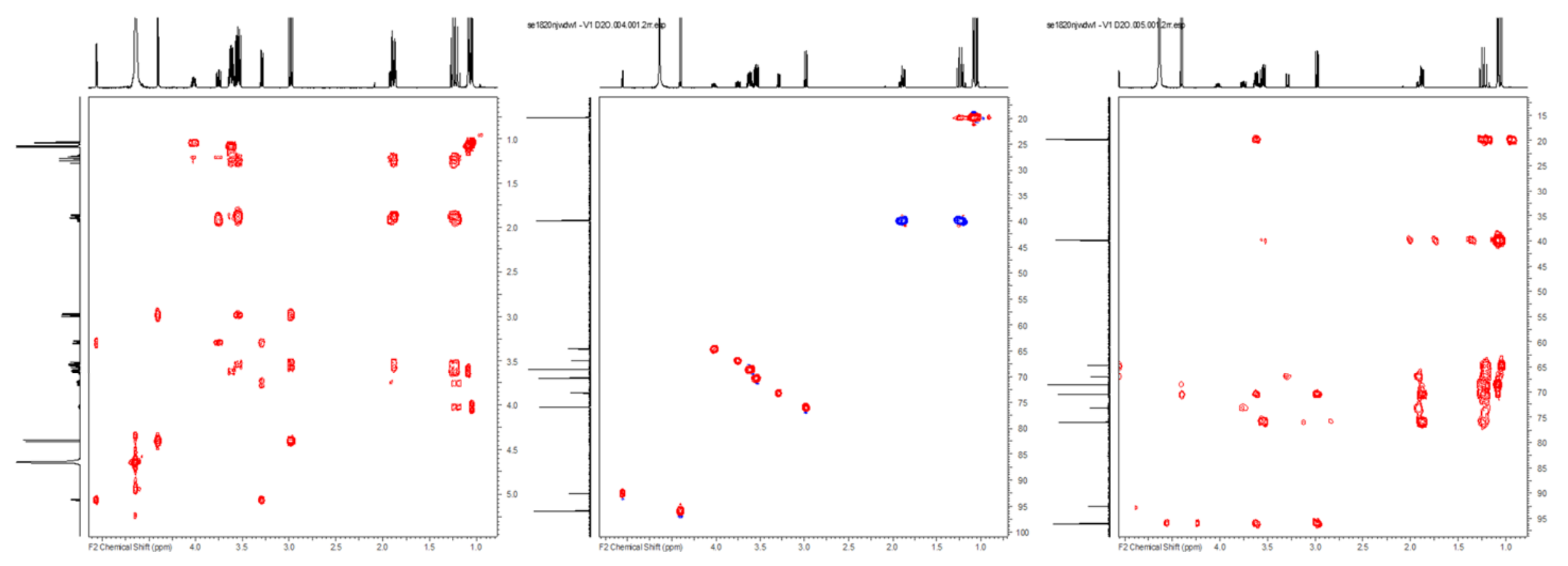


4.11.4. ${ }^{1} \mathrm{H}$ qNMR $\left(500 \mathrm{MHz}, \mathrm{D}_{2} \mathrm{O}\right)$

Duplicate \#1

DW/4,6/V1 D2O $500 \mathrm{MHz}$

Quant. proton

Longest T1 $4.0 \mathrm{~s}$ (delay set to $45 \mathrm{~s}$ for full relaxation)
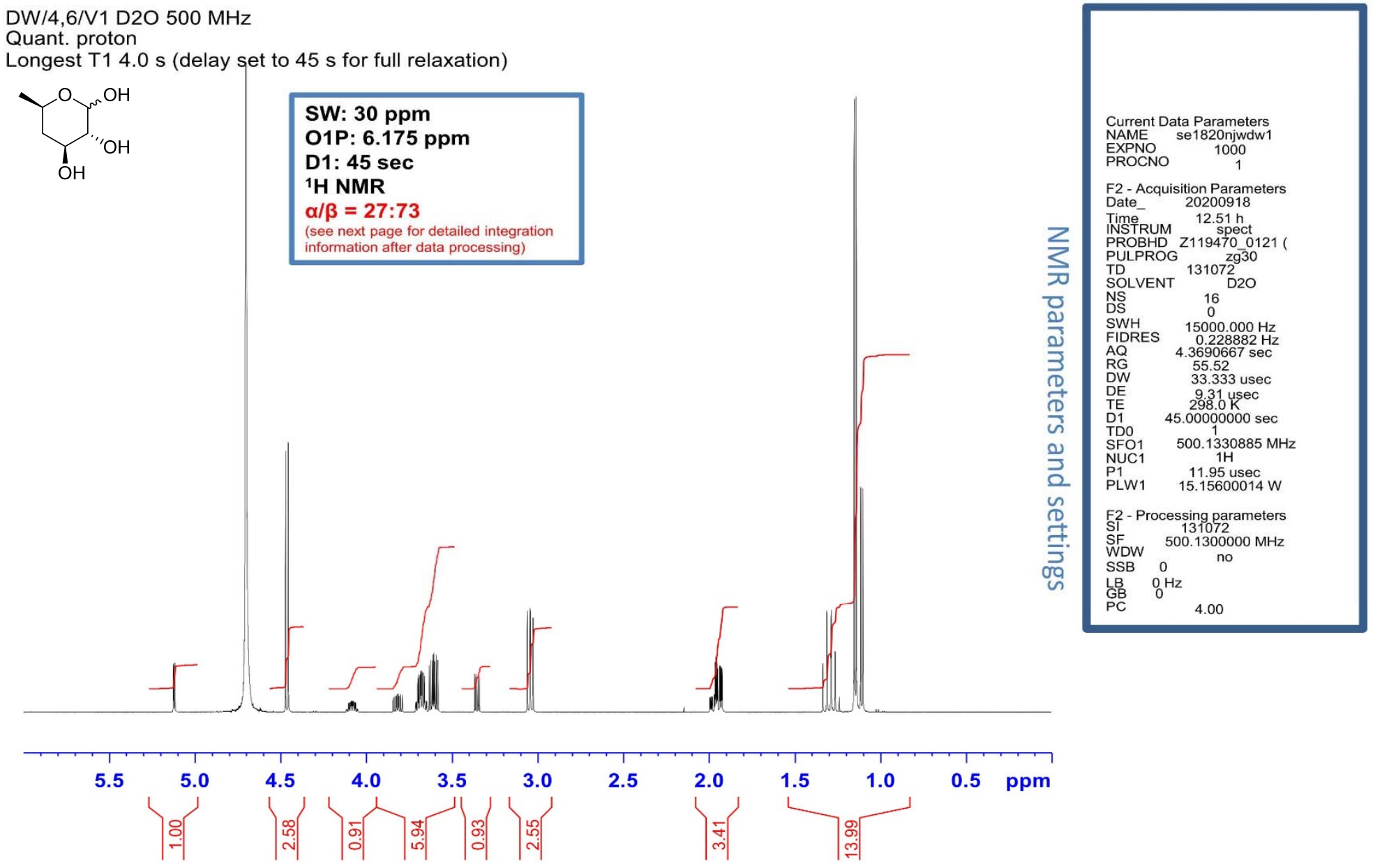
Duplicate \#1

SW: 30 ppm

1P: $6.175 \mathrm{ppm}$

D1: $45 \mathrm{sec}$

${ }^{1} \mathrm{H}$ NMR

M01(dd)

$\alpha / \beta=27: 73$
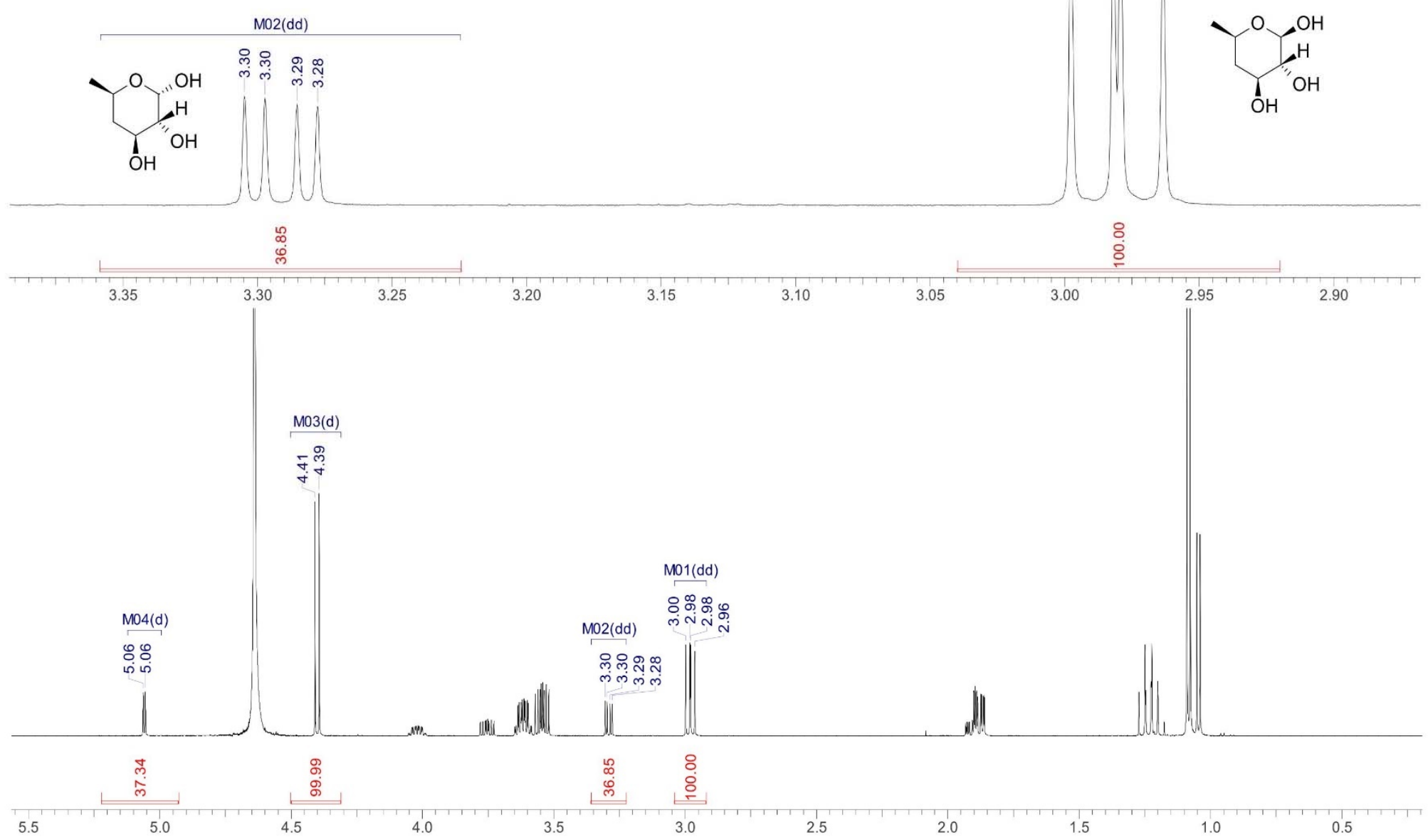


\section{Duplicate \#2}

DW/4,6/V1 $500 \mathrm{MHz}$

Quant. proton (qNMR 2)

Longest T1 $3.9 \mathrm{~s}$ (delay set to $45 \mathrm{~s}$ for full relaxation)

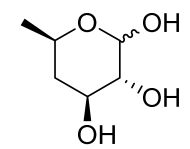

SW: 30 ppm

01P: 6.175 ppm

D1: 45 sec

${ }^{1} \mathrm{H}$ NMR

$\alpha / \beta=27: 73$

Ior detailed integratio

formation after data processing)
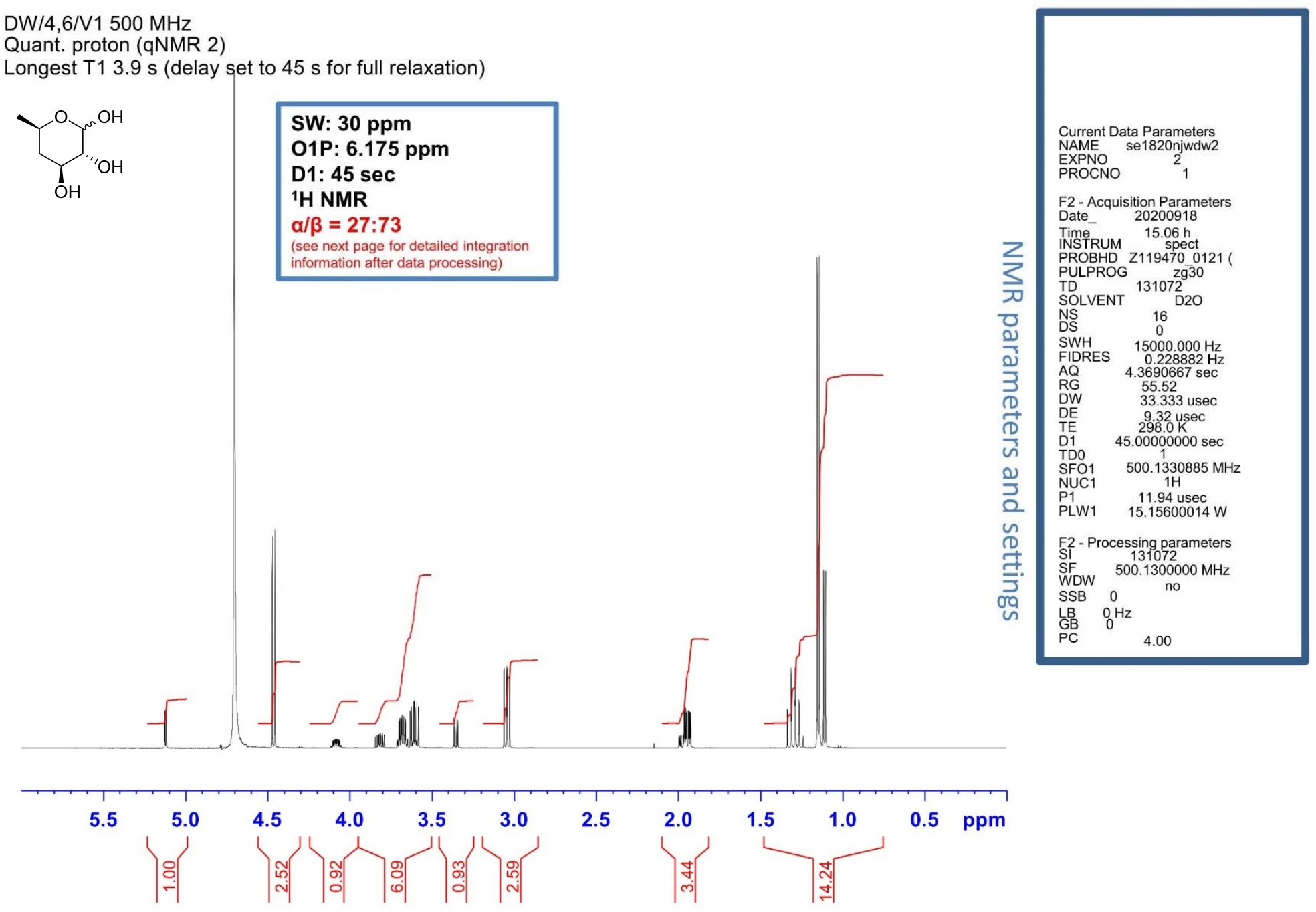
Duplicate \#2

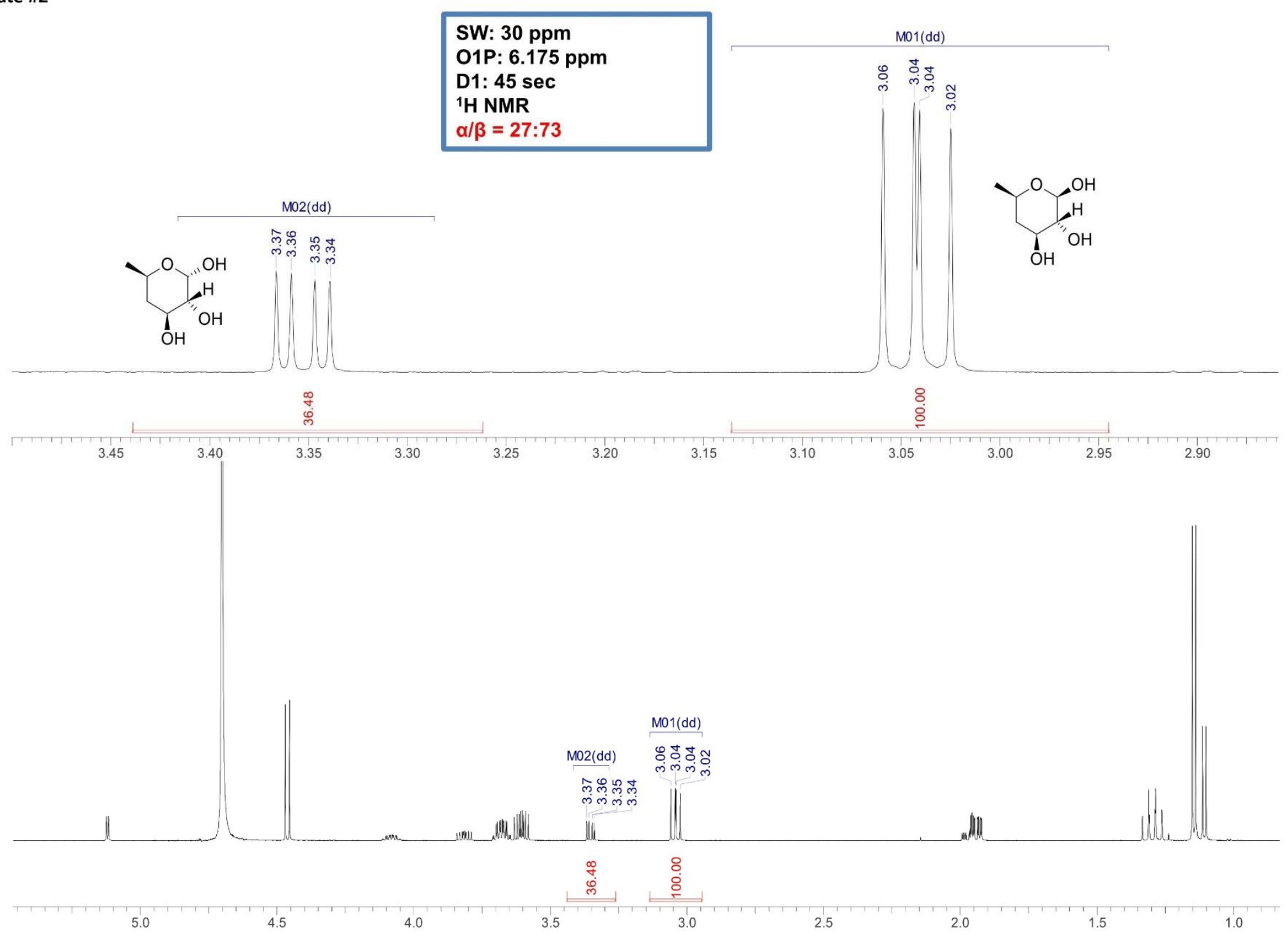


4.12. 4-Deoxy-4-fluoro-D-glucose (87)

4.12.1. ${ }^{1} \mathrm{H}$ NMR $\left(500 \mathrm{MHz}, \mathrm{D}_{2} \mathrm{O}\right)$
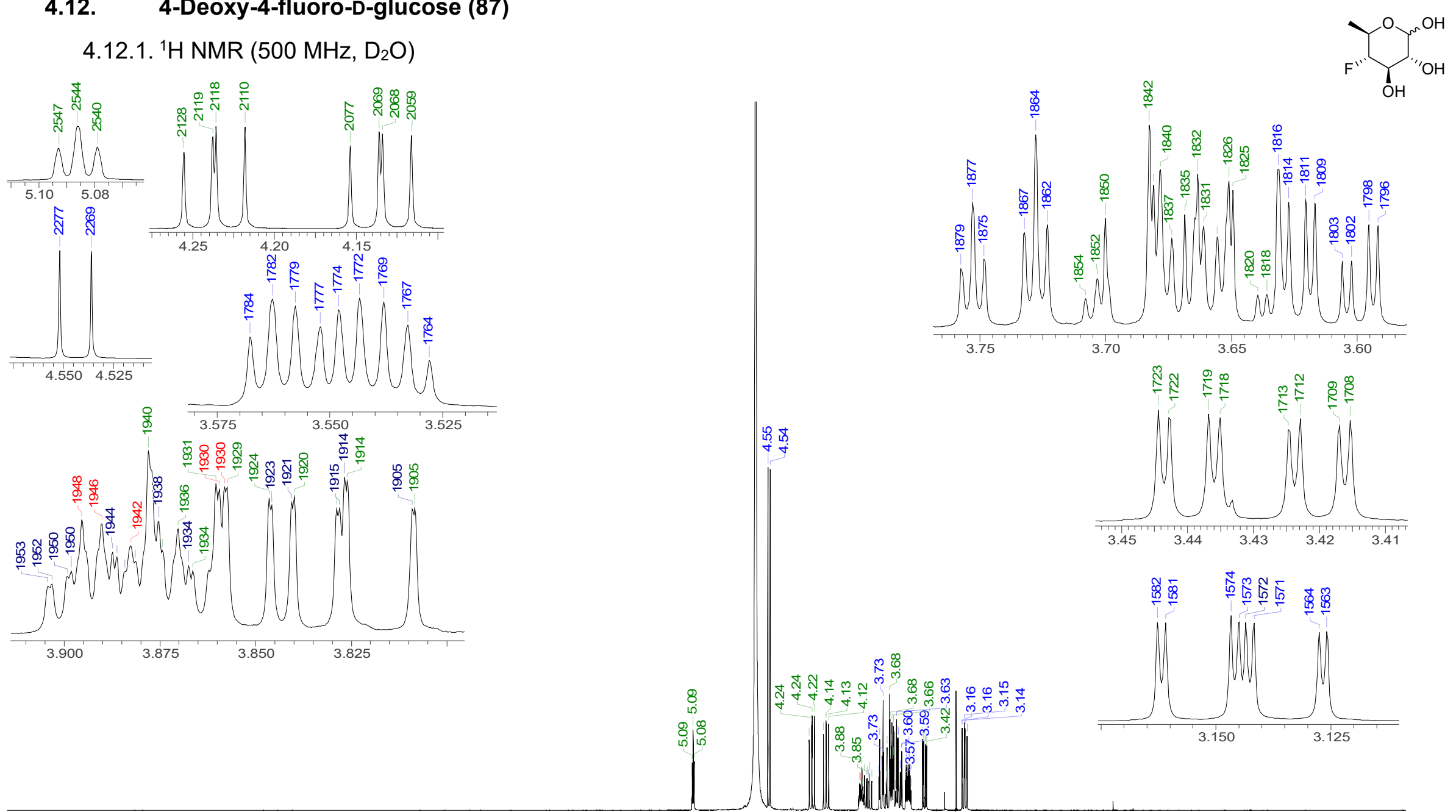

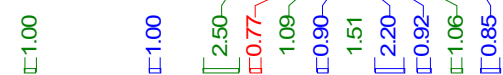

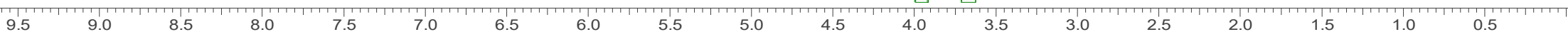


4.12.2. ${ }^{1} \mathrm{H}\left\{{ }^{19} \mathrm{~F}\right\} \mathrm{NMR}\left(500 \mathrm{MHz}, \mathrm{D}_{2} \mathrm{O}\right)$
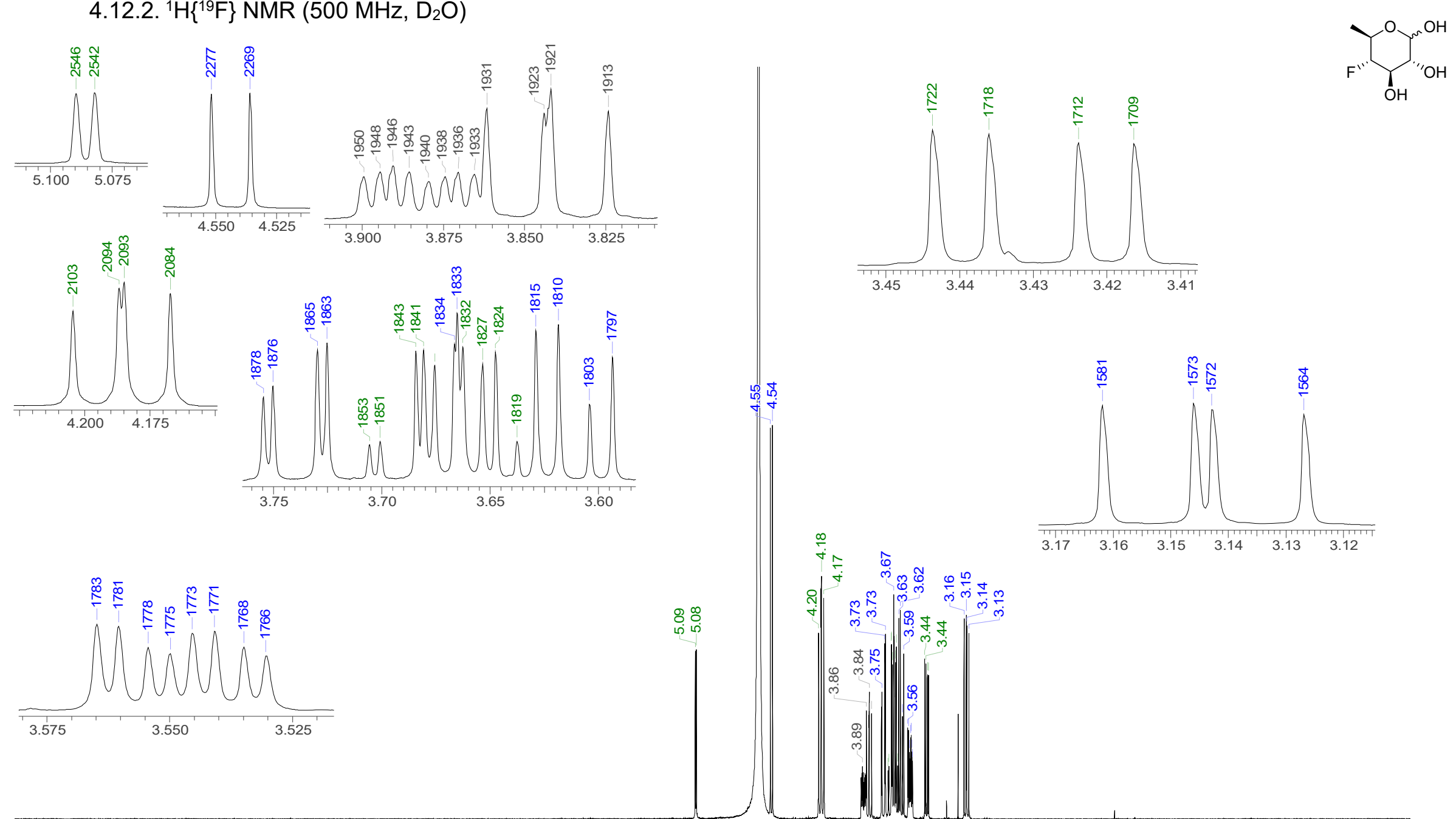

$\mathrm{OH}_{\mathrm{OH}}^{\mathrm{O}}$

(1)


4.12.3. ${ }^{13} \mathrm{C}\left\{{ }^{1} \mathrm{H}\right\}$ NMR $\left(126 \mathrm{MHz}, \mathrm{D}_{2} \mathrm{O}\right)$

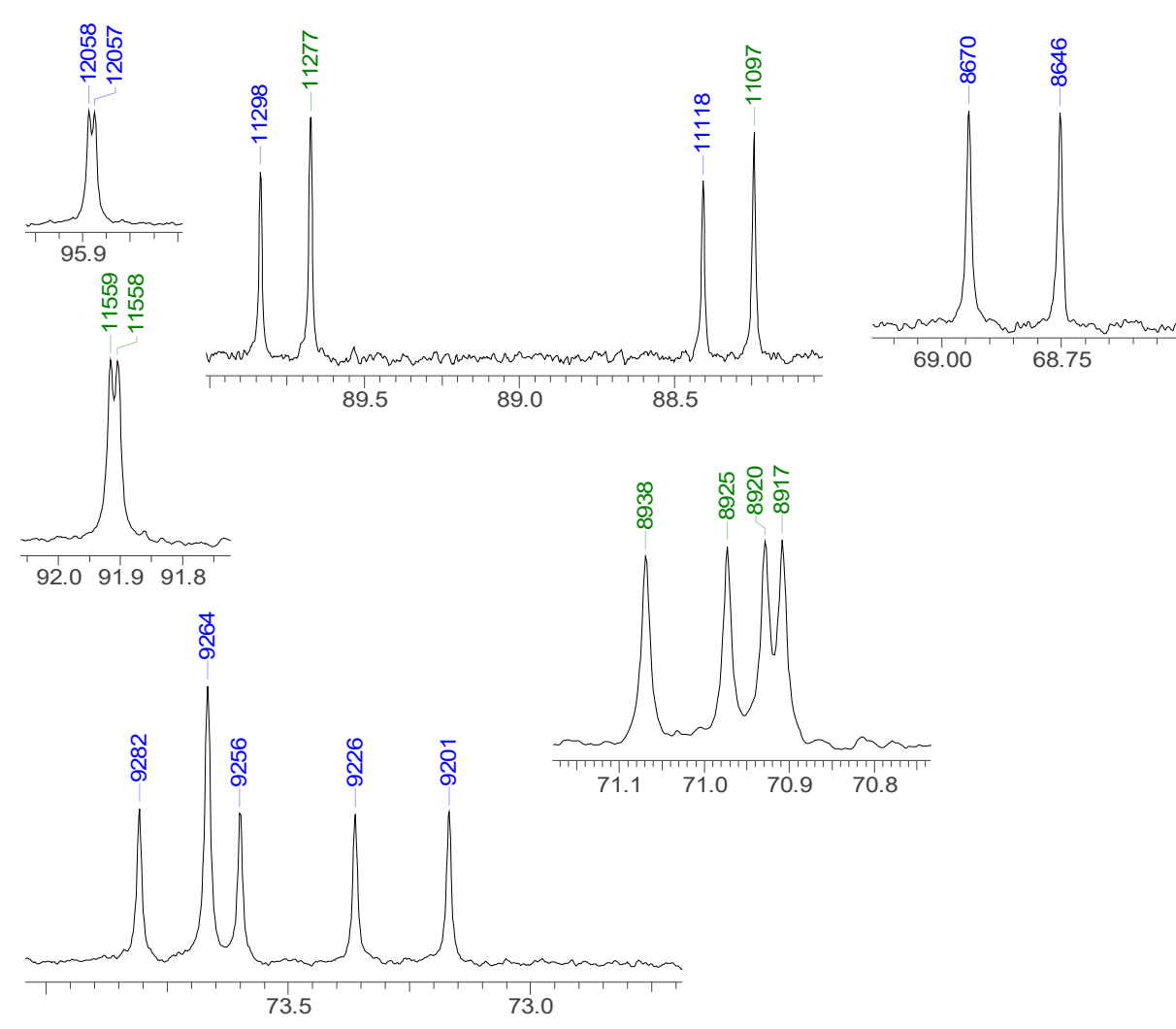

$\infty$
\[ \infty \]
\[ \]

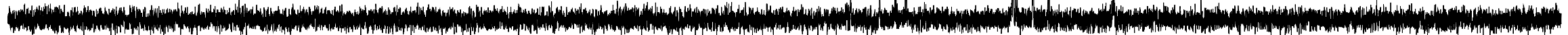

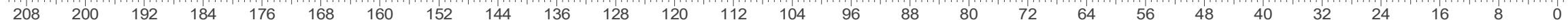


4.12.4. COSY, HSQC-DEPT \& HMBC $\left(D_{2} \mathrm{O}\right)$

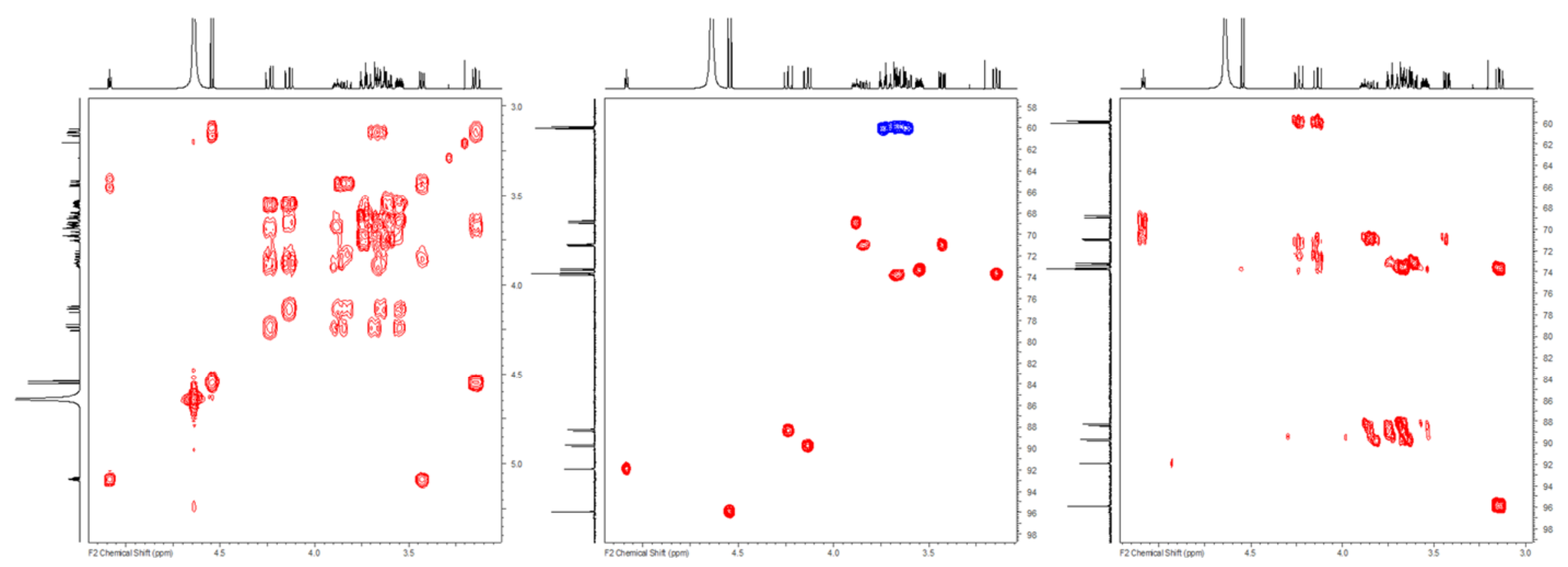


4.12.5. ${ }^{19} \mathrm{~F}$ NMR $\left(470 \mathrm{MHz}, \mathrm{D}_{2} \mathrm{O}\right)$

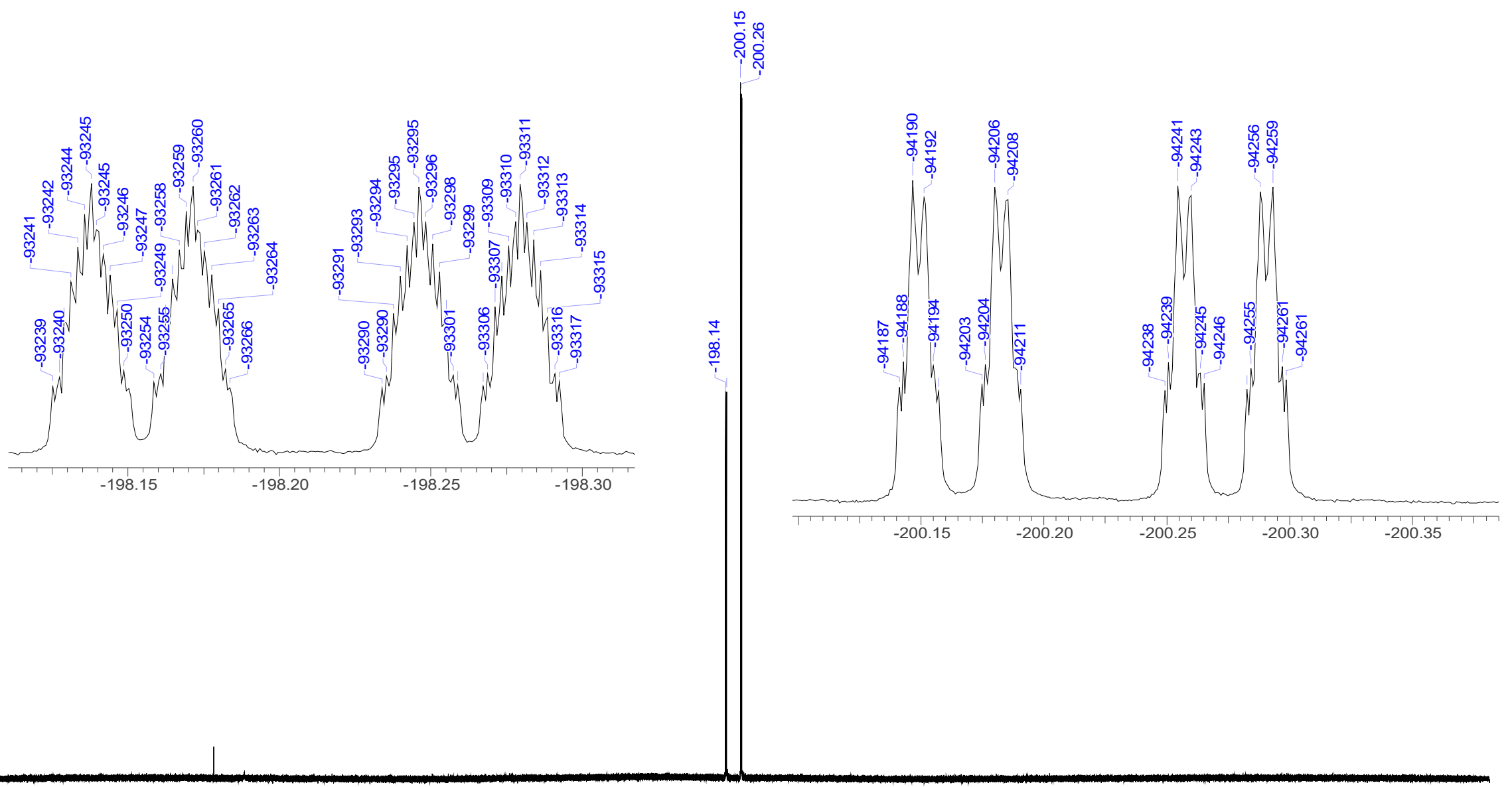

$8 \stackrel{\circ}{\circ}$

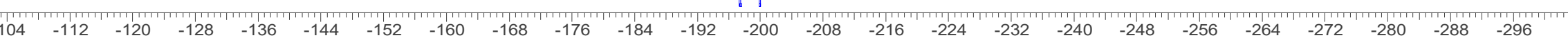


4.12.6. ${ }^{19} \mathrm{~F}\left\{{ }^{1} \mathrm{H}\right\} \mathrm{NMR}\left(470 \mathrm{MHz}, \mathrm{D}_{2} \mathrm{O}\right)$
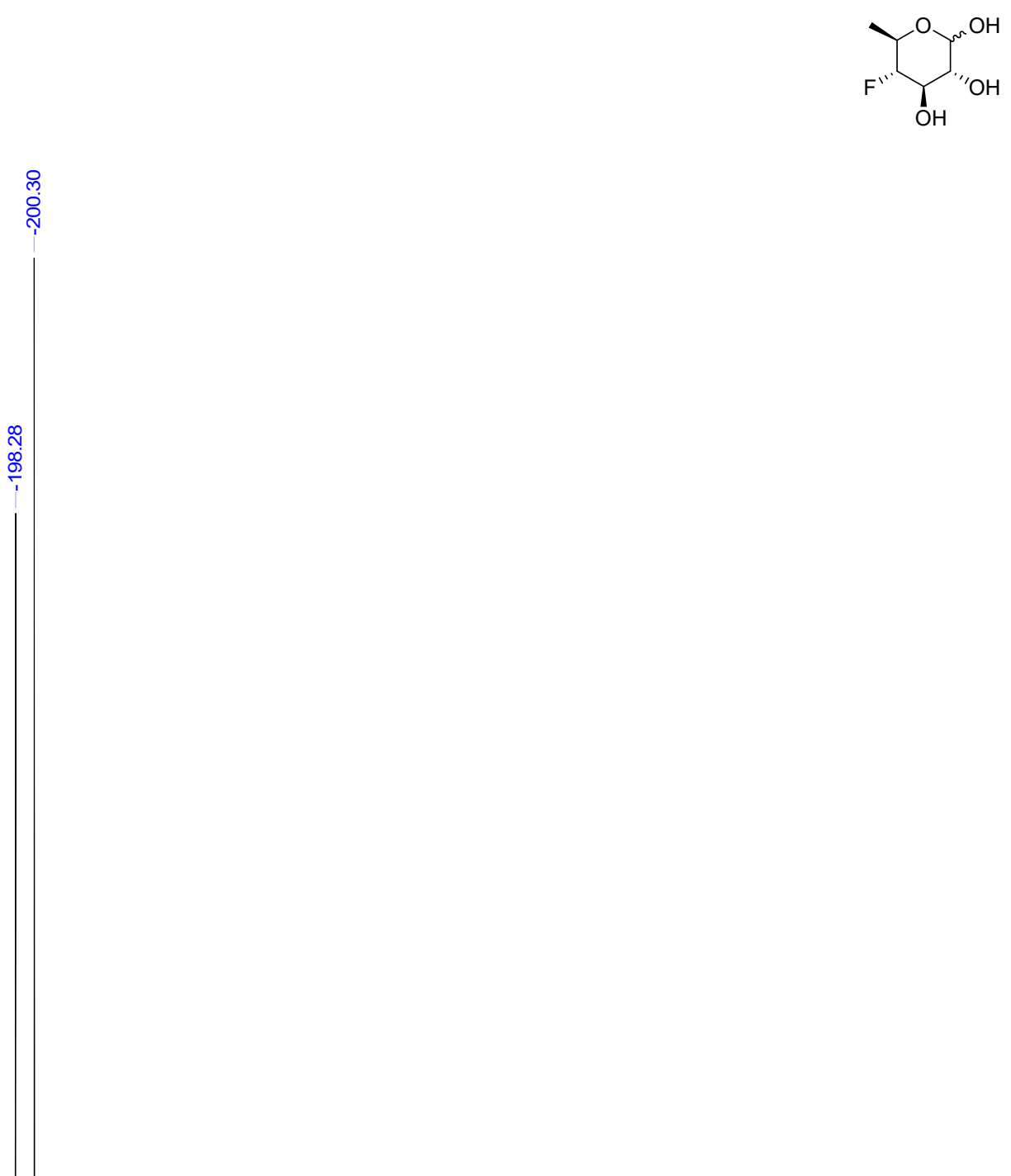

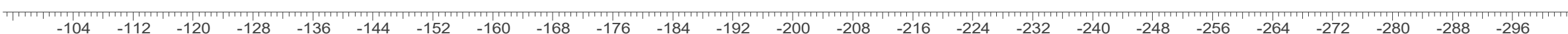


4.12.7. ${ }^{19} \mathrm{~F}$ qNMR $\left(500 \mathrm{MHz}, \mathrm{D}_{2} \mathrm{O}\right)$

Duplicate \#1

DW/4,6 4FDG D2O F\{\{H\}An1 010820 qNMR

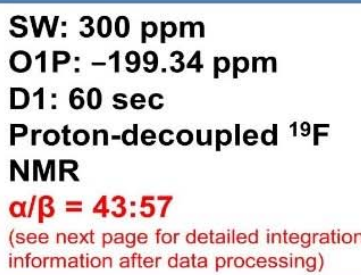

Proton-decoupled ${ }^{19} \mathrm{~F}$

NMR

$\alpha / \beta=43: 57$

57 dor

information after data processing)
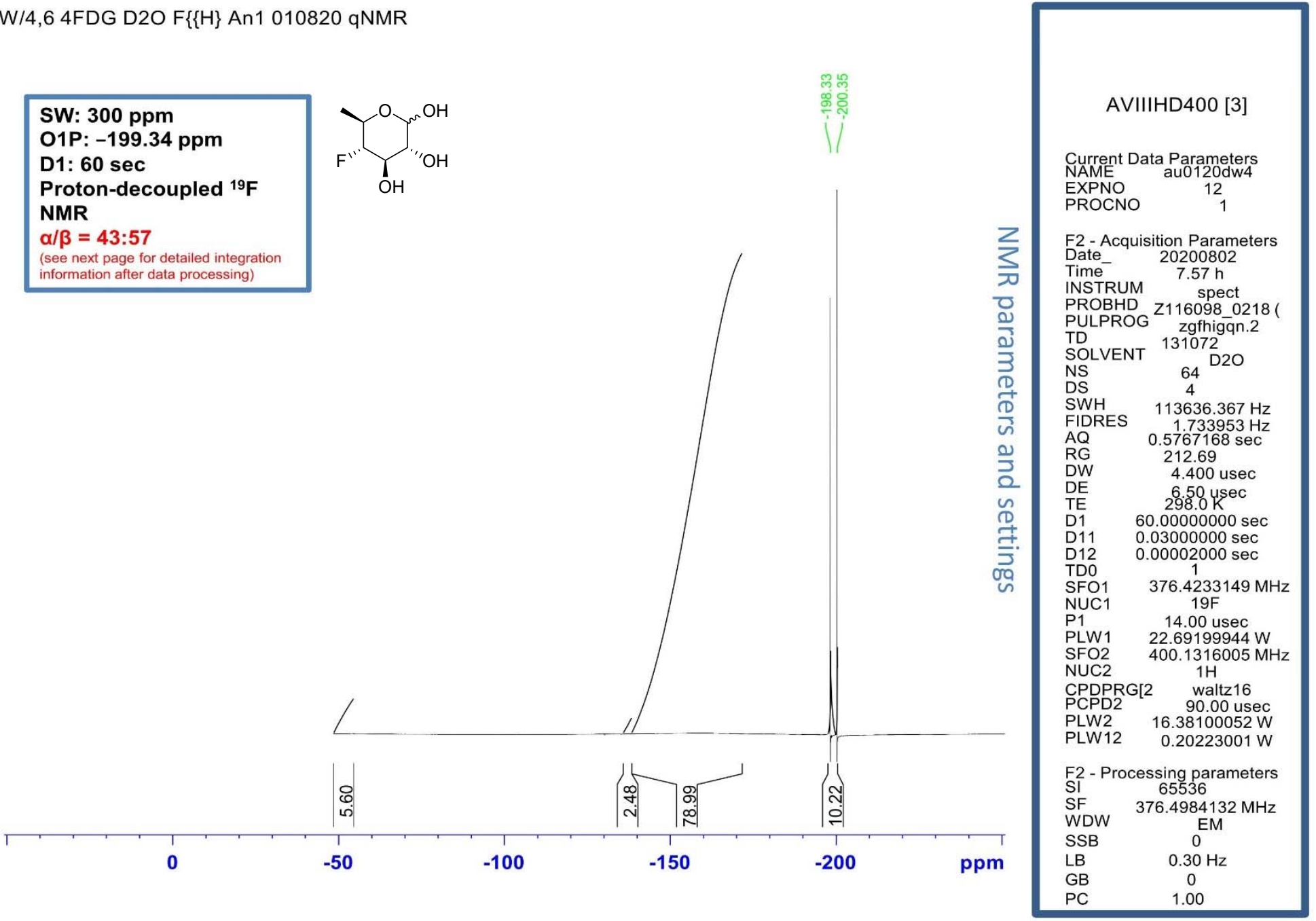
Duplicate \#1

SW: 300 ppm

1P: -199.34 ppm

1: 60 sec

Proton-decoupled ${ }^{19} \mathrm{~F}$

NMR

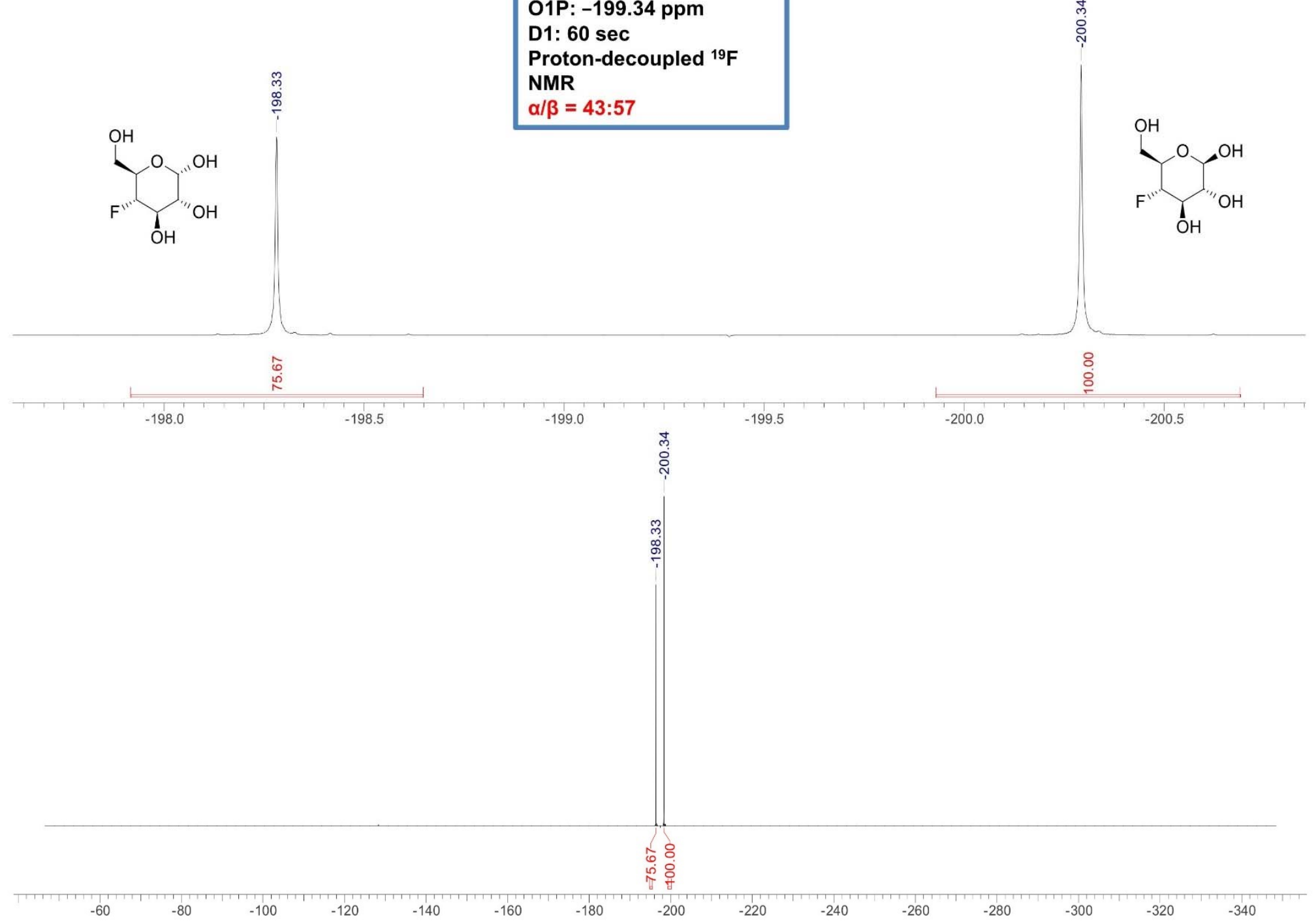




\section{Duplicate \#2}

DW/4,6 4FDG D2O F\{\{H\} An2 010820 qNMR

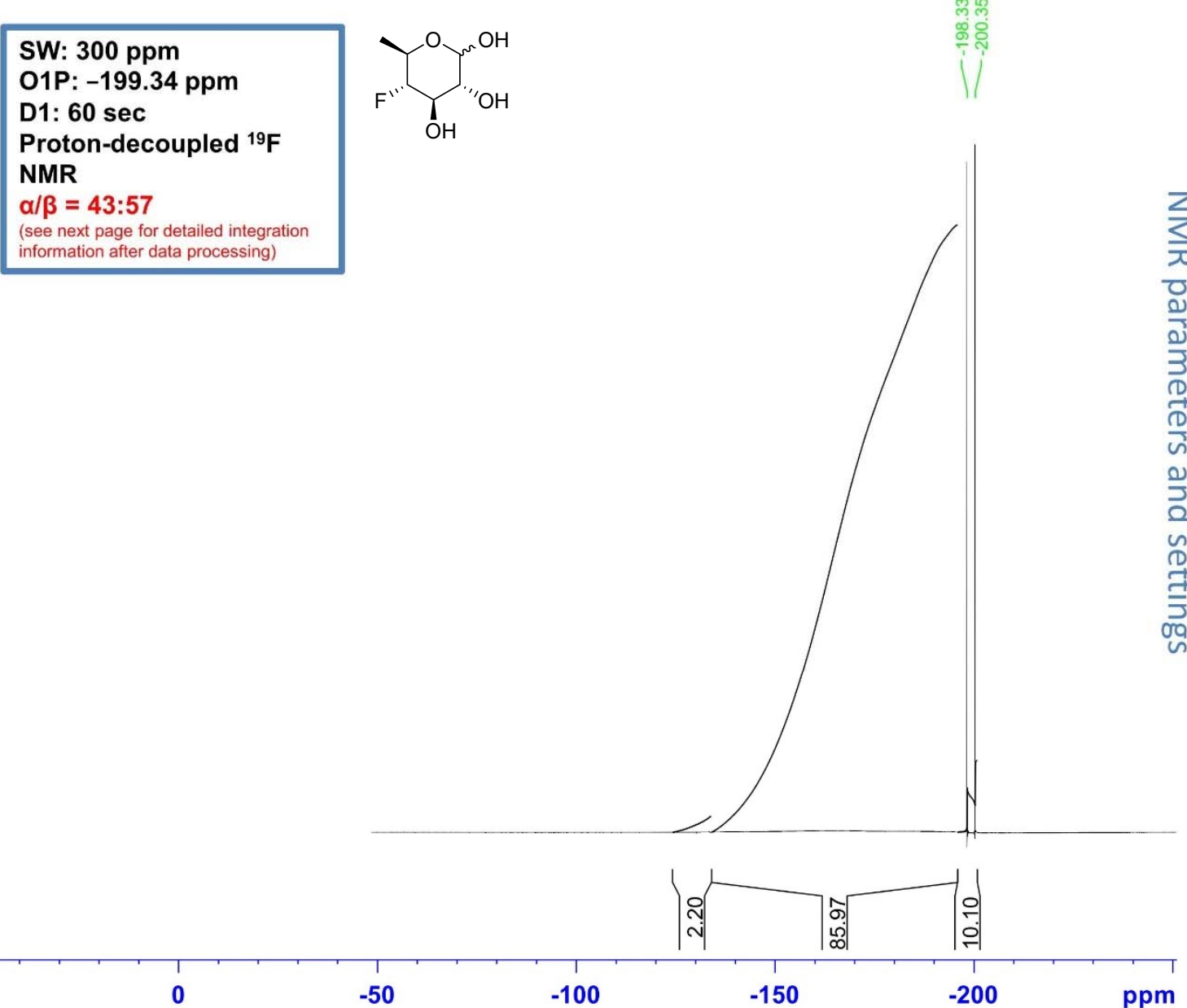

AVIIIHD400 [3]

Current Data Parameters
NAME au0120dw5

$\begin{array}{ll}\text { EXPNO } & 12 \\ \text { PROCNO } & 1\end{array}$

12

F2 - Acquisition Parameters

$\begin{array}{lc}\text { Date } & 20200802 \\ \text { Time } & 23.47 \mathrm{~h}\end{array}$

Time $23.47 \mathrm{~h}$

PROBHD Z116098_0218(

PULPROG Z116098_0218

TD 131072

NS -64 D2O

DS

${ }_{4}^{64}$

FIDRES $113636.367 \mathrm{~Hz}$

AO $1.733953 \mathrm{~Hz}$

RG $\quad 212.69$

DW $\quad 4.400$ usec

DE $\quad 6.50$ usec

TE $\quad 298.0 \mathrm{~K}$

D1 $\quad 60.00000000 \mathrm{sec}$

D12 $0.0002000 \mathrm{sec}$

TDO

SFO1 $376.4233149 \mathrm{MHz}$

NUC1 $19 \mathrm{~F}$

$\begin{array}{ll}\text { P1 } & 14.00 \text { usec } \\ \text { PLW1 } & 22.69199944 \mathrm{~W}\end{array}$

SFO2 $400.1316005 \mathrm{MHz}$

CPDPRG[2 waltz16

16.38.00 usec

$\begin{array}{lr}\text { PLW12 } & 0.20223001 \mathrm{~W}\end{array}$

F2 - Processing parameters

$\begin{array}{lc}\text { SI } & 65536 \\ \text { SF } & 376.4984132 \mathrm{MHz}\end{array}$

WDW $\begin{array}{ll}376.4984132 \\ \text { EM }\end{array}$

$\begin{array}{lc}\text { SSB } & 0 \\ \text { LB } & 0.30 \mathrm{~Hz}\end{array}$

$0-50$
$0-100$

$-150$

ppm

$\begin{array}{lc}\text { GB } & 0 \\ \text { PC } & 1.00\end{array}$ 
Duplicate \#2

SW: 300 ppm

01P: -199.34 ppm

D1: $60 \mathrm{sec}$

Proton-decoupled ${ }^{19} \mathrm{~F}$

NMR

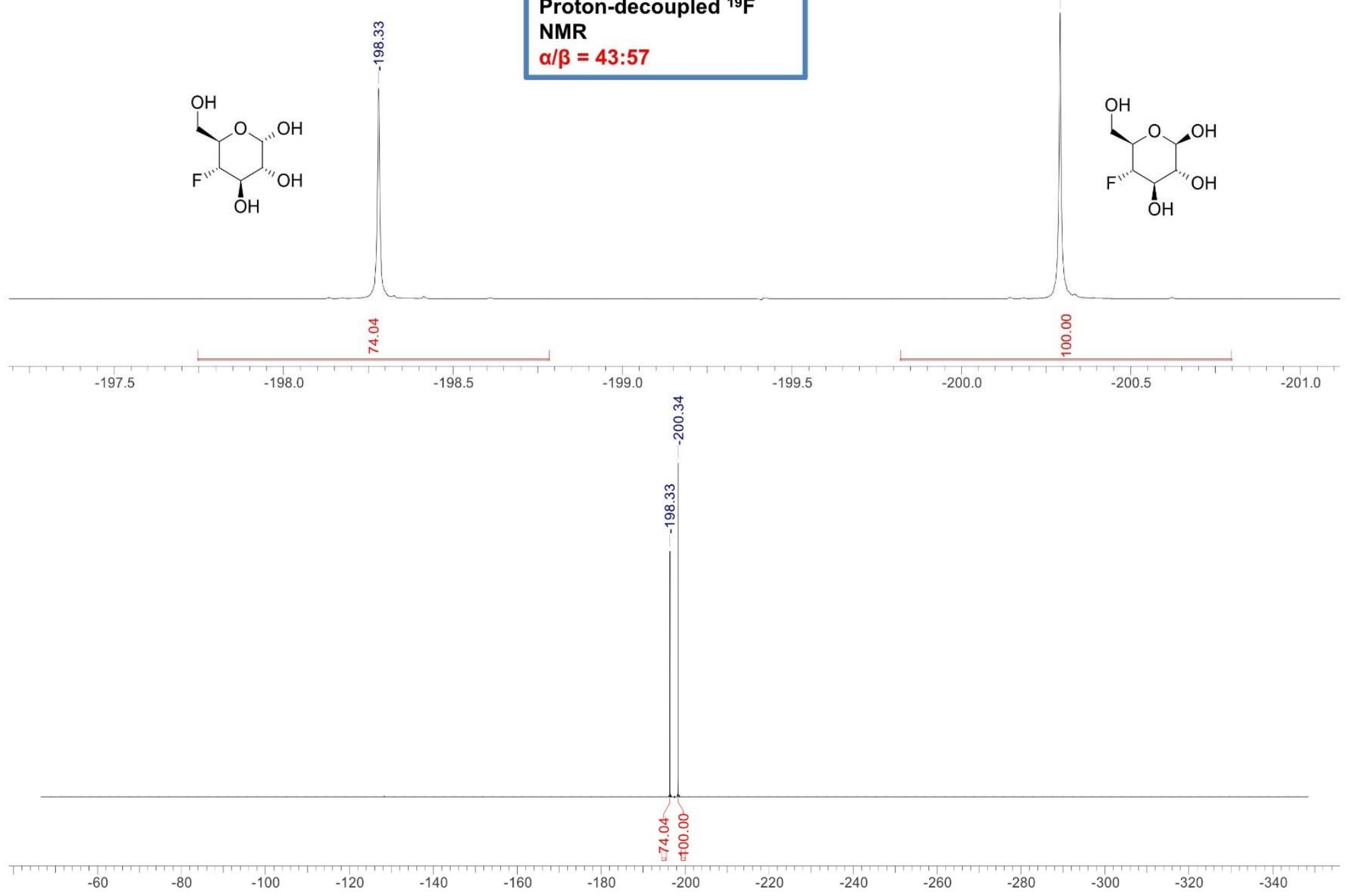




\subsection{D-Quinovose (89)}

4.13.1. ${ }^{1} \mathrm{H}$ NMR $\left(500 \mathrm{MHz}, \mathrm{D}_{2} \mathrm{O}\right)$
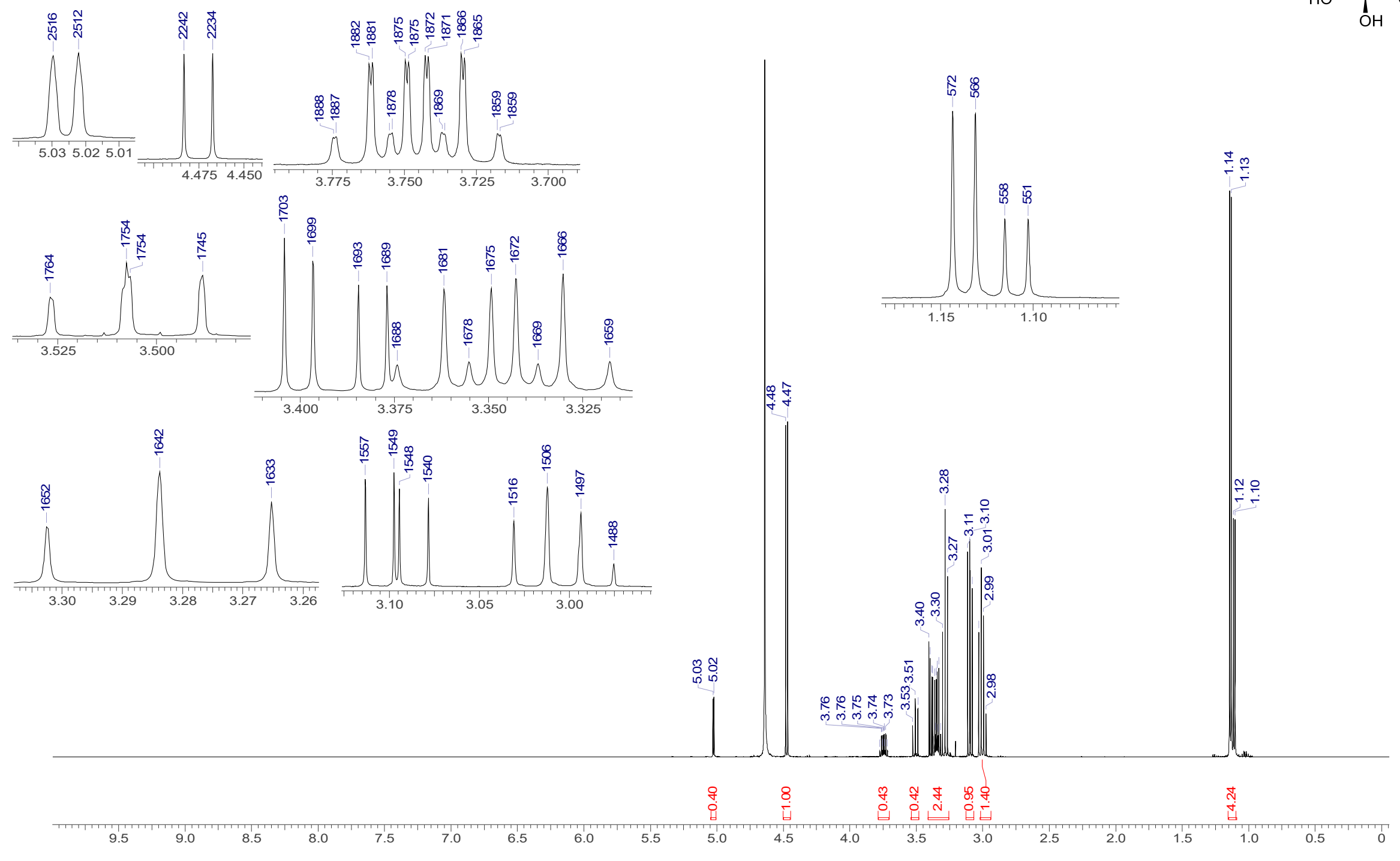
4.13.2. ${ }^{13} \mathrm{C}\left\{{ }^{1} \mathrm{H}\right\}$ NMR $\left(126 \mathrm{MHz}, \mathrm{D}_{2} \mathrm{O}\right)$
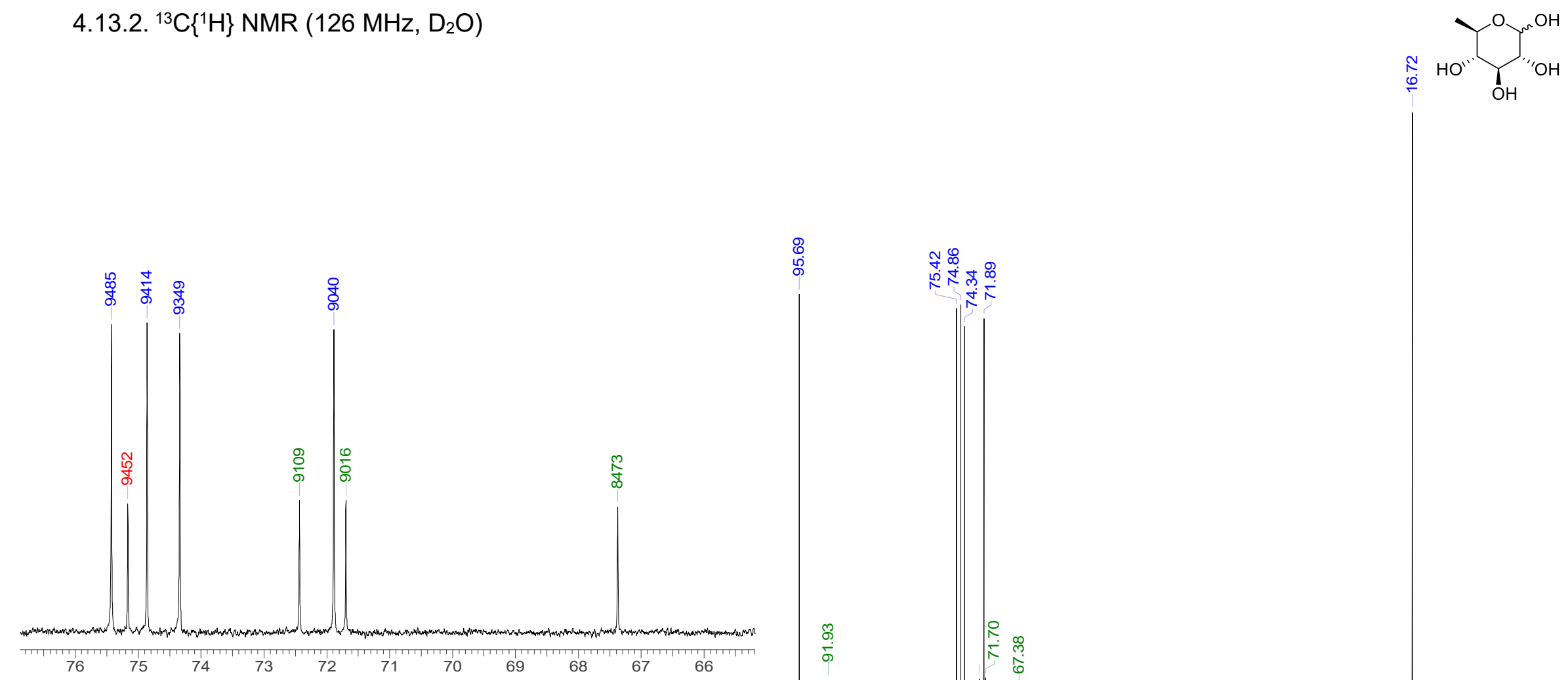

:

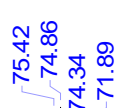

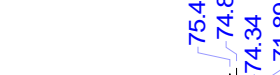

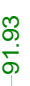


4.13.3. COSY, HSQC-DEPT \& HMBC $\left(D_{2} O\right)$

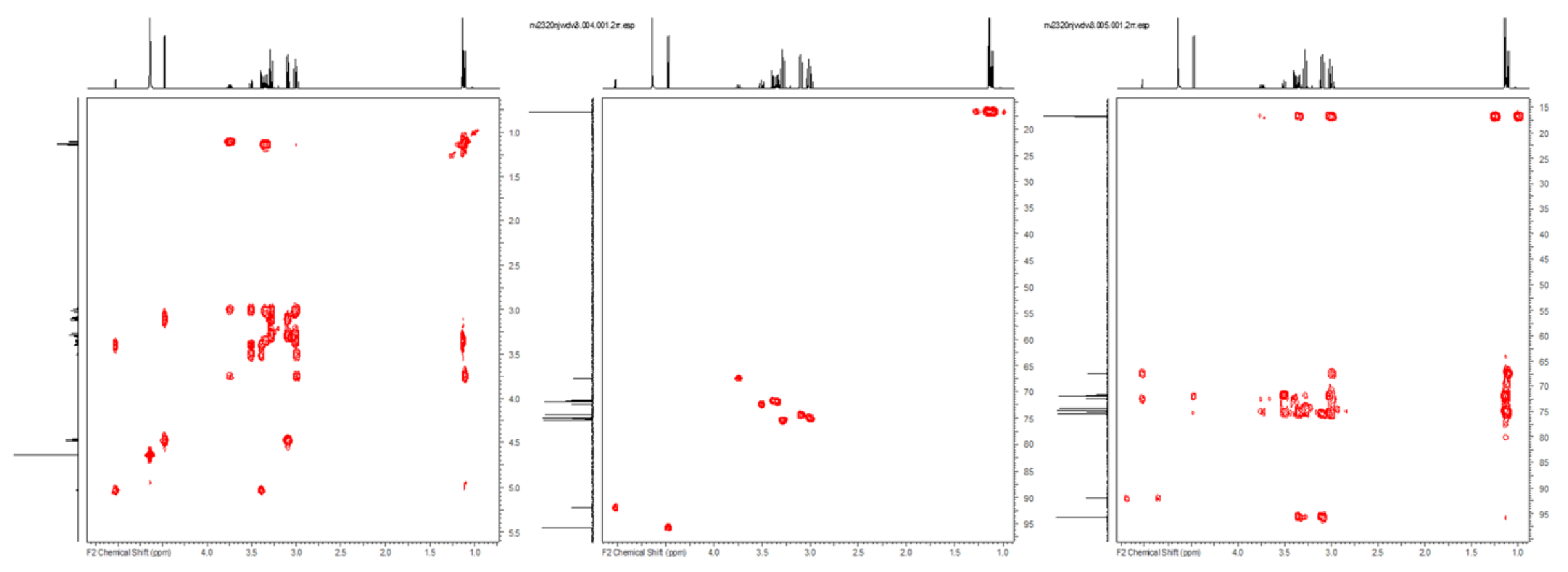


4.13.4. ${ }^{1} \mathrm{H}$ qNMR $\left(500 \mathrm{MHz}, \mathrm{D}_{2} \mathrm{O}\right)$

Duplicate \#1

DW/4,6/D-Quin D2O $500 \mathrm{MHz}$ (1)

Quant. proton

Longest T1 $3.1 \mathrm{~s}$ (delay set to $30 \mathrm{~s}$ for full relaxation)

$$
\mathrm{O}_{\mathrm{OH}}^{\mathrm{O}} \mathrm{OH}
$$

SW: $30 \mathrm{ppm}$

O1P: $6.175 \mathrm{ppm}$

D1: 30 sec

${ }^{1} \mathrm{H}$ NMR

$\alpha / \beta=31: 69$

(see nexl page for detailed integration
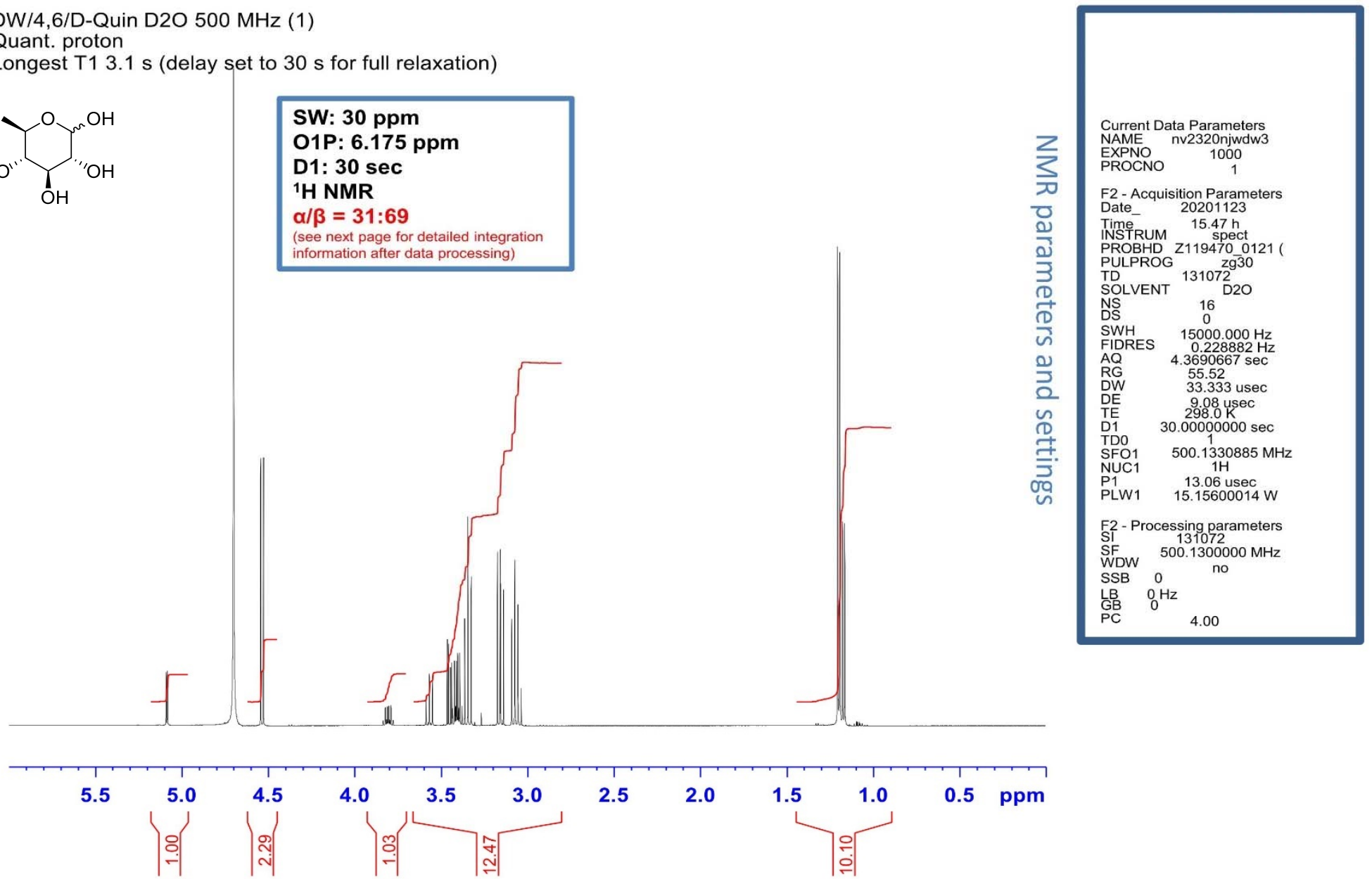


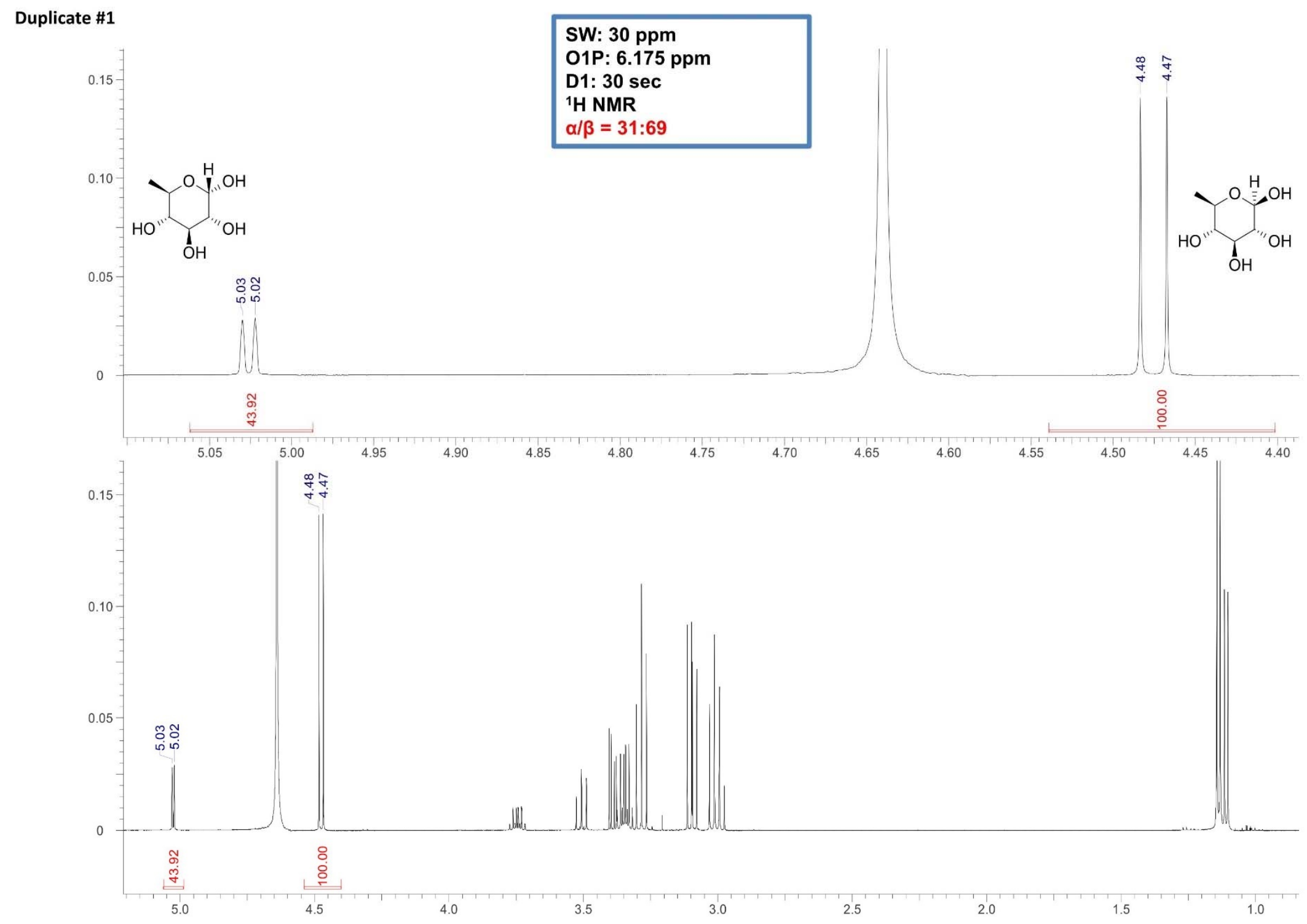




\section{Duplicate \#2}

DW/4,6/D-Quin D2O $500 \mathrm{MHz}(2)$

Quant. proton
Longest T1 $3.1 \mathrm{~s}$ (delay set to $30 \mathrm{~s}$ for full relaxation)

$$
\mathrm{O}_{\mathrm{OH}}^{\mathrm{O}}
$$

SW: 30 ppm

O1P: $6.175 \mathrm{ppm}$

D1: 30 sec

1H NMR

$\alpha / \beta=31: 69$

in for detailed integration

formation after data processing)

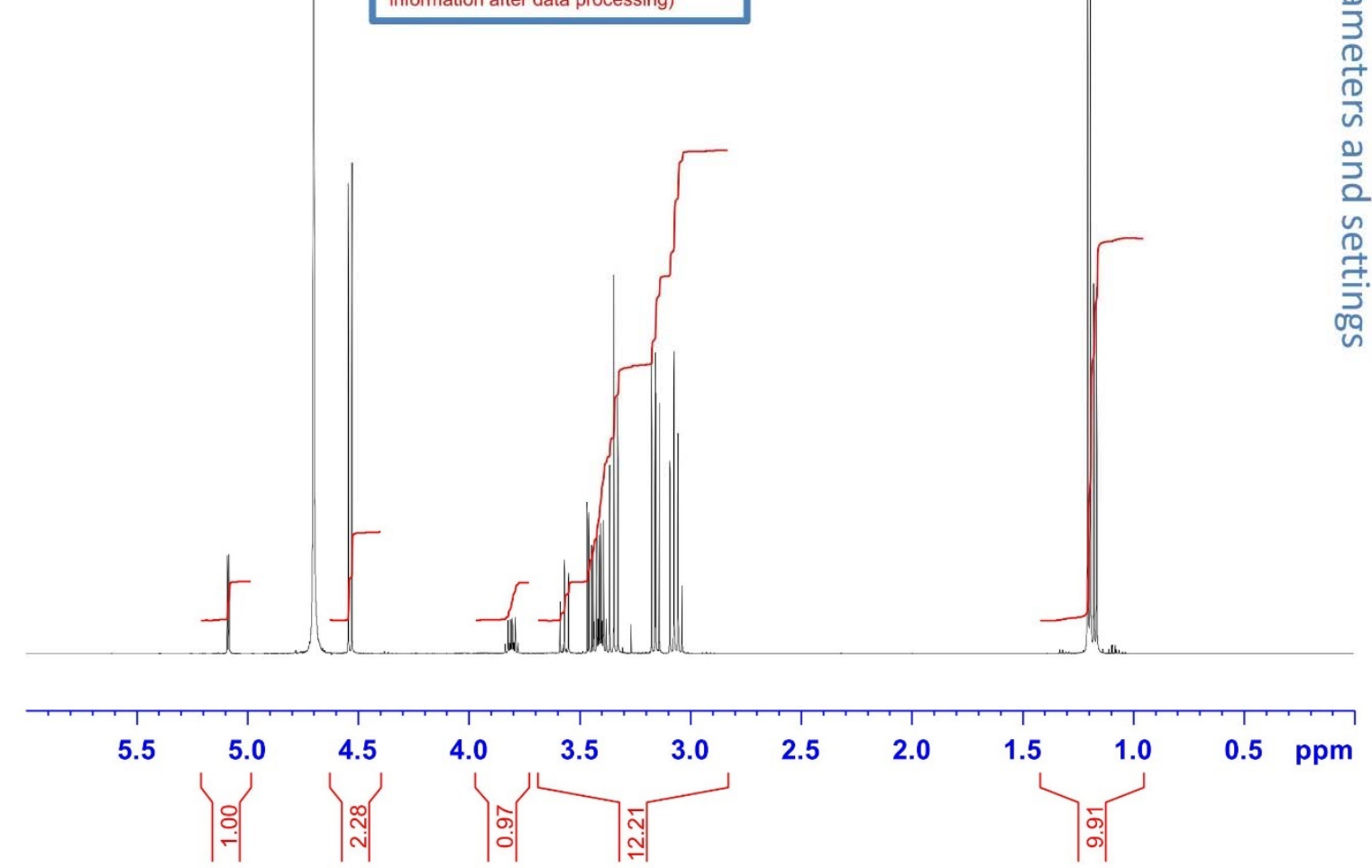

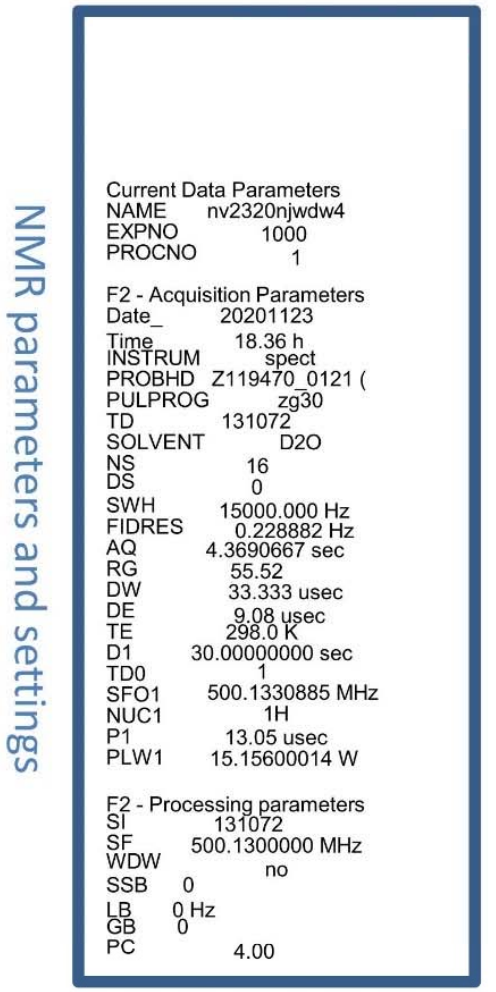


Duplicate \#2

SW: 30 ppm

01P: $6.175 \mathrm{ppm}$

D1: 30 sec

${ }^{1} \mathrm{H}$ NMR

$\overbrace{\mathrm{OH}}^{\mathrm{V}}{ }_{\mathrm{HOH}}^{\mathrm{H}} \mathrm{OH}$

$\alpha / \beta=31: 69$
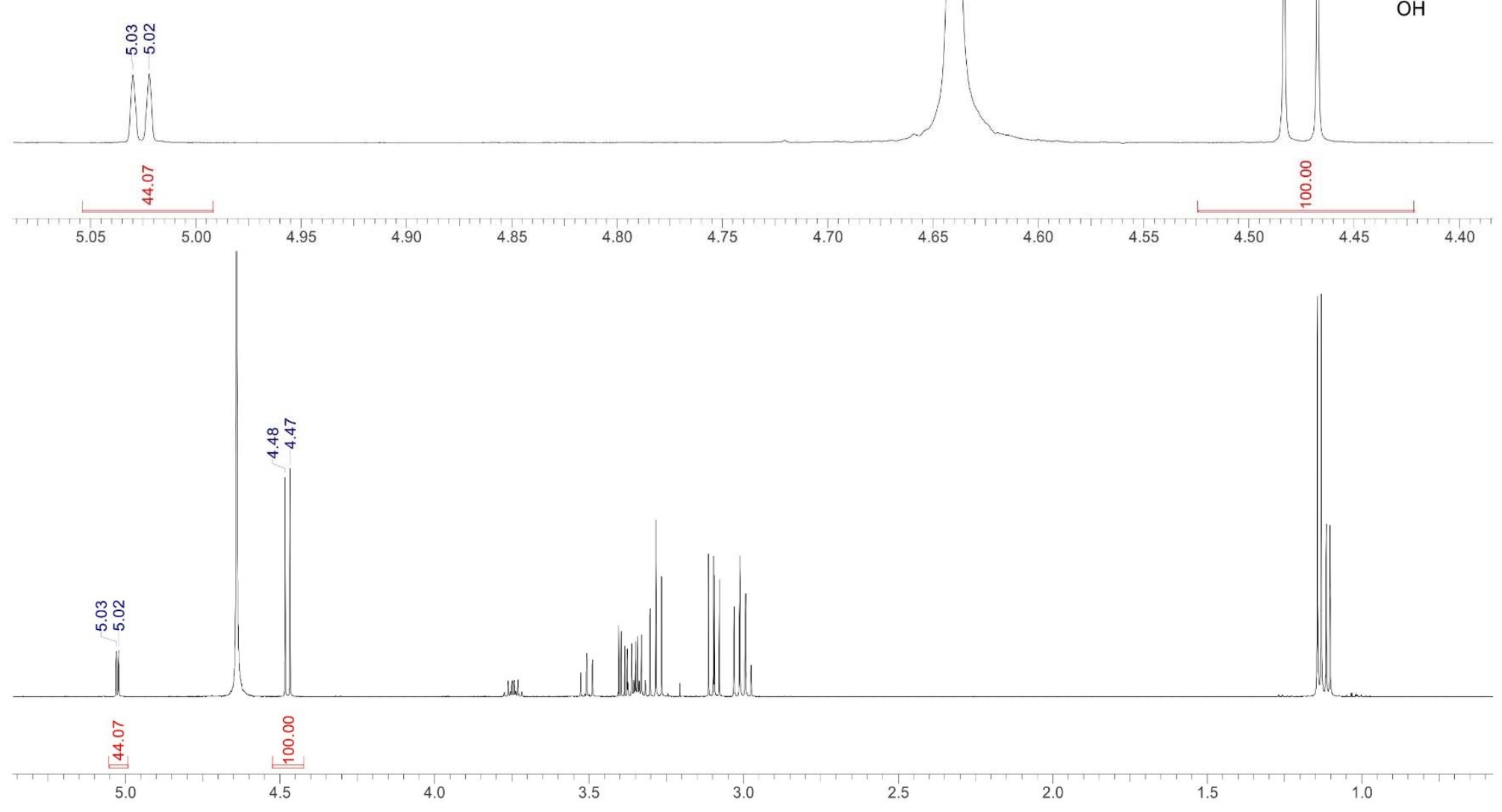


\subsection{D-Galactose (92)}

4.14.1. ${ }^{1} \mathrm{H}$ NMR $\left(500 \mathrm{MHz}, \mathrm{D}_{2} \mathrm{O}\right)$
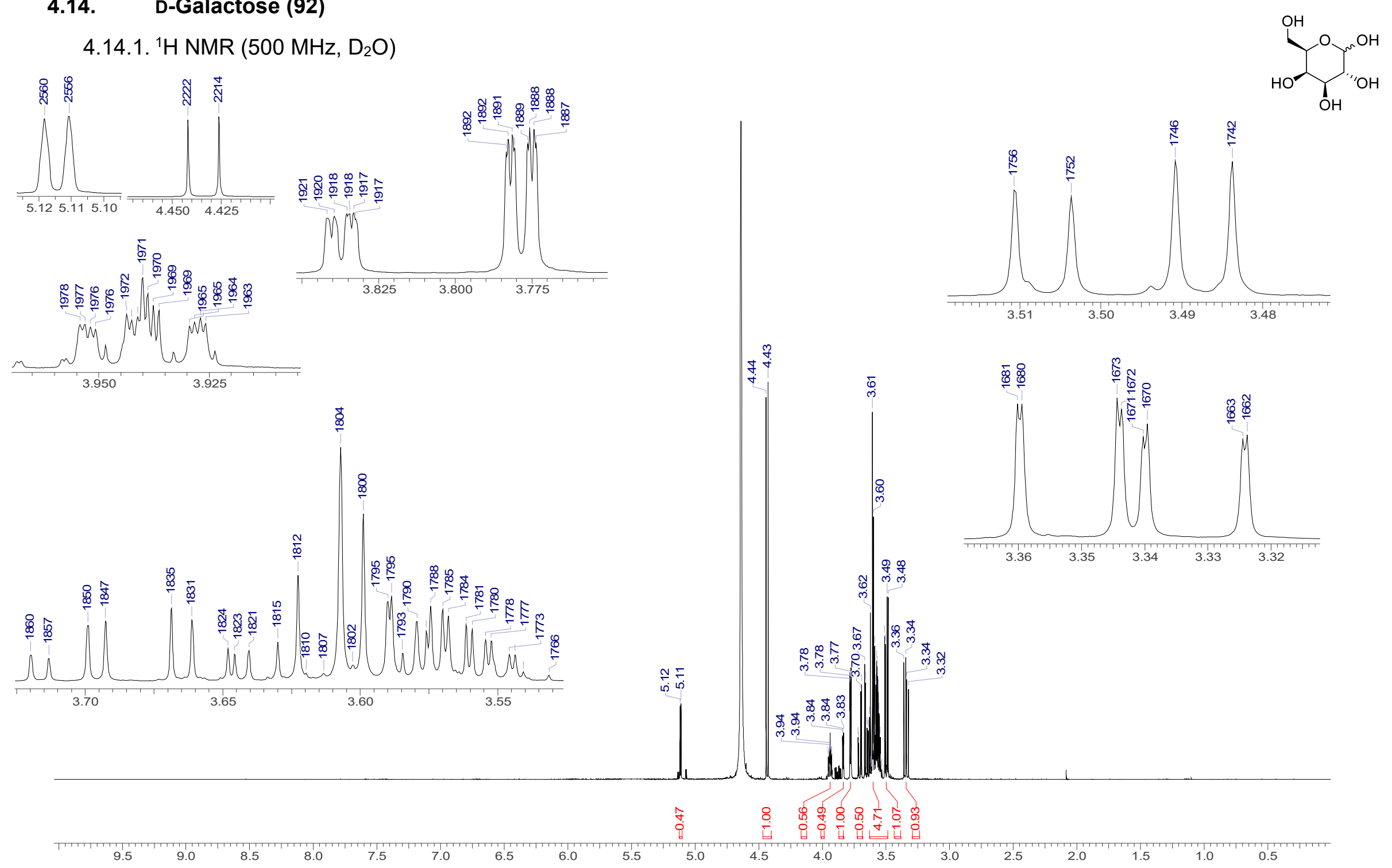
4.14.2. ${ }^{13} \mathrm{C}\left\{{ }^{1} \mathrm{H}\right\}$ NMR $\left(126 \mathrm{MHz}, \mathrm{D}_{2} \mathrm{O}\right)$

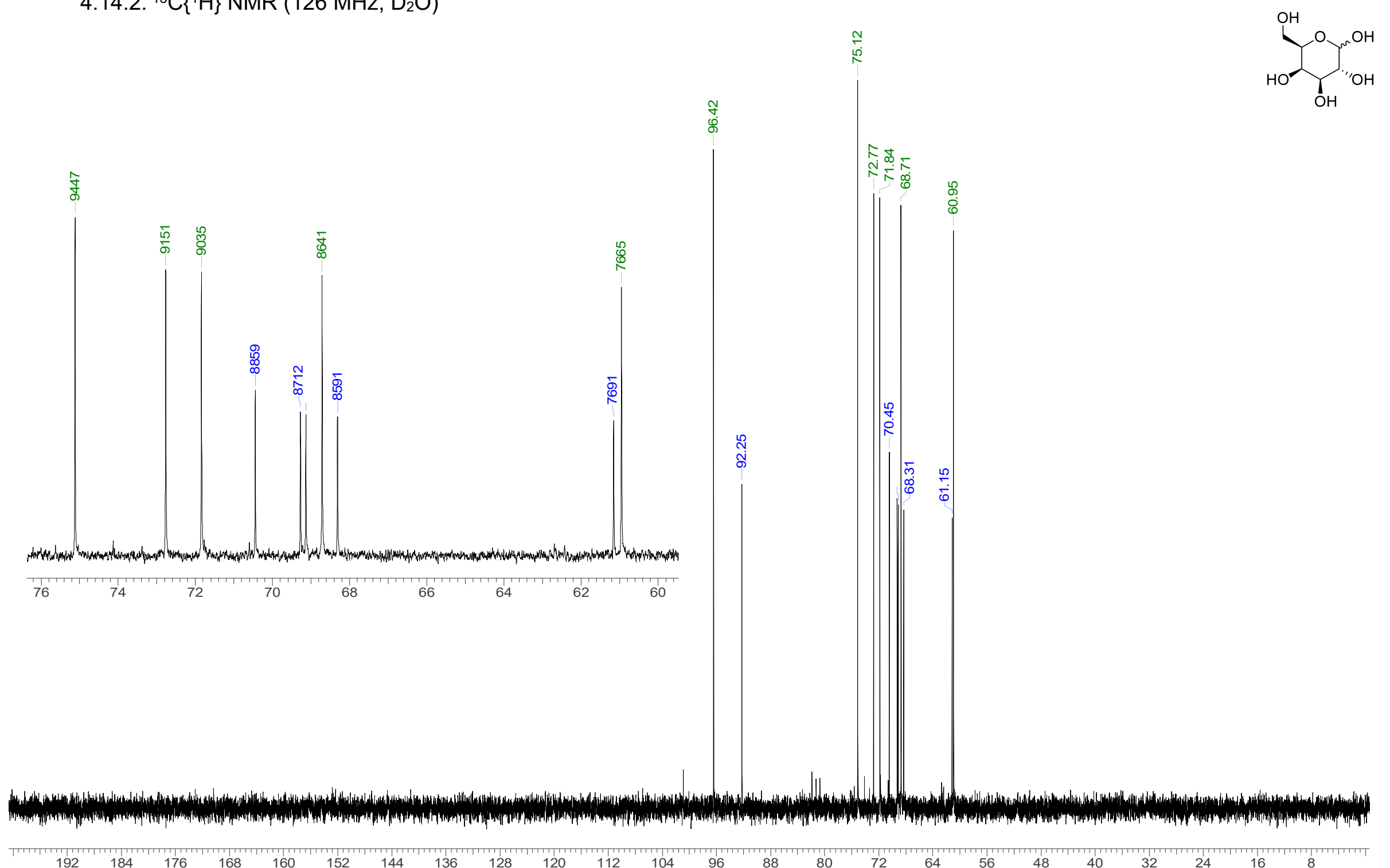


4.14.3. COSY, HSQC-DEPT \& HMBC $\left(D_{2} \mathrm{O}\right)$

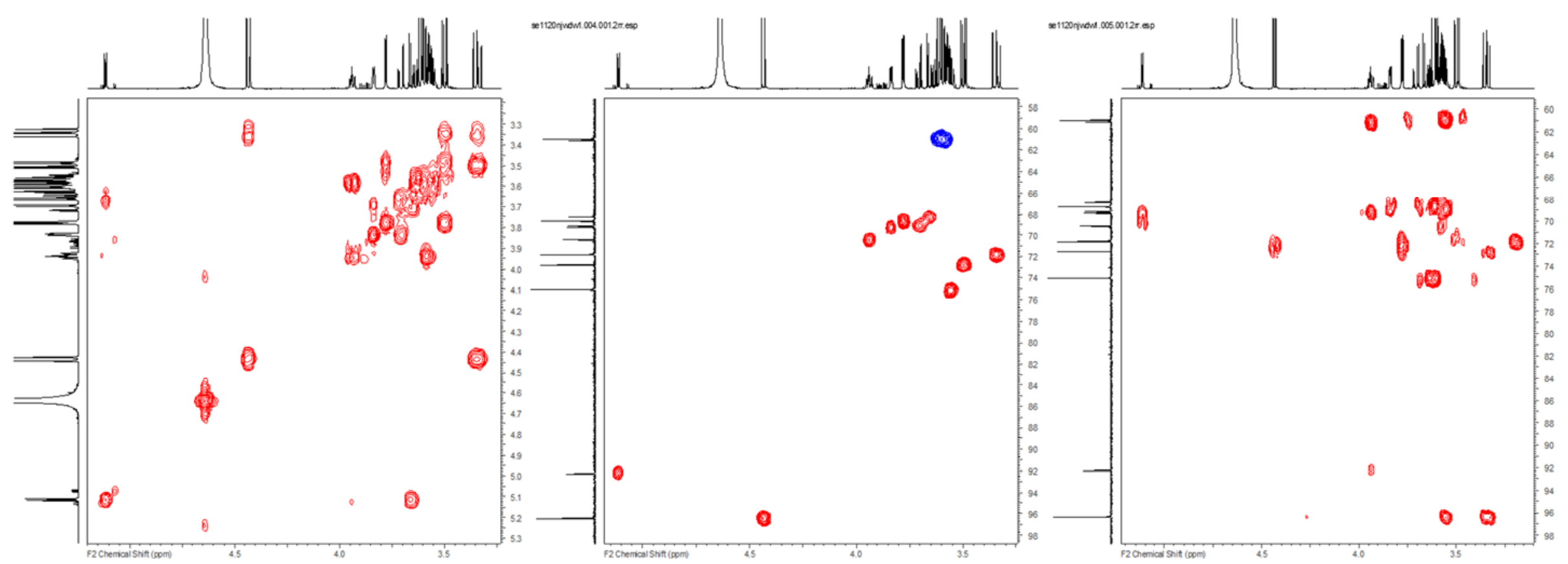


4.14.4. ${ }^{1} \mathrm{H}$ qNMR $\left(500 \mathrm{MHz}, \mathrm{D}_{2} \mathrm{O}\right)$

Duplicate \#1

DW/4,6 Gal D2O $500 \mathrm{MHz}$

Quant. proton

Longest T1 $3.7 \mathrm{~s}$ (delay set to $30 \mathrm{~s}$ for full relaxation)

$$
\overbrace{\mathrm{OH}}^{\mathrm{OH}}
$$

SW: 30 ppm

01P: 6.175 ppm

D1: $30 \mathrm{sec}$

${ }^{1} \mathrm{H}$ NMR

$\alpha / \beta=33: 67$

(see next page for detailed integration
information after data processing)
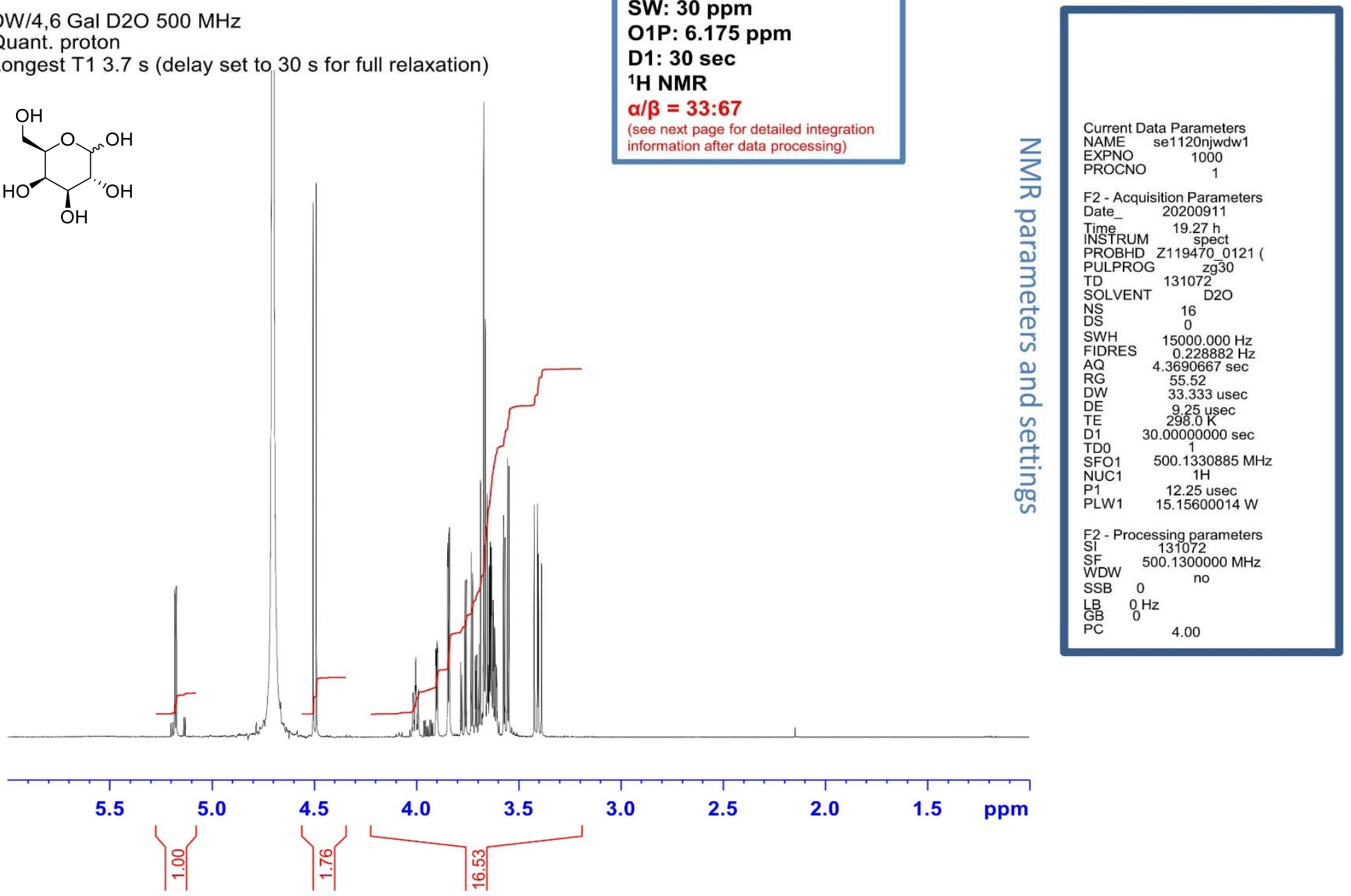


\section{Duplicate \#1}

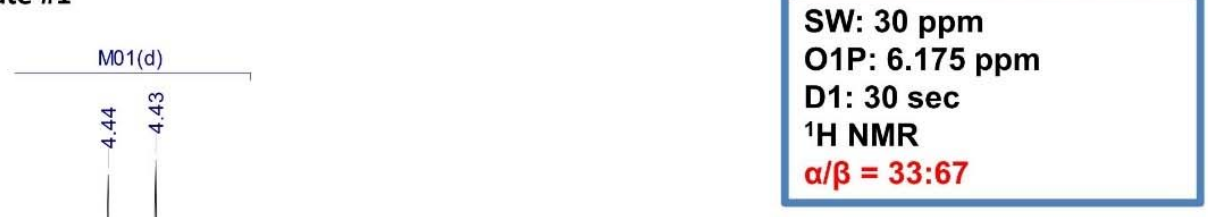

SW: 30 ppm

: $6.175 \mathrm{ppm}$

$0 \mathrm{~s}$

$\alpha / \beta=33: 6$

$$
\underbrace{\mathrm{OH}}_{=\mathrm{OH}}
$$
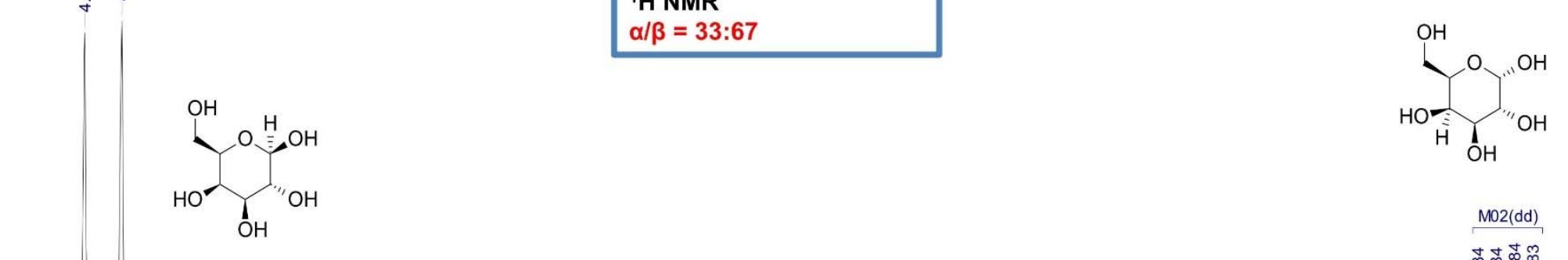

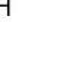
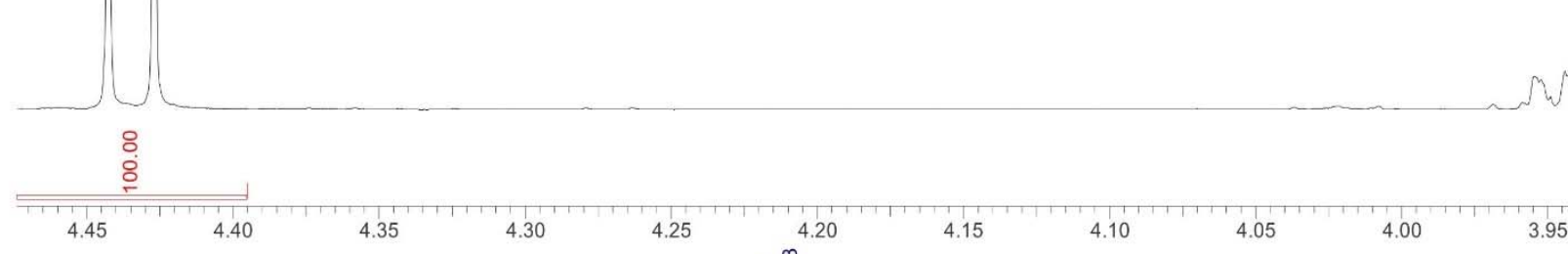

MO2(da)

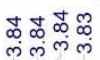

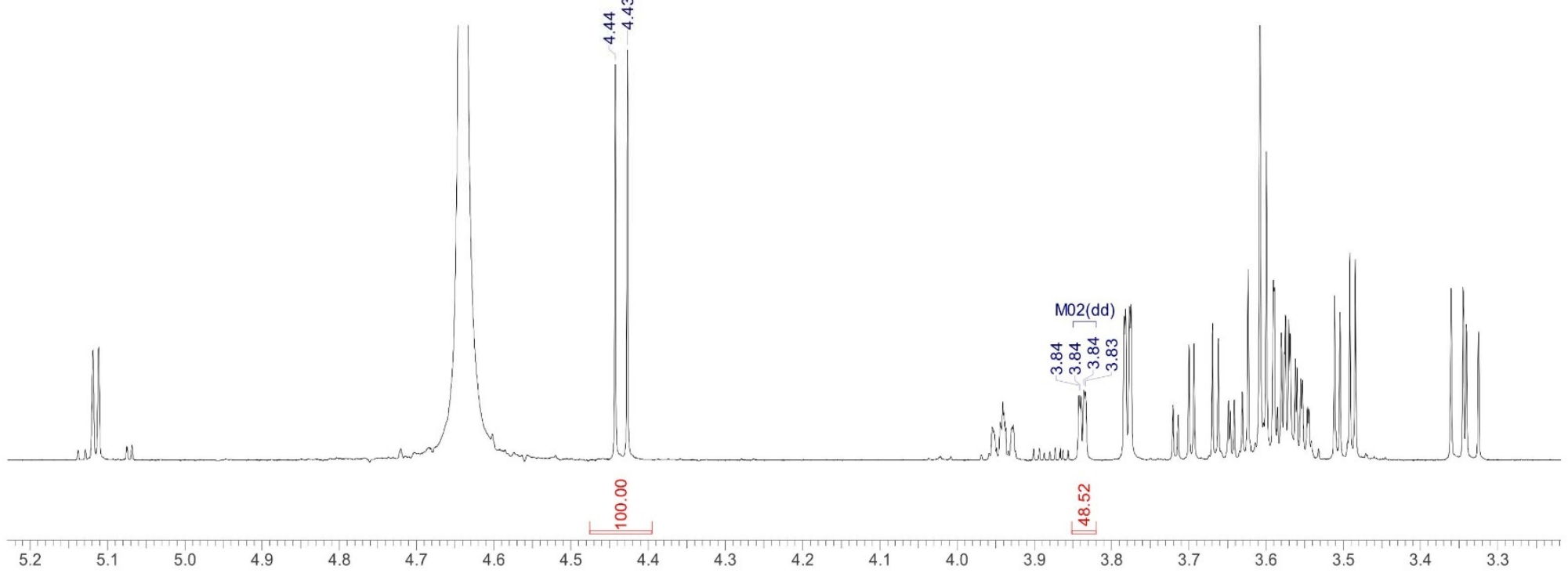




\section{Duplicate \#2}

DW/4,6 Gal $500 \mathrm{MHz}$

Quant. proton (qNMR 2)

Longest T1 $3.4 \mathrm{~s}$ (delay set to $45 \mathrm{~s}$ for full relaxation)

$$
\overbrace{\mathrm{OH}}^{\mathrm{OH}}
$$

SW: 30 ppm

01P: $6.175 \mathrm{ppm}$

D1: $30 \mathrm{sec}$

${ }^{1} \mathrm{H}$ NMR

$\alpha / \beta=33: 67$

etailed integration

information after data proce

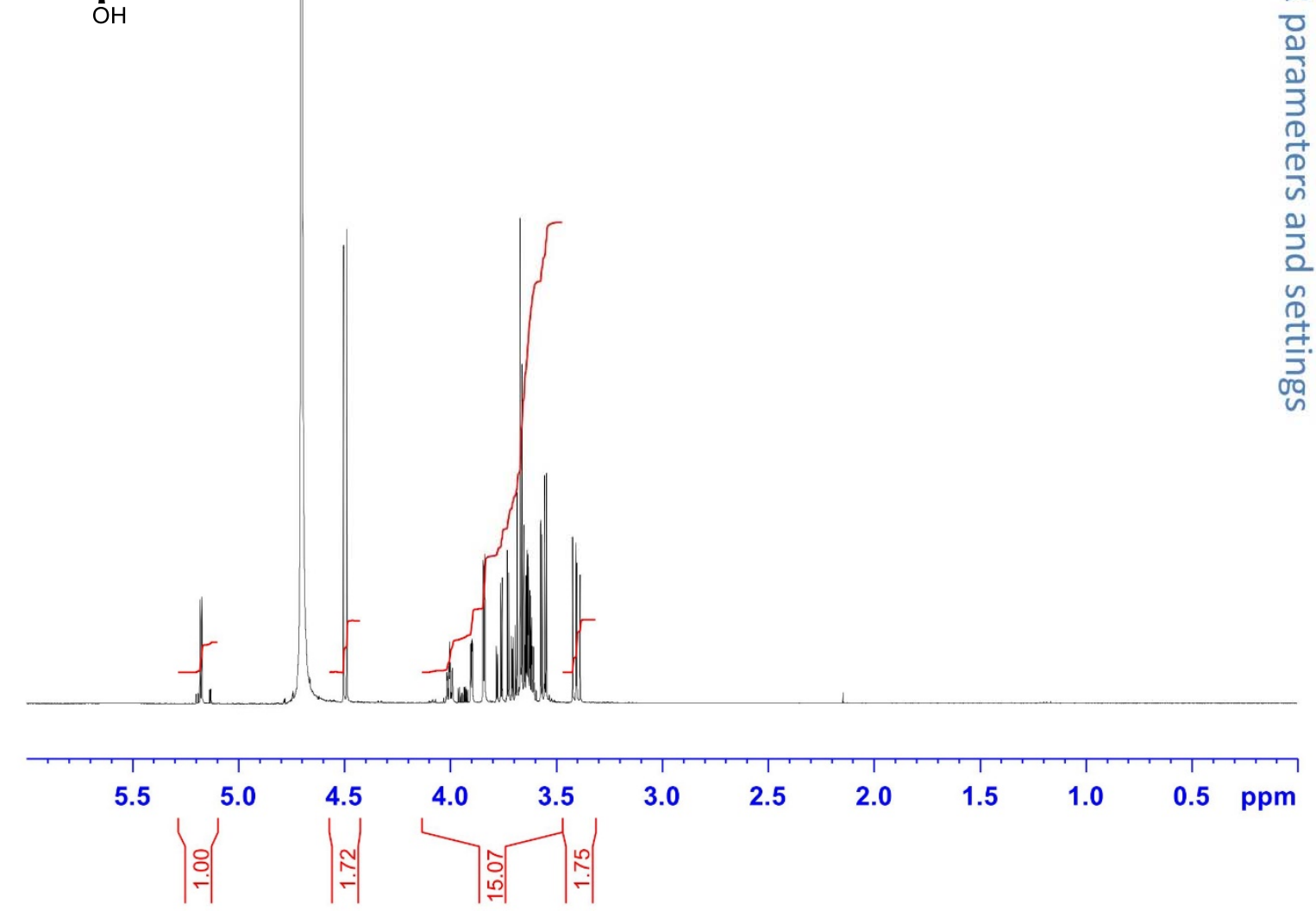

Current Data Parameters

$\begin{array}{ll}\text { EXPNO } & 2 \\ \text { PROCNO } & \end{array}$

F2 - Acquisition Parameters

Time- $8.58 \mathrm{~h}$

spect

PULPROG 279

SOLVENT ${ }^{131072}$ D2O

$\begin{array}{lll}\mathrm{NS} & 16 \\ \mathrm{DS} & 0\end{array}$

SWH $15000.000 \mathrm{~Hz}$

$\begin{array}{ll}A Q & 4.3690667 \mathrm{sec}\end{array}$

$\begin{array}{ll}\mathrm{RW} & 55.52 \\ \mathrm{DW} & 33.33 \mathrm{usec}\end{array}$

$\begin{array}{ll}\text { DE } & 9.29 \text { usec } \\ \text { TE } & 298.0 \mathrm{~K}\end{array}$

D1 $45.00000000 \mathrm{sec}$

$\begin{array}{lll}\text { TDO } & 1 \\ \text { SFO1 } & 500.1330885 \mathrm{MH}\end{array}$

$\begin{array}{lc}\text { SFO1 } & 500.1330885 \mathrm{MH} \\ \text { NUC1 } & 1 \mathrm{H}\end{array}$

$\begin{array}{ll}\text { P1 } & 12.06 \text { usec } \\ \text { PLW1 } & 15.15600014 \mathrm{~W}\end{array}$

F2 - Processing parameters

$\begin{array}{ll}\text { F2 - Processing parameters } \\ \text { SI } \\ \text { SF } & 5001072 \\ \text { SF } & 500.1300000 \mathrm{MHz}\end{array}$

NSB 0 no

$\begin{array}{ll}\text { SSB } & 0 \\ L B & 0 \mathrm{H}\end{array}$

$\begin{array}{lll}\mathrm{SG} & \mathrm{O}^{\mathrm{H}} \\ \mathrm{PC} & & 4.00\end{array}$ 
Duplicate \#2

SW: 30 ppm

01P: $6.175 \mathrm{ppm}$

D1: 30 sec

${ }^{1} \mathrm{H}$ NMR

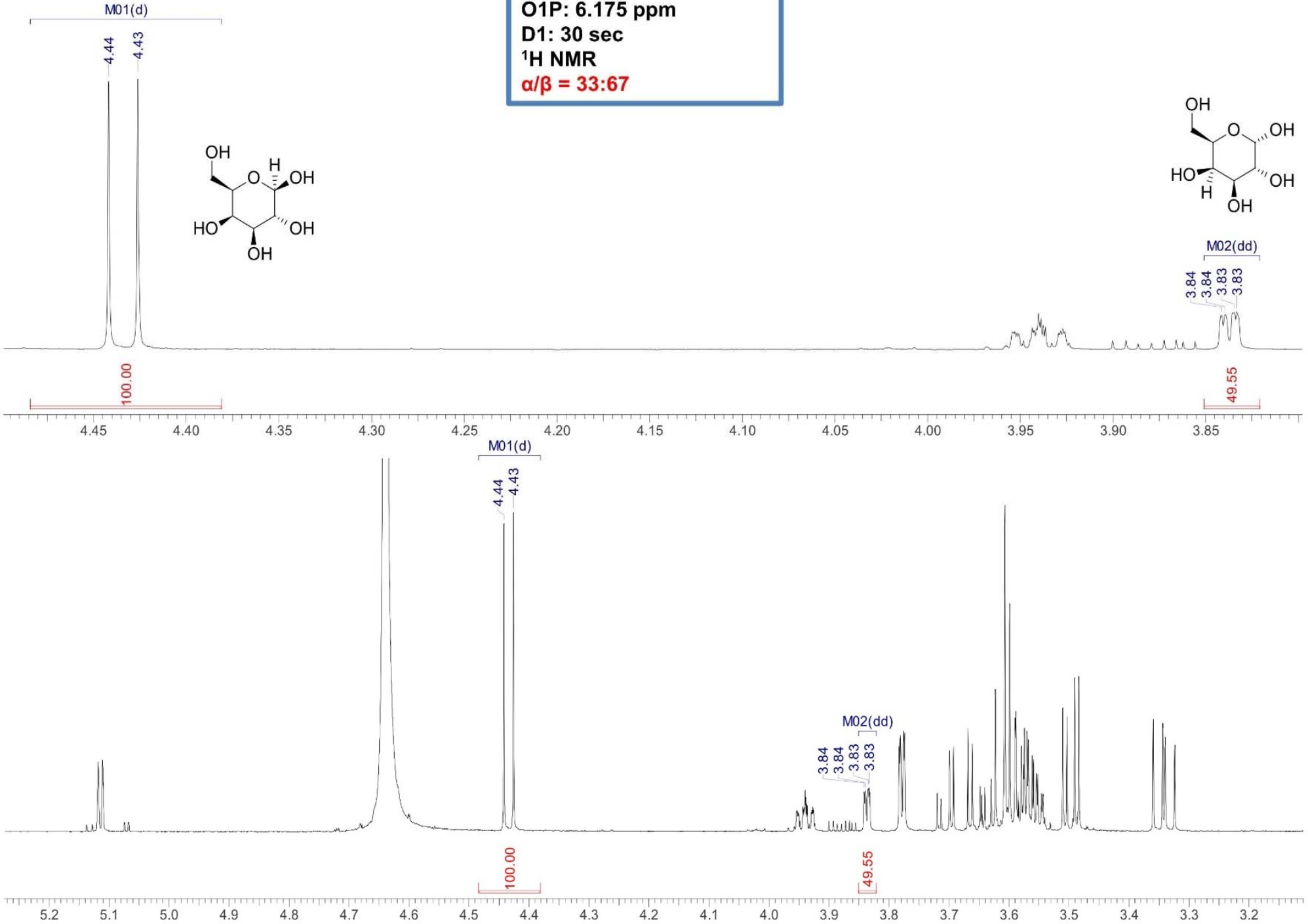


4.15. 4-Deoxy-D-xylo-hexopyranose (93)

4.15.1. ${ }^{1} \mathrm{H}$ NMR $\left(500 \mathrm{MHz}, \mathrm{D}_{2} \mathrm{O}\right)$

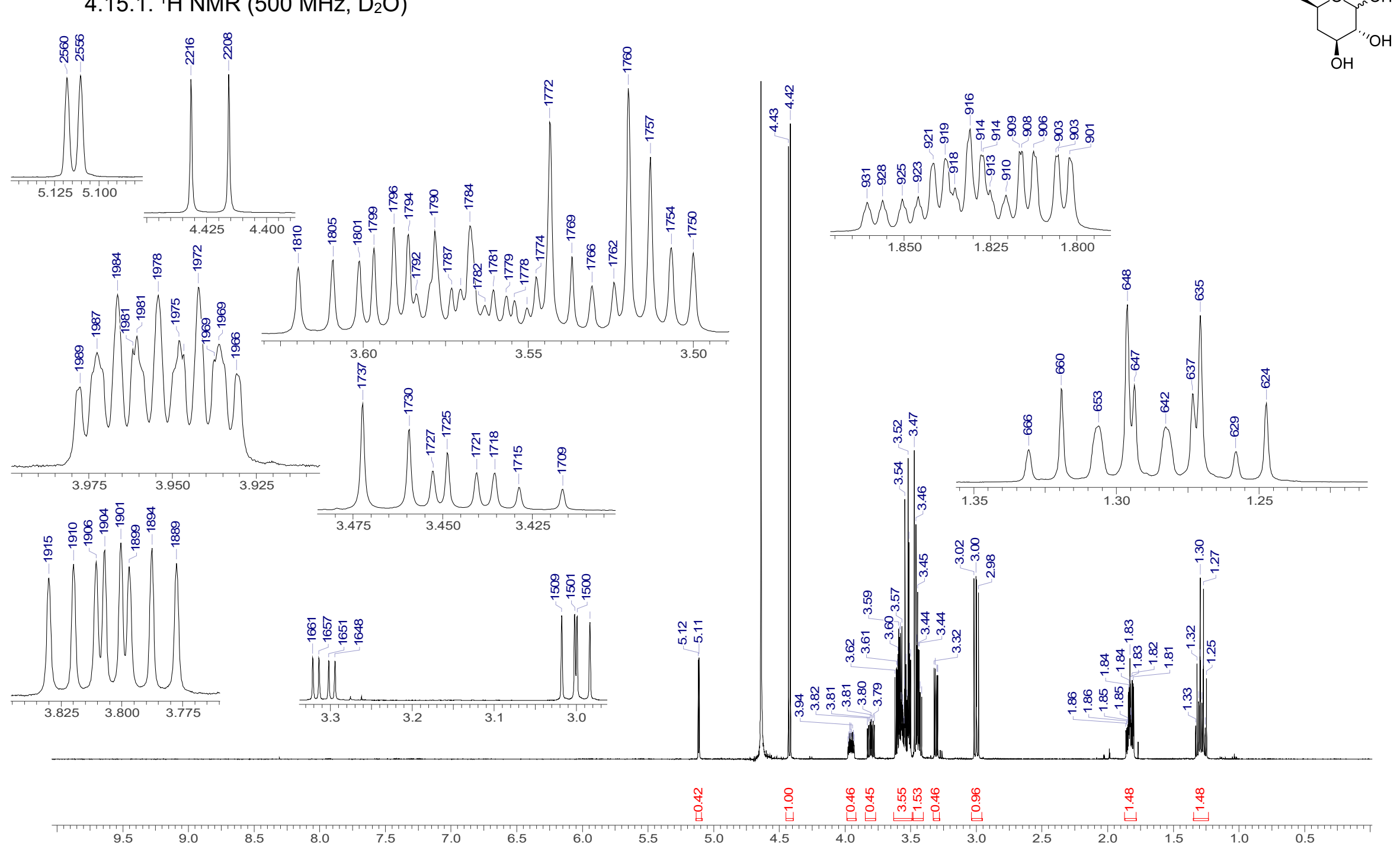


4.15.2. ${ }^{13} \mathrm{C}\left\{{ }^{1} \mathrm{H}\right\}$ NMR $\left(126 \mathrm{MHz}, \mathrm{D}_{2} \mathrm{O}\right)$
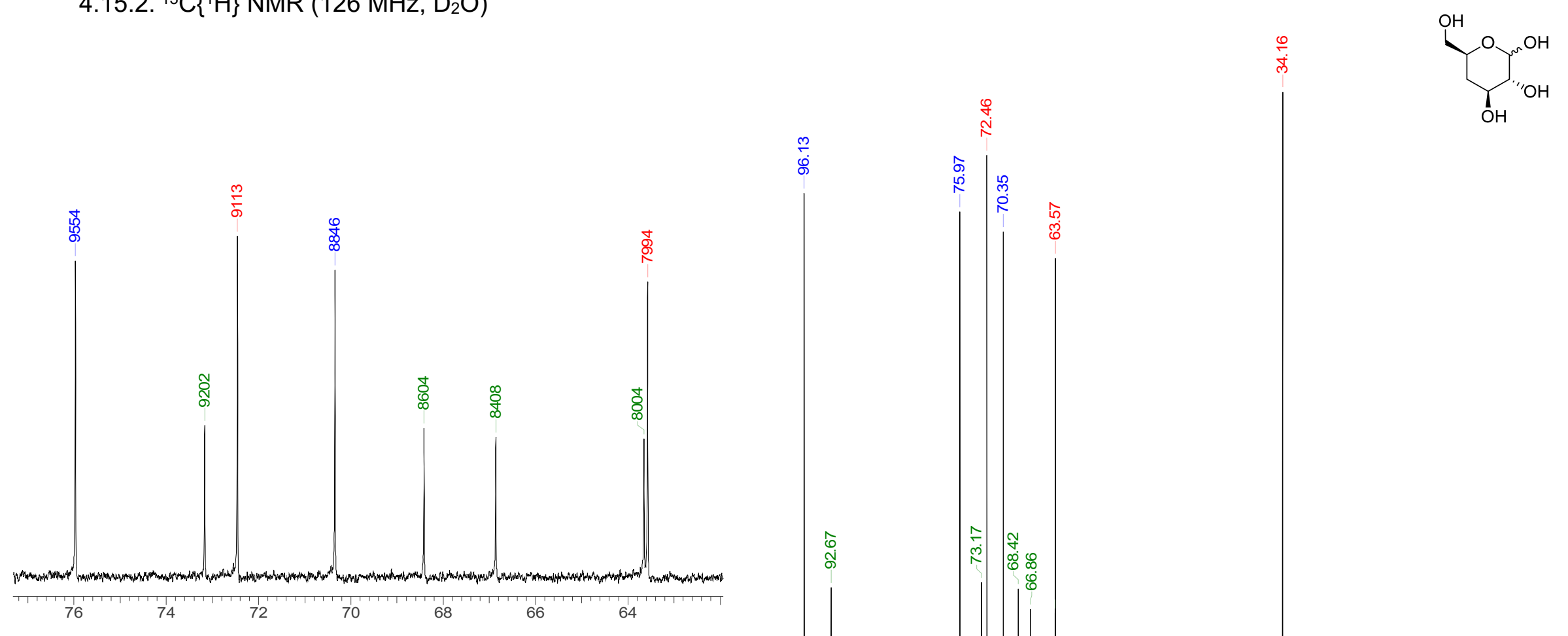

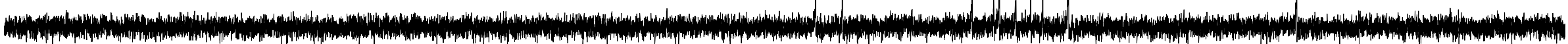

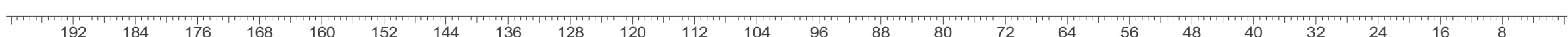




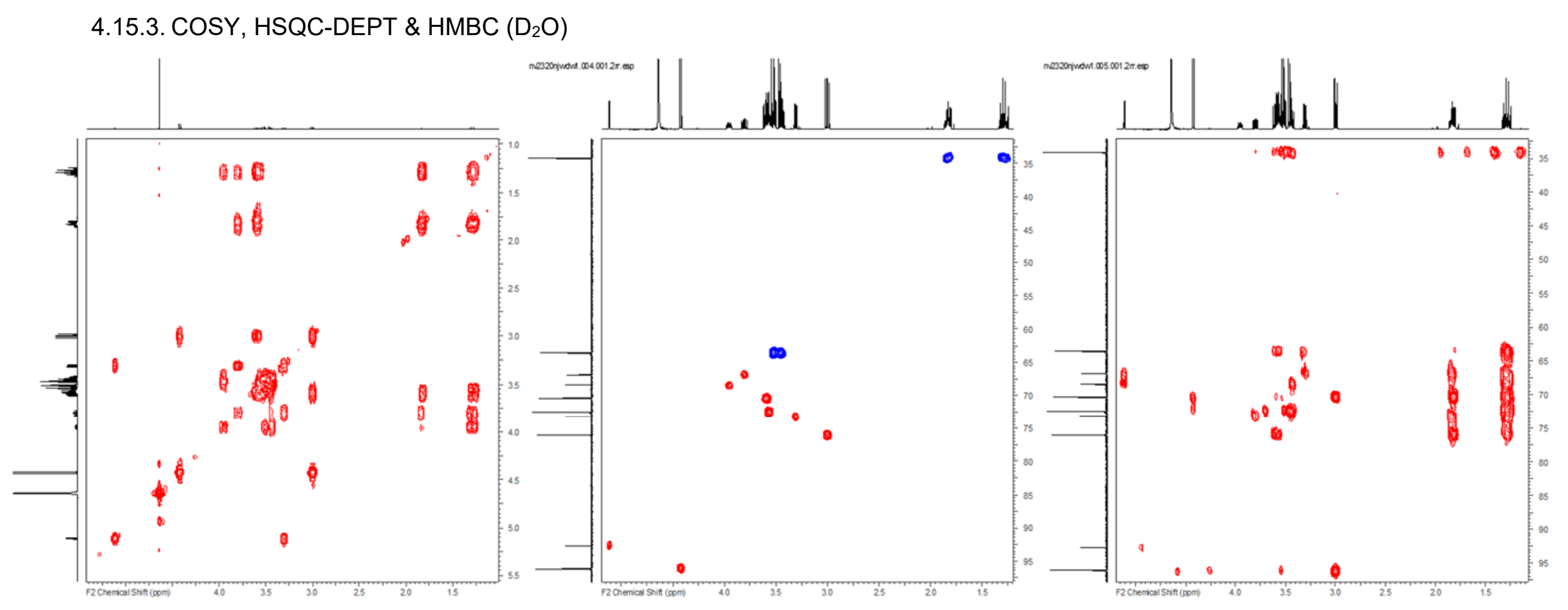


4.15.4. ${ }^{1} \mathrm{H}$ qNMR $\left(500 \mathrm{MHz}, \mathrm{D}_{2} \mathrm{O}\right)$

Duplicate \#1

DW/4,6/4DG D2O $500 \mathrm{MHz}$ (1)

Quant. proton

Longest T1 $3.2 \mathrm{~s}$ (delay set to $30 \mathrm{~s}$ for full relaxation)

$$
{ }_{\mathrm{OH}}^{\mathrm{OH}}
$$

SW: $30 \mathrm{ppm}$

O1P: $6.175 \mathrm{ppm}$

D1: 30 sec

${ }^{1} \mathrm{H}$ NMR

$\alpha / \beta=31: 69$

(see next page for detailed integration

information after data processing)
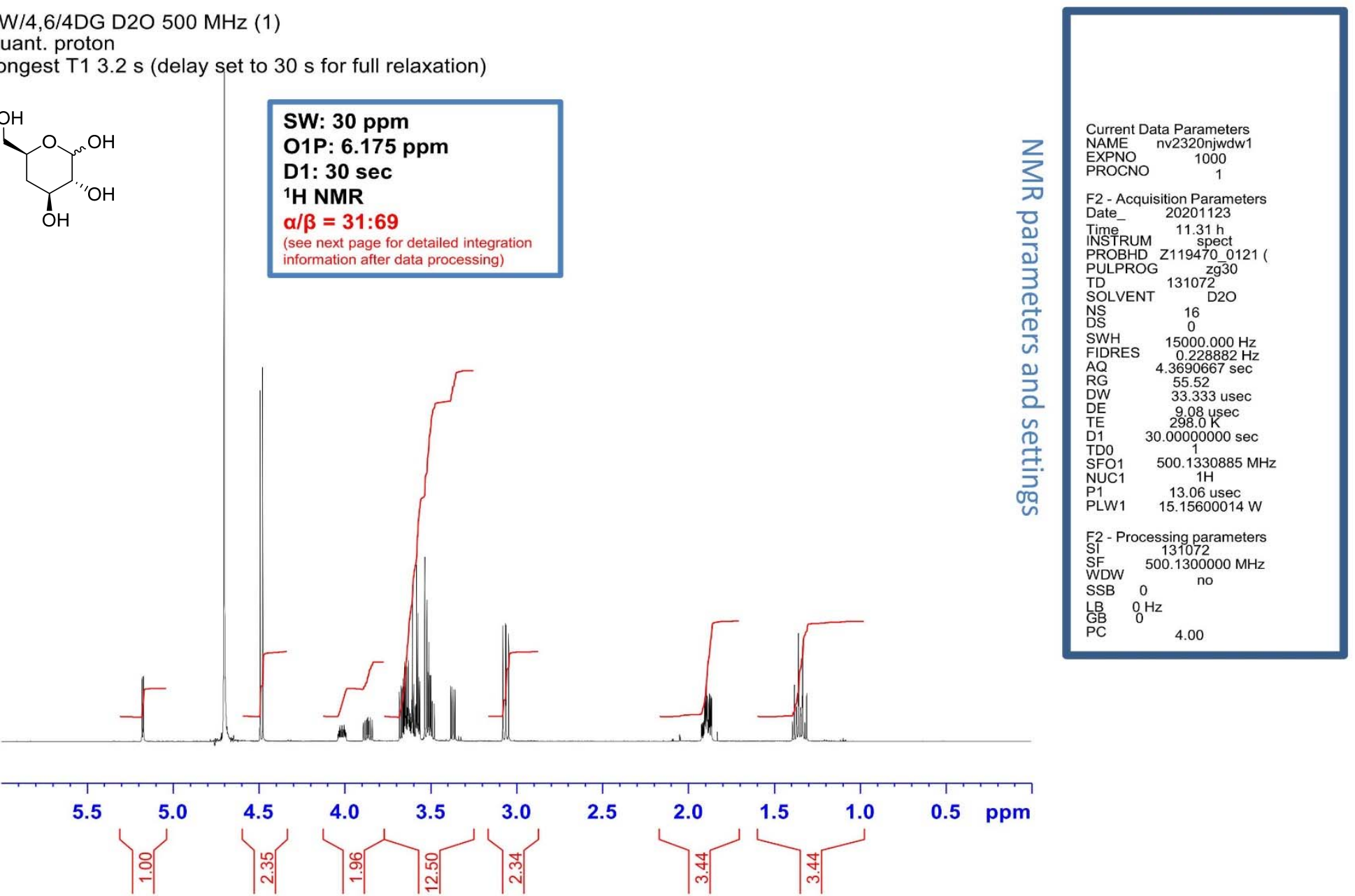
Duplicate \#1

SW: 30 ppm

1P: $6.175 \mathrm{ppm}$

D1: 30 sec

${ }^{1} \mathrm{H}$ NMR

$$
{ }_{\mathrm{OH}}^{\mathrm{OH}} \mathrm{OH}
$$
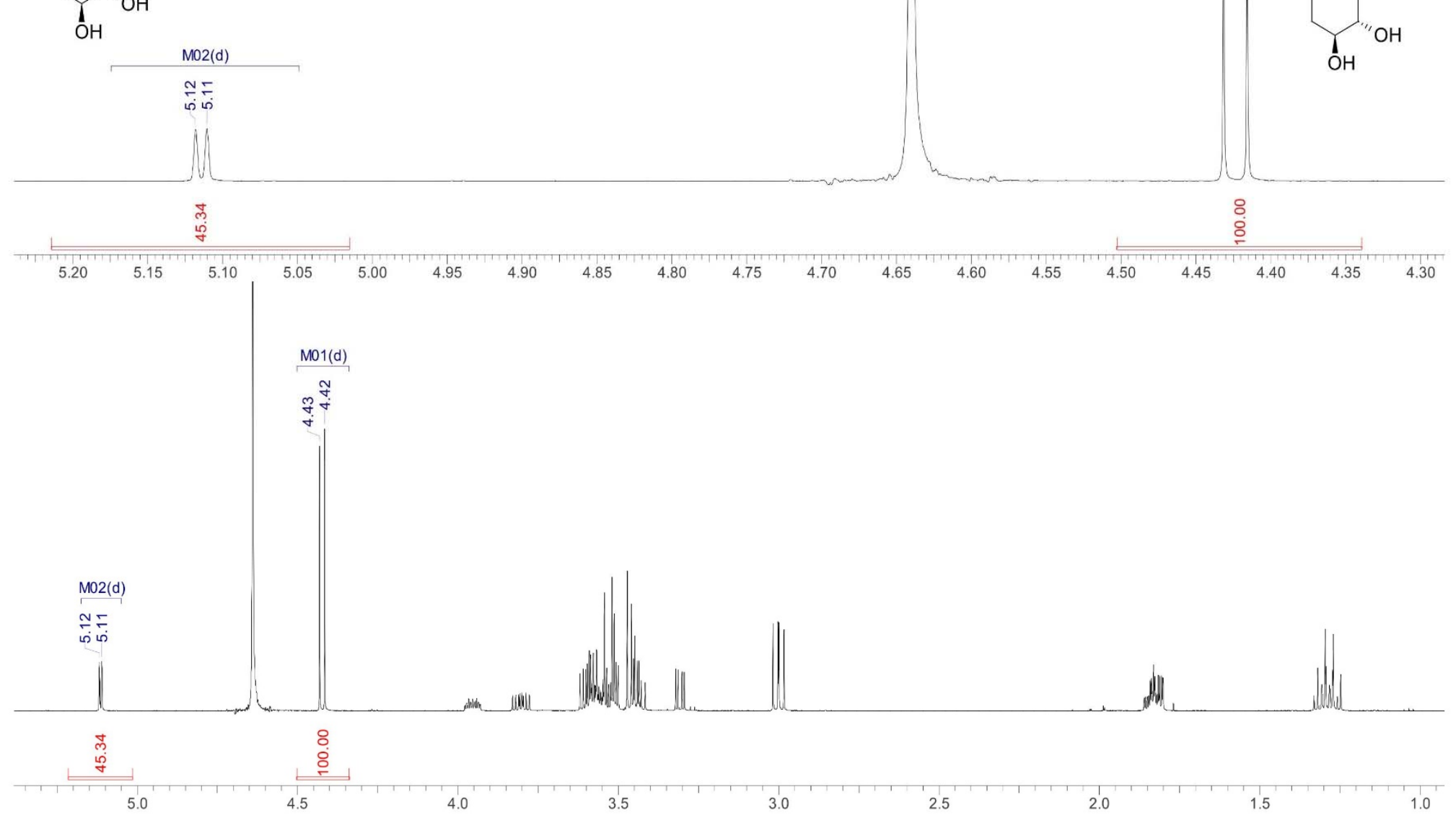


\section{Duplicate \#2}

DW/4,6/4DG D2O $500 \mathrm{MHz}(2)$

Quant. proton

Longest T1 $3.4 \mathrm{~s}$ (delay set to $30 \mathrm{~s}$ for full relaxation)

$$
\underbrace{\mathrm{OH}}_{\mathrm{OH}}
$$

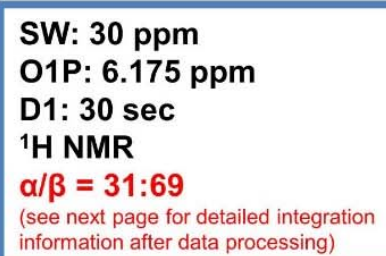

SW: $30 \mathrm{ppm}$

O1P: $6.175 \mathrm{ppm}$

D1: 30 sec

${ }^{1} \mathrm{H}$ NMR

$\alpha / \beta=31: 69$

tailed integration

(a)
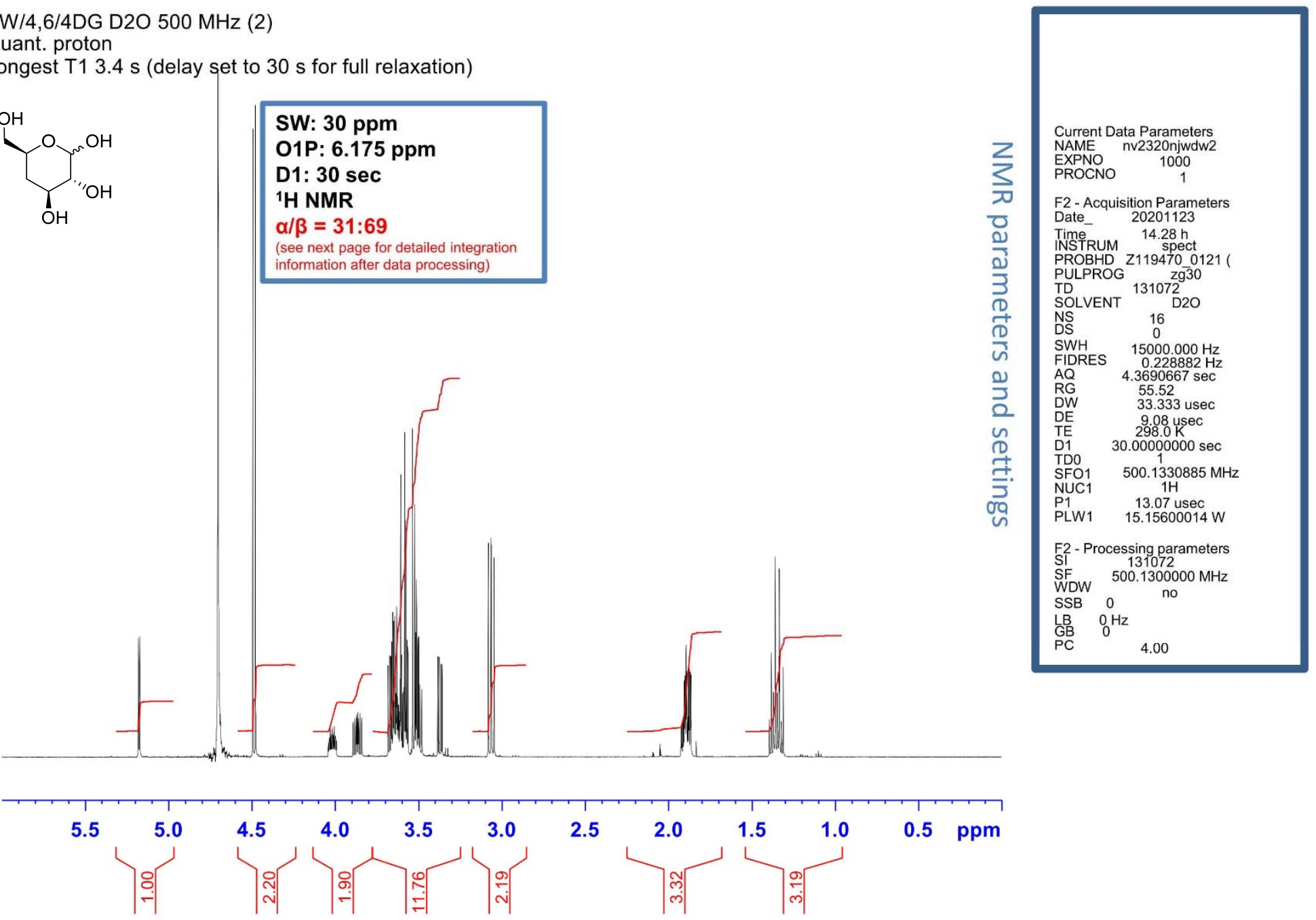
Duplicate \#2

SW: 30 ppm

01P: 6.175 ppm

D1: $30 \mathrm{sec}$

${ }^{1} \mathrm{H}$ NMR

M01(d)

$\alpha / \beta=31: 69$

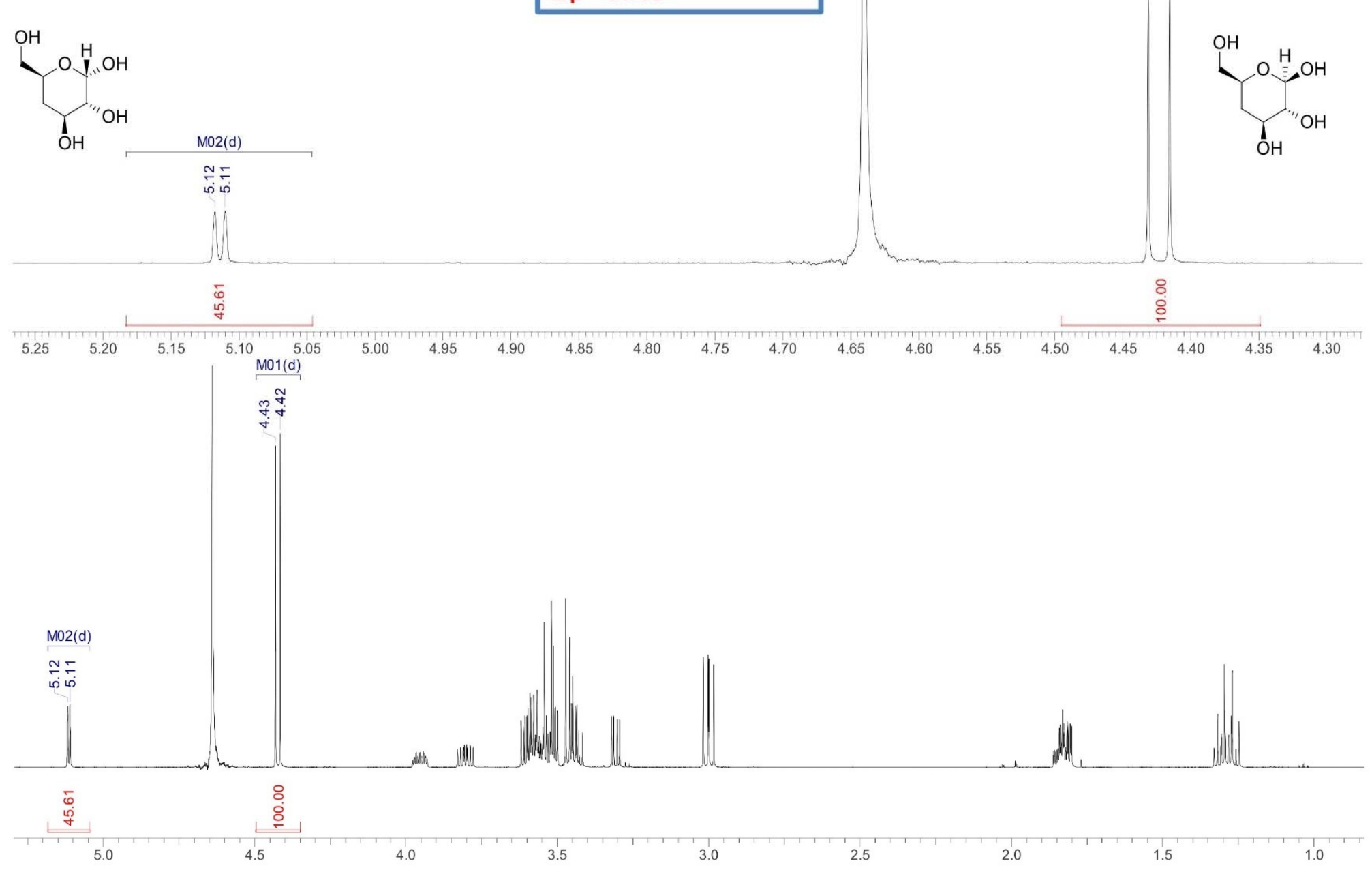


S 391

5. Crystallographic data

5.1. 4,6-Dideoxy-6-fluoro- $\alpha-D-g l u c o p y r a n o s e ~(1 a)$

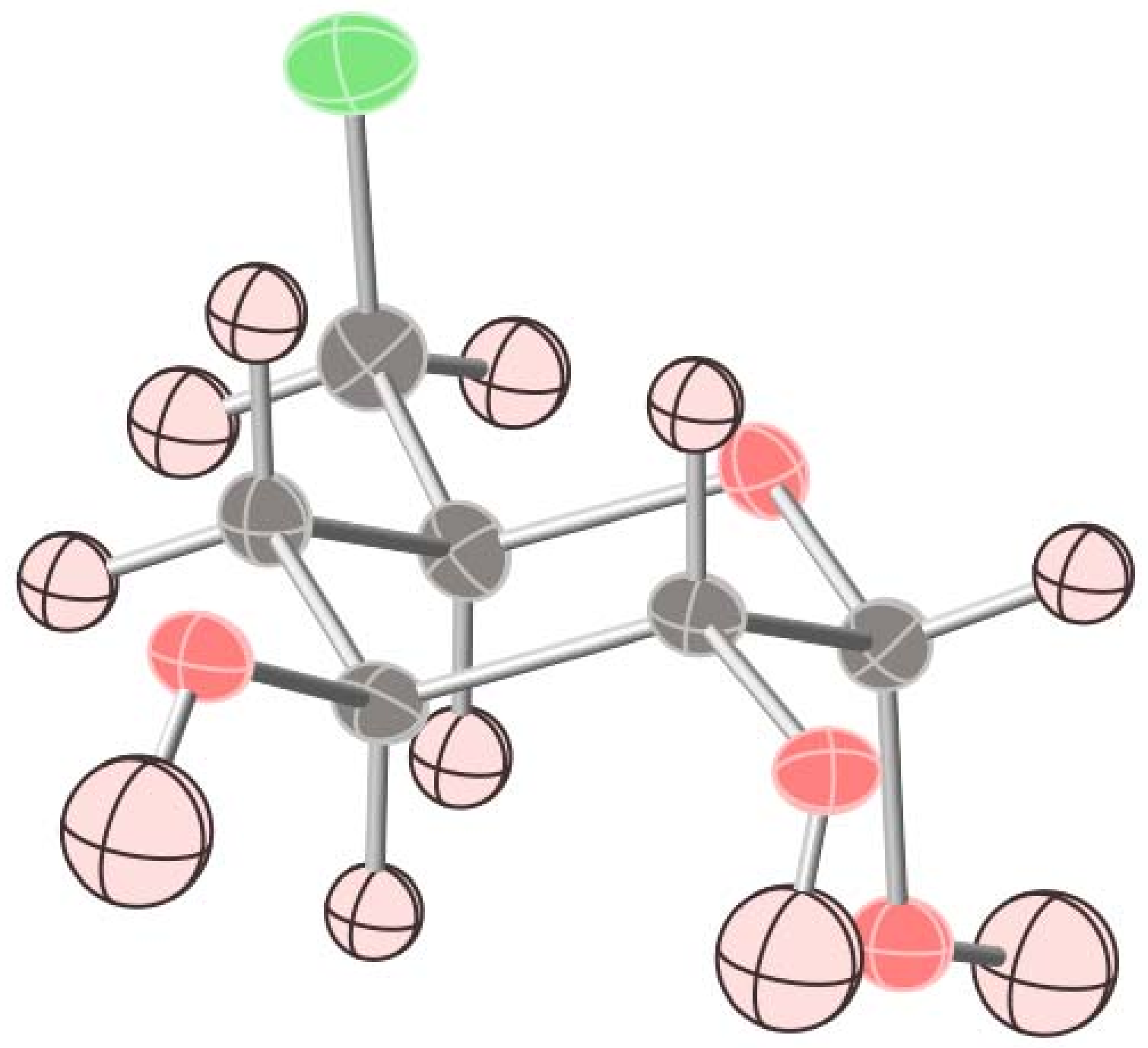

Figure S6: 4,6-Dideoxy-4-fluoro- $\alpha$-D-glucopyranose (Ia). Thermal ellipsoids drawn at the $50 \%$ probability level.

Experimental: Single clear colourless prism-shaped crystals of (2016sot0089_K1_100K) were recrystallised from a mixture of methanol and acetone by slow evaporation. A suitable crystal $(0.41 \times 0.10 \times 0.02) \mathrm{mm}^{3}$ was selected and mounted on a MITIGEN holder silicon oil on a Rigaku AFC12 FRE-HF diffractometer. The crystal was kept at $T=100$ (2) $\mathrm{K}$ during data collection. Using Olex $2,{ }^{7}$ the structure was solved with the ShelXT structure solution program, ${ }^{8}$ using the Intrinsic Phasing solution method. The model was refined with version 2016/6 of ShelXL using Least Squares minimisation. ${ }^{9}$

Crystal data: $\mathrm{C}_{6} \mathrm{H}_{11} \mathrm{FO}_{4}, M_{r}=166.15$, monoclinic, $\mathrm{P} 2_{1}$

(No. 4), $a=7.6278(2) \AA, b=4.73430(10) \AA, c=$ 10.1943(2) $\AA$, 回 $=107.665(3)^{\circ}$, 回 = 回 $=90^{\circ}, V=$ $350.781(15) \AA^{3}, T=100(2) K, Z=2, Z^{\prime}=1$, 回 $\left(M_{0} K_{\text {回}}\right)=$ $0.147,4047$ reflections measured, 1776 unique $\left(R_{\text {int }}=\right.$ $0.0215)$ which were used in all calculations. The final $w R_{2}$ was 0.1037 (all data) and $R_{1}$ was $0.0383(\mathrm{I}>2(\mathrm{I}))$. 


\subsection{4-Deoxy-4-fluoro- $\alpha$-D-fucopyranose (2a)}

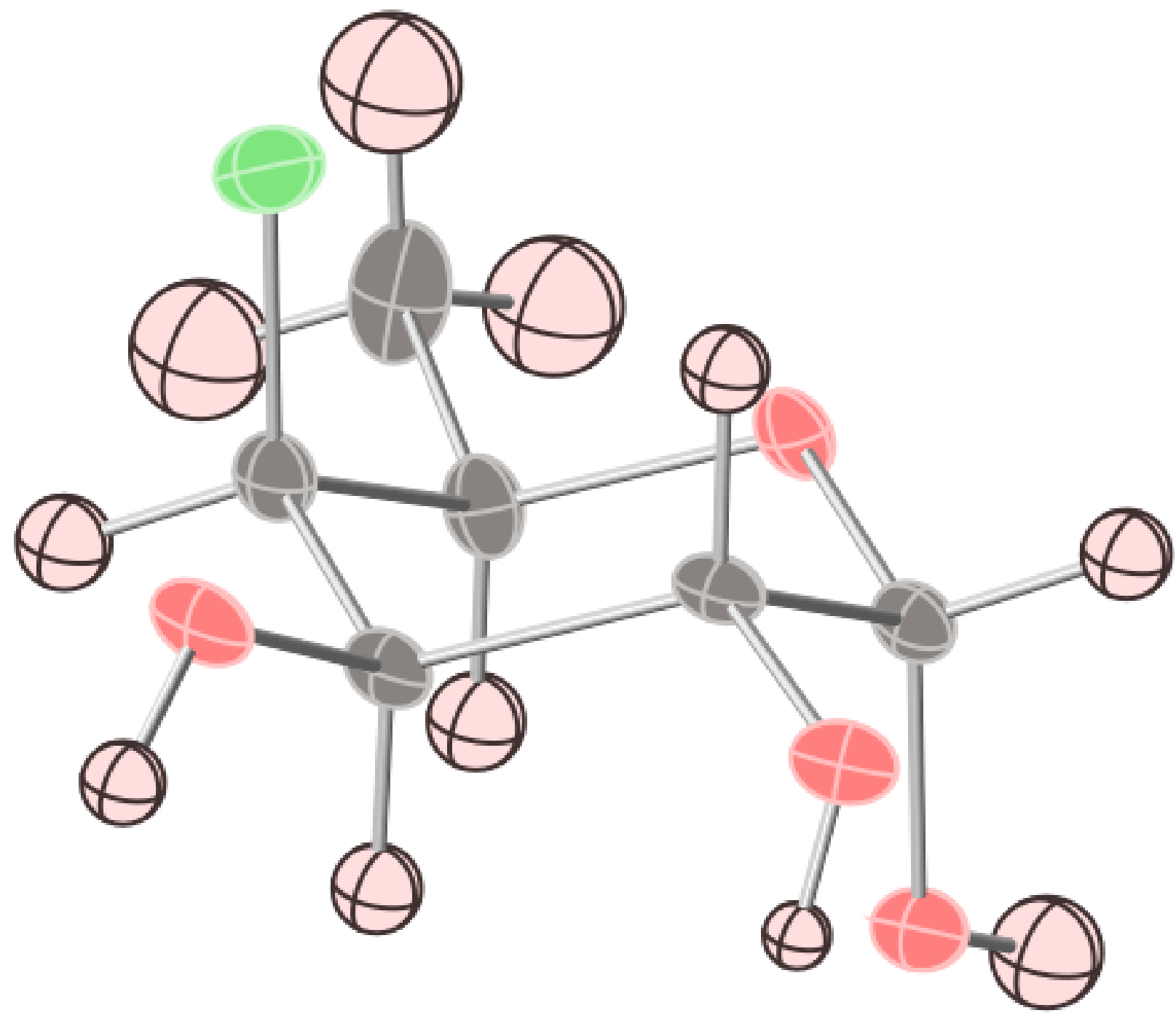

Figure S7: Deoxy-4-fluoro- $\alpha$-D-fucopyranose

(2a). Thermal ellipsoids drawn at the $50 \%$ probability level.

Experimental: Single clear colourless prismshaped crystals of (RK8071-17) were recrystallised from methanol by slow evaporation. A suitable crystal $(0.34 \times 0.12 \times 0.03) \mathrm{mm}^{3}$ was selected and mounted on a MITIGEN holder with silicon oil on a Rigaku AFC12 FRE-HF diffractometer. The crystal was kept at $T=$ 120(2) K during data collection. Using Olex2, ${ }^{7}$ the structure was solved with the ShelXT structure solution program, ${ }^{8}$ using the Intrinsic Phasing solution method. The model was refined with version 2016/6 of ShelXL using Least Squares minimisation.

Crystal data: $\mathrm{C}_{6} \mathrm{H}_{11} \mathrm{FO}_{4}, M_{r}=166.15$, monoclinic, $P 2_{1}$ (No. 4), a = 7.7574(4) $\mathrm{A}, \mathrm{b}=$ 4.7580(2) $\AA, \mathrm{c}=10.1042(4) \AA, b=$ 106.004(5) $, a=g=90^{\circ}, V=358.49(3) \AA^{3}, T=$ $120(2) \mathrm{K}, Z=2, Z^{\prime}=1, m\left(\mathrm{MoK}_{a}\right)=0.144$, 5518 reflections measured, 1806 unique $\left(R_{\text {int }}=0.0236\right)$ which were used in all calculations. The final $w R_{2}$ was 0.0795 (all data) and $R_{1}$ was $0.0314(\mathrm{I}>2(\mathrm{I}))$. 


\subsection{4,6-Dideoxy-4,6-difluoro- $\alpha$-D-glucopyranose (5a)}

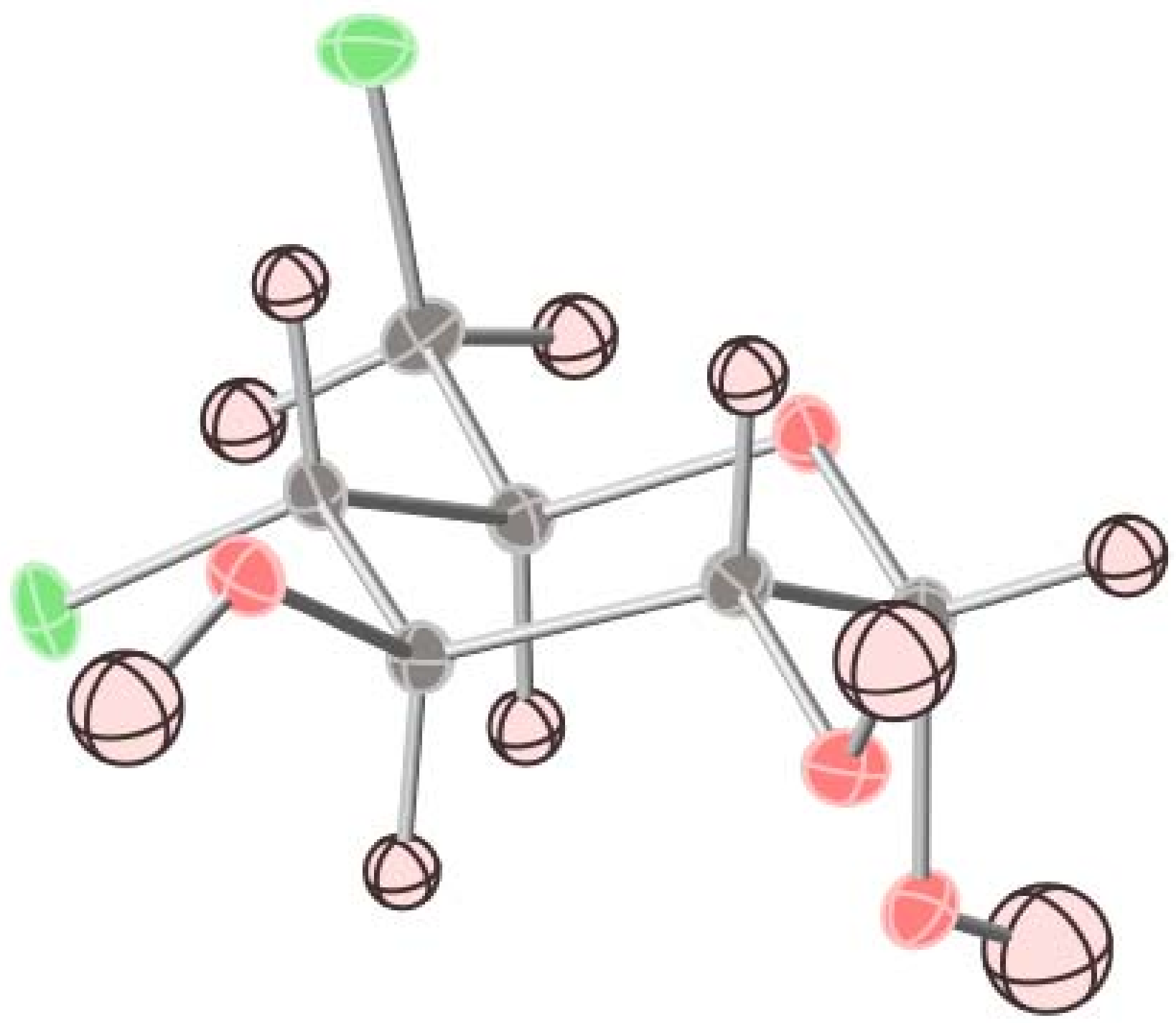

Figure S8: 4,6-Dideoxy-4,6-difluoro- $\alpha$-Dglucopyranose (5a). Thermal ellipsoids drawn at the $50 \%$ probability level.

Experimental: Single clear colourless prism-shaped crystals of (2016sot0072_R1_100K) were

recrystallised from a mixture of $\mathrm{CHCl}_{3}$ and acetone by slow evaporation. A suitable crystal $(0.09 \times 0.08 \times 0.06) \mathrm{mm}^{3}$ was selected and mounted on a MITIGEN holder with silicon oil on a Rigaku AFC12 FRE-VHF diffractometer. The crystal was kept at $T=100$ (2) $\mathrm{K}$ during data collection. Using Olex2, ${ }^{7}$ the structure was solved with the ShelXT structure solution program, ${ }^{8}$ using the Intrinsic Phasing solution method. The model was refined with version 2014/7 of ShelXL using Least Squares minimisation. ${ }^{9}$

Crystal data: $\mathrm{C}_{6} \mathrm{H}_{10} \mathrm{~F}_{2} \mathrm{O}_{4}, M_{r}=184.14$, orthorhombic, $\mathrm{P} 2{ }_{1} 2_{1} 2_{1}$ (No. 19), $\mathrm{a}=$ 6.2146(2) $\AA, b=7.4986(3) \AA, c=$ 15.5149(7) $\AA, a=b=g=90^{\circ}, V=$ 723.01(5) $\AA^{3}, T=100(2) \mathrm{K}, Z=4, Z^{\prime}=1$, $m\left(\mathrm{MoK}_{a}\right)=0.171,10226$ reflections measured, 1873 unique $\left(R_{\text {int }}=0.0182\right)$ which were used in all calculations. The final $w R_{2}$ was 0.0671 (all data) and $R_{1}$ was $0.0245(I>2(I))$. 


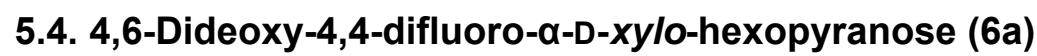

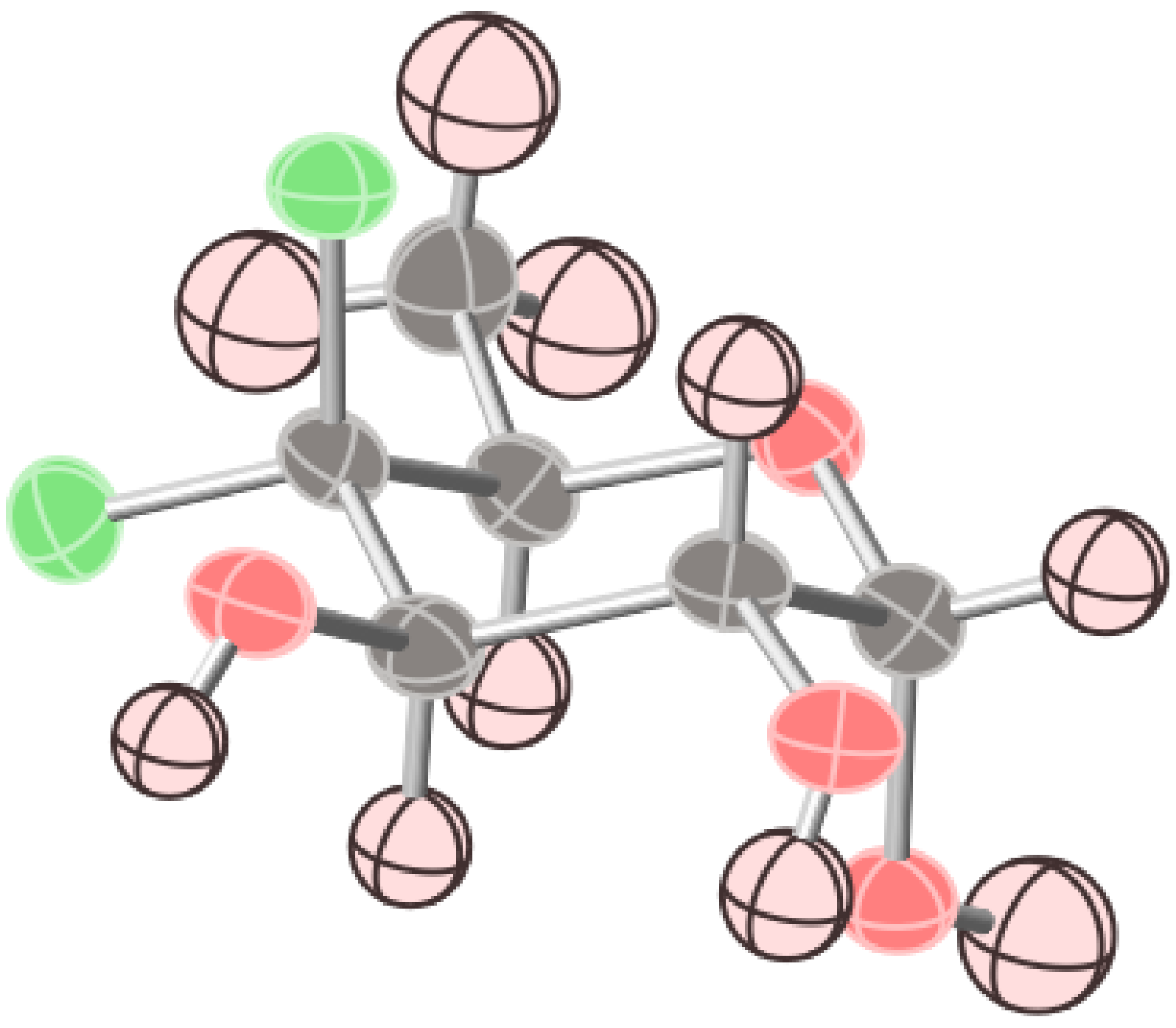

Figure S9: 4,6-Dideoxy-4,4-difluoro- $\alpha$-Dxylo-hexopyranose (6a). Thermal ellipsoids drawn at the $50 \%$ probability level.

Experimental: Single clear colourless prism-shaped crystals of

\section{(2016sot0038_S1_100K) were}

recrystallised from a mixture of $\mathrm{Et}_{2} \mathrm{O}$ and EtOAc by slow evaporation. A suitable crystal $(0.62 \times 0.18 \times 0.16) \mathrm{mm}^{3}$ was selected and mounted on a MITIGEN holder with silicon oil on a Rigaku R-AXIS Spider diffractometer. The crystal was kept at $T=$ 100 (2) $\mathrm{K}$ during data collection. Using Olex2, ${ }^{7}$ the structure was solved with the SheIXT structure solution program, ${ }^{8}$ using the Direct Methods solution method. The model was refined with version $2014 / 7$ of ShelXL using Least Squares minimisation. ${ }^{9}$

Crystal data: $\mathrm{C}_{6} \mathrm{H}_{10} \mathrm{~F}_{2} \mathrm{O}_{4}, \mathrm{M}_{r}=184.14$, orthorhombic, $\mathrm{P} 2{ }_{1} 2_{1} 2_{1}$ (No. 19), $\mathrm{a}=$ 4.8548(2) $\AA, b=10.0844(5) \AA, c=$ 15.8197(8) $\AA, a=b=g=90^{\circ}, V=$ 774.50(6) $\AA^{3}, T=100$ (2) $K, Z=4, Z^{\prime}=1$, $m\left(\mathrm{CuK}_{a}\right)=1.424,8962$ reflections measured, 1486 unique ( $\left.R_{\text {int }}=0.1027\right)$ which were used in all calculations. The final $w R_{2}$ was 0.1016 (all data) and $R_{1}$ was $0.0399(\mathrm{I}>2(\mathrm{I}))$ 


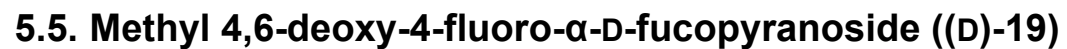

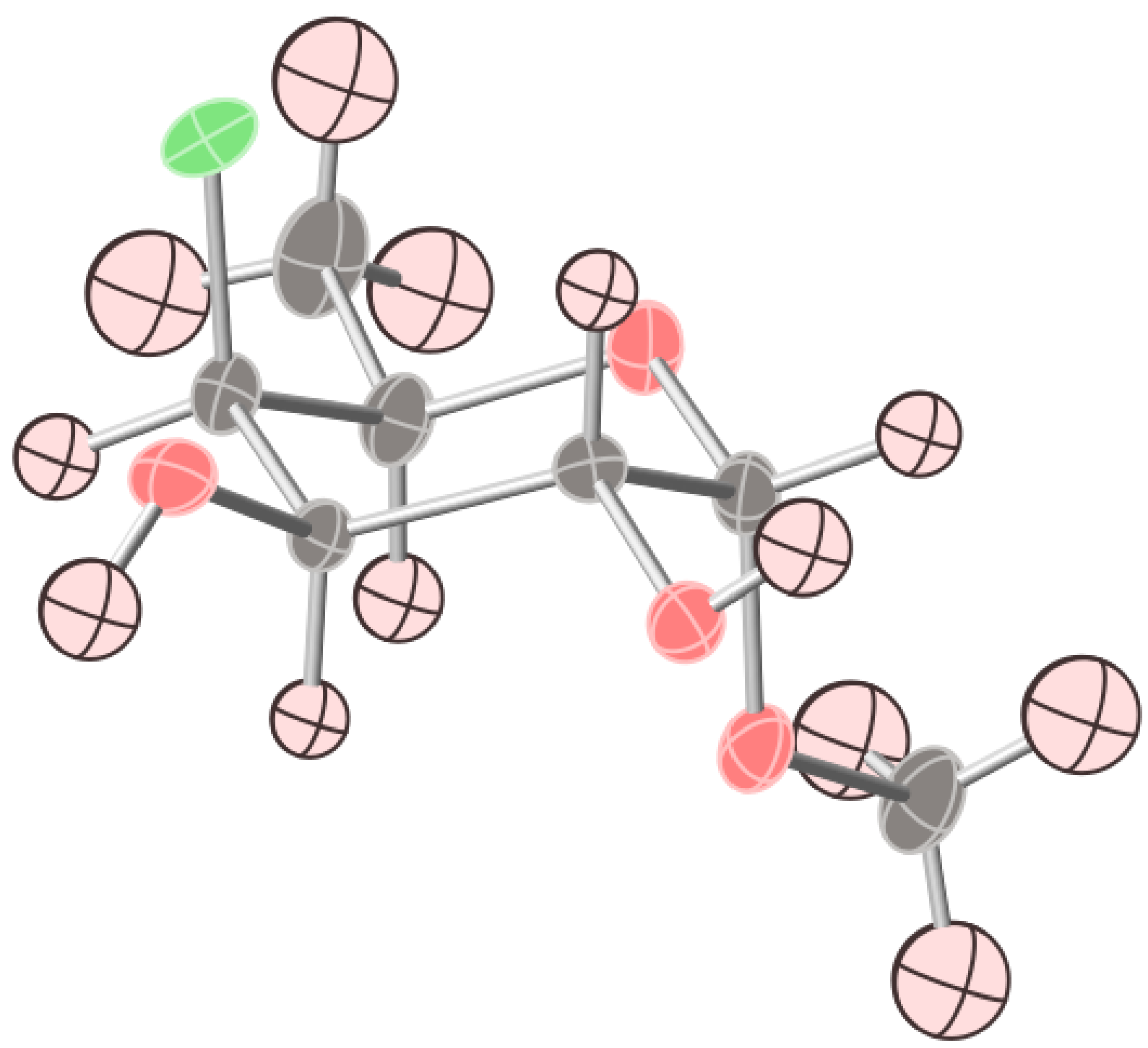

Figure S10: Methyl 4,6-deoxy-4-fluoro- $\alpha$-Dfucopyranoside ((D)-19). Thermal ellipsoids drawn at the $50 \%$ probability level.

Experimental: Single clear colourless plate crystals of 2017sot0063_K1_120K recrystallised from $\mathrm{CHCl} 3$ by slow evaporation. A suitable crystal with dimensions $0.23 \times 0.20 \times$ $0.05 \mathrm{~mm}^{3}$ was selected and mounted on a MITIGEN holder with silicon oil on a Rigaku AFC12 FRE-HF diffractometer. The crystal was kept at a steady $T=120(2) \mathrm{K}$ during data collection. Using Olex $2{ }^{7}$ the structure was solved with the ShelXT structure solution program, ${ }^{8}$ using the Direct Methods solution method. The model was refined with version 2014/7 of ShelXL using Least Squares minimisation. ${ }^{9}$

Crystal data: $\mathrm{C}_{7} \mathrm{H}_{13} \mathrm{FO}_{4}, M_{r}=180.17$, monoclinic, $P 2{ }_{1}$ (No. 4), $a=4.7200$ (3) $\AA, b=8.0247$ (5) $\AA, c=$ 11.1003(6) $\AA$, $b=91.373(6)^{\circ}, a=g=90^{\circ}, V=$ 420.32(4) $\AA^{3}, T=120(2) K, Z=2, Z^{\prime}=1$,

$m\left(\mathrm{MoK}_{a}\right)=0.129,3064$ reflections measured, 1835 unique $\left(R_{\text {int }}=0.0402\right)$ which were used in all calculations. The final $w R_{2}$ was 0.1540 (all data) and $R_{1}$ was $0.0567(I \geq 2 s(I))$. 


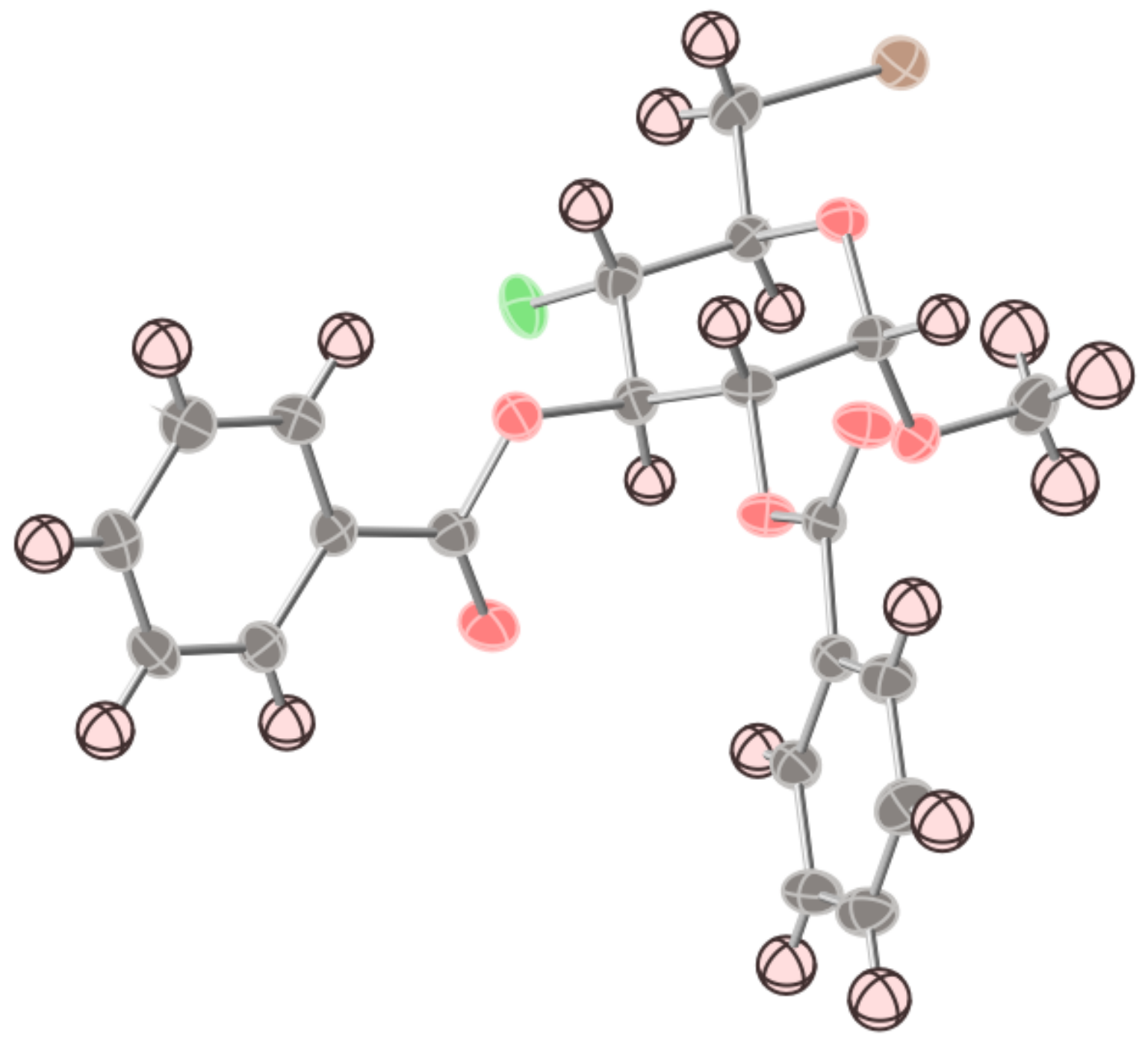

Figure S11: Methyl 4,6-dideoxy-2,3-di-O-benzoyl-6bromo-4-fluoro- $\alpha$-D-glucopyranoside (69). Thermal ellipsoids drawn at the $50 \%$ probability level.

Experimental: Single clear colourless rod-shaped crystals of (2016sot0007-K-100K) were recrystallised from a mixture of $\mathrm{CHCl}_{3}$ and petroleum ether by slow evaporation. A suitable crystal

$(0.13 \times 0.03 \times 0.03) \mathrm{mm}^{3}$ was selected and mounted on a MITIGEN holder in perfluoroether oil on a Rigaku AFC12 FRE-HF diffractometer. The crystal was kept at $T=100$ (2) K during data collection. Using Olex2, ${ }^{7}$ the structure was solved with the ShelXT structure solution program, ${ }^{8}$ using the Direct Methods solution method. The model was refined with ShelXL using Least Squares minimisation. ${ }^{9}$

Crystal Data: $\mathrm{C}_{21} \mathrm{H}_{20} \mathrm{BrFO}_{6}, \mathrm{M}_{r}=467.28$, trigonal, $\mathrm{P}_{2}$ (No. 145), $a=10.9826(2) \AA, b=10.9826(2) \AA, c=$ 14.6281(3) $\AA$, 回 $=90^{\circ}$, 回 $=90^{\circ}$, 回 $=120^{\circ}, V=$ 1528.03(7) $\AA^{3}, T=100(2) K, Z=3, Z^{\prime}=1$, 回 $\left(M^{2} K_{\Xi}\right)=$ $2.062,10544$ reflections measured, 5239 unique $\left(R_{\text {int }}=0.0203\right)$ which were used in all calculations. The final $w R_{2}$ was 0.0605 (all data) and $R_{1}$ was $0.0269(\mathrm{I}>2(\mathrm{I}))$. 
5.7. Methyl 4,6-dideoxy-4-fluoro-a-D-glucopyranoside (71)

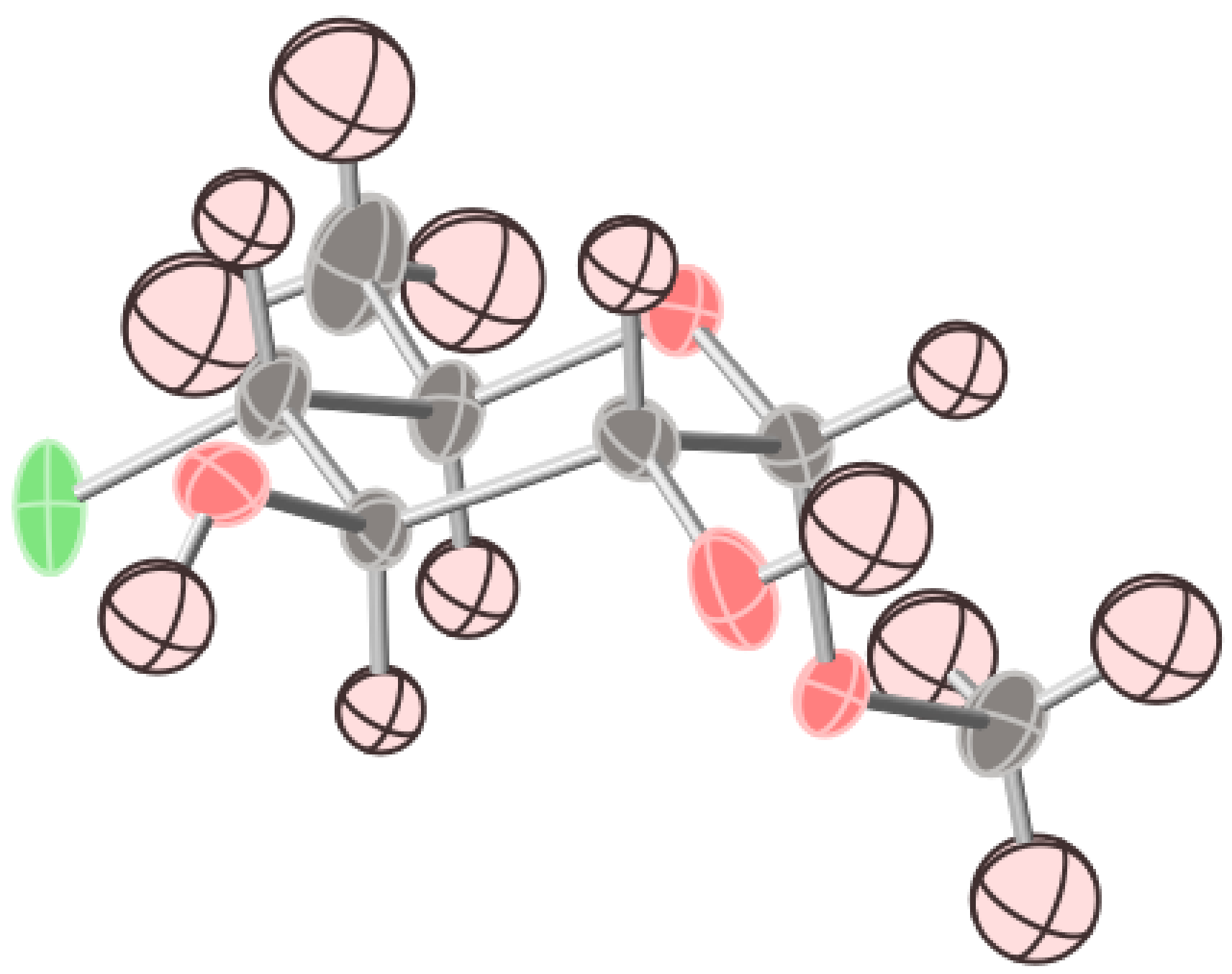

final $w R_{2}$ was 0.1593 (all data) and $R_{1}$ was $0.0563(\mathrm{I}>2(\mathrm{I}))$.
Figure S12: Methyl 4,6-dideoxy-4-fluoro$\alpha$-D-glucopyranoside (71). Thermal ellipsoids drawn at the $50 \%$ probability level.

Experimental: Single clear colourless plate-shaped crystals of

\section{(2016sot0026-K-100K) were}

recrystallised from a mixture of $\mathrm{CHCl}_{3}$ and petroleum ether by slow evaporation. A suitable crystal $(0.29 \times 0.21 \times 0.05) \mathrm{mm}^{3}$ was selected and mounted on a MITIGEN holder in perfluoroether oil on a Rigaku AFC12 FRE-HF diffractometer. The crystal was kept at $T=100(2) \mathrm{K}$ during data collection. Using Olex $2,{ }^{7}$ the structure was solved with the ShelXT structure solution program, ${ }^{8}$ using the Direct Methods solution method. The model was refined with ShelXL using Least Squares minimisation. ${ }^{9}$

Crystal Data: $\mathrm{C}_{7} \mathrm{H}_{13} \mathrm{FO}_{4}, M_{r}=180.17$, orthorhombic, $\mathrm{P} 2{ }_{1} 2_{1} 2_{1}$ (No. 19), $\mathrm{a}=$ 5.0069(3) $\AA, b=8.2607(6) \AA, c=$ 21.0238(14) $\AA, a=b=g=90^{\circ}, V=$ 869.55(10) $\AA^{3}, T=100(2) \mathrm{K}, Z=4, Z^{\prime}=1$, $m\left(\mathrm{MoK}_{a}\right)=0.124,3495$ reflections measured, 2203 unique ( $R_{\text {int }}=0.0303$ ) which were used in all calculations. The 
5.8. Methyl 4,6-dideoxy-4,4-difluoro- $\alpha-D-x y l o-h e x o p y r a n o s i d e ~(85)$

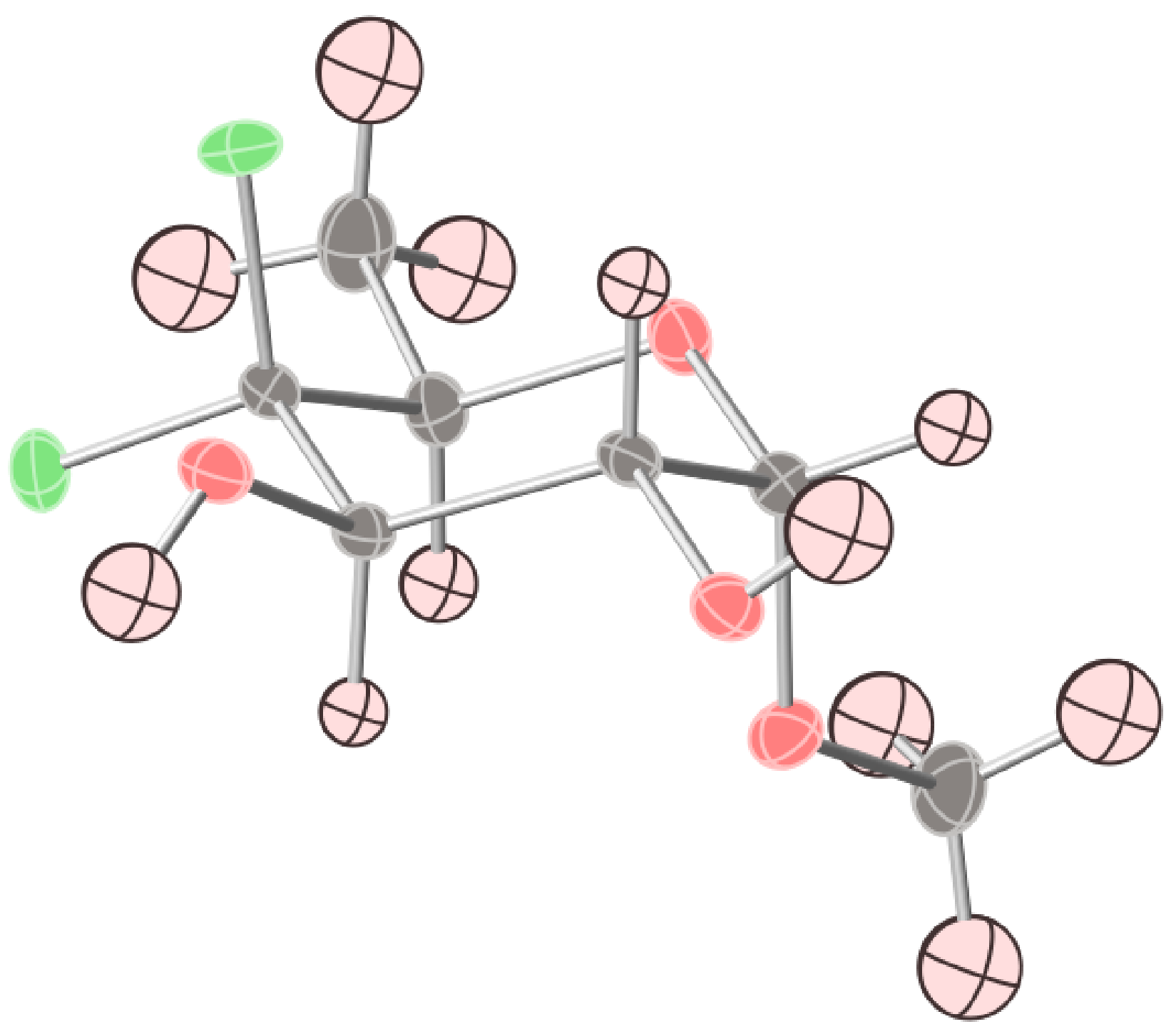

Figure S13: Methyl 4,6-dideoxy-4,4-difluoro$\alpha$-D-xylo-hexopyranoside (85). Thermal

ellipsoids drawn at the $50 \%$ probability level.

Experimental: Single clear colourless prism crystals of 2018sot0035_R1_100K

recrystallised from methanol by slow evaporation. A suitable crystal with dimensions $0.44 \times 0.16 \times 0.14 \mathrm{~mm}^{3}$ was selected and mounted on a MITIGEN holder silicon oil on a Rigaku AFC12 FRE-VHF diffractometer. The crystal was kept at a steady $T=100(2) \mathrm{K}$ during data collection. The structure was solved with the ShelXT 2014/5 (Sheldrick, 2014) solution program, ${ }^{8}$ using dual methods and by using Olex $2^{7}$ as the graphical interface. The model was refined with ShelXL 2016/6 (Sheldrick, 2015) using full matrix least squares minimisation on $\boldsymbol{F}^{2} .^{9}$

Crystal Data: $\mathrm{C}_{7} \mathrm{H}_{12} \mathrm{~F}_{2} \mathrm{O}_{4}, M_{r}=198.17$, monoclinic, $P 2_{1}$ (No. 4), a $=4.7938(3) \AA$, $b=$ 8.1697(5) $\AA, c=10.8283(9) \AA, b=91.410(6)^{\circ}$, $a=g=90^{\circ}, V=423.95(5) \AA^{3}, T=100(2) \mathrm{K}, Z=$ $2, Z^{\prime}=1, m\left(\mathrm{MoK}_{a}\right)=0.151,5582$ reflections measured, 2421 unique $\left(R_{\text {int }}=0.0243\right)$ which were used in all calculations. The final $w R_{2}$ was 0.1150 (all data) and $R_{1}$ was 0.0379 (I $\geq 2$ $s(I))$. 


\section{References}

(1) Kerins, L.; Byrne, S.; Gabba, A.; Murphy, P. V. J. Org. Chem. 2018, 83, 7714.

(2) Linclau, B.; Wang, Z.; Compain, G.; Paumelle, V.; Fontenelle, C. Q.; Wells, N.; Weymouth-Wilson, A. Angew. Chem. Int. Ed. 2016, $55,674$.

(3) Wang, Z.; Jeffries, B. F.; Felstead, H. R.; Wells, N. J.; Chiarparin, E.; Linclau, B. JoVE 2019, e58567.

(4) Claridge, T. High-Resolution NMR Techniques in Organic Chemistry.; Pergamon, 1999.

(5) Derome, A. E. Modern NMR Techniques for Chemistry Research, 6th ed.; Pergamon: Oxford, 1997.

(6) Zhang, F.-F.; Jiang, M.-H.; Sun, L.-L.; Zheng, F.; Dong, L.; Shah, V.; Shen, W.-B.; Ding, Y. Analyst 2015, 140, 280.

(7) Dolomanov, O. V.; Bourhis, L. J.; Gildea, R. J.; Howard, J. A. K.; Puschmann, H. J. Appl. Crystallogr. 2009, 42, 339.

(8) Sheldrick, G. Acta Crystallographica Section A 2015, 71, 3.

(9) TetrahedronSheldrick, G. Acta Crystallographica Section C 2015, 71, 3. 